\title{
Selektiver Mutismus bei mehrsprachigen Kindern
}

Eine Längsschnittstudie zum Einfluss kindlicher Ängste, Sprachkompetenzen und elterlicher Akkulturation auf die Entwicklung des Schweigens

vorgelegt von

Anja Starke

als Dissertation zur Erlangung des Grades einer Doktorin

der Philosophie (Dr. phil.)

in der

Fakultät Rehabilitationswissenschaften

der Technischen Universität Dortmund

Dortmund

2014

Betreuerin: Priv.-Doz. Dr. Katja Subellok

Betreuer: Prof. Dr. Christoph de Oliveira Käppler 



\section{Danksagung}

An erster Stelle möchte ich allen teilnehmenden Kindern, ihren Eltern und ihren Erzieherinnen danken, ohne die dieses Projekt nicht hätte durchgeführt werden können.

Mein besonderer Dank gilt meiner Erstbetreuerin Katja Subellok, die mir jederzeit inhaltlich und emotional zur Seite gestanden hat. Durch ihre große Begeisterung am Thema und den Glauben an mich und meine Arbeit hat sie es immer wieder geschafft mich zu motivieren.

Christoph de Oliveira Käppler danke ich für die Betreuung und Begutachtung meiner Arbeit. Die gemeinsamen Diskussionen und seine Ratschläge zur methodischen Umsetzung meiner Fragestellung haben zum Gelingen des Projektes beigetragen.

Mein besonderer Dank gilt ebenso Ute Ritterfeld, die mich in meiner Promotionsphase als Mentorin immer unterstützt hat. Sie hat meine Persönlichkeitsentwicklung und die weitere Planung meiner Karriere maßgeblich geprägt.

Der Fakultät Rehabilitationswissenschaften der TU Dortmund möchte ich dafür danken, dass sie mir die Rahmenbedingungen für die Durchführung und den erfolgreichen Abschluss des Projektes ermöglicht hat. Dem Verein zur Förderung des Sprachtherapeutischen Ambulatoriums der Technischen Universität Dortmund e.V. möchte ich für die finanzielle Unterstützung für die Vergabe von Probandengelder danken.

Ein besonderer Dank gilt meiner guten Freundin und zugleich Bürokollegin Carina Lüke für ihre Unterstützung und Freundschaft. Durch das gemeinsame Durchlaufen der Promotionsphasen konnten wir uns immer wieder gegenseitig unterstützen und motivieren. Für ihre große Begeisterung, ihr Durchhaltevermögen und die Diskussionsbereitschaft möchte ich ihr herzlich danken. Auch möchte ich an dieser Stelle Juliane Mühlhaus für das Korrekturlesen dieser Arbeit und ihre konstruktiven Kommentare danken.

Herzlich danken möchte ich zudem allen Studierenden, die in den verschiedensten Phasen des Projektes aktiv mitgearbeitet haben. Ohne die tatkräftige Unterstützung zahlreicher Personen hätten die Datenerhebungen des Projektes nicht realisiert werden können. Im Besonderen möchte ich hier meiner studentischen Hilfskraft Kathrin Hofmann danken, die viele Stunden mit mir gemeinsam Daten erhoben und ausgewertet hat.

Als letztes möchte ich meinem Mann, meiner Familie und meinen Freunden danken, die mich in der Promotionsphase in vielerlei Hinsicht unterstützt haben. 



\section{Zusammenfassung}

Mehrsprachige Kinder sind etwa vier Mal häufiger von einem selektiven Mutismus (SM) betroffen als einsprachige Kinder. Bislang fehlen jedoch Befunde, welche diese erhöhte Vulnerabilität mehrsprachiger Kinder erklären können. Hinweise gibt es darauf, dass ihr Schweigen vor allem vor dem Hintergrund sprachlicher Unsicherheiten im Zuge des Zweitspracherwerbs und sozialen Ängsten erklärt werden kann. Ein weiterer bedeutsamer Faktor bei mehrsprachigen Kindern ist die multikulturelle Lebenswelt der Kinder. Der Umgang der Eltern mit den verschiedenen Kulturen, mit denen die Familie konfrontiert ist, kann Einfluss auf die sprachliche und sozial-emotionale Entwicklung der Kinder nehmen. Ziel der vorliegenden Studie ist es, die Bedeutsamkeit von kindlichen Ängsten, Sprachkompetenzen in der Mehrheitssprache Deutsch sowie der elterlichen Akkulturation auf die Entwicklung eines SM bei mehrsprachigen Kindern zu untersuchen.

Es wurde eine Längsschnittstudie mit insgesamt 30 ein- und mehrsprachigen Kindern im Alter von 3;0 bis 5;8 durchgeführt. Rekrutiert wurden schweigende Kinder sowie sprechende Kontrollkinder, um mögliche Risikofaktoren für die Entwicklung des SM zu identifizieren. $\mathrm{Zu}$ Beginn und zum Ende der Studie wurden die kindliche Angst, die rezeptiven und produktiven Sprachkompetenzen im Deutschen sowie die Akkulturation der Eltern erfasst. Als abhängige Variable wurden alle drei Monate das Sprechverhalten der Kinder im familiären und öffentlichen sowie im Kindergarten-Kontext über Fragebögen und Beobachtungen erhoben.

Die Angst der Kinder erwies sich als bester Prädiktor für die Entwicklung des Sprechverhaltens. Ein Einfluss der Mehrsprachigkeit zeigte sich nicht. Im Kindergartenkontext waren neben der Angst vor allem auch der sozioökonomische Hintergrund der Familie sowie die sprachlichen Kompetenzen der Kinder bedeutsam. Für die mehrsprachigen Kinder zeigten sich erste Hinweise auf den Einfluss der Akkulturation der Eltern auf das Sprechverhalten der Kinder.

Eine erhöhte Angst könnte vor allem bei mehrsprachigen Kindern als früher Indikator für das Risiko einen SM zu entwickeln dienen. Die Studie stellte weiterhin heraus, dass die Bedeutsamkeit der Sprachkompetenzen und der elterlichen Akkulturation im Zusammenhang mit SM zukünftig noch intensiv untersucht werden muss.

Schlïsselwörter: Selektiver Mutismus, Mehrsprachigkeit, Migrationshintergrund, Angst, Sprachkompetenzen, Akkulturation 



\section{Inhaltsverzeichnis}

1 EINLEITUNG $\quad 13$

2 FORSCHUNGSSTAND $\quad 15$

$\begin{array}{lll}2.1 & \text { Selektiver Mutismus } & 15\end{array}$

2.2 Risikofaktoren und komorbide Phänomene 17

2.2.1 Genetische Prädisposition und familiäre Lernumgebung 19

2.2.2 Soziale Ängstlichkeit 21

2.2.3 Sprachkompetenzen 25

2.2.4 Einschneidende Lebensereignisse 28

2.2.5 Zusammenfassung: Entwicklung des selektiven Mutismus bei einsprachigen Kinder 29

2.3 Begriffsbestimmungen: Mehrsprachigkeit, Migrationshintergrund und Kultur 30

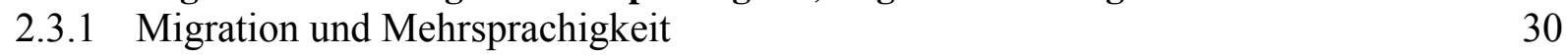

2.3.2 Mehrsprachige Entwicklung 31

$\begin{array}{lll}2.3 .3 & \text { Kultur } & 35\end{array}$

2.4 Selektiver Mutismus bei mehrsprachigen Kindern 36

2.4.1 Kulturelle Einflüsse auf die Entwicklung sozio-emotionaler und sprachlicher
Fähigkeiten

2.4.2 Akkulturation $\quad 43$

2.4.3 Einfluss der kulturellen Adaptation der Eltern auf die kindliche Entwicklung 44

2.4.4 Entwicklung des SM bei mehrsprachigen Kindern 46

2.5 Fragestellung und Hypothesen $\quad 50$

3 METHODE

$\begin{array}{lll}3.1 & \text { Studiendesign } & 53\end{array}$

3.2 Konstruktion und Auswahl der Instrumente $\quad \mathbf{5 4}$

3.2.1 Anamnestische und soziodemografische Daten 55

$\begin{array}{ll}\text { 3.2.2 Messung des Sprechverhaltens } & 57\end{array}$

$\begin{array}{lll}\text { 3.2.3 Messung des Interaktionsverhaltens } & 62\end{array}$

$\begin{array}{lll}3.2 .4 & \text { Sprachliche Fähigkeiten } & 68\end{array}$

3.2.5 Messung der kindlichen Ängstlichkeit $\quad 74$

3.2.6 Erfassung der kulturellen Adaptation der Eltern 76

$\begin{array}{lll}3.3 & \text { Pilotstudie } & \mathbf{7 6}\end{array}$

$\begin{array}{lll}3.3 .1 & \text { Probanden } & 77\end{array}$

$\begin{array}{lll}3.3 .2 & \text { Vorgehen } & 78\end{array}$

$\begin{array}{lll}3.3 .3 & \text { Instrumente } & 79\end{array}$

3.3.4 Auswertung $\quad 80$

$\begin{array}{llr}3.3 .5 & \text { Ergebnisse } & 80\end{array}$

$\begin{array}{lll}3.3 .6 & \text { Diskussion } & 85\end{array}$

$\begin{array}{llr}3.4 & \text { Hauptstudie } & \mathbf{8 8}\end{array}$

$\begin{array}{lll}3.4 .1 & \text { Rekrutierung } & 88\end{array}$

$\begin{array}{ll}3.4 .2 & \text { Probanden }\end{array}$

$\begin{array}{lll}3.4 .3 & \text { Vorgehen } & 96\end{array}$

$\begin{array}{ll}3.4 .4 & \text { Instrumente } \\ 3.4 .5 & 99\end{array}$

$\begin{array}{lll}3.4 .5 & \text { Auswertung } & 103\end{array}$ 
4 ERGEBNISSE $\quad 108$

4.1 Deskriptive Analysen 108

4.1.1 Interkorrelationen der Outcomevariablen 109

4.1.2 Interkorrelationen der Prädiktorvariablen 114

4.1.3 Korrelationen zwischen Prädiktoren und Outcomevariablen 116

4.1.4 Anteil der selektiv mutistischen Kinder zum ersten Messzeitpunkt 120

4.1.5 Anteil selektiv mutistischer Kindern zum vierten Messzeitpunkt 123

4.1.6 Entwicklung des Sprech- und Interaktionsverhaltens 125

4.1.7 Deskriptive Ergebnisse zu den Prädiktorvariablen 128

4.1.8 Profile der selektiv mutistischen Kinder $\quad 132$

$\begin{array}{lll}4.2 & \text { Hypothesenprüfung } & 138\end{array}$

4.2.1 Ängste 141

4.2.2 Kulturelle Adaptation der Eltern 143

4.2.3 Mehrebenenmodell - Outcome Elternfragebogen 145

4.2.4 Mehrebenenmodell - Outcome DortMuS-Kita 151

5 DISKUSSION 157

$\begin{array}{lll}5.1 & \text { Vorhersage der Entwicklung des Sprechverhaltens } & 157\end{array}$

$\begin{array}{lll}\text { 5.2 Entwicklungsverläufe der Kinder mit SM } & 164\end{array}$

5.2.1 Entwicklung des Interaktions- und Sprechverhaltens 164

5.2.2 Anteil der Kinder mit SM 165

5.3 Einfluss der Mehrsprachigkeit auf die Entwicklung eines SM 169

5.3.1 Kulturelle Adaptation der Eltern $\quad 169$

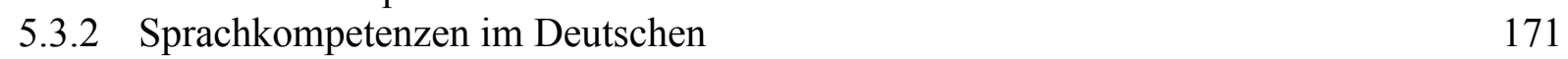

$\begin{array}{lll}\text { 5.3.3 Ängstlichkeit } & 173\end{array}$

5.3.4 Bedeutung der Ergebnisse im Diathese-Stress-Modell 175

$\begin{array}{lll}5.4 & \text { Grenzen der Studie } & 177\end{array}$

$6 \quad$ SCHLUSSFOLGERUNG UND AUSBLICK 180

$7 \quad$ LITERATURVERZEICHNIS 183

$\begin{array}{ll}\text { ANHANG } & 202\end{array}$

$\begin{array}{ll}\text { Anhang A: Tabellen } & 203\end{array}$

Anhang B: Elternfragebogen $\quad 219$

Anhang C: DortMuS-Kita Pilotversion 233

Anhang D: Beobachtungsbögen und Beobachtungsmanual 239

Anhang E: Beschreibung der Interaktionssettings $\quad 260$

Anhang F: Anleitung zur Anfertigung der Spontansprachaufnahme 265

$\begin{array}{ll}\text { Anhang G: Informationen zur Studie für Kitas und Eltern } & 267\end{array}$

Anhang H: Erstinformation für die Kitas 279

Anhang I: Kodierschema Spontansprache $\quad 284$ 


\section{Tabellenverzeichnis}

Tabelle 1 Diagnosen bei Kindern mit SM und Kontrollkindern in der Studie von Kristensen (2000)

Tabelle 2 Überblick über die erhobenen Daten zu den vier Messzeitpunkten (T)__ 54

Tabelle 3 Übersicht über die genutzten und neu konstruierten Instrumente ___ 55

Tabelle 4 Skalen, Beispielitems und interne Konsistenz für den Elternfragebogen _ 59

Tabelle 5 Übersicht über die Subskalen der Pilotversion von DortMuS-Kita _ 61

Tabelle 6 Skalen, Beispielitems und interne Konsistenzen für DortMuS-Kita___ 61

Tabelle 7 Überblick über die Kategorien der Beobachtungsbögen ___ 65

Tabelle 8 Methode der Beobachtungen

Tabelle 9 Probanden der Pilotstudie 78

Tabelle 10 Verwendete Instrumente in der Pilotstudie __ 80

Tabelle 11 Verteilung der Probanden auf die Gruppen mit den Merkmalen schweigend +/und mehrsprachig +/- 90

Tabelle 12 Schüchternheit, Ängste und Schweigen bei nahen Familienmitgliedern __ 95

Tabelle 13 Verwendete Instrumente im Rahmen der Hauptstudie _ 99

Tabelle 14 Interrater-Reliabilitäten für die Spontansprachkodierungen 102

Tabelle 15 Operationalisierung von Prädiktor und Outcomevariablen für die Hauptanalysen

Tabelle 16 Übersicht zu fehlenden Werten innerhalb der Prädiktor- und Outcomevariablen zu allen vier Messzeitpunkten

Tabelle 17 Interkorrelationen zwischen den Outcomevariablen zum ersten Messzeitpunkt 109

Tabelle 18 Interkorrelationen der Outcomevariablen zum zweiten Messzeitpunkt__ 111

Tabelle 19 Interkorrelationen der Outcomevariablen zum dritten Messzeitpunkt___ 112

Tabelle 20 Interkorrelationen der Outcomevariablen zum vierten Messzeitpunkt __ 113

Tabelle 21 Interkorrelationen der Prädiktoren zum ersten Messzeitpunkt 115

Tabelle 22 Korrelationen zwischen den Prädiktoren und den Werten auf der Subskala Schweigen des Elternfragebogens zu allen vier Messzeitpunkten

Tabelle 23 Korrelationen zwischen den Prädiktoren und den Werten auf der Subskala Schweigen und Bedürfnisäußerung des DortMuS-Kita zu allen vier Messzeitpunkten

Tabelle 24 Korrelationen zwischen den Prädiktoren und der Gesamtzahl der Interaktionen in der Freispielsituation

Tabelle 25 Korrelationen zwischen den Prädiktoren und der Gesamtzahl der verbalen Interaktionen in der Freispielsituation

Tabelle 26 Mediane und Interquartilsabstände zu den Outcome-Variablen zum ersten Messzeitpunkt

Tabelle 27 Kinder mit selektivem Mutismus zu T4 im Vergleich zu Kindern mit positiver Entwicklung und Kontrollen in den Outcomevariablen zum vierten Messzeitpunkt

Tabelle 28 Ergebnisse der Jonckheere-Terpstra-Tests für die Outcomevariablen für den zweiten bis vierten Messzeitpunkt

Tabelle 29 Mediane und Interquartilsabstände zu den Prädiktorvariablen zum ersten und vierten Messzeitpunkt

Tabelle 30 Mediane und Interquartilsabstände in den Prädiktorvariablen für ein- und mehrsprachige Kinder

Tabelle 31 Deskriptive Ergebnisse und Gruppenunterschiede zur kulturellen Adaption der Eltern zum ersten Messzeitpunkt

Tabelle 32 Alter, Geschlecht sowie Informationen zur Mehrsprachigkeit der selektiv mutistischen Kinder 
Tabelle 33 Prädiktorvariablen zum ersten Messzeitpunkt für Kinder mit der Diagnose SM

Tabelle 34 Prädiktorvariablen zum vierten Messzeitpunkt für die Kinder mit Diagnose SM

Tabelle 35 Korrelationen zwischen den Sprachvariablen zu T1 und T4 und der kulturellen Adaptation der Eltern zum vierten Messzeitpunkt

Tabelle 36 Korrelationen zwischen den Sprachvariablen und der kulturellen Adaptation der Eltern zum vierten Messzeitpunkt

Tabelle 37 Fit-Maße der angepassten Modelle mit dem Summenwert auf der Subskala Schweigen des Elternfragebogens als Outcomevariable

Tabelle 38 Modellparameter des endgültigen Modells 1.4 zum Einfluss von

Mehrsprachigkeit, Gesamtangst und Grammatikverständnis auf das Schweigen der Kinder in familiären und öffentlichen Kontexten

Tabelle 39 Fit-Maße der angepassten Modelle für den Einfluss der produktiven Sprachkompetenzen auf das Schweigen der Kinder in familiären und öffentlichen Kontexten

Tabelle 40 Modellparameter für das Mehrebenenmodell 2.3 mit dem Summenwert auf der Subskala Schweigen des Elternfragebogens als Outcomevariable

Tabelle 41 Fit-Maße der angepassten Modelle zum Einfluss der kulturellen Adaptation auf die Entwicklung des Schweigens

Tabelle 42 Modellparameter für das Mehrebenemodell 3.3 mit dem Summenwert auf der Subskala Schweigen im Elternfragebogen als Outcomevariable

Tabelle 43 Modellparameter für das Mehrebenenmodell 3.4 mit dem Summenwert auf der Subskala Schweigen im Elternfragebogen als Outcomevariable

Tabelle 44 Fit-Maße der angepassten Modelle mit dem Summenwert auf der Subskala Schweigen und Bedürfnisäußerung von DortMuS-Kita als Outcomevariable _ 152

Tabelle 45 Modellparameter des endgültigen Modells 4.4 zum Einfluss von Mehrsprachigkeit, Gesamtangst und Grammatikverständnis auf das Schweigen der Kinder in der Kita

Tabelle 46 Fit-Maße der angepassten Modelle zum Einfluss der produktiven Sprachkompetenzen auf die Entwicklung des Schweigens in der Kita

Tabelle 47 Modellparameter für das Mehrebenenmodell 5.3 zum Einfluss der produktiven Sprachkompetenzen auf das Schweigen der Kinder in der Kita

Tabelle 48 Fit-Maße der angepassten Modelle zum Einfluss der kulturellen Adaptation der Eltern auf die Entwicklung des Schweigens der Kinder in der Kita

Tabelle 49 Modellparameter für das Mehrebenenmodell 6.3 zum Einfluss der kulturellen Adaptation der Eltern auf das Schweigen der Kinder in der Kita 156

Tabelle 50 Modellparameter für das Mehrebenenmodell 6.4 zum Einfluss der kulturellen Adaptation der Eltern auf das Schweigen der Kinder in der Kita 156 


\section{Abbildungsverzeichnis}

Abbildung 1. Mögliche Wege der Entwicklung eines SM bei Kindern

(Cohan, Price et al., 2006, S. 352)

Abbildung 2. Vier Strategien der kulturellen Adaptation in Anlehnung an Berry (2003) _ 44

Abbildung 3. Erklärungsmodell zur Entstehung des selektiven Mutismus bei mehrsprachigen Kindern

Abbildung 4. Ablauf der Beobachtungen in der Pilotstudie ___ 78

Abbildung 5. Ablauf der Pilotstudie

Abbildung 6. Häufigkeiten komorbider Störungen___ 93

Abbildung 7. Fehlende Werte in den Prädiktorvariablen zum ersten Messzeitpunkt__ 106

Abbildung 8. Fehlende Werte in den Prädiktorvariablen zum vierten Messzeitpunkt__ 106

Abbildung 9. Fehlende Werte in den Outcomevariablen zum vierten Messzeitpunkt. FS = Freispielsituation, GS = Gruppensituation, ES = Essenssituation __ 107

Abbildung 10. Fehlende Werte in den Outcomevariablen zum ersten Messzeitpunkt. $\mathrm{FS}=$ Freispielsituation, GS = Gruppensituation, ES = Essenssituation

Abbildung 11. Fehlende Werte in den Outcomevariablen zum dritten Messzeitpunkt. . $\mathrm{FS}=$ Freispielsituation, GS = Gruppensituation, $\mathrm{ES}=$ Essenssituation 107

Abbildung 12. Fehlende Werte in den Outcomevariablen zum vierten Messzeitpunkt. $\overline{\mathrm{FS}}=$ Freispielsituation, GS = Gruppensituation, ES = Essenssituation

Abbildung 13. Mittlere Summenwerte (Mediane) auf der Skala Schweigen des Elternfragebogens für Kinder mit Diagnose SM zu T4, zu T4 sprechenden

Kindern und Kontrollen

Abbildung 14. Mittlere Summenwerte (Mediane) auf der Skala Schweigen und Bedürfnisäußerung von DortMuS-Kita für Kinder mit Diagnose SM zu T4, zu T4 sprechende und Kontrollen

Abbildung 15. Mittlere Anzahl (Mediane) verbaler Interaktionen in der Freispielsituation 127

Abbildung 16. Mittlere Anzahl (Mediane) nonverbaler Interaktionen in der Freispielsituation

Abbildung 17. Mittlere Anzahl (Mediane) aller Interaktionen zwischen dem Kind und einemKind bzw. mehreren Kindern

Abbildung 18. Datenstruktur im Mehrebenenmodell bei Längsschnittdaten ___ 139

Abbildung 19. Vorgehen bei der Spezifikation der Mehrebenenmodelle___ 140

Abbildung 20. Gesamtangst der Kinder zum ersten Messzeitpunkt 142 



\section{Einleitung}

Sprache ist eine bedeutsame Ressource für gesellschaftliches Handeln (Hoffmann, Leimbrink \& Quasthoff, 2011). Über sie können Menschen ihre Gedanken, ihr Wissen, ihre Gefühle und ihre Werte teilen. Erst über sie wird kooperatives Handeln möglich (Tomasello, 2008), in dem Ziele diskutiert, Handlungen koordiniert und geplant werden. Sprache ist somit eine bedeutsame Fähigkeit, welche eine gesellschaftliche Teilhabe ermöglicht. So hängen auch die sprachlichen Fähigkeiten mit den sozialen Fähigkeiten im Kindesalter zusammen (Bat-Chava, Martin \& Imperatore, 2014; Hart, Fujiki, Brinton \& Hart, 2004). Kinder mit umschriebenen Sprachentwicklungsstörungen zeigen entsprechend häufiger Auffälligkeiten in ihrem sozial-emotionalen Verhalten, haben größere Probleme sich in Gruppenkontexten zu integrieren und werden häufiger von ihren Peers zurückgewiesen (Hart et al., 2004; McCabe, 2005). Personen, denen Sprache nicht zur Verfügung steht oder bei denen sie massiv beeinträchtigt ist, haben somit ein erhöhtes Risiko von der Gesellschaft ausgeschlossen zu sein.

Manche Kinder besitzen zwar angemessene Sprachkompetenzen, um sich in sozialen Kontexten zu äußern, nutzen diese jedoch in spezifischen Situationen nicht. Sie schweigen beispielsweise im Kindergarten oder in der Schule, obwohl sie zum Sprechen in der Lage wären. Dieses Phänomen wird selektiver Mutismus (SM) genannt und gehört zu den Angststörungen der frühen Kindheit (American Psychiatric Association, 2013). Mit bislang angenommenen Prävalenzraten von 0.7\% (Bergman, Piacentini \& McCracken, 2002; Elizur \& Perednik, 2003) bis 1.9\% (Kopp \& Gilberg, 1997) kommt der SM nur selten vor. Mädchen sind dabei häufiger betroffen als Jungen. Das Geschlechterverhältnis beträgt etwa 1.6:1 (Mädchen : Jungen), (Bahr, 2006; Dummit et al., 1997; Steinhausen \& Juzi, 1996).

Für die wachsende Population der mehrsprachigen Kinder weisen erste Studien darauf hin, dass diese ein erhöhtes Risiko für die Entwicklung eines SM haben (Elizur \& Perednik, 2003). Zugleich ist die Identifikation des SM bei zweisprachig aufwachsenden Kindern vor allem für Fachkräfte in Kindergarten und Schule deutlich erschwert. So liegt bei diesen Kindern zunächst die Interpretation nahe, dass ihr Schweigen vor dem Hintergrund des Zweitspracherwerbs $\mathrm{zu}$ erklären ist. $\mathrm{Zu}$ der bei einsprachigen Kindern häufigen Interpretation des Schweigens als extreme Form der Schüchternheit (Kopp \& Gilberg, 1997; Schwartz, Freedy \& Sheridan, 2006) kommt somit zusätzlich die Erklärung der noch unzureichenden Sprachkompetenzen hinzu. Eine hohe Dunkelziffer wird daher sowohl für ein- und im Besonderen für mehrsprachige Kinder vermutet (Starke \& Subellok, 2012). Die späte oder fehlende Identifikation des SM führt unter anderem dazu, dass Kinder mit SM erst relativ spät eine spezifi- 
sche Therapie erhalten. Obwohl das Schweigen zwischen dem dritten und vierten Lebensjahr das erste Mal auftritt (Dummit et al., 1997; Steinhausen \& Juzi, 1996), werden die Kinder erst mit durchschnittlich acht Jahren einer mutismusspezifischen Therapie zugeführt (Remschmidt, Poller, Herpertz-Dahlmann, Henninghausen \& Gutenbrunner, 2001). 40\% der Kinder bleiben sogar unversorgt (Black \& Uhde, 1995; Kumpulainen, Räsänen, Raaska \& Somppi, 1998). Ein Teil der Betroffenen überwindet ihr Schweigen auch ohne spezifische Therapie (Ford, Sladeczeck, Carlson \& Kratochwill, 1998). Studien zeigen jedoch, dass bei Überdauern des Schweigens über das zehnte Lebensjahr langfristige Folgen in zahlreichen Entwicklungsbereichen bei den Kindern bzw. Jugendlichen zu erwarten sind (Kolvin \& Fundudis, 1981; Remschmidt et al., 2001). Eine frühzeitige Diagnostik und Intervention erscheint gerade für die Gruppe der mehrsprachigen Kinder hoch bedeutsam (Mayworm, Dowdy, Knights \& Rebelez, 2014), um Kindern frühzeitig ausreichend Möglichkeiten für den Zweitspracherwerb zu bieten. Der Kindergarten ist der Kontext, in dem viele mehrsprachige Kinder die Mehrheitssprache am häufigsten gebrauchen und in dem sie vorrangig ihre produktiven Sprachkompetenzen erwerben. Ein konsequentes Schweigen in diesem Kontext, wie es mit einem SM einhergeht, reduziert die Anzahl der Übungskontexte für den Zweitspracherwerb deutlich. Keller, Troesch und Grob (2013) konnten für schüchterne Kinder einen deutlich langsameren Erwerb der Zweitsprache abzeichnen als bei nicht-schüchternen Kindern gleichen Alters. Bereits oben wurde auf die Bedeutsamkeit der sprachlichen Kompetenzen für die soziale Teilhabe hingewiesen. Die frühe Identifikation mehrsprachiger Kinder mit SM ist vor diesem Aspekt besonders relevant. Bislang gibt es jedoch nur wenige Befunde zum SM bei mehrsprachigen Kindern, auf denen eine frühzeitige Differentialdiagnose aufgebaut werden könnte. Unklar ist, welche Faktoren mehrsprachige Kinder besonders vulnerabel für die Entwicklung des SM machen und anhand welcher Merkmale sie frühzeitig von solchen Kindern differenziert werden können, die tatsächlich noch nicht über angemessene Sprachkompetenzen in der Mehrheitssprache verfügen. Ziel der vorliegenden Studie ist es, mögliche Risikofaktoren für die Entwicklung eines SM bei mehrsprachigen Kindern zunächst theoretisch herzuleiten und anschließend in einer Längsschnittstudie zu prüfen. 


\section{Forschungsstand}

\subsection{Selektiver Mutismus}

Selektiver Mutismus (SM) ist eine Kommunikationsstörung, bei der Kinder in spezifischen sozialen Situationen schweigen, obwohl sie grundsätzlich über angemessene sprachliche Fähigkeiten verfügen (Cohan, Chavira \& Stein, 2006). Charakteristisch für den SM ist die große Diskrepanz zwischen einem unbefangenen Sprechen im familiären Kontext und einem Schweigen in öffentlichen oder Bildungskontexten (Bergman et al., 2002; Ford et al., 1998). Die am stärksten vom Kernsymptom Schweigen betroffenen Kontexte sind die Bildungseinrichtungen Kindertagesstätte (Kita) und Schule (Ford et al., 1998). Die Systematik des Schweigens, welche sich über Personen und Kontexte konstituiert, mit bzw. in denen das Kind schweigt, kann individuell sehr unterschiedlich sein. So gibt es Kinder, die mit ihren Eltern sprechen, aber in Gegenwart weiterer Personen schweigen. Andere schweigen lediglich in Gegenwart von wenig vertrauten bzw. fremden Erwachsenen, sprechen jedoch mit Kindern. Wieder andere schweigen in der Schule konsequent mit Mitschülern, Freunden und Lehrerkräften, obwohl sie etwa zuhause mit ihren Freunden völlig unbefangen sprechen. Viele Eltern berichten sogar, dass ihre Kinder zuhause übermäßig viel sprächen (Edison et al., 2011; Kearney, 2010).

Das DSM-IV (Diagnostisches und Statistisches Manual psychischer Störungen Version 4; Saß, Wittchen, Zaudig \& Houben, 2003) beschreibt folgende Diagnosekriterien für den SM:

A) Andauernde Unfähigkeit, in bestimmten Situationen zu sprechen (in denen das Sprechen erwartet wird, z.B. in der Schule), wobei in anderen Situationen normale Sprechfähigkeit besteht.

B) Die Störung behindert die schulischen oder beruflichen Leistungen oder die soziale Kommunikation.

C) Die Störung dauert mindestens einen Monat (und ist nicht auf den ersten Monat nach Schulbeginn beschränkt).

D) Die Unfähigkeit zu sprechen ist nicht durch fehlende Kenntnisse der gesprochenen Sprache bedingt, die in der sozialen Situation benötigt werden oder dadurch, dass der Betroffene sich in dieser Sprache nicht wohl fühlt.

E) Die Störung kann nicht besser durch eine Kommunikationsstörung (z.B. Stottern) erklärt werden und tritt nicht ausschließlich im Verlauf einer tiefgreifen- 
den Entwicklungsstörung, Schizophrenie oder einer anderen Psychotischen Störung auf. (Saß et al., 2003, S. 73f)

Mit SM häufig assoziierte Temperamentsmerkmale sind Schüchternheit und sozialer Rückzug (Ford et al., 1998; Rösler, 1981; Steinhausen \& Juzi, 1996). Der Begriff sozialer Rückzug ist ein Oberbegriff für zahlreiche Verhaltensphänomene, welche durch eine Einsamkeit der Person aufgrund unterschiedlichster Ursachen charakterisiert sind (Rubin, Coplan \& Bowker, 2009). Sozialer Rückzug stellt ein Risiko für die Entwicklung von Angststörungen und anderen internalisierenden Auffälligkeiten dar bzw. steht häufig mit ihnen im Zusammenhang (Coplan, Arbeau \& Armer, 2008; Rubin et al., 2009). In den Klassifikationssystem DSM-V (American Psychiatric Association, 2013) und ICD-10 (Deutsches Institut für Medizinische Dokumentation und Information [DIMDI], 2014) wird (sozialer) Rückzug als bedeutsames Symptom bzw. Merkmal bspw. von Angststörungen, Phobien oder Depressionen sowie bei SM angeführt.

Schüchternheit stellt eine Form des sozialen Rückzugs dar. Rubin und Coplan (2004) beschreiben Schüchternheit als einen internen Konflikt, bei dem eine hohe Motivation zu sozialer Nähe von einer Vorsicht vor der Initiation sozialer Interaktionen begleitet wird. Schüchterne Kinder sehnen sich nach sozialen Kontakten, verspüren gleichzeitig jedoch Angst in sozialen Situationen, die zur Vermeidung sozialer Kontakte führt (Coplan, Prakash, O'Neil \& Armer, 2004). Entsprechend der Konzeptualisierung der Begriffe Schüchternheit und sozialer Rückzug initiieren schüchterne zurückgezogene Kinder nur selten Kontakte mit ihren Peers bzw. benötigen deutlich länger als ihre nicht-schüchternen Peers, um ein Gespräch aufzubauen. Asendorpf und Meier (1993) konnten in ihrer Studie situative Unterschiede in den Sprechanteilen der Kinder innerhalb von Gesprächen feststellen. In gewohnten Situationen sprachen die schüchternen Kinder genauso viel wie ihre nicht-schüchternen Peers. Unterschiede zwischen den Gruppen zeigten sich vor allem in unvertrauten Situationen. Auch wenn die Sprechanteile der Kinder im vertrauten Kontext mit denen ihrer nicht-schüchternen Peers vergleichbar sind, konnten Studien zeigen, dass sich schüchterne von nicht-schüchternen Kindern in ihren linguistischen Fähigkeiten unterscheiden (z.B. Crozier \& Badawood, 2009; Evans, 1996; Spere, Schmidt, Theall-Honey \& Martin-Chang, 2004). Schüchterne Kinder schnitten deutlich schlechter in standardisierten Testverfahren zur Messung der rezeptiven und expressiven Fähigkeiten ab als ihre nicht-schüchternen Altersgenossen (Crozier \& Badawood, 2009; Crozier \& Perkins, 2002; Evans, 1996; Spere et al., 2004). Fraglich ist jedoch, ob diese Leistungsunterschiede eher einem tatsächlichen Kompetenzdefizit zuzuschreiben oder vielmehr ein Ergebnis von Unsicherheiten und Stresserleben der schüchternen Kinder in der Testsitua- 
tion sind. Crozier und Hostettler (2003) konnten dazu zeigen, dass die Leistungsunterschiede zwischen schüchternen und nicht-schüchternen Kindern in einer Gruppentestung deutlich kleiner waren als die in einer Einzeltestung.

Unterstützung für die Vermutung geringerer Sprachkompetenzen bei schüchternen Kindern findet sich ebenso in der Studie von Reynolds und Evans (2009), welche die narrativen Fähigkeiten der Kinder anhand von Bilderbuchsituationen mit einem Elternteil im häuslichen Kontext der Familie untersuchten. Die Leistungen der schüchternen und nichtschüchternen Kinder unterschieden sich signifikant hinsichtlich der formal linguistischen Komplexität und der Erzählstruktur. Die schüchternen Kinder produzierten weniger Wörter, nutzten weniger unterschiedliche Attribute und zeigten häufiger unvollständige Äußerungen. Ihre Erzählungen enthielten weniger bedeutungsvollen Inhalt. Schüchterne, zurückgezogene Kinder werden zudem häufiger von ihren Peers abgelehnt, was die Ängstlichkeit in sozialen Situationen aufrechterhalten kann (Gazelle \& Ladd, 2003).

\subsection{Risikofaktoren und komorbide Phänomene}

Wie die bisherigen Ausführungen bereits andeuten zeigen Kinder mit SM neben dem Schweigen noch weitere komorbide Auffälligkeiten, zu denen vor allem Angststörungen zählen (Dummit et al., 1997; Ford et al., 1998; Rösler, 1981; Steinhausen \& Juzi, 1996; für einen Überblick siehe Cohan, Price \& Stein, 2006). Kristensen (2000) konnte in ihrer Studie mit 54 selektiv mutistischen Kindern und 108 sprechenden Kontrollkindern zeigen, dass Kinder mit SM signifikant häufiger eine weitere Auffälligkeit zeigen als die Kontrollkinder. Ein großer Teil der Kinder mit SM erfüllte Diagnosekriterien für zwei (24.1\%) oder mehr Diagnosen (55.4\%). Die Kombination einer Entwicklungsstörung und einer Angststörung war dabei am häufigsten (46.3\%). Tabelle 1 zeigt die Ergebnisse von (Kristensen, 2000) im Überblick.

SM ist demnach kein isoliertes Phänomen und lässt sich nicht auf das alleinige Schweigen des Kindes reduzieren. Vielmehr zeigen betroffene Kinder ein breites Spektrum auffälliger Verhaltensweisen, denen in Förderung und Therapie begegnet werden muss. Konsequenterweise lässt sich ebenso nicht die eine Ursache für das Schweigen benennen, sondern es wird von einer multifaktoriellen Ätiologie ausgegangen (Cohan, Price et al., 2006). 
Tabelle 1

Diagnosen bei Kindern mit SM und Kontrollkindern in der Studie von Kristensen (2000)

\begin{tabular}{lcccccc}
\hline & \multicolumn{2}{c}{ Kinder mit SM $(\mathrm{n}=54)$} & & \multicolumn{2}{c}{ Kontrollkinder $(\mathrm{n}=108)$} \\
\cline { 2 - 3 } \cline { 5 - 6 } Diagnose & $\mathrm{n}$ & $\%$ & & $\mathrm{n}$ & $\%$ \\
\hline irgendeine Diagnose & 53 & 98.1 & & 28 & 25.9 \\
Entwicklungsstörung & 37 & 68.5 & & 14 & 13 \\
Störung der Ausscheidungsorgane & 17 & 31.5 & & 10 & 9.3 \\
Angststörung & 40 & 74.1 & & 8 & 7.4 \\
Sprachstörung (n=52/104) & 26 & 50 & & 12 & 11.5 \\
\hline
\end{tabular}

Zur Erklärung des SM ziehen einige Autoren ein Diathese-Stress-Modell heran (Bahr, 2006; Cohan, Chavira et al., 2006; Cohan, Price et al., 2006; Elizur \& Perednik, 2003; Hartmann, 1997). Diathese-Stress-Modelle nehmen eine Prädisposition oder Vulnerabilität für eine bestimmte Störung an-die so genannte Diathese (Ingram \& Luxton, 2005). Diese kann aus einem oder aus mehreren Faktoren bestehen, wie zum Beispiel genetischen, soziostrukturellen oder psychischen Faktoren. Ingram und Luxton (2005) sehen die Diathese als ein latentes Persönlichkeitsmerkmal an, welches relativ stabil und dennoch veränderbar ist. Letzteres hängt vor allem davon ab, in welchem Ausmaß das Störungsbild durch genetische oder biologische Faktoren beeinflusst wird. Dem transaktionalen Stressmodell von Lazarus folgend (Lazarus \& Folkman, 1984) wird unter Stress eine spezifische Beziehung zwischen Umwelt und Person gefasst, welche von der Person als außergewöhnlich anstrengend bewertet wird. $\mathrm{Zu}$ den Stressoren - also Umweltfaktoren, die Stress in der Person auslösen-können einschneidende Lebensereignisse, eine Anhäufung vieler, kleiner Ärgernisse oder auch sozioökonomische Faktoren zählen (Ingram \& Luxton, 2005). Die meisten Modelle gehen davon aus, dass jeder Mensch ein gewisses Diatheselevel für alle möglichen Arten psychischer Störungen besitzt (Monroe \& Simons, 1991). Ob sich eine spezifische Störung ausprägt, hängt von der Interaktion zwischen individueller Vulnerabilität und wahrgenommenem Stress der Person in einer spezifischen (Lebens-)Situation ab. Für die Interaktion von Diathese und Stress werden drei Modellformen angenommen: (1) additive Modelle (2) ipsative Modelle und (3) MegaDiathese-Stress Modelle (Ingram \& Luxton, 2005). Bei additiven Modellen geht man davon aus, dass sich die Störung durch die Kombination des Ausmaßes der Vulnerabilität und dem Effekt des Stressors ausprägt. Für Personen mit einer hohen Diathese reicht bereits geringes Stresserleben aus, um die Störung hervorzurufen. Entsprechend können sich Störungen bei Personen mit geringer Vulnerabilität auch durch ein massives Stresserleben ausprägen. Ipsati- 
ve Modelle gehen von einer reziproken Beziehung zwischen Diathese und Stress aus. Diese Form von Modellen betont die gemeinsame Verursachung der Störung durch Vulnerabilitätsund Stressfaktoren. Je mehr Vulnerabilität vorhanden ist, desto weniger Stresserleben ist nötig, um die Störung auszuprägen und umgekehrt. Eine dritte Form von Diathese-StressModellen postuliert, dass Störungen dann ausgelöst werden, wenn sowohl eine erhöhte Vulnerabilität als auch ein erhebliches Stresserleben zusammenkommen (Ingram \& Luxton, 2005). In der Literatur finden sich zahlreiche Diathese-Stress-Modelle zur Erklärung verschiedenster psychischer Erkrankungen (siehe etwa Braet, van Vlierberghe, Vandevivere, Theuwis \& Bosmans, 2013; Kotsiubinskii, 2002; Patten, 2013) und auch Kommunikationsstörungen (siehe etwa Walden et al., 2012). Den jeweiligen Störungen entsprechend werden spezifische Vulnerabilitäts- bzw. Risikofaktoren angenommen, die in Kombination mit spezifischen Stressoren die Störung hervorrufen können. Für die Erklärung der Entwicklung des SM ist ein Diathese-Stress-Modell deswegen hilfreich, weil es die Kombination vieler Risikofaktorensowohl innerhalb des Kindes als auch innerhalb seiner Umgebung - mit Lebenssituationen, welche das Kind selbst als stresshaft und überfordernd erlebt, ermöglicht. Das Schweigen kann dann als Reaktion auf die Überforderung des Kindes angesehen werden (Katz-Bernstein, 2011). In einem Diathese-Stress-Modell können zudem verschiedene Entwicklungsverläufe nachgezeichnet werden, so dass der Heterogenität der Lebensgeschichten selektiv mutistischer Kinder Rechnung getragen werden kann. Im Folgenden werden für den SM bedeutsame Risikofaktoren einzeln betrachtet, um im Anschluss mögliche Entwicklungsverläufe des SM zu skizzieren.

\subsubsection{Genetische Prädisposition und familiäre Lernumgebung}

Als Basis für die Entwicklung eines SM wird von einer grundsätzlichen Schüchternheit bzw. einem gehemmten Verhalten in unvertrauten Situationen und gegenüber unvertrauten Personen ausgegangen (Black \& Uhde, 1995; Dummit et al., 1997; Ford et al., 1998). Forschungsergebnisse weisen darauf hin, dass in Familien selektiv mutistischer Kinder gehäuft auch weitere Familienmitglieder internalisierende Störungen-wie etwa Depressionen oder soziale Phobien - aufweisen (siehe im Überblick Cohan, Price et al., 2006) und somit von einem genetischen Faktor bei der Entstehung des SM ausgegangen werden kann. Insbesondere bei Familienmitgliedern ersten Grades finden sich soziale Phobien, emotionale Störungen mit Vermeidungsverhalten oder ein SM. In der Stichprobe von Kolvin und Fundudis (1981) zeigte sich bei $42 \%$ der Familien schweigender Kinder mindestens bei einem Elternteil eine auffällige Persönlichkeit. Die häufigsten psychischen Probleme waren dabei schwere Neuro- 
sen und Depressionen. In der Stichprobe von Black und Uhde (1995) zeigten bei $70 \%$ der Kinder mit SM Familienmitglieder ersten Grades eine soziale Phobie oder Vermeidungsverhalten, bei 37\% lag bzw. hat bei einem weiteren Familienmitglied ein SM vorgelegen. In einem Viertel der Fälle waren beide Elternteile von einer sozialen Phobie betroffen. Chavira, Shipon-Blum, Hitchcock, Cohan und Murray (2007) untersuchten insgesamt 70 Elternpaare schweigender Kinder sowie 31 Elternpaare sprechender Kinder hinsichtlich psychiatrischer Diagnosen und Persönlichkeitsmerkmalen. Signifikante Unterschiede ergaben sich zwischen den Elterngruppen lediglich für die soziale Phobie und die emotionale Störung mit Vermeidungsverhalten. 37\% der Eltern schweigender Kinder im Vergleich zu 14.1\% der Kontrolleltern waren von einer sozialen Phobie betroffen, 17.5\% der Eltern schweigender Kinder gegenüber $4.7 \%$ der Kontrolleltern von einer emotionalen Störung mit Vermeidungsverhalten. Die deutlich niedrigere Prozentzahl der sozialen Phobien unter den Eltern selektiv mutistischer Kinder im Vergleich zu den Ergebnissen von Black und Uhde (1995) resultiert wahrscheinlich aus den beiden unterschiedlichen Klassifikationssystemen, die für die Diagnosestellung angelegt wurden. Black und Uhde (1995) zogen das DSM-III, Chavira et al. (2007) das DSM-IV heran. Auch Kristensen und Torgersen (2001) konnten zeigen, dass Mütter und Väter selektiv mutistischer Kinder signifikant häufiger eine Schüchternheit aufweisen (38.9\% der Mütter, 31.5\% der Väter) als Eltern sprechender Kinder (3.7\% der Mütter, 0.9\% der Väter). Signifikante Unterschiede zwischen den Müttern schweigender und sprechender Kinder zeigten sich zudem auf den Skalen schizoid, vermeidend und schizotypisch eines multiaxialen diagnostischen Interviewverfahrens, wobei die Mütter der selektiv mutistischen Kinder im Mittel höhere, das heißt klinisch auffälligere Werte erreichten als die Kontrollmütter. Die Väter unterschieden sich signifikant auf den Skalen schizoid und ängstlich, wobei auch hier die Väter der schweigenden Kinder jeweils auffälligere Werte aufwiesen als die Kontrollväter. Steinhausen und Adamek (1997) betrachteten die familiäre Belastung mit psychischen Störungen, SM und sprachlichen Auffälligkeiten mit familiendiagnostischen Verfahren. Ihre Stichprobe bestand aus 38 Kindern mit SM und einer phänotypisch ähnlichen Kontrollgruppe von 31 Kindern mit einer emotionalen Störung des Kindesalters bzw. einer Sprachentwicklungsproblematik. Mit Hilfe eines Genogramms wurden zunächst alle bedeutsamen Familienmitglieder und -beziehungen erfasst. Daraufhin wurden für jedes Mitglied die Aspekte Schweigsamkeit (definiert als wenig bis gar keine spontanen Sprachäußerungen sowie langen Perioden ausbleibender verbaler Kommunikation), psychische Erkrankungen, SM und sprachliche Auffälligkeiten abgefragt. Auch hier waren die Mütter der Kinder mit SM signifikant häufiger von einer psychischen Störung betroffen als die Kontrollmütter. Die Schweigsamkeit 
der Familienmitglieder ersten bis dritten Grades stellte sich jedoch als der Aspekt heraus, der am besten zwischen den schweigenden und sprechenden Kindern differenzierte.

Unterstützung für einen genetischen Einfluss bei der Entstehung des SM findet sich bei einer doch erheblichen Anzahl schweigender Zwillingspaare (Subellok, Kresse \& Bahrfeck-Wichitill, 2010). Innerhalb der internationalen Fachliteratur finden sich zahlreiche Falldarstellungen von Zwillingspaaren mit SM (Balla-Jaeger, 1971; Gray, Jordan, Ziegler \& Livingston, 2002; Jaeger \& Metzker, 1968; Kroppenberg, 1987; Mora, DeVault \& Schopler, 1962; Segal, 2003; Sharkey \& McNicholas, 2006). Betrachtet man zusätzlich größere phänomenologische Studien zum SM finden sich in diesen Stichproben überdurchschnittlich viele Zwillinge - bei Ford et al. (1998) 15 von 153 Kindern, bei Dummit et al. (1997) 6 von 50. Segal (1999) weist zudem darauf hin, dass bei eineiigen Zwillingen häufig beide Kinder schweigen, bei zweieiigen eher nur einer von beiden betroffen ist. Die Befunde zur familiären Häufung von Schüchternheit, Angststörungen und SM sowie zum Auftreten des SM bei Zwillingen deuten klar auf einen genetischen Einfluss bei der Entstehung des Schweigens hin.

Neben den genannten psychischen und persönlichkeitsbezogenen Aspekten spielt ebenso das familiäre Lernumfeld eine Rolle. Kinder lernen neue Verhaltensweisen häufig über die Imitation des Verhaltens anderer Personen ihres Umfeldes (Niebuhr-Siebert \& Chilla, 2012; Tomasello, 2008). In frühen Jahren sind dies vor allem nahe Familienmitglieder. So werden auch grundlegende Kommunikationsmuster zunächst über Imitation des elterlichen Verhaltens erworben. Sind Mutter und Vater schüchtern oder extrem ängstlich, werden sie in sozialen Situationen eher zurückhaltendes Verhalten zeigen. Das Kind wiederum kann dieses Verhalten als situationsangemessen interpretieren und über die Nachahmung in sein eigenes Verhaltensrepertoire aufnehmen (Melfsen \& Warnke, 2007). Dobslaff (2005) berichtet zudem von einer gehäuften sozialen Isolierung der Familien, z.B. bedingt durch eine sehr ländliche Wohnsituation. Dies führt dazu, dass Kinder kaum Kontakt zu anderen Erwachsenen oder Kindern außerhalb der Familie haben. Der Input kommunikativer Verhaltensweisen bleibt maßgeblich auf die Familie beschränkt. Ist das Kommunikationsverhalten der Eltern bedingt durch extreme Schüchternheit oder internalisierende psychische Erkrankungen deutlich eingeschränkt, wird auch das Kind zunächst nur ein wenig flexibles Verhaltensrepertoire aufbauen. Dies erschwert dem Kind den Einstieg in soziale Gruppen, wie etwa bei Eintritt in die Kita.

\subsubsection{Soziale Ängstlichkeit}

Soziale Ängstlichkeit ist das wohl am häufigsten mit SM assoziierten psychische Phänomen (siehe etwa Black \& Uhde, 1995; Cohan et al., 2008; Dummit et al., 1997; Kristensen, 
2000). Ob es sich dabei um einen den SM verursachenden oder aus ihm resultierenden Faktor handelt oder ob diese lediglich als komorbide Phänomene anzusehen sind, ist bislang nicht geklärt. Zahlreiche Studien belegen zunächst, dass ein großer Teil der Kinder mit SM zusätzlich eine Angststörung aufweisen, wobei die soziale Phobie bzw. soziale Ängstlichkeit ${ }^{1}$ die häufigste Diagnose ist (Andersson \& Thomsen, 1998; Bergman et al., 2002; Black \& Uhde, 1995; Chavira et al., 2007; Cunningham, McHolm \& Boyle, 2006; Kristensen, 2000; Manassis et al., 2007; Vecchio \& Kearney, 2005; Yeganeh, Beidel \& Turner, 2006). In welchem Ausmaß Kinder mit SM zusätzlich von sozialer Ängstlichkeit betroffen sind, ist jedoch bislang unklar. Vecchio und Kearney (2005) konnten in ihrer Studie bei allen 15 schweigenden Kindern im Alter von vier bis zehn Jahren auf Grundlage eines strukturierten Elterninterviews zusätzlich eine soziale Ängstlichkeit diagnostizieren. Black und Uhde (1995) fanden ähnlich hohe Komorbiditätsraten von sozialen Phobien bei 30 schweigenden Kindern im Alter von fünf bis zwölf Jahren. Hier konnte bei 27 der Kinder mit SM zusätzlich eine soziale Phobie nach DSM-III-R-Kriterien diagnostiziert werden. Deutlich niedrigere Raten fand Kristensen (2000) in ihrer Untersuchung von 54 Kindern mit SM im Alter von 3 bis 16 Jahren. Hier konnte nur bei $67.9 \%$ der Kinder die Diagnose soziale Phobie nach DSM-IV-Kriterien bestätigt werden.

Die Arbeitsgruppe um Yeganeh befasste sich in zwei Studien mit dem Ausmaß sozialer Ängstlichkeit bei Kindern mit SM im Vergleich zu Kindern mit isolierter sozialer Phobie (Yeganeh et al., 2006; Yeganeh, Beidel, Turner, Pina \& Silverman, 2003). In einer ersten Studie (Yeganeh et al., 2003) verglichen sie 23 Kinder mit sozialer Phobie und SM mit 23 Kontrollkindern mit isolierter sozialer Phobie im Alter von 5 bis 13 Jahren hinsichtlich des Ausmaßes ihrer sozialen Ängste. Sie nutzten dafür Kind- und Elternbefragungen sowie Einschätzungen einer klinischen Fachperson. Die beiden Gruppen unterschieden sich nicht signifikant hinsichtlich des Ausmaßes der sozialen Ängste, erhoben durch Selbstbeurteilungen der Kinder. Jedoch schätzten die klinischen Fachpersonen die Angst der Kinder mit sozialer Phobie und SM als signifikant höher ein als bei den Kindern mit isolierter sozialer Phobie. Bei der

\footnotetext{
${ }^{1}$ Die Begriffe soziale Phobie und soziale Ängstlichkeit bezeichnen phänomenologisch das gleiche Symptombild, wobei der Begriff der sozialen Ängstlichkeit eher im Kindesalter verwendet wird. Mit den Begriffen wird eine „dauerhafte, unangemessene Furcht vor sozialen oder Leistungssituationen gekennzeichnet“ (Melfsen \& Warnke, 2004, S. 166). Für die Klassifizierung von Angststörungen dieser Art im Kindesalter verwendet das ICD-10 den Begriff der Störungen mit sozialer Ängstlichkeit des Kindesalters, für den Jugend- und Erwachsenenbereich den Begriff der sozialen Phobie (Deutsches Institut für Medizinische Dokumentation und Information [DIMDI], 2014). Im DSM V wird für alle der Begriff der sozialen Ängstlichkeit verwendet (American Psychiatric Association, 2013). Vor dem Hintergrund der in der vorliegenden Studie betrachteten Probandengruppe von drei- bis fünfjährigen Kindern wird in dieser Arbeit vor allem der Begriff soziale Ängstlichkeit verwendet, um auf eine pathologische Angst innerhalb sozialer Situationen im Kindesalter zu verweisen. Der Begriff der sozialen Phobie wird dann verwendet, wenn in den zitierten Studien eben dieses Konstrukt erhoben wurde.
} 
Beobachtung der Kinder in einer standardisierten Situation mit einem Peer gleichen Alters schätzten verblindete Beobachter die schweigenden Kinder als deutlich ängstlicher und weniger sozialkompetent ein als die Kinder mit isolierter sozialer Phobie. Somit zeigte sich in dieser Studie, dass die Kinder mit sozialer Phobie und SM selbst keine massiveren Ängste in sozialen Situationen empfinden. Ihr Schweigen stellt bereits eine Problemlösestrategie dar, die womöglich ihre Angst abmildert. Erwachsene scheinen das Schweigen als massive Angstreaktion zu interpretieren. Es wird nicht als Strategie angesehen, die soziale Situation angenehmer zu machen, sondern wirkt auf den Erwachsenen als ob das Kind vor lauter Angst erstarre.

In einer zweiten Studie zum Ausmaß der sozialen Ängstlichkeit bei Kindern mit SM im Vergleich zu Kindern mit isolierter sozialer Phobie untersuchten (Yeganeh et al., 2006) 3 Gruppen mit jeweils 21 Kindern im Alter von 7 bis 15 Jahren: (1) Kinder mit SM, (2) Kinder mit sozialer Phobie und (3) sprechende, psychiatrisch unauffällige Kinder. Auch hier unterschieden sich die Kinder mit SM und sozialer Phobie in den Selbstbeurteilungsverfahren nicht signifikant voneinander. Die Kinder mit SM wurden jedoch von klinischen Fachpersonen signifikant ängstlicher eingeschätzt als die beiden Vergleichsgruppen. Die Kinder mit SM und die Kinder mit sozialer Phobie unterschieden sich erwartungsgemäß hochsignifikant von den unauffälligen Kontrollkindern hinsichtlich ihrer Ängstlichkeit. Carbone et al. (2010) konnten in ihrer Studie mit 44 Kindern mit SM, 65 Kindern mit Angststörungen sowie einer unauffälligen Kontrollgruppe mit 49 Kindern denselben Effekt auch bei Einschätzungen durch Lehrer und Eltern finden. Auch hier beurteilten Lehrer und Eltern die schweigenden Kinder als signifikant sozial ängstlicher als die Kinder mit Angststörungen. In den Selbstbeurteilungen durch die Kinder ergaben sich jedoch keine signifikanten Unterschiede. Carbone et al. (2010) untersuchten neben der Ängstlichkeit zusätzlich die sozialen Kompetenzen der Kinder auf verbaler und nonverbaler Ebene über Eltern- und Lehrerfragebögen. Die schweigenden Kinder wurden sowohl von ihren Eltern als auch den Lehrern signifikant schlechter in ihren verbalen Sozialkompetenzen eingeschätzt als die Kinder mit Angststörungen. Die schweigenden Kinder unterschieden sich zudem signifikant von den Kontrollkindern in ihren nonverbalen Sozialkompetenzen. Zwischen den schweigenden und den ängstlichen Kindern ergab sich im nonverbalen Sozialverhalten kein signifikanter Unterschied.

Manassis et al. (2003) fanden in ihrer Studie mit 23 Kindern im Alter von 7 bis 15 Jahren (14 Kinder mit SM, 9 Kinder mit sozialer Phobie) ähnliche Ergebnisse. Auch hier unterschieden sich beide Gruppen hinsichtlich des Ausmaßes sozialer Ängstlichkeit nicht signifikant voneinander. Ein statistischer Trend zur höheren Ängstlichkeit zeigte sich jedoch in 
einem Selbstbeurteilungsverfahren für die Kinder mit sozialer Phobie. In einer nachfolgenden Studie mit 44 Kindern mit SM, 28 Kindern mit Angststörungen und 19 unauffälligen Kontrollkindern jeweils im Alter von sechs bis zehn Jahren (Manassis et al., 2007) gaben die schweigenden Kinder im Vergleich zu beiden Vergleichsgruppen in einem Selbstbeurteilungsverfahren ein signifikant größeres Ausmaß der sozialen Ängstlichkeit an. Hierbei muss jedoch bedacht werden, dass in dieser Studie nicht wie zuvor schweigende Kinder mit Kindern mit isolierter sozialer Phobie verglichen wurden, sondern Kinder mit allen Arten von Angststörungen als Vergleichskinder herangezogen wurden. Somit wird hier besonders deutlich, dass der SM stark mit sozialer Ängstlichkeit und weniger mit anderen Angststörungen assoziiert ist.

In einer Untersuchung von insgesamt 341 Schulkindern mit psychiatrischen Störungen konnten Melfsen, Walitza und Warnke (2006) für alle Kinder mit SM (n = 9, 9 bis 16 Jahre) klinisch auffällige Werte in einem Selbstbeurteilungsverfahren für soziale Ängstlichkeit nachweisen. Im Vergleich der schweigenden mit den sozial phobischen Kindern ergab sich ein signifikanter Unterschied im Ausmaß der sozialen Ängstlichkeit, wobei die sozial phobischen Kinder höhere Werte als die Kinder mit SM aufwiesen und somit mehr Angst empfanden.

In allen Studien wird deutlich, dass soziale Ängstlichkeit bei einer Vielzahl von Kindern mit SM ein komorbides Phänomen darstellt. Entgegen früheren Hypothesen, dass der SM eine extreme Form der sozialen Ängstlichkeit sei (Black \& Uhde, 1995), zeigen aktuelle Forschungsergebnisse, dass schweigende Kinder im Vergleich zu Kindern mit isolierter sozialer Ängstlichkeit nicht massiver von sozialen Ängsten betroffen sind (Yeganeh et al., 2006; Yeganeh et al., 2003). Die Ergebnisse von Melfsen et al. (2006) weisen sogar darauf hin, dass schweigende Kinder ein geringeres Ausmaß sozialer Ängste zeigen.

Cohan et al. (2008) untersuchten in einer USA-weiten Studie 130 schweigende Kinder im Alter von fünf bis zwölf Jahren hinsichtlich der Aspekte sozialer Ängstlichkeit, sozialemotionalen und sprachlichen Auffälligkeiten. Mithilfe einer latenten Profilanalyse fanden sie drei Subgruppen selektiv mutistischer Kinder, die als gemeinsames Merkmal klinisch relevante Werte für soziale Ängstlichkeit aufwiesen. Unterschiede ergaben sich zwischen den Gruppen im Ausmaß der sprachlichen und sozial-emotionalen Probleme. Die größte Gruppe (44.6\% der Kinder) zeichnete sich zusätzlich durch grenzwertig klinische Werte bei sozialemotionalen Auffälligkeiten sowie im Bereich syntaktischer Fähigkeiten aus. Die geringfügig kleinere, zweite Gruppe (43.1\%) weist neben den klinisch auffälligen Werten für soziale Ängstlichkeit ebenso klinisch auffällige Werte im Bereich Syntax sowie Werte im Grenzbe- 
reich zur klinischen Auffälligkeiten im Bereich Sprechen (d.h. Aussprachefähigkeiten) auf. Die kleinste Gruppe (12.3\%) umfasste die Kinder, die sich einzig durch klinisch auffällige Werte für soziale Ängstlichkeit auszeichneten. Die Forschungsergebnisse dieser Arbeitsgruppe machen deutlich, dass SM mehr als nur soziale Ängstlichkeit ist. Knapp 88\% der Kinder dieser Stichprobe zeigten auf der syntaktischen Sprachebene laut Elternangaben Unsicherheiten bis massive Schwierigkeiten. Dies weist daraufhin, dass neben der Ängstlichkeit der Kinder vor allem auch die Sprachkompetenzen dieser betrachtet werden sollten, da sprachliche Unsicherheiten oder Entwicklungsstörungen eine bedeutsame Rolle in der Entstehung des SM spielen können.

\subsubsection{Sprachkompetenzen}

Sprachliche Auffälligkeiten finden sich bei etwa einem Drittel bis der Hälfte der Kinder mit SM (Andersson \& Thomsen, 1998; Kristensen, 2000; Kumpulainen et al., 1998; Manassis et al., 2003; Steinhausen \& Juzi, 1996). In der Studie von Kristensen (2000) zeigten $50 \%$ der Kinder mit SM eine Kommunikationsstörung nach DSM-IV-Kriterien ${ }^{2}$. In dieser Diagnosekategorie unterschieden sich die schweigenden Kinder hochsignifikant von ihren sprechenden Kontrollkindern. Sowohl bei den schweigenden als auch bei den sprechenden Kindern war die phonologische Störung die häufigste sprachliche Diagnose, wobei die schweigenden Kinder mit 42.6\% im Vergleich zu 10\% bei den sprechenden Kindern auch hier signifikant häufiger betroffen waren. Expressive Sprachstörungen zeigten sich bei $11.5 \%$ der schweigenden Kinder, rezeptiv-expressive Störungen bei 17.5\%. Stottern kam in dieser Stichprobe weder bei den schweigenden noch bei den sprechenden Kindern vor. Bei einer Teilstichprobe von 32 schweigenden Kindern und 62 Kontrollen untersuchten Kristensen und Oerbeck (2006) zusätzlich die Leistungen des phonologischen Arbeitsgedächtnisses. Auch hier unterschieden sich die Kinder mit SM signifikant von ihren Kontrollen. Die Leistungen des phonologischen Arbeitsgedächtnisses gelten als bester Prädiktor für einen erfolgreichen Spracherwerb (Gathercole, 2006) und werden bei der Diagnostik von umschriebenen Sprachentwicklungsstörungen im Kindergarten- und Vorschulalter als bedeutsamer Marker mit einbezogen (Coady \& Evans, 2008). So ist es nicht verwunderlich, dass sich die Kinder mit SM, die in dieser Stichprobe signifikant häufiger von Spracherwerbsproblemen betroffen waren, auch innerhalb ihrer Leistungen im phonologischen Arbeitsgedächtnis signifikant von den Kontrollkindern unterschieden. Kristensen und Torgersen (2002) betrachteten die Familien

\footnotetext{
${ }^{2}$ Das DSM-IV unterteilt die Kommunikationsstörungen in expressive und rezeptiv-expressive Sprachstörungen sowie phonologische Störungen und Stottern.
} 
schweigender Kinder mit und ohne zusätzlichen Sprachstörungen hinsichtlich emotionaler Aspekte. Die schweigenden Kinder mit sprachlichen Auffälligkeiten waren emotional stabiler und sozial kompetenter als die sprachlich unauffälligen Kinder mit SM. Die Eltern beider Gruppen unterschieden sich jedoch nicht signifikant hinsichtlich von Temperamentsmerkmalen. Neben dem Vergleich der sozialen Ängstlichkeit bei Kindern mit und ohne SM oder sozialen Phobien haben sich bereits zahlreiche Forschungsgruppen mit den sprachlichen Kompetenzen der Kinder beschäftigt. Die Arbeitsgruppe um Manassis hat in zwei Studien die Sprachkompetenzen von schweigenden, ängstlichen und unauffälligen Kontrollkindern untersucht (Manassis et al., 2003; Manassis et al., 2007). In einer ersten Studie mit Kindern mit SM und sozialer Phobie zeigten die schweigenden Kinder signifikant schlechtere Leistungen in der Lautdiskrimination und verfügten tendenziell über einen kleineren rezeptiven Wortschatz als die Kinder mit sozialer Phobie (Manassis et al., 2003). Beide Fähigkeitsbereiche wurden über standardisierte Testverfahren erfasst, in denen von den Kindern lediglich nonverbale Antworten erwartet wurden. In den Elternbeurteilungen der Sprachentwicklung über die Child-Communication-Checklist (Bishop, 1998) ergaben sich keine signifikanten Unterschiede zu den Kindern mit sozialer Phobie. Ähnliche Ergebnisse konnte die Arbeitsgruppe in einer zweiten Studien bei der standardisierten Testung der sprachlichen Fähigkeiten schweigender Kinder im Vergleich zu ängstlichen und unauffälligen Kontrollen finden (Manassis et al., 2007). Auch in dieser Studie zeigten die Kinder mit SM signifikant schlechtere Leistungen in der Lautdiskrimination, im rezeptiven Wortschatz sowie im Verständnis grammatischer Strukturen. Der Anteil klinisch auffälliger Testergebnisse war bei den schweigenden Kindern signifikant größer als bei den ängstlichen und psychisch unauffälligen Kontrollkindern. Nowakowski et al. (2009) betrachteten die rezeptiven sprachlichen Fähigkeiten von knapp über 100 schweigenden und ängstlichen Kindern (Altersrange sechs bis zehn) differenziert nach Geschlecht. Die Mädchen mit SM $(n=16)$ oder einer Angststörung $(n=22)$ zeigten dabei signifikant schlechtere Leistungen in einem rezeptiven Wortschatztest als die Mädchen der Kontrollgruppe. Die Jungen unterschieden sich nicht signifikant voneinander. Entgegen der bisherigen Ergebnisse (Manassis et al., 2003; Manassis et al., 2007) konnten in dieser Studie keine Unterschiede in den rezeptiven sprachlichen Fähigkeiten schweigender im Vergleich zu ängstlichen Kindern festgestellt werden.

Wahrscheinlich spielen jedoch nicht die rezeptiven, sondern vor allem die produktiven Sprachkompetenzen der Kinder eine bedeutsame Rolle für die Entwicklung eines SM. McInnes, Fung, Manassis, Fiksenbaum und Tannock (2004) konnten in ihrer Studie Unterschiede in den produktiven sprachlichen Fähigkeiten bei gleichzeitig ähnlichen rezeptiven Kompeten- 
zen schweigender gegenüber sozial phobischer Kinder nachweisen. Sie betrachteten je sieben Kinder mit SM und sozialer Phobie aus der Stichprobe von Manassis et al. (2003) hinsichtlich ihrer narrativen Fähigkeiten. Sie ließen die Kinder drei Geschichten nacherzählen, die über eine Audioaufnahme präsentiert und mit Bildern unterstützt wurde. Die Kinder mit SM produzierten dabei kürzere, linguistisch einfachere und weniger detaillierte Erzählungen als die Kinder mit sozialer Phobie. Sie zeigten vor allem Unsicherheiten in der Bildung von komplexen Nebensätzen. Die Ergebnisse von McInnes et al. (2004) sind konsistent mit den Befunden von Cohan et al. (2008), die ebenfalls neben den sozialen Ängsten expressive sprachliche Unsicherheiten als bedeutsame Merkmale eines großen Teils der schweigenden Kinder identifizieren konnten.

Wie genau diese Aspekte bei der Entstehung des Schweigens zusammenwirken ist bis dato nicht geklärt. Insbesondere zur Genese der sozialen Ängste und auch der sprachlichen Unsicherheiten ist wenig bekannt. Schweigt das Kind weil es massive Ängste in sozialen Situationen verspürt? Haben sich die Ängste durch die stresshaften und frustrierenden Erlebnisse in sozialen Situationen, in denen das Kind es nicht geschafft hat zu sprechen, entwickelt? Schweigt es, weil es Angst hat oder es ihm peinlich ist seine sprachlichen Unsicherheiten in öffentlichen Situationen zu zeigen? Haben sich die sprachlichen Unsicherheiten erst durch das Fehlen sozialer Interaktionen, das aus dem Schweigen heraus resultiert, ausgeprägt? Womöglich treffen alle Erklärungen auf Kinder mit SM zu, da eher von einer multifaktoriellen Entstehung - wie es Cohan und Chavira et al. (2006) vermuten (siehe Abbildung 1) — und vor allem sich gegenseitig bedingenden und aufrechterhaltenden Prozessen ausgegangen werden kann.

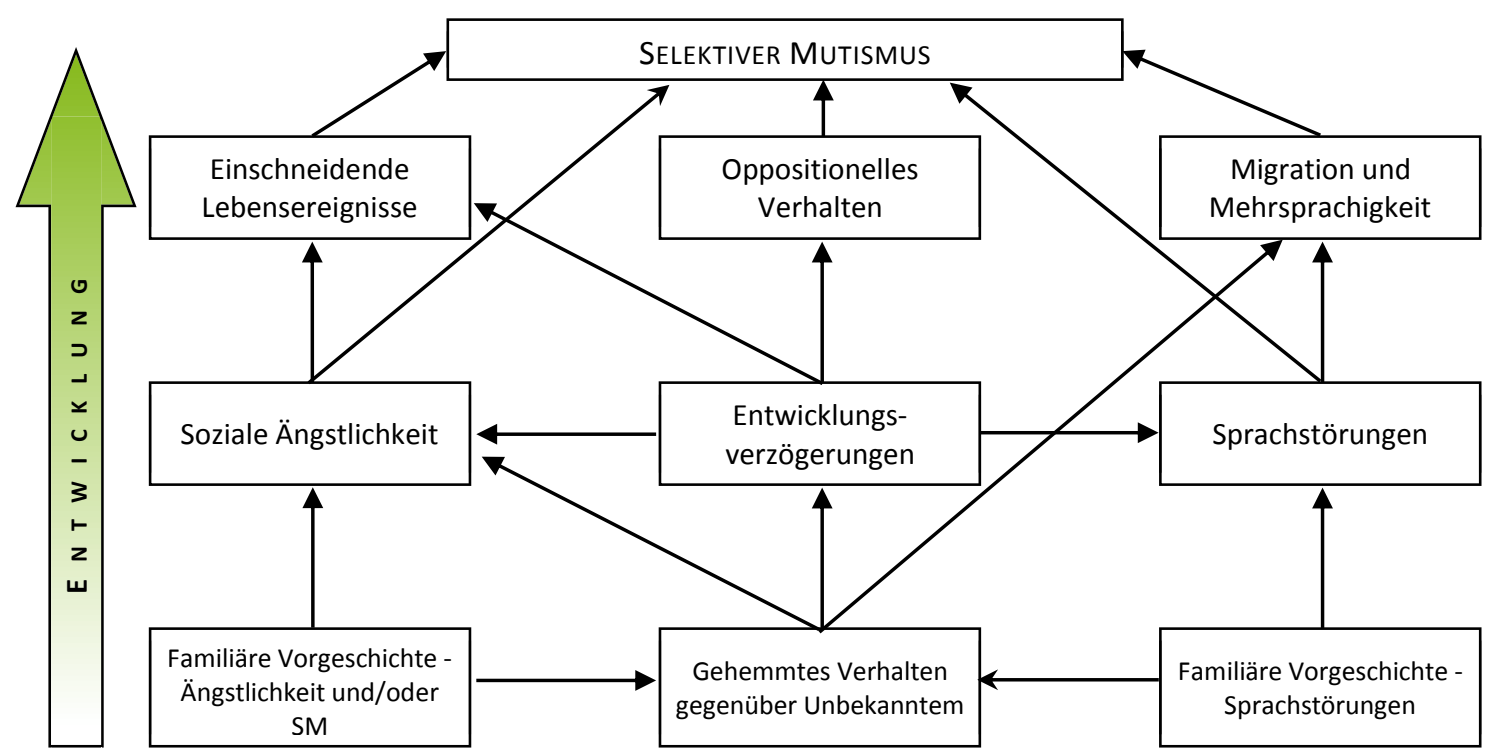

Abbildung 1. Mögliche Wege der Entwicklung eines SM bei Kindern (Cohan, Price et al., 2006, S. 352) 


\subsubsection{Einschneidende Lebensereignisse}

Für einen Teil der Kinder mit SM werden einschneidende oder traumatische Lebensereignisse vor der Ausprägung des SM berichtet (siehe im Überblick Cohan et al., 2006). Steinhausen und Juzi (1996) sowie Andersson und Thomsen (1998) berichten für jeweils etwa ein Drittel der Kinder mit SM traumatische oder einschneidende Lebensereignisse, welche im Zusammenhang mit dem Auftreten des SM standen. Dabei wurden beispielsweise die Trennung der Eltern, die Geburt eines Geschwisterkindes, der Verlust einer Bezugsperson, eine ernsthafte Erkrankung eines Familienmitgliedes oder auch körperliche Gewalt genannt. Körperlicher und sexueller Missbrauch werden für einen kleinen Teil der Kinder mit SM berichtet. In der Stichprobe von Black und Uhde (1995) waren es 13 \% der Kinder mit SM, die körperlichen oder sexuellen Missbrauch erlebt haben.

Deutlich häufiger finden sich bedeutsame Veränderungen im Kontext des Kindes—ob verursacht durch Umzüge und Schulwechsel (Ford et al., 1998; Kristensen, 2000) oder etwa durch die Trennung oder Scheidung der Eltern (Black \& Uhde, 1995). Ein Drittel der Kinder in der Studie von Kristensen (2000) hatten bereits einen Wechsel des Umfeldes miterlebt im Vergleich zu $18.5 \%$ der Kontrollkinder. Die Eltern von $23 \%$ der Kinder in der Stichprobe von Black und Uhde (1995) waren getrennt, $13 \%$ bereits geschieden. Die Eltern sahen die Trennungen oder Scheidungen jedoch nicht im Zusammenhang mit dem SM, auch wenn bei insgesamt vier der sieben Kinder mit getrennten Elternpaaren der SM zeitgleich zur oder nach der Trennung begann. Auch Elizur und Perednik (2003) konnten massivere Ehekonflikte in den Familien der selektiv mutistischen Kinder nachweisen als in den Kontrollfamilien. In der Studie von Kumpulainen et al. (1998) berichteten die Lehrpersonen für fast die Hälfte der Kinder stresshafte Situationen, darunter den Tod einer für das Kind bedeutsamen Person, Alkoholismus innerhalb der Familie oder einen Schulwechsel. Bei $16 \%$ der Kinder trat das jeweilige Lebensereignis kurz vor Beginn der Störung auf. Ob ein kausaler Zusammenhang zwischen solchen Lebensereignissen und dem Störungsbeginn besteht, ist auf der Grundlage bisheriger Studien jedoch nicht möglich. Es fehlen häufig entsprechende Kontrollgruppen oder längsschnittliche Untersuchungen, die Analysen kausaler Zusammenhänge zulassen würden. Deutlich wird jedoch, dass innerhalb zahlreicher Lebensgeschichten selektiv mutistischer Kinder bedeutsame Lebensereignisse stehen, die das Kind als besonders stresshaft und überfordernd erlebt haben kann. Dieses Stresserleben und Überforderung in Verbindung mit einer bestehenden Vulnerabilität kann die Entwicklung eines SM begünstigen. 


\subsubsection{Zusammenfassung: Entwicklung des selektiven Mutismus bei einsprachigen Kinder}

Für die Entwicklung eines SM bei einsprachigen Kindern wird dem aktuellen Trend folgend ein Diathese-Stress-Modell als Erklärungsmodell herangezogen. Die Diathese—also die grundlegende Vulnerabilität des Kindes für die Ausprägung des Störungsbildes - wurzelt in einer genetischen Disposition für die Temperamentsmerkmale Schüchternheit, sozialer Rückzug und soziale Ängstlichkeit (siehe Kapitel 2.2.1). Durch die familiäre Lernumgebung kann sich das zurückhaltende und sozial ängstliche Verhalten des Kindes manifestieren. Bei einem großen Teil der Kinder mit SM erschweren sprachliche Unsicherheiten oder Spracherwerbsstörungen die soziale Interaktion des Kindes (siehe Kapitel 2.2.3). Eine Vulnerabilität ergibt sich damit in den Bereichen sozial-emotionale und sprachliche Fähigkeiten.

Als Stressoren können einschneidende und traumatische Lebensereignisse oder auch typische Übergänge vom Elternhaus in die Kita angesehen werden (siehe dazu Kapitel 2.2.4). Unfälle, eine plötzliche Trennung oder der Tod einer Bezugsperson stellen extreme Stressoren dar, welche die emotionalen Kompetenzen des Kindes enorm beanspruchen. Ein Rückzug und Schweigen als Reaktion auf diese Überforderung im emotionalen Bereich ist nachvollziehbar. Eine solch extreme Entwicklungsgeschichte findet sich jedoch in den seltensten Fällen bei Kindern mit SM. Viel häufiger sind es die kleinen aber regelmäßig vorkommenden Stressoren wie etwa die sprachliche Überforderung in sozialen Situationen oder der Übergang vom Elternhaus in die Kita (Katz-Bernstein, 2011) und die damit verbundene regelmäßige Trennung von der Bezugsperson. Ein schüchternes, leicht sozial ängstliches Kind mit einer Aussprachestörung kann im Kita-Kontext beispielsweise plötzlich vor große Herausforderungen gestellt werden, wenn Kinder und Erwachsenene das Kind nicht verstehen oder häufig missverstehen. Frustrations- und Misserfolgserleben kann bei einer solchen Prädisposition dazu führen, dass sich das Kind weiter zurückzieht und schließlich als Reaktion auf die Überforderung schweigt. Erwirbt das Kind keine weiteren Strategien im Umgang mit schwierigen Interaktionssituationen, kann sich das Schweigen manifestieren und ein SM entstehen. 


\subsection{Begriffsbestimmungen: Mehrsprachigkeit, Migrationshintergrund und Kultur}

Bevor auf den Zusammenhang von SM und Mehrsprachigkeit bzw. einem Migrationshintergrund eingegangen wird, werden diese beiden Begriffe sowie das Konstrukt Kultur zunächst näher bestimmt.

\subsubsection{Migration und Mehrsprachigkeit}

Ein beachtlicher Teil der Bevölkerung in Deutschland hat nach der Definition des Statistischen Bundesamtes einen Migrationshintergrund. In der Gesamtbevölkerung liegt dieser bei 20\%, unter den 0-15 Jährigen sogar bei 30 bis 35\% (Statistisches Bundesamt [Destatis], 2013). Damit sind ,,alle nach 1949 auf das heutige Gebiet der Bundesrepublik Deutschland Zugewanderten, sowie alle in Deutschland geborenen Ausländer und alle in Deutschland als Deutsch Geborenen mit zumindest einem zugewanderten oder als Ausländer in Deutschland geborenen Elternteil“ (Destatis, 2013, S. 6) gemeint. Eine ähnliche Definition wurde auch von der OECD für die PISA-Studie verwendet. Hier wurden alle Jugendlichen als Schüler mit Migrationshintergrund bezeichnet, die selbst oder deren Eltern im Ausland geboren wurden (OECD, 2013). Beide Definitionen zusammenbringend werden in der vorliegenden Arbeit solche Kinder als Kinder mit Migrationshintergrund bezeichnet, die entweder selbst im Ausland geboren und somit selbst immigriert sind oder die mindestens ein im Ausland geborenes Elternteil haben.

Auch wenn ein Migrationshintergrund häufig auch den Erwerb zweier oder mehrerer Sprache nach sich zieht, sind beide Phänomene deutlich voneinander zu unterscheiden. Nicht jedes Kind mit einem Migrationshintergrund wächst auch mehrsprachig auf - und umgekehrt hat nicht jedes mehrsprachige Kind einen Migrationshintergrund. So finden sich nicht wenige Familien, in denen ein Elternteil im Ausland geboren wurde und als Erstsprache eine nichtdeutsche erworben hat und dennoch nur Deutsch in der Familie gesprochen wird. Das Kind bzw. die Kinder hätten demnach einen Migrationshintergrund, wüchsen zunächst jedoch nur einsprachig auf. Der entgegengesetzte Fall-mehrsprachig aber ohne Migrationshintergrund - könnte etwa daraus resultieren, dass die Familie berufsbedingt eine Zeit lang im Ausland gelebt und das Kind bzw. die Kinder dort eine zweite nicht-deutsche Sprache erworben haben und die Familie wieder in ihr Herkunftsland zurückkehrt. Streng genommen hätte die Familie in diesem Land keinen Migrationshintergrund, wäre jedoch mehrsprachig.

Der Begriff Mehrsprachigkeit wird in der Fachliteratur sehr unterschiedlich definiert. Fraglich ist dabei, ab welchem Grad der Sprachkompetenz in zwei oder mehreren Sprachen 
eine Person als mehrsprachig gilt. Bialystok (2001) stellt in dieser Diskussion die beiden extremen Sichtweisen von Bloomfield (1933, zit. nach Bialystok, 2001) und Grosjean (1989) gegenüber. Bloomfield (1933, zit. nach Bialystok, 2001) definiert Mehrsprachigkeit über eine vollständige Sprachkompetenz des Sprechers in beiden Sprachen-im Sinne von zwei einsprachigen Personen in einer Person. Es wird davon ausgegangen, dass die beiden Sprachsysteme einer zweisprachigen Person voneinander unabhängig sind und beide Systeme jenem System eines monolingualen Sprechers gleichen. Diskutiert wird hierbei jedoch, was unter muttersprachlichen Kompetenzen zu verstehen ist, da unter monolingualen Sprechern ebenso eine große Varianz innerhalb der Sprachkompetenzen besteht (Tracy, 2014). Dementsprechend kritisierte Grosjean (1989) diese Sichtweise und betont die Interaktion beider oder ggf. mehrerer Sprachsysteme. Er stellt heraus, dass die einzelnen Sprachen unter Umständen in ganz unterschiedlichen Situationen genutzt werden und entsprechend unterschiedlich aufgebaut sein können. Das Level der Sprachkompetenz hängt von der Notwendigkeit der Sprache im Alltag der Person zusammen. Benötige ich die Sprache in sehr vielen Situationen in meinem Alltag, sind die Sprachkompetenzen in dieser wahrscheinlich eher hoch ausgeprägt. Eine Sprache, die man jedoch nur einmal die Woche für das Gespräch mit einer ganz bestimmten Person benötigt, ist wahrscheinlich deutlich schlechter ausgeprägt als erstere. Für Grosjean (1989) spielt in der Definition von Mehrsprachigkeit der kommunikative Aspekt eine viel größere Rolle als der Kompetenzaspekt. Sobald sich eine Person in mehreren Sprachen abhängig von den jeweiligen Anforderungen sprachlich-kommunikativ äußern kann (z.B. Alltagsgespräche führen) und mehr als eine Sprache regelmäßig nutzt, gilt sie als mehrsprachig. Eine solch breit gefasste Definition fokussiert eine Personengruppe mit recht unterschiedlichen sprachlichen Kompetenzniveaus. Dies bietet den Vorteil, dass auch Kinder im laufenden Zweitspracherwerb als mehrsprachig bezeichnet werden können. Dieser Aspekt ist für die vorliegende Studie von großer Bedeutung, da zum Teil Kinder im frühen Stadium des Zweitspracherwerbs mit eingeschlossen wurden. Wenn von mehrsprachigen Kindern gesprochen wird, sind in der vorliegenden Arbeit all jene Kinder gemeint, die mindestens zwei Sprachen erwerben und einen regelmäßigen Input in den entsprechenden Sprachen erhalten.

\subsubsection{Mehrsprachige Entwicklung}

Mehrsprachigkeit kann auf ganz unterschiedlichen Wegen entstehen: Kinder können zwei Sprachen von Geburt an erwerben, wenn beide Elternteile mit dem Kind eine andere Sprache sprechen. Dies wird als simultaner Erwerb zweier Sprachen oder auch doppelter Erstspracherwerb bezeichnet. Viele Kinder erwerben ihre Erstsprache (L1) im Elternhaus und 
beginnen erst mit dem Eintritt in die Kita die zweite Sprache (L2) zu lernen. Dies wird als kindlicher Zweitspracherwerb oder sukzessiver Erwerb zweier Sprachen bezeichnet (Baker, 2006; Chilla, Rothweiler \& Barbur, 2010; Thoma \& Tracy, 2006). Beide Formen des Erwerbs sind durch ein primär informelles Lernumfeld innerhalb alltäglicher Interaktionen gekennzeichnet. Lernen Kinder oder Erwachsene eine Sprache in der Schule oder anderen Bildungseinrichtungen durch direkte Instruktion spricht man von Fremdsprachlernen (Baker, 2006). Im Folgenden wird näher auf den simultanen und sukzessiven Erwerb zweier Sprachen eingegangen. Die durch Fremdsprachlernen entstehende Mehrsprachigkeit ist für die vorliegende Studie nicht von Bedeutung.

Von einem simultanen Erwerb zweier Sprachen spricht man dann, wenn der Erwerb der L2 bis zum Alter von zwei Jahren begonnen hat (Chilla et al., 2010). Dabei ist es nicht notwendig, dass die beiden Sprachen innerhalb der häuslichen Umgebung des Kindes von Geburt an präsent sind. Romaine (1995) schlägt eine Unterteilung des simultanen Erwerbs in vier Varianten vor: (1) One Person-One Language, (2) Familiensprache unterscheidet sich von Umgebungssprache, (3) gemischte Sprachen und (4) verzögerte Einführung der zweiten Sprache. Die wohl häufigsten Varianten sind die ersten beiden (Baker, 2006). Bei der One person-One language Konstellation spricht jeweils ein Elternteil eine Sprache mit dem Kind (Baker, 2006; Chilla et al., 2010). Es erhält damit einen ausreichenden Input in beiden Sprachen von Geburt an. Durch eine frühe Betreuung des Kindes im außerfamiliären Kontext ergibt sich Variante zwei-die Familiensprache unterscheidet sich von der Umgebungssprache. Das Kind erwirbt eine Sprache im häuslichen und eine weitere Sprache außerhalb von Zuhause, beispielsweise durch die Betreuung bei einer Tagesmutter oder in der Kita. Beginnt die Betreuung und damit der sprachliche Input in der L2 vor dem zweiten Lebensjahr, wird auch diese Form als simultaner Erwerb zweier Sprachen bezeichnet. Variante drei gemischte Sprachen ergibt sich häufig in Regionen, in denen zwei oder mehr Sprachen von fast allen Mitgliedern der Gesellschaft gesprochen werden. So beschreibt Baker (2006) etwa eine maltesische Familie, bei der beide Elternteile Maltesisch und Englisch mit dem Kind sprechen und ebenso Maltesisch und Englisch innerhalb der Gesellschaft gesprochen werden. Das Kind erhält einen gemischten Sprachinput sowohl innerhalb als auch außerhalb der Familie. Bei der letzten Variante verzögerte Einführung der zweiten Sprache sprechen Eltern zunächst nur eine (häufig Minderheiten-)Sprache mit dem Kind und führen die Umgebungssprache, obwohl beide diese beherrschen, erst später ein.

Der parallele Erwerb zweier Sprachen führt nicht automatisch zu einem auffälligen Erwerb in einer Sprache oder in beiden Sprachen. Kinder sind von Geburt an dazu in der La- 
ge, zwei Sprachen zu erwerben. Sie sind dazu fähig zwei oder mehr Sprachen voneinander zu differenzieren, zu speichern und produktiv zu nutzen (Serratrice, 2013). Eine metalinguistische Bewusstheit ist bei mehrsprachigen Kindern bereits früh beobachtbar. Das explizite Sprechen über Sprache zeigt sich etwa im Beispiel von Tracy (2014, S. 14): „Mutter zu ihrer Tochter; In the Kita they call it Frühstück, don't they? Hannah (2;4): Und du heißt das Breakfast.". Hannah ist sich bereits darüber bewusst, dass sie zwei Sprachen lernt und dass es für Dinge oder Situationen meist in jeder Sprache ein Wort gibt. Der Erwerb phonetischphonologischer und syntaktisch-morphologischer Fähigkeiten verläuft bei Kindern mit doppeltem Erstspracherwerb vergleichbar zu dem monolingualer Kinder. Ebenso erfolgt der Erwerb von Wörtern nach den gleichen Strategien wie bei monolingualen Kindern (Genesee \& Nicoladis, 2007). Der Wortschatzumfang in einer Sprache kann sich bei einem bilingualen Kind jedoch von dem eines monolingualen Kindes unterscheiden (Barac \& Bialystok, 2012; Bialystok, Luk, Peets \& Yang, 2010; Oller, Pearson \& Cobo-Lewis, 2007) und hängt vor allem vom Input in der jeweiligen Sprache ab (Bialystok et al., 2010; Duursma et al., 2007). Je mehr Input die Kinder in einer Sprache haben, desto größer ist in der Regel der Wortschatz in eben selber Sprache.

Die Sprachverwendung im häuslichen Kontext hat einen bedeutsamen Einfluss auf die Sprachkompetenzen der Kinder. Duursma et al. (2007) konnten für spanisch-englisch aufwachsende Kinder zeigen, dass eine Präferenz der Eltern zuhause Englisch zu sprechen einen positiven Effekt auf die Englischkompetenzen und einen negativen auf die Spanischkompetenzen hat. Eine Präferenz des Spanischen war entsprechend mit höheren Spanischkompetenzen assoziiert. Ebenso spielt der Kontext, in dem eine Sprache gelernt wird, eine bedeutsame Rolle im Wortschatzerwerb. Bialystok et al. (2010) konnten zeigen, dass sich die rezeptiven Wortschatzleistungen mehrsprachiger Kinder hinsichtlich von Wörtern aus dem Schulkontext-eben jener, in dem ein- und mehrsprachige Kinder gemeinsam lernen und damit den gleichen Input erhalten—nicht von denen einsprachiger Kinder unterscheiden. Die niedrigeren Wortverständnisleistungen der mehrsprachigen Kinder resultierten vor allem aus einem Wortschatzdefizit im Bereich häuslicher Begriffe_-jener Bereich, in dem die mehrsprachigen Kinder in den meisten Fällen die Nicht-Umgebungssprache erwerben.

Obwohl simultan zweisprachig aufwachsende Kinder beide Sprachen von Geburt an erwerben, entwickeln sich beide Sprachen nicht synchron. Häufig zeigt das Kind in einer Sprache vergleichsweise bessere Sprachkompetenzen. Diese Sprache wird als dominante Sprache bezeichnet (Paradis, Genesee \& Crago, 2010). Die Ausprägung der Sprachdominanz hängt wiederum von der Menge des Inputs ab (Gathercole \& Thomas, 2009). Pearson, 
Fernandez, Lewedeg und Oller (1997) konnten in ihrer Studie mit 8 bis 30 Monate alten Kinder zeigen, dass mindestens $25 \%$ des Inputs in einer Sprache benötigt wird, um einen entsprechenden Wortschatz aufbauen und sich aufbauend kommunikativ äußern zu können. Ein geringerer Input kann dazu führen, dass die Sprache gar nicht oder lediglich rezeptiv beherrscht wird.

Der sukzessive Erwerb zweier Sprachen ist dadurch gekennzeichnet, dass die Konfrontation mit der zweiten Sprache erst dann erfolgt, wenn die Erstsprache der Kinder bereits gut entwickelt ist (Paradis et al., 2010). Bereits erworbene Kompetenzen in der Erstsprache können den Erwerb der Zweitsprache deutlich beeinflussen. Zweitsprachlerner können auf bereits bestehende Konzepte und Strukturen der Erstsprache aufbauen. Vor allem Sprachlernstrategien und metalinguistische Fähigkeiten können den Zweitspracherwerb positiv beeinflussen. Tabors (2008) beschreibt vier Phasen im kindlichen Zweitspracherwerb. In der ersten Phase home language use nutzen Kinder ihre L1 im Kontext der L2-Sprecher. Diese Phase umfasst häufig nur wenige Tage. Die Kinder testen zunächst, ob sie mit ihren bisherigen Sprachkompetenzen zurechtkommen, bemerken jedoch schnell, dass die anderen Kinder und Erwachsenen die Sprache nicht verstehen. Die zweite Phase nonverbal period ist geprägt durch vorwiegendes Schweigen in der L2. Die Kinder erwerben in dieser Phase vor allem rezeptive Fähigkeiten und sprechen nur wenige bis keine Wörter in der neuen Sprache. Viele Kinder entwickeln während dieser Phase ein nonverbales Kommunikationssystem (z.B. durch die Nutzung von Gesten), um sich mitzuteilen. Die soziale Interaktion mit Peers ist ein bedeutsamer Faktor im Erwerb der L2, der einerseits ein gewisses Ausmaß des Inputs in der L2 gewährleistet und andererseits die Motivation des Kindes, in der L2 zu sprechen, steigern kann (Paradis et al., 2010). Diese nonverbale Phase kann einige Wochen bis Monate andauern und ist typischerweise länger für jüngere Kinder. In der dritten Phase formulaic language use beginnen die Kinder erste Wörter und kurze Äußerungen in der L2 zu produzieren. Diese sind meist imitativ und mit wenig Inhalt. Die Kinder begeben sich dadurch in einen verbalen sozialen Austausch und sichern den Input in der L2. Im Übergang zur vierten Phase beginnen die Kinder Sätze bereits flexibler zu konstruieren. Diese bestehen nun zunehmend aus vorformulierten Satzteilen, an welche das Kind flexibel weitere Satzteile anhängt (z.B. „Ich mache + Nomen“, oder „Ich möchte + Nomen“) (Paradis et al., 2010). Phase vier productive language use ist schließlich durch einen flexiblen Sprachgebrauch geprägt. Die Kinder nutzen ein breites Spektrum von Wörtern und Satzteilen und können sich zunehmend variantenreicher ausdrücken. Typischerweise erreichen L2-Lerner diese Phase innerhalb ihres ersten Jahres mit regelmäßigem Kontakt in der L2. 


\subsubsection{Kultur}

Je nach Fachdisziplin ergeben sich erhebliche Unterschiede hinsichtlich des Kulturbegriffes. Anthropologische Definitionen heben etwa die Inhalte, die eine Kultur ausmachen, hervor. Sie definieren Kultur anhand gemeinsamer symbolischer Verhaltensweisen, Bräuchen, Werten, sozialen Regeln oder auch Kunst und Artefakten. White (1947) betont in seiner Definition von Kultur beispielsweise die Sprache als bedeutsames Symbolsystem.

Culture is an organization of phenomena - acts (patterns of behavior), objects (tools; things made with tools), ideas (belief, knowledge), and sentiments (attitudes, „values“) - that is dependent upon the use of symbols. Culture began when man as an articulate, symbol-using primate, began. Because of its symbolic character, which has its most important expression in articulate speech, culture is easily and readily transmitted from one human organism to another. (White, 1947, S. 693)

Eine zentrale Funktion von Kultur ist die Abgrenzung einer Gruppe von einer anderen bzw. die Kohäsion der kulturellen Gruppe. Behavioristische Ansätze fokussieren entsprechend ihrer Disziplin gemeinsame Verhaltensweisen der Gruppenmitglieder, welche durch soziale Normen bestimmt werden. Barnlund und Araki (1985) postulieren, dass Kultur gar nicht existiere, solange sie sich nicht im Verhalten von Personen manifestiere. Marsella (2005) schlägt zwar eine breite, für die vorliegende Studie jedoch nützliche Definition von Kultur vor.

Culture is shared learned behavior and meanings that are socially transferred in various life-activity settings for purposes of individual and collective adjustment and adaptation. Cultures can be (1) transitory (i.e. situational even for a few minutes), (2) enduring (e.g., ethnocultural life styles), and in all instances are (3) dynamic (i.e., constantly subject of change and modification). Cultures are represented (4) internally (i.e., values, beliefs, attitudes, axioms, orientations, epistemologies, consciousness levels, perceptions, expectations, personhood), and (5) externally (i.e., artifacts, roles, institutions, social structures). Cultures (6) shape and construct our realities (i.e., they contribute to our world views, perceptions, orientations) and with this ideas, morals and preferences. (Marsella, 2005, S. 657) 
Marsella (2005) betont in dieser Definition zunächst, dass Kultur in verschiedensten sozialen Settings gelerntes Verhalten und Wissen ist. Beides wird für eine individuelle und kollektive Anpassung benötigt. Dieser Aspekt erscheint vor allem vor dem Hintergrund der Entwicklung von Kindern mit Migrationshintergrund, welche in zwei oder unter Umstände mehreren Kulturen aufwachsen, bedeutsam. Kinder erlernen gesellschaftlich akzeptables Verhalten und kulturell bedeutsames Wissen in ihrem Alltag in Familie, Schule und Kita. Gehören diese Kontexte zu unterschiedlichen Kulturen, bedeutet dies für das Kind mit Migrationshintergrund eine doppelte Herausforderung: es muss einerseits verschiedene Kulturen erlernen und andererseits kulturangemessen im jeweiligen Kontext handeln, um in dieser kulturellen Gruppe akzeptiert zu werden und partizipieren zu können. Der Prozess der Anpassung an verschiedene Kulturen wird kulturelle Adaptation genannt und in Kapitel 2.4.2 näher betrachtet. Ein weiterer bedeutsamer Aspekt der Definition von Kultur für die vorliegende Studie ist die Differenzierung zwischen inneren und äußeren Anteilen von Kultur. Die inneren Aspekte umfassen vor allem Werte und Einstellungen, die wiederum Wahrnehmung und Erwartungen innerhalb sozialer Interaktionen beeinflussen. Je nachdem wie unterschiedlich die Kulturen sind, in denen ein Kind mit Migrationshintergrund aufwächst, können in der Familie erlernte Verhaltensweisen der Heimatkultur im Kontext der Kita als unpassend, nicht altersangemessen oder sogar als Anzeichen für eine Entwicklungsauffälligkeit wahrgenommen werden. Kultur beeinflusst ganz entscheidend unsere Realität, in dem sie sowohl die äußeren Strukturen in unserer Gesellschaft mitbestimmt als auch auf unsere Wahrnehmung und Einstellung wirkt. Je unterschiedlicher Heimat- und Umgebungskultur für Familien mit Migrationshintergrund sind, desto fremdartiger können etwa Institutionen und sozialen Strukturen der Umgebungskultur erscheinen und Ängste hervorrufen. Verschiedene Wahrnehmungen der Angemessenheit von Verhaltensweisen können zu Irritationen in der sozialen Interaktion führen. Ängste, Irritationen und Verunsicherungen in der Interaktion stellen bedeutsame Risikofaktoren für die Entwicklung eines SM dar, so dass bereits jetzt die Bedeutsamkeit kultureller Aspekte in der Betrachtung des SM bei Kindern mit Migrationshintergrund deutlich wird.

\subsection{Selektiver Mutismus bei mehrsprachigen Kindern}

Bislang liegen nur wenige Studien zum Zusammenhang von Mehrsprachigkeit oder einem Migrationshintergrund und SM (Bradley \& Sloman, 1975; Cline \& Kysel, 1987; Elizur \& Perednik, 2003; Zelenko \& Shaw, 2000) sowie einige Falldarstellungen mehrsprachiger Kinder bzw. Kindern mit Migrationshintergrund vor (Atlas, 1993; Bauermeister \& Jemail, 
1975; Clemente, Brafman \& Cohen, 1986; Holmbeck \& Lavigne, 1992; Mayworm et al., 2014; Meijer, 1979; Meyers, 1984; Rosenbaum \& Kellman, 1973; Shaw, 1971; Wernitznig, 1993; Youngerman, 1979; Zelenko \& Shaw, 2000). In letzteren liegt der Fokus auf therapeutischen Aspekten und weniger auf der Erklärung des SM bei mehrsprachigen Kindern. Zahlreiche Studien schließen mehrsprachige Kinder aus ihren Analysen aus (siehe etwa Dummit et al., 1997; Kristensen, 2000; Manassis et al., 2007), da differentialdiagnostische Unsicherheiten eine klare SM-Diagnose erschweren. Nach den DSM-V Kriterien wird ein Wohlfühlen sowie angemessene Sprachkompetenzen in der Umgebungssprache erwartet, um die Diagnose SM zu stellen (American Psychiatric Association, 2013). Bei mehrsprachigen Kindern ist dieses Diagnosekriterium sehr bedeutsam, um ein Schweigen als Symptom des SM von einem entwicklungstypischen Schweigen im Rahmen des Zweitspracherwerbs zu unterscheiden. $\mathrm{Zu}$ Beginn des Erwerbs einer neuen Sprache können schweigende Phasen von bis zu sechs Monaten auftreten (Toppelberg, Tabors, Coggins, Lum \& Burger, 2005; siehe auch Kapitel 2.3.2). Vor allem bei schüchternen Kindern sollte eine solch schweigende Phase im Zweitspracherwerb sichtbar werden. Ihre generelle Zurückhaltung in unvertrauten Kontext kann dazu führen, dass sie sich in den ersten Monaten innerhalb der Kita eher zurückhalten, sich in die neue Sprache „einhören“ und deutlich später mit dem Sprechen der Umgebungssprache anfangen als nicht schüchterne Kinder. Ihr Verhalten ist jedoch von außen betrachtet zunächst vergleichbar mit dem von selektiv mutistischen Kindern. Vor allem in den ersten sechs Monaten innerhalb des neuen Sprachkontextes ist eine Sprachdiagnostik daher hochbedeutsam. Da Kinder mit SM typischerweise mit fremden Personen im unvertrauten Kontext schweigen, gestaltet sich die Überprüfung der sprachlichen Fähigkeiten—vor allem der expressivenmeist schwierig (Cleator \& Hand, 2001; Klein, Armstrong \& Shipon-Blum, 2013; McInnes et al., 2004). Eine Diagnosestellung bei mehrsprachigen Kindern ist demnach vor allem im beginnenden Zweitspracherwerb deutlich erschwert.

Die Studien, die mehrsprachige Kinder nicht explizit ausgeschlossen haben, berichten einheitlich von einem hohen Anteil mehrsprachiger Kinder oder von Kindern mit Migrationshintergrund (siehe etwa Bradley \& Sloman, 1975; Steinhausen \& Juzi, 1996 Dummit et al., 1997; Starke \& Subellok, 2012). Die Autoren vermuten einheitlich, dass Kinder mit Migrationshintergrund eine erhöhte Vulnerabilität für die Entwicklung eines SM haben (ElizaldeUtnick, 2007). Bislang ist jedoch nur wenig darüber bekannt, welche Faktoren bei mehrsprachigen Kindern bzw. Kindern mit Migrationshintergrund zu dieser erhöhten Vulnerabilität führen. 
Elizur und Perednik (2003) untersuchten als erste Forschungsgruppe systematisch die Prävalenz des SM bei mehrsprachigen Kindergartenkindern sowie mögliche Ursachenfaktoren. In ihrer repräsentativen Studie in West-Jerusalem fanden sie eine vierfach erhöhte Prävalenzrate von $2.2 \%$ bei mehrsprachigen Kindern im Vergleich zu $0.47 \%$ bei Einsprachigen. Ebenso unterschieden sich beide Gruppen signifikant hinsichtlich des Onsets des SM, wobei die Mehrsprachigen fast ein Jahr später anfingen zu schweigen.

Gerade der Umstand, in zwei oder mehr Kulturen zu leben und zwei oder mehrere Sprachen zu lernen, wird als ein bedeutsamer Einflussfaktor bei der Entwicklung eines SM bei mehrsprachigen Kindern angesehen (Bradley \& Sloman, 1975; Steinhausen \& Juzi, 1996). Nach Bradley und Sloman (1975) erleben Familien und Kinder bei der Migration in ein neues Land einen „Kulturschock“. In der neuen Kultur fehlen die gewohnten Hinweise, die eine Orientierung für das eigene Verhalten bieten (siehe dazu auch Kapitel 2.3.3 und 2.4.1). Dies kann massiven Stress auslösen und in der Folge zu Ängstlichkeit und Depression führen. Zelenko und Shaw (2000) berichten in ihrem Fallbeispiel von einer mexikanischen Familie, die illegal in die USA eingewandert ist. Neben einer eher symbiotischen Beziehung zwischen Kind und Eltern wurde die dramatische Migrationserfahrung der Familie als bedeutsamer Ursachenfaktoren für die Entwicklung des SM herangezogen. Die anschließende Unsicherheit der gesamten Lebenssituation der Familie und die geringen Möglichkeiten sich angemessen an die neue Kultur anzupassen haben, so vermuten Zelenko und Shaw (2000), zur Isolation der Familie und zu Ängsten der Eltern geführt—beides Risikofaktoren für die Entwicklung eines SM beim Kind (siehe Kapitel 2.2).

Elizur und Perednik (2003) ziehen zur Erklärung des SM bei Kindern mit Migrationshintergrund das Diathese-Stress-Modell heran. Sie gehen von einer Interaktion zwischen einer grundsätzlichen Vulnerabilität für eine Verunsicherung in der Schule, ausgelöst durch die Migration der Familie, und einer Disposition zu sozialer Ängstlichkeit aus. Diese kann zu sprachlichen Problemen oder Sprechangst führen und in der Folge einen SM auslösen. Dem folgend verglichen sie ein- und mehrsprachige Kinder hinsichtlich internalisierender und externalisierender Verhaltensweisen, sozialer Kompetenzen, sozialer Ängstlichkeit und Entwicklungsverzögerungen. Die beiden Gruppen unterschieden sich signifikant hinsichtlich des Ausmaßes sozialer Ängstlichkeit, sozialer Kompetenzen und dem Vorliegen von Entwicklungsverzögerungen. Mehrsprachige Kinder zeigten ein erhöhtes Maß sozialer Ängstlichkeit bei jedoch insgesamt adäquaten sozialen Kompetenzen. Einsprachige Kinder wiesen hingegen deutlich häufiger Entwicklungsverzögerungen sowie unangemessene soziale Kompetenzen auf. Die Autoren führen dies darauf zurück, dass bei einsprachigen Kindern häufiger eine an- 
geborene Vulnerabilität in Verbindung mit der Entwicklung eines SM steht. Bei Mehrsprachigen spielen vor allem Umgebungsfaktoren wie kulturelle Unterschiede zwischen familiärem und Schulkontext eine Rolle, die eine soziale Ängstlichkeit auslösen und in der Folge zu einem Schweigen in bestimmten sozialen Situationen führen kann. Unklar bleibt jedoch, welche spezifischen Umgebungsfaktoren die Entwicklung eines SM begünstigen und welche sogar als Schutzfaktoren dienen können.

\subsubsection{Kulturelle Einflüsse auf die Entwicklung sozio-emotionaler und sprachlicher Fä- higkeiten}

Für die Entwicklung des SM sind vor allem sozial-emotionale sowie sprachlichkommunikative Aspekte bedeutsam. Sowohl die Entwicklung sozial-emotionaler als auch sprachlicher Fähigkeiten unterliegen kulturellen Einflüssen. Kulturelle Variationen zeigen sich in Einstellungen zur Kindererziehung sowie im entsprechenden Verhalten. Eltern bemühen sich, solche Verhaltensweisen und Merkmale beim Kind zu fördern, die einerseits Kompetenzentwicklung und andererseits eine erfolgreiche Eingliederung in die Kultur der Familie ermöglichen (Deater-Deckard et al., 2011). Demnach kann das elterliche Erziehungsverhalten in Familien mit Migrationshintergrund stark von der kulturellen Adaptation der Eltern beeinflusst sein (siehe dazu auch Calzada, Brotman, Huang, Bat-Chava \& Kingston, 2009).

Als Beispiele für sozial-emotionale Aspekte sind Einstellungen zum Gefühlsausdruck sowie zur Individualität und Selbstständigkeit von Kindern und Jugendlichen zu nennen. Viele europäische und nordamerikanische Kulturen können als individualistisch geprägt beschrieben werden. Unabhängigkeit, soziales Durchsetzungsvermögen, Neugierde und eine gewisse Risikobereitschaft werden in diesen Gesellschaften als eher positiv bewertet. Entsprechende Verhaltensweisen werden von Eltern dieser Gesellschaften bei ihren Kindern eher verstärkt (Majoribanks, 1994). Vorsichtiges, zurückhaltendes Verhalten wird folglich als sozial unreifer, inkompetenter und psychologisch maladaptiver angesehen (Rubin, Coplan, Bowker \& Menzer, 2011). Schüchternes, vorsichtiges und zurückhaltendes Verhalten steht in solchen Gesellschaften zudem häufig in Zusammenhang mit Zurückweisungen durch Peers (Gazelle \& Ladd, 2003) oder der Entwicklung einer negativen Eigenwahrnehmung sozialer Fähigkeiten sowie internalisierenden Auffälligkeiten (Chen, He et al., 2004; Prior, Smart, Sanson \& Oberklaid, 2000). In asiatischen Kulturen hingegen werden externalisierende Verhaltensweisen sanktioniert und Wert auf eine allgemeine Zurückhaltung, Selbstbeherrschung und Kooperationsbereitschaft gelegt (Essau, 2003). Die Aufrechterhaltung der sozialen Ordnung und der zwischenmenschlichen Harmonie steht über den individuellen Bedürfnissen. 
Kinder lernen früh, ihre Bedürfnisse und Emotionen zu unterdrücken, um soziale Zurückweisung zu vermeiden (Rubin et al., 2006). Schüchterne, zurückhaltende Kinder asiatischer Kulturen nehmen sich - anders als schüchterne Kinder westlicher Kulturen - als sozial kompetent wahr (Chen, Zappulla et al., 2004) und werden von ihren Peers akzeptiert (Chen \& Tse, 2008).

Kultur hat demnach einerseits Einfluss auf die Verstärkung bestimmter sozialer Verhaltensweisen bei Kindern und andererseits auf die Wahrnehmung dieser. Je nach Kombinationen zweier Kulturen, in denen Kinder mit Migrationshintergrund aufwachsen, kann dies zu deutlichen Unterschieden in der Bewertung bestimmter Verhaltensweisen des Kindes führen. Ein Kind asiatischer Eltern könnte etwa innerhalb seiner Familie bestärkt werden, sich zurückhaltend zu verhalten. In einem westlich orientierten Kontext würde dieses Verhalten auBerhalb der Familie jedoch eher als besorgniserregend eingestuft. Im Kindergartenkontext würde das Kind womöglich auf Schwierigkeiten stoßen, Freundschaften mit anderen Kindern zu schließen.

Hinsichtlich der sprachlich-kommunikativen Entwicklung sind bei Kindern mit Migrationshintergrund mindestens zwei Aspekte zu beachten: einerseits sind kommunikative Handlungen ebenso wie die bereits oben thematisierten allgemeinen Verhaltensweisen kulturell geprägt, andererseits erwerben Kinder mit Migrationshintergrund häufig mehrere Sprache, was vor allem für schüchterne Kinder ein mögliches Hindernis darstellen kann.

Kulturelle Variationen können sich in unterschiedlichen Konventionen für Interaktionen (z.B. Begrüßungs- und Abschiedsrituale) zeigen. Ebenso werden Werte, Normen und Einstellungen einer Kultur über Sprache vermittelt und drücken sich in Sprache aus (Schieffelin \& Eisenberg, 1984). So zeigt sich die Einstellung zum Kind als Interaktionspartner für Erwachsene in kulturell unterschiedlichen Eltern-Kind-Interaktionen (Bavin, 1995; Hoff, 2006; Nicoladis \& Genesee, 1997). Nordamerikanische oder auch europäische Mütter beginnen bereits früh, mit ihren Säuglingen zu sprechen und aus kindlichen Geräuschen erste Konversationen zu konstruieren (Snow, 1977). Kinder aus sesothischen Sprachfamiliengesprochen in Lesotho und angrenzenden Teilen Südafrikas-werden extrem früh über Frageund Aufforderungsroutinen in die Konversationen der Familie einbezogen, selbst wenn sie selbst noch nicht sprechen können (Demuth, 1992). Im Gegensatz dazu werden Vokalisationen und erste Protowörter von Kindern in Walpiri-einer Sprache der Aborigines - nicht als Sprache interpretiert. Dementsprechend erfolgen keine Wiederholungen, Interpretationen oder Expansionen des Gesagten durch Bezugsperson oder ältere Geschwister (Bavin, 1991). Auch in Mayafamilien werden Kleinkinder nicht als potenzielle Konversationspartner angesehen 
und somit deutlich seltener angesprochen als gleichaltrige nordamerikanische Kinder. Sie erwerben Sprache zunächst vor allem durch das Anhören von Gesprächen anderer Personen (Shneidman \& Goldin-Meadow, 2012). Bis zum Alter von drei Jahren erhöht sich der Anteil der direkt an das Kind gerichteten Sprache deutlich und ist im Alter von 35 Monaten vergleichbar zu dem von US-amerikanischen Kindern. Unterschiede zeigen sich jedoch in den Kommunikationspartnern der Kinder. Die US-amerikanischen Kinder erhielten den direkt an sie gerichteten sprachlichen Input zum größten Teil von Erwachsenen. Die direkten Anteile der Mayakinder kamen zu 85 \% von anderen Kindern unter elf Jahren (Shneidman \& GoldinMeadow, 2012).

Wie bereits oben für einsprachige Kinder beschrieben, ist Schüchternheit mit sprachlichen Unsicherheiten assoziiert (Crozier \& Badawood, 2009; Crozier \& Perkins, 2002) und stellt damit einen Risikofaktor für Sprachentwicklungsverzögerungen dar (Keller et al., 2013). Der Zweitspracherwerb eines Kindes mit Migrationshintergrund-hier vor allem der sukzessive-findet in den meisten Fällen nicht im vertrauten familiären Kontext statt, sondern beispielsweise im Kontext der Kita im Kontakt mit Gleichaltrigen und Erwachsenen (Hoff, 2006). Schüchterne Kinder tendieren jedoch dazu, vor allem in diesen Kontexten nur wenig zu sprechen (Evans, 1996). Wie bereits in Kapitel 2.1 beschrieben neigen sie zudem dazu, nicht selbstständig eine Interaktion mit Peers aufzubauen (Asendorpf \& Meier, 1993). Für den hier fokussierten Zweitspracherwerb mit primärem Input in der Kita spielen PeerInteraktionen jedoch eine bedeutsame Rolle. Dyadische Erwachsenen-Kind-Interaktionen ergeben sich im Kita-Alltag nur selten. Layzer, Goodson und Moss (1993) dokumentierten in ihrer Beobachtungsstudie in 119 Kitas, dass zwar insgesamt 70 \% der Zeit im Alltag der Erzieherinnen $^{3}$ aktiv mit den Kindern der Gruppe agiert wurde. Jedoch wird nur etwa $10 \%$ der Zeit mit einzelnen Kindern verbracht. Die meiste Zeit wird dazu benötigt, die Gruppe zu organisieren, anzuleiten und Wissen zu vermitteln. Nur ein geringer Anteil der ErzieherinnenKind-Interaktionen werden zur Erweiterung der kindlichen Äußerungen oder zum Aufbau eines Gespräches genutzt (Albers, 2009). Für den Erstspracherwerb gibt es Hinweise darauf, dass die Menge der direkt an das Kind gerichteten Sprache durch eine pädagogische Fachkraft ein positiver Prädiktor für den Spracherwerb von Kindern im Alter von eineinviertel bis drei Jahren ist (National Institute of Child Health and Human Development Early Child Care Research Network, 2000). Eine deutlich höhere Bedeutsamkeit kommt der Erzieherinnen-KindInteraktion im Kontext des Zweitspracherwerbs zu, wenn die Zweitsprache nicht durch einen

\footnotetext{
${ }^{3}$ Zur besseren Lesbarkeit wird aufgrund der überwiegend weiblichen pädagogischen Fachkräfte in Kitas im Folgenden die weibliche Form Erzieherinnen verwendet.
} 
qualitativ hochwertigen familiären Input unterstützt wird. Kein bzw. ein reduzierter Input durch einen kompetenten Sprecher kann zu einem verlangsamten Spracherwerb führen (National Institute of Child Health and Human Development Early Child Care Research Network, 2002). Auch wenn Peer-Interaktionen ein reduziertes Ausmaß von Interaktionen mit kompetenten, erwachsenen Sprechern nicht ersetzen können, können sie dennoch eine bedeutsame Ressource für den Zweitspracherwerb darstellen. In den so genannten Freispielphasen in der Kita finden potenziell die intensivsten Kontakte zwischen den Kindern statt (Booren, Downer \& Vitiello, 2012). In diesen spielerischen Interaktionen ergeben sich zahlreiche Möglichkeiten, die eigenen sprachlich-kommunikativen und sozialen Fähigkeiten einzusetzen und weiterzuentwickeln (Pellegrini, Galda, Flor, Bartini \& Charak, 1997).

Zurückhaltendes, schüchternes Verhalten stellt womöglich ein noch größeres Risiko für den Zweit- als für den Erstspracherwerb dar. Tong, Ting und McBride-Chang (2011) konnten zeigen, dass die sprachlichen Kompetenzen von sukzessiv zweisprachig aufwachsenden Kindergartenkindern sowohl für die L1 als auch die L2 negativ mit dem Ausmaß ihrer Schüchternheit zusammenhängen. In der Studie von Strand, Pula, Parks und Cerna (2011) konnten keine zeitparallelen Zusammenhänge zwischen der Schüchternheit der Kinder und den Sprachverständnisleistungen in der Umgebungssprache gefunden werden. Jedoch konnte das Ausmaß der Schüchternheit im Alter von vier Jahren den rezeptiven Wortschatz sechs Monate später vorhersagen. Auch in der Studie von Keller et al. (2013) zeigten die schüchternen Kinder mit Migrationshintergrund schwächere rezeptive wie auch produktive Sprachkompetenzen im Vergleich zu ihren nicht-schüchternen Peers. Zusätzlich konnten sie zeigen, dass die schüchternen Kinder deutlich geringere Fortschritte in ihrem Zweitspracherwerb innerhalb von 16 Monaten vollzogen. Damit stellt Schüchternheit ein nicht zu vernachlässigendes Risiko für den Zweitspracherwerb dar. Gerade schüchterne, sensible Kinder können auf diese sprachlichen Unsicherheiten mit Rückzugsverhalten reagieren. Meijer (1979) berichtet beispielsweise von einem achtjährigen, einsprachigen Mädchen mit Ausspracheproblemen, welches anfing zu schweigen, nachdem Kinder sich über sie lustig gemacht hatten. Eine gewisse Besorgnis über negative Konsequenzen des Sprechens außerhalb des vertrauten Kontextes ist häufig mit SM assoziiert (Kearney, 2010). Die Angst, etwas Falsches zu sagen oder sich nicht gut genug ausdrücken zu können, kann zu vermehrten Schweigen in unvertrauten Kontexten führen und sich infolgedessen zu einem SM manifestieren. 


\subsubsection{Akkulturation}

Der Begriff der Akkulturation beschreibt-je nach Perspektive-kulturelle Anpassungsprozesse auf Individual-, Gruppen- und Strukturebene (Zick, 2010). In der psychologisch orientierten Akkulturationsforschung geht es vor allem um die Anpassung des Individuums an eine neue Kultur-typischerweise nach der Immigration in ein neues Land. Dabei stehen vor allem Verhaltensänderungen, Stresserleben und gesundheitliche Risiken im Fokus (Berry, 2003; Zick, 2010). Auf der Gruppenebene werden Veränderungsprozesse innerhalb größerer kultureller Gruppen betrachtet. Diese können einerseits spezifisch aus Mitgliedern einer Ethnie bestehen oder eher universal (Zuwanderer, Neuankömmling o.ä.) bestimmt sein (Zick, 2010). Auf Strukturebene werden darüber liegende Phänomene des kulturellen Wandels von Systemen betrachtet. Auch wenn für das tiefgehende Verständnis individueller Verhaltensweisen einer Person mit Migrationserfahrung auch die Betrachtung der Akkulturation auf Gruppen- und Strukturebene bedeutsam sein kann (Berry, 2003) — etwa die grundlegende Beziehung zwischen Heimat- und Umgebungskultur oder der aus dem Kontakt beider resultierende kulturelle Wandel in beiden Systemen - wird im Folgenden ein Fokus auf die individuellen Prozesse gelegt. Für die Entwicklung des SM bei einem Kind kann der kulturelle Adaptationsprozess der Eltern eine bedeutsame Rolle spielen. Je nach Kombination und den damit einhergehenden Unterschieden von Heimat- und Umgebungskultur kann die Akkulturation der Eltern einen maßgeblichen Einfluss auf die Erziehung (Ispa et al., 2004) und damit auch auf die Entwicklung der Kinder nehmen (García Coll, Akerman \& Cicchetti, 2000).

Anfänglich wurde Akkulturation als Prozess der Assimilation eines Individuums an die Kultur des Zuwanderungslandes angesehen (Zick, 2010). Akkulturation wäre demnach ein eindimensionales Konstrukt, bei dem sich eine Person im Verlauf der Zeit von einem Pol (Lebensweise ihrer Heimatkultur) zu einem anderen (Lebensweise der Umgebungskultur/ Mehrheitskultur) bewegt (Berry, 2003). Neuere Theorien gehen jedoch davon aus, dass Akkulturation aus mindestens zwei Prozessen besteht: (1) der Auseinandersetzung mit der Frage, in welchem Ausmaß die eigene Heimatkultur beibehalten werden soll und (2) der Anpassung an die Umgebungskultur (Berry, 1997). Calzada et al. (2009) verwenden den Begriff der kulturellen Adaptation, welcher die beiden Konstrukte der Akkulturation (als Anpassung an die Mehrheitskultur) und der Enkulturation (als Beibehaltung der Heimatkultur) umfasst. Je nach Ausmaß der Akkulturation und Enkulturation werden grob vier Adaptationsstrategien unterschieden (Berry, 2003; siehe Abbildung 2): (1) Assimilation entsteht durch Unterdrückung der Heimatkultur (niedrige Enkulturation) und Eingliederung in die Umgebungskultur (hohe Akkulturation), (2) Separation durch die Zurückweisung der Umgebungskultur (niedrige Akkul- 
turation) bei gleichzeitiger Beibehaltung der Heimatkultur, (3) Marginalisierung durch eine Zurückweisung von Heimat- und Umgebungskultur (niedrige En- und Akkulturation) und (4) Integration bzw. Bikulturalität durch ein simultanes Beibehalten von Heimat- und Eingliedern in die Umgebungskultur (hohe En- und Akkulturation). Bikulturalität spiegelt ein hohes Maß kultureller Adaptation wider und wird als möglicher Schutzfaktor innerhalb der kindlichen Entwicklung angesehen, der soziodemografische Risiken ausgleichen kann (Calzada et al., 2009).

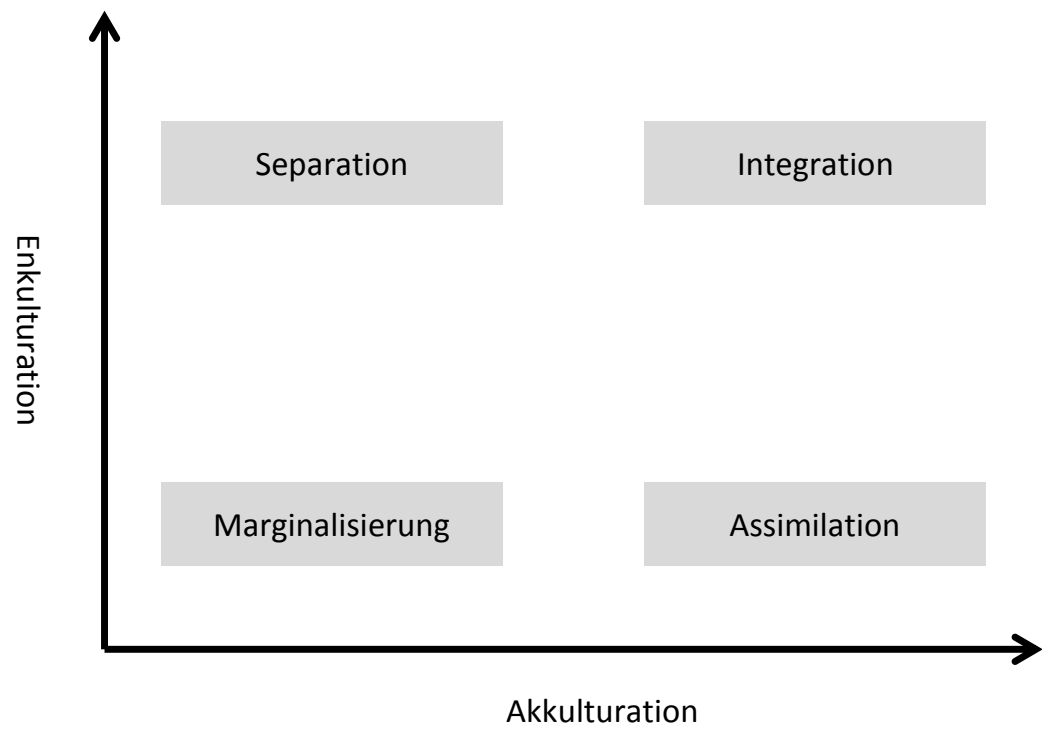

Abbildung 2. Vier Strategien der kulturellen Adaptation in Anlehnung an Berry (2003)

\subsubsection{Einfluss der kulturellen Adaptation der Eltern auf die kindliche Entwicklung}

Die kulturelle Adaptation der Eltern nimmt vor allem über das Erziehungsverhalten Einfluss auf die kindliche Entwicklung. Sind die Eltern eher an der Heimatkultur orientiert, werden sie bspw. Verhaltensweisen beim Kind fördern, die eine Eingliederung dieser in die Heimatgesellschaft ermöglicht, sowie Werte und Einstellungen der Heimatkultur dem Kind vermitteln. $\mathrm{Xu}$ und Krieg (2014) konnten zeigen, dass die Orientierung von asiatischamerikanischen Eltern an ihrer Heimatkultur positiv mit der Schüchternheit des Kindes zusammenhängt (siehe dazu auch Kapitel 2.4.1). Sind die Eltern eher an der Umgebungskultur orientiert, wird das Kind sowohl im häuslichen als auch familiären Kontext ähnliche Rückmeldungen auf sein Verhalten erhalten. Die Eltern werden solche Verhaltensweisen beim Kind verstärken, die in der Umgebungskultur als angemessen gelten. Das Kind wird mit hoher Wahrscheinlichkeit auch innerhalb der Familie in Anteilen die Sprache der Umgebungskultur erwerben und die Eltern als insgesamt positiv gegenüber der Umgebungskultur eingestellt 
erleben. Das Ausmaß der kulturellen Adaptation der Eltern beeinflusst demnach die Diskrepanz, die das Kind hinsichtlich der Rückmeldungen auf sein Verhalten oder auch der Vermittlung von Werten in der Familie und in den Bildungsinstitutionen erlebt.

Pawliuk et al. (1996) zeigten in ihrer Studie, dass Kinder von Eltern mit einem assimilierten Adaptationsstil über ein höheres Maß sozialer Kompetenzen verfügen als Kinder von Eltern aller anderen Adaptationsstile. Assimilation spiegelt die nahezu vollständige Eingliederung in die Umgebungskultur wider. Eltern, die sich stark an der Umgebungskultur orientieren, bieten den Kindern ein Lernmodell für soziales Verhalten der Umgebungskultur. Ergänzend dazu werden Eltern auch solches Sozialverhalten bei ihren Kinder belohnen, welches von der Mehrheitskultur als angemessen angesehen wird, und eher solches bestrafen, welches als nicht adäquat gilt. Elterliches Verhalten, welches eine Akzeptanz der Umgebungskultur widerspiegelt, kann somit einen positiven Einfluss auf soziale Kompetenzen und die psychische Gesundheit der Kinder haben. Die Kinder in der Studie von Pawliuk et al. (1996) zeigten entsprechend weniger internalisierende Probleme als Kinder von Eltern mit klar abweisendem Verhalten gegenüber der Umgebungskultur. Calzada et al. (2009) konnten für Kinder bikultureller Eltern signifikant weniger internalisierende Auffälligkeiten nachweisen als für Kinder nicht-bikultureller Eltern. Ein Unterschied zwischen den Gruppen ergab sich jedoch nicht für externalisierende Auffälligkeiten.

Ähnliches zeigt sich auch für die Sprachkompetenzen von Kindern mit Migrationshintergrund. Oades-Sese und Li (2011) fanden in ihrer Studie mit Familien lateinamerikanischer Herkunft in den USA, dass Kinder von Eltern mit einem niedrigen Akkulturationslevel (Separation oder Marginalisierung) oder einem bikulturellen Adaptationsstil geringere Sprachkompetenzen im Englischen aufwiesen als Kinder von Eltern mit einem hohen Akkulturationslevel (Assimilation). Auch hier spielt vermutlich der Input, den die Kinder erhalten, die entscheidende Rolle (siehe dazu auch Kapitel 2.3.2). Je mehr Input die Kinder von ihren Eltern in der entsprechenden Sprache (Herkunfts- oder Umgebungssprache) bekommen, desto besser sind die jeweiligen Sprachkompetenzen der Kinder (Prevoo et al., 2014). Eltern mit niedrigem Akkulturationslevel werden mit ihren Kindern zuhause eher ihre Herkunftssprache sprechen, so dass die Kinder vor allem außerhalb des Elternhauses die Umgebungssprache erwerben. Auf mögliche Schwierigkeiten im Erwerb der Zweitsprache in der Kita-vor allem für schüchterne Kinder-wurde bereits in Kapitel 2.4.1 eingegangen. Auch bikulturelle Eltern mit guten Kompetenzen in der Umgebungssprache werden mit ihren Kindern auch zu einem groBen Teil die Herkunftssprache sprechen, so dass auch hier von einem primären Input in der Umgebungssprache außerhalb der Familie auszugehen ist. Assimilierte Eltern hingegen spre- 
chen mit hoher Wahrscheinlichkeit auch im häuslichen Bereich die Umgebungssprache, so dass das Kind einen deutlich größeren sprachlichen Input in der Umgebungssprache erhält und ggf. sogar komplett einsprachig aufwächst.

Schlussfolgernd stellt der Stil der kulturellen Adaptation der Eltern einen potenziellen Faktor dar, der in der Entwicklung des SM bei mehrsprachigen Kindern eine bedeutsame Rolle spielen kann. Dies bezieht sich sowohl auf die Bedeutung von Sprachkompetenzen als auch auf die Tendenz zu internalisierendem Verhalten. Durch die vorangegangene Darstellung wird deutlich, dass ein hohes Maß der Anpassung an die Umgebungskultur als möglicher Schutzfaktor vor der Entwicklung eines SM wirken kann. Hingegen könnte eine geringe Anpassung an die Umgebungskultur und starke Orientierung an der Herkunftskultur einen Risikofaktor für SM darstellen.

\subsubsection{Entwicklung des SM bei mehrsprachigen Kindern}

Als Konsequenz der vorangegangenen und umfassenden Ausführungen wird ein Erklärungsmodell zur Entstehung des SM bei mehrsprachigen Kindern im Sinne eines DiatheseStress-Modells entwickelt (siehe Abbildung 2).

Basis der Diathese bildet eine genetische Prädisposition für (soziale) Ängstlichkeit. Wie in Kapitel 2.1 beschrieben, sind Schüchternheit und sozialer Rückzug häufig mit SM assoziierte Temperamentsmerkmale (Black \& Uhde, 1995; Dummit et al., 1997; Ford et al., 1998), welche wiederum ein Risiko für die Entwicklung von sozialer Ängstlichkeit darstellen (Coplan et al., 2008; Rubin et al., 2009). Diese genetische Prädisposition wird durch die familiäre Lernumgebung zusätzlich verstärkt (Melfsen \& Warnke, 2007). Kinder erwerben anfangs soziales und sprachlich-kommunikatives Verhalten zu großen Teilen durch Modelllernen anhand ihrer Bezugspersonen (Niebuhr-Siebert \& Chilla, 2012). Sind die Eltern selbst schüchtern oder sozial ängstlich, werden sie in sozialen Situationen eher zurückhaltendes, vermeidendes Verhalten zeigen. Das Kind wird dieses Verhalten als sozial angemessen einschätzen und abspeichern. Die genetische Disposition für Schüchternheit und soziale Ängstlichkeit kann sich somit ausprägen und zu Unsicherheiten des Kindes in sozialen Situationen—vor allem in unvertrauten Kontext und mit fremden Personen-führen. 


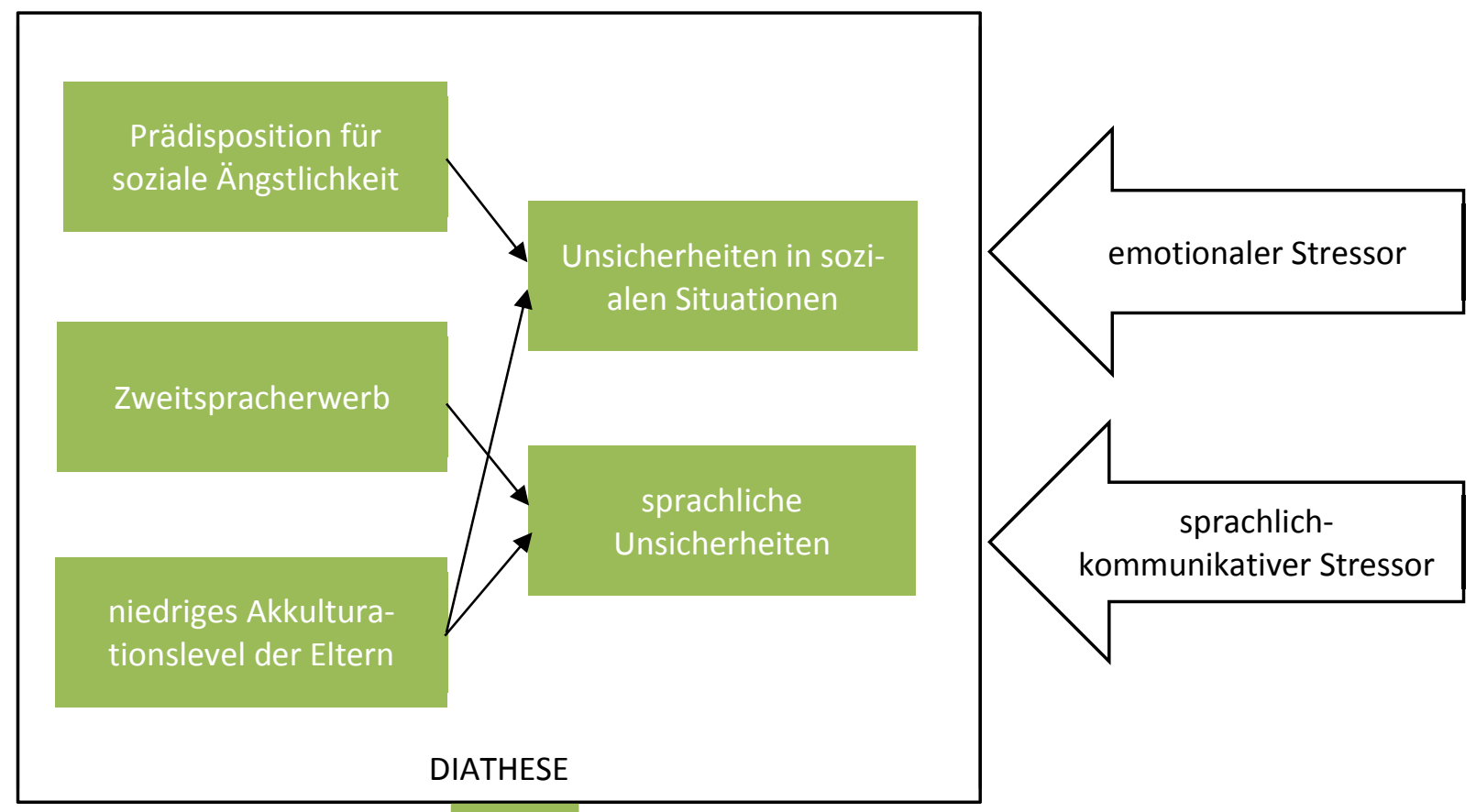

\section{SELEKTIVER MUTISMUS}

Abbildung 3. Erklärungsmodell zur Entstehung des selektiven Mutismus bei mehrsprachigen Kindern

Bei mehrsprachigen Kindern kommt der Aspekt der kulturellen Adaptation der Eltern hinzu, welche einen Einfluss sowohl auf die sozial-emotionale als auch sprachliche Entwicklung der Kinder haben kann (siehe Kapitel 2.4.3). Ein niedriges Akkulturationslevel der Eltern, d.h. eine geringe Anpassung an die Umgebungskultur, stellt eine Risiko für die Entwicklung internalisierender Auffälligkeiten, zu denen auch der SM zählt, (Calzada et al., 2009; Pawliuk et al., 1996) sowie für einen verlangsamten Zweitspracherwerb (Oades-Sese \& Li, 2011) dar. Ein niedriges Akkulturationslevel der Eltern kann somit soziale wie auch sprachliche Unsicherheiten der Kinder befördern. Innerhalb des Zweitspracherwerbs-insbesondere beim sukzessiven-kommt es unweigerlich zu Phasen, in denen das Kind sprachliche Unsicherheiten in der neu zu erwerbenden Sprache zeigt. Diese sind zwar entwicklungstypisch, können jedoch zugleich die Vulnerabilität des Kindes für den SM erhöhen.

Sprachliche und sozial-emotionale Unsicherheiten alleine reichen jedoch noch nicht aus, um einen SM auszulösen. Vielmehr entwickelt sich das Schweigen durch die Interaktion 
von Vulnerabilitätsfaktoren auf der einen und Stressoren auf der anderen Seite. Für die Stressoren wird eine grobe Unterteilung in sprachliche und emotionale Stressoren vorgenommen. Unter sprachlichen Stressoren werden vor allem sprachliche Anforderungen verstanden, die sich zum Beispiel in der Notwendigkeit verschiedene Sprachcodes einzusetzen (Alltags- vs. Bildungssprache) oder auch hinsichtlich der Spontanität der Äußerung unterscheiden können. Unter emotionalen Stressoren werden zum Beispiel Kontextwechsel wie der Übergang vom Elternhaus in die Kita und die damit verbundenen Unterschiede zwischen diesen Kontexten verstanden. Ebenso können besondere Lebensereignisse (z.B. längere Trennung von der Bezugsperson), wie sie häufig innerhalb der Lebensgeschichte der Kinder mit SM zu finden sind (siehe Kapitel 2.2.4), emotionale Stressoren darstellen.

Je nach Ausmaß der sprachlichen und sozial-emotionalen Unsicherheiten des Kindes können wenige kleine Stressoren ausreichen, um einen SM auszulösen. Für ein mehrsprachiges Kind könnte ein typischer Verlauf der Entwicklung eines SM etwa so aussehen: Das Kind wächst in einer nicht-deutschen Herkunftsfamilie auf. Die Familie lebt relativ isoliert von der Umgebungskultur in einem Stadtteil mit zahlreichen Familien der gleichen Herkunftskultur. Die Eltern sprechen beide kaum Deutsch. Das Kind wächst somit vor dem Eintritt in die Kita vorwiegend einsprachig mit der Herkunftssprache auf. Die Eltern haben zwar ein grundsätzliches Interesse an der Umgebungskultur, ihnen gelingt es jedoch aufgrund der fehlenden Deutschkenntnisse und der allgemeinen Zurückgezogenheit der Familienmitglieder nicht Anschluss an Familien der Umgebungskultur zu finden. Das Kind selbst ist eher schüchtern und sensibel. Bei Familienfeiern bleibt es lieber bei den Erwachsenen und nimmt nur selten selbstständig Kontakt zu anderen Kindern auf. Der Eintritt in die Kita mit drei Jahren fällt dem Kind sehr schwer. Es kann sich anfangs kaum von seiner Mutter trennen und sitzt über Tag häufig alleine in der Malecke, malt ein Bild und beobachtet die anderen Kinder von weitem. Das Kind erhält in der Kita zum ersten Mal intensiven Input in der Umgebungssprache, versteht anfangs nur sehr wenig, ist irritiert und verunsichert, was von ihm im Alltag erwartet wird. Das Kind erlebt eine starke Überforderung in der Situation und reagiert darauf mit Schweigen und Erstarrung, da ihm die nötigen sprachlichen und sozialen Kompetenzen fehlen, um sich Hilfe bei den Erzieherinnen zu holen. Obwohl das Kind im Laufe der Zeit Deutsch erwirbt, fängt es nicht an mit den anderen zu sprechen. Das Schweigen hat sich manifestiert, ein SM hat sich entwickelt.

In diesem Fall konstituiert sich die Diathese aus einer grundsätzlichen Schüchternheit des Kindes und den engsten Familienmitgliedern, der Herausforderung des Zweitspracherwerbs und dem niedrigen Akkulturationslevel der Eltern. Die daraus resultierenden Unsicher- 
heiten auf sprachlicher wie auch sozial-emotionaler Ebene erschweren dem Kind den KitaEintritt. Dieser stellt im Fall dieses mehrsprachigen Kindes sowohl einen sprachlichen als auch emotionalen Stressor dar. Auf emotionaler Ebene sind zunächst die Wechsel von der engen Familie in den größeren Kontext der Kita und für das mehrsprachige Kind im Speziellen von der Herkunftskultur in die Umgebungskultur als Stressoren zu nennen. Die Kontexte Familie und Kita unterscheiden sich in zweierlei Hinsicht: (1) ganz basal in der Anzahl der Personen und den sozialen Strukturen sowie (2) den kulturellen Rahmenbedingungen, welche zahlreiche Aspekte im Alltag beeinflussen könne (siehe Kapitel 2.4.1). Der Kitaeintritt übersteigt damit die Ressourcen dieses Kindes. Als Reaktion auf diese Überforderung reagiert das Kind mit Schweigen, welches sich im Verlauf der Zeit zu einem SM manifestiert.

Dieser recht einfach skizzierte Entwicklungsverlauf eines SM bei einem mehrsprachigen Kind sei nur als Beispiel zu verstehen. Wie auch bei einsprachigen Kindern dürften sich die Entwicklungsgeschichten bei mehrsprachigen Kindern als sehr heterogen darstellen-vor allem vor dem Hintergrund der verschiedensten Kulturkombinationen und dem unterschiedlichen Ausmaß kultureller Adaptation der Eltern. Hervorzuheben bei der Entwicklung des SM bei mehrsprachigen Kinder sind die Herausforderungen des Zweitspracherwerbs, welche gerade für schüchterne Kinder deutlich erschwert sein können (Keller et al., 2013), sowie die kulturellen Unterschiede zwischen familiärem Kontext und dem Kontext der Bildungseinrichtung, mit denen das Kind umgehen lernen muss. 


\subsection{Fragestellung und Hypothesen}

Ziel dieser Studie ist es, das scheinbar erhöhte Risiko von mehrsprachigen Kindern bzw. Kindern mit Migrationshintergrund für die Entwicklung eines SM zu untersuchen. Dabei wird der zentralen Frage nachgegangen, inwiefern das Ausmaß von Ängsten-insbesondere sozialen Ängsten-, die sprachlichen Kompetenzen in der Umgebungssprache sowie die kulturelle Adaptation der Eltern die Entwicklung eines SM begünstigen können.

Im Einzelnen wird angenommen, dass das Ausmaß der Angst der stärkste Prädiktor für den SM sowohl bei einsprachigen als auch bei mehrsprachigen Kindern ist. Wie bereits oben ausführlich erläutert, sind es allgemeine und im Speziellen soziale Ängste, welche sehr häufig komorbid zu einem SM auftreten (siehe dazu etwa Black \& Uhde, 1995; Chavira et al., 2007; Kristensen, 2000 sowie Kapitel 2.2.2) und als potenzielle Einflussfaktoren für die Entstehung und Aufrechterhaltung des Schweigens angesehen werden. Elizur und Perednik (2003) folgend zeigen Kinder mit Migrationshintergrund und einem SM deutlich höhere soziale Ängste als selektiv mutistische Kinder ohne Migrationshintergrund. Sie vermuten, dass bei selektiv mutistischen Kindern ohne Migrationshintergrund eine größere angeborene Vulnerabilität für soziale Ängstlichkeit und Entwicklungsstörungen Grundlage für das Schweigen bildet. Bei Kindern mit Migrationshintergrund nehmen sie hingegen an, dass aufbauend auf einer grundsätzlichen Vulnerabilität für soziale Ängstlichkeit vor allem sprachliche Unsicherheiten den SM auslösen und die sozialen Ängste verstärken. Vor allem soziale Situationen sind es, in denen Kinder mit Migrationshintergrund mit ihren sprachlichen Unsicherheiten konfrontiert werden. Eine häufige Konfrontation mit den eigenen Schwächen in sozialen Interaktionen könnten die sozialen Ängste intensivieren. Den Ausführungen entsprechend werden die folgenden vier Hypothesen (H) zur Ängstlichkeit der Kinder aufgestellt:

H1: Das Ausmaß der Angst ist der stärkste Prädiktor für die Entwicklung eines selektiven Mutismus.

H2: Kinder, die zum letzten Messzeitpunkt selektiv mutistisch sind, zeigen höhere Werte im Angstfragebogen als Kinder, die im Verlauf der Studie angefangen haben zu sprechen, sowie von Beginn an sprechende Kontrollkinder.

H3: Die mehrsprachigen Kinder mit SM zeigen zum ersten Messzeitpunkt höhere Werte im Angstfragebogen als einsprachige Kinder mit SM.

H4: Das Ausmaß der Angst hat sich bei mehrsprachigen Kindern mit SM vom ersten zum letzten Messzeitpunkt erhöht. 
Wie sich bereits in der Studie von Elizur und Perednik (2003) für die Entwicklung des SM bei Kindern mit Migrationshintergrund andeutet, könnten sprachliche Unsicherheiten aufbauend auf einer grundlegenden Ängstlichkeit in sozialen Situationen das Schweigen beeinflussen. Die Kombinationen von Ängstlichkeit und sprachlichen Problemen findet sich auch in den drei Subgruppen nach Cohan et al. (2008). Diese ähneln sich hinsichtlich des Ausmaßes sozialer Ängste, unterscheiden sich jedoch hinsichtlich zusätzlicher Sprach- und Verhaltensprobleme. Insgesamt $43.1 \%$ der Probanden konnten der Subgruppe ängstlichsprachlich verzögert zugeordnet werden. Eine gemeinsame Beeinflussung von sprachlichen Problemen und Ängstlichkeit in der Entstehung und Aufrechterhaltung des Schweigens liegt somit nahe.

H5: Neben dem Ausmaß der Angst sind die Sprachkompetenzen des Kindes in der Umgebungssprache ein bedeutsamer Prädiktor für die Entwicklung des Sprechverhaltens. Schwache Sprachkompetenzen im Deutschen stehen mit einem höheren Ausmaß des Schweigens in Verbindung.

Spezifisch für die Kinder mit Migrationshintergrund wird untersucht, ob sich das Ausmaß der kulturellen Adaptation der Eltern auf die Entwicklung des Schweigens auswirkt. Den Studienergebnissen von Calzada et al. (2009) folgend wird erwartet, dass die Eltern der Kinder mit SM ein niedrigeres Akkulturationslevel zeigen als die Eltern von Kindern, die sich positiv zum Sprechen entwickeln bzw. bereits von Beginn an gesprochen haben. Unterstützt wird diese Annahme etwa auch von Pawliuk et al. (1996). Die Autoren weisen darauf hin, dass sich eine Anpassung an die Umgebungskultur positiv auf die soziale und psychische Entwicklung auswirkt. Für den Bereich der kulturellen Adaptation der Eltern werden die folgenden zwei Hypothesen aufgestellt:

H6: Eltern von Kindern mit SM weisen ein niedrigeres Anpassungslevel an die Umgebungskultur auf als Eltern von Kindern ohne SM.

H7: Eltern von Kindern mit SM weisen ein höheres Maß an Beibehaltung ihrer Herkunftskultur auf als Eltern von Kindern ohne SM.

Darauf aufbauend wird angenommen, dass es einen Zusammenhang zwischen der kulturellen Adaptation der Eltern und den sprachlichen Kompetenzen der Kinder in der Umgebungssprache gibt. Dazu haben Oades-Sese und Li (2011) in ihrer Studie herausgefunden, 
dass Kinder von assimilierten Eltern über signifikant bessere Sprachkompetenzen verfügen als Kinder von Eltern anderer Akkulturationsstile. Diese Eltern waren zu einem großen Teil in die Umgebungskultur integriert, konnten sich mit dieser identifizieren und sprachen vermutlich zuhause eher die Umgebungssprache. So erhielten die Kinder mit hoher Wahrscheinlichkeit einen größeren Input in der Umgebungssprache als Kinder von Eltern mit einer starken Orientierung an der Herkunftskultur, welche zuhause eher die Herkunftssprache sprechen. Daraus folgend wird zum Zusammenhang von Sprachkompetenzen im Deutschen und kultureller Adaptation der Eltern die folgende Hypothese aufgestellt:

H8: Die Sprachkompetenzen des Kindes im Deutschen hängen positiv mit dem Akkulturationslevel der Eltern zusammen. Je mehr die Eltern an die Umgebungskultur angepasst sind, desto besser beherrschen die Kinder die Umgebungssprache. 


\section{Methode}

\subsection{Studiendesign}

Zur Untersuchung des Einflusses von sprachlichen Fähigkeiten in der Umgebungssprache, der Ängstlichkeit sowie der kulturellen Adaptation der Eltern auf die Entwicklung eines SM wurde eine quasiexperimentelle Längsschnittstudie mit insgesamt vier Probandengruppen durchgeführt. Ein- und mehrsprachige schweigende und sprechende Kindergartenkinder wurden vier Mal im Abstand von jeweils drei Monaten hinsichtlich ihrer kommunikativen Entwicklung in der Kita sowie in familiären und öffentlichen Situationen untersucht. Die Kita stellt den Kontext im Alltag des Kindes dar, in dem es neben seinem Zuhause am meisten Zeit verbringt. Für den SM ist bereits ausreichend dokumentiert, dass in eben solchen Bildungsinstitutionen wie Kita und Schule die Kernsymptomatik des Schweigens am deutlichsten erkennbar ist (siehe etwa Bergman et al., 2002; Ford et al., 1998). Eine kommunikative Öffnung des Kindes in diesem Kontext spiegelt entsprechend eine Abnahme der Symptomatik des SM wider. So sollten anhand von Veränderungen im Interaktions- und Sprechverhalten der Kinder auch Veränderungen des Symptombildes des SM dokumentierbar sein. Für mehrsprachige Kinder ist die Kita zudem der Kontext, in dem sie typischerweise zum ersten Mal mit der Mehrheitssprache und Mehrheitskultur in bedeutsamen Maße konfrontiert werden (Chilla et al., 2010). Wie bereits in Kapitel 2.3.2 erläutert wird eine entwicklungstypische Phase des Schweigens von ca. sechs Monaten im Zweitspracherwerb angenommen (siehe dazu auch Tabors, 2008). Diese sollte sich typischerweise in den Bildungsinstitutionen Kita und Schule zeigen. Mit Zunahme der Sprachkompetenzen des Kindes in der Mehrheitssprache wird ebenso eine Zunahme sprachlicher Äußerungen erwartet. Demnach wird vermutet, dass nach etwa sechs Monaten regelmäßigem Kita-Besuch ein Kind im beginnenden Zweitspracherwerb die Phase des Schweigens verlässt und mit dem Sprechen beginnen sollte. An diesem Punkt sollten sich idealtypisch schweigende mehrsprachige Kinder in zwei Gruppen teilen: (1) in eine Gruppe von tatsächlich selektiv mutistischen Kindern und (2) in eine Gruppe mit entwicklungstypischem Schweigen. Diese Unterscheidung sollte sich im Verlauf der Längsschnittstudie vor allem im Interaktionsverhalten der Kinder in der Kita zeigen. Die erste Gruppe müsste idealtypisch schweigend und eher zurückhaltend bleiben. Die zweite Gruppe sollte sich durch eine Zunahme interaktiver Verhaltensweisen ausweisen. Ähnliches sollte für extrem schüchterne, einsprachige Kinder gelten, die zu Beginn ihres Kita-Besuchs aufgrund der extremen Schüchternheit mutismusähnliche Verhaltensweisen zeigen. Auch sie sollten 
sich im Verlauf der Zeit durch eine Zunahme interaktiver Handlungen im Kita-Kontext auszeichnen.

Um diese Entwicklung sichtbar zu machen, werden das Interaktions- und vor allem das Sprechverhalten der Kinder aus verschiedenen Perspektiven betrachtet. Über alle Messzeitpunkte sollen sowohl Eltern als auch Erzieherinnen über Fragebögen das aktuelle (Sprech-)Verhalten der Kinder einschätzen. Zusätzlich werden im Kita-Kontext Beobachtungen durch Studierende der TU Dortmund in alltagstypischen Situationen durchgeführt.

Um mögliche Einflussfaktoren auf die Entwicklung der Kinder zu untersuchen werden zusätzlich zu Beginn und zum Ende der Längsschnittstudie die sprachlichen Fähigkeiten über ein standardisiertes Testverfahren und Spontansprachaufnahmen sowie die Ängstlichkeit der Kinder und die kulturelle Adaptation der Eltern jeweils durch Elternfragebögen erfasst. Tabelle 2 gibt einen groben Überblick über den Ablauf und die Inhalte der Hauptstudie.

Tabelle 2

Überblick über die erhobenen Daten zu den vier Messzeitpunkten (T)

\begin{tabular}{llccc}
\hline & T 1 & T 2 & T 3 & T 4 \\
\hline Anamnese & X & & & \\
Mehrsprachenkontext & X & & & \\
Sprechverhalten der Kinder & X & X & X & X \\
Ängstlichkeit der Kinder & X & & X & X \\
Interaktionsverhalten der Kinder & X & X & & X \\
sprachliche Kompetenzen (Deutsch) & X & & & X \\
Akkulturation der Eltern & X & & & \\
\hline
\end{tabular}

Aufgrund der besonderen Klientel, die in dieser Studie untersucht wurde, mussten vorab zahlreiche Instrumente neu entwickelt bzw. modelliert werden. Aus diesem Grund werden im Folgenden zunächst die Konstruktion der neu entwickelten Instrumente beschrieben sowie die Ergebnisse der Erprobung dieser im Rahmen der Pilotstudie berichtet.

\subsection{Konstruktion und Auswahl der Instrumente}

Im Folgenden wird die Konstruktion der neu entwickelten bzw. die Auswahl bestehender Instrumente zur Erfassung der unterschiedlichen Konstrukte in dieser Studie erläutert. Einen Überblick über die eingesetzten Erhebungsinstrumente und deren Quellen gibt Tabelle 3. 
Tabelle 3

Übersicht über die genutzten und neu konstruierten Instrumente

\begin{tabular}{l}
\hline Instrument \\
\hline Elternfragebogen \\
(1) anamnestische und \\
soziodemografische \\
Daten \\
(2) (Sprech-)Verhalten \\
der Kinder
\end{tabular}

Mehrsprachenkontext*

\section{Erfassungsbereich}

(1) Entwicklung des Kindes und bereits erhaltene Therapien, Einschätzung der Sprachkompetenzen in der L1 und L2*, besondere Lebensereignisse, Informationen zu den Eltern (Alter, Bildungsstand, Berufstätigkeit, Aufenthaltsdauer in Deutschland, schüchternes, ängstliches oder schweigendes Verhalten), Wohnsituation der Familie (2) Schweigedauer, eingeschätzte Sprachkompetenzen im Deutschen, 59 (*69) Items zum (Sprech-)Verhalten der Kinder in familiären Kontexten

Instrument zur Erfassung der gesprochenen Sprachen innerhalb der Familien

79 Items zum (Sprech-)Verhalten des Kindes im Kontext der Kita

26 Items zur Erfassung kindlicher Ängste

BAV 3-11 Elternfragebogen

DortMuS-Kita

FRAKK20*

TROG-D

Beobachtungsbögen standardisiertes Testverfahren zur Erfassung des Grammatikverständnisses im Deutschen
Beobachtungsbögen zur Erfassung des In-

\section{Quelle}

selbst entwickelt

Mehrsprachen-Kontexte (Ritterfeld \& Lüke, 2012)

selbst entwickelt

Bochumer Angstverfahren für Kinder im Vorschulund Grundschulalter (Mackowiak \& Lengning, 2010)

Frankfurter Akkulturationsskala (Bongard, Kelava, Sabic, Aazami-Gilan \& Kim, 2007)

Test zur Überprüfung des Grammatikverständnisses (Fox, 2007)

selbst entwickelt teraktionsverhaltens in den Situationen Freispiel, Gruppen- und Essenssituation sowie Begrüßung im Kontext der Kita

* nur bei mehrsprachigen Kindern/Familien

\subsubsection{Anamnestische und soziodemografische Daten}

Über einen Elternfragebogen (siehe Anhang B) wurden bedeutsame anamnestische und soziodemografische Daten der Kinder erfasst. Die in dieser Studie verwendete Version ist eine Weiterentwicklung des Dortmunder Mutismus Inventars (Kresse, 2011). Der Fragebogen 
gliedert sich in insgesamt sechs Teile: (A) Allgemeine Informationen zum Kind, (B) Sprechen und Schweigen, (C) Allgemeine Entwicklung, (D) Sprachliche Entwicklung, (E) weitere Hintergrundinformationen und (F) Informationen zur Familie und zum Haushalt.

In Teil A werden über eine Mischung aus geschlossenen und halboffenen Fragen das Geburtsdatum, Geschlecht, bereits erfolgte oder aktuell laufende Therapien sowie Informationen zum Besuch einer Kita (Eintrittsalter, Schwierigkeiten bei der Eingewöhnung, regelmäßiger Besuch, Freude des Kindes am Besuch) erfasst. Teil B fokussiert das (Sprech-)Verhalten des Kindes und wird in Kapitel 3.2.2 genauer beschrieben. In Teil C werden zunächst Angaben zur Schwangerschaft und Geburt (Komplikationen, Schwangerschaftswoche bei der Geburt, Geburtsgewicht, Mehrlingsgeburt) und wichtige motorische und sprachliche Meilensteine in der Entwicklung der Kinder erfragt. Weiter wird erfasst, ob bei den Kindern bereits Entwicklungsverzögerungen oder -störungen im motorischen, kognitiven oder sprachlichen Bereich diagnostiziert worden sind und ob beim Kind zeitweilig Hörprobleme (als bedeutsamer Risikofaktor für eine umschriebene Sprachentwicklungsstörung) aufgetreten sind. Die bei SM häufig komorbid auftretenden Auffälligkeiten wie zum Beispiel Enuresis und Enkorpresis, zwanghaftes Verhalten oder Tics (siehe dazu auch Kapitel 2.2) werden nachfolgend abgefragt. Aufgrund der großen Häufung sprachlicher Auffälligkeiten bei SM wird in Teil D die sprachliche Entwicklung mit insgesamt sieben Fragen bei mehrsprachigen Kindern und drei Fragen bei einsprachigen Kindern beleuchtet. Für die mehrsprachigen Kinder werden die gesprochenen Sprachen des Kindes erfasst und nachfolgend erfragt, welche von diesen die dominante (d.h. am häufigsten und am liebsten gesprochene) Sprache des Kindes ist. Ferner werden die Eltern gebeten, Einschätzungen zu den Sprachkompetenzen des Kindes in den jeweiligen Sprachen zu machen. Als Hilfestellung sollen die Eltern sich ein gleichaltriges Kind mit der gleichen Erstsprache vorstellen und einschätzen, ob ihr Kind die deutsche und nicht-deutsche Sprache besser, genauso gut, etwas schlechter oder viel schlechter beherrscht. Zusätzlich wird erfragt, ob das Kind regelmäßig andere Institutionen besucht, in denen die nicht-deutsche Sprache gesprochen wird und ggf. nach der Anzahl der Stunden pro Woche gefragt. Sowohl bei ein- als auch bei mehrsprachigen Kindern wird erfasst, ob bereits sprachliche Probleme folgender Art aufgetreten bzw. aktuell noch vorhanden sind: später Sprechbeginn, Auffälligkeiten auf der phonetisch-phonologischen, syntaktisch-morphologischen oder semantisch-lexikalischen Sprachebene, Stottern und Poltern. Das Schweigen auslösende Ereignisse wie lange Trennungen von der Bezugspersonen oder Umzüge in eine andere Stadt bzw. in ein anderes Land werden in Teil E erfasst. Im abschließenden Teil F geht es um Informationen zum Haushalt und zur Familie des Kindes. Für Mutter und Vater wird jeweils 
Alter, Erstsprache, Aufenthaltsdauer in Deutschland, der höchste Bildungsabschluss sowie erlernter Beruf und aktuelle Berufstätigkeit erfasst. Darüber hinaus wird nach Geschwistern des Kindes, deren Geschlecht und Alter gefragt. Aufgrund der familiären Häufung von Schüchternheit, Ängsten und SM werden die Eltern zudem gebeten, sich selbst und die Geschwister des Kindes hinsichtlich typischer Verhaltensweisen auf einer dreistufigen Ratingskala von 1 (trifft gar nicht zu) bis 3 (trifft genau zu) einzuschätzen. Die drei Bereiche werden mit vier bzw. fünf Items grob erfasst. Zur Einschätzung des sozioökonomischen Status werden der höchste Bildungsabschluss, die berufliche Ausbildung sowie aktuelle berufliche Situation beider Elternteile, das Einkommen des Haushaltes und die aktuelle Wohnsituation der Familie erfragt. Für die späteren Analysen wird aus diesen Informationen ein SchichtIndex berechnet (siehe dazu Lampert \& Kroll, 2009). Dazu werden die Angaben jedes Elternteils zu schulischer und beruflicher Bildung, Einkommen und beruflicher Stellung in Zahlenwerte kodiert und zu einem Index aufsummiert. Dieser kann einen Wertebereich von 3 bis 21 annehmen und laut Lampert und Kroll (2009) als metrische Variable in weitere Analysen einbezogen werden. Der Index wird zunächst für beide Eltern einzeln berechnet und für die Gesamtfamilie der höchste der beiden Werte übernommen. Bei getrennt lebenden Elternteilen wird der Wert des betreuenden Elternteils für das Kind herangezogen. Schließlich wird im Fragebogen über die Bücheraufgabe von Paulus (2009) das kulturelle Kapital erfragt. Dabei sollen die Familien auf einer fünfstufigen Ratingskala (keine oder nur sehr wenige bis über 200 Bücher) mit visueller Unterstützung durch Fotos von wenig bis stark gefüllten Bücherregalen einschätzen, wie viele Bücher im Haushalt vorhanden sind.

\subsubsection{Messung des Sprechverhaltens}

Um für den SM typisches Verhalten in verschiedenen Kontexten zu erfassen, wurden in der Arbeitsgruppe „DortMuS“ (Dortmunder Mutismus-Screening) an der TU Dortmund insgesamt drei Fragebögen entwickelt: (1) DortMuS-Schule, (2) DortMuS-Kita und (3) Elternfragebogen. Ähnlich wie das bislang einzige, existierende Verfahren zur Messung des Schweregrades des SM-der SMQ (Selective Mutism Questionnaire; Bergman, Keller, Piacentini \& Bergman, 2008), bzw. die deutsche Übersetzung FEM (Fragebogen zur Erfassung des Elektiven Mutismus; Steinhausen, 2010)—beinhalten alle drei entwickelten Bögen Items zum Ausmaß des Schweigens in unterschiedlichsten Kontexten. Als Kernsymptom sollte sich das schweigende Verhalten der Kinder als am geeignetsten zur Differenzierung zwischen SM und ähnlichen Phänomenen wie der sozialen Ängstlichkeit erweisen. Zusätzlich werden in DortMuS noch weitere vom SM beeinträchtige Bereiche erfasst, zum Beispiel das nonverbale 
Kommunikationsverhalten oder die Emotionsregulation. Zur Verminderung der Akquieszenz wurden bei beiden Fragebögen auf eine Ausgewogenheit von positiv und negativ formulierten Items geachtet (siehe dazu auch Jonkisz, Moosbrugger \& Brandt, 2012).

\section{Elternfragebogen}

Der Elternfragebogen besteht in seiner Pilotversion (siehe Anhang B) aus insgesamt 59 Items bei einsprachigen Kindern bzw. 69 Items bei mehrsprachigen Kindern. Die Items verteilen sich insgesamt auf fünf Skalen: (1) Schweigen, (2) Partizipation, (3) nonverbales Kommunikationsverhalten, (3) Emotionsregulation sowie (4) öffentliche Situationen und ggf. (5) Mehrsprachigkeit. Die Einschätzung des Verhaltens erfolgt über eine fünfstufige Ratingskala von 1 (trifft gar nicht $z u$ ) bis 5 (trifft genau $z u$ ), wobei die Werte für alle weiteren Analysen in der vorliegenden Studie auf das Spektrum von 0 bis 4 umkodiert werden. Hohe Werte stehen für das Vorhandensein von Verhaltensweisen, welche für den SM typisch sind. Das heißt zum Beispiel Schweigen in einer bestimmten Situation oder auch Rückzugsverhalten in Konfliktsituationen. Da einige Items nicht unbedingt auf alle Familien zutrifft (wenn das Kind zum Beispiel keine Geschwister hat), wird den Eltern zusätzlich die Möglichkeit gegeben ,weiß nicht“" anzukreuzen.

Tabelle 4 gibt eine Übersicht über die Anzahl der Items auf den jeweiligen Skalen, Beispielitems und Skalenkennwerte für die Hauptstudie. Im Rahmen dieser wurden die einzelnen Skalen hinsichtlich ihrer internen Konsistenz überprüft. Items, die sich zum ersten Messzeitpunkt als wenig trennscharf in der Stichprobe erwiesen (korrigierte ItemTrennschärfe <.30), wurden aus allen weiteren Analysen ausgeschlossen. Insgesamt zwölf Items wurden anhand dieser Kriterien herausgefiltert (neun aus dem Fragebogen für alle Kinder und drei Items für mehrsprachige Kinder). Eine genaue Übersicht über die Itemkennwerte findet sich in Tabelle A-1 im Anhang A.

Die Skalen Schweigen, Partizipation, Emotionsregulation sowie öffentliche Situationen erreichten auch bei allen drei weiteren Messzeitpunkten akzeptable bis sehr gute Werte in der internen Konsistenz. Die Werte für die Skalen nonverbale Kommunikation und Mehrsprachigkeit schwankten über die weiteren drei Messzeitpunkte sehr stark und erreichten mit einem Cronbachs Alpha unter .700 keine zufriedenstellenden Werte mehr, so dass sie in den Analysen der Hauptstudie nicht mit einbezogen wurden. 
Tabelle 4

Skalen, Beispielitems und interne Konsistenz für den Elternfragebogen

\begin{tabular}{|c|c|c|c|}
\hline Skala & Itemanzahl & Beispiele & Interne Konsistenz \\
\hline Schweigen & 18 & $\begin{array}{l}\text { Mein Kind schweigt in Gegenwart seiner } \\
\text { Großeltern } \\
\text { Bei Familienfesten spricht mein Kind mit an- } \\
\text { deren Kindern. (negativ) } \\
\text { Mit kleinen Kindern der Verwandtschaft } \\
\text { spricht mein Kind. }\end{array}$ & $\alpha=.946$ \\
\hline Partizipation & 9 & $\begin{array}{l}\text { Mein Kind spielt bei Familienfesten mit ande- } \\
\text { ren Kindern. (negativ) } \\
\text { Auf dem Spielplatz spielt mein Kind alleine. } \\
\text { Mein Kind spielt nur nach Aufforderung mit } \\
\text { anderen Kindern. }\end{array}$ & $\alpha=.854$ \\
\hline $\begin{array}{l}\text { Emotions- } \\
\text { regulation }\end{array}$ & 10 & $\begin{array}{l}\text { Mein Kind zieht sich zurück, wenn Besuch } \\
\text { kommt (z.B. geht in sein Zimmer, hört auf zu } \\
\text { sprechen). } \\
\text { Wenn wir in der Stadt Bekannte treffen, hält } \\
\text { unser Kind Körperkontakt zu uns (z.B. bleibt } \\
\text { an der Hand, hält meine Jacke fest). } \\
\text { Mit Veränderungen des gewohnten Tagesab- } \\
\text { laufs geht mein Kind problemlos um. (negativ) }\end{array}$ & $\alpha=.854$ \\
\hline $\begin{array}{l}\text { Öffentliche } \\
\text { Situationen }\end{array}$ & 7 & $\begin{array}{l}\text { Mein Kind kauft selbstständig z.B. Spielzeug, } \\
\text { Aufkleber oder Süßigkeiten ein. (negativ) } \\
\text { Beim Zahnarzt öffnet mein Kind den Mund. } \\
\text { (negativ) } \\
\text { Mein Kind äußert seine Wünsche und Bedürf- } \\
\text { nisse nicht in der Öffentlichkeit. }\end{array}$ & $\alpha=.714$ \\
\hline $\begin{array}{l}\text { Nonverbale } \\
\text { Kommuni- } \\
\text { kation }\end{array}$ & 4 & $\begin{array}{l}\text { Ja und Nein drückt mein Kind durch Kopf- } \\
\text { schütteln und Nicken aus. } \\
\text { Mein Kind nimmt Blickkontakt auf, wenn es } \\
\text { angesprochen wird. } \\
\text { In Gegenwart fremder Personen vermeidet } \\
\text { mein Kind Husten, Niesen oder Räuspern. }\end{array}$ & $\alpha=.731$ \\
\hline $\begin{array}{l}\text { Mehrspra- } \\
\text { chigkeit }\end{array}$ & 7 & $\begin{array}{l}\text { Mein Kind spricht mit Kindern seiner Mutter- } \\
\text { sprache. (negativ) } \\
\text { Mein Kind schweigt, wenn es von Personen in } \\
\text { seiner Muttersprache angesprochen wird. } \\
\text { Mein Kind spricht im Urlaub in unserem Hei- } \\
\text { matland mit seinen Verwandten. (negativ) }\end{array}$ & $\alpha=.865$ \\
\hline
\end{tabular}

Für die Analysen wurden für die einzelnen Skalen Summenwerte aus allen Itemantworten (nach Umkodierung der negativ gepolten Items) gebildet und entsprechend ein Gesamtwert für den Elternfragebogen aus der Summe aller Itemwerte auf den Skalen Schweigen, Partizipation, Emotionsregulation und öffentliche Situationen berechnet. 


\section{Fragebogen für die Kindertageseinrichtung}

Der Itempool der Pilotversion von DortMuS-Kita (siehe Anhang C) besteht aus 79 Items, die sich ebenfalls auf die oben erwähnten Skalen verteilen (zur Itemanzahl und zu Beispielitems siehe Tabelle 5). Zusätzlich wurde der Bereich des Hilfe und Unterstützung-Holens in der Kita-Version berücksichtigt. Im familiären Kontext dürfte es für ein Kind mit SM keine Schwierigkeit sein sich Unterstützung bei seinen Eltern zu holen, wenn es etwa seine Jacke schließen möchte. Im Kontext von Bildungseinrichtungen halten sich selektiv mutistische Kinder jedoch eher zurück (Starke \& Subellok, 2012). Erfahrungsgemäß holen sie sich nur selten bis nie Unterstützung bei anderen Kindern oder Erwachsenen. Bereits die Mitteilung von schönen Erlebnissen fällt den Kindern phänomenbedingt schwer. Das Zeigen von Schwäche, welches durch das Bitten um Unterstützung ausgedrückt wird, vermeiden die Kinder. Auf zusätzliche Items für mehrsprachige Kinder wurde verzichtet, da der Kita-Kontext in der Regel durch die deutsche Sprache und deutsche Kultur geprägt ist und somit valide Einschätzungen zu ggf. unterschiedlichen Verhaltensweisen in der nicht-deutschen Sprache von den Erzieherinnen nicht zu erwarten sind.

Auch bei DortMuS-Kita erfolgt die Einschätzung der jeweiligen Verhaltensweisen auf einer fünfstufigen Ratingskala von 1 (trifft gar nicht zu) bis 5 (trifft genau zu). Auch hier wurden die Itemantworten für die Analysen auf den Bereich 0 bis 4 umkodiert. Hohe Werte spiegeln wiederum das Vorliegen von mutismustypischen Verhaltensweisen wider. Ebenso wird den Erzieherinnen die Möglichkeit gegeben „weiß nicht“" anzukreuzen.

Im Rahmen des ersten Messzeitpunktes der Hauptstudie sowie in parallelen Erhebungen in weiteren Kitas im Ruhrgebiet wurde der Bogen anhand von sprechenden und schweigenden Kindern evaluiert und zu einer 17-Itemversion gekürzt. Das genaue Vorgehen kann bei Starke, Subellok und Käppler (in Vorb.) nachgelesen werden.

Gleichwohl die Vorversion mit 79 Items in der Hauptstudie zu allen Messzeitpunkten an die Erzieherinnen der Einrichtungen ausgegeben wurde, wird für die weiteren Analysen lediglich auf die 17-Itemversion zurückgegriffen. Die 17 Items verteilen sich auf zwei Skalen: (1) Schweigen und Bedürfnisäußerung sowie (2) Gruppensituationen. Tabelle 6 gibt einen Überblick über die Anzahl der Items auf den Skalen sowie die internen Konsistenzen in der Stichprobe der Hauptstudie zum ersten Messzeitpunkt. In Tabelle A-2 im Anhang sind zusätzlich alle Items mit Itemschwierigkeiten und Trennschärfen (siehe dazu auch Starke et al., in Vorb.) aufgeführt. 
Tabelle 5

Übersicht über die Subskalen der Pilotversion von DortMuS-Kita

\begin{tabular}{|c|c|c|}
\hline Skala & Itemanzahl & Beispiele \\
\hline $\begin{array}{l}\text { Sprechen und } \\
\text { Schweigen }\end{array}$ & 27 & $\begin{array}{l}\text { Das Kind schweigt auf dem Hof gegenüber Erziehern. } \\
\text { Bei Ansprache der Erzieherin schweigt das Kind. } \\
\text { Ich habe das Kind schon sprechen gehört. (negativ) }\end{array}$ \\
\hline $\begin{array}{l}\text { Emotionaler Aus- } \\
\text { druck }\end{array}$ & 11 & $\begin{array}{l}\text { Das Kind entzieht sich Konfliktsituationen. } \\
\text { In lustigen Situationen bleibt das Kind regungslos. } \\
\text { In Konfliktsituationen verteidigt sich das Kind. (negativ) }\end{array}$ \\
\hline Partizipation & 21 & $\begin{array}{l}\text { Auf dem Hof spielt das Kind alleine. } \\
\text { Das Kind singt bei Liedern in der Gruppe mit. (negativ) } \\
\text { In der Gruppe nimmt das Kind die Rolle des Zuschauers ein. }\end{array}$ \\
\hline Bedürfnisse & 8 & $\begin{array}{l}\text { Das Kind äußert Bedürfnisse, z.B. Durst oder Toilettengang, in } \\
\text { irgendeiner Form. (negativ) } \\
\text { Das Kind signalisiert gegenüber der Erzieherin, dass es z.B. Durst } \\
\text { hat. (negativ) } \\
\text { Im Kindergarten geht das Kind nicht zur Toilette. }\end{array}$ \\
\hline $\begin{array}{l}\text { Nonverbale } \\
\text { Kommunikation }\end{array}$ & 4 & $\begin{array}{l}\text { Das Kind reagiert bei direkter Ansprache nichtsprachlich. } \\
\text { Möchte das Kind etwa haben, zeigt es auf die Sache. } \\
\text { Bei Ansprache der Erzieherin nimmt das Kind Blickkontakt auf. } \\
\text { (negativ) }\end{array}$ \\
\hline $\begin{array}{l}\text { Hilfe/ Unterstüt- } \\
\text { zung }\end{array}$ & 8 & $\begin{array}{l}\text { Das Kind holt sich keine Unterstützung von der Erzieherin, wenn } \\
\text { es Hilfe benötigt. } \\
\text { Das Kind bittet andere Kinder beim Basteln um Materialien, an } \\
\text { die es selbst nicht heran kommt. (negativ) } \\
\text { Das Kind äußert verbal, wenn es Hilfe benötigt. (negativ) }\end{array}$ \\
\hline
\end{tabular}

Tabelle 6

Skalen, Beispielitems und interne Konsistenzen für DortMuS-Kita

\begin{tabular}{|c|c|c|c|}
\hline Skala & Itemanzahl & Beispiele & $\begin{array}{l}\text { Interne Kon- } \\
\quad \text { sistenz }\end{array}$ \\
\hline $\begin{array}{l}\text { Schweigen } \\
\text { und Bedürf- } \\
\text { nisäußerung }\end{array}$ & 12 & $\begin{array}{l}\text { Bedürfnisse werden von dem Kind nicht geäußert. } \\
\text { Das Kind spricht mit Erziehern aus anderen Gruppen. } \\
\text { (negativ) } \\
\text { Das Kind signalisiert gegenüber der Erzieherin, dass es } \\
\text { z.B. Durst hat. (negativ) }\end{array}$ & $\alpha=.943$ \\
\hline $\begin{array}{l}\text { Gruppensitu- } \\
\text { ationen }\end{array}$ & 5 & $\begin{array}{l}\text { Das Kind beteiligt sich an angeleiteten Spielangeboten } \\
\text { in der Großgruppe. (negativ) } \\
\text { Das Kind zieht sich bei Spielen in der Großgruppe } \\
\text { zurück. } \\
\text { Werden Lieder in der Gruppe gesungen, sitzt das Kind } \\
\text { „teilnahmslos“ dabei. }\end{array}$ & $\alpha=.893$ \\
\hline
\end{tabular}


Über alle Messzeitpunkte der Hauptstudie hinweg erreichten die beiden Skalen mit Cronbachs Alpha von .840 bis .950 gute bis sehr gute Werte.

\subsubsection{Messung des Interaktionsverhaltens}

\section{Beobachtungsbögen}

Zur Beobachtung des Interaktionsverhaltens im Kontext der Kindertageseinrichtungen wurden vier Beobachtungsbögen für die Situationen Begrüßung, Freispiel, Essen und Gruppenangebot entwickelt (siehe Anhang D für die Versionen der Hauptstudie). Diese Situationen sind Elemente eines typischen Kita-Alltages und stellen ganz unterschiedliche Anforderungen an das interaktive Verhalten der Kinder. Die Begrüßungssituation bietet zum einen die Möglichkeit, bedeutsame Gesprächsregeln zu erwerben und zu üben. Zudem ist sie häufig die einzige Situation im Alltag des Kindes, in der eine dyadische Interaktion zwischen Kind und Erzieherin überhaupt stattfindet. Dies war in Kombination mit den weiteren drei Beobachtungssituationen relevant, um Unterschiede in der Interaktion zwischen dem Kind und anderen Kindern und dem Kind und den Erwachsenen der Institution festhalten zu können.

Intensive Interaktionen zwischen Kindern ergeben sich vor allem in Freispielsituationen. Durch die freie Spielwahl sollten sich hier Präferenzen der Kinder für Spielverhalten und Interaktionspartner zeigen. In Gruppensituationen werden von einer erwachsenen Person strukturierte Angebote gemacht—entweder in der Groß- oder Kleingruppe. Je nach Angebot können solche Situationen für das Kind sehr niedrige Anforderungen erzeugen-z.B. bietet gemeinsames Singen die Möglichkeit verdeckt durch die Stimmen der anderen leise mitzusingen oder zumindest den Mund mitzubewegen—oder sehr hohe Anforderungen, wenn z.B. im Erzählkreis jeder von seinem schönsten Wochenenderlebnis erzählen soll. Zudem bieten gerade Gruppenspiele häufig die Möglichkeit auch nonverbal aktiv zu partizipieren. Das Ausmaß des SM sollte somit innerhalb von Gruppensituationen vor allem über die Art der Partizipation des Kindes beobachtbar sein. In Essensituationen besteht unter Umständen die Notwendigkeit, eigene Bedürfnisse und Wünsche klar zu äußern, wenn z.B. zwischen Getränken oder Speisen gewählt werden soll oder das Kind Nachschlag haben möchte.

Allen Bögen gemeinsam waren die Beobachtungskategorien im Bereich Interaktion. Hier wurde grundsätzlich zwischen verbalen und nonverbalen Interaktionsformen unterschieden. Verbal umfasste hier jegliche sprachliche Äußerung. Wenn möglich wurde dokumentiert, ob die Äußerung auf Wort- oder Satzebene war. Unter nonverbalen Interaktionsformen wurden körperliche Ausdrucksformen wie Mimik, Gestik oder Berührungen gefasst. Zusätzlich 
wurde nach initiierenden und reagierenden Verhaltensweisen differenziert. Begann ein Kind eine Interaktion mit einer anderen Person - egal ob verbal oder nonverbal—, wurde dies als Initiation gewertet. Alle Reaktionen eines Kindes auf einen Impuls einer anderen Person wurden entsprechend als Reaktion kategorisiert. Beispiel für eine verbale Initiation wäre etwa die Frage „Möchtest du mit mir Memory spielen?“ des Kindes. Eine nonverbale Initiation wäre etwa das Anstupsen der Erzieherin, um ihre Aufmerksamkeit zu erhalten. Entsprechend wäre eine verbale Reaktion des Kindes beispielsweise eine Antwort auf eine Frage eines anderen Kindes. Eine nonverbale Reaktion könnte etwa ein Kopfnicken auf eine Ja-Nein-Frage einer Erzieherin sein.

Es wurde weiter danach differenziert, ob das entsprechende Verhalten zwischen Kind und einem anderen Kind oder zwischen dem Kind und einer erwachsenen Person (im Beobachtungsbogen als Erzieher kategorisiert) gezeigt wurde. Für die Begrüßungssituation spezifisch wurde der Bereich Kind-Bezugsperson dokumentiert. Zusätzlich konnte in der Kategorie „Wechsel des Interaktionspartners“ festgehalten werden, ob das Kind eine Interaktion mit einer bislang nicht beteiligten Person eingegangen ist. Sollte das Interaktionsverhalten des Kindes einmal nicht beurteilbar gewesen sein-zum Beispiel wenn sich das Kind hinter einem Möbelstück versteckt hatte oder durch andere Personen gerade verdeckt wurde-konnte auch dies unter ,nicht beurteilbar“ dokumentiert werden.

Da der Blickkontakt ein bedeutsamer Aspekt der nonverbalen Kommunikation ist und vor allem bei schweigenden Kindern einen Großteil des kommunikativen Verhaltens ausmachen kann, wurde auch dies in den Beobachtungsbögen aufgenommen. In der Kategorie Blickkontakt wurde dokumentiert, ob dieser flüchtig, nicht vorhanden oder derzeit nicht beurteilbar war. Wurde im Bogen nicht angekreuzt, galt der Blickkontakt als unauffällig.

Typisch für selektiv mutistische Kinder ist das intensive beobachtende Verhalten (Garbani Ballnik, 2009). Saßen die Kinder beispielsweise im Raum und beobachteten das Spiel der anderen Kinder, konnte dies in der Kategorie Beobachtung festgehalten werden. Auch dies wurde in allen Bögen dokumentiert.

In den Situationen Freispiel und Essen wurde zudem beobachtet, in welcher Form der sozialen Konstellation-Einzel, Dyade oder Gruppe — sich das Kind derzeit befand. Hierbei genügte jedoch nicht die bloße Anwesenheit der Person. Vielmehr sollte der wechselseitige Bezug vom Kind mit anderen Personen berücksichtigt werden. Das heißt, dass das Kind in der Essenssituation zwar mit anderen Kindern am Tisch sitzen, jedoch völlig mit sich und seinem Frühstück beschäftigt sein konnte. Bezog es sich nicht auf die anderen Kinder am Tisch, wurde dies als Einzelsituation dokumentiert. Ein Beispiel für eine dyadische Situation war das 
Spiel des Kindes mit einer Erzieherin. Eine Gruppensituation war etwa ein Fangenspielen von mindestens drei Kindern.

In der Begrüßungssituation wurde die Kategorie Trennungssituation zusätzlich betrachtet. In dieser wurde festgehalten, ob das Trennungsverhalten auffällig oder unauffällig erschien. Als auffällig galt solches Verhalten des Kindes, was offensichtlich zeigte, dass es sich nur schwer von seiner Bezugsperson trennen konnte (z.B. Weinen, Anklammern, Rückzug oder Abwehrverhalten).

Für die Freispielsituation wurde das Spielverhalten des Kindes dokumentiert. Hier wurden die verschiedenen Spielformen Parallel-, Symbol-, Rollen-, Regel- und Bewegungsspiel sowie spielbegleitendes Sprechen unterschieden. Alle weiteren Beschäftigungsformen konnten mit dem Label „weitere Spielform“ kategorisiert werden.

Für die Gruppensituation wurde spezifisch die Partizipation des Kindes beobachtet. Hier wurde festgehalten, ob das Kind verbal oder nonverbal am Gruppenangebot teilnahm, ob es sich völlig herauszog und nicht mitmachte. Eine verbale Partizipation am Gruppenangebot konnte etwa auch das Mitsingen sein. Eine nonverbale Partizipation war etwa das Mitklatschen bei einem Lied.

In allen Bögen stand zusätzlich eine Kommentarzeile zur Verfügung, um Besonderheiten in der Beobachtung festhalten zu können (z.B. Unterbrechung der Beobachtung; Kind verlässt kurzzeitig den Raum).

Mit den Beobachtungsbögen als Dokumentationssystem wurde eine nichtteilnehmende, offene Beobachtung durchgeführt. Für die Situationen Freispiel, Essen und Gruppenangebot wurde jeweils die Methode der Zeitstichprobe gewählt. In der Freispielsituation wurde in einem Zeitintervall von 20 Sekunden zwischen Beobachtung und Dokumentation gewechselt. Das heißt, es wurde zunächst 20 Sekunden beobachtet, woraufhin 20 Sekunden Dokumentationszeit zur Verfügung standen. Es wurden insgesamt 30 Minuten beobachtet und dokumentiert-das heißt, 45 Beobachtungssequenzen wurden insgesamt im Freispiel bewertet. In der Essens- und Gruppensituation wurde aufgrund der geringeren Komplexität ein Zeitintervall von 15 Sekunden gewählt. 15 Sekunden Dokumentationszeit wurden als ausreichend erachtet. Es wurde der gesamte Zeitraum der jeweiligen Situation dokumentiert, maximal jedoch 30 Minuten. Aufgrund der Kürze der Zeit der Begrüßungssituation wurde dabei das Verfahren der Ereignisstichprobe angewendet. Tabelle 8 gibt einen Überblick über die gewählten Methoden, Zeitintervalle und die Gesamtzeit der Beobachtung für die vier Situationen. 
Tabelle 7

Überblick über die Kategorien der Beobachtungsbögen

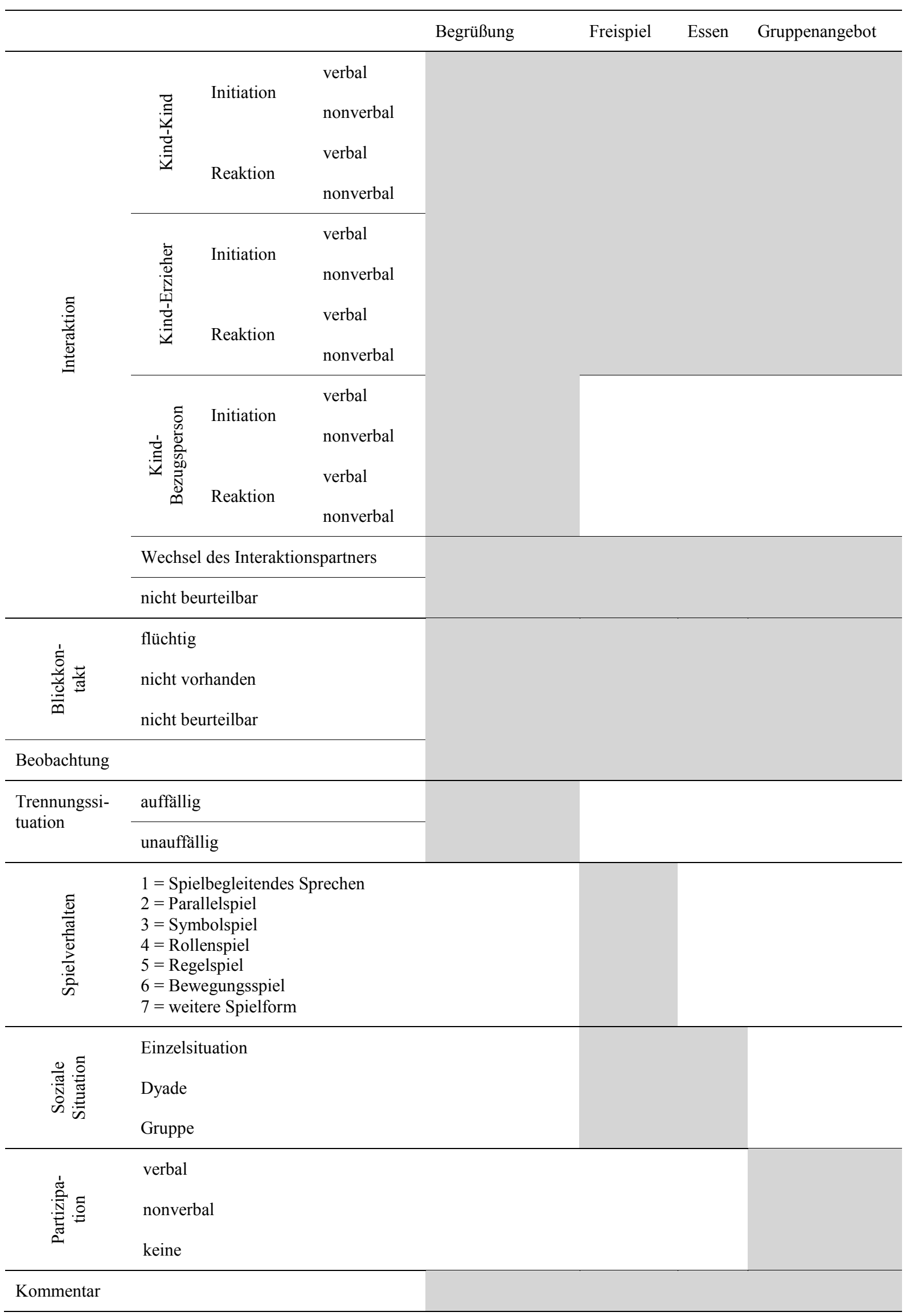


Grundsätzlich wurde in allen Situationen mit Zeitstichprobe pro Sequenz dokumentiert, ob das jeweilige Verhalten (siehe Kategorien in Tabelle 7) aufgetreten ist oder nicht. Das Auftreten einer Verhaltensweise wurde mit einem Kreuz markiert. Pro Kategorie war pro Sequenz nur ein Kreuz möglich. In einigen Kategorien (verbale Interaktionen und Spielverhalten) wurden zusätzlich Zahlen eingetragen, um die Art des Verhaltens zu konkretisieren.

Tabelle 8

Methode der Beobachtungen

\begin{tabular}{llll}
\hline & Beobachtungsplan & Zeitintervall & \multicolumn{1}{c}{ Beobachtungszeit } \\
\hline Begrüßungssituation & Ereignisstichprobe & - & gesamter Zeitraum \\
Freispiel & Zeitstichprobe & 20 Sekunden & 30 Minuten \\
Essen & Zeitstichprobe & 15 Sekunden & $\begin{array}{l}\text { gesamter Zeitraum } \\
\text { maximal jedoch 30 Minuten }\end{array}$ \\
Gruppenangebot & Zeitstichprobe & 15 Sekunden & $\begin{array}{l}\text { gesamter Zeitraum } \\
\text { maximal jedoch 30 Minuten }\end{array}$ \\
& & & \\
\hline
\end{tabular}

Als Zeitmesser wurden zwei Varianten gewählt. Je nach technischer Ausstattung der beobachtenden Studierenden wurde entweder die Smartphone-App „Repeat Timer Pro“ (Artem Lapitski, 2013) oder eine Audiodatei mit wiederkehrendem Audiosignal, die über ein MP3-Abspielgerät und In-Ear-Kopfhörer abgespielt wurde, verwendet. So wurden die Beobachterinnen $^{4}$ alle 20 (Freispiel) bzw. 15 Sekunden (Essen und Gruppenangebot) über einen Vibrationsalarm (App) oder das Audiosignal über den Wechsel von Beobachtungs- und Dokumentationsphase benachrichtigt.

Die Erläuterungen zu den einzelnen Beobachtungskategorien und zur Durchführung der Beobachtungen wurden in einem Manual festgehalten, um eine Basis für Beobachterschulungen zu haben. Dieses Manual wurde im Verlauf der Pilotstudie laufend angepasst (siehe Kapitel 3.3.5.2).

\section{Interaktionssettings}

Zur Erfassung des Interaktionsverhaltens mit einer unvertrauten Person wurden drei Untersuchungssettings mit unterschiedlichen Anforderungen entwickelt. Durch bestimmte Interaktionsangebote seitens der Testleiterin sollte ein spezifisches Interaktionsverhalten beim

\footnotetext{
${ }^{4}$ In Pilot- und Hauptstudie standen nur weibliche Studierende als Beobachterinnen zur Verfügung. Vor diesem Hintergrund wird im Folgenden lediglich die weibliche Form genutzt.
} 
Kind ausgelöst werden. Drei Anforderungsebenen wurden dabei abgedeckt: (1) einfache Reaktion, (2) Aufklären eines Missverständnisses und (3) Einfordern eines Spielzeuges, das heißt Initiation einer Interaktion. Um die Kinder zum Mitmachen zu motivieren, waren die Settings in das Rahmenthema Zoo eingebunden (für den genauen Ablauf der Interaktionssettings siehe Anhang E).

Setting 1 - einfache Reaktion: In diesem Spielsetting gab die Testleiterin den Impuls des Ballrollens. Es wurde eine einfache Reaktion—das Zurückrollen des Balls-vom Kind erwartet. Diese Anforderung war eingebettet in ein Seehundspiel. Testleiterin und Kind verwandelten sich in Seehunde und machten Kunststücke. Zur Unterstützung wurde dem Kind ein gemaltes Bild von einem Seehund gezeigt. In diesem Fall bestand das Kunststück aus dem Rollen des Balles mit der Flosse (entsprechend der Hand). Für jede gelungene Ausführung des Kunststückes erhielten Kind und Testleiterin einen Fisch als Belohnung (einlaminierte Papierfische).

Setting 2 - Missverständnissituation: In diesem Setting sollte eine Reaktion des Kindes auf ein von der Testleiterin erzeugtes Missverständnis evoziert werden. Als Rahmenhandlung diente hier der Ausbruch verschiedener Tiere aus ihren Gehegen. Dazu wurden insgesamt vier Tierarten ausgewählt, die möglichst gut visuell differenzierbar sind (Zebra, Eisbär, Giraffe und Nilpferde). Fünf Tiere jeder Art—-das heißt insgesamt 20 Tiere—standen anfangs wild durcheinander vor ihren Gehegen. Die Gehege waren so ausgestaltet und mit Fotos an der Vorderseite versehen, dass gut erkennbar war, welches Gehege zu welcher Tierart gehörte. Zudem wurde vor Beginn des eigentlichen Settings dem Kind genau erklärt, welches Tier in welches Gehege kommen sollte und welche besonderen Merkmale Tiere und entsprechende Gehege hatten. Nach dieser Erläuterung begann die Testleiterin mit dem Einsortieren der Tiere in die Gehege. Zunächst sortierte sie ein bis zwei Tiere korrekt ein. Anschließend wurde bewusst ein Fehler eingebaut (das Zebra wird in das Eisbärgehege gestellt). Insgesamt drei Fehler wurden im Verlauf des Settings gemacht: (1) Zebra in Eisbärgehege, (2) Giraffe in Nilpferdgehege und (3) Nilpferd in Giraffengehege. Über diese deutlichen Fehler sollte beim Kind eine Reaktion in dem Sinne ausgelöst werden, dass es in irgendeiner Weise auf den Fehler der Testleiterin aufmerksam machte.

Setting 3 - Actionfigur: Mithilfe einer so genannten Actionfigur-ein elektronisches Stoffspielzeug, welches auf Knopfdruck eine lustige Aktion macht—sollte das Kind zur Initiation einer Interaktion angeregt werden. Dazu wurde die Funktion der Actionfigur zu Beginn der Untersuchungssitzung einmal gezeigt. Das Spielzeug stand bei den Materialien für Setting 1. Vor dem eigentlichen Beginn des ersten Spielsettings machte die Testleiterin das Kind auf 
das Spielzeug aufmerksam und löste - für das Kind versehentlich—den Mechanismus der Figur aus. So sollte sichergestellt sein, dass das Kind die Funktion des Spielzeuges kannte und motiviert wurde, dies selbst noch einmal auszuprobieren. Die Figur wurde jedoch zunächst beiseite gestellt, verblieb aber im Sichtfeld des Kindes. Darüber sollte das Kind angeregt werden, in irgendeiner Weise der Testleiterin selbstinitiativ mitzuteilen, dass es noch einmal mit der Figur spielen möchte.

Die Durchführung der drei Untersuchungssettings erfolgte halbstandardisiert. Das heißt, dass der Ablauf und die grundlegenden Handlungen der Testleiterin sowie eine Vorlage für die sprachlichen Erläuterungen festgelegt waren. Da es sich hier um die Untersuchung des Interaktionsverhaltens handelte, wurde jedoch explizit auf Impulse des Kindes eingegangen, um keine Irritationen und Störung in der Interaktion hervorzurufen.

Die Analyse des kindlichen Verhaltens innerhalb der Interaktionssettings erfolgte im Rahmen der Hauptstudie anhand der aufgenommenen Videos lediglich grob hinsichtlich der zu evozierenden Verhaltensweisen. Eine ausführliche Analyse des Interaktionsverhaltens der Kinder ist geplant, jedoch nicht Inhalt dieser Studie. Für Setting 1 wurde kodiert, ob das Kind den Ball zurückgerollt hat oder nicht. Für Setting 2 wurde dokumentiert, ob, wie häufig und in welcher Form — verbal, nonverbal oder über eine direkte Handlung des Kindes — das Kind das Missverständnis aufgeklärt hat. Bei Setting 3 wurde kodiert, ob das Kind die Actionfigur eingefordert hat. Zusätzlich wurde dokumentiert, ob das Kind innerhalb der drei Interaktionssettings mit der Testleiterin gesprochen hat. Die so gewonnenen Informationen wurden vor allem für die Diagnosestellung des SM zum ersten und vierten Messzeitpunkt verwendet.

\subsubsection{Sprachliche Fähigkeiten}

Das Forschungsvorhaben verband zwei Aspekte miteinander: (1) den selektiven Mutismus und (2) die Mehrsprachigkeit, die beide für sich alleine bereits große Herausforderungen an den Untersucher darstellen. Die Kernproblematik selektiv mutistischer Kinder besteht darin, dass sie gegenüber fremden Personen nicht sprechen und zum Teil ihre Mitarbeit völlig einstellen. Viele Testverfahren zur Messung der kindlichen Kompetenzen erfordern jedoch eine deutliche Mitarbeit und häufig verbale Äußerungen des Kindes. Gerade innerhalb der Sprachdiagnostik besteht das Problem, dass zahlreiche Fähigkeiten nur über die Produktion der Kinder zu beurteilen sind. Eine Sprachproduktion der Kinder ist in einer Testsituation von einem selektiv mutistischen Kind jedoch nicht zu erwarten. Zusätzlich kam in diesem Forschungsvorhaben hinzu, dass ein Teil der Kinder mehrsprachig aufwächst. Für diese Kindergruppe liegen bislang nur sehr wenige Testverfahren vor, um die sprachlichen Fähigkeiten zu 
erfassen (Lüke, 2011). Die meisten standardisierten Verfahren, die für die deutsche Sprache vorliegen, sind nur an einsprachig deutschen Kindern erprobt und normiert, so dass keine Vergleichswerte für mehrsprachige Kinder vorliegen. Somit mussten für die Durchführung des Vorhabens zwei Probleme gelöst werden:

(1) Wie können sprachlich-kommunikative Fähigkeiten selektiv mutistischer Kinder sowohl für den Bereich der Rezeption als auch der Produktion erfasst werden?

(2) In welcher Form können die Deutschkompetenzen der mehrsprachig aufwachsenden Kinder beurteilt werden?

\subsubsection{Erfassung der rezeptiven Sprachfähigkeiten}

Zur Überprüfung der rezeptiven Sprachfähigkeiten liegen bereits zahlreiche standardisierte Testverfahren bzw. Untertests in umfassenderen Sprachtests zur Verfügung, zum Beispiel der TROG-D (Test zur Überprüfung des Grammatikverständnisses, Fox, 2007), der Untertest zum rezeptiven Wortschatz der Patholinguistischen Diagnostik (PDSS; Kauschke \& Siegmüller, 2009) oder der Untertest Sprachverstehen aus dem SETK 3-5 (Sprachentwicklungstest für drei- bis fünfjährige Kinder; Grimm, 2010). Als Aufgabe werden Zeige- oder Handlungsreaktionen des Kindes auf einen vorgegebenen Stimulus (Wort oder Satz) gefordert. Bei einfachen Bildauswahlverfahren auf Wortebene werden die Kinder dazu aufgefordert das passende Bild zu einem vorgesprochenen Wort zu zeigen. Damit wird der rezeptive Wortschatz des Kindes auf Wortebene überprüft. Bei Bildauswahlverfahren auf Satzebene, wie es der TROG-D ist, kommt neben der semantisch-lexikalischen Sprachebene eine syntaktisch-morphologische Dimension hinzu. Hier muss das Kind zu einem vorgesprochenen Satz das passende Bild auswählen. Zusätzlich zum reinen Wortwissen muss das Kind in dieser Aufgabe grammatikalisches Wissen anwenden und die Wörter in Beziehung zueinander bringen, um den Sinn des Satzes zu erfassen. Bei Aufgaben, die ein Ausagieren fordern (z.B. im Untertest Verstehen von Satzstrukturen der PDSS), werden dem Kind Handlungsanweisungen (Satzebene) vorgesprochen und es wird aufgefordert diese mit einem vorgegebenen Material nachzustellen. Auch hier werden wiederum über das reine Wortwissen hinaus syntaktischmorphologische Fähigkeiten des Kindes mit einbezogen.

Für die Diagnostik rezeptiver Sprachfähigkeiten von Kindern mit SM musste neben der validen Messung der formal linguistischen Fähigkeiten zusätzlich der Aspekt der Mitarbeit bzw. möglichen Verweigerung des Kindes in der Testsituation bedacht werden. Ein Testverfahren, welches das Ausagieren einer Handlungsanweisung erfordert, erschien somit wenig praktikabel. Hier war das Risiko hoch, dass das Kind nicht die sprachliche Leistung zeigen 
konnte, zu der es tatsächlich fähig wäre (siehe auch Kompetenz-Performanz-Diskussion zu sprachlichen Fähigkeiten bei schüchternen Kindern, Kapitel 2.1). In Forschung und therapeutischer Praxis wurden Bildauswahlverfahren zur Überprüfung des Sprachverstehens bereits erfolgreich bei Kindern mit SM eingesetzt (siehe etwa Cleator \& Hand, 2001; Kristensen, 2000; McInnes et al., 2004). Die Anforderung, lediglich ein Bild zu zeigen, scheint für die Kinder auch in Gegenwart einer wenig vertrauten Person sowohl im häuslichen (Cleator \& Hand, 2001) als auch im klinischen Kontext (McInnes et al., 2004) möglich und erzeugt keine Unterschiede in den Testergebnissen im Vergleich einer Testung durch die Eltern oder einen fremden Testleiter (Klein et al., 2013). Da in diesem Projekt nicht nur der rezeptive Wortschatz der Kindern, sondern vielmehr das allgemeine Sprachverständnis der Kinder erfasst werden sollte, wurde ein Testverfahren mit Bildauswahlaufgaben auf Satzebene benötigt. Der TROG-D (Fox, 2007) stellt hier das einzige Verfahren für das Deutsche dar, welches einerseits früh im Alter von 3;0 Jahren einsetzbar ist und andererseits einen breiten Altersbereich abdeckt. So können die Verbesserungen des Sprachverständnisses der Kinder auch im Längsschnitt abgebildet werden.

Das Testverfahren besteht aus 84 Items in 21 Fragenblöcken. Jeder Fragenblock überprüft eine gezielte grammatische Struktur des Deutschen-abhängig von Funktionswörtern, Flexionen oder Wortanordnungen im Satz. Die Testitems werden dem Kind auditiv vorgegeben. Aus vier Bildern muss es das jeweils Passende auswählen. Die drei Ablenker unterscheiden sich nur minimal in ihrer grammatischen oder lexikalischen Form vom Zielitem. Bezüglich des Wortschatzes wird im gesamten Test auf einfaches Wortmaterial zurückgegriffen. Über eine vorherige informelle Überprüfung des im Test verwendeten Vokabulars kann individuell überprüft werden, ob das Kind die Wörter rezeptiv und/oder produktiv beherrscht.

Die Fragenblöcke werden solange vorgegeben bis das Kind in fünf Blöcke aufeinanderfolgend mindestens einen Fehler gemacht hat. Der TROG-D wurde an monolingual Deutsch aufwachsenden Kindern standardisiert und normiert. Für mehrsprachige Kinder können somit die Normwerte des Testverfahrens nicht unkommentiert übernommen werden. Die Reliabilität des Testverfahrens ist mit einem Cronbach's alpha von .90 und einer Testhalbierungsreliabilität von .91 als sehr gut zu bewerten.

\subsubsection{Erfassung der produktiven Sprachfähigkeiten}

Die produktiven Sprachfähigkeiten würden optimaler Weise mit einem standardisierten Testverfahren geprüft, welches mehrere linguistische Ebenen umfasst und damit einen fundierten Überblick über die Sprachkompetenzen des Kindes gibt. Ein solches Verfahren 
arbeitet mit der Methode der Elizitierung sprachlicher Äußerungen des Kindes (siehe dazu auch Kauschke, 2012). Darüber können bestimmte sprachliche Strukturen gezielt überprüft werden. Beispiele für derartige Elizitierungsverfahren sind etwa Tests zur Überprüfung der Aussprache oder des produktiven Wortschatzes (z.B. Untertests Phonologie und Sprachproduktion der PDSS, Kauschke \& Siegmüller, 2009). Hier werden über das Benennen von Bildern entsprechende Sprachproduktionen des Kindes erhoben und je nach linguistischer Ebene ausgewertet.

Im klinischen Kontext ist jedoch die Wahrscheinlichkeit niedrig, dass Kinder mit SM bei einem solchen Testverfahren mitarbeiten (siehe etwa Kristensen, 2000). Die Durchführung eines standardisierten Testverfahrens durch einen Elternteil kann eine gute Möglichkeit sein, um tatsächlich die gewollten Sprachproduktionen des Kindes zu erhalten (Klein et al., 2013). Jedoch benötigt die Durchführung eines solchen Testverfahrens einerseits ein Wissen um Testdiagnostik und die Bedeutsamkeit der standardisierten Durchführung. Andererseits erfordert sie Vertrautheit mit dem Testmaterial und Übung in der Testdurchführung. Dies sind hohe Anforderungen an Eltern. Insbesondere im Hinblick auf die mehrsprachigen Familien, die am Projekt teilnahmen, erschien diese Variante nicht sinnvoll einsetzbar.

Eine andere Möglichkeit der Beurteilung kindlicher Sprachproduktionen ist die Spontansprachanalyse. Gegenstand einer solchen Analyse sind natürliche Äußerungen eines Kindes. In einer alltäglichen Situation werden dazu Audio- oder Videoaufnahmen des Kindes gemacht. Im Nachhinein können diese transkribiert und hinsichtlich bestimmter linguistischer Parameter ausgewertet werden. Je nach zu untersuchendem Phänomen sollte Länge und Häufigkeit der Spontansprachaufnahmen festgelegt werden. Unregelmäßige oder einmalige Aufnahmen sind nicht repräsentativ für die sprachlichen Kompetenzen des Kindes (Tomasello \& Stahl, 2004). Sie decken nur einen geringen Anteil der spontansprachlichen Äußerungen eines Kindes im Gesamt ab. Seltene Phänomene tauchen eventuell in den Aufnahmen gar nicht auf. Die Abwesenheit einer bestimmten linguistischen Struktur kann jedoch nicht dahingehend interpretiert werden, dass das Kind diese noch nicht produzieren kann. Vielmehr hängen die sprachlichen Produktionen des Kindes stark von der Situation (zum Beispiel verwendetes Material, Spielkontext etc.) sowie den Sprachproduktionen des Erwachsenen ab. Um einen repräsentative Einschätzung der sprachlichen Kompetenzen des Kindes zu erlangen, wird die Erstellung dichter Korpora empfohlen (Kauschke, 2012). Das heißt es werden über einen langen Zeitraum häufig Aufnahmen erstellt, um ein möglichst lückenloses Bild der sprachlichen Fähigkeiten und Entwicklung des Kindes zu erhalten. Auch wenn dichte Korpora eine bessere Einschätzung der sprachlichen Fähigkeiten der Kinder ermöglichen, muss die aufzuwendende 
Transkriptionszeit bedacht werden (10 bis 20 Stunden je einer Stunde aufgenommenem Sprachmaterial; Tomasello \& Stahl, 2004). Für die Festlegung der Häufigkeit und Länge der zu analysierenden Spontansprachaufnahmen müssen somit folgende Kriterien beachtet werden: (1) zu untersuchendes linguistisches Phänomen, (2) Häufigkeit des Auftretens dieses Phänomens in der kindlichen Sprache und (3) Transkriptionszeit. Gerade in der Zusammenarbeit mit Eltern muss jedoch noch ein weiterer Aspekt berücksichtigt: die Realisierbarkeit der Anforderung durch die Eltern und die Integration dieser in den Familienalltag.

Trotz der zahlreichen Schwierigkeiten und weniger aussagekräftigen Daten im Vergleich zu Ergebnissen aus standardisierten und normierten Sprachtests erschien die Spontansprachanalyse im Kontext des SM praktikabel. Sie bot einen entscheidenden Vorteil: die Aufnahmen könnten leicht von den Eltern oder anderen vertrauten Personen im häuslichen Kontext des Kindes gemacht werden. Im Wohnumfeld sprechen selektiv mutistische Kinder mit vertrauten Personen unbefangen und können ihre tatsächlichen sprachlichen Kompetenzen zeigen. Im Gegensatz zur Durchführung standardisierter Testverfahren durch die Eltern benötigen diese bei einer Spontansprachaufnahme keine aufwändige Einarbeitung in den Test. Die Anforderung an die Eltern können somit relativ gering gehalten werden.

Um die Spontansprachaufnahmen der Kinder möglichst vergleichbar zu machen, wurde eine Spielsituation konstruiert, in denen spontane Äußerungen der Kinder durch die Eltern evoziert werden können. Es wurde ein entwicklungstypisches Spielformat—das Rollenspiel— gewählt. Als Spielthema wurde „Arzt“ ausgesucht, da dies einerseits eine von allen Kindern bereits real erlebte Situation sein sollte und andererseits relativ geschlechtsunabhängig ist. Ausgangspunkt der Geschichte waren für alle Kinder die Bauchschmerzen des Bären „Bodo“, der von den Eltern gespielt bzw. als Bezugsperson begleitet werden sollte. Die Rolle des Arztes sollte vom Kind übernommen werden, so dass die Eltern die Möglichkeit hatten, das Kind nach seinen Handlungen zu fragen (z.B. „Was machst du denn jetzt?“, „Warum muss der Bär die Medizin nehmen?“). Insgesamt 15 Minuten des Spiels sollten über ein Diktiergerät von den Eltern aufgezeichnet werden.

Zur Durchführung der Spontansprachaufnahmen im häuslichen Rahmen erhielten die Eltern beim ersten Termin an der Universität alle notwendigen Materialien: die Spielmaterialien Teddybär und Arztkoffer sowie ein digitales Aufnahmegerät (Sony ICD-PX312). Zusätzlich lag schriftliches und videobasiertes Anleitungsmaterial bei. Auf einer DIN A4-Seite wurde genau erläutert, mit welchen Materialien und wie die spontansprachlichen Äußerungen evoziert und aufgezeichnet werden sollten. Es wurden beispielhaft Fragen für die Situation aufgeführt, die entsprechende Äußerungen des Kindes erzeugen sollten (siehe Anhang F). 
Andererseits wurde ein Beispielvideo mit einem dreijährigen Mädchen gedreht, welches auf DVD den Eltern bereitgestellt wurde. Hier wurden beispielhaft der Ablauf der Spielsituation sowie der Umgang mit Problemen dargestellt (hier vor allem das Entdecken des Aufnahmegerätes durch das Kind). Über beide Anleitungsvarianten — schriftlich und videogestützt—sollte sichergestellt werden, dass die Eltern die Aufgabenstellung der Situation nachvollziehen und sicher umsetzen konnten. Bei den mehrsprachigen Familien wurde jeweils mit den Eltern überlegt, welches Familienmitglied über ausreichende Deutschkompetenzen verfügte, um die Aufgabe mit dem Kind durchzuführen.

\subsubsection{Sprachdiagnostik bei mehrsprachigen Kindern}

Die Sprachdiagnostik bei mehrsprachigen Kindern gestaltet sich insgesamt schwierig, da es bislang keine standardisierten Testverfahren gibt, welche die sprachlichen Fähigkeiten bi- oder multilingualer Kinder valide messen können (Bedore \& Peña, 2008; Lüke, 2011). Dies resultiert vor allem aus der großen Heterogenität mehrsprachiger Entwicklungsverläufe, die durch unterschiedliche Sprachkombinationen, simultanen und sukzessiven Erwerbsprozessen und der Menge der zu erwerbenden Sprachen bedingt sind. Sprachtests werden nahezu ausschließlich an einsprachig aufwachsenden Kindern normiert, was einen Vergleich bi- oder multilingualer Kinder mit der Normierungsstichprobe nur bedingt zulässt. So können durchschnittliche Testwerte etwa dahingehend interpretiert werden, dass ein mehrsprachiges Kind in jedem Fall keine Auffälligkeiten in der deutschen Sprache zeigt. Unterdurchschnittliche Testwerte können hingegen nicht uneingeschränkt auf eine auffällige Sprachentwicklung zurückführt werden. Für die Abgrenzung mangelnder Deutschkenntnisse aufgrund eines geringen Inputs und manifesten sprachlichen Auffälligkeiten müssen die sprachlichen Fähigkeiten

der anderen Sprache berücksichtigt werden. Eine umschriebene Sprachentwicklungsstörung zeigt sich gleichermaßen in Erst- und Zweitsprache (Bedore \& Peña, 2008). Ebenso muss die Quantität und Qualität des sprachlichen Inputs in den jeweiligen Sprachen berücksichtigt werden, um die sprachlichen Leistungen des Kindes interpretieren zu können. Die Sprachkompetenzen des Kindes im Deutschen müssen immer in Relation zum Entwicklungskontext gesetzt werden. Damit kommt der Anamnese innerhalb der Sprachdiagnostik bei mehrsprachigen Kindern eine besondere Rolle zu.

\section{Mehrsprachen-Kontexte}

$\mathrm{Zu}$ Erfassung des sprachlichen Inputs der mehrsprachigen Kinder wurde der Mehrsprachen-Kontext (Ritterfeld \& Lüke, 2012) genutzt. Dies ist ein Verfahren zur Erfassung der 
Inputbedingungen von mehrsprachig aufwachsenden Kindern. Über eine grafische Darstellung werden im Gespräch mit den Eltern die sprachlichen Sozialisationsbedingungen des Kindes erhoben. Sowohl für den familiären als auch für den institutionellen Kontext soll erfasst werden, mit welchen Sprachen das Kind in Kontakt kommt und vor allem welche Sprachen es selbst verwendet. Dazu werden zunächst für die Familie bzw. den Haushalt für das Kind relevante Personen eingetragen. Es wird jeweils abgefragt, welche Sprache/n der Interaktionspartner mit dem Kind spricht und in welcher/n Sprache/n das Kind antwortet. Werden zwischen zwei Interaktionspartnern zwei Sprachen gesprochen, kann grafisch der ungefähre Anteil der jeweiligen Sprache dargestellt werden. Zusätzlich wird für die vom Kind besuchten Institutionen (z.B. Kita, Schule, Kulturverein) sowie die nähere Wohnumgebung festgehalten werden, welches Sprachangebot das Kind dort jeweils erhält. Abschließend wird erfragt welche Medien (Bücher, Hörspiele, Fernsehen usw.) und in welcher/n Sprache/n das Kind diese Angebote nutzt. Für die vorliegende Studie werden einerseits die vom Kind gesprochenen bzw. sich gerade im Erwerb befindenden Sprachen sowie die Sprache, in welcher das Kind den größten Input erhält, ermittelt. Zusätzlich wurde über das Interview mit den Eltern der Erwerbsbeginn der einzelnen Sprachen erfasst, um aufbauend die Kinder nach simultanem und sukzessivem Zweitspracherwerb zu klassifizieren (siehe dazu auch Kapitel 2.3.2).

\section{Beurteilung der rezeptiven Sprachfähigkeiten mehrsprachiger Kinder}

Wie oben bereits erläutert wurden die rezeptiven Sprachfähigkeiten der Kinder mit dem TROG-D (Fox, 2007) erhoben. Auch dieses Testverfahren wurde ausschließlich an monolingual Deutsch aufwachsenden Kindern normiert. Dennoch wurden zur Vergleichbarkeit der Kinder die Altersnormen auch bei den mehrsprachigen Kindern angelegt. Es ging in der vorliegenden Studie nicht darum, Spracherwerbsprobleme bei den mehrsprachigen Kindern zu diagnostizieren, so dass unterdurchschnittliche Leistungen nicht als Störung der rezeptiven Sprachfähigkeiten des Kindes klassifiziert wurden. Vielmehr sollte über den Vergleich mit der Altersnorm ggf. die Diskrepanz der sprachlichen Fähigkeiten der mehrsprachigen Kinder im Vergleich zu ihren monolingualen Peers aufgezeigt werden.

\subsubsection{Messung der kindlichen Ängstlichkeit}

Die kindlichen Ängste wurden mit dem Bochumer Angstverfahren für Kinder im Vorschul- und Grundschulalter (BAV 3-11, Mackowiak \& Lengning, 2010) erfasst. Das BAV ist ein standardisiertes Verfahren, welches über Selbst- und Fremdeinschätzungen entwicklungstypische Ängste (soziale Ängste, kognitive Ängste, Angst vor Verletzung und Phobien) bei 
Kindern im Alter von 3 bis 11 Jahren erhebt. Die Selbsteinschätzung der Kinder erfolgt über eine mündliche Befragung und kann ab dem Alter von vier Jahren durchgeführt werden. Die schriftliche Elternbefragung kann bereits bei Kindern im Alter von drei Jahren eingesetzt werden, so dass diese für die vorliegende Studie ausgewählt wurde. Von einer Selbsteinschätzung der Kinder wurde abgesehen, da diese (1) nicht bei allen Probanden aufgrund des Altersbereiches hätte durchgeführt werden können und (2) sie verbale Reaktionen der Kinder gefordert hätte. Beide Bögen sind unabhängig voneinander einsetz- und auswertbar. Im Folgenden wird lediglich auf den Elternfragebogen des BAV eingegangen.

Der Elternfragebogen besteht aus 26 Items, die typische Situationen beschreiben, in denen Kinder sich ängstlich zeigen können. Auf einer fünfstufigen Ratingskala von 1 (positiv) bis 5 (sehr ängstlich) sollen die Eltern zunächst einschätzen, wie sich ihr Kind in der Situation fühlt. In einem zweiten Schritt werden die Eltern gebeten, eine kurze Beschreibung zum Verhalten der Kinder in dieser Situation zu geben. Am Ende des Fragebogens werden die Eltern zudem gefragt, welche körperlichen Angstreaktionen ihr Kind zeigt. Ihnen stehen dafür 14 vorgegebene Möglichkeiten (z.B. Zittern, Herzklopfen, Weinen) zur Verfügung.

Zur Auswertung werden zunächst die Einschätzungen der Eltern auf der Ratingskala umkodiert. Die Stufen positiv und neutral werden zu 0 umkodiert. Lediglich die angstbezogenen Stufen erhalten einen numerischen Wert $(1=$ ein wenig ängstlich, $2=$ etwas ängstlich, $3=$ sehr ängstlich). Der Elternfragebogen kann skalenweise nach Angsttypen (soziale Ängste, kognitive Ängste, Angst vor Verletzung und Phobien) sowie im Gesamt ausgewertet werden. Es werden für beide Geschlechter und für verschiedene Altersgruppen Normwerte in Form von Prozenträngen und T-Werten bereitgestellt. Hohe Werte spiegeln dabei ein erhöhtes Maß der Ängstlichkeit wider.

Die Handlungsbeschreibungen der Eltern können zudem hinsichtlich verschiedener Regulationsstrategien qualitativ ausgewertet werden. Da diese im Rahmen der vorliegenden Studie nicht fokussiert wurden, wird das Auswertungsverfahren nicht näher beschrieben und auf das Manual des BAV verwiesen (Mackowiak \& Lengning, 2010).

Die interne Konsistenz der Gesamtversion des Elternfragebogens ist für den Kindergartenbereich in der Normierungsstichprobe mit einem Cronbachs alpha von .72 als zufriedenstellend zu bewerten. Auf den Einzelskalen wurden Werte von unter .70 erreicht, so dass die Subskalen eine fragliche Reliabilität aufweisen. In der Stichprobe der Hauptstudie ergab sich zum ersten Messzeitpunkt für den Gesamtbogen eine vergleichbare interne Konsistenz mit einem Cronbachs Alpha von .74. Die Subskalen erreichten auch in dieser Stichprobe keine zufriedenstellenden Werte zum ersten Messzeitpunkt, so dass für die Analysen nur der 
Gesamtwert des BAV einbezogen und von einer Auswertung auf Subskalenebene abgesehen wurde.

\subsubsection{Erfassung der kulturellen Adaptation der Eltern}

Die kulturelle Adaptation der Eltern wurde mit der Frankfurter Akkulturationsskala (FRAKK20; Bongard, o.J.; Bongard et al., 2007) erfasst. Die FRAKK20 ist ein Selbstbeurteilungsfragebogen für Erwachsene. Auf einer siebenstufigen Likert-Skala von 0 (trifft überhaupt nicht zu) bis 6 (trifft vollständig zu) soll die Person einschätzen, wie sehr sie der jeweiligen Aussage zustimmt. Das Instrument basiert auf dem Konstrukt der Akkulturation nach Berry (2003; siehe Kapitel 2.4.2). Aus diesem Grund werden sowohl Items zur Heimat- als auch zur Umgebungskultur vorgegeben, um für beide Kulturen die individuelle Bedeutsamkeit im Leben der Person erfassen zu können. Entsprechend kann der Fragebogen auf zwei Skalen-(1) Orientierung an der Herkunftskultur (HK) und (2) Orientierung an der Aufnahmekultur (AK) — sowie einem Gesamtwert, dem so genannten Akkulturationsindex (AI) ausgewertet werden. Die beiden Einzelskalen spiegeln die Rolle der jeweiligen Kultur im Leben der Person wider. Über den AI lässt sich eine Aussage zum Gesamtmaß des kulturellen Wandels der Person treffen. Bislang liegen noch keine Normwerte vor. Jedoch wurden von Bongard (o.J.) Vergleichswerte unterschiedlicher Migrationsgruppen (iranisch, südkoreanisch, bosnisch, türkisch) zur Verfügung gestellt. Sowohl die Einzelskalen als auch der Gesamtbogen erreichen laut Bongard (o.J.) gute interne Konsistenzen (Cronbachs Alpha zwischen .85 und .89).

Für den Fragebogen liegen bereits Versionen in acht Sprachen (Deutsch, Chinesisch, Englisch, Französisch, Spanisch, Polnisch, Russisch und Türkisch) vor, so dass den Eltern bei nicht ausreichenden Deutschkompetenzen ggf. eine muttersprachliche Version des Bogens vorgegeben werden konnte.

\subsection{Pilotstudie}

Ziel der Pilotstudie war es, die neu entwickelten Instrumente sowie die Testung mit den Kindern auf ihre Durchführbarkeit hin zu überprüfen. Alle Datenerhebungen der Pilotstudie fanden im Zeitraum von Ende Juni bis Ende August 2012 statt. 


\subsubsection{Probanden}

Die Rekrutierung der Probanden für die Pilotstudie fand einerseits über das Sprachtherapeutische Ambulatorium $(\mathrm{SpA})$ der TU Dortmund sowie über Kitas aus Dortmund statt, welche bereits mit dem SpA zusammengearbeitet haben. Die Sprachtherapeutinnen des SpA wurden danach gefragt, ob sie derzeit Kindergartenkinder mit SM hatten, die zum Zeitpunkt der Studie in der Kita noch konsequent schwiegen. Zusätzlich wurde nach mutismuserfahrenen Kitas in Dortmund gefragt. So sollte vorab sichergestellt werden, dass die Mitarbeiterinnen der Einrichtungen zumindest den Verdacht auf einen SM stellen konnten. Die so ermittelten zehn Kitas wurden daraufhin telefonisch von der Autorin kontaktiert und über Ziele und Inhalte der Pilotstudie aufgeklärt. Insgesamt drei Kitas erklärten sich zur Teilnahme an der Studie bereit. In jeder Einrichtung wurde zum Zeitpunkt der Pilotstudie ein schweigendes Kind betreut. $\mathrm{Zu}$ diesem Kind wurde von der Bezugserzieherin des schweigenden Kindes ein sprechendes Kontrollkind ausgewählt, welches möglichst gleichen Geschlechtes und entsprechend ein- oder mehrsprachig war. Den Kitas wurden Elterninformationen für alle potenziellen Familien zur Verfügung gestellt, in denen die Eltern über die Inhalte der Pilotstudie aufgeklärt und in einem ersten Schritt um ihr Einverständnis zur Beobachtung der Kinder in der Kita sowie der Kontaktaufnahme durch die Autorin zwecks weiterer Terminabsprache gebeten wurden. In einem zweiten Schritt wurden die Eltern telefonisch kontaktiert und ein Termin zur Testung der Kinder in den Räumlichkeiten der TU Dortmund ausgemacht.

Über diesen Rekrutierungsprozess konnten insgesamt sechs Kinder-drei schweigende und drei sprechende Kontrollkinder-für die Pilotierung der Beobachtungsbögen rekrutiert werden. Vier dieser Familien willigten zusätzlich in die Testung der Kinder in den Räumlichkeiten des SpA ein. Ein weiteres schweigendes Kind konnte über eine Diagnostikanfrage im SpA gewonnen werden. Aufgrund der relativ großen räumlichen Distanz vom Wohnort zu Dortmund konnte zu diesem Kind jedoch kein Kontrollkind in der gleichen Kita gewonnen werden.

An der Pilotstudie nahmen somit insgesamt sieben Kinder — vier schweigende und drei sprechende-im Alter von 3;8 bis 5;3 teil. Vier Kinder (S2, S4, K2 und K4) wuchsen mehrsprachig auf. Für zwei Kinder (S4 und K4) lagen keine weiteren Informationen zu den gesprochenen Sprachen vor, da es beiden Familien nicht möglich war Termine an der Universität wahrzunehmen. Hier war lediglich durch die Aussagen der Erzieherinnen bekannt, dass die Kinder zuhause eine andere Sprache als Deutsch sprachen. Die beiden anderen Kinder wuchsen mit den Kombinationen Tamil-Deutsch (S2) und Deutsch-Arabisch-Albanisch (K2) auf. Die restlichen drei Kinder (S1, S3 und K1) waren einsprachig Deutsch. Ein Kind (S1) befand 
sich zum Zeitpunkt der Pilotstudie aufgrund des SM in sprachtherapeutischer Behandlung. Bei zwei Kindern (S2 und S3) fanden die Abklärung des SM sowie eine ausführliche Elternberatung im Rahmen der Pilotstudie statt. Bei beiden Kindern konnte der SM nach DSM-IVKriterien (siehe Kapitel 2.1) bestätigt werden. Da beim vierten schweigenden Kind (S4) keine Testung stattfand, konnte ein SM weder bestätigt noch ausgeschlossen werden. Tabelle 9 zeigt alle bedeutsamen Informationen zu den Probanden der Pilotstudie im Überblick.

Tabelle 9

Probanden der Pilotstudie

\begin{tabular}{lccccccc}
\hline & S1 & K1 & S2 & K2 & S3 & S4 & K4 \\
\hline Alter & $5 ; 0$ & $5 ; 3$ & $4 ; 1$ & $4 ; 2$ & $3 ; 8$ & $4 ; 4$ & $4 ; 6$ \\
Geschlecht & weiblich & weiblich & weiblich & weiblich & weiblich & männlich & weiblich \\
$\begin{array}{l}\text { gesproche- } \\
\text { ne Spra- } \\
\text { chen }\end{array}$ & Deutsch & Deutsch & $\begin{array}{c}\text { L1: Tamil } \\
\text { L2: } \\
\text { Deutsch }\end{array}$ & $\begin{array}{c}\text { L1: Deutsch } \\
\text { L2: Arabisch } \\
\text { L3: Alba- } \\
\text { nisch }\end{array}$ & Deutsch & mehr- & mehr- \\
Diagnose & ja & nein & ja & nein & ja & sprachig \\
SM & & & & & & & nein \\
\hline
\end{tabular}

\subsubsection{Vorgehen}

Die Pilotstudie bestand aus insgesamt zwei Teilen: (1) die Beobachtungen in den Kitas und (2) die Testungen der Kinder mit anschließender Elternberatung. Der Ablauf der beiden Studienteile ist in Abbildung 3 und Abbildung 4 im Überblick dargestellt.
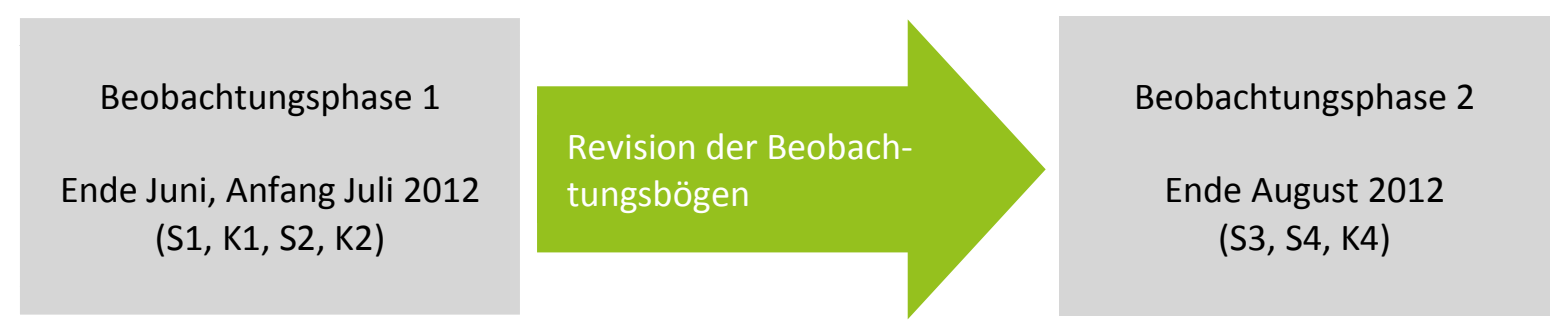

Abbildung 4. Ablauf der Beobachtungen in der Pilotstudie

Die Beobachtungen fanden in zwei Phasen statt. In der ersten Phase wurden insgesamt vier Kinder innerhalb von zwei Wochen Ende Juni bzw. Anfang Juli 2012 beobachtet. Anschließend fand eine erste Überarbeitung der Beobachtungsbögen statt. Die zweite Phase folgte Ende August 2012. Die Kinder wurden in sechs Fällen von zwei geschulten Studentinnen, 
in einem Fall aus organisatorischen Gründen nur von einer Studentin an einem Vormittag in der Einrichtung beobachtet. In den Beobachtungsphasen wurden in allen Kitas zudem DortMuS-Kita an die Bezugserzieherinnen verteilt und diese gebeten die Bögen auszufüllen und sie an die Studierenden beim Beobachtungstermin wieder abzugeben.

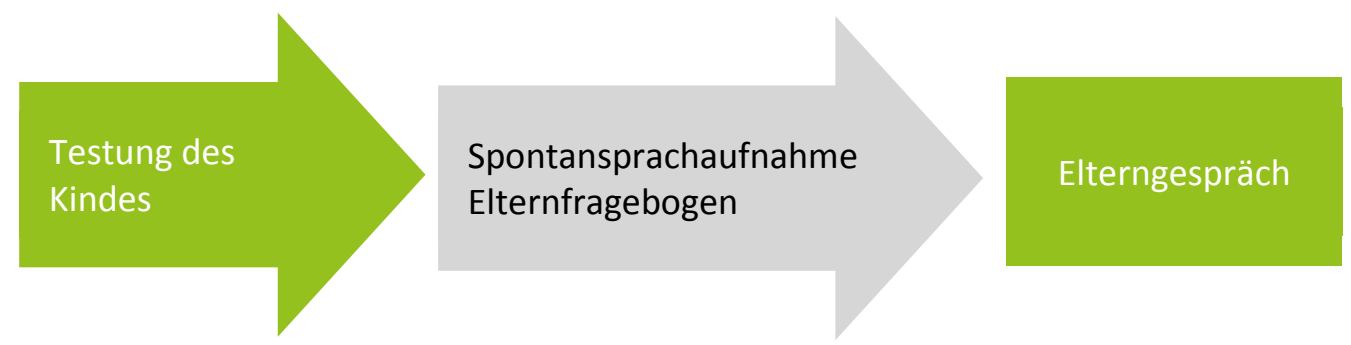

Abbildung 5. Ablauf der Pilotstudie

Die Testungen der Kinder fanden in Einzelsitzungen in den Räumlichkeiten des SpAs an der TU Dortmund statt. Die etwa einstündigen Sitzungen wurden mit zwei Kameras mit Wissen und Einverständnis der Eltern aufgezeichnet. Falls notwendig war ein Elternteil im Testraum anwesend. Die Eltern wurden jedoch vorab gebeten, sich so zurückhaltend wie möglich zu verhalten und nicht in die Testsituation einzugreifen. Die Sitzung mit dem Kind setzte sich aus zwei Teilen zusammen. Zunächst wurden die so genannten Interaktionssettings (siehe Kapitel 3.2.3) durchgeführt, in denen das Interaktionsverhalten der Kinder gegenüber einer unvertrauten Person erfasst wurde. Im zweiten Teil wurde der TROG-D durchgeführt.

Im Anschluss an die Sitzung wurde ein zweiter Termin mit den Eltern für eine abschließende Elternberatung vereinbart. Zusätzlich wurden die Eltern gebeten in der Zwischenzeit zuhause den Elternfragebogen auszufüllen sowie die Spontansprachaufnahmen des Kindes anzufertigen. Dazu wurde den Eltern entsprechendes Material (Spielmaterial und ein Aufnahmegerät, siehe Kapitel 3.2.4.2) mitgegeben. Im abschließenden Elterngespräch wurde mit den mehrsprachigen Familien der Mehrsprachenkontext ausgefüllt. Alle Eltern erhielten ausführliche Informationen zum beobachteten Verhalten ihres Kindes sowie zu den Ergebnissen des TROG-D. Gegebenenfalls wurden die Eltern hinsichtlich erforderlicher therapeutischer Maßnahmen beraten und an entsprechende Einrichtungen weiterverwiesen. Im Anschluss wurden die Eltern zur Durchführbarkeit der Spontansprachaufnahmen im häuslichen Bereich sowie zur Angemessenheit des Aufwands zum Ausfüllen des Elternfragebogens befragt.

\subsubsection{Instrumente}

Tabelle 10 gibt einen Überblick über die in der Pilotstudie verwendeten Instrumente. Für eine ausführliche Beschreibung der einzelnen Verfahren wird auf Kapitel 3.2 verwiesen. 
Tabelle 10

Verwendete Instrumente in der Pilotstudie

\begin{tabular}{lll}
\hline \multicolumn{1}{c}{ Instrument } & \multicolumn{1}{c}{ Erfassungsbereich } & \multicolumn{1}{c}{ Quelle } \\
\hline Elternfragebogen & $\begin{array}{l}\text { (1) anamnestische und soziodemografische } \\
\text { Daten } \\
(2) \text { (Sprech-)Verhalten der Kinder }\end{array}$ & selbst entwickelt \\
$\begin{array}{l}\text { Mehrsprachenkon- } \\
\text { text* }\end{array}$ & sprachliche Inputbedingungen & $\begin{array}{l}\text { Mehrsprachen-Kontexte } \\
\text { (Ritterfeld \& Lüke, 2012) }\end{array}$ \\
DortMuS-Kita & $\begin{array}{l}\text { (Sprech-)Verhalten des Kindes im Kontext der } \\
\text { Kita }\end{array}$ & selbst entwickelt \\
TROG-D & rezeptives Grammatikverständnis im Deutschen & $\begin{array}{l}\text { Test zur Überprüfung des } \\
\text { Grammatikverständnisses }\end{array}$ \\
& & $\begin{array}{l}\text { (Fox, 2007) } \\
\text { selbst entwickelt }\end{array}$ \\
$\begin{array}{l}\text { Beobachtungsbö- } \\
\text { gen }\end{array}$ & $\begin{array}{l}\text { Interaktionsverhaltens in den Situationen Frei- } \\
\text { spiel, Gruppen- und Essenssituation sowie Be- } \\
\text { grüßung im Kontext Kita }\end{array}$ & \\
\hline *nur bei mehrsprachigen Familien &
\end{tabular}

*nur bei mehrsprachigen Familien

\subsubsection{Auswertung}

Ziel der Pilotstudie war es vor allem, die neu entwickelten Instrumente sowie die Untersuchungssituation mit dem Kind auf ihre Durchführbarkeit hin zu analysieren. Aus diesem Grunde werden die Rückmeldungen der beteiligten Personen beschreibend dargestellt.

Die Auswertung der Spontansprachaufnahmen erfolgte mit dem Annotationsprogramm ELAN (Max Planck Institute for Psycholinguistics, The Language Archive, 2013; Wittenburg, Brugman, Russel, Klassmann \& Sloetjes, 2006). Für die Spontansprache wurden die Gesamtanzahl der Äußerungen des Kindes sowie die mittlere Äußerungslänge (MLU) ermittelt. Aufgrund der kleinen Stichprobe wurden keine weiteren statistischen Analysen durchgeführt. Es werden lediglich deskriptive Werte für die einzelnen Kinder berichtet.

Für die Beobachtungsbögen zum Interaktionsverhalten der Kinder im Kita-Kontext wurde die Interrater-Reliabilität mit dem ungewichteten Cohens Kappa berechnet.

\subsubsection{Ergebnisse}

Die Ergebnisdarstellung erfolgt strukturiert nach den beiden Phasen der Pilotstudie zunächst für die Beobachtungen in der Kita sowie die Erzieherfragebögen DortMuS-Kita. Darauf folgend werden die Ergebnisse der direkten Untersuchungen des Kindes sowie der Spontansprachaufnahmen und des Elternfragebogens dargestellt. 


\subsubsection{Beobachterübereinstimmung}

Die Beobachterübereinstimmung konnte für die Beobachtungen von insgesamt sechs der sieben Kinder für alle Beobachtungssituationen berechnet werden. Bei allen Beobachtungsbögen konnten gute bis sehr gute Werte der Interrater-Reliabilität erreicht werden. Mit einem $K=0.68$ ist die Interrater-Reliabilität in der Begrüßungssituation am geringsten ausgefallen. In der Freispielsituation konnte eine Beobachterübereinstimmung von $K=0.72$ er-

reicht werden. Die Werte der Gruppen- und Essenssituation sind mit $K_{\text {Gruppe }}=0.83$ und $K_{\text {Essen }}$ $=0.87$ als sehr gut einzustufen. Auch wenn die Interrater-Reliabilität der Begrüßungssituation als durchaus gut einzustufen ist, fällt in der Feinanalyse der einzelnen Beobachtungskategorien auf, dass sich die Urteile der beiden Beobachterinnen deutlich voneinander unterschieden. Einzelne Kategorien erreichten lediglich leichte bis mittelmäßige Werte von $K=0.26$ bis $K=0.56$. Darunter waren vor allem die Kategorien der Interaktion zwischen Kind und Bezugsperson sowie die Bewertung des Trennungsverhaltens in auffällig vs. unauffällig, die besonders spezifisch für die Begrüßungssituation war.

Auch in der Freispielsituation erreichten einzelne Beobachtungskategorien nur mittelmäßige Übereinstimmungen von $K=0.49$ bis $K=0.54$. Hier zeigte sich im differenzierten Vergleich der beiden Beobachtungsphasen, dass sich die Interrater-Reliabilität durch die Veränderungen der Beobachtungsbögen bzw. Beobachtungsanweisungen nach der ersten Beobachtungsphase (siehe dazu später Kapitel 3.3.5.2 und 3.3.6.1) verbesserte. Als Beispiel sei hier die Kategorie verbale Reaktion zwischen zwei Kindern genannt. In Phase 1 wurde hier eine Interrater-Reliabilität von $K=0.44$ erreicht. In Phase 2 konnte diese auf einen guten Wert von $K=0.67$ gesteigert werden. Ähnliches gilt für die Gruppen- und Essensituation. Für eine ausführliche Darstellung der Interrater-Reliabilität aller Beobachtungskategorien siehe Tabelle A-3 im Anhang sowie Bieganski (2012) und Rehr (2012).

\subsubsection{Durchführbarkeit und Fehlerquellen der Beobachtungen}

Die beobachtenden Studierenden berichteten von einer insgesamt guten Durchführbarkeit der Beobachtungen im Kita-Kontext. Innerhalb der ersten Beobachtungsphase konnten Fehlerquellen oder schwer beobachtbare Kategorien identifiziert und die Beobachtungsbögen dahingehend verändert werden. Beispielhaft ist die Kategorie Blickkontakt zu nennen. Die Beobachtung des Blickkontaktes war stark abhängig von der Positionierung des Beobachters in Relation zum Kind. Zum Teil fanden sehr schnelle Wechsel des Blickkontaktes statt, welche die Beobachterin unter Umständen aus ihrer Position heraus nicht sehen konnte. Dies hat- 
te zur Folge, dass die Kategorie Blickkontakt nicht valide eingeschätzt werden konnte und in Phase 2 sowie in der Hauptstudie aus allen Beobachtungsbögen entfernt wurde.

Dagegen wurde für alle Bögen die Kategorie typisches Verhalten hinzugefügt. Aus pragmatischen Gründen war die Beobachtung der Kinder nur an einem Vormittag möglich, so dass immer die Frage im Raum stand, ob das an diesem Tag gezeigte Verhalten des Kindes repräsentativ war. Aus diesem Grunde wurde eine anwesende Erzieherin nach der jeweiligen Beobachtungssituation gefragt, ob das Verhalten des Kindes typisch oder eher untypisch war. Im Falle eines für das Kind untypischen Verhaltens sollte zusätzlich erfragt werden, ob das Kind eher aufgeschlossener oder eher zurückhaltender war.

In der Begrüßungssituation sind vor allem Schwierigkeiten bei der Positionierung der Beobachter aufgetreten. Die Begrüßung des Kindes durch die Erzieherin erfolgte in allen Fällen im Flurbereich der Einrichtungen. Aufgrund der Enge war es für die Beobachterinnen nicht möglich, sich möglichst unauffällig zu positionieren. Hier bekamen die Kinder häufig schon zu Beginn des Vormittags mit, dass sie von den beiden Studierenden beobachtet wurden.

Beobachtungserfahrungen aus Phase 1 verdeutlichten, dass die Orientierung auf den Beobachtungsbögen während der Dokumentation der komplexen Beobachtungssituation schwer fiel. Daraus resultierend fand eine Anpassung des Layouts der Bögen statt. Zeilen wurden abwechselnd weiß und grau gefärbt und den einzelnen Beobachtungskategorien wurde jeweils eine Farbe zugewiesen. Durch die Markierung der Zeilen wurde ein Rutschen in die nachfolgende oder darüber liegende Zeile verhindert. Die spaltenweise Markierung der Beobachtungskategorien ermöglichte zudem ein schnelleres Auffinden der gesuchten Variablen innerhalb der Dokumentationszeit.

Zudem merkten die Beobachterinnen an, dass die unterschiedlichen Phasen nicht einzuhalten waren und häufig bereits vor dem Signal für den Phasenwechsel wieder hochgeschaut wurde. Dokumentations- und Beobachtungsphasen waren somit nicht strikt getrennt. Aus diesem Grund wurde eine weitere Beobachtungsregel in das Manual aufgenommen: die Blickrichtung sollte während der gesamten Dokumentationsphase auf dem Bogen belassen werden.

Für den Freispielbogen ergab sich lediglich eine Ergänzung im Bereich der Spielformen. Zwei der sechs beobachteten Kinder zeigten während der Freispielphasen ein ausgiebiges Funktionsspiel, welches bislang auf dem Bogen noch nicht als einzeln aufgeführte Spielart dokumentiert werden konnte. Aus diesem Grunde wurde die Spielart Funktionsspiel in die Liste mit aufgenommen. Zusätzlich war es beim Freispiel häufig nicht möglich, die geforder- 
ten 30 Minuten in einem Block zu beobachten. Im Manual wurde deswegen der Hinweis hinzugefügt, dass die Beobachtungen im Freispiel auch unterbrochen werden können. In jedem Fall sollen jedoch insgesamt 30 Minuten (entsprechend 45 Sequenzen) bei jedem Kind beobachtet werden.

\subsubsection{Erzieherfragebogen - DortMuS-Kita}

Die Erzieherinnen von sechs der sieben Kinder füllten DortMuS-Kita aus. Zwei Kitas gaben zudem eine kurze schriftliche Rückmeldung. Die Bearbeitungsdauer des Gesamtbogens wurde mit etwa 15 bis 20 Minuten angegeben und war somit noch im Rahmen einer möglichen Bearbeitungszeit. Instruktionen und Items wurden als sehr gut verständlich und eindeutig beschrieben. Dies zeigte sich ebenso in der kompletten Bearbeitung der Items. Auf eine statistische Analyse der Güte des Bogens wurde aufgrund der kleinen Stichprobe von sechs Kindern in der Pilotstudie verzichtet.

\subsubsection{Durchführbarkeit der direkten Untersuchung}

Insgesamt fünf Kinder, darunter zwei mehrsprachige, nahmen an den Pilotierungen der direkten Untersuchungssituation in den Räumlichkeiten des SpAs teil. Die reine Testzeit belief sich bei allen Kindern auf maximal 40 Minuten. Durch die Mischung aus spielerischen Testungen (Interaktionssettings) und direkter Testung der sprachlichen Fähigkeiten (TROGD) konnte die Aufmerksamkeit aller Kinder über die gesamte Zeit aufrechterhalten werden. Bei allen Kindern konnte die Untersuchung vollständig durchgeführt werden. Bei vier der fünf Kinder begleiteten die Eltern die Kinder in den Raum. Alle Eltern hielten sich an die vorherige Anweisung möglichst nicht in die Untersuchungssituation einzugreifen.

Für Ebene 1 (Ball rollen) und Ebene 2 (Tiere sortieren) konnten die gewünschten Reaktionen der Kinder evoziert werden. Die zwei Kontrollkinder sowie ein Kind mit SM rollten den Ball von Beginn des Spieles an problemlos zurück. Die zwei übrigen Kinder mit SM benötigten eine deutliche Unterstützung durch die Testleiterin oder ihre Bezugsperson. Die Fehler der Testleiterin beim Tiere sortieren wurden von beiden Kontrollkindern verbal oder nonverbal aufgeklärt. Die schweigenden Kinder reagierten sehr heterogen auf diese Aufgabe. Ein Kind klärte die Fehler gar nicht auf, sondern beobachtete lediglich die Testleiterin beim Sortieren. Das zweite Kind reagierte mit einem Grinsen auf die Fehler der Testleiterin, klärte sie jedoch nicht über eine nachfolgende Handlung auf. Das dritte schweigende Kind handelte wie die Kontrollkinder. Es half beim Tiere einsortieren und klärte zudem verbal und nonverbal die 
Fehler auf. Die Symptomatik des SM bei diesem Kind zeigte sich vor allem in Gegenwart von Kindern, nicht aber bei zugewandten Erwachsenen.

Auf der dritten Ebene (Actionfigur) zeigte eines der schweigenden Kinder eine Reaktion. Es schaute mehrmals nach dem Wegstellen der Figur nochmals zu ihr, so dass die Testleiterin fragte, ob es noch einmal damit spielen wolle. Alle anderen Kinder forderten die Actionfigur nicht nochmal ein. Die bereits nach der zweiten Pilotierungseinheit vorgenommenen Änderungen in diesem Setting — den zusätzlichen Hinweis, dass man an einem späteren Zeitpunkt noch einmal mit der Figur spielen könne — brachten bei einem Kind, welches als letztes zur Testung kam, den erwünschten Effekt. Zudem wurden die Testmaterialien des TROG-D auf den Schrank neben die Figur gelegt, um nach den Interaktionssettings die Aufmerksamkeit nochmals explizit auf den Schrank und die Actionfigur zu lenken.

Bei der Durchführung des TROG-D an einem Kindertisch fiel auf, dass die meisten Kinder für die Stühle immer noch zu klein waren und die Füße keinen Bodenkontakt hatten. So rutschten die Kinder immer wieder auf dem Stuhl hin und her, so dass ihre Aufmerksamkeit nicht vollständig bei der Testleiterin und der Testung war. Es wurden ggf. Schaumstoffsteine unter die Füße des Kindes gestellt. Mit diesen spielten viele Kinder mit den Füßen, so dass dadurch keine gesteigerte Aufmerksamkeit und Ruhe in die Testsituation gebracht werden konnte. Letztlich wurde der TROG-D auf dem Boden sitzend durchgeführt. Als Orientierung für das Kind wurden zwei Kissen - eines für die Testleiterin, eines für das Kind — auf dem Boden bereit gelegt. Allen Kindern gelang es, auch den schweigenden, bei der Testung mit dem TROG-D auf Bilder zu zeigen.

\subsubsection{Elternfragebögen}

Von sieben Familien, die an den unterschiedlichen Teilen der Pilotstudie teilnahmen, gaben fünf Eltern die Elternfragebögen zurück. Vier von ihnen gelang es, den Bogen selbstständig zuhause auszufüllen. Bei einer Familie half aufgrund massiver sprachlicher Schwierigkeiten eine Erzieherin. Dabei konnte nicht vollständig davon ausgegangen werden, die Sichtweise der Eltern zu erfassen. Insbesondere bei der Einschätzung des (Sprech-)Verhaltens des Kindes könnte die Erzieherin Einfluss auf die Bewertung genommen haben.

Der Aufwand für das Ausfüllen der Bögen wurde von vier Eltern als angemessen für die Studie bewertet. Im Fall der tamilischen Familie konnte aufgrund massiver sprachlicher Schwierigkeiten kein Feedback zum Elternfragebogen eingeholt werden. Der Bogen schien für diese Familie jedoch aufgrund der schwachen Deutschkompetenzen und einer nicht vorliegenden Übersetzung eigenständig nicht zu bearbeiten gewesen zu sein. 


\subsubsection{Spontansprachaufnahmen}

Vier der fünf Familien, die zu den direkten Untersuchungen an der TU Dortmund waren, gelang es die geforderten Spontansprachaufnahmen zuhause anzufertigen. Die tamilische Familie lieferte Daten ab, die wegen Unvollständigkeit jedoch nicht ausgewertet werden konnten.

Die gewonnenen Aufnahmen über die Rollenspielsituation waren mindestens 10 und maximal 14 Minuten lang. Im Mittel wurden darüber 119 Äußerungen des Kindes gewonnen. Darin enthalten waren auch solche Äußerungen, die keine Aussage über die tatsächlichen sprachlichen Fähigkeiten der Kinder zuließen (z.B. [ja] oder Repetitionen der elterlichen ÄuBerung). Dies wurde bei der Kodierung der Spontansprachaufnahmen der Pilotierung nicht berücksichtigt. Die MLU der Kinder lag bei 3.5 bis 4 Wörtern pro Äußerung. Den Eltern gelang es recht gut, über das Spiel mit dem Bären angemessene Äußerungen der Kinder zu evozieren, die sich zur groben Einschätzung der produktiven sprachlichen Fähigkeiten der Kinder eignen. Alle Audioaufnahmen waren für die Transkription geeignet.

\subsubsection{Diskussion}

Insgesamt haben sich alle neu entwickelten Instrumente sowie die Gestaltung der Testsituation mit dem Kind als durchführbar herausgestellt. Im Folgenden werden die Ergebnisse der Pilotierung hinsichtlich möglicher Fehlerursachen und Änderungsmöglichkeiten diskutiert.

\subsubsection{Beobachtungen}

Wie bereits in den Ergebnissen (Kapitel 3.3.5.1) berichtet, war die InterraterReliabilität für alle Beobachtungssituationen als gut bis sehr gut zu kategorisieren. Die Begrüßungssituation hat dabei am schlechtesten-wenn auch für den gesamten Bogen immer noch im guten Bereich—abgeschnitten. In der Feinanalyse der einzelnen Kategorien zeigten sich jedoch vor allem bei der Begrüßungssituation deutliche Schwachstellen. Aufgrund der räumlichen Gegebenheiten war eine unauffällige und gleiche Positionierung der Beobachterinnen in der Mehrzahl der Einrichtungen nicht möglich, so dass sich ggf. unterschiedliche Einschätzungen ergeben haben. Andererseits und weit bedeutsamer ist die Einschätzung des Trennungsverhaltens hinsichtlich seiner Auf- bzw. Unauffälligkeit. Trotz der Spezifizierungen im Beobachtermanual schätzten hier die Beobachterinnen sehr unterschiedlich und damit eher subjektiv ein. Eine genaue Grenze von unauffällig zu auffällig ist nur schwer zu ziehen und objektiv in einem Manual nicht ausreichend festlegbar. Eine gute Beobachterüberein- 
stimmung ist hier wahrscheinlich nur schwer zu erreichen. Aufgrund der Schwierigkeiten und Schwachstellen der Beobachtung in der Begrüßungssituation, welche zu einer unzureichenden Beobachterübereinstimmung führten, wurde diese für die Hauptstudie nicht mehr verwendet.

Die Beobachtungen der restlichen drei Situationen haben sich als objektiv gezeigt und wurden somit für die Hauptstudie mit den oben beschriebenen Veränderungen verwendet (siehe Kapitel 3.3.5.2). Zusätzlich zur Veränderung des Layouts zur besseren Orientierung auf dem Beobachtungsbogen sollte der Umgang mit dem Bogen vor Beginn der eigentlichen Beobachtung geübt werden. Innerhalb der Pilotierungen hat sich eine Sicherheit der Beobachterinnen in ihren Einschätzungen ergeben und zeigte sich in einer Verbesserung der Beobachterübereinstimmung in der zweiten Beobachtungsphase. Vor diesem Hintergrund wurden alle Beobachterinnen vor den Beobachtungen im Rahmen der Hauptstudie intensiv geschult (siehe dazu später Kapitel 3.4.4.1).

\subsubsection{Testsituation}

Auch die Testsituation hat sich im Rahmen der Pilotstudie als durchführbar und angemessen für die Aufmerksamkeitsspanne von drei- bis vierjährigen Kindern erwiesen. Bei den neu entwickelten Interaktionssettings haben die Kinder sehr heterogenes Verhalten gezeigt. In Setting 1 sollte über das Zurollen des Balles das Zurückrollen durch das Kind evoziert werden. Hier wurde erwartet, dass die Kontrollkinder keinerlei Zögern zeigen und den Ball problemlos zurückrollen. Dies zeigte sich erwartungsgemäß bei beiden Kontrollkindern. Die Kinder mit SM zeigten erwartungsgemäß abhängig vom Schweregrad ihrer Störung unterschiedliche Reaktionen. Zwei Kinder benötigten Unterstützung von ihrer Bezugsperson, um das erste Ballrollen umzusetzen. Neben dem Symptom des Schweigens zeigen Kinder mit SM häufig allgemein gehemmtes Verhalten (Ford et al., 1998; Rösler, 1981; Steinhausen \& Juzi, 1996), welches sich eben auch in einem Nicht-Rollen des Balles bzw. in der Notwendigkeit der Unterstützung einer Bezugsperson zeigen kann. Das dritte schweigende Kind rollte den Ball sofort selbstständig zur Testleiterin zurück. Letzteres zeigte sich in der gesamten Testsituation sehr offen und sprach von Beginn an mit der Testleiterin. Den Angaben der Eltern zufolge schwieg es zum Zeitpunkt der Pilotstudie vor allem in Gegenwart von Kindern, so dass sein Verhalten auch dessen Symptombild widerspiegelte. Ähnliches zeigte sich in Setting 2 beim Aufklären der Missverständnisse. Auch hier klärten die Kontrollkinder erwartungsgemäß die Fehler der Testleiterin direkt und eindeutig durch Umsortieren oder verbale Anweisungen auf. Die Kinder mit SM reagierten heterogen, entsprechend ihrer individuellen Symptomatik. Eines der Kinder beobachtete die Testleiterin beim Sortieren, zeigte keinerlei 
sichtbare Reaktionen auf die Missverständnisse und sortierte nicht mit. Ein weiteres zeigte sich offener und fing bei Entdecken der Missverständnisse an zu grinsen. Eine zusätzliche Reaktion oder Handlung vom Kind folgte jedoch nicht. Das dritte Kind nahm aktiv an der Situation teil, sortierte selbst ein und klärte die Missverständnisse durch Handlungen und verbale Äußerungen auf. Die für die grobe Auswertung beschriebenen Kategorien (siehe Kapitel 3.2.3) erschienen somit sinnvoll.

Bei Setting 3 zeigte eines der fünf Kinder die erwünschte Reaktion—das selbstinitiative Einfordern der Actionfigur. Der Grund für das geringe Einfordern der Figur über alle Kinder hinweg blieb unklar. Alle Kinder zeigten generell Interesse an der Figur. Solange die Figuren angeschaltet und im Blickfeld der Kinder standen, beobachteten sie diese, lächelten oder schauten immer wieder interessiert von der Figur zur Testleiterin. Eine Attraktivität des Spielzeuges konnte demnach angenommen werden. Bei den Kindern mit SM kann das Nichteinfordern einerseits darauf zurückgeführt werden, dass sie symptombedingt bei wenig vertrauten Personen keine eigeninitiativen Handlungen zeigen und somit ihren Wunsch nicht deutlich gegenüber der Person mitteilen. Dies wäre die gewünschte Nicht-Reaktion der Kinder im Vergleich zu einem Einfordern bei den Kontrollkindern. Da Kontrollkinder dieses Verhalten jedoch ebenso nicht gezeigt haben, blieb fraglich, ob die beiden verwendeten Figuren interessant und anregend genug waren, um das Verhalten bei möglichst vielen Kindern sicher evozieren zu können. Ein kurzfristiges Interesse am Spielzeug zeigten alle Kinder. Durch das Wegstellen der Figur auf den Schrank war sie nicht mehr im unmittelbaren Blickfeld der Kinder. Womöglich wurde das Interesse an der Figur durch die nachfolgenden Spielangebote der Testleiterin überlagert. Für die Hauptstudie blieb das Setting dennoch bestehen, da nach Änderung der Materialanordnung und dem deutlichen Hinweis auf die Möglichkeit des Spielens mit der Figur eines von drei Kindern die Figur einforderte. Bei der groben Kodierung des Settings, wie sie für die Hauptstudie vorgesehen ist, kann ein Nicht-Einfordern jedoch nicht als fehlende Eigeninitiative interpretiert werden. Hier wurde bereits deutlich, dass für den Anteil des initiativen und reaktiven Verhaltens der Kinder vielmehr die gesamte Interaktionssituation zwischen Kind und Testleiterin detailliert betrachtet werden muss. Eine detaillierte Analyse des kindlichen Verhaltens war im Rahmen der vorliegenden Studie jedoch nicht möglich. Eine Re-Analyse der Daten ist jedoch vorgesehen. 


\subsection{Hauptstudie}

Die Hauptstudie umfasste eine Längsschnittstudie mit vier Messzeitpunkten im Abstand von drei Monaten, in welcher ein- und mehrsprachige Kinder hinsichtlich der Entwicklung des Sprech- und Interaktionsverhaltens untersucht wurden (für einen Überblick zum Studiendesign siehe Kapitel 3.1). Ziel war es, den Einfluss der Sprachkompetenzen in der Mehrheitssprache, der kindlichen Ängste sowie der kulturellen Adaptation der Eltern auf die Entwicklung des SM zu untersuchen.

\subsubsection{Rekrutierung}

Die Rekrutierung der Probanden fand zwischen September 2012 und März 2013 statt. Anfangs war lediglich eine Rekrutierungsphase von ein bis zwei Monaten geplant. Da sich die Rekrutierung insbesondere von mehrsprachigen Kindern als sehr schwierig erwies, wurde die Rekrutierungsphase verlängert sowie die Einschlusskriterien gelockert. Anfangs wurden lediglich schweigende Kinder, welche gerade in die Kita gekommen sind, gesucht. Letztlich wurden jedoch auch Kinder im zweiten Kindergartenjahr in die Studie aufgenommen, um die geplante Stichprobengröße von etwa zehn Kindern pro Gruppe zu erreichen. Einschlusskriterien für die schweigenden Kinder waren (1) ein konsequentes Schweigen mit Erwachsenen und/oder Kindern und (2) ein allgemein zurückhaltendes Verhalten in der Kita sowie (3) ein unbefangenes Sprechen mit Eltern und/oder Geschwistern im häuslichen Kontext. Ausschlusskriterium waren entsprechend der DSM-IV Kriterien (Saß et al., 2003) Kinder mit diagnostizierter Autismus-Spektrum-Störung, Schizophrenie oder anderen psychotischen Störungen.

Es wurden flächendeckend alle Kitas im Dortmunder Stadtgebiet sowie verkehrsgünstig gelegene Einrichtungen in Bochum (insgesamt knapp 330 Einrichtungen) zunächst postalisch angeschrieben (siehe Anhang G) und innerhalb der nächsten zwei bis vier Wochen von der Studienleiterin telefonisch kontaktiert. Das Anschreiben an die Einrichtungen erhielt Informationen zur Studie sowie eine Checkliste zur Auswahl potenzieller Kinder. Im Telefongespräch mit einer Ansprechpartnerin in der Kita wurde geklärt, ob das über die Checkliste ausgewählte Kind die Einschlusskriterien der Studie tatsächlich erfüllte. Ansprechpartnerinnen waren die Leiterin der Kita-Gruppe oder die Bezugserzieherin des schweigenden Kindes. Waren die Einschlusskriterien erfüllt wurde die Ansprechpartnerin gebeten, ein Kontrollkind gleichen Geschlechts, gleichen Alters und entsprechend ein- oder mehrsprachig in der gleichen Gruppe oder ggf. in einer anderen Gruppe der Einrichtung auszuwählen. Parallel erhiel- 
ten die Einrichtungen eine entsprechende Anzahl gedruckter Elterninformationen und ein Kontaktformular, welche an die Eltern der ausgewählten Kinder ausgegeben und von der Erzieherin wieder eingesammelt werden sollten. Sobald die Kontaktdaten der Eltern als erste Interessensbekundung zur Teilnahme an der Studie vorlagen, wurden die Familien von der Studienleiterin telefonisch kontaktiert und zu einem ersten Termin in der Universität eingeladen. In diesem ersten Termin wurden die Eltern mündlich und/oder schriftlich über den Ablauf der Studie sowie über bedeutsame Aspekte des Datenschutzes aufgeklärt (siehe Anhang G). Erst dann sollten sich die Eltern endgültig zur Teilnahme an der Studie entscheiden und die Einverständniserklärung unterschreiben.

\subsubsection{Probanden}

An der Studie nahmen insgesamt 30 Kinder im Alter von 3;0 bis 5;8 aus 14 Kitas teil, von denen 18 anfangs schweigende Kinder und 12 sprechende Kontrollkinder waren. Tabelle 11 zeigt die Verteilung der Kinder auf die rekrutierten Gruppen mit den Merkmalen mehrsprachig +/- und schweigend +/- zum ersten Messzeitpunkt.

Insgesamt 15 Kinder-11 davon schweigend und 4 sprechend-wuchsen mehrsprachig auf. Für acht Kinder gaben die Eltern an, dass die dominante Sprache Deutsch sei. Bei den anderen sieben Kindern waren die dominanten Sprachen Türkisch $(\mathrm{n}=2)$ und Arabisch (n $=2)$, Englisch $(n=1)$, Polnisch $(n=1)$ und Kurdisch $(n=1)$. Als zweite Sprache der Kinder gaben die Eltern in vier Fällen Türkisch an und bei je einem Spanisch, Griechisch, Arabisch und Kroatisch. Fünf der mehrsprachigen Kinder (33.3 \%) wuchsen simultan, zehn Kinder sukzessiv zweisprachig (66.7 \%) auf. Im Mittel (hier Median) hatten die sukzessiv zweisprachig aufwachsenden Kinder seit 16 Monaten Input im Deutschen $(I Q R=14.0$; Range: 2 - 19). Die Verteilung der Formen der Zweisprachigkeit (sukzessiv vs. simultan) unterschied sich nicht signifikant zwischen den schweigenden und den Kontrollkindern, $X^{2}(1)=0.409$, n.s.

Zwei der einsprachigen Kinder hatten zusätzlich einen Migrationshintergrund. Eines der Kinder hatte eine afrikanische Mutter. Zuhause und in der Kita erhielt es jedoch ausschließlich einen deutschen Sprachinput. Die Mutter des zweiten Kindes kam aus Osteuropa. Auch hier wurde sowohl in Familie als auch in der Kita ausschließlich Deutsch mit dem Kind gesprochen. 
Tabelle 11

Verteilung der Probanden auf die Gruppen mit den Merkmalen schweigend +/- und mehrsprachig +/-

\begin{tabular}{|c|c|c|c|c|}
\hline & \multicolumn{2}{|c|}{ Schweigende Kinder } & \multicolumn{2}{|c|}{ Kontrollkinder } \\
\hline & $\begin{array}{c}\text { mehrsprachig } \\
\mathrm{n}=11\end{array}$ & $\begin{array}{c}\text { einsprachig } \\
n=7\end{array}$ & $\begin{array}{c}\text { mehrsprachig } \\
\mathrm{n}=4\end{array}$ & $\begin{array}{l}\text { einsprachig } \\
\quad \mathrm{n}=8\end{array}$ \\
\hline Geschlecht & $\begin{array}{l}8 \mathrm{w}(72.7 \%) \\
3 \mathrm{~m}(27.3 \%)\end{array}$ & $\begin{array}{l}5 \mathrm{w}(71.4 \%) \\
2 \mathrm{~m}(28.6 \%)\end{array}$ & $\begin{array}{l}2 \mathrm{w}(50.0 \%) \\
2 \mathrm{~m}(50.0 \%)\end{array}$ & $\begin{array}{l}7 \mathrm{w}(87.5 \%) \\
1 \mathrm{~m}(12.5 \%)\end{array}$ \\
\hline Alter in Monaten & $\begin{array}{c}51.00 \\
(I Q R=10.00)\end{array}$ & $\begin{array}{c}41.00 \\
(I Q R=13.00)\end{array}$ & $\begin{array}{c}52.50 \\
(I Q R=19.50)\end{array}$ & $\begin{array}{c}37.25 \\
(I Q R=16.50)\end{array}$ \\
\hline $\begin{array}{l}\text { Form des Zweit- } \\
\text { spracherwerbs }\end{array}$ & $\begin{array}{l}3 \text { simultan }(27.3 \%) \\
8 \text { sukzessiv }(72.7 \%)\end{array}$ & & $\begin{array}{l}2 \text { simultan }(50.0 \%) \\
2 \text { sukzessiv }(50.0 \%)\end{array}$ & \\
\hline $\begin{array}{l}\text { Hauptinput } \\
\text { im Deutschen } \\
\text { in anderer } \\
\text { Sprache }\end{array}$ & $\begin{array}{l}6(54.5 \%) \\
5(45.5 \%)\end{array}$ & & $\begin{array}{l}3(75.0 \%) \\
1(25.0 \%)\end{array}$ & \\
\hline $\begin{array}{l}\text { Anzahl der Per- } \\
\text { sonen im Haus- } \\
\text { halt }\end{array}$ & $\begin{array}{c}4.00 \\
(I Q R=1.00)\end{array}$ & $\begin{array}{c}3.00 \\
(I Q R=1.00)\end{array}$ & $\begin{array}{c}3.50 \\
(I Q R=1.00)\end{array}$ & $\begin{array}{c}4.00 \\
(I Q R=0.75)\end{array}$ \\
\hline $\begin{array}{l}\text { Anzahl der } \\
\text { Geschwister }\end{array}$ & $\begin{array}{c}1.00 \\
(I Q R=1.00)\end{array}$ & $\begin{array}{c}0.00 \\
(I Q R=1.00)\end{array}$ & $\begin{array}{c}1.00 \\
(I Q R=0.75)\end{array}$ & $\begin{array}{c}1.00 \\
(I Q R=0.75)\end{array}$ \\
\hline $\begin{array}{l}\text { Kulturelles } \\
\text { Kapital }\end{array}$ & $\begin{array}{c}2.00 \\
(I Q R=1.00)\end{array}$ & $\begin{array}{c}3.00 \\
(I Q R=2.00)\end{array}$ & $\begin{array}{c}4.00 \\
(I Q R=0.75)\end{array}$ & $\begin{array}{c}4.00 \\
(I Q R=1.00)\end{array}$ \\
\hline Schicht-Index & $\begin{array}{c}9.00 \\
(I Q R=5.00)\end{array}$ & $\begin{array}{c}7.00 \\
(I Q R=6.00)\end{array}$ & $\begin{array}{c}9.50 \\
(I Q R=11.75)\end{array}$ & $\begin{array}{c}16.50 \\
(I Q R=6.25)\end{array}$ \\
\hline
\end{tabular}

Anmerkungen $: \mathrm{w}=$ weiblich, $\mathrm{m}=$ männlich

Zur Überprüfung von Gruppenunterschieden in den demografischen Daten wurden Chi-Quadrat-Tests sowie aufgrund der kleinen und ungleichen Zellbesetzung Kruskal-WallisTests mit anschließenden paarweisen Vergleichen mit angepassten $p$-Werten gerechnet. Die vier Gruppen unterschieden sich nicht signifikant hinsichtlich

- Alter: $H(3)=1.78$, n.s.,

- Geschlecht: $X^{2}(3)=1.95$, n.s.,

- Anzahl der Personen im Haushalt: $H(3)=6.32$, n.s.,

- Anzahl der Geschwister: $H(3)=4.86$, n.s.

Hinsichtlich des kulturellen Kapitals ergab sich jedoch ein signifikanter Unterschied zwischen den Gruppen, $H(3)=11.541, p=.009$, wobei sich lediglich die mehrsprachig schweigenden von den einsprachig sprechenden Kindern signifikant $(p=.029, r=-.65)$ und von den mehrsprachig sprechenden Kindern tendenziell $(p=.053, r=-.68)$ unterschieden. Ebenso unterschieden sich die vier Gruppen signifikant hinsichtlich des Schicht-Indexes, $H(3)$ $=12.272, p=.007$. Die paarweisen Vergleiche ergaben, dass sich die beiden schweigenden 
Gruppen von den einsprachig sprechenden Kindern unterschieden (mehrsprachig schweigend $p=.017, r=-.69$; einsprachig schweigend $p=.013, r=-.79)$. Die Ergebnisse der weiteren Gruppenvergleiche für die Variablen Kulturelles Kapital und Schicht-Index finden sich in den Tabellen A-4 und A-5 im Anhang.

\section{Schwangerschaft und Geburt}

Bei neun Kindern (30.0\%, eine fehlende Angabe) gab es während der Schwangerschaft Komplikationen. Zwei (6.7 \%) der insgesamt 30 Kinder (je ein ein- und mehrsprachig schweigendes) kamen als Frühgeburt zwischen der 28. und 37. Schwangerschaftswoche (Klassifikation nach ICD-10, Deutsches Institut für Medizinische Dokumentation und Information [DIMDI], 2014) zur Welt. Im Mittel waren die Kinder zur Geburt $3170.1 \mathrm{~g}$ schwer $(S D=576.0)$. Ein Kind war mit einem Geburtsgewicht von $1365 \mathrm{~g}$ als sehr kleines Frühgeborenes zu kategorisieren. Zwei Kinder (6.7 \%) hatten mit einem Geburtsgewicht von $\leq 2500 \mathrm{~g}$ ein niedriges Geburtsgewicht. Bei 12 Kindern (40.0\%) gab es Komplikationen während der Entbindung. Die Gruppen unterschieden sich jedoch nicht signifikant hinsichtlich der Häufigkeiten von Komplikationen in Schwangerschaft, $X^{2}(3)=0.249$, n.s., oder während der Entbindung, $X^{2}(3)=0.942$, n.s.. Eines der mehrsprachig schweigenden Kinder hatte ein Zwillingsgeschwister, welches jedoch bereits während der Schwangerschaft verstarb.

\section{Komorbide (Entwicklungs-)Auffälligkeiten}

In Bezug auf zurückliegende und/oder bestehende Entwicklungsverzögerungen/störungen ergaben sich zwischen den Gruppen keine Unterschiede (siehe Tabelle A-6 im Anhang). Sprachliche Entwicklungsverzögerung bzw. -störungen waren laut Elternangaben die häufigsten in der Stichprobe (siehe Abbildung 5). Vier Probanden (13.3 \%) zeigten zum Zeitpunkt der Studie bzw. in der Vergangenheit sprachliche Auffälligkeiten. Sieben Eltern (23.3 \%) berichteten von einem verspäteten Sprechbeginn, die Hälfte der Eltern (50.0 \%) von Ausspracheschwierigkeiten, sechs Eltern (20.0\%) von morphologischen und zwei Eltern (6.7 \%) von syntaktischen Schwierigkeiten ihrer Kinder. Für jeweils drei Kinder (10.0\%) gaben die Eltern ein zeitweiliges bzw. noch bestehendes Stottern oder eine erhöhte Sprechgeschwindigkeit mit starker Unverständlichkeit an. Unterschiede in der Auftretenshäufigkeit der berichteten sprachlichen Auffälligkeiten bestanden nicht zwischen den Gruppen (siehe dazu Tabelle A-6 im Anhang). Für 26 (86.7\%) Kinder gaben die Eltern an, dass sich die Kinder in der deutschen Sprache verständigen können. Bei jeweils zwei Kindern (6.7\%) berichteten die Eltern von eingeschränkten oder nicht vorhandenen Sprachkompetenzen im Deutschen. Von 
den 15 mehrsprachigen Kindern gaben 13 Eltern (86.7\%) an, dass sich die Kinder in ihrer dominanten Sprache (davon 8 Kinder im Deutschen) verständigen können, bei jeweils einem Kind (6.7\%) bestanden laut Eltern zu Beginn der Studie eingeschränkte oder nicht ausreichende Sprachkompetenzen. Auch hier ergaben sich keine signifikanten Unterschiede zwischen den Gruppen, $X_{\text {Deutsch }}^{2}(6)=7.972$, n.s., $X_{\text {dominant }}^{2}(2)=0.839$, n.s., wobei die vier Kinder mit eingeschränkten und nicht vorhandenen Sprachkompetenzen im Deutschen der Gruppe mehrsprachig schweigend zugehörten. Für die mehrsprachigen Kinder schätzten die Eltern zusätzlich ein, wie gut die Kinder sowohl die Muttersprache als auch die deutsche Sprache im Vergleich zu gleichaltrigen Kindern derselben Muttersprache sprechen können. Für 10 Kinder $(66.7 \%)$ berichteten die Eltern gleich gute oder bessere Sprachkompetenzen in der Muttersprache, für 4 Kinder (26.7\%) etwas oder viel schlechtere. Die deutsche Sprache beherrschten 8 Kinder (53.4\%) laut Eltern gleich gut oder besser, 5 Kinder (33.3\%) etwas oder viel schlechter. Zwei Eltern machten keine Angabe. Auch hier ergaben sich keine signifikanten Unterschiede zwischen den schweigenden und sprechenden Kinder, $X_{\text {Deutsch }}^{2}(3)=2.567$, n.s., $X_{L 1}^{2}(3)=3.343$, n.s.. Zwei Kinder $(13.3 \%)$-je ein schweigendes und ein sprechendes Kind-besuchten regelmäßig für ein bis zwei Stunden eine kulturelle Institution. Motorische Probleme traten bei insgesamt zwei Probanden (6.7 \%) auf ebenso wie zeitweilige Hörprobleme (meist verursacht durch Mittelohrentzündungen). Bei drei Kindern (10.0\%) gaben die Eltern zusätzliche Auffälligkeiten an, wobei hier jeweils einmal eine chronische Bronchitis, Herznebengeräusche und Paukenröhrchen genannt wurden. Letztere standen in Zusammenhang mit zeitweiligen Hörproblemen des Kindes.

Hinsichtlich weiterer komorbider Auffälligkeiten unterschieden sich die Gruppen tendenziell bei den Störungen der Ausscheidungsorgane (Enuresis: $X^{2}(3)=7.35, p=.061$, Enkorpresis: $\left.X^{2}(3)=7.04, p=.071\right)$ sowie im aggressiven Verhalten $\left(X^{2}(3)=7.04, p=.071\right)$. Das Einnässen sowie Auffälligkeiten im Essverhalten waren die häufigsten Auffälligkeiten innerhalb der Stichprobe (jeweils $20 \%$ aller Kinder) (siehe dazu auch Tabelle A-7 im Anhang). 


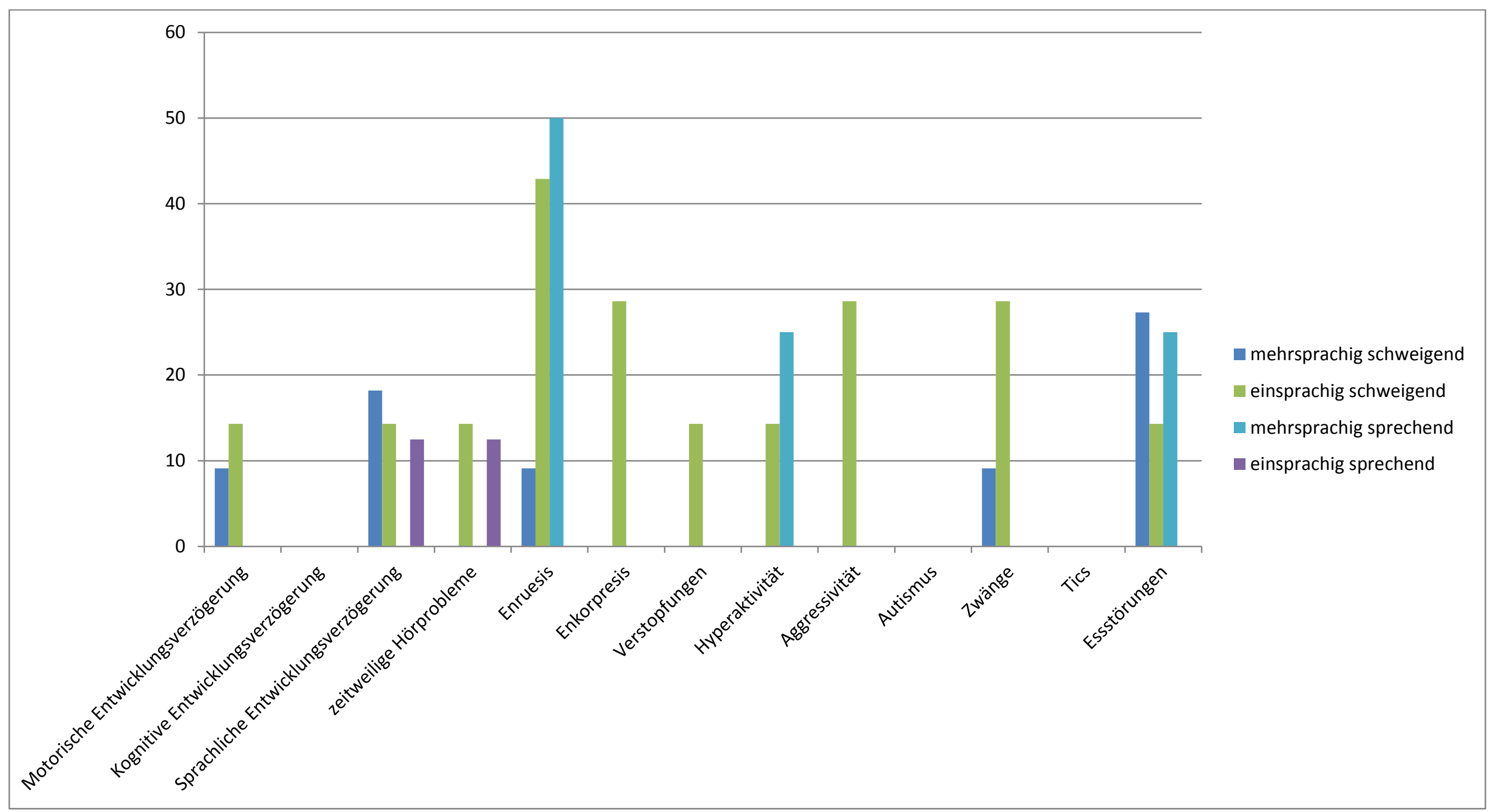

Abbildung 6. Häufigkeiten komorbider Störungen 


\section{Therapien}

Die Kinder der Studie erhielten insgesamt wenige Therapien. Bei den mehrsprachigen Kindern bekamen zum ersten Messzeitpunkt noch ein Kind (6.7 \%) Frühförderung und ein weiteres (6.7\%) Sprachtherapie. Bei den einsprachig schweigenden Kindern erhielt ein Kind (6.7\%) zum ersten Messzeitpunkt Sprachtherapie sowie heilpädagogisches Reiten. Zwei weitere Kinder (13.3\%) hatten in ihrer Entwicklung bereits Physiotherapie erhalten, ein Kind (6.7 \%) Frühförderung. Die Verteilung der Therapieformen unterschied sich zwischen den Gruppen nicht signifikant (für ausführliche Informationen siehe Tabelle A-8 im Anhang). Im Verlauf der Studie wurden bei vier der mehrsprachigen Kinder (26.7\%) und einem einsprachigen Kind $(6.7 \%)$ eine Sprachtherapie begonnen - für zwei der mehrsprachigen Kinder (13.3\%) ab Messzeitpunkt drei, für die weiteren zwei mehrsprachigen (13.3\%) und das einsprachige Kind (6.7 \%) ab Messzeitpunkt vier (siehe dazu auch Kapitel 3.4.3.6).

\section{Einschneidende Lebensereignisse}

Von den sprechenden Kindern berichtete nur 1 der 12 Elternpaare (8.3\%) von Schwierigkeiten der Kinder in der Eingewöhnung in die Kita, im Gegensatz zu 12 von 18 $(66.7 \%)$ der schweigenden Kinder. Entsprechend ergab sich ein signifikanter Unterschied zwischen den Gruppen, $X^{2}(3)=10.762, p=.013$. Nur 2 der insgesamt 30 Kinder $(6.7 \%)$ gingen zum ersten Messzeitpunkt eher ungern in die Kita-je ein schweigendes und ein sprechendes einsprachiges Kind. Ein Unterschied zwischen den Gruppen zeigte sich nicht, $X^{2}(3)=$ 2.162, n.s.. Trennungen von den Bezugspersonen wurden für zwei Kinder berichtet. Bei einem mehrsprachigen Kind (3.3\%) lebte der Vater bis kurz vor Beginn der Studie noch in seinem Heimatland, bei einem einsprachigen Kind (3.3 \%) war die Mutter zeitweise aufgrund einer psychischen Erkrankung in stationärer Behandlung. Insgesamt acht Kinder $(26.7 \%)$ waren bereits in eine andere Stadt umgezogen (fünf mehrsprachig schweigende, ein einsprachig schweigendes sowie jeweils ein mehr- und einsprachiges Kontrollkind). Drei der mehrsprachigen schweigenden Kinder waren bereits zwei Mal in eine andere Stadt umgezogen, das einsprachig schweigende Kind ebenso. Die Kontrollkinder waren jeweils nur einmal umgezogen. Zusätzlich hatte ein mehrsprachig schweigendes Kind (3.3\%) einen Umzug in ein anderes Land (in diesem Fall aus dem Heimatland nach Deutschland) erlebt. Die Gruppen unterschieden sich jedoch nicht signifikant hinsichtlich ihrer Umzugserfahrung, $X_{\text {stadt }}^{2}(3)=3.36$, n.s., $X_{\text {land }}^{2}(3)=1.79$, n.s..

Insgesamt sechs Kinder (20\%) hatten bereits die Trennung ihrer Eltern miterlebt (drei mehrsprachig schweigende, ein einsprachig schweigendes sowie zwei mehrsprachig spre- 
chende). Der Anteil der Kinder unterschied sich zwischen den Gruppen nicht signifikant, $X^{2}(3)=4.76$, n.s.. Vier der schweigenden Kinder (22.2\%) (je zwei einsprachige und mehrsprachige) waren bereits einem traumatischen Erlebnis ausgesetzt. Für drei Kinder berichteten die Eltern von medizinischen Ereignissen (Verbrennungen, Krankenhausaufenthalte), bei einem weiteren Kind wies die Mutter auf ihre eigene Depression und ihr damit einhergehendes selbstverletzendes Verhalten hin.

\section{Schüchternheit, Ängste und Schweigen in der Kernfamilie}

Die Ausprägung von Schüchternheit, Ängsten und schweigsamem Verhalten bei den nahen Familienmitgliedern der Kinder unterschied sich nicht signifikant zwischen den Gruppen (siehe Tabelle 12). Lediglich bei den Ängsten der Väter zeigte sich eine statistische Tendenz, $H(3)=6.88, p=.076$, wobei die Väter der schweigenden einsprachigen Kinder die stärksten Ängste aufwiesen.

Tabelle 12

Schüchternheit, Ängste und Schweigen bei nahen Familienmitgliedern

\begin{tabular}{rccccccc}
\hline & $\begin{array}{r}\text { mehrsprachig } \\
\text { schweigend }\end{array}$ & $\begin{array}{c}\text { einsprachig } \\
\text { schweigend }\end{array}$ & $\begin{array}{c}\text { mehrsprachig } \\
\text { sprechend }\end{array}$ & $\begin{array}{c}\text { einsprachig } \\
\text { sprechend }\end{array}$ & $H$ & $d f$ & $p$ \\
\hline Schüchternheit & & & & & & \\
Mutter & $10.0(3.0)$ & $11.0(5.0)$ & $7.0(0.0)$ & $9.0(1.75)$ & 3.54 & 3 & .315 \\
Vater & $8.0(3.0)$ & $10.0(2.0)$ & $10.0(2.0)$ & $7.5(2.75)$ & 2.66 & 3 & .447 \\
Geschwister & $11.0(5.0)$ & $10.0(3.0)$ & $10.0(0.0)$ & $8.0(4.5)$ & .66 & 3 & .882 \\
Ängste & & & & & & \\
Mutter & $7.0(2.0)$ & $7.0(3.0)$ & $5.0(0.0)$ & $6.5(2.0)$ & 4.46 & 3 & .216 \\
Vater & $5.0(1.0)$ & $7.0(4.0)$ & $7.0(1.0)$ & $4.5(1.0)$ & 6.88 & 3 & .076 \\
Geschwister & $6.5(5.0)$ & $7.0(2.0)$ & $4.0(2.0)$ & $4.0(4.5)$ & 2.98 & 3 & .395 \\
Schweigen & & & & & & \\
Mutter & $9.0(3.0)$ & $9.0(5.0)$ & $9.0(1.0)$ & $8.5(1.0)$ & 1.42 & 3 & .699 \\
Vater & $8.0(2.0)$ & $9.5(3.75)$ & $13.0(5.0)$ & $8.0(0.0)$ & 5.43 & 3 & .143 \\
Geschwister & $10.5(5.5)$ & $10.0(0.0)$ & $12.0(0.0)$ & $8.0(1.5)$ & 5.18 & 3 & .159 \\
\hline
\end{tabular}

Anmerkung. Mehrsprachig schweigend: $\mathrm{n}_{\text {Eltern }}=11, \mathrm{n}_{\text {Geschwister }}=6$; einsprachig schweigend: $\mathrm{n}_{\text {Mutter }}=7$, $\mathrm{n}_{\text {Vater }}=6, \mathrm{n}_{\text {Geschwister }}=3$; mehrsprachig sprechend: $\mathrm{n}_{\text {Eltern }}=3, \mathrm{n}_{\text {Geschwister }}=2$; einsprachig sprechend: $\mathrm{n}_{\text {El- }}$ tern $=8, \mathrm{n}_{\text {Geschwister }}=5$ 


\subsubsection{Vorgehen}

Wie bereits in Kapitel 3.1 skizziert wurde ein längsschnittliches Design mit vier Messzeitpunkten im Abstand von drei Monaten durchgeführt.

\subsubsection{Testzeitpunkt 1}

Der erste Messzeitpunkt umfasste insgesamt drei Termine, von denen zwei Termine in den Räumlichkeiten des SpA an der TU Dortmund stattfanden sowie ein Beobachtungstermin in der Kita. Zunächst wurden die Eltern zu einem Gespräch in die Universität eingeladen. Dieses erste Gespräch diente der Information der Eltern über den Ablauf der Studie. Erklärten sich die Eltern zur Teilnahme an der Studie schriftlich bereit, erhielten Sie die Elternfragebögen zur Erfassung der kindlichen Ängste (BAV 3-11), den Fragebogen zur Erfassung der anamnestischen Daten (siehe Kapitel 3.2.1) und des Sprechverhaltens der Kinder im häuslichen und öffentlichen Kontext (siehe Kapitel 3.2.2). Familien mit Migrationshintergrund erhielten zusätzlich den FRAKK zur Erfassung der kulturellen Adaptation der Eltern. Die Fragebögen wurden von allen Eltern möglichst selbstständig vor Ort ausgefüllt. Die Testleiterin war anwesend, um vor allem die mehrsprachigen Familien ggf. beim Verständnis von Fragen unterstützen zu können. Im gemeinsamen Gespräch wurden zudem mit allen mehrsprachigen Familien die Mehrsprachen-Kontexte durchgeführt. Am Ende des Gespräches erhielten die Eltern die Materialien zur Erstellung der Spontansprachaufnahmen (siehe Kapitel 3.2.4.2). Sie wurden mündlich dazu instruiert und das Aufnahmegerät wurde erklärt. Die Spontansprachaufnahme sollte zuhause von den Eltern bis zum zweiten Termin erstellt und die Materialien mit zur Universität gebracht werden. Im Rahmen des zweiten Termins an der TU Dortmund, der gemeinsam mit einer Bezugsperson und dem Kind stattfand, wurden in einem ersten Schritt die Interaktionssettings mit dem Kind und im zweiten der TROG-D durchgeführt. Falls das Kind nicht alleine in den Raum kommen wollte, begleitete eine Bezugsperson das Kind. Diese wurde vorab instruiert, sich im Hintergrund zu halten und nicht in die Testsituation einzugreifen. Im Anschluss an die Testung erhielt das Kind ein Wasserspielzeug sowie die Eltern eine Aufwandsentschädigung von 20 Euro. Der dritte Termin fand in der Kita des Kindes zur Beobachtung des Interaktionsverhaltens statt. Vorab wurde den Einrichtungen eine entsprechende Anzahl Fragebögen (DortMuS-Kita) postalisch zugesandt, die von den Bezugserzieherinnen bis zum Beobachtungstermin ausgefüllt und den Beobachterinnen mitgegeben werden sollten. Die Beobachtungen wurden von im Vorfeld geschulte Studierende (siehe Kapitel 3.4.4.1) durchgeführt. 


\subsubsection{Testzeitpunkte 2 und 3}

Das Vorgehen zum zweiten und dritten Messzeitpunkt war identisch. Die Eltern erhielten postalisch einen Elternfragebogen, um das aktuelle Sprechverhalten der Kinder im familiären und öffentlichen Kontext zu erfassen. In einem frankierten Rückumschlag konnte dieser zurückgesandt werden. Die Kinder wurden wiederum in der Kita hinsichtlich ihres Interaktionsverhaltens beobachtet. Vorab erhielten die Bezugserzieherinnen den Fragebogen DortMuS-Kita, um das aktuelle Sprechverhalten der Kinder im Kita-Kontext zu dokumentieren. Dieser Fragebogen wurde wiederum von den Studierenden im Rahmen des Beobachtungstermins eingesammelt oder ggf. von der Einrichtung postalisch an die Universität zurückgeschickt.

\subsubsection{Testzeitpunkt 4}

Der letzte Testzeitpunkt bestand—ähnlich zum ersten — aus drei Terminen, von denen zwei in den Räumlichkeiten der TU Dortmund und ein Termin in der Kita stattfanden. Anders als zu Testzeitpunkt 1 fand der Termin mit dem Kind, in dem die Interaktionssettings sowie der TROG-D durchgeführt wurden, vor dem Elterntermin statt. Der Ablauf der Testsituation war identisch zu Testzeitpunkt 1. Einzig die Actionfigur (zu Testzeitpunkt 1 „Tickle me Elmo“) wurde zum vierten Testzeitpunkt ausgetauscht (,Tanzendes Schaf"), um wiederum ein möglichst großes Interesse der Kinder an der Figur zu erzeugen. Am Ende der Testung erhielten die Kinder ein Dankeschön (Seifenblasen). Zur Erfassung der kindlichen Ängste, des aktuellen Sprechverhaltens der Kinder sowie der kulturellen Adaptation wurde den Eltern am Ende der Testung der BAV 3-11, der FRAKK sowie der Elternfragebogen zur Erfassung des Sprechverhaltens gemeinsam mit dem Material zur Erstellung der Spontansprachaufnahme ausgehändigt. Bis zum abschließenden Elterngespräch hatten die Eltern zuhause Zeit die Fragebögen auszufüllen sowie spontansprachliche Äußerungen des Kindes aufzunehmen. Im Elterngespräch wurde die Familie über die Entwicklung ihres Kindes ausführlich informiert sowie ggf. hinsichtlich weiterer Interventionsmaßnahmen beraten. Am Ende des Elterngesprächs erhielten die Eltern nochmals eine Aufwandsentschädigung von 20 Euro. Auch zum letzten Messzeitpunkt fand wiederum die Beobachtung in der Kita statt, ebenso wie die Bezugserzieherinnen das aktuelle Sprechverhalten der Kinder in der Kita über DortMuS-Kita einschätzten. 


\subsubsection{Diagnosestellung}

Die Diagnosestellung erfolgte auf Grundlage der DSM-IV Kriterien ( Saß et al., 2003, siehe auch Kapitel 2.1). Spätestens zum vierten Messzeitpunkt wurde von der Testleiterin für alle Probanden anhand der aktuellen Daten geprüft, ob ein SM vorliegt oder nicht. Konkret musste das Kind in mindestens einer spezifischen sozialen Situation konsequent schweigen. Dies wurde anhand der Testsituation mit dem Kind sowie den Eltern- und Erzieherfragebögen analysiert. Die Sprachkompetenzen der Kinder-hier vor allem die produktiven Sprachkompetenzen, nur bei nicht vorliegenden Spontansprachaufnahmen die rezeptiven - sollten für eine Alltagskommunikation ausreichend sein. Für den produktiven Bereich wurde eine MLU von mindestens 1.75 als Kriterium festgelegt. Eine reine Kommunikation über Ein-WortÄußerungen wurde nicht als angemessene Sprachkompetenz für die Kommunikation vor allem im Kita-Kontext angesehen. Falls keine Spontansprachaufnahme für das Kind vorlag, wurden die Ergebnisse des TROG-D herangezogen. Hier sollte das Kind mindestens einen TWert von 35 erreichen (d.h. maximal 1.5 Standardabweichungen vom Mittelwert abweichen). Werte unter 35 wurden als nicht ausreichend angesehen. Bei nicht ausreichend vorhandenen Sprachkompetenzen wurde die Diagnose SM nicht gestellt.

\subsubsection{Kitainformationen}

Die teilnehmenden Einrichtungen erhielten die Möglichkeit Fachinformationen zur Studie und zum SM zu erhalten. An einem ersten Termin wurden die Einrichtungen über die Hintergründe sowie den Ablauf der Studie informiert und erhielten einige grundlegende Hinweise zum Umgang mit schweigenden Kindern (siehe Anhang H). Der zweite Termin war für die Kitas optional und fand zum Ende der Studie statt. Bei Einverständnis der Eltern wurde eine Fallbesprechung zu den schweigenden und sprechenden Kindern angeboten, in der die Einrichtungen zunächst Informationen zu ersten Ergebnissen der Studie und anschließend zur Entwicklung der Kinder ihrer Einrichtung erhielten. Sollten die Kinder der Einrichtung einen SM entwickelt haben, wurden die Fachkräfte zum weiteren Umgang mit diesen im KitaAlltag beraten.

\subsubsection{Beratung und Therapieeinleitung bei SM}

Eine möglichst frühe Intervention ist im Kindergartenalter am erfolgversprechendsten und kann vor Langzeitfolgen wie generalisierten Angststörungen oder Depression schützen (Subellok \& Starke, 2012). Aus ethischen Gründen wurden die Eltern hinsichtlich Interventi- 
onsmöglichkeiten beraten, sobald die Diagnose SM beim Kind gestellt wurde. Ggf. wurden sie an mit dem SpA kooperierende sprachtherapeutische Praxen verwiesen. Die Kinder verblieben auch bei Aufnahme einer Therapie in der Studie, da eine Verbesserung der Symptomatik innerhalb von drei bis sechs Monaten eher selten ist (Cohan, Chavira et al., 2006). Der Einfluss der Intervention auf die Entwicklung der schweigenden Kinder muss in der Interpretation der Ergebnisse entsprechend berücksichtigt werden.

\subsubsection{Instrumente}

Tabelle 13 gibt einen Überblick über die in der Hauptstudie verwendeten Instrumente und zu deren Einsatz zu den verschiedenen Messzeitpunkten. Im Folgenden werden zunächst Informationen zur Schulung der Beobachterinnen gegeben. Anschließend wird näher auf die Spontansprachaufnahmen, vor allem auf die Kodierung dieser sowie die InterraterReliabilität, eingegangen. Für die Erläuterung aller weiteren Instrumente wird auf Kapitel 3.2 verwiesen.

Tabelle 13

Verwendete Instrumente im Rahmen der Hauptstudie

\begin{tabular}{|c|c|c|c|c|c|}
\hline & & $\mathrm{T} 1$ & $\mathrm{~T} 2$ & T3 & $\mathrm{T} 4$ \\
\hline \multirow{4}{*}{ 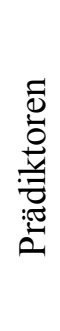 } & Angst & BAV 3-11 & & & BAV 3-11 \\
\hline & Sprache & Rezeption: TROG-D & & & Rezeption: TROG-D \\
\hline & & $\begin{array}{l}\text { Produktion: Spontan- } \\
\text { sprache }\end{array}$ & & & $\begin{array}{l}\text { Produktion: Spontan- } \\
\text { sprache }\end{array}$ \\
\hline & Kulturelle Adaptation & FRAKK & & & FRAKK \\
\hline \multirow{4}{*}{ 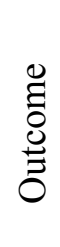 } & Sprechverhalten & \multicolumn{4}{|c|}{ Elternfragebogen } \\
\hline & & \multicolumn{4}{|c|}{ DortMuS-Kita } \\
\hline & Interaktionsverhalten & \multicolumn{4}{|c|}{ Verhaltensbeobachtungen (Freispielsituation) } \\
\hline & & Interaktionssettings & & & Interaktionssettings \\
\hline
\end{tabular}

\subsubsection{Beobachterschulung}

Um eine möglichst objektive Beobachtung durch die Studierenden zu gewährleisten, wurde mit allen Beobachterinnen vorab eine 90 bis maximal 180 minütige Schulung durchgeführt. Die Studierenden stammten aus den das Forschungsprojekt begleitenden Projektgruppen an der TU Dortmund. Für die Schulung wurde eine Freispielsituation im Hochschulkindergarten der TU Dortmund auf Video aufgezeichnet. Insgesamt vier Kinder nahmen nach 
Einverständnis der Eltern an den Filmaufnahmen teil. Aus dem Videomaterial wurden insgesamt drei 10 minütige Ausschnitte ausgewählt, die das Basismaterial für das Training darstellten. Vor der Beobachterschulung erhielten die teilnehmenden Studierenden zur Einarbeitung die Beobachtungsbögen sowie das Manual (siehe Anhang D). Im Rahmen der Schulung wurden zunächst die einzelnen Variablen auf den Beobachtungsbögen erläutert und Beispiele für mögliche Verhaltensweisen der Kinder gegeben. Anschließend erfolgten insgesamt drei Übungsphasen anhand der drei Videoausschnitte. Dabei wurde die Komplexität der Beobachtungen langsam gesteigert. In den ersten beiden Phasen lag der Fokus lediglich auf den Kategorien zum Interaktionsverhalten der Kinder gegenüber anderen Kindern und Erwachsenen. Nach der ersten Übungsphase wurden Fragen und kritische Verhaltensweisen der Kinder diskutiert, um in der zweiten Phase erneut die Interaktionsvariablen zu beobachten. In der dritten Phase wurden schließlich alle Kategorien beobachtet. Nach jeder Übungsphase wurden die Ergebnisse der Studierenden verglichen und Unstimmigkeiten untereinander diskutiert, um eine möglichst ähnliche Einschätzung der kindlichen Verhaltensweisen über alle Beobachterinnen hinweg zu erreichen.

\subsubsection{Spontansprachaufnahmen}

Zur Einschätzung der produktiven Sprachfähigkeiten der Kinder im Deutschen wurden—wie oben beschrieben (siehe Kapitel 3.2.4.2)—Spontansprachaufnahmen von den Eltern im Rollenspiel „Der Bär beim Arzt“ angefertigt. Anschließend wurden alle vorhandenen Spontansprachaufnahmen (T1: $\mathrm{n}=23, \mathrm{~T} 4: \mathrm{n}=23$ ) von zwei geschulten Masterstudierenden der TU Dortmund nach einem festgelegten Schema mit Hilfe des Annotationsprogramms ELAN (Max Planck Institute for Psycholinguistics, The Language Archive, 2013) transkribiert und kodiert (für das Kodierschema siehe Anhang I). Ziel der Spontansprachanalysen war es, den Sprachentwicklungsstand der Kinder im Deutschen einzuschätzen. Als grobes Maß für den Sprachentwicklungsstand und die grammatische Komplexität der kindlichen Sprachäußerung wird die durchschnittliche Äußerungslänge (mean length of utterances, MLU) angesehen (Kauschke, 2012). Einsprachige Kinder haben mit drei Jahren eine MLU von ca. drei Wörtern, mit vier Jahren steigt diese auf etwa vier an (Kauschke, 2000; Rice et al., 2010).

Die MLU ist recht anfällig bei unterschiedlichen Samplegrößen. Gavin und Giles (1996) untersuchten die Reliabilität verschiedener Sprachmaße-unter anderem auch die MLU—in Abhängigkeit von der Größe der Spontansprachprobe. Hier zeigte sich, dass die Reliabilität des MLU-Maßes mit der Größe der Spontansprachprobe ansteigt. Berechnungen mit Proben von unter 50 Äußerungen erreichten dabei keine gute Reliabilität. Eine Aufnah- 
melänge von mindestens 12 Minuten mit mindestens 100 Äußerungen wird als Mindestmaß angesehen.

Wenngleich die Eltern in der Studie die Anweisung erhielten, eine Aufnahme von 10 bis 15 Minuten zu erstellen, waren die Aufnahmen häufig deutlich kürzer und variierten enorm hinsichtlich der Anzahl der kindlichen Äußerungen. Zwei Aufnahmen waren hinsichtlich zeitlicher Länge und Anzahl der Äußerungen nicht ausreichend für weitere Analysen. Die Aufnahmen waren unter drei Minuten und umfassten lediglich 9 bzw. 13 analysierbare Äußerungen ${ }^{5}$ In allen weiteren Aufnahmen konnten mindestens 30 analysierbare Äußerungen identifiziert werden. Um eine Vergleichbarkeit zwischen den Kindern zu erreichen, wurden aus diesem Grund die ersten 30 analysierbaren Äußerungen des Transkriptes der Kinder als Basis für alle zu berechnenden Sprachmaße herangezogen. Von einer zeitlichen Begrenzung des Transkriptes wurde abgesehen, da in einzelnen Aufnahmen die Anfangsphasen noch durch zahlreiche Störungen des Spiels zwischen Eltern und Kind geprägt waren (z.B. verließen einige Kinder den Raum, um weiteres Spielzeug zu holen). Aufbauend auf den Kodierungen der 30 Äußerungen wurden für die späteren Analysen die MLU berechnet.

Zur Berechnung der Interrater-Reliabilität wurden zehn Prozent der Daten $(\mathrm{n}=6)$ von beiden Kodiererinnen bearbeitet. Als Maß für die Interrater-Reliabilität wurde der Krippendorffs Alpha gewählt (Krippendorff, 2004) und für die Variablen MLU, jeweils Types (Anzahl verschiedener Wörter) und Token (Gesamtzahl der Wörter) für die einzelnen Wortarten sowie Satzarten (satzwertige Äußerungen, Ellipsen, Satzabbrüche) und kommunikative Funktionen berechnet. Tabelle 14 führt alle Interrater-Reliabilitäten auf.

Nach Krippendorff (2004) gelten Daten mit einem $\alpha>.800$ als reliabel. Daten mit einem $\alpha>.667$ und $<.800$ sollten nur unter großem Vorbehalt in weitere Analysen einbezogen werden. Daten mit einem $\alpha<.667$ gelten als nicht reliabel und sollten demnach ausgeschlossen werden.

\footnotetext{
${ }^{5}$ Als nicht analysierbar galten unverständliche Äußerungen, einfache Äußerungen wie ,ja“ oder „,nein“, Imitationen und Echolalien sowie Floskeln und Stereotypien. All diese Äußerungen haben keinen bedeutsamen Informationsgehalt zu den sprachlichen Fähigkeiten der Kinder, sondern spiegeln zum Beispiel die Nachsprechleistungen des Kindes wider. Siehe dazu auch Anhang G.
} 
Tabelle 14

Interrater-Reliabilitäten für die Spontansprachkodierungen

\begin{tabular}{lc}
\hline & Krippendorffs $\alpha$ \\
\hline Äußerungslänge & .890 \\
Types Nomen & .940 \\
Token Nomen & .967 \\
Types Verben & .954 \\
Token Verben & .969 \\
Types Adjektive & .923 \\
Token Adjektive & .955 \\
Types Pronomen & .946 \\
Token Pronomen & .963 \\
Types Präpositionen & .454 \\
Token Präpositionen & .737 \\
Types Adverbien & .853 \\
Token Adverbien & .856 \\
Types Lautmalereien & .414 \\
Token Lautmalereien & .385 \\
Types interaktive Wörter & .533 \\
Token interaktive Wörter & .302 \\
Types sonstige Funktionswörter & .448 \\
Token sonstige Funktionswörter & .598 \\
Kommurten & \\
\hline
\end{tabular}

In den vorliegenden Daten konnte eine gute bis sehr gute Reliabilität für die Variablen MLU sowie Types und Token für die Wortarten Nomen, Verben, Adjektive, Pronomen und Adverbien erreicht werden. Die Variablen Token Präpositionen sowie kommunikative Funktionen könnten nach den Konventionen von Krippendorff (2004) in die Analysen mit einbezogen werden. Deutliche Unterschiede in den Kodierungen zeigten sich besonders in den Variablen der „kleinen Wörter“ (Präpositionen, interaktive Wörter, Lautmalereien und sonstige Funktionswörter). In der qualitativen Betrachtung zeigte sich, dass bei beiden Kodiererinnen Unsicherheiten in der Einordnung der Wörter in die einzelnen Wortartenkategorien bestanden. Gleiches galt für die Variable Satzarten. Hier zeigte sich, dass die Definition der Kategorien „satzwertige Äußerung“ und „Ellipse“ nicht ausreichend war, um eine eindeutige Zuord- 
nung durch die beiden Kodiererinnen zu erreichen. Eine zeigte eine deutliche Tendenz zum Kodieren von „Ellipsen“, die andere eine Tendenz zu „satzwertigen Äußerungen“. Die Übereinstimmung der Kodiererinnen in der Variable Kommunikative Funktionen kann als mäßig eingeschätzt werden. Der Einflussfaktor Subjektivität konnte somit bei der Bewertung der Äußerung in ihrer kommunikativen Funktion nicht ausgeschlossen werden.

\subsubsection{Auswertung}

Zum besseren Verständnis werden die Methoden der statistischen Analysen im Ergebniskapitel für (1) die deskriptiven Analysen (siehe Kapitel 4.1) und (2) für die Hypothesenprüfung (siehe Kapitel 4.2) erläutert. Die gesamte Datenauswertung erfolgte mit SPSS 22.0.

\subsubsection{Prädiktor- und Outcomevariablen}

In der Studie wurde eine Vielzahl von Variablen erhoben. Für die Hauptanalysen der Studie, welche die Entwicklung des SM fokussieren, wurden als Outcomevariablen die Subskalen Schweigen des Elternfragebogens sowie Schweigen und Bedürfnisäußerung von DortMuS-Kita und die Interaktionsvariablen aus den Beobachtungen innerhalb der Freispielsituation verwendet. Darüber ist ein großer Anteil des Alltags der Kinder abgedeckt. Da Kinder mit SM vor allem in den Bildungsinstitutionen schweigen (Ford et al., 1998), sollte die Entwicklung zum Sprechen dort gut sichtbar sein und sich in den Einschätzungen der Erzieherinnen wie auch in den Beobachtungen widerspiegeln. Die Verhaltenskodierungen der Interaktionssettings wurden nicht in die Hauptanalysen der Studie einbezogen, da sich die Dummykodierung der Variablen nicht als Outcomevariable eignete. Diese wurden vor allem zur Diagnosestellung herangezogen.

Der Prädiktor Angst wurde über den T-Wert des BAV Gesamtwertes operationalisiert. Die Sprachkompetenzen unterteilten sich in rezeptive und produktive Sprachkompetenzen. Als Maß für die rezeptiven Sprachkompetenzen wurde der T-Wert des TROG-Ds verwendet, als Maß für die produktiven Sprachkompetenzen die MLU, die im Rahmen der Spontansprachanalysen ermittelt wurde und als grobes Maß der gesamten Sprachentwicklung herangezogen werden kann (siehe Kapitel 3.4.4.2). Die kulturelle Adaptation der Eltern wurde mithilfe des FRAKK gemessen. Als Variablen standen hier für die Betrachtung der einzelnen Kulturen die Subskalen Orientierung an Herkunftskultur (HK) und Orientierung an Aufnahmekultur (AK) zur Verfügung. Das Ausmaß des kulturellen Wandels wurde über den Akkulturationsindex (AI), welcher die Ergebnisse beider Subskalen vereint, operationalisiert. 
Tabelle 15

Operationalisierung von Prädiktor und Outcomevariablen für die Hauptanalysen

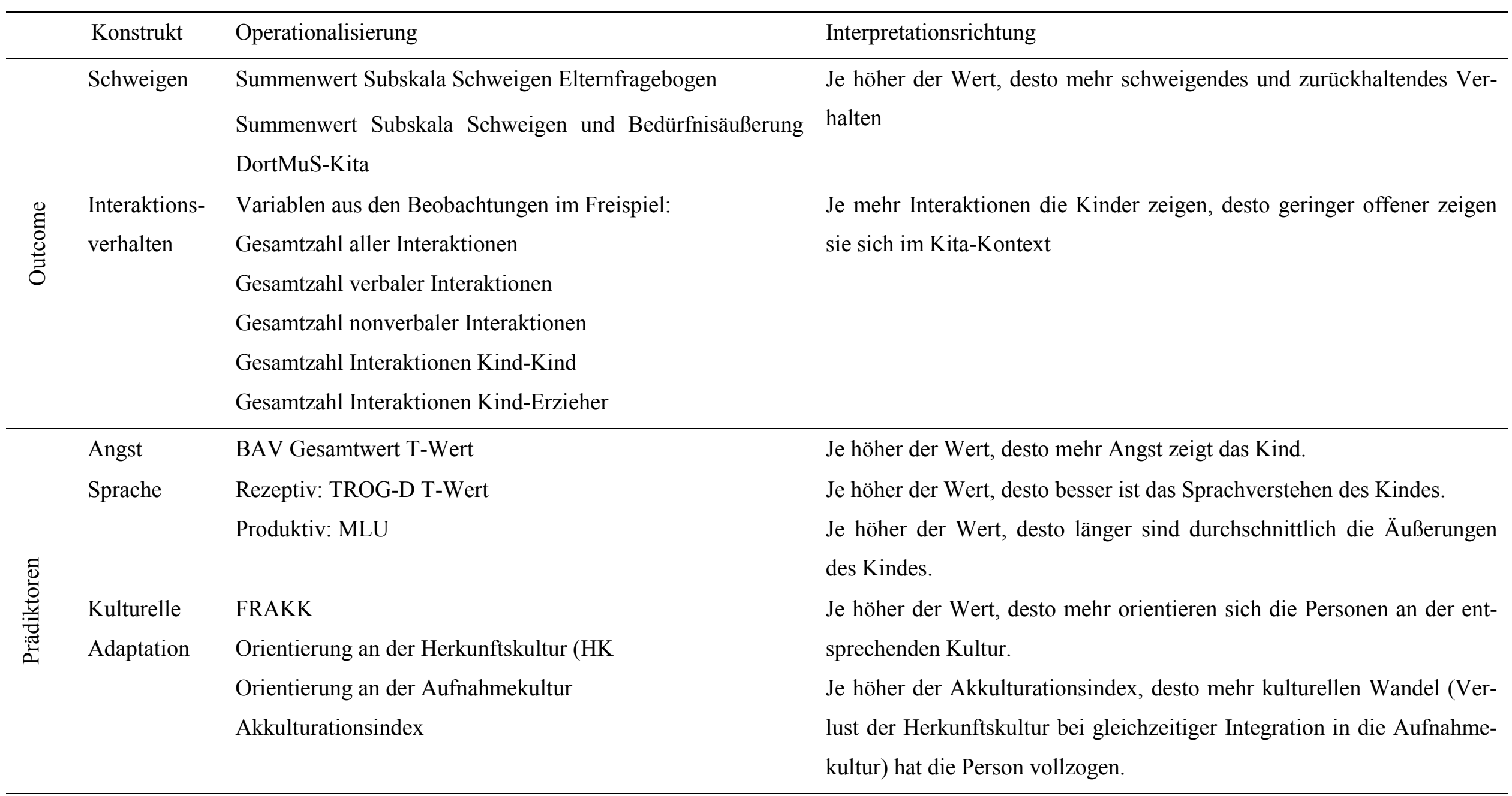


Für eine Übersicht zur Operationalisierung der Prädiktor- und Outcomevariablen sowie Interpretation der entsprechenden Werte siehe Tabelle 15.

\section{Analyse fehlender Werte}

Im Folgenden wird für Prädiktor- und Outcomevariablen der Anteil der fehlenden Werte berichtet. Dazu wird zwischen Item-Nonresponse und Wave-Nonresponse unterschieden. Eine Item-Nonresponse bedeutet in diesem Fall, dass für jeweils mindestens eine Prädiktor- oder Outcomevariable Werte zum entsprechenden Probanden vorlagen. Bei einer WaveNonresponse lagen für den entsprechenden Messzeitpunkt keinerlei Daten für die Prädiktoroder Outcomevariablen vor. Tabelle 16 gibt einen Überblick über die Anzahl der vollständigen Datensätze sowie Item- und Wave-Nonresponses zu allen vier Messzeitpunkten.

Tabelle 16

Übersicht zu fehlenden Werten innerhalb der Prädiktor- und Outcomevariablen zu allen vier Messzeitpunkten

\begin{tabular}{llccc}
\hline & & $\begin{array}{c}\text { vollständige Datensätze } \\
\mathrm{n}(\%)\end{array}$ & $\begin{array}{c}\text { Item-Nonresponse } \\
\mathrm{n}(\%)\end{array}$ & $\begin{array}{c}\text { Wave-Nonresponse } \\
\mathrm{n}(\%)\end{array}$ \\
\hline \multirow{2}{*}{$\mathrm{T} 1$} & Prädiktoren & $18(60)$ & $12(40)$ & $0(0)$ \\
& Outcome & $24(80)$ & $6(20)$ & $0(0)$ \\
$\mathrm{T} 2$ & Outcome & $18(60)$ & $11(37)$ & $1(3)$ \\
$\mathrm{T} 3$ & Outcome & $14(47)$ & $15(50)$ & $1(3)$ \\
& Prädiktoren & $19(63)$ & $9(30)$ & $2(7)$ \\
$\mathrm{T} 4$ & Outcome & $20(67)$ & $8(26)$ & $2(7)$ \\
\hline
\end{tabular}

Insgesamt zeigte sich, dass es eine Vielzahl von Item-Nonresponses gab und somit Daten zu der jeweiligen Variablengruppe vorlagen, einzelne Variablen jedoch mit fehlenden Werten gefüllt waren. Als Beispiel sei hier genannt, dass etwa die Beobachtungen in der Einrichtung beim Kind durchgeführt werden konnten, jedoch kein Fragebogen von den Erzieherinnen vorlag.

Im nächsten Schritt wurden die fehlenden Daten auf den einzelnen Variablen genauer betrachtet. In Abbildung 6 bis Abbildung 11 sind die Anteile vollständiger und nichtvollständiger Daten bezogen auf die einzelnen Variablen dargestellt. Wave-Nonresponses für die einzelnen Variablen bedeuten nun, dass die jeweilige Informationsquelle zum Messzeitpunkt nicht verfügbar war. Eine Item-Nonresponse kam dann zustande, wenn etwa der Angstfragebogen von den Eltern ausgefüllt worden ist, jedoch zu viele Items unbeantwortet blieben, 
so dass die Auswertung des Bogens nicht möglich war. Insbesondere bei den Beobachtungen kam es zu Item-Nonresponses, wenn etwa die Beobachtung der Freispielsituation möglich war, jedoch eine oder beide weiteren Situationen am Vormittag nicht vorkamen (z.B. wenn das Kind bereits zuhause gefrühstückt hatte).

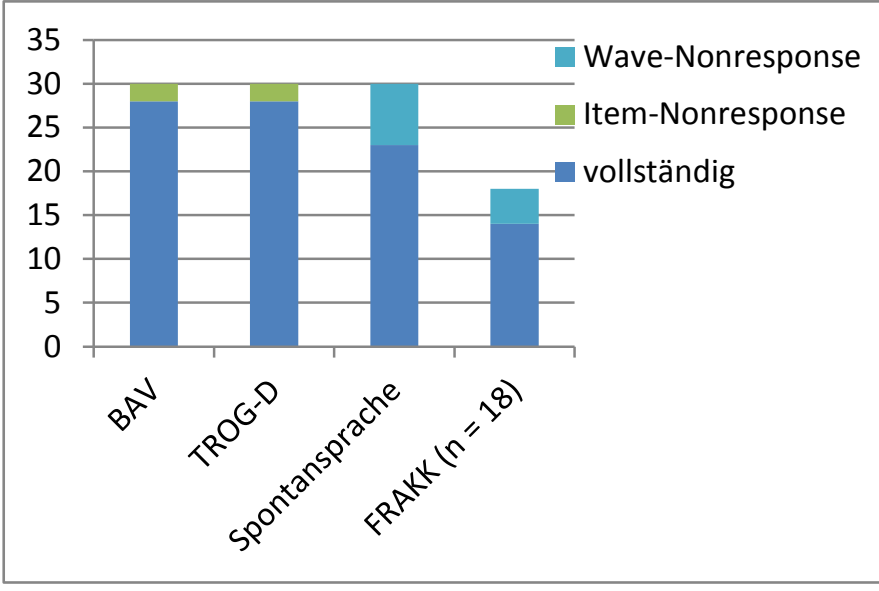

Abbildung 7. Fehlende Werte in den Prädiktorvariablen zum ersten Messzeitpunkt

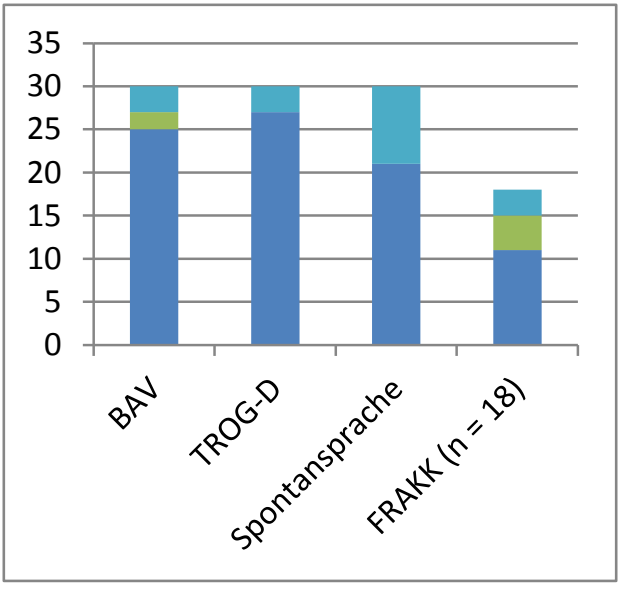

Abbildung 8. Fehlende Werte in den Prädiktorvariablen zum vierten

Messzeitpunkt

Die Nonresponse im Bereich der Kitas (sowohl für die Erzieherfragebögen als auch die Beobachtungen) war zum dritten Messzeitpunkt am höchsten, verursacht durch Kitawechsel von Probanden, einen Brand in einer Einrichtung sowie Schließzeiten in den Ferien. Zum vierten Messzeitpunkt sind zwei Kinder der Kontrollgruppen vollständig aus der Studie ausgeschieden-ein Kind war zum Testzeitpunkt schwer erkrankt, konnte die Termine an der Universität entsprechend nicht wahrnehmen und war längere Zeit nicht in der Kita; eine weitere Familie war telefonisch nicht mehr erreichbar. Bei einem weiteren Kind haben zwar die Testung in der Universität sowie die Beobachtungen in der Kita stattgefunden, jedoch hat die Familie weder Fragebögen noch Spontansprachaufnahme wieder abgegeben. Sie waren telefonisch nicht mehr erreichbar, so dass letztlich auf diese Daten verzichtet werden musste. 


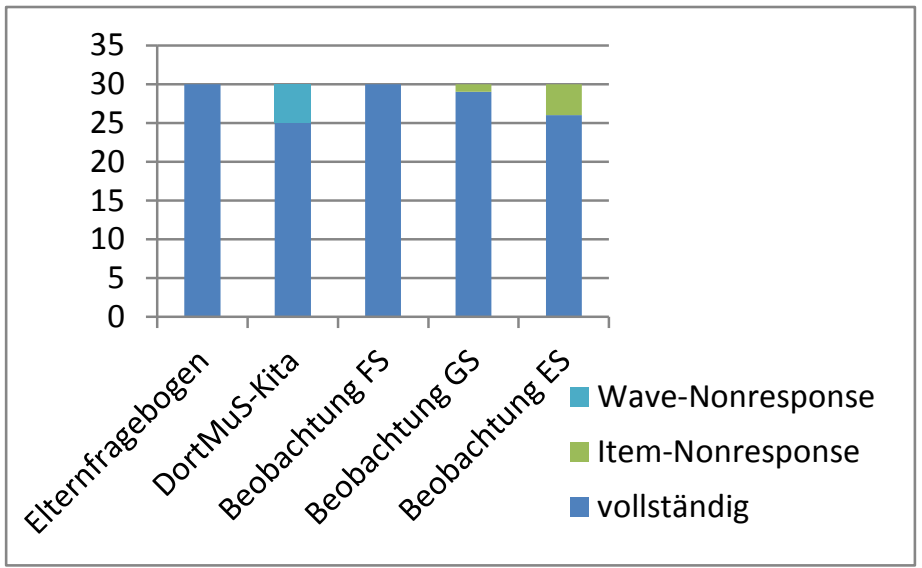

Abbildung 9. Fehlende Werte in den Outcomevariablen zum ersten Messzeitpunkt. FS = Freispielsituation, GS = Gruppensituation, ES = Essenssituation

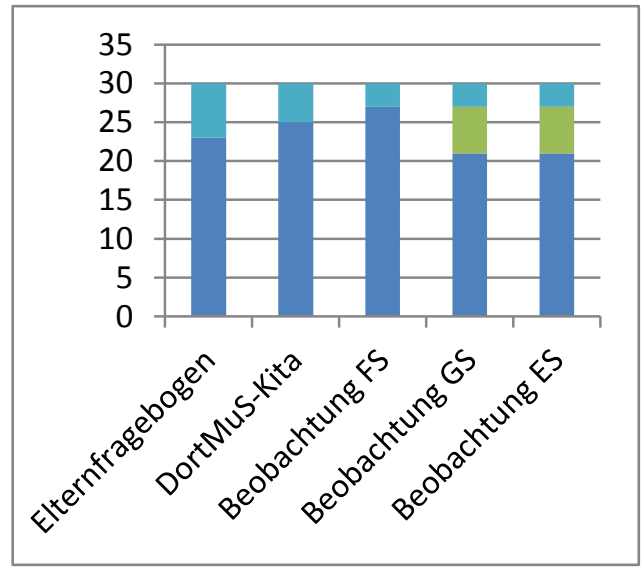

Abbildung 10. Fehlende Werte in den Outcomevariablen zum vierten Messzeitpunkt. FS = Freispielsituation, GS = Gruppensituation, ES = Essenssituation

Bei den Beobachtungen war die Freispielsituation diejenıge, die zu allen Messzeitpunkten beobachtet werden konnte. Gruppen- und Essensituation fanden häufiger nicht statt. Vor dem Hintergrund der Vergleichbarkeit des Interaktionsverhaltens aller Kinder werden somit vorrangig die Daten der Freispielsituation in die nachfolgenden Analysen einbezogen.

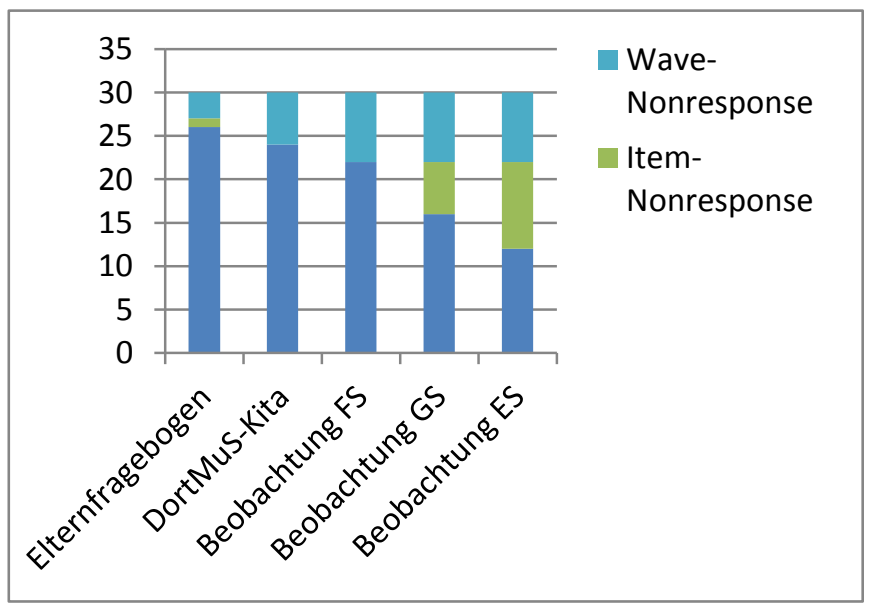

Abbildung 121. Fehlende Werte in den Outcomevariablen zum dritten Messzeitpunkt. FS = Freispielsituation, $\mathrm{GS}=$ Gruppensituation, $\mathrm{ES}=$ Essenssituation

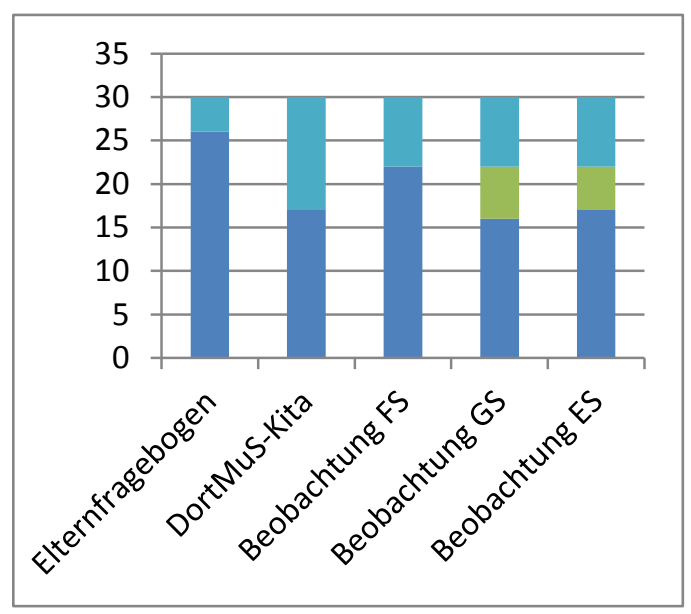

Abbildung 112. Fehlende Werte in den Outcomevariablen zum vierten Messzeitpunkt. $\mathrm{FS}=$ Freispielsituation, GS = Gruppensituation, $\mathrm{ES}=$ Essenssituation 


\section{Ergebnisse}

Im Folgenden werden die Ergebnisse der Hauptstudie präsentiert. Zunächst werden deskriptive Ergebnisse für die Outcomevariablen-Elternfragebogen, Erzieherfragebogen sowie Beobachtungen-dargestellt. Anschließend werden die in Kapitel 2.5 aufgestellten Hypothesen mit entsprechenden inferenzstatistischen Verfahren überprüft. In der Regel wird vor jedem Unterkapitel kurz erläutert, welche statistischen Methoden genutzt wurden.

\subsection{Deskriptive Analysen}

Die Überprüfung auf Normalverteilung der Daten erfolgte mithilfe von P-PDiagrammen und dem Kolmogorov-Smirnov-Test. Die Ergebnisse der Kolmogorov-SmirnovTests für die Prädiktoren und Outcomevariablen finden sich in den Tabellen A-9 und A-10 im Anhang. Als Maß für die zentrale Tendenz der einzelnen Variablen wurde einheitlich der Median $(M d)$ gewählt, da einerseits die Untergruppen teilweise sehr klein und andererseits die Daten innerhalb einzelner Variablen nicht normalverteilt waren. Entsprechend wurde als Streuungsmaß jeweils der Interquartilsabstand (IQR) berichtet. Dieser Logik der Daten folgend wurden zur Analyse von Zusammenhängen der Kendalls-Tau als Korrelationskoeffizient berechnet und zur Überprüfung von Gruppenunterschieden innerhalb der Prädiktoren und Outcomevariablen entweder der Kruskal-Wallis-Test (bei mehr als zwei Gruppen) oder der Mann-Whitney-U Test (bei zwei Gruppen) genutzt. Zusätzlich wurden Tendenzen in den Daten mithilfe des Jonckheere-Terpstra-Test untersucht. Dieser Test überprüft, ob eine statistisch bedeutungsvolle Rangfolge der einzelnen Gruppen besteht. Alle $p$-Werte wurden zweiseitig berechnet und $p$-Werte unter .05 als signifikant berichtet. Zusätzlich zu diesen Signifikanzwerten wurden für die Gruppenunterschiede bzw. Tendenzen jeweils die Effektstärke $r$ nach der Konvention von Rosenthal (1991) berechnet. Demnach ergibt sich $r$ aus folgender Formel:

$$
r=\frac{Z}{\sqrt{N}}
$$

in der $z$ die standardisierte Teststatistik und $N$ die Anzahl der Beobachtungen, auf denen $z$ basiert, ist.

Zunächst werden die Interkorrelationen der Prädiktoren und Outcomevariablen sowie die Korrelationen zwischen beiden berichtet. Anschließend werden deskriptive Ergebnisse zu selbigen für die einzelnen Untergruppen der Stichprobe präsentiert. 


\subsubsection{Interkorrelationen der Outcomevariablen}

Zur Überprüfung von Zusammenhängen zwischen den Outcomevariablen zu den einzelnen Messzeitpunkten wurden Korrelationsanalysen mit den Variablen Gesamtwert Skala Schweigen im Eltern- wie auch Erzieherfragebogen sowie den Variablen der Beobachtungen in der Freispielsituation durchgeführt. Es wurde erwartet, dass sich zwischen den Outcomevariablen zu allen vier Messzeitpunkten mehr oder weniger starke Zusammenhänge ergeben, da alle das Sprech- und Interaktionsverhalten der Kinder erfassen sollten. Der Erzieherfragebogen DortMuS-Kita sollte damit deutlich stärker mit den Beobachtungsvariablen zusammenhängen als der Elternfragebogen, da jeweils der Kontext Kita betrachtet wird. Für eine Übersicht zu den Interkorrelationen der Outcomevariablen siehe Tabelle 17 bis Tabelle 20.

Tabelle 17

Interkorrelationen zwischen den Outcomevariablen zum ersten Messzeitpunkt

\begin{tabular}{|c|c|c|c|c|c|c|}
\hline & 2 & 3 & 4 & 5 & 6 & 7 \\
\hline 1. Elternfragebogen & $.620^{* * *}$ & $-.322 *$ & $-.322 *$ & $-.322 *$ & $-.322 *$ & $-.322 *$ \\
\hline$(\mathrm{n}=30)$ & {$[.422, .798]$} & {$[-.577,-.075]$} & {$[-.577,-.075]$} & {$[-.577,-.075]$} & {$[-.577,-.075]$} & {$[-.577,-.075]$} \\
\hline 2. DortMuS-Kita $(n=25)$ & & $-.514 * * *$ & $-.649 * * *$ & $.497 * *$ & $-.559 * * *$ & -.031 \\
\hline & & {$[-.736,-.233]$} & {$[-.818,-.447]$} & {$[.249, .690]$} & {$[-.724,-.314]$} & {$[-.304, .252]$} \\
\hline 3. Gesamtzahl Interaktionen & & \multirow{2}{*}{ - } & $.769^{* * *}$ & -.240 & $.796^{* * *}$ & $.275^{*}$ \\
\hline$(\mathrm{n}=30)$ & & & {$[.604, .906]$} & {$[.-.565, .091]$} & {$[.647, .922]$} & {$[.003, .503]$} \\
\hline 4. Gesamtzahl verbaler & & & \multirow{2}{*}{-} & $-.509 * * *$ & $.699 * * *$ & .162 \\
\hline Interaktionen & & & & {$[-.704,-.270]$} & {$[.556, .824]$} & {$[-.147, .439]$} \\
\hline 5. Gesamtzahl nonverbale & & & & & -.212 & -.025 \\
\hline Interaktionen & & & & & {$[-.479, .061]$} & {$[-.359, .311]$} \\
\hline 6. Gesamtzahl Interaktionen & & & & & & .034 \\
\hline Kind-Kind & & & & & & {$[-.258, .280]$} \\
\hline 7. Gesamtzahl Interaktionen & & & & & & \\
\hline Kind-Erzieher & & & & & & \\
\hline
\end{tabular}

Anmerkungen. $* p<.05,{ }^{* *} p<.01, * * * p<.001,95 \%$ Konfidenzintervall in eckigen Klammern

Erwartungsgemäß korrelierten die beiden Fragebögen auf ihren Skalen zum Ausmaß des schweigenden Verhaltens signifikant positiv miteinander, $\tau=.620, p<.001$. Hohe Werte im Elternfragebogen gingen demnach auch mit hohen Werten im Erzieherfragebogen einher, ebenso niedrige Werte im Elternfragebogen mit niedrigen Werten im Erzieherfragebogen. Erwartungsgemäß zeigte sich ein negativer Zusammenhang zwischen den Werten in den Fragebögen und der Gesamtzahl der Interaktionen der Kinder in der Freispielsituation, d.h. je mehr die Kinder schwiegen, desto weniger interagierten sie insgesamt in der Kita. Der Zusammenhang war für den Erzieherfragebogen erwartungsgemäß stärker, $\tau=-.514, p<.001$, als für den Elternfragebogen, $\tau=-.363, p=.013$. Die Einschätzungen der Eltern und Erzieher hingen negativ mit der Gesamtzahl der verbalen Interaktionen der Kinder zusammen, wobei 
auch hier wiederum der Zusammenhang für den Erzieherfragebogen stärker war, $\tau=-.649, p$ $<.001$, als für den Elternfragebogen, $\tau=-.535, p<.001$. Je größer das Ausmaß des Schweigens der Kinder war, desto weniger interagierten sie verbal in der Freispielsituation mit anderen Personen. Ein positiver Zusammenhang zeigte sich zwischen den Werten der Fragebögen und der Gesamtzahl der nonverbalen Interaktionen, $\tau_{\text {Eltern }}=.448, p_{\text {Eltern }}=.003, \tau_{\text {Erzieher }}=.497$, $p_{\text {Erzieher }}=.001$. Je mehr die Kinder schwiegen, desto mehr nonverbale Interaktionen zeigten sie in der Freispielsituation in der Kita. Für die getrennte Betrachtung der Interaktionen zwischen Kindern untereinander sowie dem Kind und einer erwachsenen Person ergaben sich nur für erstere signifikante Zusammenhänge. Entsprechend der vorherigen Ergebnisse zeigte sich zwischen den Werten in den Fragebögen und der Gesamtzahl der Interaktionen zwischen zwei Kindern ein signifikant negativer Zusammenhang. Je mehr die Kinder schwiegen, desto weniger interagierten sie demnach mit einem oder mehreren anderen Kindern in der Kita. Auch hier war der Zusammenhang für den Erzieherfragebogen, $\tau=-.559, p<.001$, wiederum deutlich stärker als der für den Elternfragebogen, $\tau=-.344, p=.018$.

Die Interkorrelationen der Beobachtungsvariablen hingen zum Teil damit zusammen, dass zwei Variablen die gleichen Anteile der Daten beinhalteten. Die Gesamtzahl der Interaktionen ergab sich zum Beispiel aus der Summe aller verbalen und nonverbalen Interaktionen. Zusammenhänge zwischen diesen Variablen waren demnach nicht überraschend. Dennoch zeigte sich ein enger positiver Zusammenhang zwischen der Gesamtzahl der Interaktionen und verbalen Formen, $\tau=.769, p<.001$, im Vergleich zu einem eher niedrigen Zusammenhang mit nonverbalen Verhaltensweisen, $\tau=-.240, p=.075$. Kinder, die insgesamt viel in der Freispielsituation interagierten, taten dies eher verbal. Kinder die weniger interagierten, taten dies tendenziell eher auf nonverbaler Ebene. Erwartungsgemäß zeigte sich ein negativer $\mathrm{Zu}$ sammenhang zwischen der Gesamtzahl der verbalen und nonverbalen Interaktionen, $\tau=-.509, p<.001$. Kinder, die verbal interagierten, nutzten entsprechend seltener nonverbale Interaktionsformen und entsprechend anders herum nutzten die selten sprechenden Kinder eher nonverbale Kommunikationsformen. 
Tabelle 18

Interkorrelationen der Outcomevariablen zum zweiten Messzeitpunkt

\begin{tabular}{|c|c|c|c|c|c|c|}
\hline & 2 & 3 & 4 & 5 & 6 & 7 \\
\hline \multirow[t]{2}{*}{ 1. Elternfragebogen $(n=23)$} & $.344^{* a}$ & $.159^{\mathrm{b}}$ & $-.148^{b}$ & $.201^{\mathrm{b}}$ & $.072^{\mathrm{b}}$ & $.089^{\mathrm{b}}$ \\
\hline & {$[-.018, .658]$} & {$[-.106, .422]$} & {$[-.511, .223]$} & {$[-.163, .496]$} & {$[-.255, .366]$} & {$[-.253, .418]$} \\
\hline \multirow[t]{2}{*}{ 2. DortMuS-Kita $(n=25)$} & \multirow{2}{*}{ - } & $-.122^{c}$ & $-.267^{\mathrm{c}}$ & $.353^{* \mathrm{c}}$ & $-.257^{\mathrm{c}}$ & $.093^{\mathrm{c}}$ \\
\hline & & {$[-.409, .171]$} & {$[-.575, .060]$} & {$[.0 .52, .580]$} & {$[-.569, .072]$} & {$[-.186, .341]$} \\
\hline 3. Gesamtzahl Interaktionen & & \multirow{2}{*}{-} & .201 & .024 & $.629^{* * *}$ & .104 \\
\hline$(\mathrm{n}=27)$ & & & {$[-.091, .450]$} & {$[-.259, .306]$} & {$[.403, .818]$} & {$[-.193, .384]$} \\
\hline 4. Gesamtzahl verbaler & & & \multirow{2}{*}{-} & -.087 & -.071 & $.492 * *$ \\
\hline Interaktionen & & & & {$[-.456, .272]$} & {$[-.411, .253]$} & {$[.173, .771]$} \\
\hline 5. Gesamtzahl nonverbaler & & & & \multirow{2}{*}{ - } & -.279 & $.513^{* * *}$ \\
\hline Interaktionen & & & & & {$[-.512,-.042]$} & {$[.274, .691]$} \\
\hline 6. Gesamtzahl Interaktionen & & & & & \multirow{2}{*}{-} & $-.292 *$ \\
\hline Kind-Kind & & & & & & {$[-.542,-.003]$} \\
\hline \multicolumn{6}{|l|}{ 7. Gesamtzahl Interaktionen } & \\
\hline Kind-Erzieher & & & & & & - \\
\hline
\end{tabular}

Anmerkungen. ${ }^{*} p<.05,{ }^{* *} p<.01,{ }^{* * *} p<.001,95 \%$ Konfidenzintervall in eckigen Klammern; ${ }^{\mathrm{a}} \mathrm{n}=$ $19,{ }^{\mathrm{b}} \mathrm{n}=21,{ }^{\mathrm{c}} \mathrm{n}=24$

Auch zum zweiten Messzeitpunkt zeigte sich ein signifikant positiver Zusammenhang zwischen den Werten im Eltern- und im Erzieherfragebogen, $\tau=.344, p=.042$. Der Zusammenhang hat im Vergleich zu Testzeitpunkt 1 deutlich abgenommen. Es ist anzumerken, dass nur für 19 Probanden sowohl Eltern- als auch Erzieherfragebogen vorlagen. Die Zusammenhänge zwischen Elternfragebogen und den Ergebnissen der Beobachtungen in der Kita haben sich im Vergleich zum ersten Messzeitpunkt massiv geändert. Es bestanden keine signifikanten Zusammenhänge zwischen dem Interaktionsverhalten der Kinder in der Kita und den Einschätzungen der Eltern zum Sprechverhalten in familiären und öffentlichen Kontexten. Ähnliches zeigte sich auch für den Erzieherfragebogen. Auch hier zeigten sich die zu Messzeitpunt 1 deutlichen Zusammenhänge zwischen dem beobachteten Verhalten der Kinder in der Freispielsituation und den Einschätzungen der Erzieherinnen nicht mehr. Der Zusammenhang zwischen den Werten im Erzieherfragebogen und der Gesamtzahl nonverbaler Interaktionen war auch hier der Einzige, der statistisch signifikant wurde, $\tau=.353, p=.022$. Für den Elternwie für den Erzieherfragebogen galt: je höher die Werte in den Fragebögen waren, desto mehr nonverbale Interaktionen zeigten die Kinder in der Freispielsituation. Die negativen Zusammenhänge zwischen Werten im Erzieherfragebogen und der Gesamtzahl verbaler Interaktionen, $\tau=-.267, p=.085$, bzw. der Gesamtzahl der Interaktionen zwischen Kindern, $\tau=-.257$, $p=.082$, erreichten lediglich eine statistische Tendenz. Ähnlich wie zu Messzeitpunkt 1 interagierten Kinder mit hohen Werten im Erzieherfragebogen eher weniger verbal sowie eher seltener mit anderen Kindern. 
Die Interkorrelationen der Beobachtungsvariablen veränderten sich leicht im Vergleich zum ersten Messzeitpunkt. Es ergab sich lediglich ein signifikant positiver Zusammenhang zwischen der Gesamtzahl der Interaktionen und den Interaktionen zwischen Kindern, $\tau=.629, p=.000$. Kinder, die insgesamt viel interagierten, taten dies vor allem mit ihren Gleichaltrigen. Es zeigte sich kein Zusammenhang zwischen der Anzahl verbaler und nonverbaler Interaktionen, $\tau=-.087, p=.561$. Beide Variablen hingen jedoch signifikant positiv mit der Anzahl der Interaktionen zwischen Kind und Erzieher zusammen, $\tau_{\text {verbal }}=.492$, $p_{\text {verbal }}=.001, \tau_{\text {nonverbal }}=.513, p_{\text {nonverbal }}=.000$. Je mehr die Kinder mit einer erwachsenen Person in der Kita interagierten, desto mehr verbales und nonverbales Interaktionsverhalten zeigten sie. Für eine Übersicht über alle Interkorrelationen der Outcomevariablen zum zweiten Messzeitpunkt wird auf Tabelle 18 verwiesen.

Tabelle 19

Interkorrelationen der Outcomevariablen zum dritten Messzeitpunkt

\begin{tabular}{|c|c|c|c|c|c|c|}
\hline & 2 & 3 & 4 & 5 & 6 & 7 \\
\hline \multirow[t]{2}{*}{ 1. Elternfragebogen $(n=26)$} & $.288^{\mathrm{a}}$ & $.000^{\mathrm{b}}$ & $-.006^{b}$ & $.096^{\mathrm{b}}$ & $-.129^{b}$ & $-.018^{b}$ \\
\hline & {$[-.116, .614]$} & {$[-.304, .318]$} & {$[-.339, .308]$} & {$[-.256, .437]$} & {$[-.449, .182]$} & {$[-.364, .381]$} \\
\hline \multirow[t]{2}{*}{ 2. DortMuS-Kita $(n=17)$} & \multirow{2}{*}{-} & $-.174^{c}$ & $-.221^{c}$ & $.183^{\mathrm{c}}$ & $-.211^{\mathrm{c}}$ & $-.049^{c}$ \\
\hline & & {$[-.591, .278]$} & {$[-.624, .254]$} & {$[-.342, .662]$} & {$[-.637, .279]$} & {$[-.505, .396]$} \\
\hline 3. Gesamtzahl Interaktionen & & & $.779 * * *$ & .038 & $.718^{* * *}$ & .262 \\
\hline$(\mathrm{n}=22)$ & & & {$[.622, .907]$} & {$[-.345, .406]$} & {$[.476, .912]$} & {$[-.135, .607]$} \\
\hline 4. Gesamtzahl verbaler & & & & -.212 & $.808^{* * *}$ & .062 \\
\hline Interaktionen & & & - & {$[-.560, .204]$} & {$[.618, .954]$} & {$[-.321, .426]$} \\
\hline 5. Gesamtzahl nonverbaler & & & & & -.193 & $.603^{* * *}$ \\
\hline Interaktionen & & & & - & {$[-.551, .194]$} & {$[.364, .786]$} \\
\hline 6. Gesamtzahl Interaktionen & & & & & & -.035 \\
\hline Kind-Kind & & & & & - & {$[-.409, .364]$} \\
\hline 7. Gesamtzahl Interaktionen & & & & & & \\
\hline Kind-Erzieher & & & & & & \\
\hline
\end{tabular}

Anmerkungen. ${ }^{*} p<.05,{ }^{* *} p<.01,{ }^{* * *} p<.001,95 \%$ Konfidenzintervall in eckigen Klammern; ${ }^{a} \mathrm{n}=$ $16,{ }^{\mathrm{b}} \mathrm{n}=19,{ }^{\mathrm{c}} \mathrm{n}=15$

Der Zusammenhang zwischen Eltern- und Erzieherfragebogen erreichte zum dritten Messzeitpunkt keine statistische Signifikanz mehr, $\tau=.288, p=.124$. Aufgrund einer hohen Anzahl fehlender Erzieherfragebögen zu diesem Messzeitpunkt basierte diese Korrelationsberechnung jedoch leidglich auf 16 Probanden. Weder die Werte im Eltern- noch im Erzieherfragebogen hingen zum dritten Messzeitpunkt mit dem Interaktionsverhalten der Kinder in der Freispielsituation zusammen. Auch hier ist wieder die ungünstige Konstellation von nicht stattgefunden Beobachtungen und fehlenden Eltern- bzw. Erzieherfragebögen anzugeben. Für 
die Kombination Elternfragebogen und Beobachtungen konnte auf die Daten von 19 Probanden zurückgegriffen werden, für den Erzieherfragebogen lediglich auf 15 Probanden.

Zum dritten Messzeitpunkt zeigte sich wiederum ein deutlicher Zusammenhang zwischen der Gesamtzahl der Interaktionen und der Gesamtzahl verbaler Interaktionen, $\tau=.799$, $p<.001$, sowie der Gesamtzahl der Interaktionen zwischen dem Kind und einem/mehreren anderen, $\tau=.718, p<.001$. Kinder, die insgesamt viel interagierten, taten dies vor allem in verbaler Art und Weise mit anderen Kindern. Entsprechend hoch war die Korrelation zwischen der Gesamtzahl verbaler Interaktionen und der Gesamtzahl der Kind-Kind Interaktionen, $\tau=.808, p<.001$. Das nonverbale Interaktionsverhalten hingegen hing signifikant positiv mit Kind-Erzieher Interaktionen zusammen, $\tau=.603, p<.001$. Demnach interagierten Kinder, die häufiger nonverbales Interaktionsverhalten zeigten, auch häufiger mit erwachsenen Personen. Eine Übersicht über alle Interkorrelationen der Outcomevariablen zum dritten Messzeitpunkt findet sich in Tabelle 19.

Tabelle 20

Interkorrelationen der Outcomevariablen zum vierten Messzeitpunkt

\begin{tabular}{|c|c|c|c|c|c|c|}
\hline & 2 & 3 & 4 & 5 & 6 & 7 \\
\hline \multirow[t]{2}{*}{ 1. Elternfragebogen $(n=27)$} & $.556^{* * * a}$ & $-.332 * b$ & $-.394 * b$ & $.299^{b}$ & $-.333^{* b}$ & $.115^{b}$ \\
\hline & {$[.328, .744]$} & {$[-.638,-.010]$} & {$[-.668,-.129]$} & {$[-.033, .644]$} & {$[-.667, .025]$} & {$[-.243, .471]$} \\
\hline \multirow[t]{2}{*}{ 2. DortMuS-Kita $(n=24)$} & \multirow{2}{*}{-} & $-.279^{b}$ & $-.341 * b$ & $.090^{\mathrm{b}}$ & $-.225^{b}$ & $.065^{\mathrm{b}}$ \\
\hline & & {$[-.548, .031]$} & {$[-.615,-.037]$} & {$[-.324, .452]$} & {$[-.492, .083]$} & {$[-.322, .416]$} \\
\hline 3. Gesamtzahl Interaktionen & & & $.728 * * *$ & .005 & $-.678 * * *$ &.-.068 \\
\hline$(\mathrm{n}=22)$ & & 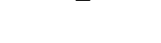 & {$[.519, .896]$} & {$[-.363, .350]$} & {$[.474, .846]$} & {$[-.429, .326]$} \\
\hline 4. Gesamtzahl verbaler & & & & -.288 & $.549 * * *$ & -.091 \\
\hline Interaktionen & & & - & {$[-.623, .062]$} & {$[.358, .716]$} & {$[-.459, .267]$} \\
\hline 5. Gesamtzahl nonverbaler & & & & & -.032 & -.060 \\
\hline Interaktionen & & & & 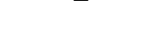 & {$[-.380, .326]$} & {$[-.439, .361]$} \\
\hline 6. Gesamtzahl Interaktionen & & & & & & $-.418 * *$ \\
\hline Kind-Kind & & & & & - & {$[-.695,-.106]$} \\
\hline 7. Gesamtzahl Interaktionen & & & & & & - \\
\hline Kind-Erzieher & & & & & & \\
\hline
\end{tabular}

Anmerkungen. ${ }^{*} p<.05,{ }^{* *} p<.01,{ }^{* * *} p<.001,95 \%$ Konfidenzintervall in eckigen Klammern; ${ }^{\mathrm{a}} \mathrm{n}=$ $23,{ }^{\mathrm{b}} \mathrm{n}=21$

Zum vierten Messzeitpunkt zeigte sich wiederum der signifikant positive Zusammenhang zwischen Werten im Eltern- und Erzieherfragebogen, $\tau=.556, p<.001$. Kinder, die in familiären und öffentlichen Situationen laut Eltern noch eher schwiegen, schwiegen den Erzieherinnen zufolge auch noch eher in der Kita. Das Interaktionsverhalten der Kinder hing zudem wieder deutlich mit den Einschätzungen der Eltern zusammen. Signifikant negative Zusammenhänge zeigten sich zwischen Werten im Elternfragebogen und der Gesamtzahl der 
Interaktionen, $\tau=-.332, p=.039$, der Gesamtzahl der verbalen Interaktionen, $\tau=-.394, p=$ .014 , sowie der Gesamtzahl der Interaktionen zwischen Kindern, $\tau=-.333, p=.039$. Statistisch tendenziell war der Zusammenhang zwischen Elternfragebogen und der Gesamtzahl nonverbaler Interaktionen, $\tau=.299, p=.067$. Kinder mit einem höheren Ausmaß des Schweigens interagierten demnach weniger in Freispielsituationen mit anderen Personen, hier vor allem mit Kindern, und zeigten eher nonverbale anstatt verbaler Verhaltensweisen.

Der Zusammenhang zwischen Erzieherfragebogen und beobachtetem Interaktionsverhalten erreichte im Gesamt nur ein statistisch tendenzielles Niveau, $\tau=-.279, p=.083$, blieb jedoch weiterhin erwartungsgemäß negativ. Ein signifikant negativer Zusammenhang zeigte sich lediglich für die Variable der Gesamtzahl verbaler Interaktionen, $\tau=-.341, p=.034$. Kinder mit hohen Werten im Erzieherfragebogen, d.h. einem hohen Ausmaß des Schweigens in der Kita, interagierten entsprechend auch weniger verbal in Freispielsituationen.

Für die Beobachtungsvariablen ergaben sich ähnliche Zusammenhänge untereinander wie zu den vergangenen Messzeitpunkten. Die Gesamtzahl der Interaktionen des Kindes in der Freispielsituation hing signifikant positiv mit der Gesamtzahl verbaler Interkationen zusammen, $\tau=.728, p<.001$, und mit der Gesamtzahl der Kind-Kind Interaktionen, $\tau=.678$, $p<.001$. Entsprechend war auch der Zusammenhang zwischen den verbalen Interaktionen und den Kind-Kind Interaktionen signifikant positiv, $\tau=.549, p<.001$. Ein signifikant negativer Zusammenhang zeigte sich zwischen der Gesamtzahl der Kind-Kind Interaktionen und den Kind-Erzieher Interaktionen, $\tau=-.418, p=.009$. Kinder, die viel mit anderen Kinder interagierten, taten dies weniger mit einer erwachsenen Person. Tabelle 20 zeigt alle Interkorrelationen der Outcome-Variablen zum vierten Messzeitpunkt im Überblick.

\subsubsection{Interkorrelationen der Prädiktorvariablen}

Im Folgenden werden die Interkorrelationen der Prädiktorvariablen präsentiert. Zusätzlich wurde kontrolliert, ob es Zusammenhänge zwischen dem sozioökonomischen Status sowie dem kulturellen Kapital und den Prädiktorvariablen gab, da sich zwischen den rekrutierten Gruppen Unterschiede in diesen soziodemografischen Variablen gezeigt haben (siehe Kapitel 3.4.2). Tabelle 21 gibt einen Überblick über die Interkorrelationen der Prädiktoren.

Die Prädiktoren korrelierten zum ersten Messzeitpunkt nur in sehr geringem Maße miteinander. Die Gesamtangst der mehrsprachigen Kinder hing signifikant negativ mit der Orientierung an der Herkunftskultur der Eltern zusammen, $\tau=-.433, p=.039$. Je stärker die Eltern sich an der Herkunftskultur orientierten, desto niedriger war die Gesamtangst der Kinder. Entsprechend zeigte sich auch ein positiver Zusammenhang zwischen der Gesamtangst 
und dem Akkulturationsindex, welcher aus den Subskalen Orientierung an Herkunftskultur (für den Gesamtwert umgepolt) und Orientierung an Aufnahmekultur besteht, $\tau=.451, p=$ .030. Je höher der Akkulturationsindex der Eltern war, desto höher war die Gesamtangst der Kinder. Diesen Berechnungen lagen Daten von 14 Probanden zugrunde.

Tabelle 21

Interkorrelationen der Prädiktoren zum ersten Messzeitpunkt

\begin{tabular}{|c|c|c|c|c|c|c|c|}
\hline & 2 & 3 & 4 & 5 & 6 & 7 & 8 \\
\hline 1. BAV & $.104^{\mathrm{a}}$ & $.112^{\mathrm{b}}$ & $-.433 * \mathrm{c}$ & $.238^{\mathrm{c}}$ & $.451 * \mathrm{c}$ & $-.174^{\mathrm{d}}$ & $-.144^{d}$ \\
\hline $\mathrm{n}=28$ & {$[-.218, .438]$} & {$[-.264, .482]$} & {$[-.757, .025]$} & {$[-.235, .745]$} & {$[.076, .787]$} & {$[-.499, .177]$} & {$[-.464, .171]$} \\
\hline 2. TROG-D & \multirow{4}{*}{-} & $.287^{\mathrm{e}}$ & $-.032^{f}$ & $.262^{\mathrm{f}}$ & $.222^{\mathrm{f}}$ & $.290^{\mathrm{d}}$ & $.244^{\mathrm{d}}$ \\
\hline $\mathrm{n}=28$ & & {$[-.028, .622]$} & {$[-.515, .458]$} & {$[-.198, .672]$} & {$[-.284, .661]$} & {$[-.051, .543]$} & {$[.010, .441]$} \\
\hline 3. MLU & & \multirow{2}{*}{ - } & $-.255^{\mathrm{g}}$ & $-.192^{\mathrm{g}}$ & $-.109^{\mathrm{g}}$ & $.044^{\mathrm{e}}$ & $.107^{\mathrm{e}}$ \\
\hline $\mathrm{n}=23$ & & & {$[-1.00, .454]$} & {$[-.889, .459]$} & {$[-.750, .858]$} & {$[-.347, .415]$} & {$[-.221, .410]$} \\
\hline 4. FRAKK HK & & & \multirow{2}{*}{-} & -.222 & $-.568 * *$ & .075 & .082 \\
\hline $\mathrm{n}=14$ & & & & {$[-.658, .337]$} & {$[-.902,-.138]$} & {$[-.452, .615]$} & {$[-.468, .551]$} \\
\hline 5. FRAKK AK & & & & \multirow{2}{*}{ - } & $.613^{* *}$ & $.472 *$ & $-.476^{*}$ \\
\hline $\mathrm{n}=14$ & & & & & {$[.278, .862]$} & {$[-.029, .887]$} & {$[-.869, .062]$} \\
\hline 6. FRAKK AI & & & & & \multirow{2}{*}{-} & .248 & -.370 \\
\hline $\mathrm{n}=14$ & & & & & & {$[-.275, .723]$} & {$[-.759, .068]$} \\
\hline \multirow[t]{2}{*}{ 7. $\mathrm{KK}$} & & & & & & - & $.175^{\mathrm{h}}$ \\
\hline & & & & & & & {$[-.214, .501]$} \\
\hline 8. SES & & & & & & & - \\
\hline
\end{tabular}

Anmerkungen. FRAKK HK = Subskala Orientierung an Herkunftskultur, FRAKK AK = Subskala Orientierung an Aufnahmekultur, FRAKK AI = Akkulturationsindex, KK = Kulturelles Kapital, SES $=$ Sozioökonomisches Status, ${ }^{*} p<.05,{ }^{* *} p<.01,{ }^{* * *} p<.001,95 \%$ Konfidenzintervall in eckigen Klammern; ${ }^{\mathrm{a}} \mathrm{n}=26,{ }^{\mathrm{b}} \mathrm{n}=21,{ }^{\mathrm{c}} \mathrm{n}=14,{ }^{\mathrm{d}} \mathrm{n}=27,{ }^{\mathrm{e}} \mathrm{n}=22,{ }^{\mathrm{f}} \mathrm{n}=12,{ }^{\mathrm{g}} \mathrm{n}=8,{ }^{\mathrm{h}} \mathrm{n}=29$

Ein statistisch tendenziell positiver Zusammenhang zeigte sich zwischen dem Grammatikverständnis und der MLU, $\tau=.287, p=.072$. Je besser die Kinder im TROG-D abschnitten, desto höher war auch ihre MLU. Für keine der Sprachvariablen zeigten sich statistisch bedeutsame Zusammenhänge mit der kulturellen Adaptation der Eltern (siehe Tabelle 21). Ein statistisch tendenzieller Zusammenhang zeigte sich zwischen den Leistungen im Grammatikverständnis und dem kulturellen Kapital der Familie (gemessen über die Anzahl der Bücher im Haushalt), $\tau=.290, p=.058$, sowie mit dem sozioökonomischen Status, $\tau=.244, p=.091$. Je höher das kulturelle Kapital bzw. der sozioökonomische Status der Familie war, desto höher waren die Leistungen der Kinder im TROG-D.

Entsprechend der Konstruktion des FRAKK zeigte sich ein signifikant negativer Zusammenhang zwischen der Subskala Orientierung an der Herkunftskultur mit dem Akkulturationsindex, $\tau=-.568, p=.006$, und ein signifikant positiver Zusammenhang zwischen der Orientierung an der Aufnahmekultur und dem Akkulturationsindex, $\tau=.613, p=.003$. Die 
Subskalen korrelierten nicht signifikant miteinander, $\tau=-.222, p=.289$. Ein signifikant positiver Zusammenhang zeigte sich zudem zwischen der Orientierung der Eltern an der Aufnahmekultur und dem kulturellen Kapital der Familie, $\tau=.472, p=.032$, sowie ein signifikant negativer Zusammenhang mit dem sozioökonomischen Status, $\tau=-.476, p=.025$. Je höher das kulturelle Kapital bzw. je niedriger der sozioökonomische Status der Familie war, desto höher war die Akkulturation der Eltern.

\subsubsection{Korrelationen zwischen Prädiktoren und Outcomevariablen}

Eine Übersicht über die Zusammenhänge zwischen Prädiktorvariablen zum ersten Messzeitpunkt und den Outcomevariablen zu allen vier Messzeitpunkten findet sich in Tabelle 22 bis Tabelle 25 .

Tabelle 22

Korrelationen zwischen den Prädiktoren und den Werten auf der Subskala Schweigen des Elternfragebogens zu allen vier Messzeitpunkten

Subskala Schweigen Elternfragebogen

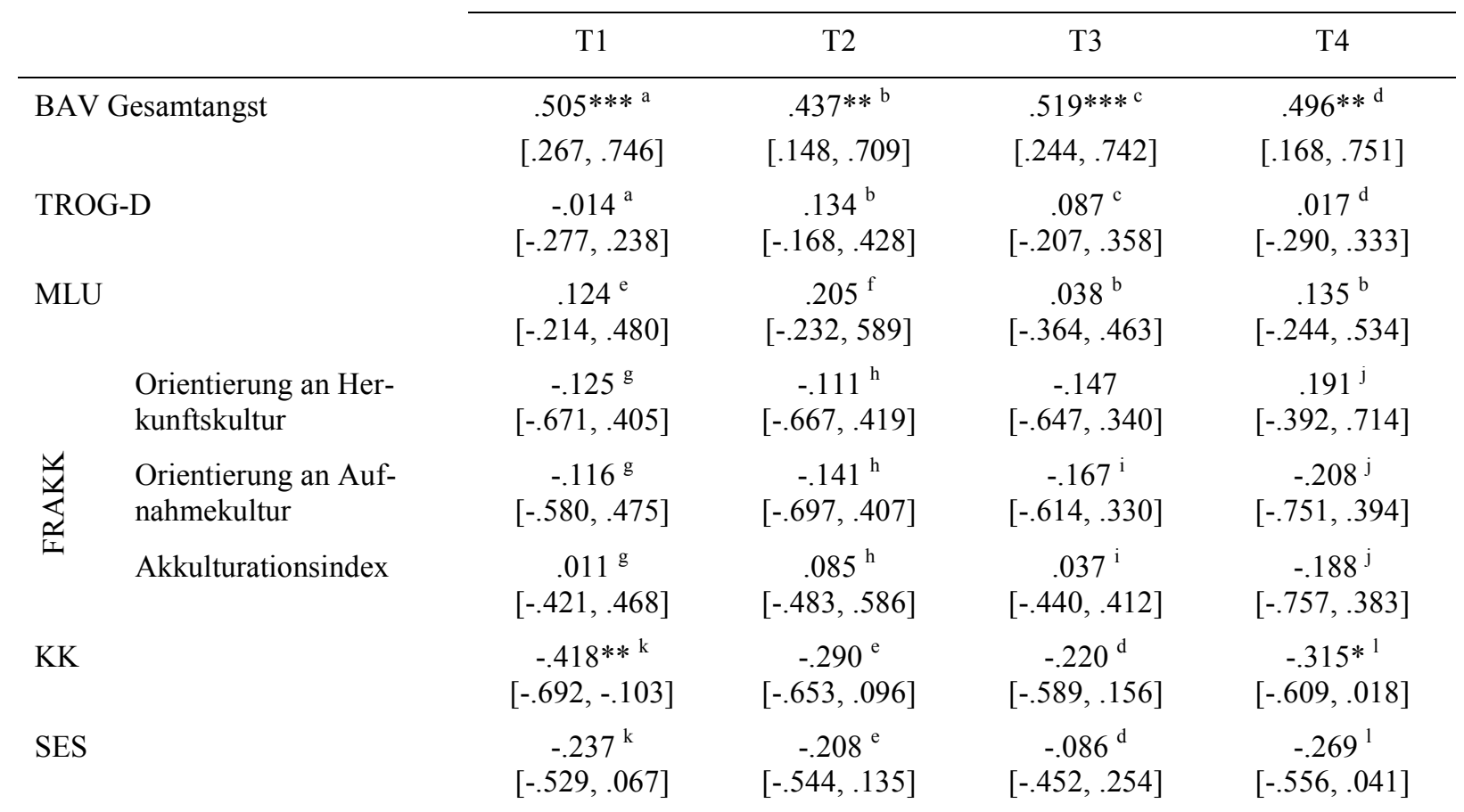

Anmerkungen. $* p<.05, * * p<.01, * * * p<.001,95 \%$ Konfidenzintervall in eckigen Klammern; ${ }^{\mathrm{a}} \mathrm{n}=$ $28,{ }^{\mathrm{b}} \mathrm{n}=21,{ }^{\mathrm{c}} \mathrm{n}=24,{ }^{\mathrm{d}} \mathrm{n}=25,{ }^{\mathrm{e}} \mathrm{n}=23,{ }^{\mathrm{f}} \mathrm{n}=18,{ }^{\mathrm{g}} \mathrm{n}=14,{ }^{\mathrm{h}} \mathrm{n}=9,{ }^{\mathrm{i}} \mathrm{n}=11,{ }^{\mathrm{j}} \mathrm{n}=12,{ }^{\mathrm{k}} \mathrm{n}=29,{ }^{1} \mathrm{n}=26$

Ein signifikant positiver Zusammenhang zwischen der Gesamtangst und dem Ausmaß des Schweigens eingeschätzt durch die Eltern zeigte sich über alle vier Messzeitpunkte hinweg, $\tau_{1}=.505, p_{1}<.001, \tau_{2}=.437, p_{2}=.006, \tau_{3}=.519, p_{3}<.001, \tau_{4}=.496, p_{4}=.001 . \mathrm{Je}$ 
mehr Angst die Kinder hatten, desto mehr schwiegen sie in familiären und öffentlichen Kontexten. Zwischen den weiteren Prädiktoren-Sprachkompetenz und elterliche Akkulturation-ergaben sich keine signifikanten Zusammenhänge mit dem Ausmaß des Schweigens (siehe Tabelle 22).

Der Zusammenhang zwischen Gesamtangst und Ausmaß des Schweigens zeigte sich für den Erzieherfragebogen nicht in konstanter Weise. Hier korrelierten die Werte des Fragebogens lediglich zum vierten Messzeitpunkt mit der Gesamtangst der Kinder, $\tau=.319$, $p=.044$. Je höher die Angst der Kinder demnach zu T1 war, desto mehr schwiegen sie zu T4. Wiederum keine Zusammenhänge zeigten sich zwischen dem Ausmaß des Schweigens und den Sprachkompetenzen der Kinder (siehe Tabelle 23). Jedoch zeigte sich für den Erzieherfragebogen ein tendenzieller bzw. teilweise signifikant negativer Zusammenhang mit der Orientierung der Eltern an der Aufnahmekultur, $\tau_{1}=-.485, p_{1}=.084, \tau_{2}=-.563, p_{2}=.013, \tau_{3}=-$ $.781, p_{3}=.015, \tau_{4}=-.506, p_{4}=.052$. Je mehr sich die Eltern demnach an der Aufnahmekultur orientierten, desto weniger schwiegen die mehrsprachigen Kinder im Kontext der Kita. Hierbei muss jedoch bedacht werden, dass die Gruppengröße über die Messzeitpunkte hinweg aufgrund von fehlenden Daten schwankte und zudem insgesamt recht klein war. Die Konfidenzintervalle waren entsprechend breit.

Für die Beobachtungsvariablen zeigten sich keine derart deutlichen und vor allem konstanten Zusammenhänge mit den Prädiktoren. Zur besseren Übersicht werden hier beispielhaft nur die Variablen Gesamtzahl der Interaktionen (siehe Tabelle 24) sowie Gesamtzahl der verbalen Interkationen in der Freispielsituation (siehe Tabelle 25) betrachtet. Die Korrelationen zwischen Prädiktoren und den übrigen Beobachtungsvariablen finden sich in den Tabellen A-11 bis A-13 im Anhang. 
Tabelle 23

Korrelationen zwischen den Prädiktoren und den Werten auf der Subskala Schweigen und Bedürfnisäußerung des DortMuS-Kita zu allen vier Messzeitpunkten

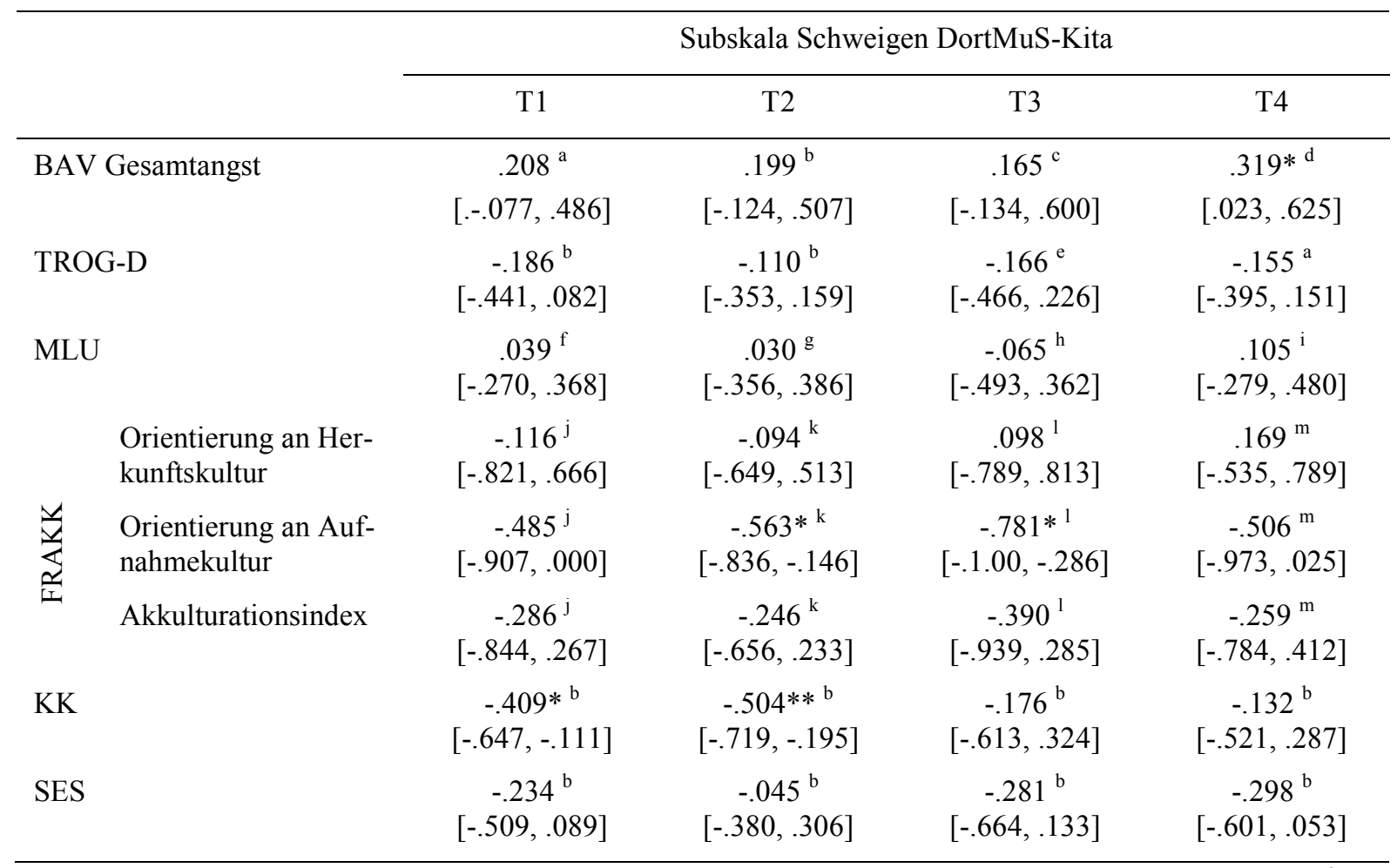

Anmerkungen. ${ }^{*} p<.05, * * p<.01, * * * p<.001,95 \%$ Konfidenzintervall in eckigen Klammern, ${ }^{a} \mathrm{n}=$ $23,{ }^{\mathrm{b}} \mathrm{n}=24,{ }^{\mathrm{c}} \mathrm{n}=15,{ }^{\mathrm{d}} \mathrm{n}=22,{ }^{\mathrm{e}} \mathrm{n}=16,{ }^{\mathrm{f}} \mathrm{n}=21,{ }^{\mathrm{g}} \mathrm{n}=19,{ }^{\mathrm{h}} \mathrm{n}=13,{ }^{\mathrm{i}} \mathrm{n}=17,{ }^{\mathrm{j}} \mathrm{n}=9,{ }^{\mathrm{k}} \mathrm{n}=12,{ }^{1} \mathrm{n}=7,{ }^{\mathrm{m}} \mathrm{n}$ $=10$

Signifikante Zusammenhänge zeigten sich nur zu vereinzelten Messzeitpunkten. Beispielsweise bestand ein signifikant positiver Zusammenhang zwischen der Gesamtzahl der Interaktionen und dem Akkulturationsindex der Eltern, $\tau=.452, p=.027$ und ein tendenziell positiver Zusammenhang mit der Orientierung der Eltern an der Aufnahmekultur, $\tau=.384$, $p=.066$, zum ersten Messzeitpunkt. Ein signifikant positiver Zusammenhang zeigte sich zudem zwischen der Gesamtzahl der Interaktionen zum ersten Messzeitpunkt und dem kulturellen Kapital der Familie, $\tau=.359, p=.013$, sowie zum zweiten Messzeitpunkt mit dem sozioökonomischen Status, $\tau=.328, p=.023$. Entsprechend hing die Gesamtzahl verbaler Interaktionen zum ersten Messzeitpunkt wiederum mit dem kulturellen Kapital zusammen, $\tau=.376$, $p=.009$. Einen tendenziellen Zusammenhang zwischen den beiden Variablen gab es zudem zum zweiten Messzeitpunkt, $\tau=.296, p=.064$. Alle weiteren Korrelationen schwankten hinsichtlich ihrer Stärke und Richtung über die vier Messzeitpunkte. Die Konfidenzintervalle waren zudem z.T. sehr groß und überschritten häufig den Nullpunkt. 
Tabelle 24

Korrelationen zwischen den Prädiktoren und der Gesamtzahl der Interaktionen in der Freispielsituation

\begin{tabular}{|c|c|c|c|c|c|}
\hline & & \multicolumn{4}{|c|}{ Gesamtzahl der Interaktionen } \\
\hline & & $\mathrm{T} 1$ & $\mathrm{~T} 2$ & $\mathrm{~T} 3$ & $\mathrm{~T} 4$ \\
\hline \multirow{2}{*}{\multicolumn{2}{|c|}{ BAV Gesamtangst }} & $-.096^{\mathrm{a}}$ & $.164^{\mathrm{b}}$ & $-.215^{c}$ & $.081^{\mathrm{c}}$ \\
\hline & & {$[-.405, .223]$} & {$[-.191, .457]$} & {$[-.542, .129]$} & {$[-.266, .457]$} \\
\hline \multicolumn{2}{|c|}{ TROG-D } & $\begin{array}{c}.092^{\mathrm{a}} \\
{[-.223, .398]}\end{array}$ & $\begin{array}{c}.039^{\mathrm{d}} \\
{[-.293, .324]}\end{array}$ & $\begin{array}{c}-.070^{\mathrm{e}} \\
{[-.391, .258]}\end{array}$ & $\begin{array}{c}.085^{\mathrm{e}} \\
{[-.347, .491]}\end{array}$ \\
\hline \multicolumn{2}{|c|}{ MLU } & $\begin{array}{c}.029^{\mathrm{f}} \\
{[-.315, .332]}\end{array}$ & $\begin{array}{c}.086^{\mathrm{c}} \\
{[-.238, .381]}\end{array}$ & $\begin{array}{c}-.329^{\mathrm{g}} \\
{[-.652, .062]}\end{array}$ & $\begin{array}{c}-.020^{\mathrm{h}} \\
{[-.448, .404]}\end{array}$ \\
\hline \multirow{3}{*}{ 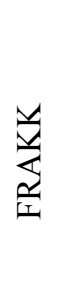 } & $\begin{array}{l}\text { Orientierung an Her- } \\
\text { kunftskultur }\end{array}$ & $\begin{array}{c}-.251^{\mathrm{i}} \\
{[-.623, .239]}\end{array}$ & $\begin{array}{c}.053^{\mathrm{j}} \\
{[-.427, .575]}\end{array}$ & $\begin{array}{c}-.019^{\mathrm{k}} \\
{[-.474, .551]}\end{array}$ & $\begin{array}{c}-.036^{1} \\
{[-.668,1.00]}\end{array}$ \\
\hline & $\begin{array}{l}\text { Orientierung an Auf- } \\
\text { nahmekultur }\end{array}$ & $\begin{array}{c}.384^{\mathrm{i}} \\
{[-.040, .722]}\end{array}$ & $\begin{array}{c}-.123^{\mathrm{j}} \\
{[-.706, .433]}\end{array}$ & $\begin{array}{c}.076^{\mathrm{k}} \\
{[-.375, .534]}\end{array}$ & $\begin{array}{c}.074^{1} \\
{[-.778,1.00]}\end{array}$ \\
\hline & Akkulturationsindex & $\begin{array}{c}.452^{* \mathrm{i}} \\
{[.025, .735]}\end{array}$ & $\begin{array}{c}-.211^{\mathrm{j}} \\
{[-.736, .368]}\end{array}$ & $\begin{array}{c}.278^{\mathrm{k}} \\
{[-.292, .800]}\end{array}$ & $\begin{array}{c}.036^{1} \\
{[-.545,1.00]}\end{array}$ \\
\hline \multicolumn{2}{|l|}{ KK } & $\begin{array}{c}.359 * \mathrm{~m} \\
{[.097, .581]}\end{array}$ & $\begin{array}{c}.148^{\mathrm{d}} \\
{[-.180, .474]}\end{array}$ & $\begin{array}{c}.086^{\mathrm{e}} \\
{[-.280, .454]}\end{array}$ & $\begin{array}{c}.189^{\mathrm{e}} \\
{[-.130, .477]}\end{array}$ \\
\hline \multicolumn{2}{|c|}{ SES } & $\begin{array}{c}.202^{\mathrm{m}} \\
{[-.084, .471]}\end{array}$ & $\begin{array}{c}.328 * \mathrm{~d} \\
{[.023, .617]}\end{array}$ & $\begin{array}{c}.005^{\mathrm{e}} \\
{[-.327, .298]}\end{array}$ & $\begin{array}{c}-.005^{\mathrm{e}} \\
{[-.294, .265]}\end{array}$ \\
\hline
\end{tabular}

Anmerkungen. ${ }^{*} p<.05, * * p<.01, * * * p<.001,95 \%$ Konfidenzintervall in eckigen Klammern, ${ }^{\mathrm{a}} \mathrm{n}=$ $28,{ }^{\mathrm{b}} \mathrm{n}=25,{ }^{\mathrm{c}} \mathrm{n}=20,{ }^{\mathrm{d}} \mathrm{n}=26,{ }^{\mathrm{e}} \mathrm{n}=21,{ }^{\mathrm{f}} \mathrm{n}=23,{ }^{\mathrm{g}} \mathrm{n}=15,{ }^{\mathrm{h}} \mathrm{n}=18,{ }^{\mathrm{i}} \mathrm{n}=14,{ }^{\mathrm{j}} \mathrm{n}=13,{ }^{\mathrm{k}} \mathrm{n}=11,{ }^{\mathrm{l}} \mathrm{n}=8,{ }^{\mathrm{m}}$ $\mathrm{n}=29$

Zusammen mit den ebenfalls stark schwankenden Korrelationen zwischen den Werten in Eltern- und Erzieherfragebogen und den Beobachtungsvariablen spricht dies eher für eine geringe Reliabilität und Validität der Beobachtungsdaten. Für die nachfolgenden komplexen statistischen Analysen wurde vor diesem Hintergrund nur auf die Daten der Eltern- und Erzieherfragebögen zurückgegriffen. 
Tabelle 25

Korrelationen zwischen den Prädiktoren und der Gesamtzahl der verbalen Interaktionen in der Freispielsituation

\begin{tabular}{|c|c|c|c|c|c|}
\hline & & \multicolumn{4}{|c|}{ Gesamtzahl der verbalen Interaktionen } \\
\hline & & $\mathrm{T} 1$ & $\mathrm{~T} 2$ & $\mathrm{~T} 3$ & $\mathrm{~T} 4$ \\
\hline \multirow{2}{*}{\multicolumn{2}{|c|}{ BAV Gesamtangst }} & $-.237^{\mathrm{a}}$ & $.033^{\mathrm{b}}$ & $-.203^{c}$ & $.059^{\mathrm{c}}$ \\
\hline & & {$[-.516, .059]$} & {$[-.308, .367]$} & {$[-.507, .139]$} & {$[-.301, .391]$} \\
\hline \multicolumn{2}{|c|}{ TROG-D } & $\begin{array}{c}.000^{\mathrm{a}} \\
{[-.298, .289]}\end{array}$ & $\begin{array}{c}.219^{\mathrm{d}} \\
{[-.073, .500]}\end{array}$ & $\begin{array}{c}.035^{\mathrm{e}} \\
{[-.292, .409]}\end{array}$ & $\begin{array}{c}-.015^{\mathrm{e}} \\
{[-.405, .352]}\end{array}$ \\
\hline \multicolumn{2}{|c|}{ MLU } & $\begin{array}{c}-.037^{\mathrm{f}} \\
{[-.380, .274]}\end{array}$ & $\begin{array}{c}.182^{\mathrm{c}} \\
{[-.219, .538]}\end{array}$ & $\begin{array}{c}-.317^{\mathrm{g}} \\
{[-.618,-.011]}\end{array}$ & $\begin{array}{c}-.053^{\mathrm{h}} \\
{[-.475, .354]}\end{array}$ \\
\hline \multirow{3}{*}{$\frac{\sqrt{2}}{2}$} & $\begin{array}{l}\text { Orientierung an Her- } \\
\text { kunftskultur }\end{array}$ & $\begin{array}{c}-.080^{\mathrm{i}} \\
{[-.535, .466]}\end{array}$ & $\begin{array}{c}-.122^{\mathrm{j}} \\
{[-.590, .390]}\end{array}$ & $\begin{array}{c}-.057^{\mathrm{k}} \\
{[-.500, .602]}\end{array}$ & $\begin{array}{c}-.222^{1} \\
{[-.668, .440]}\end{array}$ \\
\hline & $\begin{array}{l}\text { Orientierung an Auf- } \\
\text { nahmekultur }\end{array}$ & $\begin{array}{c}.370^{\mathrm{i}} \\
{[-.065, .759]}\end{array}$ & $\begin{array}{c}.280^{\mathrm{j}} \\
{[-.323, .738]}\end{array}$ & $\begin{array}{c}.191^{\mathrm{k}} \\
{[-.333, .666]}\end{array}$ & $\begin{array}{c}.189^{1} \\
{[-.637, .860]}\end{array}$ \\
\hline & Akkulturationsindex & $\begin{array}{c}.281^{\mathrm{i}} \\
{[-.202, .643]}\end{array}$ & $\begin{array}{c}.030^{\mathrm{j}} \\
{[-.545, .542]}\end{array}$ & $\begin{array}{c}.315^{\mathrm{k}} \\
{[-.231, .787]}\end{array}$ & $\begin{array}{c}.074^{1} \\
{[-.652, .635]}\end{array}$ \\
\hline \multicolumn{2}{|l|}{ KK } & $\begin{array}{c}.376^{* * \mathrm{~m}} \\
{[.127, .594]}\end{array}$ & $\begin{array}{c}.296^{\mathrm{d}} \\
{[-.034, .607]}\end{array}$ & $\begin{array}{c}.135^{\mathrm{e}} \\
{[-.186, .484]}\end{array}$ & $\begin{array}{c}.221^{\mathrm{e}} \\
{[-.117, .554]}\end{array}$ \\
\hline \multicolumn{2}{|c|}{ SES } & $\begin{array}{c}.093^{\mathrm{m}} \\
{[-.177, .357]}\end{array}$ & $\begin{array}{c}.154^{\mathrm{d}} \\
{[-.201, .474]}\end{array}$ & $\begin{array}{c}.055^{\mathrm{e}} \\
{[-.277, .366]}\end{array}$ & $\begin{array}{c}.130^{\mathrm{e}} \\
{[-.178, .434]}\end{array}$ \\
\hline
\end{tabular}

Anmerkungen. ${ }^{*} p<.05,{ }^{*} p<.01,{ }^{*} * * p<.001,95 \%$ Konfidenzintervall in eckigen Klammern, ${ }^{\mathrm{a}} \mathrm{n}=$ $28,{ }^{\mathrm{b}} \mathrm{n}=25,{ }^{\mathrm{c}} \mathrm{n}=20,{ }^{\mathrm{d}} \mathrm{n}=26,{ }^{\mathrm{e}} \mathrm{n}=21,{ }^{\mathrm{f}} \mathrm{n}=23,{ }^{\mathrm{g}} \mathrm{n}=15,{ }^{\mathrm{h}} \mathrm{n}=18,{ }^{\mathrm{i}} \mathrm{n}=14,{ }^{\mathrm{j}} \mathrm{n}=13,{ }^{\mathrm{k}} \mathrm{n}=11,{ }^{1} \mathrm{n}=8,{ }^{\mathrm{m}}$ $\mathrm{n}=29$

\subsubsection{Anteil der selektiv mutistischen Kinder zum ersten Messzeitpunkt}

Insgesamt 30 Kinder (15 mehrsprachige und 15 einsprachige) nahmen an der Studie teil. Zum ersten Messzeitpunkt schwiegen davon 18 Kinder (11 mehrsprachige, 7 einsprachige). Wie bereits in Kapitel 3.4.2 dargestellt konnte für acht dieser Kinder zum ersten Messzeitpunkt die Diagnose selektiver Mutismus nach DSM-IV Kriterien gestellt werden. Bei zehn Kindern war die Diagnose aus den verschiedensten Gründen unsicher. Die mehrsprachigen Kinder verfügten meist noch nicht über ausreichende Sprachkompetenzen im Deutschen (gemessen über den TROG-D sowie die Spontansprachaufnahmen), so dass unklar war, ob die Kinder aufgrund der fehlenden Sprachkenntnisse oder als Symptom des SM schwiegen. Die einsprachigen Kinder mit unsicherer Diagnose zeigten häufig ein untypisches Symptombild. Sie schwiegen zwar in bestimmten Situationen im Kontext der Kindertageseinrichtung, zeigten sich jedoch in der Diagnostiksituation in den Räumlichkeiten der Universität sehr kommunikativ, sprachen mit der Testleiterin und zeigten keinerlei Scheu in der wenig vertrauten Situation. Tabelle 26 gibt einen Überblick über die Einschätzungen der Eltern und Erziehe- 
rinnen über das Sprechverhalten der Kinder sowie die Beobachtungen des Interaktionsverhaltens in einer Freispielsituation zum ersten Messzeitpunkt.

Tabelle 26

Mediane und Interquartilsabstände zu den Outcome-Variablen zum ersten Messzeitpunkt

\begin{tabular}{|c|c|c|c|c|}
\hline & \multicolumn{2}{|c|}{ Schweigende Kinder } & \multirow{2}{*}{$\begin{array}{l}\text { Kontrollkinder } \\
\qquad \mathrm{n}=12\end{array}$} & \multirow{2}{*}{$\begin{array}{l}\text { Gesamt } \\
\mathrm{N}=30\end{array}$} \\
\hline & $\begin{array}{l}\text { Diagnose SM } \\
\quad \mathrm{n}=8\end{array}$ & $\begin{array}{l}\text { unsicher } \\
\mathrm{n}=10\end{array}$ & & \\
\hline einsprachig $(n=15)$ & $2(13.3 \%)$ & $5(33.3 \%)$ & $8(53.4 \%)$ & $15(100.0 \%)$ \\
\hline mehrsprachig $(\mathrm{n}=15)$ & $6(40.0 \%)$ & $5(33.3 \%)$ & $4(26.7 \%)$ & $15(100.0 \%)$ \\
\hline \multicolumn{5}{|l|}{ Elternfragebogen } \\
\hline Schweigen & $\begin{array}{c}45.00 \\
(I Q R=15.50)\end{array}$ & $\begin{array}{c}31.50 \\
(I Q R=14.25)\end{array}$ & $\begin{array}{c}10.50 \\
(\mathrm{IQR}=14.25)\end{array}$ & $\begin{array}{c}28.00 \\
(I Q R=27.25)\end{array}$ \\
\hline Partizipation & $\begin{array}{c}17.00 \\
(I Q R=13.75)\end{array}$ & $\begin{array}{c}15.50 \\
(I Q R=8.25)\end{array}$ & $\begin{array}{c}9.00 \\
(I Q R=6.75)\end{array}$ & $\begin{array}{c}13.00 \\
(I Q R=8.25)\end{array}$ \\
\hline Emotionsregulation & $\begin{array}{c}20.00 \\
(I Q R=14.25)\end{array}$ & $\begin{array}{c}15.50 \\
(I Q R=6.75)\end{array}$ & $\begin{array}{c}9.00 \\
(I Q R=5.50)\end{array}$ & $\begin{array}{c}13.50 \\
(I Q R=10.50)\end{array}$ \\
\hline Öffentliche Situationen & $\begin{array}{c}11.50 \\
(I Q R=7.50)\end{array}$ & $\begin{array}{c}8.00 \\
(I Q R=6.75)\end{array}$ & $\begin{array}{c}5.00 \\
(I Q R=5.00)\end{array}$ & $\begin{array}{c}7.50 \\
(I Q R=8.25)\end{array}$ \\
\hline $\begin{array}{r}\text { DortMuS Kita } \\
\text { Schweigen und } \\
\text { Bedürfnisäußerung }\end{array}$ & $\begin{array}{c}41.00 \\
(I Q R=11.25)\end{array}$ & $\begin{array}{c}31.00 \\
(I Q R=13.50)\end{array}$ & $\begin{array}{c}6.50 \\
(I Q R=15)\end{array}$ & $\begin{array}{c}25.00 \\
(I Q R=25.00)\end{array}$ \\
\hline Gruppe & $\begin{array}{c}11.50 \\
(I Q R=11.25)\end{array}$ & $\begin{array}{c}13.00 \\
(I Q R=6.00)\end{array}$ & $\begin{array}{c}2.00 \\
(I Q R=9.25)\end{array}$ & $\begin{array}{c}10.00 \\
(I Q R=11.00)\end{array}$ \\
\hline $\begin{array}{l}\text { Gesamtanzahl der } \\
\text { Interaktionen }\end{array}$ & $\begin{array}{c}17.00 \\
(I Q R=22.00)\end{array}$ & $\begin{array}{c}14.50 \\
(I Q R=14.75)\end{array}$ & $\begin{array}{c}32.00 \\
(I Q R=14.00)\end{array}$ & $\begin{array}{c}22.00 \\
(I Q R=21.50)\end{array}$ \\
\hline $\begin{array}{l}\text { Gesamtanzahl verbaler } \\
\text { Interaktionen }\end{array}$ & $\begin{array}{c}0.50 \\
(I Q R=15.50)\end{array}$ & $\begin{array}{c}3.50 \\
(I Q R=7.50)\end{array}$ & $\begin{array}{c}29.50 \\
(I Q R=19.75)\end{array}$ & $\begin{array}{c}13.50 \\
(I Q R=27.00)\end{array}$ \\
\hline $\begin{array}{l}\text { Gesamtanzahl nonverbaler } \\
\text { Interaktionen }\end{array}$ & $\begin{array}{c}10.50 \\
(I Q R=11.75)\end{array}$ & $\begin{array}{c}6.00 \\
(I Q R=13.25)\end{array}$ & $\begin{array}{c}3.50 \\
(I Q R=4.50)\end{array}$ & $\begin{array}{c}5.00 \\
(I Q R=11.50)\end{array}$ \\
\hline $\begin{array}{l}\text { Gesamtanzahl Interaktionen } \\
\text { zwischen Kindern }\end{array}$ & $\begin{array}{c}6.50 \\
(I Q R=22.75)\end{array}$ & $\begin{array}{c}9.00 \\
(I Q R=9.50)\end{array}$ & $\begin{array}{c}27.50 \\
(I Q R=20.25)\end{array}$ & $\begin{array}{c}13.50 \\
(I Q R=21.75)\end{array}$ \\
\hline $\begin{array}{l}\text { Gesamtanzahl Interaktionen } \\
\text { zwischen Kind und Erzieherin }\end{array}$ & $\begin{array}{c}4.50 \\
(I Q R=5.50)\end{array}$ & $\begin{array}{c}2.00 \\
(I Q R=7.50)\end{array}$ & $\begin{array}{c}6.00 \\
(I Q R=11.50)\end{array}$ & $\begin{array}{c}4.50 \\
(I Q R=5.50)\end{array}$ \\
\hline
\end{tabular}

Die drei Gruppen unterschieden sich zum ersten Messzeitpunkt signifikant hinsichtlich ihres Sprechverhaltens sowohl im familiären als auch im Kita-Kontext, $H(2)_{\text {Eltern }}=20.93$, $p_{\text {Eltern }}<.001, H(2)_{\text {Erzieher }}=16.96, p_{\text {Erzieher }}<.001, H(2)_{\text {Gruppe }}=9.85, p_{\text {Gruppe }}=.007$. Der Jonckheere-Terpstra-Test bestätigte einen signifikanten Trend in den Daten, wobei das Ausmaß des Schweigens gemessen an den Einschätzungen der Eltern und Erzieher von der Gruppe Diag- 
nose $\mathrm{SM}$ bis zu den Kontrollkindern kontinuierlich abnahm, $J_{\text {Eltern }}=20.00, z_{\text {Eltern }}=-4.89$, $p_{\text {Eltern }}<.001, r_{\text {Eltern }}=-.89 ; J_{\text {Erzieher }}=14.50, z_{\text {Erzieher }}=-4.40, p_{\text {Erzieher }}<.001, r_{\text {Erzieher }}=-.88, J_{\text {Grup- }}$ $p e=46.50, z_{\text {Gruppe }}=-2.80, p_{\text {Gruppe }}=.005, r_{\text {Gruppe }}=-.56$. Auch auf den weiteren Skalen des Elternfragebogens unterschieden sich die drei Gruppen signifikant voneinander, $H(2)_{\text {Partizipation }}=$ $16.22, p_{\text {Partizipation }}<.001, H(2)_{\text {Emotion }}=10.28, p_{\text {Emotion }}=.006, H(2)_{\text {öffentlich }}=11.19, p_{\text {öffentlich }}=$ .004. Für allen drei Skalen zeigte sich wiederum ein signifikanter Trend, wobei jeweils die Kinder mit SM im Mittel die höchsten Werte hatten, gefolgt von den Kindern mit unsicherer Diagnose und den Kontrollkindern, $J_{\text {Partizipation }}=47.00, z_{\text {Partizipation }}=-3.87, p_{\text {Partizipation }}<.001$, $r_{\text {Partizipation }}=-.71 ; J_{\text {Emotion }}=62.50, z_{\text {Emotion }}=-3.28, p_{\text {Emotion }}=.001, r_{\text {Emotion }}=-.60, J_{\text {öffentlich }}=$ $58.50, z_{\text {öffentlich }}=-3.44, p_{\text {öffentlich }}=.001, r_{\text {öffentlich }}=-.63$. Die schweigenden Kinder spielten demnach weniger mit anderen Kindern, zeigten häufiger Vermeidungsverhalten bei Problemsituationen und zogen sich in öffentlichen Situationen eher zurück, schwiegen oder forderten Hilfe bei ihren Eltern ein.

Signifikante Unterschiede zwischen den Gruppen zeigten sich zudem in vier von fünf der Beobachtungsvariablen zum ersten Messzeitpunkt, $H(2)_{\text {Gesamt }}=12.14, p_{\text {Gesamt }}=.002$, $H(2)_{\text {verbal }}=14.06, p_{\text {verbal }}=.001, H(2)_{\text {nonverbal }}=8.21, p_{\text {nonverbal }}=.017, H(2)_{\text {Kind-Kind }}=9.22$, $p_{\text {Kind-Kind }}=.010$. Auch hier ließ sich ein signifikanter Trend nachweisen, wobei die Anzahl der Interaktionen von selektiv mutistischen über Kinder mit unsicherer Diagnose hin zu den Kontrollkindern - ausgenommen der Variable nonverbale Interaktionen-stieg, $J_{\text {Gesamt }}=221.50$, $z_{\text {Gesamt }}=2.82, p_{\text {Gesamt }}=.005 ; r_{\text {Gesamt }}=.51, J_{\text {verbal }}=245.50, z_{\text {verbal }}=3.74, p_{\text {verbal }}<.001 ; r_{\text {verbal }}=$ $68, J_{\text {Kind-Kind }}=225.00, z_{\text {Kind-Kind }}=2.95, p_{\text {Kind-Kind }}=.003, r_{\text {Kind-Kind }}=.54$. Bei den nonverbalen Interaktionsformen ließ sich ein umgekehrter Trend nachweisen. Kinder mit der Diagnose SM nutzten nonverbale Interaktionsformen häufiger als Kinder mit unsicherer Diagnose oder Kontrollkinder, $J_{\text {nonverbal }}=75.00, z_{\text {nonverbal }}=-2.81, p_{\text {nonverbal }}=.005, r_{\text {nonverbal }}=-.51$. Anzumerken ist hier jedoch, dass bei der Kodierung der Beobachtung nur solche Verhaltensweisen als nonverbale Interaktion bewertet wurden, bei denen die Kinder ausschließlich nonverbal handelten. Gleichzeitiges verbales und nonverbales Handeln wurde ausschließlich unter verbal kodiert, weil der Fokus bei der Datenerhebung auf dem Sprechverhalten lag. Bei den Interaktionen zwischen Kind und Erzieherin ergab sich kein signifikanter Unterschied zwischen den Gruppen, $H(2)_{\text {Kind-Erzieher }}=2.65, p_{\text {Kind-Erzieher }}=.266$. 


\subsubsection{Anteil selektiv mutistischer Kindern zum vierten Messzeitpunkt}

Zum vierten Messzeitpunkt zeigten insgesamt sieben Kinder (zwei einsprachige, fünf mehrsprachige) einen SM. Von den unsicheren Kindern hatte nur ein mehrsprachiges Kind letztlich einen SM ausgeprägt. Von den Kindern mit Diagnose SM zum ersten Messzeitpunkt haben sich zwei positiv entwickelt. Sie sprachen zum vierten Messzeitpunkt in nahezu allen Kontexten unbefangen und waren damit als nicht mehr selektiv mutistisch zu klassifizieren. Eines der Kinder zeigte jedoch nun eine ausgeprägte Stottersymptomatik. Tabelle 27 gibt einen Überblick über die Werte der Kinder zum vierten Messzeitpunkt.

Die Kinder mit Diagnose SM zum vierten Messzeitpunkt, die zu T4 sprechenden sowie die Kontrollkinder unterschieden sich zum Ende der Studie signifikant im Ausmaß des Schweigens in familiären und öffentlichen Situationen, $H(2)=15.88, p<.001$. Anschließende paarweise Vergleiche mit angepassten p-Werten zeigen einen signifikanten Unterschied zwischen den schweigenden Kindern und den Kontrollen $(p<.001, r=.91)$ sowie einen tendenziellen zwischen den zu T4 sprechenden Kindern und den Kontrollen ( $p=.075, r=.47)$. Zwischen den Kindern mit Diagnose SM und den zu T4 sprechenden ergab sich kein signifikanter Unterschied ( $p=.165, r=.45)$. Ein tendenzieller Unterschied zeigte sich zwischen den Gruppen auf der Skala Partizipation des Elternfragebogens, $H(2)=4.67, p=.097$, sowie kein Unterschied auf der Skala Emotionsregulation, $H(2)=4.38, p=.112$. Für beide Skalen bestätigte der Jonckheere-Terpstra-Test jedoch einen signifikanten Trend, wobei die Kinder mit Diagnose SM jeweils die höchsten Werte zeigten, gefolgt von den zu T4 sprechenden und den Kontrollkindern, $J_{\text {Partizipation }}=71.00, z_{\text {Partizipation }}=-2.20, p_{\text {Partizipation }}=.028, r_{\text {Partizipation }}=-.42$; $J_{\text {Emotion }}=72.50, z_{\text {Emotion }}=-2.13, p_{\text {Emotion }}=.033, r_{\text {Emotion }}=-.43$. Auf der Skala öffentliche Situationen ergab sich zum vierten Messzeitpunkt ein signifikanter Unterschied zwischen den Gruppen, $H(2)=10.00, p=.007$. Die paarweisen Vergleich mit angepassten $p-W e r t e n$ zeigten signifikante Unterschiede zwischen den Kontrollkindern und den zu T4 sprechenden ( $p=$ $.017, r=58)$ sowie den Kinder mit Diagnose SM $(p=.027, r=.60)$. Die schweigenden und zu T4 sprechenden Kinder unterschieden sich nicht voneinander $(p=1.000, r=.00)$. Der Jonckheere-Terpstra-Test bestätigte zusätzlich einen signifikanten Trend, wobei die Kinder mit Diagnose SM die höchsten Werte zeigten, gefolgt von den zu T4 sprechenden Kindern und den Kontrollen, $J=54.50, z=-2.97, p=.003, r=-.57$. 
Tabelle 27

Kinder mit selektivem Mutismus zu T4 im Vergleich zu Kindern mit positiver Entwicklung und Kontrollen in den Outcomevariablen zum vierten Messzeitpunkt

\begin{tabular}{|c|c|c|c|}
\hline & $\begin{array}{l}\text { selektiv mutistisch } \\
\mathrm{n}=7\end{array}$ & $\begin{array}{l}\text { sprechend zu T4 } \\
\mathrm{n}=11\end{array}$ & $\begin{array}{l}\text { Kontrollen } \\
\mathrm{n}=12\end{array}$ \\
\hline einsprachig & $2(28.6 \%)$ & $5(45.5 \%)$ & $8(66.7 \%)$ \\
\hline mehrsprachig & $5(71.4 \%)$ & $6(54.5 \%)$ & $4(33.3 \%)$ \\
\hline Mutismusdiagnose zu T1 & $6(85.7 \%)$ & $2(18.2 \%)$ & $0(0 \%)$ \\
\hline Elternfragebogen & $\begin{array}{c}35.0 \\
(I Q R=29.0)\end{array}$ & $\begin{array}{c}23.5 \\
(I Q R=14.0)\end{array}$ & $\begin{array}{c}12.0 \\
(I Q R=10.0)\end{array}$ \\
\hline Partizipation & $\begin{array}{c}14.0 \\
(I Q R=13.0)\end{array}$ & $\begin{array}{c}11.0 \\
(I Q R=6.75)\end{array}$ & $\begin{array}{c}7.5 \\
(I Q R=9.0)\end{array}$ \\
\hline Emotionsregulation & $\begin{array}{c}16.0 \\
(I Q R=17.0)\end{array}$ & $\begin{array}{c}9.5 \\
(I Q R=9.25)\end{array}$ & $\begin{array}{c}6.0 \\
(I Q R=8.5)\end{array}$ \\
\hline Öffentliche Situationen & $\begin{array}{c}8.0 \\
(I Q R=9.0)\end{array}$ & $\begin{array}{c}7.0 \\
(I Q R=4.5)\end{array}$ & $\begin{array}{c}0.0 \\
(I Q R=3.25)\end{array}$ \\
\hline $\begin{array}{r}\text { DortMuS-Kita } \\
\text { Schweigen und } \\
\text { Bedürfnisäußerung }\end{array}$ & $\begin{array}{c}15.5 \\
(I Q R=18.25)\end{array}$ & $\begin{array}{c}15.5 \\
(I Q R=5.25)\end{array}$ & $\begin{array}{c}1.5 \\
(I Q R=3.0)\end{array}$ \\
\hline Gruppe & $\begin{array}{c}4.0 \\
(I Q R=5.0)\end{array}$ & $\begin{array}{c}5.0 \\
(I Q R=8.25)\end{array}$ & $\begin{array}{c}0.5 \\
(I Q R=3.5)\end{array}$ \\
\hline Gesamtanzahl der Interaktionen & $\begin{array}{c}18.0 \\
(I Q R=18.0)\end{array}$ & $\begin{array}{c}19.0 \\
(I Q R=24.75)\end{array}$ & $\begin{array}{c}34.0 \\
(I Q R=28.0)\end{array}$ \\
\hline $\begin{array}{l}\text { Gesamtanzahl verbaler } \\
\text { Interaktionen }\end{array}$ & $\begin{array}{c}3.0 \\
(I Q R=23.0)\end{array}$ & $\begin{array}{c}15.0 \\
(I Q R=25.25)\end{array}$ & $\begin{array}{c}29.0 \\
(I Q R=11.0)\end{array}$ \\
\hline $\begin{array}{l}\text { Gesamtanzahl nonverbaler } \\
\text { Interaktionen }\end{array}$ & $\begin{array}{c}10.0 \\
(I Q R=8.5)\end{array}$ & $\begin{array}{c}3.5 \\
(I Q R=3.5)\end{array}$ & $\begin{array}{c}3.0 \\
(I Q R=5.0)\end{array}$ \\
\hline $\begin{array}{l}\text { Gesamtanzahl Interaktionen } \\
\text { zwischen Kindern }\end{array}$ & $\begin{array}{c}5.0 \\
(I Q R=24.5)\end{array}$ & $\begin{array}{c}9.5 \\
(I Q R=21.75)\end{array}$ & $\begin{array}{c}27.0 \\
(I Q R=34.0)\end{array}$ \\
\hline $\begin{array}{l}\text { Gesamtanzahl Interaktionen } \\
\text { zwischen Kind und Erzieherin }\end{array}$ & $\begin{array}{c}12.0 \\
(I Q R=11.0)\end{array}$ & $\begin{array}{c}4.0 \\
(I Q R=6.0)\end{array}$ & $\begin{array}{c}3.0 \\
(I Q R=22.0)\end{array}$ \\
\hline
\end{tabular}

Für den Erzieherfragebogen zeigte sich ein ähnliches Bild. Auch hier unterschieden sich die drei Gruppen zum vierten Messzeitpunkt auf der Skala Schweigen und BedürfnisäuBerung, $H(2)=9.42, p=.009$. Signifikante Unterschiede zeigten sich zwischen den Kontrollkindern und den zu T4 sprechenden $(p=.037, r=.52)$ und den Kinder mit Diagnose SM ( $p=$ $.017, r=.64)$. Ein Unterschied zwischen den schweigenden und zu T4 sprechenden Kinder ergab sich nicht $(p=1.000, r=.00)$. Der Jonckheere-Terpstra-Test bestätigte wiederum einen signifikanten Trend in den Daten, $J=38.50, z=-2.97, p=.003, r=-.61$. Auf der Skala Gruppe unterschieden sich die drei Gruppen nur tendenziell voneinander, $H(2)=5.58, p=.062$. 
Ein statistisch signifikanter Trend war nicht nachweisbar, $J=69.00, z=-1.35, p=.177$, $r=-.28$.

Eine Analyse der Entwicklung des Interaktionsverhaltens innerhalb der verschiedenen Kontexte erfolgt im nächsten Kapitel. Dabei werden unter anderem Unterschiede innerhalb der Beobachtungsvariablen zwischen den drei Gruppen untersucht. Aus diesem Grund werden Gruppenunterschieds- und Trendanalysen für diese Variablen nicht aufgeführt, sondern auf die nachfolgenden Ausführungen und die Ergebnisübersicht in Tabelle 28 verwiesen.

\subsubsection{Entwicklung des Sprech- und Interaktionsverhaltens}

Insgesamt zeigten alle drei Gruppen eine positive Entwicklung hinsichtlich des Sprechens. Sowohl im Eltern- als auch im Erzieherfragebogen nahmen die durchschnittlichen Werte auf den Skalen Schweigen (Elternfragebogen) sowie Schweigen und Bedürfnisäußerung (DortMuS-Kita) ab (siehe Abbildung 12 und Abbildung 13). Dennoch unterschieden sich die drei Gruppen zu allen Messzeitpunkten signifikant im Elternfragebogen, $H(2)_{T 1}=20.97$, $p_{T 1}<.001, H(2)_{T 2}=9.74, p_{T 2}=.008, H(2)_{T 3}=10.03, p_{T 3}=.007, H(2)_{T 4}=15.88, p_{T 4}<.001$, sowie im Erzieherfragebogen, $H(2)_{T 1}=16.94, p_{T 1}<.001, H(2)_{T 2}=14.01, p_{T 2}=.001, H(2)_{T 3}=$ $10.02, p_{T 3}=.007, H(2)_{T 4}=9.42, p_{T 4}=.009$. Der signifikante Trend blieb über alle Messzeitpunkte stabil (siehe Tabelle 28). Die Kinder mit der Diagnose SM zu T4 schwiegen im Mittel mehr als die Kinder mit positiver Entwicklung und die Kontrollkinder. Hervorzuheben ist, dass die selektiv mutistischen Kinder zum vierten Messzeitpunkt im Erzieherfragebogen im Mittel etwa gleiche Werte erreichten wie die zu T4 sprechenden Kinder. Die Varianz innerhalb der Gruppe der schweigenden Kinder war mit einem Interquartilsabstand von 18.25 (Minimum 13, Maximum 38) jedoch deutlich größer als die innerhalb der Gruppe der zu T4 sprechenden Kinder $(I Q R=5.25$, Minimum 4, Maximum 28).

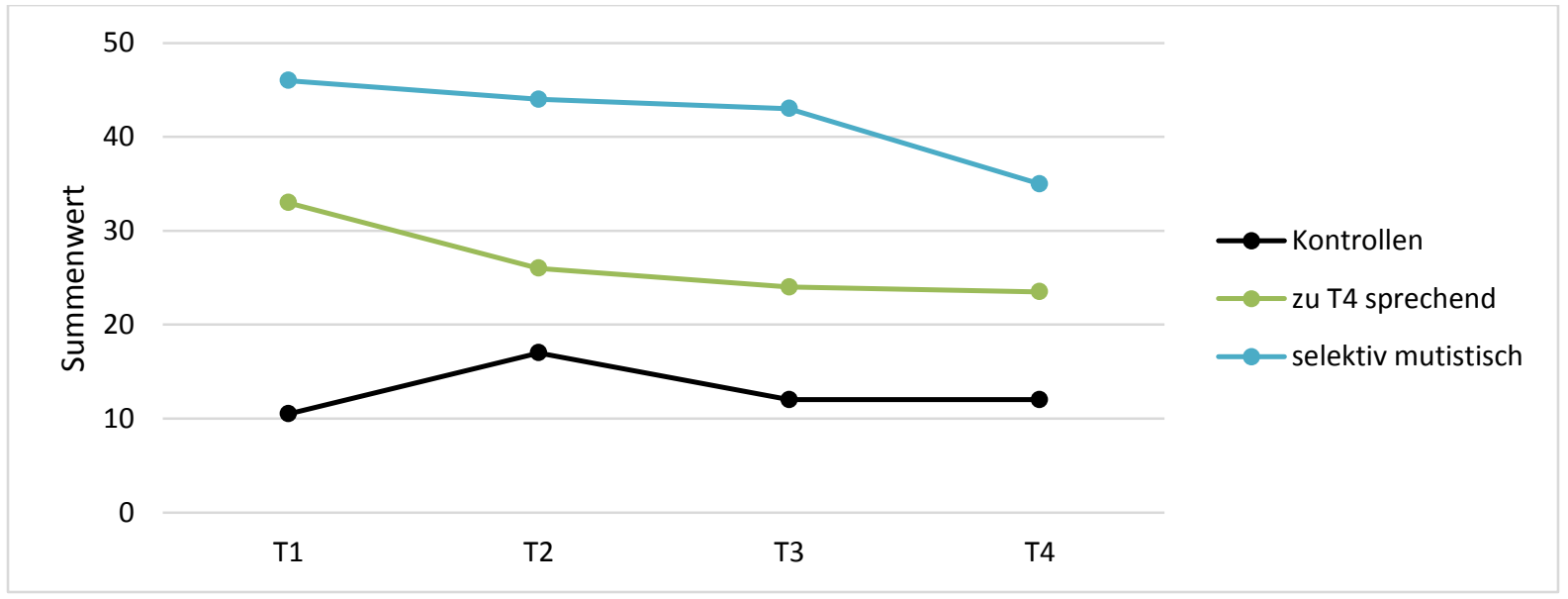

Abbildung 13. Mittlere Summenwerte (Mediane) auf der Skala Schweigen des Elternfragebogens für Kinder mit Diagnose SM zu T4, zu T4 sprechenden Kindern und Kontrollen 


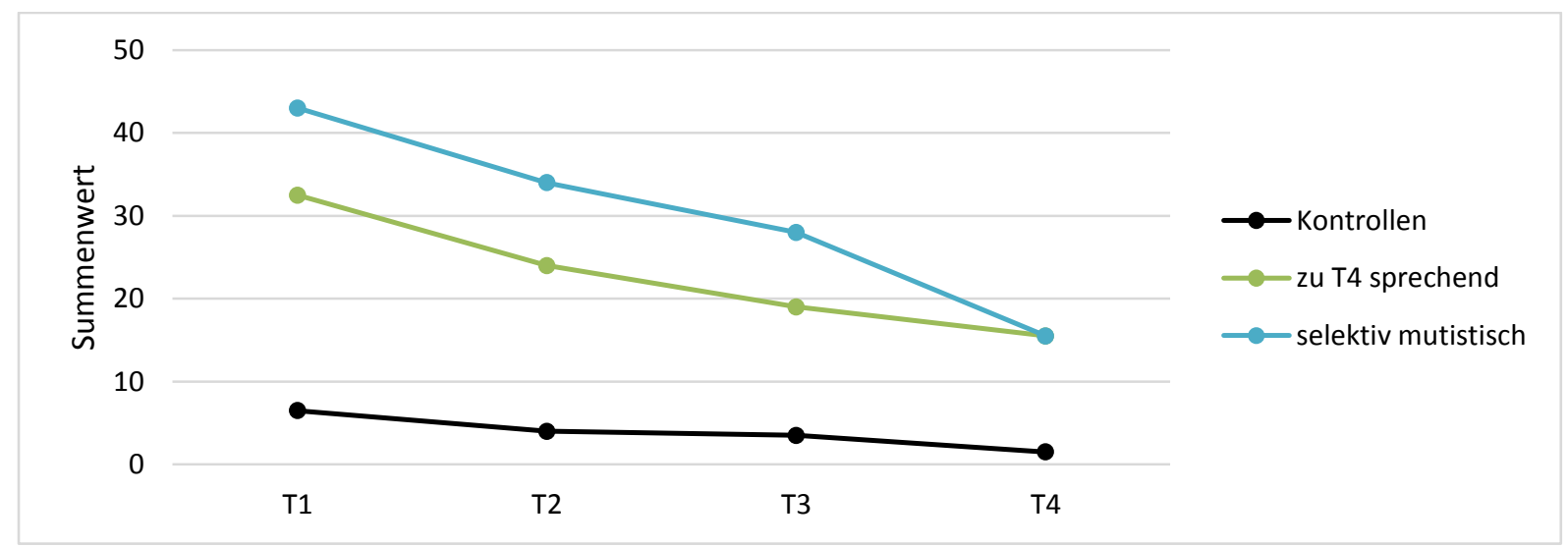

Abbildung 14. Mittlere Summenwerte (Mediane) auf der Skala Schweigen und Bedürfnisäußerung von DortMuS-Kita für Kinder mit Diagnose SM zu T4, zu T4 sprechende und Kontrollen

Tabelle 28

Ergebnisse der Jonckheere-Terpstra-Tests für die Outcomevariablen für den zweiten bis vierten Messzeitpunkt

\begin{tabular}{|c|c|c|c|c|c|c|c|c|c|c|c|c|}
\hline & \multicolumn{4}{|c|}{$\mathrm{T} 2$} & \multicolumn{4}{|c|}{$\mathrm{T} 3$} & \multicolumn{4}{|c|}{$\mathrm{T} 4$} \\
\hline & $J$ & $z$ & $p$ & $r$ & $J$ & $z$ & $p$ & $r$ & $J$ & $z$ & $p$ & $r$ \\
\hline Elternfragebogen & 29.00 & -3.22 & .001 & -.67 & 36.50 & -3.36 & .001 & -.66 & 24.50 & -4.27 & .000 & -.82 \\
\hline DortMuS-KiTa & 27.00 & -3.81 & .000 & -.76 & 10.50 & -3.25 & .001 & -.79 & 38.50 & -2.97 & .003 & -.61 \\
\hline $\begin{array}{l}\text { Gesamtanzahl Inter- } \\
\text { aktionen }\end{array}$ & 147.50 & 1.25 & .210 & .24 & 96.50 & 1.10 & .274 & .23 & 101.00 & 1.44 & .150 & .31 \\
\hline $\begin{array}{l}\text { Gesamtzahl verbaler } \\
\text { Interaktionen }\end{array}$ & 172.00 & 2.39 & .017 & .46 & 107.00 & 1.73 & .083 & .37 & 112.50 & 2.14 & .032 & .46 \\
\hline $\begin{array}{l}\text { Gesamtzahl nonver- } \\
\text { baler Interaktionen }\end{array}$ & 69.50 & -2.26 & .024 & -.44 & 47.00 & -1.96 & .050 & -.42 & 45.00 & -2.00 & .046 & -.43 \\
\hline $\begin{array}{l}\text { Gesamtzahl Interak- } \\
\text { tionen Kind-Kind }\end{array}$ & 158.00 & 1.72 & .085 & .33 & 102.50 & 1.46 & .145 & .31 & 106.00 & 1.75 & .081 & .37 \\
\hline $\begin{array}{l}\text { Gesamtzahl Interak- } \\
\text { tionen Kind-Erzieher }\end{array}$ & 116.50 & -0.14 & .893 & -.03 & 75.50 & -0.18 & .855 & -.04 & 63.00 & -0.89 & .372 & -.19 \\
\hline
\end{tabular}

Im Interaktionsverhalten in der Freispielsituation unterschieden sich die Gruppen lediglich in ihrem verbalen Interaktionsverhalten zum zweiten Messzeitpunkt signifikant voneinander, $H(2)=6.06, p=.048$. Ein signifikanter Trend ist über alle Messzeitpunkte hinweg nachweisbar (siehe Tabelle 28). Die Kinder mit SM interagierten im Mittel zu den ersten beiden Messzeitpunkten nicht verbal mit anderen Personen, zum dritten und vierten Messzeitpunkt war eine leichte Steigerung verbaler Interaktionen zu verzeichnen. Auch die zu T4 sprechenden Kinder interagierten im Mittel zu den ersten beiden Messzeitpunkten nur sehr wenig verbal mit anderen Personen in der Kita und steigerten ihre Sprechanteile deutlich ab dem dritten Messzeitpunkt. Die Kontrollkinder sprachen sehr häufig mit anderen Personen 
und lagen zahlenmäßig im Mittel über den beiden Gruppen, wobei auch bei ihnen zum zweiten Messzeitpunkt eine vergleichsweise geringe Anzahl verbaler Interaktionen zu beobachten war (siehe Abbildung 14).

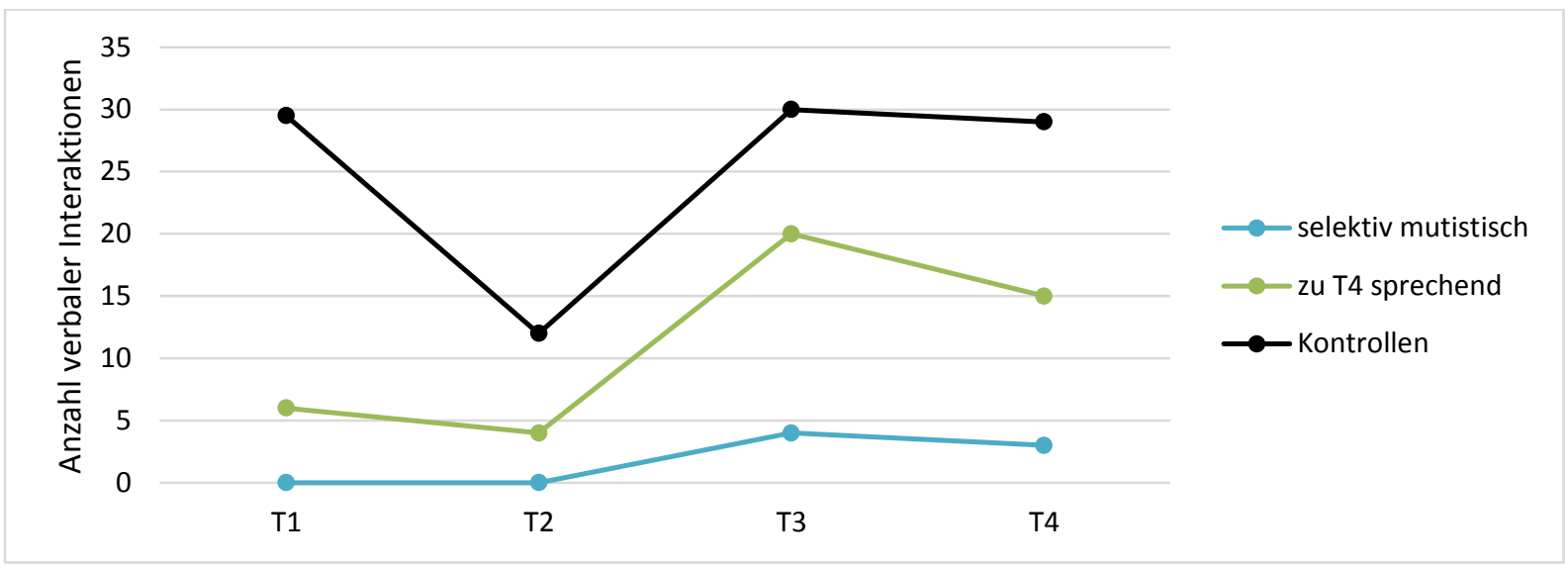

Abbildung 15. Mittlere Anzahl (Mediane) verbaler Interaktionen in der Freispielsituation

Ein signifikanter Unterschied zwischen den Gruppen ergab sich zudem für das nonverbale Interaktionsverhalten zum ersten und vierten Messzeitpunkt, $H(2)_{T 1}=9.53$, $p_{T 1}=.009, H(2)_{T 4}=8.56, p_{T 4}=.014$, sowie ein tendenzieller zum zweiten Messzeitpunkt, $H(2)_{T 2}=5.85, p_{T 2}=.054$. Eine signifikanter bzw. tendenzieller Trend war über alle Messzeitpunkte sichtbar (siehe Tabelle 28). Die Kinder mit SM interagierten deutlich häufiger nonverbal als die zu T4 sprechenden Kinder sowie die Kontrollen (siehe auch Abbildung 15).

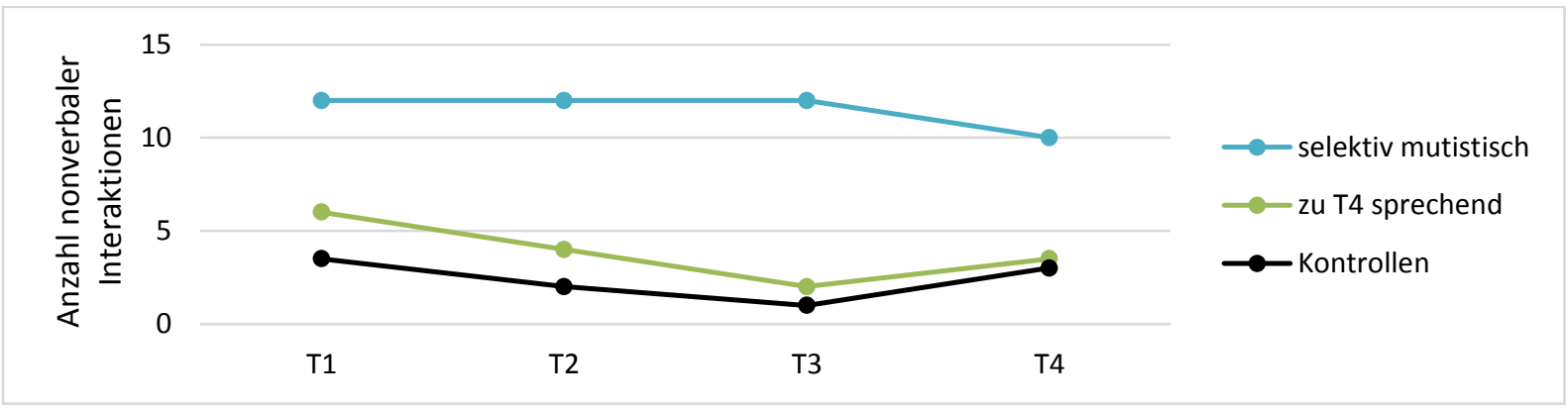

Abbildung 16. Mittlere Anzahl (Mediane) nonverbaler Interaktionen in der Freispielsituation 


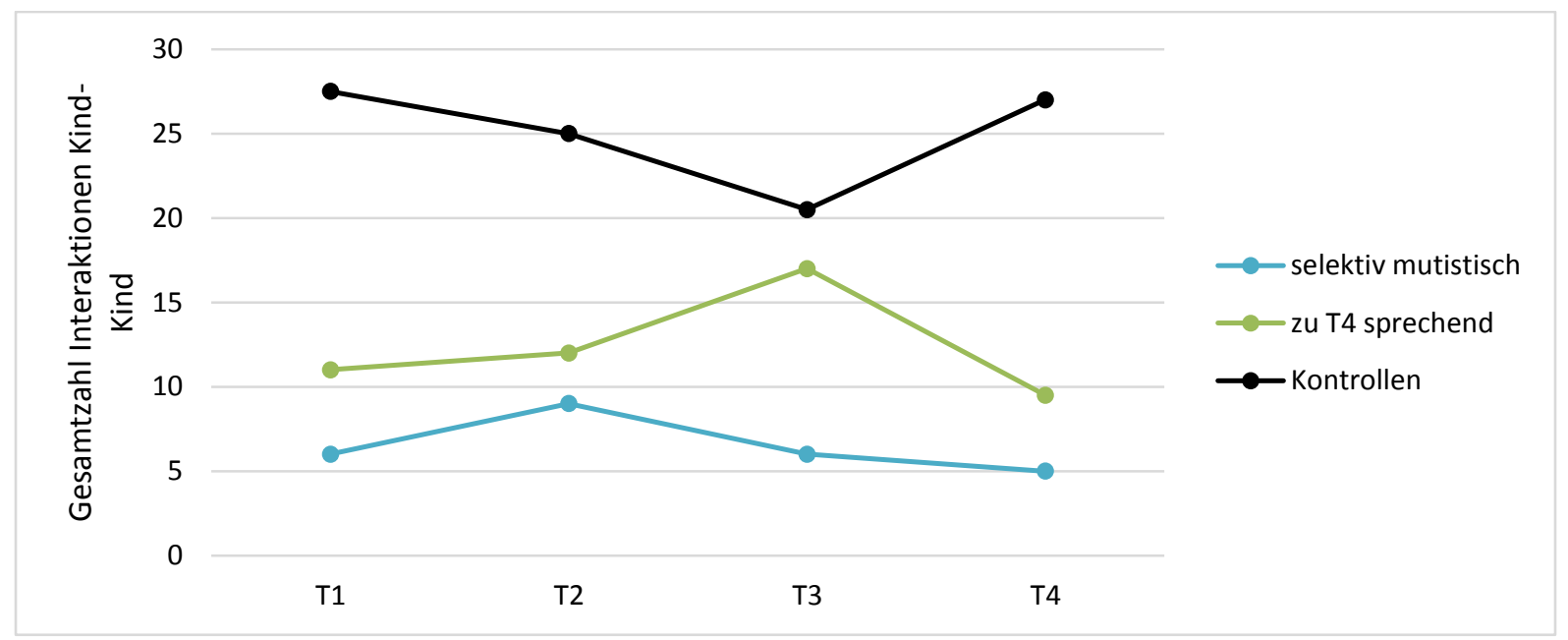

Abbildung 17. Mittlere Anzahl (Mediane) aller Interaktionen zwischen dem Kind und einemKind bzw. mehreren Kindern

\subsubsection{Deskriptive Ergebnisse zu den Prädiktorvariablen}

Im Folgenden werden zunächst die deskriptiven Ergebnisse zu den Prädiktorvariablen (Ängste, Sprache und Akkulturation) für die Gesamtstichprobe sowie für die Gruppeneinteilung nach der Mutismusdiagnose zum vierten Messzeitpunkt vorgestellt (siehe dazu im Überblick Tabelle 29). Es werden zunächst alle Kinder innerhalb der Diagnosegruppen gemeinsam betrachtet und nicht zwischen ein- und mehrsprachigen Kindern unterschieden. Eine Unterscheidung würde vor allem die Gruppengröße für Kinder mit SM (zwei einsprachige und fünf mehrsprachige Kinder) reduzieren und weitere statistische Analysen nicht möglich machen. Eine gesonderte Betrachtung von ein- und mehrsprachigen Kindern mit SM erfolgt in Kapitel 4.1.8. Aufgrund der kleinen Stichprobe-insbesondere bei gesonderter Betrachtung der Einzelgruppen - sowie der tendenziell nicht vorliegenden Normalverteilung innerhalb der Variablen der kulturellen Adaptation (siehe dazu Tabelle A-9 im Anhang) wird auch hier wiederum einheitlich als Maß der zentralen Tendenz der Median und als Streuungsmaß der Interquartilsabstand berichtet.

Hinsichtlich der Ängstlichkeit der Kinder ergab sich ein signifikanter Unterschied zwischen den Gruppen zum ersten Messzeitpunkt, $H(2)=7.07, p=.029$. Paarweise Vergleiche mit angepasstem p-Wert ergaben lediglich einen signifikanten Unterscheid zwischen den Kindern mit SM und den Kontrollen $(p=.024, r=.61)$. Der Jonckheere-Terpstra-Test bestätigte zusätzlich einen signifikanten Trend in den Daten, wobei die Gesamtangst bei Kindern mit SM am höchsten ausgeprägt war und über die Kinder mit unsicherer Diagnose bis hin zu den Kontrollkindern deutlich sank, $J=65.00, z=-2.63, p=.009, r=-.50$. Mit einem durchschnittlichen T-Wert von 60 im Angstfragebogen war das Ausmaß der Angst der selektiv mu- 
tistischen Kinder an der Grenze zur überdurchschnittlich hohen Ausprägung. Hervorzuheben ist jedoch hier die große Varianz in den Daten $(I Q R=26.0)$. Die zum vierten Messzeitpunkt sprechenden Kinder sowie die Kontrollkinder zeigten im Mittel eine durchschnittlich ausgeprägte Angst.

Tabelle 29

Mediane und Interquartilsabstände zu den Prädiktorvariablen zum ersten und vierten Messzeitpunkt

\begin{tabular}{|c|c|c|c|c|c|}
\hline & & $\begin{array}{c}\text { Diagnose SM } \\
n=7\end{array}$ & $\begin{array}{c}\mathrm{zu} \text { T4 sprechend } \\
\mathrm{n}=11\end{array}$ & $\begin{array}{c}\text { Kontrollkinder } \\
\mathrm{n}_{\mathrm{T} 1}=12 \\
\mathrm{n}_{\mathrm{T} 4}=10\end{array}$ & $\begin{array}{c}\text { Gesamt } \\
\mathrm{N}_{\mathrm{T} 1}=30 \\
\mathrm{~N}_{\mathrm{T} 4}=28\end{array}$ \\
\hline \multirow{2}{*}{$\begin{array}{l}\text { BAV Gesamt } \\
\text { T-Wert }\end{array}$} & $\mathrm{T} 1$ & $\begin{array}{c}60.00 \\
(I Q R=26.0)\end{array}$ & $\begin{array}{c}50.0 \\
(I Q R=20.0)\end{array}$ & $\begin{array}{c}43.0 \\
(I Q R=15.0)\end{array}$ & $\begin{array}{c}48.5 \\
(I Q R=24.0)\end{array}$ \\
\hline & $\mathrm{T} 4$ & $\begin{array}{c}61.0 \\
(I Q R=34.25)\end{array}$ & $\begin{array}{c}47.0 \\
(I Q R=14.8)\end{array}$ & $\begin{array}{c}40.0 \\
(I Q R=21.0)\end{array}$ & $\begin{array}{c}48.0 \\
(I Q R=21.0)\end{array}$ \\
\hline \multirow{2}{*}{$\begin{array}{l}\text { TROG-D } \\
\text { T-Wert }\end{array}$} & $\mathrm{T} 1$ & $\begin{array}{c}45.00 \\
(I Q R=19.0)\end{array}$ & $\begin{array}{c}35.0 \\
(I Q R=22.0)\end{array}$ & $\begin{array}{c}50.0 \\
(I Q R=14.0)\end{array}$ & $\begin{array}{c}45.00 \\
(I Q R=15.0)\end{array}$ \\
\hline & $\mathrm{T} 4$ & $\begin{array}{c}45.0 \\
(I Q R=25.0)\end{array}$ & $\begin{array}{c}35.5 \\
(I Q R=5.8)\end{array}$ & $\begin{array}{c}50.5 \\
(I Q R=12.0)\end{array}$ & $\begin{array}{c}45.0 \\
(I Q R=22.0)\end{array}$ \\
\hline \multirow{2}{*}{ MLU } & $\mathrm{T} 1$ & $\begin{array}{c}3.8 \\
(I Q R=1.8)\end{array}$ & $\begin{array}{c}2.9 \\
(I Q R=1.1)\end{array}$ & $\begin{array}{c}3.7 \\
(I Q R=2.1)\end{array}$ & $\begin{array}{c}3.2 \\
(I Q R=1.8)\end{array}$ \\
\hline & $\mathrm{T} 4$ & $\begin{array}{c}3.6 \\
(I Q R=1.3)\end{array}$ & $\begin{array}{c}3.4 \\
(I Q R=0.6)\end{array}$ & $\begin{array}{c}3.4 \\
(I Q R=1.3)\end{array}$ & $\begin{array}{c}3.4 \\
(I Q R=0.6)\end{array}$ \\
\hline
\end{tabular}

Zum vierten Messzeitpunkt unterschieden sich die Gruppen nicht mehr signifikant voneinander, $H(2)=3.64, p=.162$. Es zeigte sich nur noch ein statistisch tendenzieller Trend hinsichtlich der Gesamtangst der Kinder, wobei auch hier die Kinder mit der Diagnose SM die höchsten Angstwerte zeigten, gefolgt von den Kindern mit positiver Entwicklung und den Kontrollkindern, $J=64.50, z=-1.89, p=.059, r=-.58$. Betrachtet man jedoch die Effektstärken, war der Effekt zum vierten Messzeitpunkt mit $r=-.58$ deutlich stärker. Die Varianz der Gesamtangst innerhalb der selektiv mutistischen Kinder $(I Q R=34.25)$ ist nochmals gestiegen.

Im Grammatikverständnis unterschieden sich die Gruppen zum ersten Messzeitpunkt tendenziell voneinander, $H(2)=4.79, p=.091$. Zum vierten Messzeitpunkt zeigte sich ein signifikanter Unterschied, $H(2)=12.08, p=.002$. In den anschließenden paarweisen Vergleichen mit angepasstem p-Wert ergab sich lediglich zwischen den zu T4 sprechenden Kindern und den Kontrollen ein signifikanter Unterschied ( $p=.002, r=-.076)$. Für beide Messzeitpunkt ließ sich ein signifikanter Trend in den Daten nachweisen, $J_{T 1}=179.50, z_{T 1}=2.18$, $p_{T 1}=.029, r_{T 1}=.41 ; J_{T 4}=199.50, z_{T 4}=3.56, p_{T 4}<.001, r_{T 4}=.69$, wobei jeweils die Kon- 
trollkinder die höchsten Leistungen zeigten, gefolgt von den Kindern mit SM. Die niedrigsten Leistungen im Grammatikverstehen zeigten die Kinder, die sich bis zum vierten Messzeitpunkt vom Schweigen zum Sprechen entwickelt haben. Zum vierten Messzeitpunkt war der Effekt deutlich größer als zum ersten Messzeitpunkt. Mit einem mittleren T-Wert von 35 zum ersten Messzeitpunkt und einem mittleren T-Wert von 35.5 zum vierten Messzeitpunkt sind die Leistungen der zu T4 sprechenden Kinder als nicht altersgemäß zu bezeichnen. Die Kinder mit SM lagen mit einem T-Wert von 45 zu beiden Messzeitpunkten im Durchschnittsbereich ebenso wie die Kontrollkinder mit einem T-Wert von $50 \mathrm{zu} \mathrm{T1}$ und $50.5 \mathrm{zu}$ T4. Auch hier ist jedoch die große Varianz-vor allem bei den schweigenden Kindern-hervorzuheben. Hinsichtlich der MLU war zu beiden Messzeitpunkten weder ein Unterschied zwischen den Gruppen, $H(2)_{T 1}=0.78, p_{T 1}=.683, H(2)_{T 4}=0.30, p_{T 4}=.862$, noch ein Trend nachweisbar, $J_{T 1}=90.50, z_{T 1}=.37, p_{T 1}=.709, r_{T 1}=.08 ; J_{T 4}=79.50, z_{T 1}=.49, p_{T 1}=.626, r_{T 1}=.11$.

\section{Tabelle 30}

Mediane und Interquartilsabstände in den Prädiktorvariablen für ein- und mehrsprachige Kinder

\begin{tabular}{lccc}
\hline & & mehrsprachig & einsprachig \\
& & $\mathrm{n}_{\mathrm{T} 1}=15, \mathrm{n}_{\mathrm{T} 4}=13$ & $\mathrm{n}=15$ \\
\hline BAV Gesamt T-Wert & $\mathrm{T} 1$ & 51.5 & 44.5 \\
& & $(I Q R=21.8)$ & $(I Q R=34.00)$ \\
& $\mathrm{T} 4$ & 45.0 & 50.0 \\
TROG-D T-Wert & $\mathrm{T} 1$ & $(I Q R=15.0)$ & $(I Q R=26.5)$ \\
& & $(I Q R=17.5)$ & 50.0 \\
& $\mathrm{~T} 4$ & 39.5 & $(I Q R=17.0)$ \\
MLU & & $(I Q R=10.0)$ & 51.0 \\
& $\mathrm{~T} 1$ & 3.0 & 3.8 \\
& & $(I Q R=1.5)$ & 3.5 \\
& $\mathrm{~T} 4$ & $(I Q R=0.6)$ & $(I Q R=0.6)$ \\
& & &
\end{tabular}

Beim Vergleich von mehr- und einsprachigen Kindern (siehe auch Tabelle 30) ergab sich ein Unterschied in den Leistungen im Grammatikverstehen sowohl zum ersten als auch zum vierten Messzeitpunkt, wobei die mehrsprachigen Kinder erwartungsgemäß schlechter abschnitten $\left(M d_{T 1}=38.00, M d_{T 4}=39.50\right)$ als die einsprachigen Kinder $\left(M d_{T 1}=50.00, M d_{T 4}\right.$ $=51.00), U_{T 1}=38.50, z_{T 1}=-2.74, p_{T 1}=.005, r_{T 1}=-.52 ; U_{T 4}=31.50, z_{T 4}=-2.86, p_{T 4}=.003$, $r_{T 4}=-.55$. Die MLU und das Ausmaß der Angst unterschieden sich nicht signifikant zwischen ein- und mehrsprachigen Kindern weder zum ersten, $U_{M L U T 1}=39.00, z_{M L U T 1}=-1.51, p_{M L U T 1}=$ $.141, r_{M L U T 1}=-.32 ; U_{B A V T 1}=118.50, z_{B A V T 1}=0.94, p_{B A V T 1}=.352, r_{B A V}=.18$, noch zum vierten 
Messzeitpunkt, $U_{M L U T 4}=42.00, z_{\text {MLUT4 }}=-0.92, p_{\text {MLUT4 }}=.387, r_{M L U T 4}=-.20 ; U_{\text {BAVT4 }}=70.00$, $z_{B A V T 1}=.-0.44, p_{B A V T 1}=.689, r_{B A V}=-.09$.

Die dritte Prädiktorvariable, die kulturelle Adaptation der Eltern gemessen über den FRAKK, wurde nur bei den mehrsprachigen Familien erhoben. Entsprechend wird im Folgenden lediglich die Teilstichprobe der mehrsprachigen Kinder betrachtet. Für den FRAKK liegen bislang keine Normwerte, sondern lediglich Vergleichswerte aus einer Studie mit 305 bosnischen, iranischen und koreanischen Personen mit Migrationshintergrund vor (Bongard, o.J.). Verglichen mit dieser Stichprobe lagen die Werte der Eltern (siehe Tabelle 31) in der Skala „Orientierung an der Herkunftskultur“ im Mittel zwischen dem 25. und 50. Perzentil, in der Skala „Orientierung an der Aufnahmekultur“ im Mittel zwischen dem 75. und 90. Perzentil sowie ebenso für den Akkulturationsindex im Mittel zwischen dem 75. und 90. Perzentil. Dies legte nahe, dass sich die Eltern der Probanden vermehrt an der Umgebungskultur, in diesem Falle der deutschen, orientierten, jedoch zu einem gewissen Maße ihre Herkunftskultur beibehielten. Innerhalb der Gesamtstichprobe der Familien mit Migrationshintergrund schien demnach eher ein assimilierter Akkulturationsstil vorzuliegen. Die drei Gruppen selektiv mutistisch, sprechend zu T4 und Kontrollen unterschieden sich nicht signifikant im Ausmaß der elterlichen Orientierung an der Herkunfts- oder Aufnahmekultur sowie im Akkulturationsindex voneinander. Ein statistischer Trend war ebenso nicht nachweisbar (siehe Tabelle 31). Der statistisch tendenzielle Trend in der Orientierung an der Herkunftskultur zum vierten Messzeitpunkt resultierte wahrscheinlich aus der ungünstigen Zellbesetzung zu diesem Messzeitpunkt. Zur Kontrolle des Unterschiedes zwischen den Kindern mit SM und den zu T4 sprechenden Kindern wurde zusätzlich ein Mann-Whitney-U Test gerechnet. Hier ergab sich kein signifikanter Unterschied in der Orientierung der Eltern an der Herkunftskultur, $U=18.00, z=1.17, p=.310, r=.37$. 
Tabelle 31

Deskriptive Ergebnisse und Gruppenunterschiede zur kulturellen Adaption der Eltern zum ersten Messzeitpunkt

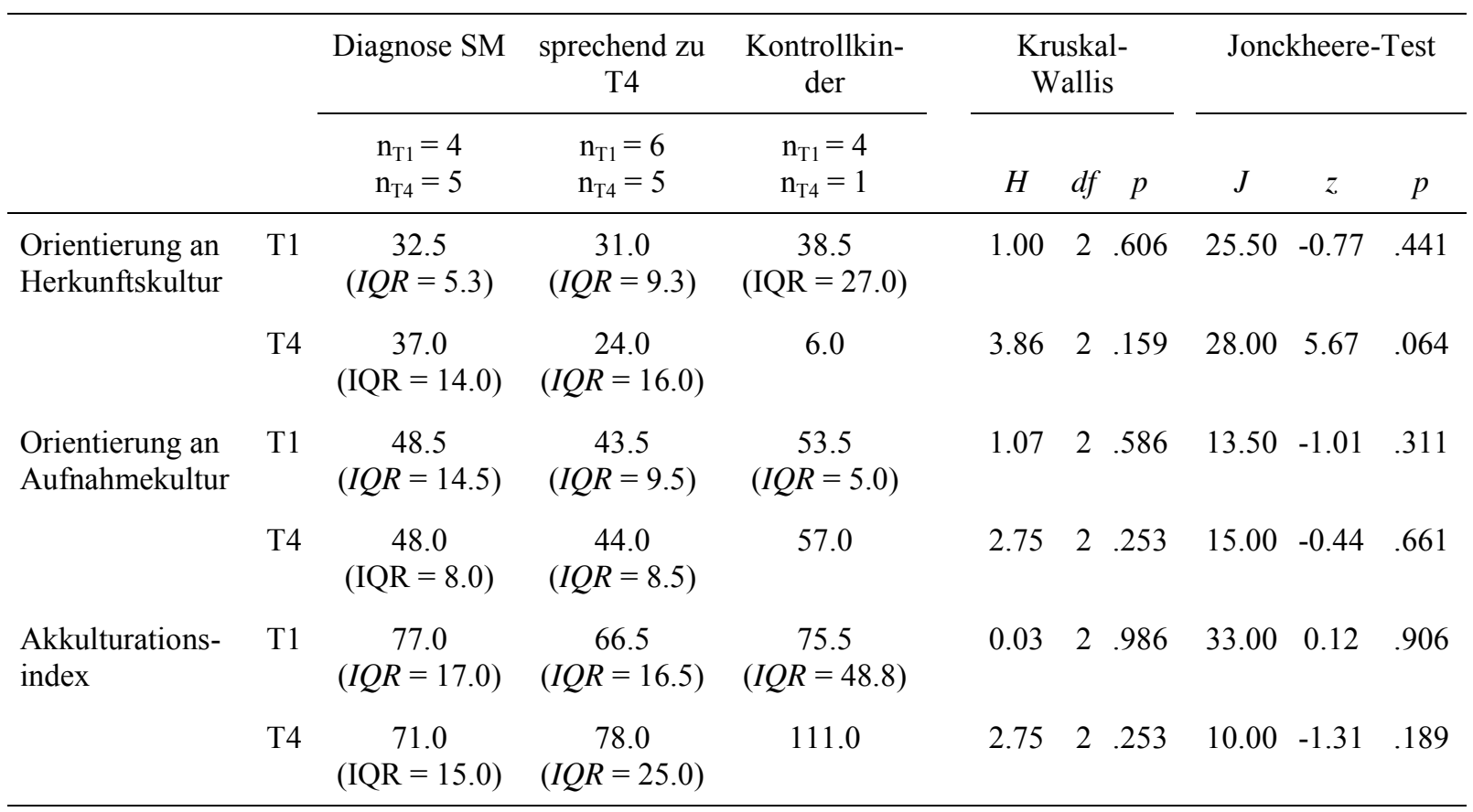

\subsubsection{Profile der selektiv mutistischen Kinder}

Aufgrund der großen Varianz innerhalb der Prädiktor- und Outcomevariablen für die selektiv mutistischen Kinder, werden im Folgenden diese sieben Kinder nochmals einzeln betrachtet und anschließend den Subgruppen nach Cohan et al. (2008) zugeordnet. Tabelle 32 gibt zunächst eine Übersicht über Alter, Geschlecht sowie Art der Mehrsprachigkeit der zum vierten Messzeitpunkt selektiv mutistischen Kinder. Die Kinder waren zu Beginn der Studie im Alter zwischen 3;5 und 5;8 Jahren (entsprechend neun Monate älter zum vierten Messzeitpunkt) sowie alle weiblichen Geschlechts. Zwei Mädchen waren einsprachig, fünf mehrsprachig. Zwei der Kinder sind sukzessiv mehrsprachig aufgewachsen, die anderen drei simultan: drei in der Kombination Türkisch-Deutsch, ein Mädchen mit Kurdisch-Deutsch sowie eines mit Arabisch-Deutsch. 
Tabelle 32

Alter, Geschlecht sowie Informationen zur Mehrsprachigkeit der selektiv mutistischen Kinder

\begin{tabular}{lccccc}
\hline Vpn & Alter zu T1 & Geschlecht & Sprachlichkeit & Zweitspracherwerb & Sprachen \\
\hline S1 & $5 ; 8$ & weiblich & einsprachig & - & Deutsch \\
S2 & $3 ; 5$ & weiblich & einsprachig & - & Deutsch \\
S6 & $3 ; 10$ & weiblich & mehrsprachig & sukzessiv & Kurdisch-Deutsch \\
S13 & $4 ; 3$ & weiblich & mehrsprachig & simultan & Deutsch-Türkisch \\
S14 & $4 ; 4$ & weiblich & mehrsprachig & sukzessiv & Deutsch-Türkisch \\
S15 & $3 ; 10$ & weiblich & mehrsprachig & simultan & Arabisch-Deutsch \\
S18 & $4 ; 9$ & weiblich & mehrsprachig & simultan & Türkisch-Deutsch \\
\hline
\end{tabular}

Anmerkung. Die jeweils dominante Sprache der Kinder ist in der Spalte Sprachen immer die zuerst Genannte.

Tabelle 33 gibt einen Überblick über die Ausprägung der Angst, der Sprachkompetenzen sowie der kulturellen Adaptation der Eltern zum ersten Messzeitpunkt. Die einsprachigen Mädchen mit SM zeichneten sich vor allem durch eine deutlich erhöhte Ängstlichkeit bei gut durchschnittlichen Sprachkompetenzen aus. Mit T-Werten von 78 und 81 im BAV war die Gesamtangst der Kinder zum ersten Messzeitpunkt überdurchschnittlich hoch im Vergleich zur Altersnorm. Die Sprachverständnisfähigkeiten der Kinder waren mit T-Werten von 57 und $58 \mathrm{im}$ gut durchschnittlichen Bereich. Eine MLU von 4.40 wies zudem daraufhin, dass die beiden Mädchen bereits über einen gewissen Wortschatz sowie syntaktische Fähigkeiten verfügten, um komplexe Sätze zu bilden. Die qualitative Analyse der Spontansprache zeigte, dass beide Mädchen keine Schwierigkeiten auf der phonetisch-phonologischen oder syntaktisch-morphologischen Sprachebene zeigten. In ihren Äußerungen verwendeten sie ein breites Spektrum der Wortarten sowie innerhalb der einzelnen Wortart verschiedene Wörter. Im Gesamten waren ihre sprachlichen Fähigkeiten damit als altersangemessen anzusehen.

S15 zeigte als einziges mehrsprachiges Kind zum ersten Messzeitpunkt eine überdurchchnittlich stark ausgeprägte Gesamtangst (T-Wert 62). S6 und S13 lagen mit T-Werten von jeweils 58 im oberen Durchschnittsbereich. S18 war mit einem T-Wert von 37 als unterdurchschnittlich ängstlich im Vergleich zur Altersnorm einzustufen. Bei S14 fehlten im Elternfragebogen zu viele Antworten, so dass kein Gesamtwert berechnet werden konnte. Für einzelne Subskalen, die aufgrund der geringen Reliabilität nicht in die weiteren Analysen mit einbezogen worden sind (siehe Kapitel 3.2.5), lagen jedoch Werte vor. Die sozialen Ängste waren mit einem T-Wert von 53 als durchschnittlich ausgeprägt anzusehen, die Angst vor Verletzung mit einem T-Wert von 34 als unterdurchschnittlich. Für die Skala kognitive Ängs- 
te lag aufgrund zahlreicher fehlender Itemantworten kein Wert vor. Die Werte auf den beiden übrigen Skalen wiesen jedoch darauf hin, dass S14 nicht übermäßig ängstlich war—vor allem im Hinblick auf für SM bedeutsame soziale Situationen.

Tabelle 33

Prädiktorvariablen zum ersten Messzeitpunkt für Kinder mit der Diagnose SM

\begin{tabular}{lcccccc}
\hline Vpn & $\begin{array}{c}\text { BAV Gesamt } \\
\text { T-Wert }\end{array}$ & $\begin{array}{c}\text { TROG-D } \\
\text { T-Wert }\end{array}$ & MLU & $\begin{array}{c}\text { FRAKK HK } \\
\text { Rohwert }\end{array}$ & $\begin{array}{c}\text { FRAKK AK } \\
\text { Rohwert }\end{array}$ & $\begin{array}{c}\text { FRAKK AI } \\
\text { Rohwert }\end{array}$ \\
\hline S1 & 78 & 57 & 4.40 & & einsprachig & \\
S2 & 81 & 58 & 4.40 & & einsprachig & \\
S6 & 58 & 45 & 1.47 & 34 & 52 & 78 \\
S13 & 58 & 38 & 4.33 & 29 & 45 & 76 \\
S14 & fehlend & 41 & 3.20 & & fehlende Angabe & \\
S15 & 62 & 50 & fehlend & 31 & 53 & 82 \\
S18 & 37 & 29 & 3.00 & 35 & 36 & 60 \\
\hline
\end{tabular}

Drei der mehrsprachigen Mädchen erreichten im TROG-D altersangemessene Leistungen. S13 und S18 zeigten mit T-Werten unter 40 keine altersadäquaten Sprachverständnisfähigkeiten, wobei S18 mit einem T-Wert von 29 die größten Schwierigkeiten im Verstehen von Sätzen aufwies. In der Spontansprache gelang es S18 zwar bereits, komplexe Äußerungen zu produzieren (MLU von 3.00). Die qualitativen Analysen zeigten jedoch, dass diese teilweise von einer starren Satzstruktur (z.B. [die hat husten] [der hat dann bauchschmerzen]), zahlreichen Satzabbrüchen (16 \% der Äußerungen in der Gesamtaufnahme zu T1) sowie syntaktisch-morphologischen Schwierigkeiten geprägt warem. Zum ersten Messzeitpunkt hatte sie damit noch deutliche Schwierigkeiten sowohl im Verstehen als auch in der Produktion im Deutschen. S6 produzierte innerhalb der Spontansprachaufnahme fast nur Ein-WortÄußerungen, wobei es vor allem Nomen und nur wenige Verben verwendete. Die produktiven Sprachkompetenzen waren im Deutschen somit noch sehr rudimentär ausgebildet bei jedoch bereits durchschnittlichen Verstehensleistungen im Vergleich zu einsprachig aufwachsenden Alterspeers.

S13 und S14 produzierten beide bereits deutlich längere Äußerungen. Beide hatten leichte Auffälligkeiten auf der phonetisch-phonologischen Sprachebene. S13 zeigte eine Vorverlagerung der Phoneme /k, g/, wobei es begann diese bereits in einigen Wörtern korrekt einzusetzen. S14 zeigte einen Sigmatismus interdentalis sowie noch leichte Schwierigkeiten das Phonem $/ \int /$ in Konsonantenclustern einzusetzen. S13 wies einen deutlich variantenreicheren Wortschatz (Typ-Token-Rate Nomen: .88, Verben: .65, Adjektive: 1.00 sowie Adverbien: 
.41) auf als S14 (Typ-Token-Rate Nomen: .50, Verben: .40, Adjektive: .80 sowie Adverbien: .80). Beide Kinder verfügten jedoch über ausreichende Sprachkompetenzen im Deutschen, um sich im Spiel und im Kita-Alltag äußern zu können.

Hinsichtlich der kulturellen Adaptation waren sich die Bezugspersonen der mehrsprachigen Kinder sehr ähnlich. Alle Elternteile orientierten sich nur noch in eher geringem Maße an ihrer Herkunftskultur. Als Vergleichsgruppe diente eine Stichprobe von 60 männlichen Personen mit türkischem Migrationshintergrund aus Frankfurt am Main (Bongard, o.J.). Die Werte der Eltern der vorliegenden Stichprobe lagen mit einem Maximum von 35 innerhalb der 5. Perzentile der Vergleichsstichprobe. Die Level der Orientierung an der Herkunftskultur waren im Vergleich zu Personen gleichen oder ähnlichen Migrationshintergrundes demnach als eher niedrig einzuschätzen. Die Eltern von S6, S13 und S15 orientierten sich sehr stark an der Umgebungskultur. Mit einem Rohwert von 52 und 53 für die Orientierung an der Aufnahmekultur warem die Akkulturationslevel der Bezugspersonen von S6 und S15 im Vergleich zur Stichprobe von Bongard (o.J.) als hoch einzustufen. Sie lagen damit innerhalb der 90. Perzentile der Vergleichsstichprobe. Die Bezugsperson von S13 lag mit einem Wert von 45 zwischen der 50. und 75. Perzentile der Vergleichsstichprobe und war damit als durchschnittlich anzusehen. Das Akkulturationslevel der Bezugsperson von S18 war mit einem Wert von 36 (25.-50. Perzentil der Vergleichsstichprobe) als vergleichsweise niedrig einzustufen. Bei vier der fünf Elternteile war der kulturelle Adaptationsstile damit als Assimilation zu kategorisieren. Die Bezugsperson von S18 befand sich zu Beginn der Studie noch auf dem Kontinuum zwischen einer Marginalisierung und der Assimilation.

Auch zum vierten Messzeitpunkt (siehe Tabelle 34 für alle Werte der Kinder im Überblick) waren die einsprachigen Kinder überdurchschnittlich ängstlich bei gleichzeitig überdurchschnittlich ausgeprägten Sprachkompetenzen. Bei den mehrsprachigen Kindern haben sich vor allem im Bereich der Gesamtangst Veränderungen zum ersten Messzeitpunkt ergeben. Die Gesamtangst von S6 hat sich über die neun Monate um 16 T-Wertpunkte gesteigert und lag zu T4 im weit überdurchschnittlichen Bereich. Die Gesamtangst von S13 und S15 hat deutlich abgenommen und war jeweils als durchschnittlich im Vergleich zur Altersnorm einzustufen. S18 zeigte weiterhin eine unterdurchschnittlich ausgeprägte Gesamtangst. Für S14 lagen wiederum nicht genügend Antworten vor, um einen Gesamtwert für den BAV errechnen zu können. Auf den Subskalen mit T-Werten von 48 (Soziale Ängste) und 36 (Angst vor Verletzung) erreichte S14 weiterhin durchschnittlich bis unterdurchschnittlich ausgeprägte Ängste. 
Tabelle 34

Prädiktorvariablen zum vierten Messzeitpunkt für die Kinder mit Diagnose SM

\begin{tabular}{lcccccc}
\hline Vpn & $\begin{array}{c}\text { BAV Gesamt } \\
\text { T-Wert }\end{array}$ & $\begin{array}{c}\text { TROG-D } \\
\text { T-Wert }\end{array}$ & MLU & $\begin{array}{c}\text { FRAKK HK } \\
\text { Rohwert }\end{array}$ & $\begin{array}{c}\text { FRAKK AK } \\
\text { Rohwert }\end{array}$ & $\begin{array}{c}\text { FRAKK AI } \\
\text { Rohwert }\end{array}$ \\
\hline S1 & 65 & 61 & fehlend & & einsprachig & \\
S2 & 81 & 65 & 3.63 & & einsprachig & \\
S6 & 74 & 45 & 1.93 & 37 & 45 & 68 \\
S13 & 44 & 43 & 3.80 & 37 & 41 & 64 \\
S14 & fehlend & 35 & 3.63 & 26 & 50 & 84 \\
S15 & 57 & 45 & fehlend & 24 & 48 & 78 \\
S18 & 34 & 29 & 2.93 & 41 & 52 & 71 \\
\hline
\end{tabular}

Drei von fünf Kindern zeigten zum vierten Messzeitpunkt angemessene Sprachverständnisleistungen im Vergleich zu ihren einsprachig deutschen Alterspeers. S14 hat sich leicht verschlechtert und lag mit einem T-Wert von 35 nun im unterdurchschnittlichen Bereich. S18 zeigte weiterhin weit unterdurchschnittliche Sprachverstehensleistungen im Deutschen. In der Sprachproduktion zeigte S6 weiterhin die geringsten Fähigkeiten. Die MLU hat sich leicht gesteigert, was sich ebenso in einer leichten Erweiterung des Wortschatzes widerspiegelte. Zum ersten Messzeitpunkt produzierte S6 innerhalb der 30 Äußerungen bspw. nur 7 Nomen (Type-Token-Rate .47) und 3 Verben (Type-Token-Rate .50), zum vierten Messzeitpunkt bereits 15 Nomen (Type-Token-Rate .65) und 5 Verben (Type-Token-Rate .45). S6 nutzte vermehrt Zwei- bis Drei-Wort-Sätze mit korrekter Satzstruktur-sowohl für Haupt- als auch Fragesätze. S13 zeigte weiterhin adäquate sprachproduktive Kompetenzen, wobei die Vorverlagerung von $/ \mathrm{k}, \mathrm{g} /$ noch nicht überwunden wurde. Auch S14 zeigte weiterhin einen Sigmatismus interdentalis und konnte ihre MLU leicht steigern. Die produktiven Sprachfähigkeiten von S18 waren zum vierten Messzeitpunkt vergleichbar zu ihren Leistungen zu Beginn der Studie. Sie produzierte vornehmlich Drei- bis Vier-Wort-Sätze, wobei sie noch vereinzelt Fehler in der Satzstellung machte.

Die Werte im FRAKK haben sich bei allen Eltern deutlich verändert. Bei drei der fünf mehrsprachigen Kinder hat die Orientierung der Eltern an der Aufnahmekultur leicht abgenommen. Mit Werten zwischen 41 und 52 lagen die Bezugspersonen der Kinder jedoch auch zum vierten Messzeitpunkt innerhalb der 50.bis 90. Perzentil der Vergleichsgruppe von Bongard (o.J.). Die Orientierung an der Herkunftskultur hat sich bei drei der fünf mehrsprachigen Familien leicht erhöht, bei einem weiteren Elternteil hat sie leicht abgenommen. Mit Werten zwischen 24 und 37 war die Orientierung der Eltern an der Herkunftskultur im Ver- 
gleich zur Stichprobe von Bongard (o.J.) als niedrig einzuschätzen (1. bis 10. Perzentile). Die Bezugsperson von S14 hat lediglich zum vierten Messzeitpunkt einen FRAKK ausgefüllt. Sie zeigte mit einem Wert von 26 die geringste Orientierung an der Herkunftskultur verglichen mit allen Elternteilen der vorliegenden Stichprobe. Die Orientierung an der Aufnahmekultur lag mit einem Wert von 50 innerhalb der 75. bis 90. Perzentile der Vergleichsgruppe. Damit sind die kulturellen Adaptationsstile der Eltern zum vierten Messzeitpunkt als Assimilation zu kategorisieren.

Die Kinder ließen sich zum vierten Messzeitpunkt somit folgenden Subgruppen nach Cohan et al. (2008) zuordnen: Die einsprachigen Kinder zeigten beide konstant eine deutlich erhöhte Ängstlichkeit bei guten Sprachkompetenzen. Sie wurden damit der Gruppe ängstliche Kinder zuzuordnen. Die beiden mehrsprachigen Kinder S6 und S15 waren ebenfalls vergleichsweise ängstlich und zeigten zusätzlich leichte (S15) bis massive (S6) sprachliche Unsicherheiten in der Umgebungssprache. Sie wurden damit der Gruppe ängstliche Kinder mit sprachlichen Unsicherheiten zugeordnet. Die übrigen drei Kinder zeigten keine übermäßige Ängstlichkeit. Bei ihnen waren jedoch leichte sprachliche Unsicherheiten festzustellen. Sie konnten damit nicht klar einer der Subgruppen von Cohan et al. (2008) zugeordnet werden. Für eines der Kinder (S13) lag zum ersten Messzeitpunkt noch eine leicht erhöhte Ängstlichkeit vor. Die Symptomatik des SM war anfangs sehr stark. Sie konnte innerhalb der neun Monat ihre Ängste deutlich und gleichzeitig auch das Ausmaß des Schweigens reduzieren. Sie gehörte demnach zum ersten Messzeitpunkt ebenso eher den ängstlichen Kindern mit sprachlichen Unsicherheiten an. Für S14 lagen nicht genügend Elternantworten vor, um die Gesamtangst des Kindes sowohl für den ersten als auch vierten Messzeitpunkt zu bestimmen. Auf Subskalenebene waren ihren sozialen Ängste sowie die Angst vor Verletzung altersentsprechend ausgeprägt. Sie zeigte jedoch vor allem zum vierten Messzeitpunkt unterdurchschnittlich ausgeprägte Leistungen im Grammatikverstehen sowie Auffälligkeiten auf der phonetisch-phonologischen Sprachebene, so dass von sprachlichen Unsicherheiten im Deutschen ausgegangen werden konnte. S18 unterschied sich von allen anderen mehrsprachigen Kindern hinsichtlich der kulturellen Adaptation der Eltern. Die Bezugsperson hat innerhalb der neun Monate eine deutliche Entwicklung hin zu einem Assimilationsstil vollzogen. Anfangs war die Orientierung an der Aufnahmekultur noch relativ gering. Zum vierten Messzeitpunkt warem ihre Werte vergleichbar mit denen der anderen Elternteile. 


\subsection{Hypothesenprüfung}

Zur Überprüfung der in Kapitel 2.5 aufgestellten Hypothesen wurden für Vergleiche zwischen zwei Gruppen der Mann-Whitney-U-Test, zur Überprüfung von Trends in den Daten der Jonckheere-Terpstra-Test und bei Vergleichen zweier Messzeitpunkte der WilcoxonVorzeichen-Rangtest für verbundene Stichproben genutzt. Zur Analyse von Zusammenhängen wurde wiederum auf den Kendalls-Tau zurückgegriffen.

Ziel der Studie war es, den Einfluss von Angst, Sprachkompetenzen im Deutschen und kultureller Adaptation der Eltern auf die Entwicklung eines SM zu untersuchen. Zu diesem Zweck wurden verschiedene Mehrebenenmodelle gerechnet. Mehrebenenmodelle gehören zu den linearen Modellen und sind zur Analyse von genesteten Daten geeignet (Snijders \& Bosker, 2012). Prinzipiell können eine Vielzahl von Ebenen in das Modell implementiert werden. Meist werden jedoch zwei bis drei Ebenen einbezogen. Die unterste Ebene (Ebene 1) umfasst in der Regel Individuen, die darüber liegenden Ebenen (Ebene 2 etc.) irgendeine Art von Gruppenzuweisung (z.B. Klassen, Schulen, Länder). Dies ermöglicht sowohl Effekte auf Individual- als auch auf Gruppenebene zu untersuchen. In der Analyse von Längsschnittdaten werden die Messzeitpunkt quasi als Individuen auf Level 1 behandelt, welche im einzelnen Probanden auf Level 2 genestet sind (Hox \& Boom, 2012). Durch diese Art der Datenstruktur ergibt sich eine besondere Flexibilität gegenüber fehlenden Werten auf Level 1 (fehlende Werte in den Outcomevariablen zu einzelnen Messzeitpunkten) im Vergleich zu Regressionsanalysen zu bestimmten Messzeitpunkten. Trotz fehlender Werte zu bestimmten Messzeitpunkten können die vorhandenen Informationen des Probanden für die Schätzung der Modellparameter genutzt werden (Snijders \& Bosker, 2012). Abbildung 17 zeigt beispielhaft die Datenstruktur des Zweiebenenmodells der vorliegenden Studie. Auf Level 1 befinden sich jeweils entsprechende Werte der Outcomevariablen sowie der Messzeitpunkt als Prädiktorvariable (kodiert von 0 bis 3). Als Outcomevariablen wurde für den familiären und öffentlichen Kontext die Subskala Schweigen des Elternfragebogens gewählt, da diese die Kernsymptomatik des SM erfasst. Für den Kitakontext wurde entsprechend die Subskala Schweigen und Bedürfnisäußerung verwendet. Die Beobachtungsvariablen wurden aufgrund der fraglichen Reliabilität und Validität der Daten nicht in die Mehrebenenanalysen einbezogen. 


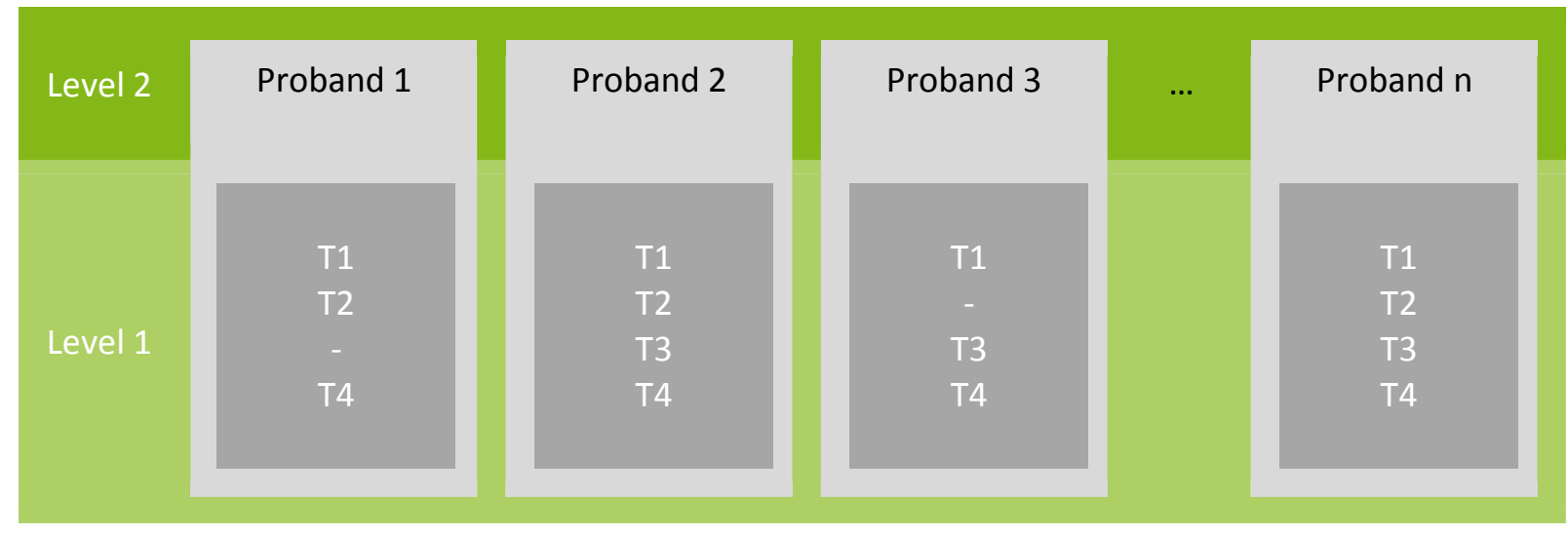

Abbildung 18. Datenstruktur im Mehrebenenmodell bei Längsschnittdaten

Auf Level 2 befinden sich der Faktor Mehrsprachigkeit (dummykodiert mit $0=$ einsprachig und $1=$ mehrsprachig) und die eigentlichen Prädiktoren Ausmaß der Angst, Sprachkompetenzen der Kinder sowie kulturelle Adaptation der Eltern. Da die Prädiktoren (außer die MLU) keinen bedeutungsvollen Nullpunkt haben (jeweils T-Werte), wurden sie zur besseren Interpretation der Effekte Median zentriert (siehe dazu auch Finch, Bolin \& Kelley, 2014). Je nach Modell würde der Nullpunkt beispielsweise ein Kind widerspiegeln, welches für die Gesamtgruppe durchschnittliche Leistungen im Grammatikverstehen und eine für die Gruppe durchschnittliche Ängstlichkeit zeigt.

Die Modellspezifikation erfolgte Raudenbush und Bryk (2002) folgend in zwei Phasen, wobei zunächst feste und zufällige Effekte auf Level 1 getestet wurden und anschließend untersucht wurde, welche Level 2 Variablen die auf Level 1 gefundene Varianz erklären. Zufällige Effekte auf den Achsenabschnitt (Random Intercept) können durch Hinzunahme von Level 2 Variablen erklärt werden, zufällige Effekte auf die Steigung der Regressionsgeraden (Random Slope) können durch Interaktionseffekte von Level 2 Prädiktoren mit Level 1 Prädiktoren erklärt werden. Für den Modellvergleich wurde zunächst ein Basismodell angepasst, welches lediglich den mittleren Wert der Outcomevariable als beste Vorhersage annimmt. Abbildung 18 zeigt im Überblick das Vorgehen bei der Modellspezifikation. 


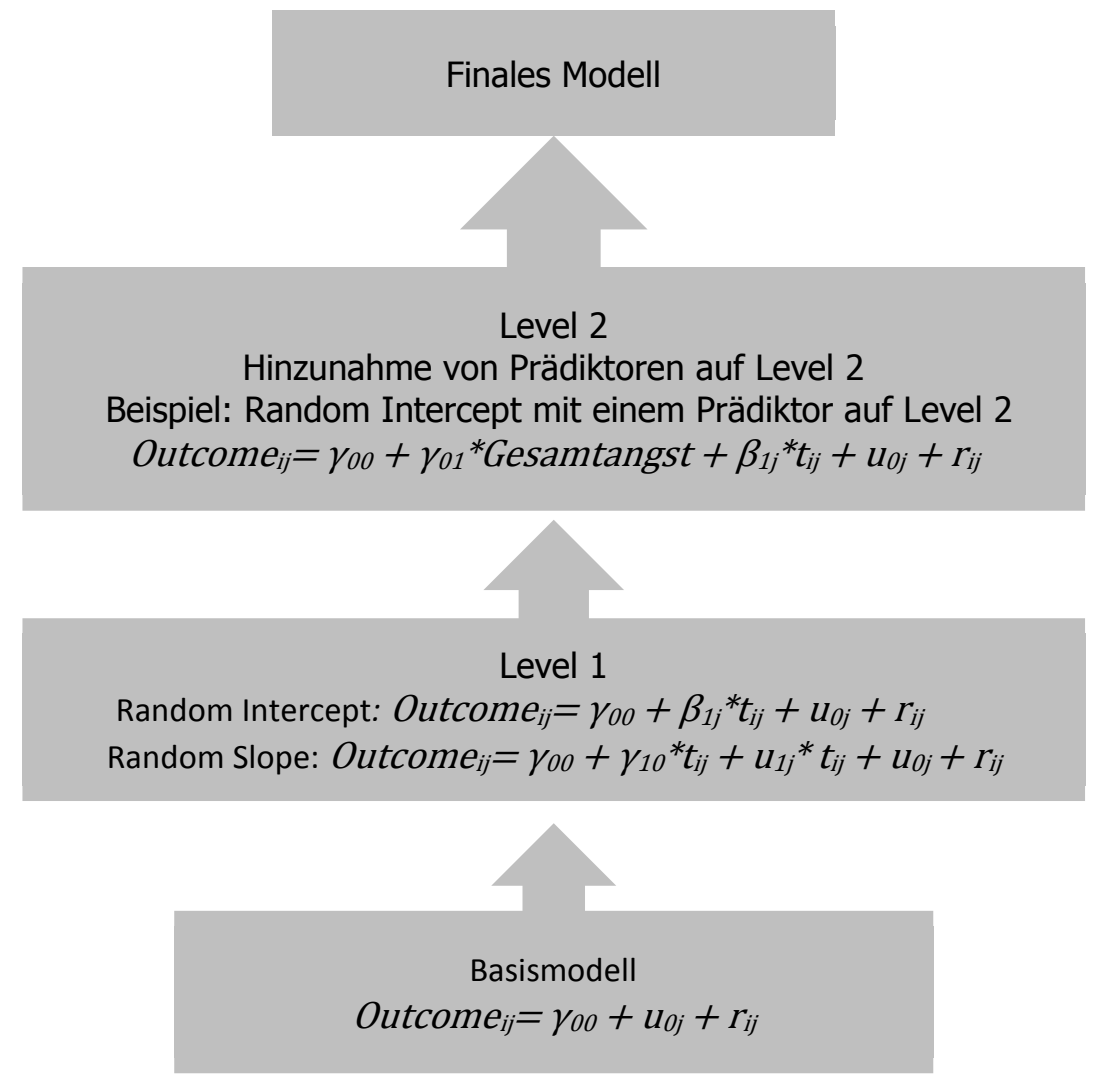

Abbildung 19. Vorgehen bei der Spezifikation der Mehrebenenmodelle

Zum Vergleich des Modellfits stehen in Mehrebenenmodellen verschiedene Fit-Maße zur Verfügung. Bei der Schätzung von Modellen über die Maximum-Likelihood-Methodewie bei Mehrebenenenmodellen-erhält man generell den so genannten Likelihood, aus welchem sich die Devianz als Badness-of-Fit-Maß errechnen lässt (Devianz $=-2 \times$ Log Likelihood). Die Devianzen zweier aufeinander aufbauenden Modelle (z.B. Basismodell im Vergleich zu Random Intercept Modell auf Level 1) können zum Vergleich des Modellfits genutzt werden. Grundsätzlich spiegelt eine kleinere Devianz immer einen besseren Fit des Modells wider. Über einen Chi-Quadrat Test der beiden Werte kann zusätzlich geprüft werden, ob sich die Devianzen signifikant voneinander unterscheiden (Snijders \& Bosker, 2012). Dazu wurden alle Modelle mit der Full-Maximum-Likelihood-Methode geschätzt. Ferner stehen weitere Informationskriterien, welche auf der Devianz beruhen, zur Verfügung. Das Akaike Informationskriterium (AIC) Ist ein Maß, welches hinsichtlich der Komplexität des Modells korrigiert ist. Das Bayesian Informationskriterium (BIC) ist vergleichbar mit dem AIC, jedoch etwas konservativer. BIC korrigiert die Devianz nach der Anzahl der geschätzten Parameter. Beide Kriterien können jedoch ähnlich wie die Devianz interpretiert werden. Kleinere Werte spiegeln jeweils einen besseren Fit des Modells wider (Field, 2013). 
Zusätzlich interessiert, wie viel Varianz in der abhängigen Variable durch das Modell erklärt werden kann. In Regressionsanalysen wird der quadrierte multiple Korrelationskoeffizient $R^{2}$ genutzt. Dieser gibt den Anteil der erklärten Varianz wider. Snijders und Bosker (1994) schlagen für Mehrebenenenmodelle ein ähnliches Maß vor, wobei das $R^{2}$ hier den Anteil der Reduktion in den geschätzten Varianzkomponenten $\sigma^{2}$ und $\tau^{2}$ durch Einbindung neuer Variablen in das Modell widergibt. Ähnlich wie bei der Überprüfung der Verbesserung des Modellfits über den Vergleich der Devianzen werden für die Berechnung von $R^{2}$ die Varianzkomponenten des Basismodells mit denen der komplexeren Modelle verglichen. $R^{2}$ auf Level 1 spiegelt die erklärte Varianz in den einzelnen Einheiten (in der vorliegenden Studie Messzeitpunkte) wider und wird folgendermaßen berechnet:

$$
R_{1}^{2}=1-\frac{\sigma_{\mathrm{M} 1}^{2}+\tau_{\mathrm{M} 1}^{2}}{\sigma_{\mathrm{M} 0}^{2}+\tau_{\mathrm{M} 0}^{2}} .
$$

Auf Level 2 steht $R^{2}$ für die erklärte Varianz auf Gruppenebene (hier auf Probandenebene) und wird mithilfe folgender Formel berechnet, wobei $B$ die durchschnittliche Größe der Level 2 Einheiten (in diesem durchschnittliche Anzahl von Messzeitpunkten pro Proband) ist:

$$
R_{1}^{2}=1-\frac{\sigma_{\mathrm{M} 1}^{2} / \mathrm{B}+\tau_{\mathrm{M} 1}^{2}}{\sigma_{\mathrm{M} 0}^{2} / \mathrm{B}+\tau_{\mathrm{M} 0}^{2}} .
$$

Für Modelle mit Random Slope ist die Berechnung des $R^{2}$ deutlich komplexer. Dem Vorgehen von Snijders und Bosker (2012) folgend werden bei Modellen mit Random Slope die $R^{2}$ auf einem gleich spezifizierten Random Intercept Modell berechnet.

In der vorliegenden Längsschnittstudie interessierte vor allem die erklärte Varianz auf Level 2 Ebene, da die individuellen Entwicklungsverläufe der Probanden und mögliche Einflussfaktoren derer untersucht wurden.

\subsection{1 Ängste}

H2: Kinder, die zum letzten Messzeitpunkt selektiv mutistisch sind, zeigen höhere Werte im Angstfragebogen als Kinder, die im Verlauf der Studie angefangen haben zu sprechen, sowie von Beginn an sprechende Kontrollkinder.

Zur Überprüfung der Hypothese zwei wurde ein Jonckheere-Terpstra-Test mit der abhängigen Variable BAV Gesamtangst T-Wert und der Gruppenvariable Mutismusdiagnose zu T4 gerechnet. Dieser ergab einen signifikanten Trend in den Daten, $J=188.00, z=2.63$, $p=.009, r=.50$. Wie vermutet zeigten die Kontrollkinder die niedrigsten Werte im Angstfragebogen $(M d=43)$, die Kinder, die zum vierten Messzeitpunkt sprechend waren, mittlere 
Werte $(M d=50)$ und die selektiv mutistischen Kinder die höchsten Werte $(M d=60)$. Siehe dazu auch Abbildung 20.

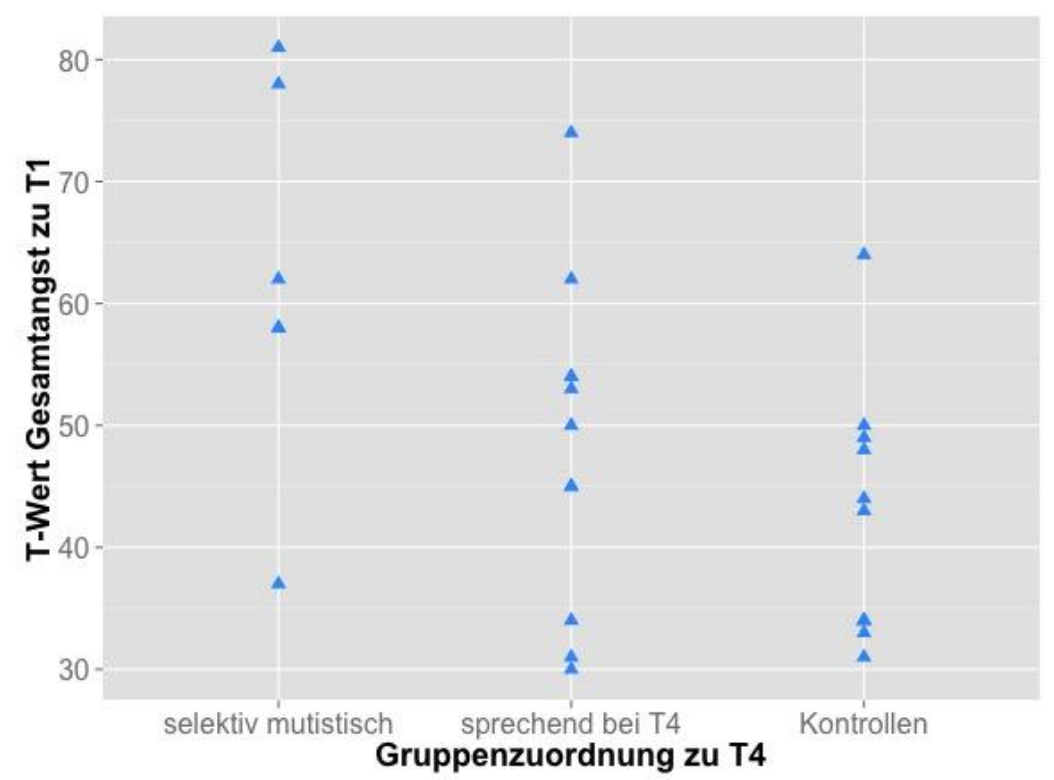

Abbildung 20. Gesamtangst der Kinder zum ersten Messzeitpunkt

H3: Die mehrsprachigen Kinder mit SM zeigen höhere Werte im Angstfragebogen zum ersten Messzeitpunkt als einsprachige Kinder mit SM.

Die Überprüfung von Hypothese drei war aufgrund der Daten nicht möglich, da sich in der Gruppe der einsprachigen, selektiv mutistischen Kinder nur zwei Probanden befanden. Beide Kinder erreichten Werte im überdurchschnittlichen Bereich (T-Wert 78 und 81), was für eine deutlich erhöhte Gesamtangst im Vergleich zu Kindern des gleichen Alters spricht. Bei den mehrsprachigen, selektiv mutistischen Kindern lagen für vier von fünf Kindern zum letzten Messzeitpunkt Ergebnisse des Angstfragebogens vor. Drei von vier Kindern lagen mit T-Werten zwischen 58 und 62 im oberen Durschnitts- bzw. überdurchschnittlichen Bereich. Das vierte Kind lag mit einem T-Wert von 37 im unterdurchschnittlichen Bereich und zeigte damit für sein Alter eher wenige Ängste.

H4: Das Ausmaß der Angst hat sich bei mehrsprachigen Kindern mit SM vom ersten zum letzten Messzeitpunkt erhöht.

Zur Überprüfung von Hypothese vier wurde ein Wilcoxon-Vorzeichen-Rang-Test durchgeführt. Dieser ergab keine signifikanten Veränderungen in der Gesamtangst der mehrsprachigen, selektiv mutistischen Kinder zwischen dem ersten $(M d=58.00)$ und vierten Messzeitpunkt $(M d=50.50), T=1.00, p=.625, r=-.25$. Lediglich bei einem der Kinder 
ergab sich ein deutlicher Zuwachs der Angst von einem T-Wert von 58 zu 74. Bei den restlichen drei Kindern, für die Fragebögen zu beiden Messzeitpunkten vorlagen, war eine leichte Abnahme der Angst von drei bis sechs T-Wertpunkten zu verzeichnen.

\subsubsection{Kulturelle Adaptation der Eltern}

H6: Eltern von Kindern mit SM weisen ein niedrigeres Anpassungslevel an die Umgebungskultur auf als Eltern von Kindern ohne SM.

Das Anpassungslevel an die Umgebungskultur unterschied sich nicht signifikant zwischen den Eltern der Kinder mit SM $\left(M d_{t 1}=48.50, M d_{t 4}=48.00\right)$ und den Eltern der sprechenden Kinder $\left(M d_{t 1}=50.00, M d_{t 4}=46.00\right)$ weder zum ersten, $U=15.50, z=-0.64$, $p=.539, r=-.17$ noch zum letzten Messzeitpunkt, $U=15, z=0.00, p=1.000, r=0$.

H7: Eltern von Kindern mit SM weisen ein höheres Maß an Beibehaltung ihrer Herkunftskultur auf als Eltern von Kindern ohne SM.

Ebenso ergab sich kein signifikanter Unterschied im Ausmaß der Beibehaltung der Herkunftskultur zwischen den Eltern der Kinder mit SM $\left(M d_{t 1}=32.50, M d_{t 4}=37.00\right)$ und den Eltern der sprechenden Kinder $\left(M d_{t 1}=33.00, M d_{t 4}=24.00\right)$, weder zum ersten Messzeitpunkt, $U_{t 1}=16.50, z_{t 1}=-0.50, p_{t 1}=.670, r_{t 1}=-.13$ noch zum letzten Messzeitpunkt, $U_{t 4}=23.00, z_{t 4}=1.48, p_{t 4}=.177, r_{t 4}=.47$.

Dementsprechend unterschieden sich die Eltern der schweigenden Kinder auch nicht hinsichtlich des Akkulturationsindexes $\left(M d_{t 1}=77.00, M d_{t 4}=71.00\right)$ von den Eltern der sprechenden Kinder $\left(M d_{t 1}=76.00, M d_{t 4}=81.00\right)$, weder zum ersten Messzeitpunkt, $U_{t 1}=21.00$, $z_{t l}=0.14, p_{t l}=1.000, r_{t 1}=.04$, noch zum letzten Messzeitpunkt, $U_{t 4}=10.00, z_{t 4}=-0.92$, $p_{t 4}=.429, r_{t 4}=-.28$.

H8: Die Sprachkompetenzen des Kindes im Deutschen hängen positiv mit dem Akkulturationslevel der Eltern zusammen. Je mehr die Eltern an die Umgebungskultur angepasst sind, desto besser beherrschen die Kinder die Umgebungssprache.

Zum ersten Messzeitpunkt zeigten sich keine signifikanten Zusammenhänge zwischen den Sprachkompetenzen der Kinder und der Akkulturation der Eltern (siehe Tabelle 35). Jedoch hing die MLU der Kinder zu T4 signifikant negativ mit der Orientierung der Eltern an der Herkunftskultur zu T1 zusammen, $\tau=-.535, p=.046$. Je geringer die Eltern an der Herkunftskultur orientiert waren, desto höher war die MLU der Kinder zum vierten Messzeitpunkt. Der signifikant negative Zusammenhang zeichnete sich ebenso zwischen der MLU 
gemessen am vierten Messzeitpunkt und der Orientierung der Eltern an ihrer Herkunftskultur $\mathrm{ab}$, erreichte jedoch kein statistisch bedeutsames Signifikanzniveau, $\tau=-.400, p=.140$. Für die weiteren Sprachvariablen ergaben sich keine signifikanten Zusammenhänge mit der kulturellen Adaption der Eltern (siehe Tabelle 36).

Tabelle 35

Korrelationen zwischen den Sprachvariablen zu T1 und T4 und der kulturellen Adaptation der Eltern zum vierten Messzeitpunkt

\begin{tabular}{cccc}
\hline & $\begin{array}{c}\text { Orientierung an Her- } \\
\text { kunftskultur }\end{array}$ & $\begin{array}{c}\text { Orientierung an } \\
\text { Aufnahmekultur }\end{array}$ & $\begin{array}{c}\text { Akkulturations- } \\
\text { index }\end{array}$ \\
\hline TROG-D $(\mathrm{n}=12)$ & -.032 & .262 & .222 \\
& {$[-.509, .446]$} & {$[-.205, .656]$} & {$[-.252, .685]$} \\
MLU $(\mathrm{n}=8)$ & -.255 & -.192 & -.109 \\
& {$[-.935, .500]$} & {$[-.810, .565]$} & {$[-.764, .653]$} \\
& -.079 & .277 & .117 \\
& {$[-.731, .573]$} & {$[-.323, .767]$} & {$[-.525, .796]$} \\
& $-.535^{*}$ & .319 & .366 \\
& {$[-.967, .000]$} & {$[-.476, .935]$} & {$[-.267, .960]$} \\
\hline
\end{tabular}

Anmerkungen. ${ }^{*} p<.05,{ }^{* *} p<.01, * * * p<.001,95 \%$ Konfidenzintervall in eckigen Klammern

Tabelle 36

Korrelationen zwischen den Sprachvariablen und der kulturellen Adaptation der Eltern zum vierten Messzeitpunkt

\begin{tabular}{lccc}
\hline & $\begin{array}{c}\text { Orientierung an Her- } \\
\text { kunftskultur }\end{array}$ & $\begin{array}{c}\text { Orientierung an } \\
\text { Aufnahmekultur }\end{array}$ & $\begin{array}{c}\text { Akkulturations- } \\
\text { index }\end{array}$ \\
\hline TROG-D $(\mathrm{n}=10)$ & -.386 & -.094 & .188 \\
MLU $(\mathrm{n}=8)$ & {$[-.880, .299]$} & {$[-.771, .632]$} & {$[-.423, .716]$} \\
& -.400 & .000 & .286 \\
& {$[-.857, .131]$} & {$[-.677, .615]$} & {$[-.339, .857]$} \\
\hline
\end{tabular}

Anmerkungen. $* p<.05, * * p<.01, * * * p<.001,95 \%$ Konfidenzintervall in eckigen Klammern 


\subsubsection{Mehrebenenmodell - Outcome Elternfragebogen}

Mithilfe der Mehrebenenmodelle wurde analysiert, welche Variablen das Ausmaß des Schweigens bei den Kindern in den einzelnen Kontexten am besten vorhersagen können. Konkret wurden dabei H1 und H5 überprüft.

H1: Das Ausmaß der Angst ist der stärkste Prädiktor für die Entwicklung eines SM.

H5: Neben dem Ausmaß der Angst sind die Sprachkompetenzen des Kindes in der Umgebungssprache ein bedeutsamer Prädiktor für die Entwicklung des Sprechverhaltens. Schwache Sprachkompetenzen im Deutschen stehen mit einem höheren Ausmaß des Schweigens in Verbindung.

Für die Modellbildung war es zunächst wichtig, solche Probanden auszuschließen, bei denen auf Prädiktorebene fehlende Werte vorlagen. Die meisten fehlenden Werte lagen in den Variablen der Spontansprachaufnahmen vor, da diese Aufgabe von den Eltern zuhause aus den unterschiedlichsten Gründen nicht durchgeführt werden konnte. Deswegen wurden zunächst Modelle für Probanden mit vollständigen Informationen zur Gesamtangst und zum Grammatikverständnis zum ersten Messzeitpunkt spezifiziert und erst im zweiten Schritt die sprachproduktiven Fähigkeiten miteinbezogen Fünf Kinder mussten aus den Analysen ausgeschlossen werden, so dass eine Gesamtstichprobe von 25 Kindern den Analysen zugrundlagen. Im Folgenden werden zunächst die Modelle mit der abhängigen Variable Summenwert auf der Skala Schweigen im Elternfragebogen vorgestellt.

\section{Tabelle 37}

Fit-Maße der angepassten Modelle mit dem Summenwert auf der Subskala Schweigen des Elternfragebogens als Outcomevariable

\begin{tabular}{|c|c|c|c|c|c|}
\hline \multicolumn{2}{|c|}{ Modell } & \multirow{2}{*}{$\begin{array}{c}A I C \\
624.81\end{array}$} & \multirow{2}{*}{$\begin{array}{c}B I C \\
632.21\end{array}$} & \multirow{2}{*}{$\begin{array}{c}\text { Devianz } \\
618.81\end{array}$} & \multirow{2}{*}{$\begin{array}{c}d f \\
3\end{array}$} \\
\hline 1.1 & Basismodell & & & & \\
\hline 1.2 & Random Intercept Level 1 & 621.59 & 631.46 & 613.59 & 4 \\
\hline 1.3 & Random Intercept und Random Slope Level 1 & 623.56 & 635.89 & 613.56 & 5 \\
\hline 1.4 & Random Intercept mit Prädiktoren auf Level 2 & 599.62 & 619.35 & 583.62 & 7 \\
\hline \multirow[t]{2}{*}{1.5} & Random Intercept mit Interaktionseffekten & 600.95 & 628.08 & 578.95 & 11 \\
\hline & MS*KK, MS*Angst und MS*GV & & & & \\
\hline \multirow[t]{2}{*}{1.6} & Random Intercept mit Interaktionseffekt & 601.46 & 623.65 & 583.46 & 8 \\
\hline & Angst*GV & & & & \\
\hline
\end{tabular}

Anmerkungen . MS = Mehrsprachigkeit, KK = Kulturelles Kapital, GV = Grammatikverständnis 
In einem ersten Schritt wurde das Basismodell berechnet, welches lediglich den Mittelwert der Outcomevariable als besten Vorhersagewert annahm. Dies diente der Beurteilung der Passung aller weiteren Modelle sowie der Berechnung von $R^{2}$.

Für das erste Mehrebenenmodell wurde zunächst ein Random-Intercept-Modell mit dem Messzeitpunkt als erklärende Variable berechnet. Der Einbezug der Zeit als Prädiktor für die Entwicklung des Schweigens ergab eine signifikante Verbesserung des Modell-Fits, $X^{2}(1)=5.22, p<.05$. Dies zeigte sich ebenso in den deutlich kleineren Werten des AIC und BIC (siehe Tabelle 37). Demnach bestand eine deutliche Varianz im Zusammenhang zwischen Messzeitpunkt und dem Schweigen über die Probanden hinweg. Die Zeit hatte einen signifikant negativen Effekt auf das Ausmaß des Schweigens, $b=-1.17, t(62.11)=-2.34$, $p=.023$. Dies bedeutet, dass von einem Messzeitpunkt zum anderen der Summenwert auf der Skala Schweigen des Elternfragebogens um durchschnittlich 1.17 Punkte abnahm-das Schweigen verringerte sich über die Zeit hinweg. In einem nächsten Schritt wurde analysiert, ob es eine deutliche Varianz in den Entwicklungsverläufen der Probanden gab, d.h. ob sich die Steigungen der Entwicklungsgerade unterschieden. Dazu wurde ein Random Intercept und Random Slope Modell mit dem Messzeitpunkt als erklärende Variable berechnet. Dies ergab keine Verbesserung des Modell-Fits, $X^{2}(1)=0.03, p>.05$. Die Steigungen variierten demnach nicht zwischen den Probanden. In den folgenden Modellen wurde somit immer ein Random Intercept Modell angenommen. Cross-Level-Interaktionen zwischen den Prädiktoren und der Zeit waren damit ausgeschlossen.

In einem nächsten Schritt sollte geklärt werden, welche Variablen auf Level 2 die Varianz in den Intercepts erklären konnten. Dazu wurden die Variablen Mehrsprachigkeit (dummykodiert 0 = einsprachig, 1 = mehrsprachig), Gesamtangst und Grammatikverständnis jeweils zum ersten Messzeitpunkt in das Modell aufgenommen. Zusätzlich wurde die Variable kulturelles Kapital in das Modell einbezogen, um die Effekte dieser auf die abhängige Variable zu kontrollieren (siehe dazu auch Kapitel 4.1.3). Die Aufnahme der Prädiktoren führte zu einer signifikanten Verbesserung des Modell-Fits im Vergleich zu Modell 2, $X^{2}(3)=29.94$, $p<.01$. Die Zeit hatte weiterhin einen signifikant negativen Effekt auf das Ausmaß des Schweigens der Kinder, $b=-1.19, t(62.90)=-2.38, p=.020$. Weiterhin hatte die Gesamtangst einen hochsignifikant positiven Effekt auf das Schweigen der Kinder, $b=.69, t(25.08)=6.36$ $p<.001$. Pro T-Wert-Punkt mehr stieg der Summenwert auf der Skala Schweigen des Elternfragebogens um durchschnittlich 0.69 Punkte. Für Mehrsprachigkeit, kulturelles Kapital und Grammatikverständnis zeigten sich keine signifikanten Haupteffekte (siehe Tabelle 38). 
Folgend wurden mögliche Interaktionseffekte auf Level 2 analysiert. Zunächst wurden Interaktionseffekte zwischen Mehrsprachigkeit und den restlichen Prädiktoren auf Level 2 hinzugefügt. Diesen Interaktionseffekten lagen die Hypothesen zugrunde, dass Angst und Sprachverstehen bei mehrsprachigen Kindern einen anderen Einfluss auf die Entwicklung des Schweigens nehmen als bei einsprachigen Kindern. Im Vergleich zum vorherigen RandomIntercept-Modell (Modell 1.4) unterschied sich der Fit dieses Modells nicht signifikant, $X^{2}(4)=4.67, p>.05$.

Als letztes wurde überprüft, ob ein Interaktionseffekt zwischen Angst und Grammatikverständnis bestand. Durch Hinzunahme des Interaktionseffektes zwischen Angst und Grammatikverständnis ergab sich keine Verbesserung des Modell-Fits, $X^{2}(1)=0.16, p>.05$. Es war kein Interaktionseffekt zwischen den beiden Variablen nachweisbar, $b=0.00$, $t(25.95)=0.40, p=.693$. Eine Übersicht über die Modellparameter aller geschätzten Modelle findet sich in den Tabellen A-14 und A-15 im Anhang.

Modell 1.4 zeigte demnach den besten Modell-Fit. Die Zeit hatte einen signifikant negativen Effekt auf die Entwicklung des Schweigens. Über die Messzeitpunkte hinweg nahm das Schweigen der Kinder ab. Die Varianz der Intercepts konnte am besten durch die Gesamtangst erklärt werden. Kinder mit hohen Werten im BAV zeigten demnach zu Beginn der Studie bereits ein höheres Maß der Ängstlichkeit. Insgesamt 69 \% der Varianz konnten durch dieses Modell auf Probandenebene aufgeklärt werden.

Tabelle 38

Modellparameter des endgültigen Modells 1.4 zum Einfluss von Mehrsprachigkeit, Gesamtangst und Grammatikverständnis auf das Schweigen der Kinder in familiären und öffentlichen Kontexten

\begin{tabular}{lccccc}
\hline & $b$ & $S E$ & $d f$ & $t$ & $p$ \\
\hline Konstanter Term & 24.73 & 2.27 & 31.42 & 10.89 & .000 \\
Zeit & -1.19 & 0.50 & 62.90 & -2.38 & .020 \\
Kulturelles Kapital & -1.83 & 1.42 & 25.07 & -1.29 & .210 \\
Mehrsprachigkeit (dummykodiert) & -0.74 & 3.48 & 25.85 & -0.21 & .832 \\
Gesamtangst (Median zentriert) & 0.69 & 0.11 & 25.08 & 6.36 & .000 \\
Grammatikverständnis (Median & 0.01 & 0.15 & 25.63 & 0.04 & .967 \\
zentriert) & & & & & \\
\hline
\end{tabular}
Approximatives $R^{2} \quad$ Level 1 $.62 \quad$ Level 2 .69

Wie bereits oben erwähnt, lagen nur für eine kleinere Teilstichprobe die Spontansprachaufnahmen zum ersten Messzeitpunkt vor. Um den Einfluss der produktiven Sprach- 
kompetenzen auf das Ausmaß des Schweigens $\mathrm{zu}$ untersuchen, wurden vergleichbare Mehrebenenmodelle mit den insgesamt 20 Probanden (8 mehrsprachige, 12 einsprachige Kinder) gerechnet, für die gültige Werte sowohl für den Angstfragebogen als auch die Spontansprachaufnahmen vorlagen. Der Anteil der mehrsprachigen Kinder war in dieser Teilstichprobe deutlich reduziert, da es für viele Familien nicht möglich war, Aufnahmen auf Deutsch im häuslichen Bereich anzufertigen.

Tabelle 39

Fit-Maße der angepassten Modelle für den Einfluss der produktiven Sprachkompetenzen auf das Schweigen der Kinder in familiären und öffentlichen Kontexten

\begin{tabular}{llcccc}
\hline Modell & AIC & BIC & Devianz & $d f$ \\
\hline 2.1 & Basismodell & 506.78 & 513.61 & 500.78 & 3 \\
2.2 & Random Intercept Level 1 & 508.23 & 517.34 & 500.23 & 4 \\
2.3 & Random Intercept mit Prädiktoren auf Level 2 & 486.25 & 504.46 & 470.25 & 8 \\
\hline
\end{tabular}

Für diese Teilstichprobe zeigte sich kein signifikanter Effekt der Zeit, was sich bereits bei der Anpassung des Random Intercept Modells mit dem Messzeitpunkt als einzigen Prädiktor zeigte. Durch Hinzunahme des Messzeitpunktes ergab sich keine Verbesserung des Modellfits im Vergleich zum Basismodell, $X^{2}(1)=0.55, p>.05$. Durch das Hinzufügen der Prädiktoren Gesamtangst und Sprachkompetenz auf Level 2 ergab sich eine signifikante Verbesserung des Modellfits im Vergleich zum Basismodell, $X^{2}(5)=30.53, p<.01$, wie auch zu Modell 2, $X^{2}(4)=29.98, p<.01$. Tabelle 40 gibt einen Überblick über die Modellparameter.

Der negative Effekte der Zeit, welcher in der Gesamtstichprobe gefunden wurde, zeigte sich nicht derart ausgeprägt, $b=-0.36, t(52.46)=-0.71, p=.481$. In dieser Teilstichprobe nahm das Schweigen der Kinder nur unbedeutsam über die Messzeitpunkt hinweg ab. Das kulturelle Kapital hatte einen statistisch tendenziellen negativen Effekt auf das Schweigen der Kinder, $b=-2.28, t(20.18)=-1.84, p=.080$. Je höher das kulturelle Kapital der Familie war, desto weniger schwiegen demnach die Kinder. Der positive Effekt der Gesamtangst auf das Ausmaß des Schweigens blieb hingegen unverändert stark, $b=0.76, t(19.94)=7.00, p<.001$. Auch in dieser Teilstichprobe galt: je größer die Angst zum ersten Messzeitpunkt war, desto mehr schwiegen die Kinder in familiären und öffentlichen Kontexten. Der Faktor Mehrsprachigkeit hatte auch hier wiederum keinen statistisch bedeutsamen Effekt, $b=1.61$, $t(20.41)=0.48, p=.635$. Ähnliches zeigte sich für die produktiven Sprachkompetenzen, $b=0-.69, t(19.95)=-0.38, p=.710$. Somit ließ sich weder für das Grammatikverständnis (siehe Modell 1.4) noch für die MLU (siehe Tabelle 40) ein Effekt auf das Ausmaß des 
Schweigens nachweisen. Die Varianzaufklärung für Modell 2.3 war mit $72 \%$ auf Level 1 und $78 \%$ auf Level 2 etwas höher als die von Modell 1.4.

Tabelle 40

Modellparameter für das Mehrebenenmodell 2.3 mit dem Summenwert auf der Subskala Schweigen des Elternfragebogens als Outcomevariable

\begin{tabular}{|c|c|c|c|c|c|}
\hline & $b$ & $S E$ & $d f$ & $t$ & $p$ \\
\hline Konstanter Term & 31.14 & 7.90 & 20.35 & 4.39 & .000 \\
\hline Zeit & -0.36 & .50 & 52.46 & -0.71 & .481 \\
\hline Kulturelles Kapital & -2.28 & 1.24 & 20.18 & -1.84 & .080 \\
\hline Mehrsprachigkeit (dummykodiert) & 1.61 & 3.33 & 20.41 & .482 & .635 \\
\hline Gesamtangst (Median zentriert) & 0.76 & 0.11 & 19.94 & 7.00 & .000 \\
\hline MLU & -0.69 & 1.84 & 19.95 & -0.38 & .710 \\
\hline Approximatives $R^{2}$ & Level 1 & .72 & & Level 2 & .78 \\
\hline
\end{tabular}

In einem letzten Schritt wurde die Stichprobe nochmals zugunsten des Aspektes Mehrsprachigkeit reduziert, um den Einfluss der Akkulturation auf das Ausmaß des Schweigens zu untersuchen. Hier wurde jedoch als Variable zur Operationalisierung der Sprachkompetenz das Grammatikverständnis gewählt, um die Teilstichprobe nicht massiv zu reduzieren. Insgesamt 12 der 15 mehrsprachigen Kinder konnten in die Analyse einbezogen werden. Neben den bekannten Prädiktoren Gesamtangst und Grammatikverständnis wurde zusätzlich der Wert auf der Subskala Orientierung an der Aufnahmekultur des FRAKK mit aufgenommen, da letztere am deutlichsten mit den Einschätzungen von Eltern und Erzieherinnen zusammenhing (siehe Kapitel 4.1.3). Da sich sowohl zwischen dem kulturellen Kapital als auch dem sozioökonomischen Status ein Zusammenhang zur Akkulturation der Eltern zeigte (siehe Kapitel 4.1.2) wurden die Modelle für beide Kontrollvariablen einzeln berechnet. 
Tabelle 41

Fit-Maße der angepassten Modelle zum Einfluss der kulturellen Adaptation auf die Entwicklung des Schweigens

\begin{tabular}{llcccc}
\hline Modell & AIC & BIC & Devianz & $d f$ \\
\hline 3.1 & Basismodell & 285.71 & 290.62 & 279.71 & 3 \\
3.2 & Random Intercept Level 1 & 277.53 & 284.08 & 269.53 & 4 \\
3.3 & Random Intercept mit Prädiktoren auf Level 2 & 269.30 & 282.40 & 253.30 & 8 \\
& Kontrollvariable: Kulturelles Kapital & & & & \\
3.4 & Random Intercept mit Prädiktoren auf Level 2 & 269.85 & 282.95 & 253.85 & 8 \\
& Kontrollvariable: Schicht-Index & & & \\
\hline
\end{tabular}

Das einfache Random Intercept Modell mit dem Prädiktor Messzeitpunkt auf Level 1 fittete signifikant besser als das Basismodell, $X^{2}(1)=10.18, p<.01$, das komplexe Modell mit allen Prädiktoren auf Level 2 wiederum besser als Modell 3.2, Modell $3.3 X^{2}(4)=16.23$, $p<.01$ und Modell $3.4 X^{2}(4)=15.68, p<.01$. Zunächst werden die Ergebnisse bei Kontrolle des kulturellen Kapitals berichtet. In der Teilstichprobe der mehrsprachigen Kinder hatte der Messzeitpunkt einen signifikant negativen Effekt auf die Entwicklung des Schweigens, $b=-2.77, t(27.07)=-3.71, p=.001$. Pro Messzeitpunkt sank der Wert auf der Subskala Schweigen des Elternfragebogens bei den mehrsprachigen Kindern demnach um durchschnittlich 2.77 Punkte. Die Gesamtangst hatte weiterhin einen signifikant positiven Effekt, $b=.90, t(12.86)=3.32, p=.006$. Pro T-Wertpunkt mehr im BAV stieg der Wert im Elternfragebogen um durchschnittlich 0.90 Punkte. Das Grammatikverständnis hatte weiterhin keinen bedeutsamen Effekt, $b=.56, t(13.13)=1.28, p=.224$. Der neu hinzugefügte Prädiktor der Akkulturation der Eltern hatte keinen signifikant Effekt auf das Schweigen der Kinder, $b=-.71, t(12.52)=-1.35, p=.202$. Modell 3.3 konnte insgesamt $71 \%$ der Varianz auf Probandenlevel (Level 2) erklären (siehe Tabelle 42).

Bei Kontrolle des sozioökonomischen Status blieben die Effekte von Messzeitpunkt und Gesamtangst bestehen (siehe Tabelle 43). Einen signifikant negativen Effekt hatte die Orientierung der Eltern an der Aufnahmekultur auf die Entwicklung des Schweigens, $b=-.98, t(12.96)=-2.54, p=.025$. Modell 3.4 konnte insgesamt $70 \%$ der Varianz auf Probandenlevel erklären. 
Tabelle 42

Modellparameter für das Mehrebenemodell 3.3 mit dem Summenwert auf der Subskala Schweigen im

Elternfragebogen als Outcomevariable

\begin{tabular}{|c|c|c|c|c|c|}
\hline & $b$ & $S E$ & $d f$ & $t$ & $p$ \\
\hline Konstanter Term & 37.62 & 11.13 & 13.56 & 3.38 & .005 \\
\hline Zeit & -2.77 & .75 & 27.07 & -3.71 & .001 \\
\hline Kulturelles Kapital & -3.30 & 4.31 & 12.93 & -.77 & .458 \\
\hline Gesamtangst (Median zentriert) & .90 & .27 & 12.86 & 3.32 & .006 \\
\hline TROG-D (Median zentriert) & .56 & .44 & 13.13 & 1.28 & .224 \\
\hline FRAKK AK (Median zentriert) & -.71 & .53 & 12.52 & -1.35 & .202 \\
\hline Approximatives $R^{2}$ & Level 1 & .65 & & Level 2 & .71 \\
\hline
\end{tabular}

Tabelle 43

Modellparameter für das Mehrebenenmodell 3.4 mit dem Summenwert auf der Subskala Schweigen im Elternfragebogen als Outcomevariable

\begin{tabular}{|c|c|c|c|c|c|}
\hline & $b$ & $S E$ & $d f$ & $t$ & $p$ \\
\hline Konstanter Term & 28.64 & 5.87 & 14.31 & 4.88 & .000 \\
\hline Zeit & -2.75 & .75 & 27.01 & -3.68 & .001 \\
\hline Schicht-Index & .08 & .55 & 13.16 & .15 & .886 \\
\hline Gesamtangst (Median zentriert) & 1.04 & .20 & 13.96 & 5.20 & .000 \\
\hline TROG-D (Median zentriert) & .28 & .26 & 12.64 & 1.08 & .302 \\
\hline FRAKK AK (Median zentriert) & -.98 & .39 & 12.96 & -2.54 & .025 \\
\hline Approximatives $R^{2}$ & Level 1 & .64 & & Level 2 & .70 \\
\hline
\end{tabular}

\subsubsection{Mehrebenenmodell - Outcome DortMuS-Kita}

Im Folgenden werden die Ergebnisse der Mehrebenenmodellierung für die Ergebnisse der Erzieherfragebögen als Outcomevariable vorgestellt. Hier stand im Fokus, in welchem Ausmaß Sprachkompetenzen (H5) und Angst (H1) die Entwicklung des Schweigens im Kontext der Kita beeinflussen. Auch hier bildete eine Gruppe von 25 Kindern die Basis der Berechnungen, da für die übrigen fünf Kinder mindestens für eine der Prädiktorvariablen bzw. für die Kontrollvariable kulturelles Kapital keine Werte vorlagen. Auch hier wurden Modelle mit zunehmender Komplexität angepasst, um das am besten passende Modell zu ermitteln. 
Tabelle 44

Fit-Maße der angepassten Modelle mit dem Summenwert auf der Subskala Schweigen und Bedürfnisäußerung von DortMuS-Kita als Outcomevariable

\begin{tabular}{llcccc}
\hline Modell & AIC & BIC & Devianz & $d f$ \\
\hline 4.1 & Basismodell & 582.98 & 589.98 & 576.98 & 3 \\
4.2 & Random Intercept Level 1 & 563.36 & 572.68 & 555.36 & 4 \\
4.3 & Random Intercept und Random Slope Level 1 & 562.47 & 574.12 & 552.47 & 5 \\
4.4 & Random Intercept mit Prädiktoren Level 2 & 553.64 & 572.29 & 537.64 & 8 \\
4.5 & Random Intercept mit Prädiktoren und Inter- & 553.42 & 579.06 & 531.42 & 11 \\
& aktionseffekten MS*KK, MS*Angst und & & & & \\
& MS*GV & & & & \\
4.6 & Random Intercept mit Prädiktoren und Inter- & 555.56 & 576.54 & 537.56 & 9 \\
& aktionseffekten Angst* GV & & & \\
\hline
\end{tabular}

Anmerkungen. $\mathrm{MS}=$ Mehrsprachigkeit, $\mathrm{KK}=$ Kulturelles Kapital, GV = Grammatikverständnis

Im Vergleich zum leeren Modell fittete das einfachste Mehrebenenmodell mit Random Intercept und dem Messzeitpunkt als erklärende Variable auf Level 1 signifikant besser, $X^{2}(1)=21.62, p<.01$. Der Messzeitpunkt hatte in diesem Modell einen signifikant negativen Einfluss auf die Entwicklung des Schweigens, $b=-3.57, t(52.35)=-5.18, p<.001$. Demnach nahm das Schweigen der Kinder über die Messzeitpunkte hinweg ab. Als nächstes wurde überprüft, ob die Entwicklungsverläufe zwischen den Kindern variierten. Dazu wurde ein Random Intercept und Random Slope Modell mit dem Messzeitpunkt als erklärende Variable auf Level 1 angepasst. Durch die Hinzunahme des zufälligen Effekts verbesserte sich der Model-Fit nicht signifikant, $X^{2}(1)=2.89, p>.05$. Demnach wurde für alle weiteren Modelle das Random Intercept Modell zugrunde gelegt.

In einem nächsten Schritt wurden die Level 2 Prädiktoren Gesamtangst eingeschätzt durch die Eltern und das Grammatikverständnis sowie die Mehrsprachigkeit in das Modell eingefügt. Der Modell-Fit verbesserte sich signifikant im Vergleich zu Modell 4.2, $X^{2}(4)=17.72, p<.01$. Der Messzeitpunkt sagte weiterhin signifikant negativ die Entwicklung des Schweigens vorher, $b=-3.76, t(55.01)=-5.55, p<.001$. Das kulturelle Kapital hatte zusätzlich einen signifikant negativen Effekt auf das Schweigen der Kinder, $b=-3.35$, $t(24.93)=-2.15, p=.041$. Die Gesamtangst der Kinder hatte einen signifikant positiven Effekt auf das Schweigen, $b=0.33, t(25.48)=2.72, p=.011$. Pro T-Wert-Punkt stieg der Wert auf der Subskala Schweigen im Erzieherfragebogen um durchschnittlich 0.33 Punkte. Zusätzlich zur Gesamtangst zeigte sich ein statistisch tendenzieller Effekt des Grammatikverständnisses, wobei sich hier ein negativer Effekt zeigte, $b=-0.34, t(26.86)=-1.98, p=.058$. Die T- 
Werte des TROG-D werden jedoch genau entgegensetzt zu den T-Werten des BAV interpretiert. Hohe T-Werte spiegeln höhere Sprachkompetenzen im Deutschen wider, niedrige TWerte schwächere Kompetenzen. Somit lässt sich der negative Effekt des Grammatikverständnisses so interpretieren, dass höhere Sprachkompetenzen eher dazu führten, dass das Schweigen der Kinder im Kita-Kontext abnahm. Entsprechend führten niedrige Verstehensleistungen eher zu einer Beibehaltung bzw. Erhöhung des Schweigens. Ein Haupteffekt der Mehrsprachigkeit konnte ähnlich wie in den Modellen zum Elternfragebogen nicht nachgewiesen werden, $b=-4.52, t(26.96)=-1.17, p=.252$ (siehe Überblickt auch Tabelle 45).

Tabelle 45

Modellparameter des endgültigen Modells 4.4 zum Einfluss von Mehrsprachigkeit, Gesamtangst und Grammatikverständnis auf das Schweigen der Kinder in der Kita

\begin{tabular}{lccccc}
\hline & $b$ & $S E$ & $d f$ & $t$ & $p$ \\
\hline Konstanter Term & 31.47 & 4.27 & 27.78 & 7.37 & .000 \\
Zeit & -3.77 & .68 & 55.01 & -5.55 & .000 \\
Kulturelles Kapital & -3.35 & 1.56 & 24.93 & -2.15 & .041 \\
Mehrsprachigkeit (dummykodiert) & -4.52 & 3.86 & 26.96 & -1.17 & .252 \\
Gesamtangst (Median zentriert) & 0.33 & 0.12 & 25.48 & 2.72 & .011 \\
Grammatikverständnis (Median & -0.34 & 0.12 & 26.86 & -1.98 & .058 \\
zentriert) & & & & &
\end{tabular}

$\begin{array}{llll}\text { Approximatives } R^{2} & \text { Level } 1 & .46 & \text { Level } 2\end{array}$

.49

In einem nächsten Schritt wurde wiederum analysiert, ob sich Interaktionseffekte zwischen Mehrsprachigkeit und der Gesamtangst bzw. dem Grammatikverständnis ergaben. Der Einbezug der Interaktionseffekte ergab keine signifikante Verbesserung des Modells, $X^{2}(3)=6.22, p>.05$.

Als letztes wurde der Interaktionseffekt zwischen der Gesamtangst und dem Grammatikverständnis ins Modell aufgenommen. Im Vergleich zu Modell 4.4 (Random-Intercept mit Haupteffekten der Prädiktoren) verbesserte sich der Modell-Fit nicht, $X^{2}(1)=0.08, p>.05$. Aufgrund des besseren Modell-Fits wurde Modell 4.4 als endgültiges Modell angenommen. Insgesamt 49 \% der Varianz auf Probandenlevel (Level 2) konnten über dieses Modell erklärt werden. Eine Übersicht über alle angepassten Modelle finden sich in den Tabellen A-16 und A-17 im Anhang.

Wie auch für den Elternfragebogen wurde der Einfluss der produktiven Sprachkompetenzen anhand einer Teilstichprobe von 20 Probanden (8 mehrsprachige, 12 einsprachige Kinder) analysiert, da für diese Kinder Daten sowohl für die Gesamtangst als auch für die 
mittlere Äußerungslänge vorlagen. Es wurden wiederum drei Modelle—das Basismodell, das einfache Random Intercept Modell mit dem Messzeitpunkt als Prädiktor sowie das komplexe Modelle mit den Prädiktoren auf Level 2-berechnet. Tabelle 46 gibt einen Überblick über die Fit-Maße der Modelle.

Tabelle 46

Fit-Maße der angepassten Modelle zum Einfluss der produktiven Sprachkompetenzen auf die Entwicklung des Schweigens in der Kita

\begin{tabular}{llcccc}
\hline Modell & AIC & BIC & Devianz & $d f$ \\
\hline 5.1 & Basismodell & 458.12 & 464.35 & 452.12 & 3 \\
5.2 & Random Intercept Level 1 & 443.72 & 452.03 & 435.72 & 4 \\
5.3 & Random Intercept mit Prädiktoren auf Level 2 & 435.53 & 452.15 & 419.53 & 8 \\
\hline
\end{tabular}

Durch Hinzunahme der Prädiktoren verbesserte sich der Fit des Modells zunehmend. Die angepassten Modelle unterschieden sich jeweils signifikant von dem vorherigen Modell: 5.1 vs. $5.2 X^{2}(1)=16.40, p<.01,5.2$ vs. $5.3 X^{2}(4)=16.19, p<.01$. Eine Übersicht über die Modellparameter von Modell 5.3 findet sich in Tabelle 47. Der Messzeitpunkt hatte auch hier wiederum einen signifikant negativen Effekt auf die Entwicklung des Schweigens, $b=-3.70$, $t(41.85)=-4.72, p<.001$. Der positive Effekt der Gesamtangst auf das Schweigen der Kinder blieb auch in dieser Teilstichprobe bestehen, $b=.36, t(20.57)=2.69, p=.014$. Kein signifikanter Effekt zeigte sich für Mehrsprachigkeit, $b=4.98, t(21.40)=1.21, p=.240$, sowie für die produktiven Sprachkompetenzen, $b=0.27, t(22.43)=0.12, p=.908$. Insgesamt $53 \%$ der Varianz auf Probandenlevel (Level 2) konnten mit dem Modell erklärt werden.

Tabelle 47

Modellparameter für das Mehrebenenmodell 5.3 zum Einfluss der produktiven Sprachkompetenzen auf das Schweigen der Kinder in der Kita

\begin{tabular}{|c|c|c|c|c|c|}
\hline & $b$ & $S E$ & $d f$ & $t$ & $p$ \\
\hline Konstanter Term & 29.24 & 9.03 & 23.70 & 3.24 & .004 \\
\hline Zeit & -3.70 & 0.78 & 41.85 & -4.72 & .000 \\
\hline Kulturelles Kapital & -4.27 & 1.53 & 21.32 & -2.79 & .011 \\
\hline Mehrsprachigkeit (dummykodiert) & 4.98 & 4.12 & 21.40 & 1.21 & .240 \\
\hline Gesamtangst (Median zentriert) & 0.36 & 0.13 & 20.57 & 2.69 & .014 \\
\hline MLU & 0.27 & 2.32 & 22.43 & 0.12 & .908 \\
\hline Approximatives $R^{2}$ & Level 1 & .49 & & Level 2 & .53 \\
\hline
\end{tabular}


In einem letzten Schritt wurde wiederum die Stichprobe zugunsten der mehrsprachigen Kinder reduziert, um den Einfluss der kulturellen Adaptation der Eltern auf die Entwicklung des Schweigens im Kontext der Kita zu analysieren. Dazu stand wiederum die Teilstichprobe von 12 mehrsprachigen Kindern zur Verfügung. Tabelle 48 gibt einen Überblick über die Fit-Maße der berechneten Modelle.

\section{Tabelle 48}

Fit-Maße der angepassten Modelle zum Einfluss der kulturellen Adaptation der Eltern auf die Entwicklung des Schweigens der Kinder in der Kita

\begin{tabular}{llcccc}
\hline Modell & AIC & BIC & Devianz & df \\
\hline 6.1 & Basismodell & 269.27 & 273.85 & 263.27 & 3 \\
6.2 & Random Intercept Level 1 & 262.81 & 268.92 & 254.81 & 4 \\
6.3 & Random Intercept mit Prädiktoren auf Level 2 & 247.50 & 259.72 & 231.50 & 8 \\
& Kontrollvariable: Kulturelles Kapital & & & & \\
6.4 & Random Intercept mit Prädiktoren auf Level 2 & 249.58 & 261.79 & 233.58 & 8 \\
& Kontrollvariable: Schicht-Index & & & & \\
\hline
\end{tabular}

Auch hier verbesserte sich der Modell-Fit durch Hinzunahme der Prädiktoren auf Level 1, $X^{2}(1)=8.46, p<.01$, und Level 2, Modell 6.3: $X^{2}(4)=23.31, p<.01$ und Modell 6.4: $X^{2}(4)=21.23, p<.01$, wobei Modell 6.3 nicht vollständig konvergiert ist und die Ergebnisse dieses Modells mit Vorsicht zu interpretieren sind (siehe dafür Tabelle 49). Im Folgenden werden vor diesem Hintergrund lediglich die Ergebnisse des Modells mit dem sozioökonomischen Status als Kontrollvariable (Modell 6.4) ausführlich berichtet (siehe dazu auch Tabelle 50). Die Zeit hatte auch innerhalb der Teilstichprobe der mehrsprachigen Kinder einen signifikant negativen Effekt auf das Schweigen der Kinder, $b=-3.97, t(25.97)=-3.24, p=.003$. Der positive Effekt der Gesamtangst blieb in diesem Modell bestehen, $b=0.46$, $t(25.42)=2.55, p=.017$. Der signifikant negative Effekt des Grammatikverständnisses zeigte sich für die mehrsprachigen Kinder nicht, $b=-0.11, t(16.86)=-0.59, p=.562$. Vielmehr hatte die Orientierung der Eltern an der Aufnahmekultur einen signifikant negativen Effekt auf das Schweigen der Kinder, $b=-1.43, t(10.95)=-5.89, p<.001$. Der sozioökonomische Status hatte keinen signifikanten Effekt auf die Entwicklung des Schweigens der mehrsprachigen Kinder, $b=0.46, t(25.42)=0.98, p=.335$. Die Varianzaufklärung dieses Modells lag bei $92 \%$ auf Probandenlevel. 
Tabelle 49

Modellparameter für das Mehrebenenmodell 6.3 zum Einfluss der kulturellen Adaptation der Eltern auf das Schweigen der Kinder in der Kita

\begin{tabular}{lccccc}
\hline & $b$ & $S E$ & $d f$ & $t$ & $p$ \\
\hline Konstanter Term & 36.67 & 7.85 & 34 & 4.67 & .000 \\
Zeit & -4.36 & 1.15 & 34 & -3.79 & .001 \\
Kulturelles Kapital & -5.45 & 3.04 & 34 & -1.79 & .082 \\
Gesamtangst (Median zentriert) & 0.31 & 0.18 & 34 & 1.69 & .100 \\
TROG-D (Median zentriert) & 0.30 & 0.30 & 34 & 1.00 & .326 \\
FRAKK AK (Median zentriert) & -0.95 & 0.37 & 34 & -2.56 & .015 \\
\hline
\end{tabular}
Approximatives $R^{2} \quad$ Level 1 Level 2

Tabelle 50

Modellparameter für das Mehrebenenmodell 6.4 zum Einfluss der kulturellen Adaptation der Eltern auf das Schweigen der Kinder in der Kita

\begin{tabular}{lccccc}
\hline & $b$ & $S E$ & $d f$ & $t$ & $p$ \\
\hline Konstanter Term & 19.39 & 5.03 & 28.16 & 3.86 & .001 \\
Zeit & -3.97 & 1.23 & 25.97 & -3.24 & .003 \\
Schicht-Index & 0.46 & 0.47 & 25.42 & 0.98 & .335 \\
Gesamtangst (Median zentriert) & 0.43 & 0.17 & 24.99 & 2.55 & .017 \\
TROG-D (Median zentriert) & -0.11 & 0.19 & 16.86 & -0.59 & .562 \\
FRAKK AK (Median zentriert) & -1.43 & 0.24 & 10.95 & -5.89 & .000 \\
\hline \multicolumn{1}{c}{ Approximatives $R^{2}$} & Level 1 & .95 & & Level 2 & .92 \\
\hline
\end{tabular}




\section{Diskussion}

Ziel der Studie war es, mögliche Einflussfaktoren auf die Entwicklung des SM bei mehrsprachigen Kindern zu untersuchen. Dabei standen die Ängstlichkeit, die Sprachkompetenzen im Deutschen und die kulturelle Adaptation der Eltern im Fokus. Durch die längsschnittliche Untersuchung sollten mögliche Risikofaktoren für die Entwicklung eines SM identifiziert werden. Die Analyse der Verlaufsdaten der Probanden konnte weder für den familiären noch für den Kita-Kontext einen signifikanten Effekt der Mehrsprachigkeit nachweisen. Demnach scheinen sich die Entwicklungsverläufe und -zusammenhänge der ein- und mehrsprachigen Kinder in dieser Stichprobe in Bezug auf Ängstlichkeit und Sprachkompetenzen nicht stark voneinander zu unterscheiden. Die Gesamtangst zeigte sich für beide Kontexte-familiär und institutionell—als bester Prädiktor für das Sprechverhalten der Kinder, wobei innerhalb des Kita-Kontextes zusätzlich tendenzielle Effekte des Sprachverständnisses auf das Ausmaß des Schweigens der Kinder sichtbar waren. Darüber hinaus zeigten sich für sozioökonomische Variablen wie den Schicht-Index und das kulturelle Kapital Effekte auf das Sprechverhalten aller, vor allem auf das der mehrsprachigen Kinder. Nachfolgend werden die zuvor dargestellten Ergebnisse im Hinblick auf die Hauptfragestellung und die Hypothesen diskutiert.

\subsection{Vorhersage der Entwicklung des Sprechverhaltens}

Sowohl für den familiären als auch den Kita-Kontext konnte die Gesamtangst, wie in Hypothese 1 erwartet, als stärkster Prädiktor für die Entwicklung des Sprechverhaltens der Kinder unabhängig einer Ein- oder Mehrsprachigkeit identifiziert werden. Ebenso konnte Hypothese 2 bestätigt werden: Kinder, die zum Ende der Studie die Diagnose SM zeigten, hatten bereits zu Beginn im Durchschnitt ein höheres Ausmaß der Gesamtangst im Vergleich zu Kindern, die sich zum Sprechen entwickelt haben, sowie zu den Kontrollkindern. Damit unterstützt diese Studie die breite Evidenz eines engen Zusammenhanges zwischen Angst und SM. Zahlreiche Studien haben bereits gezeigt, dass Kinder mit SM ängstlicher sind bzw. von Eltern und Lehrkräften als ängstlicher eingeschätzt werden als ihre altersparallelen Kontrollen (siehe etwa Cunningham et al., 2006; Cunningham, McHolm, Boyle \& Patel, 2004; Kristensen, 2000; Steinhausen \& Juzi, 1996). Black und Uhde (1995) konnten in ihrer Studie mit selektiv mutistischen Kindern einen engen positiven Zusammenhang zwischen der Einschätzung des Schweregrades des SM durch die Eltern und der allgemeinen Ängstlichkeit der Kinder nachweisen. Ähnlich hohe Zusammenhänge zwischen den Elterneinschätzungen und der 
Gesamtangst zeigten sich auch in der vorliegenden Studie zu allen vier Messzeitpunkten. Einen negativen Effekt hoher Ängstlichkeit auf den Schweregrad des SM konnten auch Manassis und Kollegen (2007) zeigen. In dieser Studie sagte die soziale Ängstlichkeit einen SM mit hohem Schweregrad am deutlichsten voraus. Die soziale Angst konnte in der vorliegenden Studie als Angstform aufgrund der unzureichenden Testgüte des BAV jedoch nicht einzeln betrachtet werden.

Der positive Zusammenhang zwischen dem Ausmaß der Angst und dem Ausmaß des Schweigens zeigte sich in der vorliegenden Studie vor allem in den Einschätzungen der Eltern. Über die Skala „Schweigen“ des Elternfragebogens wurde erfasst, in welchen familiären und öffentlichen Situationen und vor allem in Gegenwart welcher Personen das Kind schwieg. Hohe Werte auf dieser Subskala spiegelten ein großes Ausmaß des Schweigens innerhalb der Verwandtschaft, Nachbarschaft und im öffentlichen Raum wider. Für alle vier Messzeitpunkte konnte für diese Skala ein enger positiver Zusammenhang mit der Gesamtangst der Kinder zu T1 nachgewiesen werden. Je ängstlicher die Kinder waren, desto mehr schwiegen sie in den familiären und öffentlichen Situationen. Entsprechend war innerhalb des Mehrebenenmodells mit den Elterneinschätzungen als Outcomevariable die Gesamtangst der stärkste und einzige Prädiktor für die Entwicklung des Sprechverhaltens.

Ford et al. (1998) konnten in ihrer Studie zeigen, dass Interaktionen mit fremden Personen, soziale Veranstaltungen, neue Situationen und Familienfeiern neben dem Kontext Schule die Situationen sind, in denen das Schweigen am häufigsten auftritt. All diese Situationen - ausgenommen dem Kontext Schule- haben ein gemeinsames Anforderungsprofil hinsichtlich sprachlich-kommunikativer und sozial-emotionaler Fähigkeiten. Neue Situationen, Interaktionen mit unvertrauten Personen oder auch Verwandten, welche die Kinder nur selten sehen, erfordern Fähigkeiten auf der Schnittstelle der sprachlich-kommunikativen und sozialemotionalen Fähigkeiten. Einerseits werden sprachlich-kommunikative Fähigkeiten zum Aufbau von Interaktionen mit anderen Kindern oder zur Reaktion auf Gesprächsangebote von Erwachsenen benötigt. Hierbei sind nicht formal linguistische Kompetenzen im engeren Sinne gemeint, sondern vielmehr pragmatische Kompetenzen, die einen selbstbestimmten Dialog mit einem Gegenüber ermöglichen. Katz-Bernstein (2011) vermutet, dass Kindern mit SM eben jene Gesprächsregeln nicht angemessen zur Verfügung stehen und es ihnen dadurch nicht gelingt, die Fremdheit in unvertrauten Situationen über diese ritualisierten Gesprächsregeln und -floskeln zu überwinden. Andererseits sind Emotionsregulationsstrategien notwendig, um dem erhöhten Stresserleben in derart unvertrauten Situationen zu begegnen. Für Kinder mit SM gibt es Hinweise auf eine vermehrte Nutzung vermeidender Regulationsstrategien 
(Sharkey \& McNicholas, 2006). Moldan (2005) wie auch Bahr (2006) interpretieren das Schweigen selbst als Angstregulation. In ihrer Untersuchung der Emotionsregulationsstrategien von Kindern mit SM fand Hoke (2012) sowohl in den Selbstbeurteilungen der Kinder als auch in den Fremdbeurteilungen durch die Eltern bei jeweils etwa der Hälfte ein Überwiegen vermeidender Regulationsstrategien (z.B. Verhaltensdesorganisation). Ähnliches berichten Lange und Tröster (2014) für Kinder mit sozialer Ängstlichkeit. Auch hier zeigten Kinder mit der Diagnose soziale Ängstlichkeit im Vergleich zu Kindern ohne soziale Ängste signifikant häufiger maladaptive Regulationsstrategien (wie z.B. Rückzug). Für den in der vorliegenden Studie identifizierten deutlichen Einfluss der Gesamtangst der Kinder auf das Sprechverhalten in wenig vertrauten, öffentlichen und familiären Kontexten sind womöglich beide Aspektedie geringen Gesprächskompetenzen in Kombination mit vorwiegend vermeidenden Regulationsstrategien — verantwortlich. Dem Diathese-Stress-Modell (siehe Kapitel 2.4.4) folgend besteht bei Kindern mit SM eine Diathese in Form einer grundsätzlichen Schüchternheit sowie Disposition für (soziale) Ängstlichkeit. Schwach ausgeprägte Gesprächskompetenzen erhöhen die Vulnerabilität des Kindes zusätzlich. Die beschriebenen Interaktionssituationen mit wenig vertrauten Personen des öffentlichen Raumes oder der Verwandtschaft und Nachbarschaft bieten zahlreiche Möglichkeiten von Stressoren. Auf sprachlich-kommunikativer Ebene werden ebene jene Gesprächskompetenzen der Kinder angesprochen, über die sie nur wenig verfügen. Auf sozial-emotionaler Ebene stehen die Kinder vor der Herausforderung, mehr oder weniger eigenständig in Interaktion vor allem mit Erwachsenen zu gehen. Übersteigen die Anforderungen der Situation die Kompetenzen des Kindes, löst dies Stresserleben aus. Hier wiederum verfügen die Kinder tendenziell über ein geringes Repertoire von Regulationsstrategien und nutzen vermehrt problemvermeidendes Verhalten, wie Rückzug oder Verhaltensdesorganisation. Erfolgt keine Änderung innerhalb des Systems, kann sich langfristig das Schweigen — als anfänglich vermeidende Problemlösestrategie - in unvertrauten Situationen manifestieren.

Für die Erzieherfragebögen, welche das Schweigen im Kontext der Kita widerspiegeln, zeigten sich deutlich geringere Korrelationen zwischen der Gesamtangst und dem Sprechverhalten der Kinder. Hier waren statistisch bedeutsame Zusammenhänge nur für den vierten Messzeitpunkt nachweisbar. Dennoch konnte im Mehrebenenmodell die Gesamtangst wiederum als bester Prädiktor für die Entwicklung des Sprechverhaltens identifiziert werden. Zusätzlich beeinflussen das Grammatikverständnis des Kindes und das kulturelle Kapital der Familie die Entwicklung des Kindes in der Kita. Hypothese 5, in welcher neben der Angst auch die Sprachkompetenzen des Kindes in der Umgebungssprache als bedeutsamer Prädiktor 
für die Entwicklung des Sprechverhaltens vermutet wurde, wird somit zum Teil bestätigt. Der Effekt des Grammatikverstehens erreichte nur ein statistisch tendenzielles Niveau und war zudem nur für den Kita-Kontext nachweisbar. Für die MLU zeigte sich weder für familiäre und öffentliche noch für den Kita-Kontext ein bedeutsamer Effekt. Der Vergleich der Ergebnisse für Eltern- und Erzieherfragebogen deutet auf eine Kontextabhängigkeit im Zusammenhang von Prädiktoren und Sprechverhalten hin.

Für das Kernsymptom Schweigen ist die Kontextabhängigkeit bereits rein definitorisch gegeben, indem eine Diskrepanz zwischen Sprechen und Schweigen in unterschiedlichen Kontexten erwartet wird (American Psychiatric Association, 2013). Innerhalb der außerfamiliären Kontexte, in denen Kinder mit SM typischerweise schweigen, ergeben sich jedoch zusätzlich graduelle Abstufungen der Symptomatik. Wie bereits oben angeführt sind es vor allem unvertraute Kontexte sowie der schulische Bereich, in dem das Schweigen primär auftritt (Dummit et al., 1997; Ford et al., 1998). Die Schulsituation, wie sie in vielen USamerikanischen Studien zur Phänomenologie des SM betrachtet wurde (Bergman et al., 2002; Dummit et al., 1997; Ford et al., 1998), ist mit der Kita-Situation in Deutschland nur bedingt vergleichbar. Die Kita-Situation ist durch eine spielerische Atmosphäre und weniger durch formale Lernsituationen geprägt (Honig, 2012). Der geringere Zusammenhang zwischen der Gesamtangst und dem Sprechverhalten der Kinder in der Kita ist durch die deutlich höhere und vor allem mit der Zeit wachsenden Vertrautheit der Kinder in diesem Kontext zu erklären. Zurückhaltende Kinder zeigen vor allem in unvertrauten Kontexten Angst und Rückzugsverhalten. In vertrauten Situationen agieren sie hingegen vergleichbar zu nichtzurückhaltenden Kontrollkindern (Laptook et al., 2008). Angst dürfte vor allem in solchen Kontexten auftreten, die von den Eltern beurteilt wurden. Der Kita-Kontext wird für die Kinder zunehmend vertrauter, so dass die Angst hier nicht der alleinige Einflussfaktor für zurückhaltendes und schweigendes Verhalten in der Kita ist.

Strukturierte Spielangebote, Kleingruppenarbeit und freie Spielzeit sind feste Bestandteile des Alltags in den meisten Kitas. Dadurch erleben die Kinder eine Vielzahl verschiedener Anforderungen an ihre-für diese Studie besonders relevanten-sprachlichkommunikativen und sozial-emotionalen Fähigkeiten. Bedeutsam sind vor allem PeerInteraktionen, die einen Großteil des Alltages von Kindern in der Kita ausmachen, was sich auch in den Beobachtungsdaten der vorliegenden Studie widerspiegelte. Interaktionen mit Erwachsenen ergeben sich nur selten und sind häufig von Verhaltensregulationen geprägt (Albers, 2009). Inhalte von Peer-Interaktionen sind vor allem Interaktionen im Rahmen von Spielsituationen. Für das in der vorliegenden Studie betrachtete Alter zwischen etwa drei und 
fünf Jahren ist vor allem das Rollenspiel entwicklungstypisch von großer Bedeutung (Schneider \& Hasselhorn, 2012). Dabei schlüpfen Kinder in verschiedene Rollen. Sie ahmen beispielsweise alltägliche Situationen in Rollenspielen wie Mutter-Vater-Kind und Einkaufen nach oder versetzen sich in kreativen Fantasiespielen in Heldenfiguren. Darüber üben sie soziale Verhaltensweise oder erproben andere Positionen, die sie im Alltag vielleicht nicht erleben. Diese Art von Spiel erfordert soziale wie auch sprachliche Kompetenzen. Die Kinder müssen bereits zu kooperativen Handlungen fähig sein. Gemeinsam muss bestimmt werden, wer welche Rollen übernimmt. Ideen müssen ins Spiel eingebracht und Entwicklungen innerhalb der Spielhandlung miteinander verhandelt werden. Dreijährige Kinder nutzen vor allem Sprache, um innerhalb von Spielsituationen Dinge und Ereignisse zu beschreiben (Lyytinen, 1991). Für Kinder mit sprachlichen Auffälligkeiten gibt es Hinweise darauf, dass sie im Vergleich zur ihren normalentwickelten, gleichaltrigen Peers weniger Zeit im Rollenspiel mit anderen verbringen (Stich, 2010). Unklar ist hier jedoch, ob dies Folge der sprachlichen Schwierigkeiten oder Symptom des gleichen zugrundeliegenden Phänomens ist. Unberührt dessen sind sprachlich unsichere Kinder weniger attraktive Spielpartner und werden häufiger von ihren Altersgenossen zurückgewiesen (McCabe, 2005). Neben den pragmatischen Fähigkeiten zum Aufbau sozialer Interaktionen können demnach im Kita-Kontext auch formal linguistische Fähigkeiten eine bedeutsame Rolle für die Partizipation der Kinder spielen.

In der vorliegenden Studie sagten entsprechend bessere Leistungen im Grammatikverstehen ein niedrigeres Ausmaß des Schweigens voraus. Hypothese 5 konnte damit teilweise bestätigt werden. Für den Kita-Kontext zeigte sich das Grammatikverstehen neben dem Ausmaß der Angst als bedeutsamer Prädiktor für das Sprechverhalten der Kinder. Diesen positiven Effekt rezeptiver Sprachkompetenzen auf das Ausmaß des Sprechens konnten auch Manassis et al. (2007) in ihrer Studie nachweisen. Neben dem Alter und der sozialen Ängstlichkeit zeigte sich eben das Sprachverstehen in ihrer Studie als bedeutsamer Prädiktor für die Erklärung des Schweregrades eines SM. Aufbauend auf der grundlegenden Schüchternheit von Kindern mit SM, die zu geringem selbstinitiativem Verhalten der Kinder führt (Asendorpf \& Meier, 1993), können sprachliche Unsicherheiten das Rückzugsverhalten der Kinder zusätzlich verstärken — sowohl auf individueller Ebene des Kindes als auch auf interindividueller Ebene in Form von Zurückweisung durch Andere. Die Kombination von Schüchternheit, sozialer Ängstlichkeit und sprachlichen Unsicherheiten ist für einen vergleichsweise großen Teil der Kind mit SM charakterisierend, wie beispielsweise die Studie von Cohan et al. (2008) zeigte. Der Einfluss des kulturellen Kapitals (operationalisiert über die Anzahl der Bücher im Haushalt) unterstützt dieses Ergebnis zusätzlich. Für dieses ließ 
sich ein negativer Effekt auf das Ausmaß des Schweigens der Kinder nachweisen. Je höher das kulturelle Kapital der Familie war, desto weniger schwiegen die Kinder im Kontext der Kita. Wenngleich die Interaktionseffekte des kulturellen Kapitals mit den restlichen Prädiktoren aufgrund der kleinen Stichprobe nicht untersucht wurden, wird ein Zusammenwirken von kulturellem Kapital und Sprachverstehen vermutet. Sowohl das kulturelle Kapital als auch der Schicht-Index als Maß für den sozioökonomischen Status der Familie hingen statistisch tendenziell positiv mit den Leistungen im TROG-D zusammen. In der Anzahl der Bücher im Haushalt spiegeln sich der Bildungshintergrund der Eltern sowie das Interesse der Familie an Literatur wider. Je größer das Interesse der Eltern selbst an Büchern und je höher ihr Bildungsgrad ist, desto eher werden sie ihr Kind bereits früh an Bücher heranführen (Haak, Downer \& Reeve, 2012). Frühes, regelmäßiges Vorlesen und gemeinsames Bilderbuchbetrachten (in der englischsprachigen Fachliteratur als early home literacy bezeichnet) haben einen positiven Einfluss auf die Sprachkompetenzen von Kindern (Frijters, Barron \& Brunello, 2000; Haak et al., 2012; Schmitt, Simpson \& Friend, 2011, Sénéchal \& LeFevre, 2014, 2014). Early home literacy ist neben der mütterlichen Bildung, dem Einkommen der Familie und kognitiven Kompetenzen des Kindes bedeutsamer Prädiktor für die Sprachkompetenzen von Vorschulkindern (Haak et al., 2012). Ein indirekter Einfluss des kulturellen Kapitals über die Sprachkompetenzen der Kinder auf die Entwicklung des Schweigens lässt sich daher vermuten.

Wie bereits oben erwähnt zeigte sich kein bedeutsamer Effekt der sprachproduktiven Fähigkeiten im Deutschen (operationalisiert über die MLU) auf die Entwicklung des Sprechverhaltens der Kinder. Zum ersten als auch vierten Messzeitpunkt, zu welchem jeweils die produktiven Sprachkompetenzen der Kinder erfasst wurden, zeigten sich weder signifikante Unterschiede noch ein signifikanter Trend innerhalb der drei Gruppen selektiv mutistisch, zu T4 sprechend und Kontrollen. Ähnliches zeigte sich auch in der Studie von McInnes et al. (2004), die keine Unterschiede in der mittleren Länge von so genannten communication units $^{6}(\mathrm{C}$-unit) zwischen Kindern mit SM und Kindern mit sozialer Ängstlichkeit fanden. In ihrer Studie zeigten sich vor allem Unterschiede in der Anzahl der Wörter und Anzahl der Cunits der gesamten Erzählungen der Kinder. Aufgabe der Kinder war es eine Geschichte nachzuerzählen, so dass die Ausgangslage für alle Kinder gleich war. Hingegen hingen in der vorliegenden Studie die kindlichen Äußerungen in ihrer Länge und Komplexität nicht nur von

\footnotetext{
${ }^{6}$ Die von den Kindern produzierten Erzählungen wurden nach den SNAP-Richtlinien nach Strong (1998) ausgewertet. Als Kodiereinheiten werden die Transkripte in so genannte communication units (C-units) segmentiert. Diese bestehen mindestens aus einem Hauptsatz (mind. Subjekt und Prädikat) und können zusätzlich Attribute oder Nebensätze beinhalten.
} 
den zugrundliegenden kindlichen Fähigkeiten, sondern vor allem von der Interaktionssituation zwischen Elternteil und Kind ab. Diese waren trotz vorgegebener Spielsituation nicht über alle Kinder vergleichbar, so dass Unterschiede in der Gesamtanzahl der Äußerungen oder Wörter der Kinder innerhalb der Spontansprachaufnahmen nicht allein auf die kindlichen Fähigkeiten zurückgeführt werden können. Fraglich ist, inwieweit die hier berechnete MLU die tatsächlichen Fähigkeiten der Kinder widerspiegelte. Aufgrund der-hinsichtlich der Aufnahmelänge und der Anzahl der kindlichen Äußerungen—sehr heterogenen Aufnahmen, konnte für alle Kinder lediglich eine Gesamtzahl von 30 Äußerungen als Grundlage für die Berechnungen genutzt werden. Wie Gavin und Giles (1996) jedoch zeigen konnten, ist die MLU bei unterschiedlichen Samplegrößen recht anfällig. In ihrer Studie erreichten Berechnungen von unter 50 Äußerungen keine gute Reliabilität. Die fragliche Güte der MLUBerechnungen wird in der vorliegenden Studie durch die geringe Korrelation der MLU mit den Ergebnissen des TROG-D unterstützt.

Ergänzend zur fraglichen Reliabilität der MLU kommt hinzu, dass nur für Zweidrittel der Probanden Spontansprachaufnahmen vorlagen. Vor allem bei den mehrsprachigen Kindern konnten häufig keine Aufnahmen angefertigt werden. Für einen Teil der Kinder berichteten die Eltern, dass die Aufnahmen auf Deutsch noch gar nicht möglich waren, da das Kind die einzelnen Utensilien im Arztkoffer noch nicht benennen konnte. Bei anderen Familien waren nach Aussage der Eltern die Deutschkompetenzen der Eltern nicht ausreichend oder die Belastung der Familie zu hoch, so dass die Aufnahmen nicht angefertigt wurden. Demnach war bei einigen Kindern die fehlende Spontansprachaufnahme zusätzlich auch eine qualitative Information zu noch fehlenden Sprachkompetenzen im Deutschen, die jedoch in die statistischen Analysen nicht mit einbezogen werden konnte. Somit bleibt fraglich, inwieweit die in dieser Studie untersuchten sprachproduktiven Fähigkeiten im Deutschen die Entwicklung des Sprechverhaltens bei den hier untersuchten ein- und vor allem bei den mehrsprachigen Kindern erklären können. 


\subsection{Entwicklungsverläufe der Kinder mit SM}

\subsubsection{Entwicklung des Interaktions- und Sprechverhaltens}

Insgesamt war innerhalb des Untersuchungszeitraumes der Studie eine positive Entwicklung bei allen Kinder zu beobachten. Sowohl im Eltern- als auch im Erzieherfragebogen war ein Absinken der Werte auf den Subskalen „Schweigen“ zu verzeichnen. Mit steigendem Alter und längerem Verbleib in der Kita steigerten sich die sprachlich-kommunikativen Anteile aller Kinder. Für alle Messzeitpunkte konnte jedoch ein signifikanter Unterschied zwischen den drei Gruppen (Kinder mit SM, Kinder mit positiver Entwicklung zu T4 und Kontrollkinder) nachgewiesen werden. Die Kinder mit SM zeigten dabei jeweils die höchsten Werte in den Fragebögen und damit über alle Messzeitpunkte hinweg ein vergleichsweise hohes Ausmaß des Schweigens. Die Ergebnisse unterstützen bisherige Befunde zum graduellen Anstieg des Sprechens über die Zeit hinweg bei Kindern mit SM (Kolvin \& Fundudis, 1981; Manassis et al., 2007; Steinhausen \& Juzi, 1996). Einen vergleichbaren Alterseffekt konnten Manassis et al. (2007) in ihrer Querschnittsstudie finden. Hier hing das Sprechverhalten der Kinder signifikant positiv mit dem Alter der Kinder zusammen.

Dieser Effekt war in der vorliegenden Studie auch in den Beobachtungsdaten nachzuweisen. Über alle vier Messzeitpunkte hinweg zeigte sich ein deutlicher, positiver Trend im verbalen Interaktionsverhalten zwischen den drei Gruppen. Die Kontrollkinder interagierten maßgeblich verbal mit anderen Personen, vor allem mit Kindern, im Kita-Kontext. Die zu T4 sprechenden Kinder sprachen zu den ersten zwei Messzeitpunkten vergleichsweise wenig in der Kita. Ihre verbalen Anteile stiegen jedoch ab dem dritten Messzeitpunkt deutlich. Die Kinder mit SM zeigten zum ersten und zweiten Messzeitpunkt im Mittel keine verbalen Interaktionen in der Freispielsituation in der Kita und steigerten ihre verbalen Anteile nur minimal zum dritten und vierten Messzeitpunkt. Die Varianz innerhalb der Sprechanteile der selektiv mutistischen Kinder war dabei jedoch relativ hoch. Drei Kinder schwiegen komplett in der Kita und zeigten damit zu allen vier Messzeitpunkten eine starke Symptomatik des SM. Die vier weiteren Kinder mit SM begannen im Verlauf der Studie mit einzelnen vertrauten Personen (Bezugserzieherin oder Freundinnen) zu sprechen. Je nach beobachteter Situation konnten bei den Kindern mit SM hohe Sprechanteile dokumentiert werden, wenngleich sie in anderen Situationen in der Kita noch konsequent schwiegen. Die Heterogenität des Sprechverhaltens von Kindern mit SM ist bereits gut dokumentiert (Dummit et al., 1997; Ford et al., 1998; Steinhausen \& Juzi, 1996) und zudem charakterisierend für das Störungsbild. Unterschiede hinsichtlich der Personen, mit denen Kinder interagieren, zeigten sich lediglich für 
Kind-Kind-Interaktionen. Auch hier waren es wiederum die Kontrollkinder, welche die meisten Interaktionen mit anderen Kindern zeigten, gefolgt von den zu T4 sprechenden Kindern. Kinder mit SM interagierten zu allen vier Messzeitpunkten vergleichsweise wenig mit anderen Kindern. Für Interaktionen zwischen Kind und Erwachsenen ergaben sich keine signifikanten Unterschiede zwischen den Gruppen, wobei insgesamt, vergleichbar mit anderen Studien (siehe dazu auch Albers, 2009; Booren et al., 2012), nur wenig direkte Interaktionen zwischen erwachsenen Personen und dem Kind beobachtbar waren.

Wenngleich Unterschiede im Interaktionsverhalten der Kinder in der Kita nachweisbar waren, zeigten sich keine einheitlichen Zusammenhänge zwischen den Prädiktoren und den Variablen aus den Beobachtungen. Die Korrelationen schwankten deutlich hinsichtlich Richtung und Stärke für alle Variablen über alle vier Messzeitpunkte, so dass diese nicht in die komplexen Berechnungen mit einbezogen werden konnten. Fraglich bleibt hier, ob die Verhaltensbeobachtungen das tatsächliche Verhalten der Kinder im Kita-Kontext erfassen konnten. Die Daten spiegelten lediglich einen kurzen Ausschnitt des täglichen Verhaltens der Kinder wider, wohingegen Eltern- und Erziehereinschätzungen auf einem größeren Zeitraum beruhten. Eine mehrmalige Beobachtung der Kinder in unterschiedlichen Spielsituationen hätte die Reliabilität der Daten erhöhen können. Dies konnte im Rahmen der Studie jedoch aufgrund des hohen personellen und zeitlichen Aufwands nicht realisiert werden. Aus pragmatischen Gründen wechselten die Beobachterinnen zum Teil zwischen den Messzeitpunkten, so dass die Daten insgesamt auf einer recht hohen Beobachterzahl beruhten. Trotz intensiver Beobachterschulung sind die Ergebnisse hier womöglich durch eine hohe Subjektivität der einzelnen Personen beeinflusst und können nicht nur auf die Entwicklung des Kindes zurückgeführt werden.

\subsubsection{Anteil der Kinder mit SM}

Eine Grundannahme der Studie war, dass ein Teil der zum ersten Messzeitpunkt schweigenden Kinder sich zum Sprechen entwickelt und ein Teil schweigend bleibt. Über die Betrachtung der unterschiedlichen Entwicklungsverläufe sollten dann potenzielle Risikofaktoren für die Entwicklung eines SM identifiziert werden. Von den 18 anfangs schweigenden Kindern entwickelten sich 11 positiv. Sie zeigten zum vierten Messzeitpunkt keinen SM. Der Anteil der Kinder mit SM am Ende der Studie war mit 7 von 18 Kindern eher gering.

Zum ersten Messzeitpunkt konnte für 8 der 18 schweigenden Kinder die Diagnose SM nach DSM-IV Kriterien gestellt werden. Davon waren zwei Kinder einsprachig (S1 und S2) und sechs mehrsprachig (S8, S13, S14, S15, S17 und S18). Die einsprachigen Kinder erfüllten 
auch zum vierten Messzeitpunkt noch alle Kriterien für die Diagnose SM. Von den sechs mehrsprachigen Kindern mit SM zum ersten Messzeitpunkt haben sich zwei Kinder positiv zum Sprechen entwickelt. Sie sprachen am Ende der Studie sowohl im Kita-Kontext als auch in unvertrauten Kontexten im öffentlichen und familiären Raum. Bei einem Kind (S8) wurde im Zuge des Sprechbeginns ein Stottern offensichtlich, welches komorbid zu SM auftreten kann (siehe etwa Steinhausen \& Juzi, 1996) und je nach Schweregrad auch als Ausschlussdiagnose bei SM gilt (American Psychiatric Association, 2013). Auf den Spontansprachaufnahmen zu T1 war bei diesem Kind jedoch keine Stottersymptomatik hörbar, so dass das Schweigen des Kindes nicht durch ein massives Stottern erklärt werden konnte. Das zweite Kind (S17) sprach zu Beginn der Studie in der Kita bereits mit ausgewählten Kindern. Im familiären Umfeld haben sich zudem im Verlauf der Studie bedeutsame Veränderungen durch die Geburt von Zwillingen ergeben. Dieses Ereignis erforderte von den weiteren Geschwistern deutlich mehr Selbstständigkeit als zuvor, da beide Elternteile mit der Pflege der Zwillinge befasst waren. Die zusätzliche sprachtherapeutische Behandlung hat diese Entwicklung vermutlich befördert (siehe dazu auch Kapitel 5.2.1). Eine ähnlich positive Entwicklung hat auch das Geschwisterkind (S15) gezeigt, welche jedoch eine deutlich stärkere Symptomatik des SM zum ersten Messzeitpunkt aufwies. Es zeigte zum vierten Messzeitpunkt weiterhin einen SM, wobei seine Sprechanteile vor allem in der Kita deutlich gestiegen sind.

Die zum ersten Messzeitpunkt zehn schweigenden Kinder mit noch nicht gesicherter Diagnose entwickelten sich über den Untersuchungszeitraum positiv. Sie steigerten durchschnittlich ab dem dritten Messzeitpunkt ihre Sprechanteile in der Kita deutlich, waren zu T4 jedoch weiterhin zurückhaltender als die sprechenden Kontrollkinder. Für die fünf einsprachigen Kinder war dieser Entwicklungsverlauf zu erwarten. Sie zeigten bereits zum ersten Messzeitpunkt kein typisches Symptombild des SM. Im Kitakontext zeigten sie zu Beginn der Studie zwar relativ vergleichbare Verhaltensweisen und wurden von den Erzieherinnen ähnlich schweigsam und zurückhaltend eingeschätzt wie die Kinder mit Diagnose SM. Im unvertrauten Kontext in der Testsituation an der Universität waren sie jedoch vergleichsweise aufgeschlossen. Alle fünf Kinder sprachen mit der Testleiterin und nahmen aktiv an den Spielsituationen teil. Von den fünf mehrsprachigen Kindern mit unsicherer Diagnose entwickelte nur ein Kind (S6) einen SM, die weiteren vier Kinder entwickelten sich ebenso positiv. Für die Entwicklung der mehrsprachigen Kinder wurde erwartet, dass spätestens nach sechs Monaten die Kinder, welche lediglich eine im Zweitspracherwerb typische Phase des Schweigens (Tabors, 2008) durchlaufen, ihre Sprechanteile vor allem im Kitakontext deutlich steigern. Die Steigerung der Sprechanteile der Kinder ab dem dritten Messzeitpunkt lässt sich vor die- 
sem Hintergrund erklären. Die Kinder, die sich bis zum vierten Messzeitpunkt zwar positiv entwickelt haben, blieben jedoch zum Ende der Studie weiterhin vergleichsweise zurückhaltend. Sie sprachen und interagierten tendenziell weniger als die Kontrollkinder und zählten vermutlich zu den eher schüchternen Kindern. Das vorwiegende Schweigen der Kinder zu Beginn der Studie ist als Übergangsphänomen zu interpretieren. Einerseits kann der Kitaeintritt als kritisches Lebensereignis angesehen werden (Ahnert, 2013), auf welches vor allem schüchterne Kinder mit Rückzug und Schweigen reagieren können. Mit zunehmender Vertrautheit der Personen und sozialen Situationen nimmt auch das Rückzugsverhalten langsam ab (siehe dazu auch Kapitel 5.1 sowie Laptook et al., 2008). Für den Zweitspracherwerb ist eine Phase des Schweigens zudem typisch (Tabors, 2008), bevor die Kinder beginnen ihre erworbenen Kenntnisse in der neuen Sprache auch produktiv zu nutzen.

Bei den Kindern mit SM zu T4 fielen im Besonderen gleichbleibende und sogar sinkende Angstwerte innerhalb der neun Monate der Studie auf. Lediglich eins der sieben Kinder mit SM (S6) hat in diesem Zeitraum eine höhere Angst entwickelt. Dieses Kind unterschied sich nicht nur in der Veränderung der Angst, sondern auch in der Ausgangslage. S6 war eines der Kinder mit unsicherer Diagnose zum ersten Messzeitpunkt. Bei den weiteren sechs Kindern konnte schon zum ersten Messzeitpunkt die Diagnose SM gestellt werden. Sie begannen im Verlauf der Studie eine sprachtherapeutische Behandlung. Die gleichbleibenden bzw. sinkenden Angstwerte sind somit vor diesem Hintergrund $\mathrm{zu}$ interpretieren (siehe dazu auch Bergman, Gonzalez, Piacentini \& Keller, 2013; Oerbeck, Johansen, Lundahl \& Kristensen, 2012). Neben diesen beiden Aspekten zeigte S6 deutliche Unsicherheiten in ihren produktiven Sprachkompetenzen in der Umgebungssprache, was sich vor allem in einer nur langsam steigenden MLU zeigte. Für sie wird vermutet, dass die Kombination aus der Prädisposition für eine Ängstlichkeit und den sprachlichen Unsicherheiten in der Umgebungssprache zu einer Beibehaltung des Schweigens in sozialen Interaktionen geführt hat. Vermehrte Misserfolgserlebnisse in sozialen Situationen durch die genannten sprachlichen Unsicherheiten haben vermutlich zu einer Erhöhung der Angst geführt. Umgekehrt kann auch die erhöhte Angst dazu geführt haben, dass das Kind sich in sozialen Situationen eher zurückgehalten hat und seine sprachproduktiven Kompetenzen nur langsam verbessern konnte (Keller et al., 2013). Eine gegenseitige Beeinflussung beider Merkmale liegt demnach nahe.

Zur Kategorisierung der unterschiedlichen Entwicklungsverläufe der sieben Kinder mit SM zum vierten Messzeitpunkt wurden sie anhand ihrer individuellen Profile den Subgruppen nach Cohan et al. (2008) zugeordnet. Cohan et al. (2008) unterscheiden insgesamt drei Subgruppen: ängstlich, ängstlich mit begleitenden sprachlichen Auffälligkeiten sowie 
ängstlich leicht oppositionell. Aufgrund des Schwerpunktes der Studie im Bereich der Ängste sowie sprachlichen Kompetenzen konnte jedoch keine Aussage zu möglichen Unsicherheiten im Sozialverhalten der Kindergemacht werden. Eine Kategorisierung der an der Studie teilnehmenden Kinder mit SM erfolgte somit lediglich zu den Gruppen ängstlich und ängstlich mit begleitenden sprachlichen Auffälligkeiten. Die beiden einsprachigen Kinder mit SM (S1 und S2) wurden der Subgruppe der ängstlichen Kinder zugeordnet. Von den fünf mehrsprachigen Kindern mit SM zeigten drei (S6, S13 und S15) ein Profil der Subgruppe ängstlich mit begleitenden sprachlichen Auffälligkeiten, wobei letztere für die mehrsprachigen Kinder vielmehr als Unsicherheiten bezeichnet werden sollten. Für die Diagnose einer Spracherwerbsstörung oder anderen sprachlichen Auffälligkeiten lagen keine ausreichenden Daten vor. Die anderen beiden mehrsprachigen Kinder aus der Gruppe der Kinder mit SM ließen sich nicht ohne weiteres einer der Subgruppen zuordnen. Ein Kind (S14) zeichnete sich vor allem durch schwache Leistungen im Sprachverstehen bei jedoch durchschnittlich ausgeprägten sozialen Ängsten aus. Es ist anzunehmen, dass neben den sprachlichen Unsicherheiten andere Faktoren, wie die enge Bindung zur Mutter und ein eher überbehütendes Verhalten dieser sowie Komplikationen in der Schwangerschaft (Tod eines Zwillings), eine bedeutsame Rolle in der Entwicklung des SM gespielt haben. Das zweite nicht klar zu kategorisierende Kind (S18) unterschied sich in seinem Profil deutlich von den anderen Kindern mit SM. Es zeigte eine unterdurchschnittlich ausgeprägte Gesamtangst und zeichnete sich vor allem durch deutliche sprachliche Unsicherheiten im Deutschen sowie eine geringe Orientierung der Eltern an der Umgebungskultur aus. Letztere stieg im Verlauf der Studie, so dass das Akkulturationslevel der Eltern zum Ende der Studie vergleichbar mit dem der anderen Elternteile war. Für dieses Kind wird ein für mehrsprachige Kinder spezifischer Entwicklungsverlauf angenommen, in dem neben sprachlichen auch kulturelle Aspekte eine Rolle spielen (siehe dazu auch Kapitel 5.3.1). 


\subsection{Einfluss der Mehrsprachigkeit auf die Entwicklung eines SM}

Die Mehrsprachigkeit eines Kindes hatte keinen Effekt auf die Entwicklung des Sprechverhaltens, weder im familiären und öffentlichen noch im Kita-Kontext. Somit unterschieden sich die Entwicklungsverläufe der Kinder dieser Stichprobe nicht deutlich voneinander. Vielmehr schien das Ausmaß der Angst, ggf. in Kombination mit den Sprachkompetenzen des Kindes und dem sozioökonomischen Hintergrund, bedeutsam für die Entwicklung des Sprechverhaltens gewesen zu sein.

\subsubsection{Kulturelle Adaptation der Eltern}

Für die mehrsprachigen Kinder wurde erwartet, dass sich die Eltern der Kinder mit SM signifikant in ihrer kulturellen Adaptation von den Eltern der sprechenden Kinder unterscheiden. Hypothesen 6 und 7, in denen Unterschiede in der Orientierung der Eltern an der Herkunfts- bzw. Aufnahmekultur vermutetet wurden, müssen verworfen werden. Die Eltern der selektiv mutistischen Kinder zeigten keine niedrigere Anpassung an die Umgebungskultur bzw. stärkere Beibehaltung der Herkunftskultur als die Eltern der sprechenden Kinder. Vielmehr waren die rekrutierten Familien bereits stark an die Umgebungskultur angepasst und nur noch zu einem geringen Maße an ihrer Herkunftskultur orientiert. Es war keine große Varianz innerhalb der Stichprobe nachweisbar. Dies ist vermutlich auf einen Stichprobeneffekt zurückzuführen. Die Teilnahme an dieser Studie erforderte von den Eltern ein gewisses Maß an Deutschkompetenzen sowie eine gewisse Sicherheit in der deutschen Kultur, um innerhalb der Gespräche mit der Projektleiterin partizipieren und die Fragebögen ausfüllen zu können. Der relativ hohe Aufwand (etwa die mehrmaligen Messzeitpunkte, Ausfüllen von Fragebögen, Wahrnehmen von Terminen an der Universität) hat die Stichprobe womöglich schon im Vorfeld vorselektiert. Dies zeigte sich vor allem in der Rekrutierung von mehrsprachigen Kontrollkindern, die sich als sehr schwierig herausstellte. Mehrsprachige Familien mit einem schweigenden Kind waren aufgrund der gewissen Besorgnis der Eltern und den Beratungsmöglichkeiten, die mit einer solchen Studie einhergehen, vermutlich motivierter als mehrsprachige Familien mit sprechenden Kindern. Bei den mehrsprachig sprechenden Kontrollkindern war der größte Dropout zum vierten Messzeitpunkt zu verzeichnen. Im Vergleich zu den einsprachig sprechenden Kontrollen wies diese Gruppe zudem einen geringeren sozioökonomischen Status auf, welcher mit einem höheren Risiko für einen Dropout einhergeht (Cotter, Burke, Loeber \& Mutchka, 2005). Somit ist die vorliegende Stichprobe bereits hinsichtlich bestimmter Variablen, hier vor allem dem Akkulturationslevel, vorselektiert. Eltern mit einer geringen Orientierung an der Umgebungskultur, welche eher mit niedrigen Sprach- 
kompetenzen in der Umgebungssprache (Laroche, Kim, Hui \& Tomiuk, 1998) und einer gewissen Skepsis gegenüber der Umgebungskultur einhergeht (Miglietta \& Tartaglia, 2009), konnten über den Rekrutierungsprozess nicht gewonnen werden.

Trotz der geringen Varianz innerhalb der Akkulturationslevel der Eltern zeigte sich ein konstanter, negativer Zusammenhang zwischen der Orientierung an der Aufnahmekultur und dem Sprechverhalten der Kinder in der Kita. Dieser erreichte aufgrund der geringen Stichprobengröße nur zum zweiten und dritten Messzeitpunkt ein statistisch signifikantes Niveau. Für das Sprechverhalten in familiären und öffentlichen Kontexten war ein solcher Zusammenhang nicht nachweisbar. Dennoch zeigte sich im Mehrebenmodell bei Kontrolle des Schicht-Indexes ein signifikanter Effekt der Orientierung an der Aufnahmekultur auf die Entwicklung des Sprechverhaltens eingeschätzt über den Elternfragebogen. Bei Kontrolle des kulturellen Kapitals war dieser Effekt jedoch nicht nachweisbar. Dies lässt sich vor allem durch den positiven Zusammenhang zwischen dem kulturellen Kapital und der Orientierung an der Aufnahmekultur erklären. Das kulturelle Kapital spiegelt den soziokulturellen Hintergrund der Familie wider und damit unter anderem das Bildungsniveau der Eltern. Den positiven Zusammenhang zwischen dem Bildungsniveau einer Person und der Adaptation an die Umgebungskultur haben bereits zahlreiche Studien bestätigt (siehe etwa Berry, 1997; Suinn, Ahuna \& Khoo, 1992). Mit steigendem Bildungsniveau steigt auch die Anpassung an die Umgebungskultur. In der vorliegenden Studie hing das kulturelle Kapital negativ mit dem Sprechverhalten der Kinder über alle Messezeitpunkt hinweg zusammen - unabhängig einer Ein- oder Mehrsprachigkeit der Kinder. Je höher der soziokulturelle Hintergrund der Familie war, desto weniger schwiegen die Kinder. Ein großer Teil des Zusammenhangs zwischen der Orientierung an der Aufnahmekultur und der Entwicklung des Sprechverhaltens der Kinder in familiären und öffentlichen Kontexten resultiert womöglich aus diesem negativen Zusammenhang zwischen soziokulturellem Hintergrund der Familie und Sprechverhalten der Kinder.

Für den Kita-Kontext zeigte sich bereits in den Korrelationsberechnungen, trotz der kleinen Stichprobengröße, ein konstant negativer Zusammenhang zwischen der Anpassung der Eltern an die Umgebungskultur und dem Ausmaß des Schweigens der Kinder. Je mehr die Eltern an der Umgebungskultur orientiert waren, desto weniger schwiegen die Kinder im Kita-Kontext. Im Mehrebenenmodell war auch bei Kontrolle des kulturellen Kapitals ein Einfluss der Orientierung der Eltern an der Umgebungskultur sichtbar. Das Modell konvergierte jedoch nicht vollständig, so dass diese Ergebnisse nur mit Einschränkungen zu betrachten sind. Insgesamt reicht die Stichprobengröße von 12 mehrsprachigen Kindern mit vollständi- 
gen Datensätzen in den drei bedeutsamen Erklärungsvariablen Angst, Sprachkompetenzen und Akkulturation nicht aus, um die Hauptfragestellung der Studie zu beantworten. Dennoch weisen die Ergebnisse auf einen Einfluss der elterlichen Akkulturation hin, wie es im Vorfeld der Studie vermutet wurde. Ein hohes Maß der Akkulturation, wie es sich in den beiden Akkulturationsstrategien der Assimilation und Bikulturalität widerspiegelt, geht mit einem geringeren Risiko für internalisierende Auffälligkeiten (Calzada et al., 2009) und höheren sozialen (Pawliuk et al., 1996) sowie sprachlichen Kompetenzen (Oades-Sese \& Li, 2011) einher. Höhere soziale und sprachliche Kompetenzen wiederum wirken sich sowohl bei ein- als auch bei mehrsprachigen Kindern positiv auf die Peer-Interaktionen aus (Grünigen, KochenderferLadd, Perren \& Alsaker, 2012), was sich in einem erhöhten Sprechverhalten in der Kita ausdrücken sollte.

\subsubsection{Sprachkompetenzen im Deutschen}

Entgegen den Erwartungen ließ sich kein Zusammenhang zwischen der kulturellen Adaptation der Eltern und den rezeptiven Sprachkompetenzen der Kinder im Deutschen nachweisen. Ein signifikant negativer Zusammenhang der Orientierung an der Herkunftskultur zum ersten Messzeitpunkt zeigte sich jedoch mit den produktiven Sprachkompetenzen (operationalisiert über die MLU) zum vierten Messzeitpunkt. Dieser Zusammenhang zeichnete sich auch zwischen beiden Variablen gemessen zum vierten Messzeitpunkt ab, erreichte jedoch kein statistisches signifikantes Niveau. Hypothese 8, in der ein positiver Zusammenhang zwischen der Anpassung der Eltern an die Umgebungskultur und den Sprachkompetenzen der Kinder im Deutschen vermutet wurde, kann somit nicht bestätigt werden. Vielmehr weisen die Ergebnisse auf einen negativen Zusammenhang zwischen der Orientierung der Eltern an der Herkunftskultur und den produktiven Sprachkompetenzen der Kinder im Deutschen hin. Ähnliches zeigten bereits Oades-Sese und Li (2011). Eine geringe Anpassung an die Umgebungskultur war auch in ihrer Studie mit einer geringen Sprachkompetenz in der Umgebungssprache assoziiert. Wie in Kapitel 2.4.3 vermutet, sprechen Eltern mit einer starken Orientierung an der Herkunftskultur im häuslichen Kontext vor allem ihre Herkunftssprache. Der Input in einer Sprache beeinflusst die Sprachkompetenzen in der jeweiligen Sprache (Prevoo et al., 2014), so dass eine starke Orientierung an der Herkunftskultur eher mit geringeren Sprachkompetenzen im Deutschen einhergeht. Der nicht bestehende Zusammenhang zwischen den rezeptiven Sprachkompetenzen und der kulturellen Adaptation der Eltern lässt sich vor dem Hintergrund von Spracherwerbsprinzipien erklären. Rezeptive Fähigkeiten übersteigen zu jedem Zeitpunkt der Sprachentwicklung — auch im Zweitspracherwerb-die pro- 
duktiven Sprachkompetenzen (Niebuhr-Siebert \& Chilla, 2012) und hängen eng mit der Quantität und Qualität des sprachlichen Inputs zusammen (Bialystok et al., 2010; Duursma et al., 2007). Für den Zweitsprachlerner ist jedoch auch der eigene Output bedeutsam, um produktive Sprachkompetenzen zu verfeinern (Niebuhr-Siebert \& Baake, 2014). Über das eigene Sprechen und Ausprobieren der neuen Sprache erhalten Kinder ggf. ein korrektives Feedback durch kompetentere Sprecher und können so ihre sprachlichen Fähigkeiten verbessern. Dieser Argumentation entsprechend gibt es für schüchterne Kinder Hinweise auf niedrigere Sprachkompetenzen im Vergleich zu ihren nicht-schüchternen Peers (Crozier \& Badawood, 2009; Crozier \& Hostettler, 2003; Evans, 1996; Spere et al., 2004), wenn diese Kinder vor allem in weniger vertrauten Situationen einen deutlich geringeren Sprechanteil zeigen als nichtschüchterne Kinder (Asendorpf \& Meier, 1993). Evans (1996) fanden Unterschiede zwischen schüchternen und nicht-schüchternen Kindern in ihren produktiven Wortschatzleistungen, nicht jedoch in ihren rezeptiven Wortschatzleistungen. Auch bei Crozier und Hostettler (2003) wiesen schüchterne Kinder einen deutlich niedrigeren produktiven Wortschatz auf als nicht-schüchterne Kinder gleichen Alters. Spere et al. (2004) konnten für schüchterne Kinder signifikant schwächere Wortschatzleistungen sowohl auf rezeptiver als auch produktiver Ebene im Vergleich zu nicht-schüchternen Kinder nachweisen, wobei in ihrer Untersuchung Extremgruppen (untertesten und obersten 15\% einer Schüchternheitsskala) verglichen wurden. In der vorliegenden Studie gehörte der Hauptteil der mehrsprachigen Kinder zur Gruppe der schweigenden Kinder, bei denen von einer grundlegenden Zurückhaltung, vor allem im Kontext der Kita, ausgegangen werden kann. Vermutlich erhielten sie einen ausreichenden Input im Deutschen, so dass die kulturelle Adaptation der Eltern keinen deutlichen Einfluss auf die rezeptiven Sprachkompetenzen der Kinder hatte. Die Zurückhaltung in Interaktionen kann jedoch zu einem verlangsamten Erwerb der produktiven Sprachfähigkeiten führen. Sind die Eltern zusätzlich stark an der Herkunftskultur orientiert, erhalten die Kinder mit hoher Wahrscheinlichkeit auch im vertrauten familiären Kontext nur wenige Möglichkeiten ihre produktiven Kompetenzen in der Umgebungssprache zu trainieren. Die kulturelle Adaptation der Eltern nimmt somit einen deutlicheren Einfluss auf die produktiven als auf die rezeptiven Sprachkompetenzen der Kinder.

Aus methodischer Sicht ist eine weitere Erklärung für den Zusammenhang der kulturellen Adaptation der Eltern mit den produktiven Sprachkompetenzen der Kinder in der Art der Messung dieser zu sehen. Die Spontansprachaufnahmen, auf deren Grundlage die MLU berechnet wurde, erfolgten in Spielsituationen mit den Eltern. Die Äußerungen der Kinder hingen somit nicht nur von den Kompetenzen des Kindes, sondern auch von denen der Eltern 
ab. Eltern mit einer geringen Orientierung an der Umgebungskultur verfügen selbst über eher niedrige Sprachkompetenzen in der Umgebungssprache (Laroche et al., 1998), so dass bei einem Teil der Eltern der Studie ebenso sprachliche Unsicherheiten im Deutschen zu erwarten waren. Die vom Kind produzierten spontansprachlichen Äußerungen hängen jedoch maßgeblich von der Interaktionssituation und vor allem vom Input der Eltern ab (Demuth, 1996). Befinden sich die Eltern beispielsweise noch in einem Erwerbsstadium, in welchem eher starre Satzstrukturen genutzt werden, evoziert dies mit hoher Wahrscheinlich auch beim Kind entsprechend starre Satzstrukturen.

Die geringen Zusammenhänge zwischen der Orientierung der Eltern an der Umgebungskultur und den sowohl rezeptiven als auch produktiven Sprachkompetenzen der Kinder im Deutschen resultiert womöglich aus der deutlich geringeren Varianz der Werte auf dieser Subskala des FRAKK im Vergleich zu den Werten auf der Skala Orientierung an der Herkunftskultur. Wie bereits oben angeführt wies der Großteil der Eltern bereits eine recht starke Anpassung an die deutsche Kultur auf, so dass der Einfluss verschiedener Anpassungslevel auf die Sprachkompetenzen der Kinder nicht untersucht werden konnte.

\subsection{3 Ängstlichkeit}

Wie bereits oben angeführt erwies sich die Gesamtangst auch für die mehrsprachigen Kinder als bester Prädiktor für die Entwicklung des Sprechverhaltens, wobei dieser Effekt für den familiären und öffentlichen Kontext deutlich stärker war als für den Kita-Kontext (siehe dazu auch Kapitel 5.1). Aufbauend auf den Ergebnissen von Elizur und Perednik (2003) wurde in der vorliegenden Studie in Hypothese 3 vermutet, dass die mehrsprachigen Kinder mit SM zum ersten Messzeitpunkt signifikant höhere Werte im Angstfragebogen zeigen als die einsprachigen Kinder mit SM. Diese Hypothese ließ sich auf der Basis der vorliegenden Daten nicht untersuchen, da die Gruppe der einsprachigen Kinder mit SM aus lediglich zwei Probanden bestand. Beide zeigten zudem die in der Stichprobe höchsten Werte im Angstfragebogen. Ein Großteil der mehrsprachigen Kinder mit SM zeigten zum ersten Messzeitpunkt, ähnlich wie die Probanden der Interventionsstudie von Oerbeck et al. (2012), leicht erhöhte, jedoch noch altersangemessene Werte im Angstfragebogen. Ein Kind lag mit einem T-Wert von $62 \mathrm{im}$ leicht überdurchschnittlichen Bereich, ein weiteres mit einem T-Wert von $37 \mathrm{im}$ leicht unterdurchschnittlichen Bereich. Hinweise auf eine deutlich erhöhte Angst bei mehrsprachigen Kindern mit SM, wie es Elizur und Perednik (2003) in ihrer Studie nachweisen konnten, zeigte sich in der vorliegenden Studie nicht. Die Stichproben beider Studien unterscheiden sich jedoch grundsätzlich hinsichtlich soziökonomischer Merkmale. Die teilneh- 
menden Familien in der Studie von Elizur und Perednik (2003) wiesen einen recht hohen sozioökonomischen Status auf. Im Durchschnitt hatten die Eltern dort mindestens einen Highschool-Abschluss. Die mehrsprachigen Familien der vorliegenden Studie hatten dagegen im Durchschnitt einen eher niedrigen sozioökonomischen Status. Der Großteil der Eltern der mehrsprachigen Kinder mit SM hat einen Haupt- oder Realschulabschluss erworben. Höhere Bildungsabschlüsse gab es vor allem bei den einsprachigen Familien, im Besonderen bei den Eltern der Kontrollkinder. Das Bildungsniveau der Eltern, vor allem der Mutter, hat einen bedeutsamen Einfluss auf die Entwicklung sprachlicher Fähigkeiten (Haak et al., 2012; Raviv, Kessenich \& Morrison, 2004), nicht jedoch auf die Entwicklung übermäßiger Ängste (Wichstrøm, Belsky \& Berg-Nielsen, 2013). Somit wird für die vorliegende Stichprobe vermutet, dass im Gegensatz zu Elizur und Perednik (2003) sprachliche Aspekte neben der Angst eine bedeutsame Rolle gespielt haben. Die Kinder der Studie von Elizur und Perednik (2003) wurden womöglich aufgrund des eher hohen Bildungsniveaus ihrer Eltern zuhause deutlich stärker gefördert und konnten insgesamt auf ein besseres sprachliches Ausgangsniveau für den Erwerb der zweiten Sprache zurückgreifen. Für sie standen vermutlich die kulturellen Unterschiede im Fokus, welche Unsicherheiten in sozialen Situationen hervorrufen können (siehe dazu Kapitel 2.4.1) und aufbauend auf einer genetischen Prädisposition zu einer deutlich höheren sozialen Ängstlichkeit geführt haben als bei den einsprachigen Kindern. Letztlich geklärt werden kann dieser Punkt jedoch nicht, da die sprachlichen Kompetenzen der Kinder in der Umgebungssprache bei Elizur und Perednik (2003) nicht erfasst wurden.

In Hypothese 4 wurde zusätzlich vermutet, dass sich das Ausmaß der Angst bei mehrsprachigen Kindern mit SM vom ersten zum vierten Messzeitpunkt erhöht. Aufgrund von potenziell bestehenden sprachlichen Unsicherheiten sowie kulturellen Unterschieden wurde angenommen, dass mehrsprachige Kinder gehäuft Misserfolgserlebnisse sowie Verunsicherungen in sozialen Situationen erleben, die zu einer erhöhten Angst in sozialen Situationen führen. Dies konnte in der vorliegenden Studie jedoch nicht bestätigt werden. Lediglich bei einem der vier Kinder, für die für beide Messzeitpunkte Werte für die Gesamtangst vorlagen, konnte ein Anstieg der Angst beobachtet werden. Bei den drei weiteren Kindern war ein leichtes Absinken der Angst zu verzeichnen. Nach Kenntnis der Autorin liegen keine Studien zur Entwicklung der Angst bei Kindern mit SM unabhängig von einer Intervention vor. Für die vorliegende Studie wird vermutet, dass die Verringerung der Angst beim Großteil der Kinder auf pädagogische und therapeutische Einflüsse zurückzuführen ist. Alle Kitas wurden zu Beginn der Studie über das Symptombild des SM aufgeklärt sowie hinsichtlich grundlegender Strategien im Umgang mit schweigenden Kindern informiert. Dies hat womöglich zu 
einer erhöhten Sensibilität seitens der Erzieherinnen geführt. Potenziell Angst- und Stress auslösende Situationen wurden vermutlich frühzeitig erkannt und vermieden sowie den Kindern entsprechende Hilfestellungen angeboten, die ihnen den Umgang mit sozialen Situationen erleichtern. Zusätzlich erhielten die Kinder mit SM in den meisten Fällen sprachtherapeutische Unterstützung. Der Beginn der Therapie hing dabei vom Zeitpunkt der Diagnosestellung ab. Der Großteil der Kinder erhielt erst ab dem dritten oder vierten Messzeitpunkt eine sprachtherapeutische Behandlung. Die Interventionen wurden allesamt durch mit dem SpA kooperierenden Therapeutinnen durchgeführt, welche nach der Dortmunder MutismusTherapie (Subellok, Katz-Bernstein, Bahrfeck-Wichitill \& Starke, 2012) arbeiten. Diese enthält Anteile kognitiv-behavioraler Interventionen, welche sich bei Angststörungen und SM als effektiv gezeigt haben (Bennett et al., 2013; Bergman et al., 2013; Ishikawa, Okajima, Matsuoka \& Sakano, 2007; King, Heyne \& Ollendick, 2005; Oerbeck et al., 2012; Oerbeck, Stein, Pripp \& Kristensen, 2014). Für Angststörungen zeigen sich bereits bei zehn und weniger therapeutischen Sitzungen mittlere Therapieeffekte, wobei Interventionen mit einer Dauer von mehr als zehn Sitzungen effektiver sind (Ishikawa et al., 2007). Bei einer kognitivbehavioralen Intervention für vier- bis achtjährige Kinder mit SM war nach drei Monaten bereits eine Verringerung der sozialen Ängste der Kinder zu verzeichnen (Bergman et al., 2013). In ihrer Pilotstudie mit sieben drei- bis fünfjährigen Kindern konnten Oerbeck et al. (2012) keine deutlichen Unterschiede im Ausmaß der Angst der Kinder ein Jahr nach Beginn der Therapie nachweisen. Die Kinder zeigten jedoch bereits zu Therapiebeginn-ähnlich wie die mehrsprachigen Kinder mit SM in der vorliegenden Studie - nur leicht erhöhte, jedoch noch altersangemessene Ängste. Eine deutliche Verringerung der Angst war somit nicht zu erwarten. Für die Entwicklungsverläufe der mehrsprachigen Kinder mit SM in der vorliegenden Studie wird davon ausgegangen, dass die Beratung der pädagogischen Fachkräfte in der Kita, die Sensibilisierung der Eltern für Problemsituationen ihrer Kinder durch die Teilnahme an der Studie sowie die sprachtherapeutische Unterstützung zum Absinken der Angst maßgeblich beigetragen haben.

\subsubsection{Bedeutung der Ergebnisse im Diathese-Stress-Modell}

In Kapitel 2.4.4 wurde zur Erklärung der Entstehung des SM bei mehrsprachigen Kindern ein Diathese-Stress-Modell entwickelt (siehe Abbildung 2). Ziel der Studie war es, aufbauend auf dem theoretisch hergeleiteten Modell erste Befunde zur Bedeutung der drei Faktoren Sprache, Angst und Akkulturation als Diathese zu ermitteln. Eine komplette, empirische Prüfung des Modells wurde nicht angestrebt, da im Vorfeld bereits von einer nicht ausrei- 
chend großen Stichprobe ausgegangen werden konnte. Für eine Annäherung an die Entwicklungszusammenhänge bei mehrsprachigen Kindern wird im Folgenden eine Einordnung der Ergebnisse in das Modell vorgenommen.

Die Prädisposition in Form der Temperamentsmerkmale „Schüchternheit“ und ,sozialer Rückzug“ wurde in der Studie nicht explizit untersucht. Vielmehr wurde das Produkt aus einer solchen Prädisposition und Umweltfaktoren (wie der familiären Lernumwelt) über die Gesamtangst des Kindes erfasst. Diese zeigte sich als bedeutsamer Prädiktor für das Sprechverhalten der Kinder in unterschiedlichen sozialen Kontexten. Für die familiäre Lernumwelt wurden keine Unterschiede hinsichtlich der Angst, Schüchternheit oder SM zwischen Familien schweigender und sprechender Kinder gefunden. Diese Aspekte wurden jedoch über wenige Items im Elternfragebogen erfasst. Auf Basis der Ergebnisse kann bei den Kindern mit SM jedoch von einer Prädisposition für eine erhöhte Ängstlichkeit ausgegangen werden, welche zu Unsicherheiten in sozialen Situationen führen kann.

Der Einfluss der kulturellen Adaptation auf die sozialen und sprachlichen Kompetenzen, welcher für mehrsprachige Kinder als spezifischer Faktor im Modell angenommen wurde, bleibt auf Basis der Ergebnisse unklar. Lediglich bei einem Kind erwies sich die Orientierung der Eltern an der Umgebungskultur zu Studienbeginn als vergleichsweise gering. Dieses Kind zeigte eine gering ausgeprägte Ängstlichkeit, vor allem jedoch sprachliche Unsicherheiten. Dies weist auf den Zusammenhang zwischen der kulturellen Adaptation der Eltern und den Sprachkompetenzen der Kinder in der Umgebungssprache (Oades-Sese \& Li, 2011) hin. Der Effekt der Akkulturation auf die sozialen Kompetenzen war (Pawliuk et al., 1996) für dieses Kind nicht sichtbar.

Für die Bedeutsamkeit der sprachlichen Kompetenzen in der Umgebungssprache gibt es in der vorliegenden Studie deutliche Hinweise. Vor allem für den Kita-Kontext deuten die Ergebnisse auf den im Modell vermuteten gemeinsamen Einfluss von sozialen und sprachlichen Unsicherheiten hin.

Die Rolle der Stressoren in der Entwicklung des SM wurde nicht explizit überprüft, da hier von einer großen Heterogenität und somit nur schwer nachweisbaren Effekten ausgegangen werden kann. Deutliche Hinweise finden sich in der Studie jedoch auf den Übergang von Elternhaus in die Kita als bedeutsamen Stressor in der Lebensgeschichte schweigender Kinder (siehe dazu auch Ahnert, 2013; Ford et al., 1998; Katz-Bernstein, 2011). Für fünf der sieben Kinder berichteten die Eltern Schwierigkeiten in der Eingewöhnung in die Kita.

Zusammenfassend finden sich für die Diathese somit folgende Hinweise in den Ergebnissen der Studie: eine erhöhte Ängstlichkeit, die zu Unsicherheiten in sozialen Situatio- 
nen führt und ein Zweitspracherwerb, welcher sprachliche Unsicherheiten mit sich bringt. Gemeinsam bilden sie die Vulnerabilität für die Entwicklung eines SM. Die Rolle der kulturellen Adaptation in der Ausprägung dieser Vulnerabilität bleibt unklar. Als Stressor scheint vor allem der Übergang vom Elternhaus in die Kita bedeutsam zu sein.

Die multifaktorielle Entstehung des SM wird in dieser Studie für alle Kinder unabhängig einer Ein- oder Mehrsprachigkeit unterstrichen (siehe dazu etwa Cohan, Price et al., 2006; Manassis, 2009). Vor allem in der Einzelfallbetrachtung wird deutlich, dass die Entwicklungsverläufe der Kinder heterogen sind und neben den bedeutsamen Variablen Angst und Sprache auch zahlreiche Drittvariablen eine Rolle zur Entwicklung des SM spielen können. Dazu zählen beispielsweise das elterliche Erziehungsverhalten, besondere Lebensereignisse oder andere Stressfaktoren in der Lebensumwelt des Kindes. Ein Einbezug all dieser Drittvariablen in die statistischen Analysen ist aufgrund der niedrigen Prävalenzrate des SM nur schwer möglich. Somit hebt diese Studie die Bedeutsamkeit der systematischen Betrachtung von Einzelfällen-insbesondere für die Betrachtung der heterogenen Gruppe der mehrsprachigen Kinder-hervor.

\subsection{Grenzen der Studie}

Die Gesamtangst der Kinder erwies sich in dieser Studie als bester Prädiktor für die Entwicklung des Sprechverhaltens sowohl im familiären und öffentlichen als auch im Kitakontext. Der Einfluss der sprachlichen Kompetenzen sowie der elterlichen Akkulturation auf die Entwicklung eines SM konnte aufgrund von zahlreichen fehlenden Werten (vor allem für die Spontansprachaufnahmen) sowie der insgesamt kleinen Stichprobe nicht abschließend geklärt werden.

Die Anforderungen der Studie an die Familien waren durch die längsschnittliche Erhebung relativ hoch. So konnte die angestrebte Stichprobengröße von $n=40$ sowie eine ausbalancierte Verteilung der Kinder auf die vier Gruppen (mehrsprachig +/-, schweigend +/-) nicht erreicht werden. Vor allem der Aspekt der Mehrsprachigkeit erschwerte die Rekrutierung deutlich. Der zusätzliche Dropout von drei mehrsprachigen Kindern, davon zwei der Kontrollkinder, sowie die zahlreichen fehlenden Werte (siehe Kapitel 3.4.5.2) beeinflussten die Validität der Studie nachteilig (Flick, 1988). Neben den genannten Faktoren Rekrutierung und Dropout ist die Heterogenität der betrachteten Kinder ein bedeutsamer Einflussfaktor auf die Ergebnisse der Studie. Innerhalb einzelner Gruppen zeigten sich große Varianzen, die wiederum bei einer insgesamt kleinen Stichprobe die Aussagekraft der Ergebnisse limitiert. 
Vor dem Hintergrund der hier betrachteten Population der Kinder mit SM lassen sich die genannten Faktoren jedoch nur schwer vermeiden. Mit einer Prävalenzrate von knapp einem Prozent (Bergman et al., 2002; Elizur \& Perednik, 2003) ist der SM sehr selten. Größere Stichproben, die hinsichtlich des Alters, sozioökonomischer oder sprachlicher Aspekte homogen sind, würden zwar eine stärkere Aussagekraft besitzen, sind aber aufgrund existierender Rahmenbedingungen (finanziell, personell und zeitlich) nur schwer realisierbar. Um die Rekrutierungsrate in nachfolgenden Untersuchungen zu erhöhen, sollte der Aufwand für die Familien deutlich geringer gehalten werden. Für eine repräsentativere Abbildung der Population der mehrsprachigen Kinder ist zudem der Einbezug von Dolmetschern wünschenswert, um auch solche Familien mit geringen Kenntnissen der Umgebungssprache einbeziehen zu können.

Die kleine Stichprobe führte vor allem in den statistischen Analysen dazu, dass Berechnungen nicht durchgeführt (z.B. Überprüfung von Hypothese 3) und Interaktionseffekte innerhalb der Mehrebenenmodelle nicht überprüft werden konnten. Die Komplexität der Zusammenhänge einzelner Risikofaktoren in der Entstehung des SM erfordert für eine empirische Überprüfung eine deutlich größere Stichprobe als in dieser Studie realisiert werden konnte.

Hinsichtlich der verwendeten Messinstrumente wurde bereits in den vorausgegangenen Ausführungen auf die vermutlich geringe Reliabilität der Beobachtungen eingegangen, welche folgend nicht in die komplexen Analysen einbezogen wurden. Der Elternfragebogen wurde im Rahmen dieser Studie neu entwickelt und ist entsprechend noch nicht ausreichend auf seine Gütekriterien hin überprüft. Für die Analysen wurden vor diesem Hintergrund nur die Skalen einbezogen, die für alle Messzeitpunkte eine ausreichende interne Konsistenz aufwiesen. Messtechnisch problematisch erwiesen sich die Spontansprachaufnahmen und -analysen. Letztere wiesen zwar eine gute Interrater-Reliabilität auf und können somit als objektiv angesehen werden. Insgesamt wiesen die Audioaufnahmen interindividuell jedoch eine große Variabilität hinsichtlich ihrer Länge auf. So konnte insgesamt nur ein geringer Teil der Aufnahmen (jeweils die ersten 30 Äußerungen der Kinder) zur Auswertung genutzt werden, so dass viele Informationen für die Analysen verloren gingen und die Güte der aus den Analysen extrahierten Variablen fraglich bleibt. Eine weitere Schwierigkeit bestand darin, dass ein nicht zu vernachlässigender Teil der mehrsprachigen Familien (T1: 6 von 15, T4: 5 von 15) keine Aufnahmen anfertigten. Bei einem Teil der Familien spiegelte die NichtAufnahme eine relevante Information wider-die nicht ausreichenden Sprachkompetenzen des Kindes im Deutschen. Zum ersten Messzeitpunkt berichteten drei Bezugspersonen expli- 
zit, dass die Aufnahmen nicht möglich gewesen seien, da das Kind bereits die einzelnen Spielzeuge nicht sicher benennen konnte. Eine der Familie führte die Aufnahme folgend in der Herkunftssprache durch. Durch den Nicht-Einbezug dieser Informationen in die Analysen bleibt die Bedeutsamkeit der produktiven Sprachkompetenzen damit innerhalb dieser Studie offen.

Insgesamt betrachtet, kann somit eine Generalisierung der Ergebnisse vor dem Hintergrund der kleinen Stichprobe, vor allem im Hinblick auf die geringe Anzahl von selektiv mutistischen Kindern zu T4, nicht erfolgen. 


\section{Schlussfolgerung und Ausblick}

Mithilfe der vorliegenden Studie wurde ein erster bedeutsamer Schritt zur Untersuchung des SM bei mehrsprachigen Kindern und dem Verstehen seiner Entwicklungsprozesse unternommen. Die Ergebnisse legen nahe, dass Angst sowohl bei ein- als auch bei mehrsprachigen Kindern maßgeblich Einfluss auf die Entstehung des SM nimmt. Ein weiteres Ziel der Studie war es, potenzielle Faktoren zu ermitteln, die eine frühzeitige Diagnose des SM bei mehrsprachigen Kindern ermöglichen. Das Ausmaß der Angst stellt hier einen Faktor dar, welcher dies leisten könnte. Im Rahmen der Diagnosestellung bei mehrsprachigen Kindern könnte die Angst des Kindes einen Hinweis auf das Risiko für die Entwicklung eines SM geben-unabhängig davon, wie gut das Kind die Umgebungssprache bereits beherrscht. Der Vorteil dieses Indikators liegt in der kind-unabängigen Ermittlung des Angstlevels, welches durch Eltern-, Erzieher- oder Lehrerfragebögen erhoben wird. Hierbei sollte jedoch berücksichtigt werden, dass Kinder mit SM von Bezugs- und pädagogischen Betreuungspersonen häufig ängstlicher eingeschätzt werden als die Kinder es selbst empfinden (Yeganeh et al., 2003). Im Schulalter sollten aus diesem Grund möglichst Selbstbeurteilungsverfahren eingesetzt werden, die den Kindern eine schriftliche Beantwortung ermöglichen. Für den hier betrachteten Altersbereich ist dies jedoch aufgrund der fehlenden Schriftsprachkompetenzen noch nicht möglich, so dass vor allem Eltern und Erzieherinnen als Informationsquellen zur Verfügung stehen. Der in der vorliegenden Studie verwendete BAV (Mackowiak \& Lengning, 2010) bietet ab einem Alter von vier Jahren die Möglichkeit, die Angst in der Eins-zuEins-Situation mit dem Kind zu erfassen. Über das verwendete kindgerechte Ratingsystem (Smileys) könnte jedoch auch in diesem Altersbereich das Angstniveau bereits erfasst werden.

Auch wenn die (sozialen) Ängste bereits in zahlreichen Studien als häufig komorbid auftretendes Phänomen bei SM ermittelt wurden (siehe etwa Black \& Uhde, 1995; Cohan et al., 2008; Kristensen, 2000), fehlen Längsschnittstudien, welche die genauen Entstehungsmechanismen untersuchen. So ist bislang noch nicht geklärt, ob Angst und vor allem soziale Ängste sich erst im Verlauf des SM aufgrund der gehäuften Misserfolgserlebnisse in sozialen Situationen entwickeln. Damit ist auch die prognostische Validität des Faktors Angst nicht eindeutig. Vor allem der Faktor soziale Angst konnte aufgrund von unzureichenden Messinstrumenten in der vorliegenden Studie nicht ausreichend untersucht werden. Hier fehlen aktuell noch gute Testverfahren, welche die Erfassung sozialer Ängste im Kindergartenalter ermöglicht. 
Der Einfluss der Sprachkompetenzen im Deutschen und der kulturellen Adaptation der Eltern auf die Entwicklung des SM bei mehrsprachigen Kindern konnte nicht abschließend geklärt werden. Für den Kita-Kontext zeigten sich erste Hinweise auf die Bedeutsamkeit des Sprachverstehens sowie der Orientierung der Eltern an der Umgebungskultur für die Entwicklung der Kinder. Hier sind jedoch noch weitere Studien erforderlich, um die Zusammenhänge zwischen Sprachkompetenzen, Akkulturation und Angst in der Entstehung des SM zu untersuchen. Die vorliegende Studie hat gezeigt, dass die Messung der einzelnen Konstrukte bei der fokussierten Klientel eine große Herausforderung darstellt. Bereits im Vorfeld wurde auf die Schwierigkeit der Sprachdiagnostik bei selektiv mutistischen Kindern hingewiesen (siehe Kapitel 3.2.4). Für einsprachige Kinder erwies sich die Erfassung der produktiven Sprachkompetenzen über die Aufnahme spontansprachlicher Äußerungen im häuslichen Bereich als gut durchführbar. Über dieses Vorgehen konnte bei den einsprachigen Kindern genügend Material gewonnen werden, um die Angemessenheit der sprachlichen Fähigkeiten des Kindes einzuschätzen. Für die mehrsprachigen Familien war dies jedoch nur bedingt möglich. Zum Teil resultierten die fehlenden Spontansprachaufnahmen aus vermutlich noch nicht ausreichenden Sprachkompetenzen der Kinder. Diese Information konnte zwar nicht in die statistischen Analysen mit einbezogen werden. Es weist jedoch auf die Bedeutsamkeit der Eltern als Informationsquelle in der Diagnosestellung bei mehrsprachigen Kindern hin. Eltern, die bereits zur zweiten Generation der Personen mit Migrationshintergrund gehören, verfügen in den meisten Fällen über ausreichende Sprachkompetenzen in der Umgebungssprache, um eine grobe Einschätzung der sprachlichen Kompetenzen der Kinder zu geben. Für Familien, in denen bei keinem Familienmitglied ausreichend Sprachkompetenzen vorliegen, gestaltet sich die gesamte Diagnostik des SM schwierig, da häufig keine entsprechenden Übersetzungen von Anamnese- und Angstfragebögen vorliegen, die Hintergrundinformationen zum Kind und damit ein Aufdecken möglicher Risikofaktoren (z.B. familiäre Häufung ähnlicher Phänomene) liefern könnten. Wenn möglich, sollten Dolmetscher oder weitere Verwandte mit einbezogen werden, welche bei der Beantwortung von Fragebögen und in der Beratung der Eltern unterstützen können.

Auch wenn die Bedeutsamkeit der sprachlichen Fähigkeiten in der vorliegenden Studie nicht ausreichend bestätigt werden konnte, spielt sie im klinischen Bereich bei der Diagnosestellung eine wichtige Rolle. Nur über den Ausschluss fehlender Sprachkompetenzen in der Umgebungssprache kann die Diagnose SM gestellt werden (American Psychiatric Association, 2013). Zugleich stellt Schüchternheit einen Risikofaktor für den Zweitspracherwerb dar (Keller et al., 2013). Das Vorliegen sprachlicher Unsicherheiten wiederum ist ein bedeutsa- 
mer Risikofaktor für den SM (siehe etwa Cohan et al., 2008). Es werden jedoch noch weitere Studien benötigt, um die Entwicklung der produktiven Sprachkompetenzen im Deutschen und deren Einfluss auf das Sprechverhalten von schüchternen Kindern in unterschiedlichen sozialen Kontexten genauer zu untersuchen.

Ein nicht zu vernachlässigender Faktor, welcher in der vorliegenden Studie aufgrund einer großen Selektivität der Stichprobe nicht ausreichend untersucht werden konnte, ist die kulturelle Adaptation der Eltern. Der Umgang der Eltern mit den zwei oder mehr Kulturen, mit denen die Familie täglich konfrontiert ist, kann einen Einfluss auf die sozial-emotionalen und sprachlichen Kompetenzen der Kinder nehmen (Oades-Sese \& Li, 2011; Pawliuk et al., 1996). Die vorliegende Studie konnte erste Hinweise auf einen Einfluss der Orientierung der Eltern an der Herkunftskultur auf die Entwicklung eines SM liefern. Eine Beibehaltung der Herkunftskultur und gleichzeitige geringe Orientierung an der Umgebungskultur könnte einen Risikofaktor für den SM darstellen. Aufgrund einer kleinen Stichprobe und einer insgesamt eher starken Orientierung der Eltern an der Umgebungskultur konnte diese Studie einen möglichen Zusammenhang von kultureller Adaptation und SM nicht umfassend aufklären. Um den Faktor Akkulturation näher untersuchen zu können, ist eine in Bezug auf die Akkulturation heterogenere Stichprobe notwendig. Aus dem bisherigen Forschungsstand sowie den Befunden der vorliegenden Studie erscheinen vor allem die Separation und Marginalisierung als für den SM bedeutsame Akkulturationsstile (siehe dazu auch Kapitel 2.4.3). Eine Rekrutierung von Familien mit entsprechend geringer Orientierung an der Umgebungskultur erfordert jedoch ein großes Maß an Wissen kultureller Hintergründe und entsprechende Strategien, um die Motivation einer Teilnahme an der Studie zu erhöhen und Skepsis vor einer Teilnahme zu vermindern. Eine Zusammenarbeit mit Vermittlern aus der entsprechenden Kultur erscheint für eine Steigerung der Rekrutierungsrate sinnvoll.

Die kulturelle Adaptation ist zudem ein sehr komplexes Konstrukt (siehe Kapitel 2.4.2), welches im Rahmen von Studien mit Kindern mit SM nur schwer in Gänze zu untersuchen ist. Aufgrund der geringen Prävalenzrate ist immer mit einer kleineren Stichprobengröße zu rechnen, so dass der Einfluss einer bestimmten Kultur oder bestimmten Kulturkonstellationen empirisch nur bedingt zu untersuchen ist. Hier können eher systematische Einzelfalluntersuchungen vorgenommen werden, die Aufschluss über mögliche Entwicklungszusammenhänge und Besonderheiten für bestimmte Kulturen bieten können. 


\section{Literaturverzeichnis}

Ahnert, L. (2013). Entwicklungs- und Sozialisationsrisiken bei jungen Kindern. In L. Fried \& L. Ahnert (Hrsg.), Handbuch Pädagogik der frühen Kindheit (Frühe Kindheit. Ausbildung \& Studium, 3., überarb. und erweiterte Aufl., S. 75-85). Berlin: Cornelsen.

Albers, T. (2009). Sprache und Interaktion im Kindergarten. Eine quantitativ-qualitative Analyse der sprachlichen und kommunikativen Kompetenzen von drei- bis sechsjährigen Kindern. Bad Heilbrunn: Klinkhardt.

American Psychiatric Association. (2013). Diagnostic and statistical manual of mental disorders, fifth edition. Arlington: American Psychiatric Publication Inc.

Andersson, C. B. \& Thomsen, P. H. (1998). Electively mute children: An analysis of 37 Danish cases. Nordic Journal of Psychiatry, 52 , 231-238.

Artem Lapitski. (2013) Repeat Timer Pro [Computer software]: Artem Lapitski.

Asendorpf, J. B. \& Meier, G. H. (1993). Personality effects on children's speech in everyday life: sociability-mediated exposure and shyness-mediated reactivity to social situations. Journal of Personality and Social Psychology, 64, 1072-1083.

Atlas, J. A. (1993). Symbol use in a case of elective mutism. Perceptual and Motor Skills, 76, 1079-1082.

Bahr, R. (2006). Schweigende Kinder verstehen: Kommunikation und Bewältigung beim selektiven Mutismus (4. Aufl.). Heidelberg: Universitätsverlag Winter.

Baker, C. (2006). Foundations of bilingual education and bilingualism (4. Aufl.). Clevedon: Multilingual Matters.

Balla-Jaeger, W. (1971). Mutismus bei eineiigen Zwillingen - ein katamnestischer Bericht. Acta Paedopsychiatrica, 38, 17-21.

Barac, R. \& Bialystok, E. (2012). Bilingual effects on cognitive and linguistic development: role of language, cultural background, and education. Child Development, 83, 413-422.

Barnlund, D. C. \& Araki, S. (1985). Intercultural encounters: the management of compliments by Japanese and Americans. Journal of Cross-Cultural Psychology, 16, 9-26.

Bat-Chava, Y., Martin, D. \& Imperatore, L. (2014). Long-term improvements in oral communication skills and quality of peer relations in children with cochlear implants: parental testimony. Child: Care, Health and Development, 40, 870-881. 
Bauermeister, J. J. \& Jemail, J. A. (1975). Modification of "elective mutism" in the classroom setting: a case study. Behavior Therapy, 6, 246-250.

Bavin, E. L. (1991). The acquisition of Walpiri kin terms. Pragmatics, 1, 319-344.

Bavin, E. L. (1995). Language acquisition in crosslinguistic perspective. Annual Review of Anthropology, 24, 373-396.

Bedore, L. M. \& Peña, E. D. (2008). Assessment of bilingual children for identification of language impairment: current findings and implications for practice. International Journal of Bilingual Education and Bilingualism, 11, 1-29.

Bennett, K., Manassis, K., Walter, S. D., Cheung, A., Wilansky-Traynor, P., Diaz-Granados, N. et al. (2013). Cognitive behavioral therapy age effects in child and adolescent anxiety: an individual patient data metaanalysis. Depression and Anxiety, 30, 829-841.

Bergman, L. R., Piacentini, J. \& McCracken, J. T. (2002). Prevalence and description of selective mutism in a school-based sample. Journal of the American Academy of Child \& Adolescent Psychiatry, 41, 938-946.

Bergman, R. L., Gonzalez, A., Piacentini, J. \& Keller, M. L. (2013). Integrated behavior Therapy for Selective Mutism: a randomized controlled pilot study. Behaviour Research and Therapy, 51, 680-689.

Bergman, R. L., Keller, M. L., Piacentini, J. \& Bergman, A. J. (2008). The development and psychometric properties of the selective mutism questionnaire. Journal of Clinical Child \& Adolescent Psychology, 37, 456-464.

Berry, J. W. (1997). Immigration, acculturation, and adaptation. Applied Psychology, 46, 568.

Berry, J. W. (2003). Conceptual approaches to acculturation. In K. Chun, P. Balls Organista \& M. Gerardo (Hrsg.), Advances in theory, measurement, and applied research (S. 17-37). Washington, DC: American Psychological Association.

Bialystok, E. (2001). Bilingualism in development: language, literacy, and cognition. New York: Cambridge University Press.

Bialystok, E., Luk, G., Peets, K. F. \& Yang, S. (2010). Receptive vocabulary differences in monolingual and bilingual children. Bilingualism: Language and Cognition, 13, 525-531.

Bieganski, W. (2012). Entwicklung und Pilotierung von Beobachtungsbögen zur Erfassung des Interaktionsverhaltens selektiv mutistischer Kinder im Kindergarten - Analyse der 
Gruppen- und Frühstücksistuation. Bachelorarbeit, Technische Universität Dortmund. Dortmund.

Bishop, D. (1998). Development of the Children's Communiation Checklist (CCC): a method for assessing qualitative aspects of communicative impairment of children. Journal of Child Psychology and Psychiatry, and allied Disciplines, 39, 879-891.

Black, B. \& Uhde, T. W. (1995). Psychiatric characteristics of children with selective mutism: a pilot study. Journal of the American Academy of Child \& Adolescent Psychiatry, 34, $847-856$.

Bongard, S. (o.J.). Frankfurter Akkulturationsskala (FRAKK20).

Bongard, S., Kelava, A., Sabic, M., Aazami-Gilan, D. \& Kim, Y. B. (2007). Akkulturation und gesundheitliche Beschwerden bei drei Migrantenstichproben in Deutschland. In $\mathrm{H}$. Eschenbeck, U. Heim-Dreger \& C. W. Kohlmann (Hrsg.), Beiträge zur Gesundheitspsychologie (S. 53). Schwäbisch Gmünd: Gmünder Hochschulreihe Band 29.

Booren, L. M., Downer, J. T. \& Vitiello, V. E. (2012). Observations of children's interactions with teachers, peers, and tasks across preschool classroom activity settings. Early Education \& Development, 23, 517-538.

Bradley, S. \& Sloman, L. (1975). Elective mutism in immigrant families, Journal of the American Academy of Child Psychiatry, 2, 510-514.

Braet, C., van Vlierberghe, L., Vandevivere, E., Theuwis, L. \& Bosmans, G. (2013). Depression in early, middle and late adolescence: differential evidence for the cognitive diathesisstress model. Clinical Psychology \& Psychotherapy, 20, 369-383.

Calzada, E. J., Brotman, L. M., Huang, K.-Y., Bat-Chava, Y. \& Kingston, S. (2009). Parent cultural adaptation and child functioning in culturally diverse, urban families of preschoolers. Journal of Applied Developmental Psychology, 30, 515-524.

Carbone, D., Schmidt, L. A., Cunningham, C. C., McHolm, A. E., Edison, S. C., St. Pierre, J. et al. (2010). Behavioral and socio-emotional functioning in children with selective mutism: a comparison with anxious and typically developing children across mutliple informants. Journal of Abnormal Psychology, 28, 1057-1067.

Chavira, D. A., Shipon-Blum, E., Hitchcock, C., Cohan, S. \& Murray, B. (2007). Selective mutism and social anxiety disorder: all in the family? Journal of the American Academy of Child \& Adolescent Psychiatry, 46, 1464-1472. 
Chen, X., He, Y., Oliveira, Ana Maria De, Lo Coco, A., Zappulla, C., Kaspar, V. et al. (2004). Loneliness and social adaptation in Brazilian, Canadian, Chinese and Italian children: a multi-national comparative study. Journal of Child Psychology and Psychiatry, and allied Disciplines, 45, 1373-1384.

Chen, X. \& Tse, H. C.-H. (2008). Social functioning and adjustment in Canadian-born children with Chinese and European backgrounds. Developmental Psychology, 44, 1184-1189.

Chen, X., Zappulla, C., Lo Coco, A., Schneider, B., Kaspar, V., De Oliveira, Ana Maria et al. (2004). Self-perceptions of competence in Brazilian, Canadian, Chinese and Italian children: Relations with social and school adjustment. International Journal of Behavioral Development, 28, 129-138.

Chilla, S., Rothweiler, M. \& Barbur, E. (2010). Kindliche Mehrsprachigkeit; Grundlagen Störungen - Diagnostik. München: Reinhardt.

Cleator, H. \& Hand, L. (2001). Selective mutism: how successful speech and language assessment really is possible. International Journal of Language \& Communication Disorders, 36 (Supplement), 126-131.

Clemente, J., Brafman, M. \& Cohen, C. H. (1986). Concurrent treatment of mother and child in resolution of multigenerational separation and individuation difficulties. Journal of Contemporary Psychotherapy, 16, 140-150.

Cline, T. \& Kysel, F. (1987). Children who refuse to speak. Ethnic background of children with special educational needs describes as elective mute. Children \& Society, 88, $327-$ 334.

Coady, J. A. \& Evans, J. L. (2008). Use and interpretations of non-word repetition tasks in children with and without specific language impairments (SLI). International Journal of Language \& Communication Disorders, 43, 1-40.

Cohan, S. L., Chavira, D. A., Shipon-Blum, E., Hitchcock, C., Roesch, S. C. \& Stein, M. B. (2008). Refining the calssification of children with selective mutism: a latent profile analysis. Journal of Clinical Child \& Adolescent Psychiatry, 37, 770-784.

Cohan, S. L., Chavira, D. A. \& Stein, M. B. (2006). Practitioner review: psychosocial interventions for children with selective mutism: a critical evaluation of the literature from 1990-2005. Journal of Child Psychology and Psychiatry, and allied Disciplines, 47, 10851097. 
Cohan, S. L., Price, J. M. \& Stein, M. B. (2006). Suffering in silence: why a developmental psychopathology perspective on selective mutism is needed. Journal of Developmental \& Behavioral Pediatrics, 27, 341-355.

Coplan, R. J., Arbeau, K. A. \& Armer, M. (2008). Don't fret, be supportive! Maternal characteristics linking child shyness to psychosocial and school adjustment in kindergarten. Journal of Abnormal Child Psychology, 36, 359-371.

Coplan, R. J., Prakash, K., O'Neil, K. \& Armer, M. (2004). Do you "want" to play? Distinguishing between conflicted shyness and social disinterest in early childhood. Developmental Psychology, 40, 244-258.

Cotter, R. B., Burke, J. D., Loeber, R. \& Mutchka, J. (2005). Predictors of contact difficulty and refusal in a longitudinal study. Criminal Behaviour and Metal Health, 1, 126-137.

Crozier, W. R. \& Badawood, A. (2009). Shyness, vocabulary and children's reticence in Saudi Arabian preschools. Infant and Child Development, 18, 255-270.

Crozier, W. R. \& Hostettler, K. (2003). The influence of shyness on children's test performance. British Journal of Educational Psychology, 73, 317-328.

Crozier, W. R. \& Perkins, P. (2002). Shyness as a factor when assessing children. Educational Psychology in Practice, 18, 239-244.

Cunningham, C. E., McHolm, A., Boyle, M. H. \& Patel, S. (2004). Behavioral and emotional adjustment, family functioning, academic performance, and social relationships in children with selective mutism. Journal of Child Psychology and Psychiatry, and allied Disciplines, $45,1363-1372$.

Cunningham, C. E., McHolm, A. E. \& Boyle, M. H. (2006). Social phobia, anxiety, oppositional behavior, social skills and self-concept in children with specific selective mutism, generelized mutism, and community controls. European Child \& Adolescent Psychiatry, 15, $245-255$.

Deater-Deckard, K., Lansford, J. E., Malone, P. S., Pena Alampay, L., Sorbring, E., Bacchini, D. et al. (2011). The association between parental warmth and control in thirteen cultural groups. Journal of Family Psychology, 25, 790-794.

Demuth, K. (1992). The acquisition of Sesotho. In D. I. Slobin (Hrsg.), The crosslinguistic study of language acquisition (S. 557-638). Hillsdale, New Jersey: Lawrence Erlbaum.

Demuth, K. (1996). Collecting spontaneous production data. In D. McDaniel, C. McKee \& H. 
S. Carins (Hrsg.), Methods for assessing children's syntax (S. 3-22). Cambridge, MA: MIT Press.

Deutsches Institut für Medizinische Dokumentation und Information [DIMDI]. (2014). Internationale statistische Klassifikation der Krankheiten und verwandter Gesundheitsprobleme. 10. Revision. German Modification. Zugriff am 27.08.2014. Verfügbar unter http://www.dimdi.de/static/de/klassi/icd-10-gm/kodesuche/onlinefassungen/htmlgm2015 /index.htm

Dobslaff, O. (2005). Mutismus in der Schule. Berlin: Edition Marhold.

Dummit, S., Klein, R., Tancer, N., Asche, B., Martin, J. \& Fairbanks, J. (1997). Systematic assesment of 50 children with selective mutism. Journal of the American Academy of Child \& Adolescent Psychiatry, 36, 653-660.

Duursma, E., Romero-Contreras, S., Szuber, A., Proctor, P., Snow, C., August, D. et al. (2007). The role of home literacy and language environment on bilinguals' English and Spanish vocabulary development. Applied Psycholinguistics, 28, 171-190.

Edison, S. C., Evans, M. A., McHolm, A. E., Cunningham, C. E., Nowakowski, M. E., Boyle, M. et al. (2011). An investigation of control among parents of selectively mute, anxious, and non-anxious children. Child Psychiatry \& Human Development, 42, 270-290.

Elizalde-Utnick, G. (2007). Young selectively mute english language learners: school-based intervention strategies. Journal of Early Childhood and Infant Psychology, 3, 141-161.

Elizur, Y. \& Perednik, R. (2003). Prevalence and description of selective mutism in immigrant and native families: a controlled study. Journal of the American Academy of Child \& Adolescent Psychiatry, 42, 1451-1459.

Essau, C. (2003). Angst bei Kindern und Jugendlichen. München: Reinhardt.

Evans, M. A. (1996). Reticent primary grade children and their more talkative peers: verbal, nonverbal, and self-concept characteristics. Journal of Educational Psychology, 88, 739749.

Field, A. (2013). Discovering statistics using IBM SPSS statistics. And sex and drugs and rock ' $n$ ' roll (4. Aufl). London: Sage.

Finch, W. H., Bolin, J. E. \& Kelley, K. (2014). Multilevel modeling using R. Boca Raton, FL: CRC Press.

Flick, S. N. (1988). Managing attrition in clinical research. Clinical Psychology Review, 8, 
$499-515$.

Ford, M. A., Sladeczeck, I. E., Carlson, J. \& Kratochwill, T. R. (1998). Selective mutism: phenomological characteristics. School Psychology Quarterly, 13, 192-227.

Fox, A. (2007). Test zur Überprüfung des Grammatikverständnisses (TROG-D) (2. Aufl.). Idstein: Schulz-Kirchner Verlag.

Frijters, J. C., Barron, R. W. \& Brunello, M. (2000). Direct and mediated influences of home literacy and literacy interest on prereaders' oral vocabulary and early written language skill. Journal of Educational Psychology, 92, 466-477.

Garbani Ballnik, O. (2009). Schweigende Kinder. Formen des Mutismus in der pädagogischen und therapeutischen Praxis. Göttingen: Vandenhoeck \& Ruprecht.

García Coll, C., Akerman, A. \& Cicchetti, D. (2000). Cultural influences on developmental processes and outcomes: Implications for the study of development and psychopathology. Development and Psychopathology, 12, 333-356.

Gathercole, S. E. (2006). Nonword repetition and word learning: the nature of the relationship. Applied Psycholinguistics, 27, 513-543.

Gathercole, V. C. M. \& Thomas, E. M. (2009). Bilingual first-language development: dominant language takeover, threatened minority language take-up. Bilingualism: Language and Cognition, 12, 213-237.

Gavin, W. J. \& Giles, L. (1996). Sample size effects on temporal reliability of language sample measures of preschool children. Journal of Speech Language and Hearing Research, 39, $1258-1262$.

Gazelle, H. \& Ladd, G. W. (2003). Anxious solitude and peer exclusion: a diathesis-stress model of internalizing trajectories in childhood. Child Development, 74, 257-278.

Genesee, F. \& Nicoladis, E. (2007). Bilingual first language acquisition. In E. Hoff \& M. Shatz (Hrsg.), Blackwell handbook of language development (S. 324-342). Oxford, UK: Blackwell Publishing Ltd.

Gray, R. M., Jordan, C. M., Ziegler, R. S. \& Livingston, R. B. (2002). Two sets of twins with selective mutism: neuropsychological findings. Child Neuropsychology, 8, 41-51.

Grimm, H. (2010). Sprachentwicklungstest für drei- bis fünfjährige Kinder (SETK 3-5). Diagnose von Sprachverarbeitungsfähigkeiten und auditiven Gedächtnisleistungen (2., überarbeitete Aufl.). Göttingen: Hogrefe. 
Grosjean, F. (1989). Neurolinguists, beware! The bilingual is not two monolinguals in one person. Brain and Language, 36, 3-15.

Grünigen, R. von, Kochenderfer-Ladd, B., Perren, S. \& Alsaker, F. D. (2012). Links between local language competence and peer relations among Swiss and immigrant children: The mediating role of social behavior. Journal of School Psychology, 50, 195-213.

Haak, J., Downer, J. \& Reeve, R. (2012). Home literacy exposure and early language and literacy skills in children who struggle with behavior and attention Problems. Early Education \& Development, 23, 728-747.

Hart, K. I., Fujiki, M., Brinton, B. \& Hart, C. (2004). The relationship between social behavior and severity of language impairment. Journal of Speech Language and Hearing Research, 47, 647-662.

Hartmann, B. (1997). Mutismus. Zur Theorie und Kasuistik des totalen und elektiven Mutismus (Schriften zur Sprachheilpädagogik, Bd. 1, 4., überarb. und erw. Aufl.). Berlin: Ed. Marhold.

Hoff, E. (2006). How social contexts support and shape language development. Developmental Review, 26, 55-88.

Hoffmann, L., Leimbrink, K. \& Quasthoff, U. (Hrsg.). (2011). Die Matrix der menschlichen Entwicklung. Berlin: de Gruyter.

Hoke, F. (2012). Erleben und Verhalten selektiv mutistischer Kinder in angstauslösenden Situationen. Eine multimethodale Erhebung. Masterarbeit, Universität Bielefeld. Bielefeld.

Holmbeck, G. N. \& Lavigne, J. V. (1992). Combining self-modeling and stimulus fading in the treatment of an electively mute child. Psychotherapy, 29, 661-667.

Honig, M.-S. (2012). Frühpädagogische Einrichtungen. In L. Fried, B. Dippelhofer-Stiem, M.-S. Honig \& L. Liegle (Hrsg.), Pädagogik der frühen Kindheit (S. 91-128). Weinheim: Beltz.

Hox, J. J. \& Boom, J. (2012). Growth curve modeling from a multilevel model perspective. In B. Laursen, T. D. Little \& N. A. Card (Hrsg.), Handbook of developmental research methods (S. 432-444). New York: Guilford Press.

Ingram, R. E. \& Luxton, D. (2005). Vulnerability-stress models. In B. L. Hankin \& Abela, J. R. Z. (Hrsg.), Development of psychopathology: a vulnerability-stress perspective (S. $32-$ 46). New York: Sage. 
Ishikawa, S.-i., Okajima, I., Matsuoka, H. \& Sakano, Y. (2007). Cognitive behavioural therapy for anxiety disorders in children and adolescents: a meta-analysis. Child and Adolescent Mental Health, 12, 164-172.

Ispa, J. M., Fine, M. A., Halgunseth, L. C., Harper, S., Robinson, J., Boyce, L. et al. (2004). Maternal intrusiveness, maternal warmth, and mother-toddler relationship outcomes: variations across low-income ethnic and acculturation groups. Child Development, 75, 16131631.

Jaeger, W. \& Metzker, H. (1968). Mutismus bei eineiigen Zwillingen. Acta Paedopsychiatrica, 35, 59-70.

Jonkisz, E., Moosbrugger, H. \& Brandt, H. (2012). Planung und Entwicklung von Tests und Fragebogen. In H. Moosbrugger \& A. Kelava (Hrsg.), Testtheorie und Fragebogenkonstruktion. Mit 66 Abbildung und 41 Tabellen (Springer-Lehrbuch, 2., aktual. und überarb. Aufl, S. 75-102). Berlin: Springer.

Katz-Bernstein, N. (2011). Selektiver Mutismus bei Kindern. Erscheinungsbilder, Diagnostik, Therapie (3. Aufl.). München: Ernst Reinhardt.

Kauschke, C. (2000). Der Erwerb des frühkindlichen Lexikons. Eine empirische Studie zur Entwicklungs des Wortschatzes im Deutschen. Tübingen: Gunter Narr Verlag.

Kauschke, C. (2012). Kindlicher Spracherwerb im Deutschen. Verläufe, Forschungsmethoden, Erklärungsansätze (Germanistischer Arbeitshefte, Bd. 45). Berlin: de Gruyter.

Kauschke, C. \& Siegmüller, J. (2009). Patholinguistische Diagnostik bei Sprachentwicklungsstörungen (2. Aufl). München: Elsevier.

Kearney, C. (2010). Helping children with selective mutism and their parents: a guide for school-based professionals (1. Aufl.). New York: Oxford University Press.

Keller, K., Troesch, L. M. \& Grob, A. (2013). Shyness as a risk factor for second language acquisition of immigrant preschoolers. Journal of Applied Developmental Psychology, 34, $328-335$.

King, N. J., Heyne, D. \& Ollendick, T. H. (2005). Cognitive-behavioral treatments for anxiety disorders in children and adolescents: a review. Behavioral Disorders, 30, 241-257.

Klein, E. R., Armstrong, S. L. \& Shipon-Blum, E. (2013). Assessing spoken language competence in children with selective mutism: using parents as test presenters. Communication Disorders Quarterly, 34, 184-195. 
Kolvin, I. \& Fundudis, T. (1981). Elective mute children: psychological development and background factors. Journal of Child Psychology and Psychiatry, and allied Disciplines, $22,219-232$.

Kopp, S. \& Gilberg, C. (1997). Selective mutism: a population-based study: a research note. Journal of Child Psychology and Psychiatry, and allied Disciplines, 38, 257-262.

Kotsiubinskii, A. P. (2002). A biopsychosocial model of schizophrenia. International Journal of Mental Health, 31 (2), 51-60.

Kresse, A. (Februar 2011). Erfassung diagnostischer Daten bei Selektivem Mutismus. Entwicklung und Erprobung eines Elternfragebogens. Masterarbeit, Universität Bielefeld. Bielefeld.

Krippendorff, K. (2004). Content analysis: an introduction to its methodology (2. Aufl). Thousand Oaks, CA: Sage.

Kristensen, H. (2000). Selective mutism and comorbidity with developmental disorder/delay, anxiety disorder, and elimination disorder. Journal of the American Academy of Child \& Adolescent Psychiatry, 39, 249-256.

Kristensen, H. \& Oerbeck, B. (2006). Is selective mutism associated with deficits in memory span and visual memory?: an exploratory case-control study. Depression and Anxiety, 23, $71-76$.

Kristensen, H. \& Torgersen, S. (2001). MCM-II personality traits and symptom traits in parents of children with selective mutism: a case-control study. Journal of Abnormal Psycho$\log y, 110,648-652$.

Kristensen, H. \& Torgersen, S. (2002). A case-control study of EAS child and parental temperaments in selectively mute children with and without a co-morbid communication disorder. Nordic Journal of Psychiatry, 56), 347-353.

Kroppenberg, D. (1987). Das dialogische Moment im menschlichen Spracherwerb mit einer kasuistischen Studie über dreizehnjährige eineiige Zwillinge mit selektivem Mutismus. In DGS-Landesgruppe Rheinland (Hrsg.), Spracherwerb und Spracherwerbsstörungen (S. 284-299). Hamburg: Wartenberg.

Kumpulainen, K., Räsänen, E., Raaska, H. \& Somppi, V. (1998). Selective mutism among second-graders in elementary school. European Child \& Adolescent Psychiatry, 7, 24-29.

Lampert, T. \& Kroll, L. E. (2009). Die Messung des sozioökonomischen Status in sozial- 
epidemiologische Studien. In M. Richter \& K. Hurrelmann (Hrsg.), Gesundheitliche Ungleichheit. Grundlagen, Probleme, Perspektiven (2., aktualisierte Aufl, S. 309-334). Wiesbaden: VS, Verl. für Sozialwiss.

Lange, S. \& Tröster, H. (2014). Strategien der Emotionsregulation bei sozial ängstlichen Kindern und Jugendlichen. Zeitschrift für Klinische Psychologie und Psychotherapie, 43, 3542.

Laptook, R. S., Klein, D. N., Durbin, C. E., Hayden, E. P., Olino, T. M. \& Carlson, G. (2008). Differentiation between low positive affectivity and behavioral inhibition in preschool-age children: a comparison of behavioral approach in novel and non-novel Contexts. Personality and Individual Differences, 44, 758-767.

Laroche, M., Kim, C., Hui, M. K. \& Tomiuk, M. A. (1998). Test of a nonlinear relationship between linguistic acculturation and ethnic Identification. Journal of Cross-Cultural Psychology, 29, 418-433.

Layzer, J. I., Goodson, B. D. \& Moss, M. (1993). Observational study of early childhood programs. Final report volume 1: life in preschool. Washington, DC: U.S. Department of Education.

Lazarus, R. S. \& Folkman, S. (1984). Stress, appraisal, and coping. New York: Springer Pub. Co.

Lüke, C. (2011). Sprachdiagnostik bei mehrsprachigen Schulkindern - Anwendbarkeit des PITPA und des SET 5-10 zur Erfassung der deutschsprachigen Kompetenzen. L.O.G.O.S. interdisziplinär, 19, 164-172.

Lyytinen, P. (1991). Developmental trends in children's pretend play. Child: Care, Health and Development, 17, 9-25.

Mackowiak, K. \& Lengning, A. (2010). Das Bochumer Angstverfahren für Kinder im Vorschul- und Grundschulalter (BAV 3-11). Bern: Verlag Hans Huber.

Majoribanks, K. (1994). Crosscultural comparisons of family environments of anglo-, greek-, and italian-australians. Psychological Reports, 74, 49-50.

Manassis, K. (2009). Silent suffering: understanding and treating children with selective mutism. Expert Review of Neurotherapeutics, 9, 235-243.

Manassis, K., Fung, D., Tannock, R., Sloman, L., Fiksenbaum, L. \& McInnes, A. (2003). Characterizing selective mutism: is it more than social anxiety? Depression and Anxiety, 
$18,153-161$.

Manassis, K., Tannock, R., Garland, E. J., Minde, K., McInnes, A. \& Clark, S. (2007). The sounds of silence: language, cognition and anxiety in selective mutism. Journal of the American Academy of Child \& Adolescent Psychiatry, 46, 1187-1195.

Marsella, A. J. (2005). Culture and conflict: understanding, negotiating, and reconciling conflicting constructions of reality. International Journal of Intercultural Relations, 29, 651673.

Max Planck Institute for Psycholinguistics, The Language Archive. (2013) ELAN - ELAN Linguistic Annotator [Computer software]. Nijmegen, The Netherlands.

Mayworm, A. M., Dowdy, E., Knights, K. \& Rebelez, J. (2014). Assessment and treatment of selective mutism with english language learners. Contemporary School Psychology, Online Veröffentlichung, doi: 10.1007/s40688-014-0035-5.

McCabe, P. C. (2005). Social and behavioral correlates of preschoolers with specific language impairment. Psychology in the Schools, 42, 373-387.

McInnes, A., Fung, D., Manassis, K., Fiksenbaum, L. \& Tannock, R. (2004). Narrative skills in children with selective mutism: an exploratory study. American Journal of SpeechLanguage Pathology, 13, 304-315.

Meijer, A. (1979). Elective mutism in children. Israel Annals of Psychiatry and related Disciplines, 17, 93-100.

Melfsen, S., Walitza, S. \& Warnke, A. (2006). The extent of social anxiety in combination with mental disorders. European Child \& Adolescent Psychiatry, 15, 111-117.

Melfsen, S. \& Warnke, A. (2004). Soziale Phobie. In S. Schneider (Hrsg.), Angststörungen bei Kindern und Jugendlichen (S. 165-195). Berlin: Springer.

Melfsen, S. \& Warnke, A. (2007). Überblick zur Behandlung des Selektiven Mutismus. Zeitschrift für Kinder- und Jugendpsychiatrie und Psychotherapie, 35, 309-409.

Meyers, S. V. (1984). Elective mutism in children: a family systems approach. The American Journal of Family Therapy, 12 (4), 39-46.

Miglietta, A. \& Tartaglia, S. (2009). The influence of stay, linguistic competence, and media exposure in immigrants' adaptation. Cross-Cultural Research, 43, 46-61.

Moldan, M. B. (2005). Selective mutism and self-regulation. Clinical Social Work Journal, 33, 291-307. 
Monroe, S. M. \& Simons, A. D. (1991). Diathesis-stress theories in the context of life stress research: implications for the depressive disorders. Psychological Bulletin, 110, 406-425.

Mora, G., DeVault, S. \& Schopler, E. (1962). Dynamics and psychotherapy of identical twins with elective mutism. Journal of Child Psychology and Psychiatry, and allied Disciplines,3, 41-52.

National Institute of Child Health and Human Development Early Child Care Research Network. (2000). The relation of child care to cognitive and language development. Child Development, 71, 960-980.

National Institute of Child Health and Human Development Early Child Care Research Network. (2002). Child-care structure -> process -> outcome: direct and indirect effects of child-care quality on young children's development. Psychological Science, 13, 199-206.

Nicoladis, E. \& Genesee, F. (1997). Language development in preschool bilingual children. Journal of Speech-Language-Pathology and Audiology, 21, 258-270.

Niebuhr-Siebert, S. \& Baake, H. (2014). Kinder mit Deutsch als Zweitsprache in der Grundschule (1. Aufl.). Stuttgart: Kohlhammer.

Niebuhr-Siebert, S. \& Chilla, S. (2012). Spracherwerb. In S. Niebuhr-Siebert \& U. Wiecha (Hrsg.), Kindliche Sprach-, Sprech-, Stimm- und Schluckstörungen. Gezielte Elternberatung (1. Aufl., S. 13-33). München: Elsevier.

Nowakowski, M. E., Cunningham, C. C., McHolm, A. E., Evans, M. A., Edison, S. C., St. Pierre, J. et al. (2009). Language and academic abilities in children with selective mutism. Infant and Child Development, 18, 271-290.

Oades-Sese, G. V. \& Li, Y. (2011). Attachment relationships as predictors of language skills for at risk bilingual preschool children. Psychology in the Schools, 48, 707-722.

OECD. (2013). PISA 2012 results: excellence through equity (volume II): OECD Publishing.

Oerbeck, B., Johansen, J., Lundahl, K. \& Kristensen, H. (2012). Selective mutism: a homeand kindergarten-based intervention for children 3-5 years: a pilot study. Clinical Child Psychology and Psychiatry, 17, 370-383.

Oerbeck, B., Stein, M. B., Pripp, A. H. \& Kristensen, H. (2014). Selective mutism: follow-up study 1 year after end of treatment. European Child \& Adolescent Psychiatry, Online Veröffentlichtung, doi: 10.1007/s00787-014-0620-1. 
Oller, D. K., Pearson, B. Z. \& Cobo-Lewis, A. B. (2007). Profile effects in early bilingual language and literacy. Applied Psycholinguistics, 28, 191-230.

Paradis, J., Genesee, F. \& Crago, M. B. (2010). Dual language development \& disorders. Baltimore: Paul H. Brooks Publishing.

Patten, S. B. (2013). Major depression epidemiology from a diathesis-stress conceptualization. BMC Psychiatry, 13, 19.

Paulus, C. (2009). Die "Bücheraufgabe" zur Bestimmung des kulturellen Kapitals bei Grundschülern, Universität des Saarlandes. Zugriff am 22.09.2014. Verfügbar unter http://psydok.sulb.uni-saarland.de/volltexte/2009/2368/

Pawliuk, N., Grizenko, N., Chan-Yip, A., Gantous, P., Mathew, J. \& Nguyen, D. (1996). Acculturation style and psychological functioning in children of immigrants. American Journal of Orthopsychiatry, 66, 111-121.

Pearson, B. Z., Fernandez, S. C., Lewedeg, V. \& Oller, D. K. (1997). The relation of input factors to lexical learning by bilingual infants. Applied Psycholinguistics, 18, 41-58.

Pellegrini, A. D., Galda, L., Flor, D., Bartini, M. \& Charak, D. (1997). Close relationships, individual differences, and early literacy learning. Journal of Experimental Child Psycho$\log y, 67,409-422$.

Prevoo, M., Malda, M., Mesman, J., Emmen, Rosanneke A. G., Yeniad, N., Van Ijzendoorn, M. H. et al. (2014). Predicting ethnic minority children's vocabulary from socioeconomic status, maternal language and home reading input: different pathways for host and ethnic language. Journal of Child Language, 41, 963-984.

Prior, M., Smart, D., Sanson, S. \& Oberklaid, F. (2000). Does shy-inhibited temperament in childhood lead to anxiety disorder in adolescence? Journal of the American Academy of Child \& Adolescent Psychiatry, 39, 461-468.

Raudenbush, S. W. \& Bryk, A. S. (2002). Hierarchical linear models: application and data analysis methods (2. Aufl.). Thousand Oaks, CA: Sage.

Raviv, T., Kessenich, M. \& Morrison, F. J. (2004). A mediational model of the association between socioeconomic status and three-year-old language abilities: the role of parenting factors. Early Childhood Research Quarterly, 19, 528-547.

Rehr, U. (2012). Entwicklung und Pilotierung eines Beobachtungsinstrumentes zur Erfassung des Interaktionsverhaltens mutistischer und nicht mutistischer Kinder - Analyse von Be- 
grïßungs- und Freispielsituation -. Bachelorarbeit, Technische Universität Dortmund. Dortmund.

Remschmidt, H., Poller, M., Herpertz-Dahlmann, B., Henninghausen, K. \& Gutenbrunner, C. (2001). A follow-up study of 45 patients with elective mutism. European Archives of Psychiatry and Clinical Neuroscience (251), 284-296.

Reynolds, K. P. \& Evans, M. A. (2009). Narrative performance and parental scaffolding of shy and nonshy children. Applied Psycholinguistics, 30, 363-384.

Rice, M. L., Smolik, F., Perpich, D., Thompson, T., Rytting, N. \& Blossom, M. (2010). Mean length of utterance levels in 6-month intervals for children 3 to 9 years with and without language impairments. Journal of Speech Language and Hearing Research, 53, 333-349.

Ritterfeld, U. \& Lüke, C. (2012). Mehrsprachen-Kontexte: Erfassung der Inputbedingungen von mehrsprachig aufwachsenden Kindern. L.O.G.O.S. interdisziplinär, 20, 24-29.

Romaine, S. (1995). Bilingualism (2. Aufl.). Oxford: Wiley-Blackwell.

Rosenbaum, E. \& Kellman, M. (1973). Treatment of a selectively mute third-grade child. Journal of School Psychology, 11, 26-29.

Rosenthal, R. (1991). Meta-analytic procedures for social research (2. Aufl). Newbury Park, CA: Sage.

Rösler, M. (1981). Findings on neurotic mutism in children. An examination of 32 mutistic children. Praxis der Kinderpsychologie und Kinderpsychiatrie, 30, 187-194.

Rubin, K. H. \& Coplan, R. J. (2004). Paying attention to an not neglecting social withdrawal and social isolation. Merrill-Palmer Quarterly, 50, 506-534.

Rubin, K. H., Coplan, R. J., Bowker, J. C. \& Menzer, M. (2011). Shyness and social withdrawal. In P. K. Smith \& C. Hart (Hrsg.), Blackwell handbook of childhood social development (2. Aufl., S. 434-452). New York: Wiley-Blackwell.

Rubin, K. H., Hemphill, S. A., Chen, X., Hastings, P., Sanson, A., Lo Coco, A. et al. (2006). A cross-cultural study of behavioral inhibition in toddlers: East-West-North-South. International Journal of Behavioral Development, 30, 219-226.

Rubin, K. H., Coplan, R. J. \& Bowker, J. C. (2009). Social withdrawal in childhood. Annual Review of Psychology, 60, 141-171.

Saß, H., Wittchen, H. U., Zaudig, M. \& Houben, I. (2003). Diagnostisches und Statistisches Manual Psychischer Störungen DSM-IV-TR (1. Aufl.). Göttingen: Hogrefe. 
Schieffelin, B. B. \& Eisenberg, A. R. (1984). Cultural variation in children's conversations. In R. L. Schiefelbusch \& J. Pickar (Hrsg.), The acquisition of communicative competence (S. 379-420). Baltimore: University Park Press.

Schmitt, S. A., Simpson, A. M. \& Friend, M. (2011). A longitudinal assessment of the home literacy environment and early language. Infant and Child Development, 20, 409-431.

Schneider, W. \& Hasselhorn, M. (2012). Frühe Kindheit (3-6 Jahre). In W. Schneider \& U. Lindenberger (Hrsg.), Entwicklungspsychologie (7. Aufl., S. 187-210). Weinheim: Beltz.

Schwartz, R. H., Freedy, A. S. \& Sheridan, M. J. (2006). Selective mutism: are primary care physicians missing the silence? Clinical Pediatrics, 45, 43-48.

Segal, N. (1999). Silent partners: twins with selective mutism. Twin Research, 22, 235-241.

Segal, N. L. (2003). 'Two' quiet: monozygotic female twins with selective mutism. Clinical Child Psychology and Psychiatry, 8, 473-488.

Sénéchal, M. \& LeFevre, J.-A. (2014). Continuity and change in the home literacy environment as predictors of growth in vocabulary and reading. Child Development, 85, 15521568.

Serratrice, L. (2013). The bilingual child. In T. K. Bhatia \& C. Ritchie (Hrsg.), The handbook of bilingualism and multilingualism (2. Aufl., S. 87-108). Chichester: Blackwell.

Sharkey, L. \& McNicholas, F. (2006). Female monozygotic twins with selective mutism-a case report. Developmental and Behavioral Pediatrics, 22, 129-133.

Shaw, W. H. (1971). Aversive control in the treatment of elective mutism. Journal of the American Academy of Child \& Adolescent Psychiatry, 10, 572-581.

Shneidman, L. A. \& Goldin-Meadow, S. (2012). Language input and acquisition in a Mayan village: how important is directed speech? Developmental Science, 15, 659-673.

Snijders, T. \& Bosker, R. J. (1994). Modeled variance in two-level models. Sociological Methods \& Research, 22, 342-363.

Snijders, T. \& Bosker, R. J. (2012). Multilevel analysis. an introduction to basic and advanced multilevel modeling (2. Aufl.). Los Angeles: Sage.

Snow, C. E. (1977). The development of conversation between mothers and babies. Journal of Child Language, 4, 1-22.

Spere, K. A., Schmidt, L. A., Theall-Honey, L. A. \& Martin-Chang, S. (2004). Expressive and 
receptive language skills of temperamentally shy preschoolers. Infant and Child Development, 13, 123-133.

Starke, A. \& Subellok, K. (2012). KiMut NRW: Eine Studie zur Identifikation von Kindern mit selektivem Mutismus im schulischen Primarbereich. Empirische Sonderpädagogik, 4, $63-77$.

Starke, A., Subellok, K. \& Käppler, C. (in Vorb.). Entwicklung und Evaluation des Dortmunder Mutismus-Screening.

Statistisches Bundesamt. (2013). Bevölkerung mit Migrationshintergrund - Ergebnisse des Mikrozensus 2012. Wiesbaden: Statistisches Bundesamt.

Steinhausen, H.-C. (2010). Fragebogen zur Erfassung des Elektiven Mutismus (FEM). In H.C. Steinhausen (Hrsg.), Psychische Störungen bei Kindern und Jugendlichen (7. Aufl, S. 558-560). München: Elsevier.

Steinhausen, H.-C. \& Adamek, R. (1997). The family history of children with elective mutism: a research report. European Child \& Adolescent Psychiatry, 6, 107-111.

Steinhausen, H.-C. \& Juzi, C. (1996). Elective mutism: an analysis of 100 cases. Journal of the American Academy of Child \& Adolescent Psychiatry, 35, 606-614.

Stich, M. (2010). Theory of mind and pretend play in children with specific language impairment. Dissertation.

Strand, P. S., Pula, K., Parks, C. D. \& Cerna, S. (2011). Shyness-anxiousness and receptive language skills development in Spanish- and English-speaking preschoolers. Journal of Applied Developmental Psychology, 32, 363-368.

Strong, C. J. (1998). The strong narrative assessment procedure. Eau Claire, WI: Thinking Publications.

Subellok, K., Katz-Bernstein, N., Bahrfeck-Wichitill, K. \& Starke, A. (2012). DortMuT (Dortmunder Mutismus-Therapie): eine (sprach-)therapeutische Konzeption für Kinder und Jugendliche mit selektivem Mutismus. L.O.G.O.S. interdisziplinär, 20, 84-96.

Subellok, K., Kresse, A. \& Bahrfeck-Wichitill, K. (2010). Gemeinsam Schweigsam: Selektiver Mutismus bei Zwillingen. Teil 1: Spezifische Risikofaktoren für die Entstehung und Aufrechterhaltung des Schweigens. Die Sprachheilarbeit, 55, 110-120.

Subellok, K. \& Starke, A. (2012). Selektiver Mutismus. In S. Niebuhr-Siebert \& U. Wiecha (Hrsg.), Kindliche Sprach-, Sprech-, Stimm- und Schluckstörungen. Gezielte Elternbera- 
tung (1. Aufl., S. 219-237). München: Elsevier.

Suinn, R. M., Ahuna, C. \& Khoo, G. (1992). The Suinn-Lew Asian self-identity acculturation scale: concurrent and factorial validation. Educational and Psychological Measurement, $52,1041-1046$.

Tabors, P. (2008). One child, two languages: a guide for preschool educators of children learning English as a second language (2. Aufl.). Baltimore: Paul H. Brooks Publishing.

Thoma, D. \& Tracy, R. (2006). Deutsch als frühe Zweitsprache: zweite Erstsprache? In B. Ahrenholz (Hrsg.), Kinder mit Migrationshintergrund - Spracherwerb und Fördermöglichkeiten (S. 58-79). Freiburg i. Br.: Fillbach.

Tomasello, M. (2008). Die Ursprünge der menschlichen Kommunikation. Frankfurt: Suhrkamp.

Tomasello, M. \& Stahl, D. (2004). Sampling childrens spontaneous speech: how much is enough? Journal of Child Language, 31, 101-121.

Tong, X., Ting, K. T. \& McBride-Chang, C. (2011). Shyness and Chinese and English vocabulary skills in Hong Kong kindergarteners. Early Education and Development, 22, 29-52.

Toppelberg, C. O., Tabors, P., Coggins, A., Lum, K. \& Burger, C. (2005). Differential diagnosis of selective mutism in bilingual children. Journal of the American Academy of Child \& Adolescent Psychiatry, 44, 592-595.

Tracy, R. (2014). Mehrsprachigkeit: Vom Störfall zum Glücksfall. In M. Krifka, J. Blasczak, A. Leßmöllmann, A. Meinunger, B. Stiebels, R. Tracy et al. (Hrsg.), Das mehrsprachige Klassenzimmer. Über die Muttersprachen unserer Schüler (S. 13-33). Berlin: Springer.

Vecchio, J. L. \& Kearney, C. A. (2005). Selective mutism in children: comparison to youths with and without anxiety disorders. Journal of Psychopathology and Behavioral Assessment, 27, 31-37.

Walden, T. A., Frankel, C. B., Buhr, A. P., Johnson, K. N., Conture, E. G. \& Karrass, J. M. (2012). Dual diathesis-stressor model of emotional and linguistic contributions to developmental stuttering. Journal of Abnormal Child Psychology, 40, 633-644.

Wernitznig, H. (1993). Stationäre Behandlung eines elektiv mutistischen Kindes - eine Fallstudie. Praxis der Kinderpsychologie und Kinderpsychiatrie, 42, 160-167.

White, L. A. (1947). Culturological vs. psychological interpretation of human behavior. American Sociological Review, 12, 686-698. 
Wichstrøm, L., Belsky, J. \& Berg-Nielsen, T. S. (2013). Preschool predictors of childhood anxiety disorders: a prospective community study. Journal of Child Psychology and Psychiatry, and allied Disciplines, 54, 1327-1336.

Wittenburg, P., Brugman, H., Russel, A., Klassmann, A. \& Sloetjes, H. (2006). ELAN: a professional framework for multimodality research. In Proceedings of language resources and evaluation conference.

Xu, Y. \& Krieg, A. (2014). Shyness in Asian American children and the relation to temperament, parents' acculturation, and psychosocial functioning. Infant and Child Development, $23,333-342$.

Yeganeh, R., Beidel, D. C. \& Turner, S. M. (2006). Selective mutism: more than social anxiety? Depression and Anxiety, 23, 117-123.

Yeganeh, R., Beidel, D. C., Turner, S. M., Pina, A. A. \& Silverman, W. K. (2003). Clinical distinctions between selective mutism and social phobia: an investigation of childhood psychopathology. Journal of the American Academy of Child \& Adolescent Psychiatry, 42, $1069-1075$.

Youngerman, J. K. (1979). The syntax of silence: electively mute therapy. International Review of Psycho-Analysis, 6, 283-295.

Zelenko, M. \& Shaw, R. (2000). Case study: selective mutism in an immigrant child. Clinical Child Psychology and Psychiatry, 5, 555-562.

Zick, A. (2010). Psychologie der Akkulturation. Wiesbaden: VS Verlag für Sozialwissenschaften. 


\section{Anhang}

Anhang A: Tabellen

Anhang B: Elternfragebogen

Anhang C: DortMuS-Kita

Anhang D: Beobachtungsbögen und Beobachtungsmanual

Anhang E: Beschreibung der Interaktionssettings

Anhang F: Anleitung zur Durchführung der Spontansprachaufnahme

Anhang G: Studieninformationen und Einverständniserklärungen

Anhang H: Erstinformation für die Kitas

Anhang I: Kodierschema Spontansprache 
Anhang A: Tabellen 
Tabelle A-1

Itemkennwerte für den Elternfragebogen

\begin{tabular}{|c|c|c|c|}
\hline Nr. & Item & korrigierte Itemtrennschärfe & Itemschwierigkeit \\
\hline \multicolumn{4}{|c|}{ Schweigen } \\
\hline 7 & Mein Kind begrüßt Verwandte ohne Aufforderung. & .60 & .49 \\
\hline 10 & Mein Kind begrüßt Nachbarn ohne Aufforderung & .81 & .53 \\
\hline 14 & Wenn mein Kind von Verwandten oder Nachbarn begrüßt wird, grüßt es zurück. & .70 & .48 \\
\hline 20 & Bei Familienfesten spricht mein Kind mit den anderen Kindern. & .58 & .28 \\
\hline 21 & Mein Kind weigert sich, Bekannte oder Verwandte zu begrüßen. & .86 & .43 \\
\hline 25 & Mein Kind bestellt z.B. in der Eisdiele selbst. & .88 & .62 \\
\hline 28 & Mein Kind spricht mit mir in der Öffentlichkeit. & .36 & .13 \\
\hline 29 & Mein Kind schweigt, wenn es von einem Verkäufer angesprochen wird. & .72 & .59 \\
\hline 30 & In Gegenwart fremder Personen flüstert mein Kind mit mir. & .54 & .48 \\
\hline 31 & Mein Kind spricht zuhause mit uns völlig normal. & .00 & .95 \\
\hline 33 & Mein Kind schweigt in Gegenwart seiner Großeltern. & .35 & .08 \\
\hline 35 & Mit seinen Geschwistern spricht mein Kind. & .00 & .72 \\
\hline 38 & Mein Kind schweigt in Gegenwart des Kinderarztes. & .80 & .56 \\
\hline 39 & Sobald wir aus dem Haus treten, schweigt mein Kind. & .48 & .14 \\
\hline 40 & Mit kleinen Kindern der Verwandtschaft spricht mein Kind. & .41 & .12 \\
\hline 42 & Mit Erwachsenen der Verwandtschaft schweigt mein Kind. & .83 & .36 \\
\hline 44 & Meinem Kind fällt es schwer, fremde Kinder anzusprechen. & .82 & .57 \\
\hline 45 & Mein Kind schweigt in Gegenwart von älteren Kindern der Nachbarschaft. & .79 & .41 \\
\hline 47 & Mit gleichaltrigen Kindern der Verwandtschaft spricht mein Kind. & .67 & .15 \\
\hline 48 & Mein Kind erstarrt völlig, wenn es schweigt. & .74 & .25 \\
\hline \multicolumn{4}{|c|}{ Partizipation } \\
\hline 8 & Mein Kind spielt bei Familienfesten mit anderen Kindern. & .53 & .30 \\
\hline 9 & Bei Familienfesten bleibt mein Kind in unserer Nähe. & .64 & .53 \\
\hline 11 & Bei Familienfesten zeigt mein Kind sein gewöhnliches Essverhalten. & .15 & .28 \\
\hline 15 & Auf dem Spielplatz spielt mein Kind mit anderen Kindern. & .63 & .43 \\
\hline 16 & Bei Familienfesten verweigert mein Kind das Essen. & .16 & .27 \\
\hline
\end{tabular}




\begin{tabular}{|c|c|c|c|}
\hline Nr. & Item & korrigierte Itemtrennschärfe & Itemschwierigkeit \\
\hline 17 & $\mathrm{Zu}$ anderen Verwandten nimmt mein Kind nur zusammen mit Geschwistern Kontakt auf. & .23 & .30 \\
\hline 37 & Mein Kind spielt mit Kindern aus der Nachbarschaft. & .47 & .38 \\
\hline 51 & Auf dem Spielplatz spielt mein Kind alleine. & .76 & .47 \\
\hline 54 & Wenn wir Bekannte in der Stadt treffen, verhält sich unser Kind unbefangen. & .47 & .43 \\
\hline 56 & In der Spielgruppe spielt mein Kind alleine. & .45 & .37 \\
\hline \multicolumn{4}{|c|}{ Nonverbale Kommunikation } \\
\hline 12 & Mein Kind gibt anderen zur Begrüßung die Hand. & .40 & 64 \\
\hline 43 & Ja und Nein drückt mein Kind durch Kopfschütteln und Nicken aus. & .45 & .48 \\
\hline 49 & Auch wenn mein Kind schweigt, zeigt es Freude durch Lächeln oder Lachen. & .01 & .26 \\
\hline 50 & In Gegenwart fremder Personen vermeidet mein Kind Husten, Niesen oder Räuspern. & .63 & .18 \\
\hline 55 & Wenn mein Kind schweigt, drückt es Wünsche oder Bedürfnisse durch Zeigen aus. & .16 & .58 \\
\hline 58 & Mein Kind nimmt keinen Blickkontakt auf, wenn es angesprochen wird. & .81 & .44 \\
\hline \multicolumn{4}{|c|}{ Emotionsregulation } \\
\hline 2 & Bei Problemen kommt mein Kind zu uns, um darüber zu reden. & -.22 & .34 \\
\hline 3 & Wenn mein Kind etwas kaputt macht oder etwas verschüttet, weint es. & .77 & .41 \\
\hline 4 & Mit Veränderungen des gewohnten Tagesablaufs geht mein Kind problemlos um. & .45 & .33 \\
\hline 5 & Über Besuch freut sich mein Kind. & .66 & .18 \\
\hline 6 & Bei Problemen zieht sich mein Kind in sein Zimmer zurück. & .49 & .30 \\
\hline 13 & Bei Familienfesten benötigt mein Kind eine Aufwärmphase. & .29 & .61 \\
\hline 18 & Mein Kind freut sich auf Familienfeste. & .51 & .15 \\
\hline 26 & Mein Kind vermeidet Menschenansammlungen (z.B. volle Räume, volle Läden). & .84 & .29 \\
\hline 27 & $\begin{array}{l}\text { Wenn wir in der Stadt Bekannte treffen, hält unser Kinder Körperkontakt zu uns (z.B. bleibt an } \\
\text { der Hand, hält meine Jacke fest). }\end{array}$ & .45 & .64 \\
\hline 36 & Mein Kind weint viel beim Arzt. & .21 & .17 \\
\hline 59 & Bei Menschenansammlungen (z.B. im Bus) klammert sich mein Kind an mich. & .36 & .28 \\
\hline
\end{tabular}




\begin{tabular}{|c|c|c|c|}
\hline & Item & korrigierte Itemtrennschärfe & Itemschwierigkeit \\
\hline \multicolumn{4}{|c|}{ Öffentliche Situationen } \\
\hline 22 & $\begin{array}{l}\text { Beim Einkaufen nimmt mein Kind kleine Geschenke von Verkäufern an (z.B. Bonbons, eine } \\
\text { Wurstscheibe). }\end{array}$ & .39 & .14 \\
\hline 23 & Mein Kind isst in Anwesenheit fremder Personen z.B. Obst, Brötchen, Schokoriegel. & .34 & .19 \\
\hline 24 & Mein Kind kauft selbstständig z.B. Spielzeug, Aufkleber oder Süßigkeiten ein. & .43 & .71 \\
\hline 32 & Beim Zahnarzt öffnet mein Kind den Mund. & .62 & .29 \\
\hline 41 & Mein Kind lässt sich vom Arzt untersuchen & .39 & .16 \\
\hline 52 & Mein Kind äußert seine Wünsche und Bedürfnisse nicht in der Öffentlichkeit. & .51 & .36 \\
\hline 53 & Mein Kind fährt gern mit dem Bus oder geht mit mir einkaufen. & .28 & .15 \\
\hline 57 & In der Öffentlichkeit äußert mein Kind seine Wünsche und Bedürfnisse. & .38 & .21 \\
\hline
\end{tabular}


Tabelle A-2

Itemkennwerte für DortMuS-Kita

\begin{tabular}{|c|c|c|c|}
\hline Nr. & Item & korrigierte Itemtrennschärfe & Itemschwierigkeit \\
\hline \multicolumn{4}{|c|}{ Schweigen und Bedürfnisäußerung } \\
\hline 13 & Das Kind äußert verbal, wenn es Hilfe benötigt. & .83 & .58 \\
\hline 21 & Das Kind schweigt auf dem Hof gegenüber Erziehern. & .71 & .53 \\
\hline 24 & Das Kind holt sich Unterstützung von anderen Kindern, wenn es Hilfe benötigt. & .65 & .52 \\
\hline 30 & Das Kind signalisiert gegenüber der Erzieherin, dass es z.B. Durst hat. & 69 & .44 \\
\hline 51 & In der Gruppe schweigt das Kind. & .84 & .58 \\
\hline 70 & In Spielsituationen spricht das Kind mit anderen Kindern. & .76 & .57 \\
\hline 71 & Bedürfnisse werden vom Kind geäußert. & .74 & .47 \\
\hline 73 & In Gesprächssituationen vermeidet das Kind sprachliche Äußerungen. & .85 & .54 \\
\hline \multicolumn{4}{|c|}{ Gruppe } \\
\hline 69 & Das Kind zieht sich bei Spielen in der Großgruppe zurück. & .71 & .49 \\
\hline
\end{tabular}


Tabelle A-3

Interrater-Reliabilität für die Beobachtungen im Rahmen der Pilotstudie

\begin{tabular}{|c|c|c|}
\hline Beobachtungskategorie & Ungewichteter Cohens Kappa & Standardfehler $(S E)$ \\
\hline \multicolumn{3}{|l|}{ Begrüßungssituation } \\
\hline Kind-Kind Initiation verbal & 1.00 & .00 \\
\hline Kind-Kind Initiation nonverbal & 1.00 & .00 \\
\hline Kind-Kind Reaktion verbal & 1.00 & .00 \\
\hline Kind-Kind Reaktion nonverbal & 1.00 & .00 \\
\hline Kind-Erzieher Initiation verbal & 1.00 & .00 \\
\hline Kind-Erzieher Initiation nonverbal & 1.00 & .00 \\
\hline Kind-Erzieher Reaktion verbal & 1.00 & .00 \\
\hline Kind-Erzieher Reaktion nonverbal & .33 & .20 \\
\hline Kind-Eltern Initiation verbal & .10 & .20 \\
\hline Kind-Eltern Initiation nonverbal & -.07 & -.20 \\
\hline Kind-Eltern Reaktion verbal & .56 & .20 \\
\hline Kind-Eltern Reaktion nonverbal & .40 & .20 \\
\hline Beobachtung & .86 & .22 \\
\hline Wechsel der Interaktionspartner & -.02 & .20 \\
\hline Interaktion nicht beobachtbar & 1.00 & .00 \\
\hline Trennungssituation auffällig & .60 & .20 \\
\hline Trennungssituation unauffällig & .26 & .20 \\
\hline \multicolumn{3}{|l|}{ Freispielsituation } \\
\hline Kind-Kind Initiation verbal & .62 & .06 \\
\hline Kind-Kind Initiation nonverbal & .50 & .06 \\
\hline Kind-Kind Reaktion verbal & .62 & .06 \\
\hline Kind-Kind Reaktion nonverbal & .39 & .06 \\
\hline Kind-Erzieher Initiation verbal & .49 & .06 \\
\hline Kind-Erzieher Initiation nonverbal & -.00 & .06 \\
\hline Kind-Erzieher Reaktion verbal & .66 & .06 \\
\hline Kind-Erzieher Reaktion nonverbal & .54 & .06 \\
\hline Wechsel der Interaktionspartner & .40 & .06 \\
\hline Interaktion nicht beobachtbar & .90 & .06 \\
\hline Beobachtung & .70 & .06 \\
\hline Spielbegleitendes Sprechen & .73 & .06 \\
\hline Parallelspiel & .68 & .06 \\
\hline Symbolspiel & .53 & .06 \\
\hline
\end{tabular}




\begin{tabular}{lcc}
\hline Beobachtungskategorie & Ungewichteter Cohens Kappa & Standardfehler (SE) \\
\hline Rollenspiel & -.01 & .06 \\
Regelspiel & 1.00 & .06 \\
Bewegungsspiel & -.03 & .06 \\
Weitere Spielformen & .65 & .06 \\
Soziale Situation Einzel & .77 & .06 \\
Soziale Situation Dyade & .55 & .06 \\
Soziale Situation Gruppe & .63 & .06
\end{tabular}

Gruppensituation

$\begin{array}{lll}\text { Kind-Kind Initiation verbal } & .00 & .70\end{array}$

$\begin{array}{lll}\text { Kind-Kind Initiation nonverbal } & .00 & .57\end{array}$

Kind-Kind Reaktion verbal $\quad 1.00 \quad 00$

$\begin{array}{lll}\text { Kind-Kind Reaktion nonverbal } & .24 & .31\end{array}$

$\begin{array}{lll}\text { Kind-Erzieher Initiation verbal } & .66 & .33\end{array}$

$\begin{array}{lll}\text { Kind-Erzieher Initiation nonverbal } & 1.00 & .00\end{array}$

$\begin{array}{lll}\text { Kind-Erzieher Reaktion verbal } & 1.00 & .00\end{array}$

$\begin{array}{lll}\text { Kind-Erzieher Reaktion nonverbal } & .29 & .23\end{array}$

$\begin{array}{lll}\text { Beobachtung } & .67 & .08\end{array}$

$\begin{array}{lll}\text { Blickkontakt } & .84 & .02\end{array}$

$\begin{array}{lll}\text { Partizipation } & .77 & .03\end{array}$

\section{Frühstückssituation}

Kind-Kind Initiation verbal $\quad .43 \quad .15$

$\begin{array}{lll}\text { Kind-Kind Initiation nonverbal } & .59 & .14\end{array}$

$\begin{array}{lll}\text { Kind-Kind Reaktion verbal } & .57 & .13\end{array}$

$\begin{array}{lll}\text { Kind-Kind Reaktion nonverbal } & .73 & .07\end{array}$

Kind-Erzieher Initiation verbal $\quad .83 \quad .12$

$\begin{array}{lll}\text { Kind-Erzieher Initiation nonverbal } & .00 & 1.00\end{array}$

$\begin{array}{lll}\text { Kind-Erzieher Reaktion verbal } & .00 & 1.00\end{array}$

$\begin{array}{lll}\text { Kind-Erzieher Reaktion nonverbal } & .58 & .15\end{array}$

$\begin{array}{lll}\text { Beobachtung } & .76 & .06\end{array}$

$\begin{array}{lll}\text { Blickkontakt } & .90 & .02\end{array}$

$\begin{array}{lll}\text { Soziale Situation } & .91 & .02\end{array}$ 
Tabelle A-4

Post hoc Vergleiche zu Gruppenunterschieden im Kulturellen Kapital

\begin{tabular}{lccccc}
\hline & $H$ & $Z$ & $p$ & angepasstes $p$ & $r$ \\
\hline ESM - MSM & -3.79 & -0.952 & .341 & 1.000 & -.22 \\
ESM - MSP & -11.22 & -2.815 & .005 & .029 & \\
ESM - ESP & -12.61 & -2.62 & .009 & .053 & \\
MSM - MSP & -7.43 & -1.686 & .092 & .551 & -.44 \\
MSM - ESP & -8.82 & -1.707 & .088 & .526 & -.51 \\
MSP - ESP & 1.39 & 0.270 & .787 & 1.000 & .08
\end{tabular}

Anmerkungen $. \mathrm{ESM}=$ Einsprachig schweigende Kinder, $\mathrm{MSM}=$ mehrsprachig schweigende Kinder, $\mathrm{ESP}=$ einsprachig sprechende Kinder, MSP = mehrsprachig sprechende Kinder

Tabelle A-5

Post hoc Vergleiche zu Gruppenunterschieden im Schicht-Index

\begin{tabular}{lccccc}
\hline & $H$ & $Z$ & $p$ & angepasstes $p$ & $r$ \\
\hline ESM - MSM & 1.71 & 0.404 & .686 & 1.000 & .10 \\
ESM - MSP & -2.84 & -0.517 & .605 & 1.000 & -.16 \\
ESM - ESP & -13.90 & -3.063 & .002 & .013 & -.79 \\
MSM - MSP & -1.13 & -0.220 & .826 & 1.000 & -.06 \\
MSM - ESP & -12.19 & -2.991 & .003 & .017 & -.69 \\
MSP - ESP & -11.06 & -2.06 & .039 & .236 & -.59
\end{tabular}

Anmerkungen $. \mathrm{ESM}=$ Einsprachig schweigende Kinder, $\mathrm{MSM}=$ mehrsprachig schweigende Kinder, $\mathrm{ESP}=$ einsprachig sprechende Kinder, MSP = mehrsprachig sprechende Kinder

Tabelle A-6

Anteil der Kinder mit Entwicklungsverzögerungen/-störungen laut Elternangaben

\begin{tabular}{|c|c|c|c|c|c|c|c|}
\hline & \multicolumn{2}{|c|}{ Schweigende Kinder } & \multicolumn{2}{|c|}{ Kontrollkinder } & \multicolumn{3}{|c|}{ Chi-Quadrat } \\
\hline & $\begin{array}{c}\text { mehrsprachig } \\
\mathrm{n}=11\end{array}$ & $\begin{array}{c}\text { einsprachig } \\
n=7\end{array}$ & $\begin{array}{c}\text { mehrsprachig } \\
n=4\end{array}$ & $\begin{array}{c}\text { einsprachig } \\
n=8\end{array}$ & $d f$ & $X^{2}$ & $p$ \\
\hline Motorik & $\begin{array}{c}1 \\
(9.1 \%)\end{array}$ & $\begin{array}{c}1 \\
(14.3 \%)\end{array}$ & $\begin{array}{c}0 \\
(0 \%)\end{array}$ & $\begin{array}{c}0 \\
(0 \%)\end{array}$ & 3 & 1.61 & .656 \\
\hline Kognition & $\begin{array}{c}0 \\
(0 \%)\end{array}$ & $\begin{array}{c}0 \\
(0 \%)\end{array}$ & $\begin{array}{c}0 \\
(0 \%)\end{array}$ & $\begin{array}{c}0 \\
(0 \%)\end{array}$ & - & - & 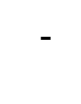 \\
\hline Sprache & $\begin{array}{c}2 \\
(18.2 \%)\end{array}$ & $\begin{array}{c}1 \\
(14.3 \%)\end{array}$ & $\begin{array}{c}0 \\
(0 \%)\end{array}$ & $\begin{array}{c}1 \\
(12.5 \%)\end{array}$ & 3 & 0.85 & .838 \\
\hline $\begin{array}{l}\text { zweitweilige } \\
\text { Hörprobleme }\end{array}$ & $\begin{array}{c}0 \\
(0 \%)\end{array}$ & $\begin{array}{c}1 \\
(14.3 \%)\end{array}$ & $\begin{array}{c}0 \\
(0 \%)\end{array}$ & $\begin{array}{c}1 \\
(12.5 \%)\end{array}$ & 3 & 2.02 & .568 \\
\hline andere & $\begin{array}{c}1 \\
(9.1 \%)\end{array}$ & $\begin{array}{c}1 \\
(14.3 \%) \\
\end{array}$ & $\begin{array}{c}0 \\
(0 \%) \\
\end{array}$ & $\begin{array}{c}1 \\
(12.5 \%) \\
\end{array}$ & 3 & 0.73 & .866 \\
\hline
\end{tabular}


Tabelle A-7

Anteil der Kinder mit komorbiden Auffälligkeiten

\begin{tabular}{lccccccc}
\hline & \multicolumn{2}{c}{ Schweigende Kinder } & \multicolumn{2}{c}{ Kontrollkinder } & \multicolumn{2}{c}{ Chi-Quadrat } \\
\cline { 2 - 7 } & $\begin{array}{l}\text { mehrsprachig } \\
\mathrm{n}=11\end{array}$ & $\begin{array}{c}\text { einsprachig } \\
\mathrm{n}=7\end{array}$ & $\begin{array}{c}\text { mehrsprachig } \\
\mathrm{n}=4\end{array}$ & $\begin{array}{c}\text { einsprachig } \\
\mathrm{n}=8\end{array}$ & $d f$ & $X^{2}$ & $p$ \\
\hline Enuresis & 1 & 3 & 2 & 0 & 3 & 7.35 & .061 \\
Enkorpresis & $(9.1 \%)$ & $(42.9 \%)$ & $(50.0 \%)$ & $(0 \%)$ & & & \\
& 0 & 2 & 0 & 0 & 3 & 7.04 & .071 \\
Verstopfungen & $(0 \%)$ & $(28.6 \%)$ & $(0 \%)$ & $(0 \%)$ & & & \\
& 0 & 1 & 0 & 0 & 3 & 3.40 & .334 \\
Hyperaktivität & $(0 \%)$ & $(14.3 \%)$ & $(0 \%)$ & $(0 \%)$ & & & \\
& 0 & 1 & 1 & 0 & 3 & 4.17 & .244 \\
Aggressivität & $(0 \%)$ & $(14.3 \%)$ & $(25.0 \%)$ & $(0 \%)$ & & & \\
& 0 & 2 & 0 & 0 & 3 & 7.04 & .071 \\
Autismus & $(0 \%)$ & $(28.6 \%)$ & $(0 \%)$ & $(0 \%)$ & & & \\
Zwanghaftes & 0 & 0 & 0 & 0 & - & - & - \\
Verhalten & $(0 \%)$ & $(0 \%)$ & $(0 \%)$ & $(0 \%)$ & & & \\
Tics & 1 & 2 & 0 & 0 & 3 & 4.03 & .259 \\
& $(9.1 \%)$ & $(28.6 \%)$ & $(0 \%)$ & $(0 \%)$ & & & \\
Essstörungen & 0 & 0 & 0 & 0 & - & - & - \\
& $(0 \%)$ & $(0 \%)$ & $(0 \%)$ & $(0 \%)$ & & & \\
& $(27.3 \%)$ & $(14.3 \%)$ & $(25.0 \%)$ & $(0 \%)$ & & & .437 \\
\hline
\end{tabular}

Tabelle A-8

Anteil der Therapieformen zum ersten Messzeitpunkt

\begin{tabular}{lccccccc}
\hline & \multicolumn{2}{c}{ Schweigende Kinder } & \multicolumn{2}{c}{ Kontrollkinder } & \multicolumn{2}{c}{ Chi-Quadrat } \\
\cline { 2 - 7 } & $\begin{array}{c}\text { mehrsprachig } \\
\mathrm{n}=11\end{array}$ & $\begin{array}{c}\text { einsprachig } \\
\mathrm{n}=7\end{array}$ & $\begin{array}{c}\text { mehrsprachig } \\
\mathrm{n}=4\end{array}$ & $\begin{array}{c}\text { einsprachig } \\
\mathrm{n}=8\end{array}$ & $d f$ & $X^{2}$ & $p$ \\
\hline Frühförderung & 2 & 0 & 0 & 0 & 3 & 3.70 & .296 \\
Sprachtherapie & $(18.2 \%)$ & $(0 \%)$ & $(0 \%)$ & $(0 \%)$ & & & \\
& 1 & 1 & 0 & 0 & 3 & 1.61 & .656 \\
Ergotherapie & $(9.1 \%)$ & $(14.3 \%)$ & $(0 \%)$ & $(0 \%)$ & & & \\
Physiotherapie & 0 & 0 & 0 & 0 & - & - & - \\
& $(0 \%)$ & $(0 \%)$ & $(0 \%)$ & $(0 \%)$ & & & \\
Psychotherapie & $(9.1 \%)$ & $(0 \%)$ & $(0 \%)$ & $(12.5 \%)$ & & & \\
& 0 & 0 & 0 & 0 & - & - & - \\
andere & $(0 \%)$ & $(0 \%)$ & $(0 \%)$ & $(0 \%)$ & & & \\
& 0 & 1 & 0 & 0 & 3 & 3.40 & .334 \\
& $(0 \%)$ & $(14.3 \%)$ & $(0 \%)$ & $(0 \%)$ & & & \\
\hline
\end{tabular}


Tabelle A-9

Ergebnisse des Kolmogorov-Smirnov-Test zur Überprüfung der Normalverteilung der Prädiktorvariablen zum ersten Messzeitpunkt

\begin{tabular}{lccc}
\hline Variable & $n$ & $D$ & $p$ \\
\hline BAV Gesamtangst T1 & 28 & .134 & .200 \\
TROG-D T1 & 28 & .116 & .200 \\
Pseudowörter T1 & 23 & .162 & .119 \\
MLU T1 & 23 & .136 & .200 \\
FRAKK Orientierung an Herkunftskultur & 14 & .203 & .122 \\
FRAKK Orientierung an Aufnahmekultur & 14 & .222 & .060 \\
FRAKK Akkulturationsindex & 14 & .217 & .072 \\
\hline
\end{tabular}


Tabelle A-10

Ergebnisse des Kolmogorov-Smirnov-Test zur Überprüfung der Normalverteilung der Outcomevariablen

\begin{tabular}{|c|c|c|c|c|}
\hline Variable & & $n$ & $D$ & $p$ \\
\hline & $\mathrm{T} 1$ & 30 & .086 & .200 \\
\hline Elternfragebogen & $\mathrm{T} 2$ & 23 & .110 & .200 \\
\hline \multirow[t]{2}{*}{ Skala Schweigen } & $\mathrm{T} 3$ & 26 & .130 & .200 \\
\hline & $\mathrm{T} 4$ & 27 & .113 & .200 \\
\hline \multirow{4}{*}{$\begin{array}{l}\text { DortMuS-Kita } \\
\text { Skala Schweigen und Bedürfnisäuße- } \\
\text { rung }\end{array}$} & $\mathrm{T} 1$ & 25 & .125 & .200 \\
\hline & $\mathrm{T} 2$ & 25 & .118 & .200 \\
\hline & $\mathrm{T} 3$ & 17 & .203 & .060 \\
\hline & $\mathrm{T} 4$ & 24 & .163 & .097 \\
\hline \multirow{4}{*}{$\begin{array}{l}\text { Gesamtzahl Interaktionen Freispielsitu- } \\
\text { ation }\end{array}$} & $\mathrm{T} 1$ & 30 & .120 & .200 \\
\hline & $\mathrm{T} 2$ & 27 & .242 & .000 \\
\hline & $\mathrm{T} 3$ & 22 & .125 & .200 \\
\hline & $\mathrm{T} 4$ & 22 & .121 & .200 \\
\hline \multirow{4}{*}{$\begin{array}{l}\text { Gesamtzahl verbaler Interaktionen Frei- } \\
\text { spielsituation }\end{array}$} & $\mathrm{T} 1$ & 30 & .188 & .008 \\
\hline & $\mathrm{T} 2$ & 27 & .247 & .000 \\
\hline & $\mathrm{T} 3$ & 22 & .176 & .073 \\
\hline & $\mathrm{T} 4$ & 22 & .159 & .152 \\
\hline \multirow{4}{*}{$\begin{array}{l}\text { Gesamtzahl nonverbaler Interaktionen } \\
\text { Freispielsituation }\end{array}$} & $\mathrm{T} 1$ & 30 & .227 & .000 \\
\hline & $\mathrm{T} 2$ & 27 & .230 & .001 \\
\hline & $\mathrm{T} 3$ & 22 & .212 & .011 \\
\hline & $\mathrm{T} 4$ & 22 & .238 & .002 \\
\hline \multirow{5}{*}{ Gesamtzahl Interaktionen Kind-Kind } & $\mathrm{T} 1$ & 30 & .161 & .046 \\
\hline & $\mathrm{T} 2$ & 27 & .189 & .015 \\
\hline & $\mathrm{T} 3$ & 22 & .152 & .200 \\
\hline & $\mathrm{T} 4$ & 22 & .233 & .003 \\
\hline & $\mathrm{T} 1$ & 30 & .254 & .000 \\
\hline \multirow{3}{*}{$\begin{array}{l}\text { Gesamtzahl Interaktionen Kind- } \\
\text { Erzieher }\end{array}$} & $\mathrm{T} 2$ & 27 & .242 & .000 \\
\hline & $\mathrm{T} 3$ & 22 & .178 & .069 \\
\hline & $\mathrm{T} 4$ & 22 & .258 & .001 \\
\hline
\end{tabular}


Tabelle A-11

Korrelationen zwischen den Prädiktoren und der Gesamtzahl der nonverbalen Interaktionen

\begin{tabular}{|c|c|c|c|c|c|}
\hline & \multicolumn{4}{|c|}{ Gesamtzahl der nonverbalen Interaktionen } \\
\hline & & $\mathrm{T} 1$ & $\mathrm{~T} 2$ & $\mathrm{~T} 3$ & $\mathrm{~T} 4$ \\
\hline \multicolumn{2}{|c|}{ BAV Gesamtangst } & $\begin{array}{c}.334^{* \mathrm{a}} \\
{[.117, .530]}\end{array}$ & $\begin{array}{c}.298 * \mathrm{~b} \\
{[-.032, .606]}\end{array}$ & $\begin{array}{c}.156^{\mathrm{c}} \\
{[-.178, .512]}\end{array}$ & $\begin{array}{c}.082^{\mathrm{c}} \\
{[-.245, .418]}\end{array}$ \\
\hline \multicolumn{2}{|c|}{ TROG-D } & {$\left[-.006^{\mathrm{a}}\right.$} & $\begin{array}{c}.058^{\mathrm{d}} \\
{[-.291, .397]}\end{array}$ & $\begin{array}{c}-.027^{\mathrm{e}} \\
{[-.453, .404]}\end{array}$ & $\begin{array}{c}.173^{\mathrm{e}} \\
{[-.110, .428]}\end{array}$ \\
\hline \multicolumn{2}{|c|}{ MLU } & $\begin{array}{c}-.062^{\mathrm{f}} \\
{[-.416, .322]}\end{array}$ & $\begin{array}{c}.350^{* c} \\
{[-.042, .696]}\end{array}$ & $\begin{array}{c}.000^{\mathrm{g}} \\
{[-.473, .507]}\end{array}$ & $\begin{array}{c}.034^{\mathrm{h}} \\
{[-.323, .417]}\end{array}$ \\
\hline \multirow{3}{*}{$\frac{v}{d}$} & $\begin{array}{l}\text { Orientierung an Her- } \\
\text { kunftskultur }\end{array}$ & $\begin{array}{c}-.047^{\mathrm{i}} \\
{[-.543, .403]}\end{array}$ & $\begin{array}{c}.069^{\mathrm{j}} \\
{[-.478, .616]}\end{array}$ & $\begin{array}{c}.333^{\mathrm{k}} \\
{[-.144, .864]}\end{array}$ & $\begin{array}{c}.444^{1} \\
{[-.048, .889]}\end{array}$ \\
\hline & $\begin{array}{l}\text { Orientierung an Auf- } \\
\text { nahmekultur }\end{array}$ & $\begin{array}{c}-.024^{\mathrm{i}} \\
{[-.383, .473]}\end{array}$ & $\begin{array}{c}-.482 * \mathrm{j} \\
{[-.832,-.018]}\end{array}$ & $\begin{array}{c}-.158^{\mathrm{k}} \\
{[-.638, .296]}\end{array}$ & $\begin{array}{c}-.340^{1} \\
{[-.934, .348]}\end{array}$ \\
\hline & Akkulturationsindex & $\begin{array}{c}.000^{\mathrm{i}} \\
{[-.354, .439]}\end{array}$ & $\begin{array}{c}-.356^{\mathrm{j}} \\
{[-.756, .070]}\end{array}$ & $\begin{array}{c}-.250^{\mathrm{k}} \\
{[-.709, .210]}\end{array}$ & $\begin{array}{c}-.148^{1} \\
{[-.810, .609]}\end{array}$ \\
\hline \multicolumn{2}{|c|}{ KK } & $\begin{array}{c}-.223^{\mathrm{m}} \\
{[-.491, .067]}\end{array}$ & $\begin{array}{c}-.290^{\mathrm{d}} \\
{[-.582, .032]}\end{array}$ & $\begin{array}{c}-.265^{\mathrm{e}} \\
{[-.615, .142]}\end{array}$ & $\begin{array}{c}-.033^{\mathrm{e}} \\
{[-.425, .374]}\end{array}$ \\
\hline \multicolumn{2}{|c|}{ SES } & $\begin{array}{c}-.016^{\mathrm{m}} \\
{[-.307, .292]}\end{array}$ & $\begin{array}{c}.094^{\mathrm{d}} \\
{[-.216, .383]}\end{array}$ & $\begin{array}{c}-.122^{\mathrm{e}} \\
{[-.409, .197]}\end{array}$ & $\begin{array}{c}-.077^{\mathrm{e}} \\
{[-.445, .280]}\end{array}$ \\
\hline
\end{tabular}

Anmerkungen. ${ }^{*} p<.05, * * p<.01, * * * p<.001,95 \%$ Konfidenzintervall in eckigen Klammern, ${ }^{\mathrm{a}} \mathrm{n}=28,{ }^{\mathrm{b}} \mathrm{n}=25,{ }^{\mathrm{c}} \mathrm{n}=20,{ }^{\mathrm{d}} \mathrm{n}=26,{ }^{\mathrm{e}} \mathrm{n}=21,{ }^{\mathrm{f}} \mathrm{n}=23,{ }^{\mathrm{g}} \mathrm{n}=15,{ }^{\mathrm{h}} \mathrm{n}=18,{ }^{\mathrm{i}} \mathrm{n}=14,{ }^{\mathrm{j}} \mathrm{n}=$ $13,{ }^{\mathrm{k}} \mathrm{n}=11,{ }^{1} \mathrm{n}=8,{ }^{\mathrm{m}} \mathrm{n}=29$ 
Tabelle A-12

Korrelationen zwischen den Prädiktoren und der Gesamtzahl der Interaktionen zwischen Kind und einem/mehreren Kind/ern

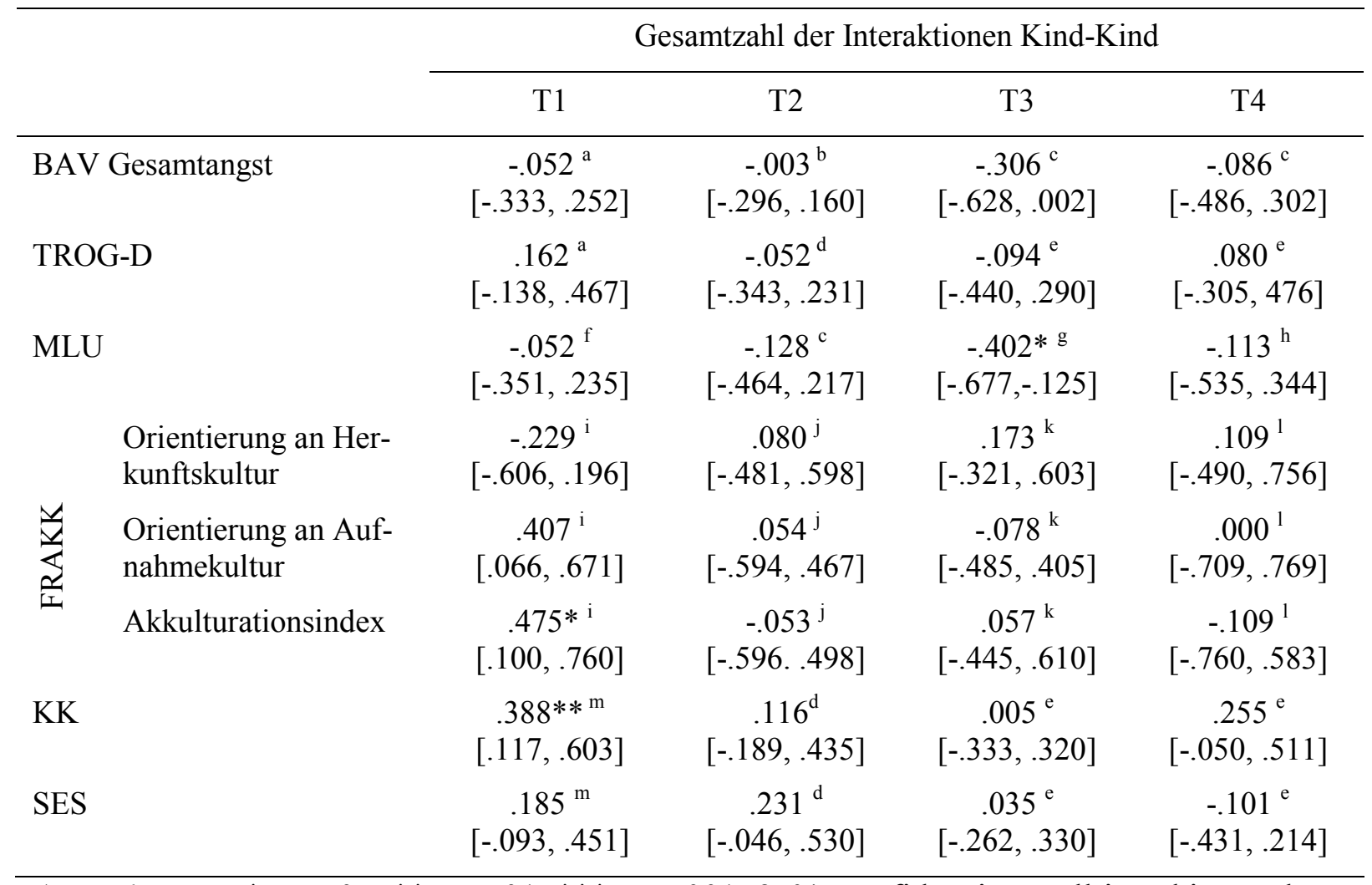

Anmerkungen. $* p<.05, * * p<.01, * * * p<.001,95 \%$ Konfidenzintervall in eckigen Klammern, ${ }^{\mathrm{a}} \mathrm{n}=28,{ }^{\mathrm{b}} \mathrm{n}=25,{ }^{\mathrm{c}} \mathrm{n}=20,{ }^{\mathrm{d}} \mathrm{n}=26,{ }^{\mathrm{e}} \mathrm{n}=21,{ }^{\mathrm{f}} \mathrm{n}=23,{ }^{\mathrm{g}} \mathrm{n}=15,{ }^{\mathrm{h}} \mathrm{n}=18,{ }^{\mathrm{i}} \mathrm{n}=14,{ }^{\mathrm{j}} \mathrm{n}=$ $13,{ }^{\mathrm{k}} \mathrm{n}=11,{ }^{1} \mathrm{n}=8,{ }^{\mathrm{m}} \mathrm{n}=29$ 
Tabelle A-13

Korrelationen zwischen den Prädiktoren und der Gesamtzahl der Interaktionen zwischen Kind und Erzieher

\begin{tabular}{|c|c|c|c|c|c|}
\hline & \multicolumn{4}{|c|}{ Gesamtzahl der Interaktionen Kind-Erzieher } \\
\hline & & $\mathrm{T} 1$ & $\mathrm{~T} 2$ & $\mathrm{~T} 3$ & $\mathrm{~T} 4$ \\
\hline \multicolumn{2}{|c|}{ BAV Gesamtangst } & $\begin{array}{c}.023^{\mathrm{a}} \\
{[-.307, .350]}\end{array}$ & $\begin{array}{c}.247^{\mathrm{b}} \\
{[-.103, .547]}\end{array}$ & $\begin{array}{c}-.158^{\mathrm{c}} \\
{[-.503, .149]}\end{array}$ & $\begin{array}{c}-.170^{\mathrm{c}} \\
{[-.146, .452]}\end{array}$ \\
\hline \multicolumn{2}{|c|}{ TROG-D } & $\begin{array}{l}-.205^{\mathrm{a}} \\
{[-.483, .070]}\end{array}$ & $\begin{array}{c}.227^{\mathrm{d}} \\
{[-.068, .522]}\end{array}$ & $\begin{array}{c}.030^{\mathrm{e}} \\
{[-.358, .399]}\end{array}$ & $\begin{array}{c}-.128^{\mathrm{e}} \\
{[-.482, .222]}\end{array}$ \\
\hline \multicolumn{2}{|c|}{ MLU } & $\begin{array}{c}.057^{\mathrm{f}} \\
{[-.301, .378]}\end{array}$ & $\begin{array}{c}.359^{* c} \\
{[.018, .661]}\end{array}$ & $\begin{array}{c}-.137^{\mathrm{g}} \\
{[-.629, .305]}\end{array}$ & $\begin{array}{c}-.182^{\mathrm{h}} \\
{[-.224, .568]}\end{array}$ \\
\hline \multirow{3}{*}{ 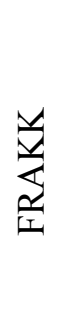 } & $\begin{array}{l}\text { Orientierung an Her- } \\
\text { kunftskultur }\end{array}$ & $\begin{array}{c}-.094^{\mathrm{i}} \\
{[-.549, .429]}\end{array}$ & $\begin{array}{c}-.135^{\mathrm{j}} \\
{[-.638, .417]}\end{array}$ & $\begin{array}{c}.289^{\mathrm{k}} \\
{[-.333, .845]}\end{array}$ & $\begin{array}{c}-.296^{1} \\
{[-.826, .333]}\end{array}$ \\
\hline & $\begin{array}{l}\text { Orientierung an Auf- } \\
\text { nahmekultur }\end{array}$ & $\begin{array}{c}.060^{\mathrm{i}} \\
{[-.437, .544]}\end{array}$ & $\begin{array}{c}-.235^{j} \\
{[-.648, .246]}\end{array}$ & $\begin{array}{c}.272^{\mathrm{k}} \\
{[-.280, .720]}\end{array}$ & $\begin{array}{c}-.189^{1} \\
{[-.780, .585]}\end{array}$ \\
\hline & Akkulturationsindex & $\begin{array}{c}.081^{\mathrm{i}} \\
{[-.375, .516]}\end{array}$ & $\begin{array}{c}-.267^{\mathrm{j}} \\
{[-.710, .216]}\end{array}$ & $\begin{array}{c}.019^{\mathrm{k}} \\
{[-.580, .651]}\end{array}$ & $\begin{array}{c}-.222^{1} \\
{[-.809, .478]}\end{array}$ \\
\hline \multicolumn{2}{|l|}{ KK } & $\begin{array}{c}.067^{\mathrm{m}} \\
{[-.260, .376]}\end{array}$ & $\begin{array}{c}.004^{\mathrm{d}} \\
{[-.308, .326]}\end{array}$ & $\begin{array}{c}.005^{\mathrm{e}} \\
{[-.350, .328]}\end{array}$ & $\begin{array}{c}-.327^{\mathrm{e}} \\
{[-.659, .006]}\end{array}$ \\
\hline \multicolumn{2}{|c|}{ SES } & $\begin{array}{c}.184^{\mathrm{m}} \\
{[-.097, .433]}\end{array}$ & $\begin{array}{c}.072^{\mathrm{d}} \\
{[-.248, .358]}\end{array}$ & $\begin{array}{c}-.035^{\mathrm{e}} \\
{[-.351, .305]}\end{array}$ & $\begin{array}{c}.149^{\mathrm{e}} \\
{[-.229, .504]}\end{array}$ \\
\hline
\end{tabular}

Anmerkungen. ${ }^{*} p<.05,{ }^{* *} p<.01, * * * p<.001,95 \%$ Konfidenzintervall in eckigen Klammern, ${ }^{\mathrm{a}} \mathrm{n}=28,{ }^{\mathrm{b}} \mathrm{n}=25,{ }^{\mathrm{c}} \mathrm{n}=20,{ }^{\mathrm{d}} \mathrm{n}=26,{ }^{\mathrm{e}} \mathrm{n}=21,{ }^{\mathrm{f}} \mathrm{n}=23,{ }^{\mathrm{g}} \mathrm{n}=15,{ }^{\mathrm{h}} \mathrm{n}=18,{ }^{\mathrm{i}} \mathrm{n}=14,{ }^{\mathrm{j}} \mathrm{n}=$ $13,{ }^{\mathrm{k}} \mathrm{n}=11,{ }^{1} \mathrm{n}=8,{ }^{\mathrm{m}} \mathrm{n}=29$ 
Tabelle A-14

Modellparameter für Zweiebenenmodelle (Modelle 1-3) mit dem Summenwert auf der Subskala Schweigen des Elternfragebogens als Outcome

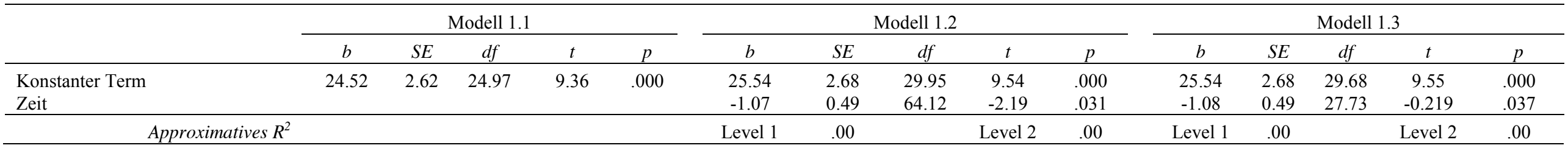

\section{Tabelle A-15}

Modellparameter für Zweiebenenmodelle (Modelle 4-6) mit dem Summenwert auf der Subskala Schweigen des Elternfragebogens als Outcome

\begin{tabular}{|c|c|c|c|c|c|c|c|c|c|c|c|c|c|c|c|}
\hline & \multicolumn{5}{|c|}{ Modell 1.4} & \multicolumn{5}{|c|}{ Modell 1.5} & \multicolumn{5}{|c|}{ Modell 1.6} \\
\hline & $b$ & $S E$ & $d f$ & $t$ & $p$ & $b$ & $S E$ & $d f$ & $t$ & $p$ & $b$ & $S E$ & $d f$ & $t$ & $p$ \\
\hline Konstanter Term & 24.73 & 2.27 & 31.42 & 10.89 & .000 & 44.42 & 8.91 & 28.68 & 4.98 & .000 & 29.34 & 3.96 & 27.15 & 7.42 & .000 \\
\hline Zeit & -1.19 & 0.50 & 62.90 & -2.38 & .020 & -1.21 & .50 & 63.12 & -2.43 & .018 & -1.18 & 0.50 & 62.79 & -2.37 & .021 \\
\hline Kulturelles Kapital & -1.83 & 1.42 & 25.07 & -1.29 & .210 & -7.22 & 3.15 & 28.00 & -2.29 & .029 & -1.86 & 1.42 & 25.03 & -1.31 & .202 \\
\hline Mehrsprachigkeit (dummykodiert) & -0.74 & 3.48 & 25.85 & -0.21 & .832 & -15.94 & 9.86 & 27.43 & -1.62 & .118 & 0.32 & 3.97 & 26.04 & 0.01 & .994 \\
\hline Gesamtangst (Median zentriert) & 0.69 & 0.11 & 25.08 & 6.36 & .000 & 0.68 & 0.23 & 27.25 & 2.90 & .007 & 0.66 & 0.13 & 25.66 & 4.99 & .000 \\
\hline $\begin{array}{l}\text { Grammatikverständnis (Median } \\
\text { zentriert) }\end{array}$ & 0.01 & 0.15 & 25.63 & 0.04 & .967 & 0.79 & 0.42 & 27.71 & 1.87 & .072 & 0.04 & 0.18 & 25.23 & 0.25 & .808 \\
\hline Mehrsprachigkeit * Kulturelles & & & & & & 6.11 & 3.54 & 27.22 & 1.73 & .095 & & & & & \\
\hline Kapital & & & & & & & & & & & & & & & \\
\hline Mehrsprachigkeit* Gesamtangst & & & & & & -0.01 & 0.26 & 26.77 & -.041 & .968 & & & & & \\
\hline $\begin{array}{l}\text { Mehrsprachigkeit* Grammatikver- } \\
\text { ständnis }\end{array}$ & & & & & & -0.86 & 0.45 & 27.49 & -1.92 & .065 & & & & & \\
\hline $\begin{array}{l}\text { Gesamtangst * Grammatikver- } \\
\text { ständnis }\end{array}$ & & & & & & & & & & & 0.00 & 0.01 & 25.95 & 0.40 & .693 \\
\hline Approximatives $R^{2}$ & Level 1 & .62 & & Level 2 & .69 & Level 1 & .67 & & Level 2 & .74 & Level 1 & .63 & & Level 2 & .70 \\
\hline
\end{tabular}


Tabelle A-16

Modellparameter für Zweiebenenmodelle (Modelle 1-3) mit dem Summenwert auf der Subskala Schweigen des Erzieherfragebogens als Outcome

\begin{tabular}{|c|c|c|c|c|c|c|c|c|c|c|c|c|c|c|c|}
\hline & \multicolumn{5}{|c|}{ Modell 1} & \multicolumn{5}{|c|}{ Modell 2} & \multicolumn{5}{|c|}{ Modell 3} \\
\hline & $b$ & $S E$ & $d f$ & $t$ & $p$ & $b$ & $S E$ & $d f$ & $t$ & $p$ & $b$ & $S E$ & $d f$ & $t$ & $p$ \\
\hline Konstanter Term & 17.73 & 2.24 & 24.18 & 7.92 & .000 & 22.55 & 2.43 & 33.13 & 9.30 & .000 & 21.40 & 2.64 & 25.76 & 8.11 & .000 \\
\hline Zeit & & & & & & -3.57 & 0.69 & 52.35 & -5.18 & .000 & -3.33 & 0.81 & 20.97 & -4.10 & .001 \\
\hline Approximatives $R^{2}$ & & & & & & Level 1 & .09 & & Level 2 & .00 & Level 1 & .09 & & Level 2 & .00 \\
\hline
\end{tabular}

Tabelle A-17

Modellparameter für Zweiebenenmodelle (Modelle 4-6) mit dem Summenwert auf der Subskala Schweigen des Erzieherfragebogens als Outcome

\begin{tabular}{|c|c|c|c|c|c|c|c|c|c|c|c|c|c|c|c|}
\hline & \multicolumn{5}{|c|}{ Modell 4} & \multicolumn{5}{|c|}{ Modell 5} & \multicolumn{5}{|c|}{ Modell 6} \\
\hline & $b$ & $S E$ & $d f$ & $t$ & $p$ & $b$ & $S E$ & $d f$ & $t$ & $p$ & $b$ & $S E$ & $d f$ & $t$ & $p$ \\
\hline Konstanter Term & 31.47 & 4.27 & 27.78 & 7.37 & .000 & 27.32 & 4.70 & 27.18 & 5.82 & .000 & 31.17 & 4.39 & 28.06 & 7.09 & .000 \\
\hline Zeit & -3.77 & 0.68 & 55.01 & -5.55 & .000 & -3.80 & 0.68 & 54.17 & -5.58 & .000 & -3.76 & 0.68 & 55.03 & -5.55 & .000 \\
\hline Kulturelles Kapital & -3.35 & 1.56 & 24.93 & -2.15 & .041 & -1.50 & 1.72 & 24.58 & -0.87 & .390 & -3.90 & 4.43 & 27.32 & -0.88 & .387 \\
\hline $\begin{array}{l}\text { Mehrsprachigkeit (dummyko- } \\
\text { diert) }\end{array}$ & -4.52 & 3.86 & 26.96 & -1.17 & .252 & 21.04 & 10.13 & 24.19 & 2.08 & .049 & -3.37 & 1.56 & 24.91 & -2.16 & .040 \\
\hline Gesamtangst (Median zentriert) & 0.33 & 0.12 & 25.48 & 2.72 & .011 & 0.38 & 0.12 & 24.09 & 3.25 & .003 & 0.30 & 0.14 & 24.70 & 2.11 & .045 \\
\hline $\begin{array}{l}\text { Grammatikverständnis (Median } \\
\text { zentriert) }\end{array}$ & -0.34 & 0.12 & 26.86 & -1.98 & .058 & -0.41 & 0.17 & 26.99 & -2.41 & .023 & -0.31 & .20 & 25.93 & -1.57 & .129 \\
\hline Mehrsprachigkeit * Kulturelles & & & & & & -9.56 & 3.62 & 23.79 & -2.64 & .015 & & & & & \\
\hline Kapital & & & & & & & & & & & & & & & \\
\hline Mehrsprachigkeit * Gesamtangst & & & & & & -0.38 & 0.27 & 26.02 & -1.36 & .187 & & & & & \\
\hline $\begin{array}{l}\text { Mehrsprachigkeit * Grammatik- } \\
\text { verständnis }\end{array}$ & & & & & & 1.00 & 0.47 & 25.56 & 2.14 & .042 & & & & & \\
\hline $\begin{array}{l}\text { Gesamtangst * Grammatikver- } \\
\text { ständnis }\end{array}$ & & & & & & & & & & & 0.00 & 0.01 & 25.33 & 0.29 & .777 \\
\hline Approximatives $R^{2}$ & Level 1 & .46 & & Level 2 & .49 & Level 1 & .54 & & Level 2 & .61 & Level 1 & .46 & & Level 2 & .50 \\
\hline
\end{tabular}


Anhang B: Elternfragebogen 


\section{Elternfragebogen}

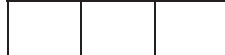

Dieser Bogen wurde am von folgender Person ausgefüllt

Mutter/ Stiefmutter/ Pflegemutter/ Adoptivmutter*

Vater/ Stiefvater/ Pflegevater/ Adoptivvater*

andere

* BITTE ZUTREFFENDES UNTERSTREICHEN

\section{Teil A Allgemeine Informationen zum Kind}

\section{Angaben zum Kind}

Name:

männlich

weiblich

Geburtsdatum:

1. Eintritt in den Kindergarten mit Jahren

2. Hat ihr Kind mehrere Kindergärten besucht?

ja, Anzahl

nein

3. Hatte Ihr Kind Schwierigkeiten bei der Eingewöhnung?

eher ja

eher nein

4. Geht Ihr Kind gerne in den Kindergarten?

eher ja

eher nein

5. Geht Ihr Kind regelmäßig in den Kindergarten?

eher ja

eher nein

6. Erhielt Ihr Kind bereits eine oder mehrere der folgenden Therapien?

MeHRFACHNENNUNGEN MÖGLICH

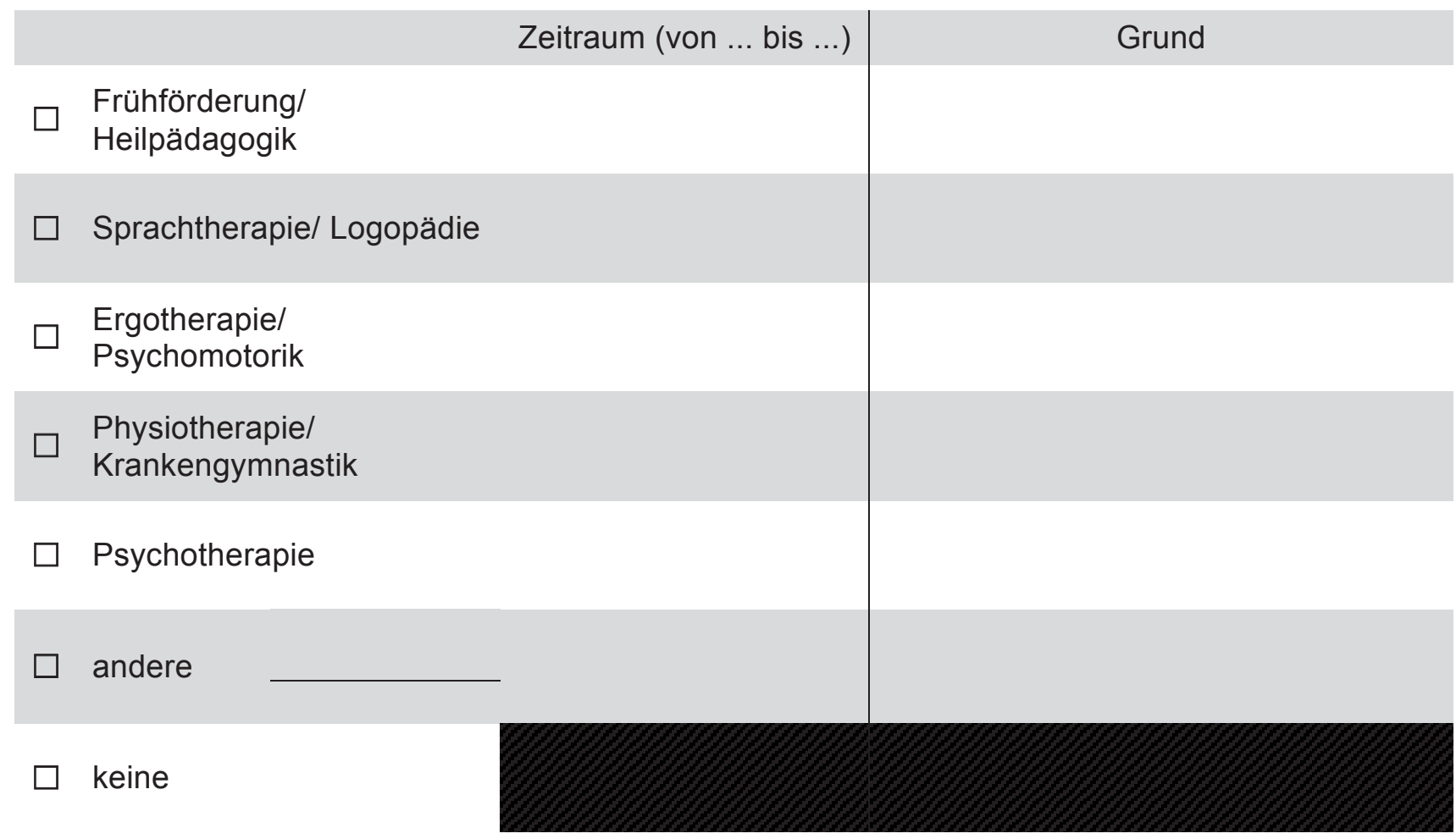




\section{Teil B Sprechen und Schweigen}

1. Mein Kind schweigt seit etwa...
1 - 6 Monaten
6 - 12 Monaten
1 - 2 Jahren
schon immer

weiß ich nicht

2. Wer hat das Schweigen Ihres Kindes als erstes angesprochen?

* BITTE ZUTREFFENDES UNTERSTREICHEN
Mutter/ Stief-/ Pflege-/ Adoptivmutter*
Vater/ Stief-/ Pflege-/ Adoptivvater
Großeltern
Freunde/ Bekannte der Eltern
Erzieher/in
Nachbarn
andere

3. Kann sich Ihr Kind in der deutschen Sprache verständigen?

ja

nein

eingeschränkt

Im Folgenden möchten wir ermitteln, welche Verhaltensweisen Ihr Kind in bestimmten Situationen zeigt. Kreuzen Sie dazu bitte für jede Aussage an, ob diese auf Ihr Kind zutrifft oder nicht (1= trifft gar nicht zu bis $5=$ trifft genau zu). Können Sie einzelne Aussagen nicht bewerten, kreuzen Sie bitte in der rechten Spalte "weiß nicht" an.

Beispiel: Bei Problemen kommt mein Kind zu uns, um darüber zu reden.

$\begin{array}{cccccc}1 & 2 & \aleph & 4 & 5 & \square \\ \begin{array}{ccc}\text { trifft } \\ \text { genau } \\ \text { zu }\end{array} & \begin{array}{c}\text { trifft } \\ \text { eher } \\ \text { nicht zu }\end{array} & \begin{array}{c}\text { teils/ } \\ \text { teils }\end{array} & \begin{array}{c}\text { trifft } \\ \text { eher } \\ \text { zu }\end{array} & \begin{array}{c}\text { trifft } \\ \text { genau } \\ \text { zu }\end{array} & \begin{array}{c}\text { weiß } \\ \text { nicht }\end{array}\end{array}$

Haben Sie bei dieser Aussage „3“ angekreuzt, bedeutet dies, dass Ihr Kind manchmal zu Ihnen kommt, um über Probleme zu reden und manchmal nicht zu Ihnen kommt, um über Probleme zu reden.

\begin{tabular}{|c|c|c|c|c|c|c|c|}
\hline & & $\begin{array}{c}\text { trifft } \\
\text { gar } \\
\text { nicht } \\
\text { zu }\end{array}$ & $\begin{array}{c}\text { trifft } \\
\text { eher } \\
\text { nicht } \\
\text { zu }\end{array}$ & teils/teils & $\begin{array}{l}\text { trifft } \\
\text { eher } \\
\text { zu }\end{array}$ & $\begin{array}{c}\text { trifft } \\
\text { genau } \\
\text { zu }\end{array}$ & $\begin{array}{l}\text { weiß } \\
\text { nicht }\end{array}$ \\
\hline 1 & $\begin{array}{l}\text { Mein Kind zieht sich zurück, wenn Besuch } \\
\text { kommt (z.B. geht in sein Zimmer, hört auf zu } \\
\text { sprechen). }\end{array}$ & 1 & 2 & 3 & 4 & 5 & $\square$ \\
\hline 2 & $\begin{array}{l}\text { Bei Problemen kommt mein Kind zu uns, um } \\
\text { darüber zu reden. }\end{array}$ & 1 & 2 & 3 & 4 & 5 & $\square$ \\
\hline 3 & $\begin{array}{l}\text { Wenn mein Kind etwas kaputt macht oder etwas } \\
\text { verschüttet, weint es. }\end{array}$ & 1 & 2 & 3 & 4 & 5 & $\square$ \\
\hline 4 & $\begin{array}{l}\text { Mit Veränderungen des gewohnten } \\
\text { Tagesablaufs geht mein Kind problemlos um. }\end{array}$ & 1 & 2 & 3 & 4 & 5 & $\square$ \\
\hline 5 & Über Besuch freut sich mein Kind. & 1 & 2 & 3 & 4 & 5 & $\square$ \\
\hline 6 & $\begin{array}{l}\text { Bei Problemen zieht sich mein Kind in sein } \\
\text { Zimmer zurück. }\end{array}$ & 1 & 2 & 3 & 4 & 5 & $\square$ \\
\hline 7 & $\begin{array}{l}\text { Mein Kind begrüßt Verwandte ohne } \\
\text { Aufforderung. }\end{array}$ & 1 & 2 & 3 & 4 & 5 & $\square$ \\
\hline 8 & $\begin{array}{l}\text { Mein Kind spielt bei Familienfesten mit anderen } \\
\text { Kindern. }\end{array}$ & 1 & 2 & 3 & 4 & 5 & $\square$ \\
\hline
\end{tabular}




\begin{tabular}{|c|c|c|c|c|c|c|c|}
\hline & & $\begin{array}{c}\text { trifft } \\
\text { gar } \\
\text { nicht } \\
\mathrm{zu}\end{array}$ & $\begin{array}{c}\text { trifft } \\
\text { eher } \\
\text { nicht } \\
\text { zu }\end{array}$ & teils/teils & $\begin{array}{l}\text { trifft } \\
\text { eher } \\
\text { zu }\end{array}$ & $\begin{array}{c}\text { trifft } \\
\text { genau } \\
\text { zu }\end{array}$ & $\begin{array}{l}\text { weiß } \\
\text { nicht }\end{array}$ \\
\hline 9 & $\begin{array}{l}\text { Bei Familienfesten bleibt mein Kind in unserer } \\
\text { Nähe. }\end{array}$ & 1 & 2 & 3 & 4 & 5 & $\square$ \\
\hline 10 & $\begin{array}{l}\text { Mein Kind begrüßt Nachbarn ohne } \\
\text { Aufforderung. }\end{array}$ & 1 & 2 & 3 & 4 & 5 & $\square$ \\
\hline 11 & $\begin{array}{l}\text { Bei Familienfesten zeigt mein Kind sein } \\
\text { gewöhnliches Essverhalten. }\end{array}$ & 1 & 2 & 3 & 4 & 5 & $\square$ \\
\hline 12 & $\begin{array}{l}\text { Mein Kind gibt anderen zur Begrüßung die } \\
\text { Hand. }\end{array}$ & 1 & 2 & 3 & 4 & 5 & $\square$ \\
\hline 13 & $\begin{array}{l}\text { Bei Familienfesten benötigt mein Kind eine } \\
\text { Aufwärmphase. }\end{array}$ & 1 & 2 & 3 & 4 & 5 & $\square$ \\
\hline 14 & $\begin{array}{l}\text { Wenn mein Kind von Verwandten oder } \\
\text { Nachbarn begrüßt wird, grüßt es zurück. }\end{array}$ & 1 & 2 & 3 & 4 & 5 & $\square$ \\
\hline 15 & $\begin{array}{l}\text { Auf dem Spielplatz spielt mein Kind mit anderen } \\
\text { Kindern. }\end{array}$ & 1 & 2 & 3 & 4 & 5 & $\square$ \\
\hline 16 & $\begin{array}{l}\text { Bei Familienfesten verweigert mein Kind das } \\
\text { Essen. }\end{array}$ & 1 & 2 & 3 & 4 & 5 & $\square$ \\
\hline 17 & $\begin{array}{l}\text { Zu anderen Verwandten nimmt mein Kind nur } \\
\text { zusammen mit Geschwistern Kontakt auf. }\end{array}$ & 1 & 2 & 3 & 4 & 5 & $\square$ \\
\hline 18 & Mein Kind freut sich auf Familienfeste. & 1 & 2 & 3 & 4 & 5 & $\square$ \\
\hline 19 & $\begin{array}{l}\text { Mein Kind spielt nur nach Aufforderung mit } \\
\text { anderen Kindern. }\end{array}$ & 1 & 2 & 3 & 4 & 5 & $\square$ \\
\hline 20 & $\begin{array}{l}\text { Bei Familienfesten spricht mein Kind mit den } \\
\text { anderen Kindern. }\end{array}$ & 1 & 2 & 3 & 4 & 5 & $\square$ \\
\hline 21 & $\begin{array}{l}\text { Mein Kind weigert sich, Bekannte oder } \\
\text { Verwandte zu begrüßen. }\end{array}$ & 1 & 2 & 3 & 4 & 5 & $\square$ \\
\hline 22 & $\begin{array}{l}\text { Beim Einkaufen nimmt mein Kind kleine } \\
\text { Geschenke von Verkäufern an (z.B. Bonbons, } \\
\text { eine Wurstscheibe). }\end{array}$ & 1 & 2 & 3 & 4 & 5 & $\square$ \\
\hline 23 & $\begin{array}{l}\text { Mein Kind isst in Anwesenheit fremder Personen } \\
\text { z.B. Obst, Brötchen, Schokoriegel. }\end{array}$ & 1 & 2 & 3 & 4 & 5 & $\square$ \\
\hline 24 & $\begin{array}{l}\text { Mein Kind kauft selbstständig z.B. Spielzeug, } \\
\text { Aufkleber oder Süßigkeiten ein. }\end{array}$ & 1 & 2 & 3 & 4 & 5 & $\square$ \\
\hline 25 & Mein Kind bestellt z.B. in der Eisdiele selbst. & 1 & 2 & 3 & 4 & 5 & $\square$ \\
\hline 26 & $\begin{array}{l}\text { Mein Kind vermeidet Menschansammlungen } \\
\text { (z.B. volle Räume, volle Läden). }\end{array}$ & 1 & 2 & 3 & 4 & 5 & $\square$ \\
\hline 27 & $\begin{array}{l}\text { Wenn wir in der Stadt Bekannte treffen, hält } \\
\text { unser Kind Körperkontakt zu uns (z.B. bleibt an } \\
\text { der Hand, hält meine Jacke fest). }\end{array}$ & 1 & 2 & 3 & 4 & 5 & $\square$ \\
\hline 28 & Mein Kind spricht mit mir in der Öffentlichkeit. & 1 & 2 & 3 & 4 & 5 & $\square$ \\
\hline 29 & $\begin{array}{l}\text { Mein Kind schweigt, wenn es von einem } \\
\text { Verkäufer angesprochen wird. }\end{array}$ & 1 & 2 & 3 & 4 & 5 & $\square$ \\
\hline 30 & $\begin{array}{l}\text { In Gegenwart fremder Personen flüstert mein } \\
\text { Kind mit mir. }\end{array}$ & 1 & 2 & 3 & 4 & 5 & $\square$ \\
\hline 31 & Mein Kind spricht zuhause mit uns völlig normal. & 1 & 2 & 3 & 4 & 5 & $\square$ \\
\hline 32 & Beim Zahnarzt öffnet mein Kind den Mund. & 1 & 2 & 3 & 4 & 5 & $\square$ \\
\hline 33 & $\begin{array}{l}\text { Mein Kind schweigt in Gegenwart seiner } \\
\text { Großeltern }\end{array}$ & 1 & 2 & 3 & 4 & 5 & $\square$ \\
\hline 34 & $\begin{array}{l}\text { Mein Kind weigert sich bereits zuhause, zum } \\
\text { Arzt zu gehen. }\end{array}$ & 1 & 2 & 3 & 4 & 5 & $\square$ \\
\hline 35 & Mit seinen Geschwistern spricht mein Kind. & 1 & 2 & 3 & 4 & 5 & $\square$ \\
\hline 36 & Mein Kind weint viel beim Arzt. & 1 & 2 & 3 & 4 & 5 & $\square$ \\
\hline
\end{tabular}




\begin{tabular}{|c|c|c|c|c|c|c|c|}
\hline & & $\begin{array}{c}\text { trifft } \\
\text { gar } \\
\text { nicht } \\
\mathbf{z u}\end{array}$ & $\begin{array}{c}\text { trifft } \\
\text { eher } \\
\text { nicht } \\
\text { zu }\end{array}$ & teils/teils & $\begin{array}{l}\text { trifft } \\
\text { eher } \\
\mathrm{zu}\end{array}$ & $\begin{array}{c}\text { trifft } \\
\text { genau } \\
\text { zu }\end{array}$ & $\begin{array}{l}\text { weiß } \\
\text { nicht }\end{array}$ \\
\hline 37 & $\begin{array}{l}\text { Mein Kind spielt mit Kindern aus der } \\
\text { Nachbarschaft. }\end{array}$ & 1 & 2 & 3 & 4 & 5 & $\square$ \\
\hline 38 & $\begin{array}{l}\text { Mein Kind schweigt in Gegenwart des } \\
\text { Kinderarztes. }\end{array}$ & 1 & 2 & 3 & 4 & 5 & $\square$ \\
\hline 39 & $\begin{array}{l}\text { Sobald wir aus dem Haus treten, schweigt mein } \\
\text { Kind. }\end{array}$ & 1 & 2 & 3 & 4 & 5 & $\square$ \\
\hline 40 & $\begin{array}{l}\text { Mit kleinen Kindern der Verwandtschaft spricht } \\
\text { mein Kind. }\end{array}$ & 1 & 2 & 3 & 4 & 5 & $\square$ \\
\hline 41 & Mein Kind lässt sich vom Arzt untersuchen. & 1 & 2 & 3 & 4 & 5 & $\square$ \\
\hline 42 & $\begin{array}{l}\text { Mit Erwachsenen der Verwandtschaft schweigt } \\
\text { mein Kind. }\end{array}$ & 1 & 2 & 3 & 4 & 5 & $\square$ \\
\hline 43 & $\begin{array}{l}\text { Ja und Nein drückt mein Kind durch } \\
\text { Kopfschütteln und Nicken aus. }\end{array}$ & 1 & 2 & 3 & 4 & 5 & $\square$ \\
\hline 44 & $\begin{array}{l}\text { Meinem Kind fällt es schwer, fremde Kinder } \\
\text { anzusprechen. }\end{array}$ & 1 & 2 & 3 & 4 & 5 & $\square$ \\
\hline 45 & $\begin{array}{l}\text { Mein Kind schweigt in Gegenwart von älteren } \\
\text { Kindern der Nachbarschaft. }\end{array}$ & 1 & 2 & 3 & 4 & 5 & $\square$ \\
\hline 46 & $\begin{array}{l}\text { In der Spielgruppe spielt mein Kind mit den } \\
\text { anderen Kindern. }\end{array}$ & 1 & 2 & 3 & 4 & 5 & $\square$ \\
\hline 47 & $\begin{array}{l}\text { Mit gleichaltrigen Kindern der Verwandtschaft } \\
\text { spricht mein Kind. }\end{array}$ & 1 & 2 & 3 & 4 & 5 & $\square$ \\
\hline 48 & Mein Kind erstarrt völlig, wenn es schweigt. & 1 & 2 & 3 & 4 & 5 & $\square$ \\
\hline 49 & $\begin{array}{l}\text { Auch wenn mein Kind schweigt, zeigt es Freude } \\
\text { durch Lächeln oder Lachen. }\end{array}$ & 1 & 2 & 3 & 4 & 5 & $\square$ \\
\hline 50 & $\begin{array}{l}\text { In Gegenwart fremder Personen vermeidet mein } \\
\text { Kind Husten, Niesen oder Räuspern. }\end{array}$ & 1 & 2 & 3 & 4 & 5 & $\square$ \\
\hline 51 & Auf dem Spielplatz spielt mein Kind alleine. & 1 & 2 & 3 & 4 & 5 & $\square$ \\
\hline 52 & $\begin{array}{l}\text { Mein Kind äußert seine Wünsche und } \\
\text { Bedürfnisse nicht in der Öffentlichkeit. }\end{array}$ & 1 & 2 & 3 & 4 & 5 & $\square$ \\
\hline 53 & $\begin{array}{l}\text { Mein Kind fährt gern mit dem Bus oder geht mit } \\
\text { mir einkaufen. }\end{array}$ & 1 & 2 & 3 & 4 & 5 & $\square$ \\
\hline 54 & $\begin{array}{l}\text { Wenn wir Bekannte in der Stadt treffen, verhält } \\
\text { sich unser Kind unbefangen. }\end{array}$ & 1 & 2 & 3 & 4 & 5 & $\square$ \\
\hline 55 & $\begin{array}{l}\text { Wenn mein Kind schweigt, drückt es Wünsche } \\
\text { oder Bedürfnisse durch Zeigen aus. }\end{array}$ & 1 & 2 & 3 & 4 & 5 & $\square$ \\
\hline 56 & In der Spielgruppe spielt mein Kind alleine. & 1 & 2 & 3 & 4 & 5 & $\square$ \\
\hline 57 & $\begin{array}{l}\text { In der Öffentlichkeit äußert mein Kind seine } \\
\text { Wünsche und Bedürfnisse. }\end{array}$ & 1 & 2 & 3 & 4 & 5 & $\square$ \\
\hline 58 & $\begin{array}{l}\text { Mein Kind nimmt keinen Blickkontakt auf, wenn } \\
\text { es angesprochen wird. }\end{array}$ & 1 & 2 & 3 & 4 & 5 & $\square$ \\
\hline 59 & $\begin{array}{l}\text { Bei Menschenansammlungen (z.B. im Bus) } \\
\text { klammert sich mein Kind an mich. }\end{array}$ & 1 & 2 & 3 & 4 & 5 & $\square$ \\
\hline
\end{tabular}




\section{Teil C Allgemeine Entwicklung}

\section{Schwangerschaft und Geburt}

EVtL. LAUt ANGaben Im MutTerpass

1. Gab es während der Schwangerschaft Komplikationen (z.B. Krankheit der Mutter, Blutungen, Unfälle, Infektionen)?

$\square$ ja $\quad \square$ nein $\quad \square$ weiß nicht

2. In der wievielten Schwangerschaftswoche wurde Ihr Kind geboren? Woche

3. Wie hoch war das Geburtsgewicht? Gramm

4. Gab es Komplikationen bei der Entbindung (z.B. Kaiserschnitt, Nabelschnurumschlingungen, Saugglocke, Zangengeburt, falsche Lage des Kindes)?
ja
nein

5. Lag eine Mehrlingsgeburt vor (Zwilling, Drilling)?
ja
nein

\section{Entwicklung}

EVTL. LAUT ANGABEN IM VoRsoRgeheFT

6. Mit wie viel Monaten konnte Ihr Kind Folgendes?

$\begin{array}{llr}\text { alleine sitzen } & \text { ca. Monate } \\ \text { krabbeln } & \text { ca. Monate } \\ \text { alleine stehen } & \text { ca. Monate } \\ \text { erste Schritte } & \text { ca. Monate } \\ \text { erste Worte („Mama“, „Papa“) } & \text { ca. Monate } \\ \text { erste Zweiwortsätze („Mama da“) } & \text { ca. Monate }\end{array}$

7. Gibt/ gab es in der Entwicklung Ihres Kindes eine oder mehrere der folgenden Auffälligkeiten?

\begin{tabular}{lll} 
motorische Entwicklungsverzögerung/-störung & $\square$ ja & $\square$ nein \\
geistige Entwicklungsverzögerung/-störung & $\square$ ja & $\square$ nein \\
sprachliche Entwicklungsverzögerung/-störung & $\square$ ja \\
\hline zeitweilige Hörprobleme (z.B. häufige Mittelohrentzündungen) & $\square$ ja \\
anderes & $\square$ ja nein \\
\hline
\end{tabular}




\section{weitere Entwicklungsprobleme}

8. Liegen bei Ihrem Kind eine oder mehrere der folgenden Probleme vor?

MEHRFACHNENNUNGEN MÖGLICH

$\begin{array}{lll}\square \text { Einnässen } & \square \quad \text { Einkoten } \\ \square \text { Verstopfungen } & \square \quad \text { Hyperaktivität } \\ \square \text { Aggressivität } & \square \quad \text { Autismus } \\ \square \quad \begin{array}{l}\text { Zwänge/ zwanghaftes Verhalten (z.B. } \\ \text { Festhalten an vertrauten Abläufen) }\end{array} & \square \quad \begin{array}{l}\text { Tics (unwillkürliche Bewegungen oder } \\ \text { Lautäußerungen) }\end{array} \\ \square \text { Essstörungen } & \square \text { keine }\end{array}$

9. Wurden bei Ihrem Kind weitere Erkrankungen diagnostiziert?

ja

nein

Wenn ja, welche

\section{Teil D Sprachliche Entwicklung}

1. Welche Sprache(n) spricht Ihr Kind?

MEHRFACHNENNUNGEN MÖGLICH

$\begin{array}{lll}\square \text { Deutsch } & \square \text { Englisch } & \square \text { Spanisch } \\ \square \text { Französisch } & \square \text { Türkisch } & \square \text { Polnisch } \\ \square \text { Russisch } & \square \text { Chinesisch } & \square \text { Japanisch } \\ \square \text { Griechisch } & \square \text { Portugiesisch } & \square \text { Italienisch } \\ \square \text { Arabisch } & \square \text { Niederländisch } & \square \text { andere }\end{array}$

2. Lagen/ liegen bei Ihrem Kind eine oder mehrere der folgenden sprachlichen Probleme vor? MeHRFACHNENNUNGEN MÖGLICH

keine

später Sprechbeginn

kann/ konnte viele Laute nicht richtig aussprechen (z.B. /k, g/, /sch/)

kann/ konnte die Mehrzahl noch nicht richtig bilden (z.B. „Vogeln“ anstatt „Vögel“)

hat/ hatte Probleme bei der Satzbildung (z.B. „Mama Auto fahren“ statt „Mama fährt Auto")

sucht/e häufig nach Wörtern

stottert/e

„nuschelt/e“ und spricht/sprach häufig zu schnell

anderes 


\section{Teil E weitere Hintergrundinformationen}

1. Was hat Ihrer Meinung nach zur Ausprägung des Schweigens bei Ihrem Kind geführt?

2. War Ihr Kind zeitweise von seiner Bezugsperson getrennt?

(z.B. durch Heim-, Internats- oder Krankenhausaufenthalte)
1. Lebensjahr
nein Grund
2. Lebensjahr
nein Grund
3. Lebensjahr
ja
nein Grund
4. Lebensjahr
ja
nein Grund

3. Ist Ihr Kind bereits ein oder mehrere Male in eine andere Stadt umgezogen?
ja
nein
Anzahl der Umzüge

Wenn ja, in welchem Lebensalter fand/en der Umzug/ die Umzüge statt?

BITTE ZUTREFFENDES ANKREUZEN

$\begin{array}{lllllll}\text { Lebensjahr } & 1 & 2 & 3 & 4 & 5 & 6\end{array}$

4. Ist Ihr Kind bereits ein oder mehrere Male in ein anderes Land umgezogen?
ja
nein
Anzahl der Umzüge

Wenn ja, in welchem Lebensalter fand/en der Umzug/ die Umzüge statt?

BITTE ZUTREFFENDES ANKREUZEN

$\begin{array}{lllllll}\text { Lebensjahr } & 1 & 2 & 3 & 4 & 5 & 6\end{array}$

5. Hat Ihr Kind eine Trennung seiner Eltern miterlebt?

ja

nein

Wenn ja, wie alt war Ihr Kind bei der Trennung?

BITTE ZUTREFFENDES ANKREUZEN

$\begin{array}{lllllll}\text { Lebensjahr } & 1 & 2 & 3 & 4 & 5 & 6\end{array}$

6. War Ihr Kind schon ein Mal einem traumatischen Erlebnis ausgesetzt?

(z.B. Autounfall, schwere Krankheit, Verlust einer nahestehenden Person, Gewalterfahrungen)
ja
nein

Wenn ja, welches

Wie alt war Ihr Kind bei diesem Erlebnis?

BITTE ZUTREFFENDES ANKREUZEN

$\begin{array}{lllllll}\text { Lebensjahr } & 1 & 2 & 3 & 4 & 5 & 6\end{array}$


7. Gibt oder gab es in der Entwicklung Ihres Kindes weitere Besonderheiten, die Sie für erwähnenswert halten?

\section{Teil F Informationen zur Familie und zum Haushalt}

1. Welche Postleitzahl hat Ihr aktueller Haushalt?

2. Wie viele Personen leben im Haushalt des Kindes? Personen

3. Wer wohnt im Haushalt des Kindes?

MEHRFACHNENNUNGEN MÖGLICH

$\begin{array}{llll}\square & \text { leibliche Mutter } & \square \text { leiblicher Vater } & \square \text { Stiefmutter } \\ \square & \text { Stiefvater } & \square \text { Pflegeeltern } & \square \text { Adoptiveltern } \\ \square \quad \text { leibliche Geschwister } & \square \text { Halbgeschwister } & \square \text { Stiefgeschwister } \\ \square \quad \text { Großeltern } & \square \text { andere } & \end{array}$

Angaben zur Mutter/ Stiefmutter/ Pflegemutter/ Adoptivmutter*

* BitTE ZUTREFFEnDES UNTERSTREICHEN

Alter:

Muttersprache

Wie lange lebt die Mutter schon in Deutschland?

$\square$ schon immer

$\square$ seit Jahren

$\square$ weiß nicht

Höchster Bildungsabschluss
$\square$ noch Schüler/in
$\square \quad$ Abschluss einer Förderschule
$\square$ Hauptschulabschluss
$\square \quad$ Realschulabschluss
abgeschlossenes
Hochschulstudium
Promotion
$\square \quad$ anderer Abschluss
Hat die Mutter einen Beruf erlernt?
$\square$ ja
ja $\quad \square$ nein
Wenn ja, welchen?
Ist die Mutter zurzeit berufstätig?

$\begin{array}{ll}\square & \text { ja, in Teilzeit mit } \\ \square \quad \text { ja, in Vollzeit } & \text { Stunden pro Woche } \\ \square & \text { nein }\end{array}$

kein Schulabschluss

$\square$ (Fach-)Hochschulreife/ (Fach-)Abitur 
Alter:

Muttersprache

Wie lange lebt der Vater schon in Deutschland?

$\square$ schon immer

$\square \quad$ seit $\_$wahren
$\square \quad$ weiß nicht

Höchster Bildungsabschluss
$\square$ noch Schüler/in
kein Schulabschluss
$\square \quad$ Abschluss einer Förderschule
$\square$ Hauptschulabschluss
$\square \quad$ Realschulabschluss
$\square$ (Fach-)Hochschulreife/ (Fach-)Abitur
$\square \quad$ abgeschlossenes
Hochschulstudium
$\square$ Promotion
$\square \quad$ anderer Abschluss

Hat der Vater einen Beruf erlernt?
$\square$ ja
$\square \quad$ nein

Wenn ja, welchen?

Ist der Vater zurzeit berufstätig?
$\square \quad$ ja, in Teilzeit mit
$\square$ ja, in Vollzeit
$\square$ nein Stunden pro Woche

\section{Angaben zu den Geschwistern/ Halbgeschwistern/ Stiefgeschwistern}

\begin{tabular}{|c|c|c|c|c|c|c|c|c|c|}
\hline Initialen & Gesc & lec & & Alter & Wohnt & eich & en $\mathrm{Ha}$ & & \\
\hline 1. & $\square \mathrm{m}$ & $\square$ & w & & $\square$ ja & $\square$ & nein & $\square$ & zeitweise \\
\hline 2. & $\square \mathrm{m}$ & $\square$ & w & & $\square$ ja & $\square$ & nein & $\square$ & zeitweise \\
\hline 3. & $\square \mathrm{m}$ & $\square$ & w & & $\square$ ja & $\square$ & nein & $\square$ & zeitweise \\
\hline 4. & $\square \mathrm{m}$ & $\square$ & w & & $\square$ ja & $\square$ & nein & $\square$ & zeitweise \\
\hline 5. & $\square \mathrm{m}$ & $\square$ & $w$ & & $\square$ ja & $\square$ & nein & $\square$ & zeitweise \\
\hline 6. & $\square \mathrm{m}$ & $\square$ & w & & $\square$ ja & $\square$ & nein & $\square$ & zeitweise \\
\hline 7. & $\square \mathrm{m}$ & $\square$ & w & & $\square$ ja & $\square$ & nein & $\square$ & zeitweise \\
\hline 8. & $\square \mathrm{m}$ & $\square$ & w & & $\square$ ja & $\square$ & nein & $\square$ & zeitweise \\
\hline
\end{tabular}

4. Wer ist/sind Ihrer Meinung nach die wichtigste/n Bezugsperson/en für Ihr Kind?
MeHRFACHNENNUNGEN MÖGLICH
* BITTE ZUTREFFEndEs UNTERSTREICHEN
Mutter/ Stiefmutter/ Pflegemutter/ Adoptivmutter*
$\square$ Oma
Vater/ Stiefvater/ Pflegevater/ Adoptivvater*
Opa
Bruder/ Schwester*
andere 
Bitte geben Sie im Folgenden an, wie sehr die jeweilige Aussage auf Mutter, Vater und Geschwister zutrifft.

Schüchternheit

\begin{tabular}{|c|c|c|c|c|c|c|c|c|c|c|c|c|}
\hline & \multirow{2}{*}{\multicolumn{4}{|c|}{ Mutter }} & & & & & & & & \\
\hline & & & & & \multicolumn{4}{|c|}{ Vater } & \multicolumn{4}{|c|}{ Geschwister } \\
\hline & $\begin{array}{l}\text { trifft } \\
\text { gar } \\
\text { nicht } \\
\text { zu }\end{array}$ & $\begin{array}{l}\text { teils/ } \\
\text { teils }\end{array}$ & $\begin{array}{c}\text { trifft } \\
\text { genau } \\
\text { zu }\end{array}$ & $\begin{array}{l}\text { weiß } \\
\text { nicht }\end{array}$ & $\begin{array}{c}\text { trifft } \\
\text { gar } \\
\text { nicht } \\
\text { zu }\end{array}$ & $\begin{array}{l}\text { teils/ } \\
\text { teils }\end{array}$ & $\begin{array}{c}\text { trifft } \\
\text { genau } \\
\text { zu }\end{array}$ & $\begin{array}{l}\text { weiß } \\
\text { nicht }\end{array}$ & $\begin{array}{c}\text { trifft } \\
\text { gar } \\
\text { nicht } \\
\text { zu }\end{array}$ & $\begin{array}{l}\text { teils/ } \\
\text { teils }\end{array}$ & $\begin{array}{c}\text { trifft } \\
\text { genau } \\
\text { zu }\end{array}$ & $\begin{array}{l}\text { weiß } \\
\text { nicht }\end{array}$ \\
\hline $\begin{array}{l}\text { Ist gegenüber } \\
\text { fremden Personen } \\
\text { ruhig und } \\
\text { zurückhaltend }\end{array}$ & 1 & 2 & 3 & $\square$ & 1 & 2 & 3 & $\square$ & 1 & 2 & 3 & $\square$ \\
\hline $\begin{array}{l}\text { geht auf } \\
\text { andere/fremde } \\
\text { Personen zu } \\
\text { (z.B. auf } \\
\text { Geburtstagsfeiern) }\end{array}$ & 1 & 2 & 3 & $\square$ & 1 & 2 & 3 & $\square$ & 1 & 2 & 3 & $\square$ \\
\hline $\begin{array}{l}\text { Äußert innerhalb der } \\
\text { Familie seine eigene } \\
\text { Meinung }\end{array}$ & 1 & 2 & 3 & $\square$ & 1 & 2 & 3 & $\square$ & 1 & 2 & 3 & $\square$ \\
\hline $\begin{array}{l}\text { Beteiligt sich } \\
\text { innerhalb der Familie } \\
\text { kaum an Gesprächen }\end{array}$ & 1 & 2 & 3 & $\square$ & 1 & 2 & 3 & $\square$ & 1 & 2 & 3 & $\square$ \\
\hline $\begin{array}{l}\text { Verhält sich } \\
\text { zurückhaltend oder } \\
\text { vorsichtig bei } \\
\text { Behördengängen/ } \\
\text { Arztbesuchen, etc. }\end{array}$ & 1 & 2 & 3 & $\square$ & 1 & 2 & 3 & $\square$ & 1 & 2 & 3 & $\square$ \\
\hline
\end{tabular}

\begin{tabular}{|c|c|c|c|c|c|c|c|c|c|c|c|c|}
\hline & \multicolumn{8}{|c|}{ Ängste } & \multirow{2}{*}{\multicolumn{4}{|c|}{ Geschwister }} \\
\hline & \multicolumn{4}{|c|}{ Mutter } & \multicolumn{4}{|c|}{ Vater } & & & & \\
\hline & $\begin{array}{l}\text { trifft } \\
\text { gar } \\
\text { nicht } \\
\text { zu }\end{array}$ & $\begin{array}{l}\text { teils/ } \\
\text { teils }\end{array}$ & $\begin{array}{c}\text { trifft } \\
\text { genau } \\
\text { zu }\end{array}$ & $\begin{array}{l}\text { weiß } \\
\text { nicht }\end{array}$ & $\begin{array}{c}\text { trifft } \\
\text { gar } \\
\text { nicht } \\
\text { zu }\end{array}$ & $\begin{array}{l}\text { teils/ } \\
\text { teils }\end{array}$ & $\begin{array}{c}\text { trifft } \\
\text { genau } \\
\text { zu }\end{array}$ & $\begin{array}{l}\text { weiß } \\
\text { nicht }\end{array}$ & $\begin{array}{l}\text { trifft } \\
\text { gar } \\
\text { nicht } \\
\text { zu }\end{array}$ & $\begin{array}{l}\text { teils/ } \\
\text { teils }\end{array}$ & $\begin{array}{c}\text { trifft } \\
\text { genau } \\
\text { zu }\end{array}$ & $\begin{array}{l}\text { weiß } \\
\text { nicht }\end{array}$ \\
\hline $\begin{array}{l}\text { Scheut sich vor } \\
\text { Neuem (z.B. Umzug } \\
\text { in eine andere Stadt, } \\
\text { neuer Arbeitsplatz, } \\
\text { Schulwechsel etc.) }\end{array}$ & 1 & 2 & 3 & $\square$ & 1 & 2 & 3 & $\square$ & 1 & 2 & 3 & $\square$ \\
\hline $\begin{array}{l}\text { Hat große Angst vor } \\
\text { bestimmten Dingen } \\
\text { oder Situationen } \\
\text { (vor Spinnen, } \\
\text { Höhenangst, engen } \\
\text { Räumen, } \\
\text { Menschenmassen, } \\
\text { etc.) }\end{array}$ & 1 & 2 & 3 & $\square$ & 1 & 2 & 3 & $\square$ & 1 & 2 & 3 & $\square$ \\
\hline $\begin{array}{l}\text { Vermeidet Kontakte } \\
\text { zu anderen Personen } \\
\text { außerhalb der Familie }\end{array}$ & 1 & 2 & 3 & $\square$ & 1 & 2 & 3 & $\square$ & 1 & 2 & 3 & $\square$ \\
\hline $\begin{array}{l}\text { Besucht gerne } \\
\text { Veranstaltungen mit } \\
\text { vielen Menschen }\end{array}$ & 1 & 2 & 3 & $\square$ & 1 & 2 & 3 & $\square$ & 1 & 2 & 3 & $\square$ \\
\hline
\end{tabular}


Selektiver Mutismus

\begin{tabular}{|c|c|c|c|c|c|c|c|c|c|c|c|c|}
\hline & \multicolumn{4}{|c|}{ Mutter } & \multicolumn{4}{|c|}{ Vater } & \multicolumn{4}{|c|}{ Geschwister } \\
\hline & $\begin{array}{c}\text { trifft } \\
\text { gar } \\
\text { nicht } \\
\text { zu }\end{array}$ & $\begin{array}{l}\text { teils/ } \\
\text { teils }\end{array}$ & $\begin{array}{c}\text { trifft } \\
\text { genau } \\
\text { zu }\end{array}$ & $\begin{array}{l}\text { weiß } \\
\text { nicht }\end{array}$ & $\begin{array}{c}\text { trifft } \\
\text { gar } \\
\text { nicht } \\
\text { zu }\end{array}$ & $\begin{array}{l}\text { teils/ } \\
\text { teils }\end{array}$ & $\begin{array}{c}\text { trifft } \\
\text { genau } \\
\text { zu }\end{array}$ & $\begin{array}{l}\text { weiß } \\
\text { nicht }\end{array}$ & $\begin{array}{c}\text { trifft } \\
\text { gar } \\
\text { nicht } \\
\text { zu }\end{array}$ & $\begin{array}{l}\text { teils/ } \\
\text { teils }\end{array}$ & $\begin{array}{c}\text { trifft } \\
\text { genau } \\
\text { zu }\end{array}$ & $\begin{array}{l}\text { weiß } \\
\text { nicht }\end{array}$ \\
\hline $\begin{array}{l}\text { Schweigt in } \\
\text { bestimmten } \\
\text { Situationen, wendet } \\
\text { sich ab oder versteckt } \\
\text { sich }\end{array}$ & 1 & 2 & 3 & $\square$ & 1 & 2 & 3 & $\square$ & 1 & 2 & 3 & $\square$ \\
\hline $\begin{array}{l}\text { Flüstert oder spricht } \\
\text { sehr leise }\end{array}$ & 1 & 2 & 3 & $\square$ & 1 & 2 & 3 & $\square$ & 1 & 2 & 3 & $\square$ \\
\hline $\begin{array}{l}\text { Spricht innerhalb der } \\
\text { Familie frei }\end{array}$ & 1 & 2 & 3 & $\square$ & 1 & 2 & 3 & $\square$ & 1 & 2 & 3 & $\square$ \\
\hline $\begin{array}{l}\text { Zieht sich in sozialen } \\
\text { Situationen zurück } \\
\text { (z.B. bei Besuch, im } \\
\text { Restaurant) }\end{array}$ & 1 & 2 & 3 & $\square$ & 1 & 2 & 3 & $\square$ & 1 & 2 & 3 & $\square$ \\
\hline $\begin{array}{l}\text { Erstarrt in manchen } \\
\text { Situationen }\end{array}$ & 1 & 2 & 3 & $\square$ & 1 & 2 & 3 & $\square$ & 1 & 2 & 3 & $\square$ \\
\hline $\begin{array}{l}\text { Kann keinen } \\
\text { Blickkontakt zu } \\
\text { fremden Personen } \\
\text { aufnehmen bzw. } \\
\text { halten }\end{array}$ & 1 & 2 & 3 & $\square$ & 1 & 2 & 3 & $\square$ & 1 & 2 & 3 & $\square$ \\
\hline
\end{tabular}

\section{Angaben zur häuslichen Situation}

Wie viel Geld hat Ihr Haushalt im Monat aus Einkommen und Zuwendungen zur Verfügung?
$\square$ bis $1.000 €$
$\square \quad 1.000 €-2.000 €$
$\square \quad 2.000 €-3.000 €$
$\square \quad 3.000 €-4.000 €$
$\square \quad 4.000 €-5.000 €$
$\square \quad 5.000 €-6.000 €$
$\square$ über $6.000 €$
$\square \quad$ keine Angabe

Bitte kreuzen Sie alles an, was auf die Wohnsituation Ihres Kindes zutrifft.

$\square \quad$ eher ländlich
$\square \quad$ zur Miete
$\square \quad$ Garten
$\square \quad$ hat eigenes Zimmer

$\square \quad$ eher städtisch
$\square \quad$ Eigentum
$\square \quad$ Spielstraße
$\square \quad$ teilt Zimmer mit
Personen 
Wie viele Bücher haben Sie Zuhause?

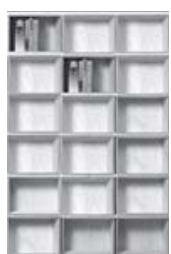

$\square$

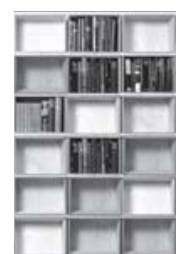

$\square$ keine oder nur sehr wenige genug um eine Regalbrett zu füllen

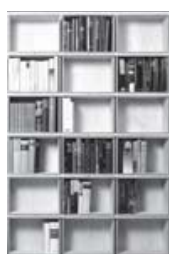

$\square$

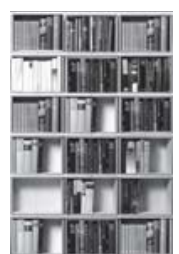

$\square$

genug um drei

Regale zu füllen

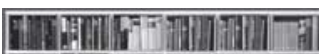

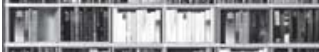

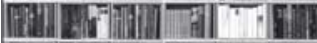
THANE TIMFH

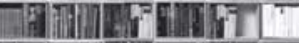
Fit genug um ein Regal zu füllen

über 200 Bücher

Hier haben Sie Platz für weitere Anmerkungen! 
Anhang C: DortMuS-Kita Pilotversion 


\section{Dortmunder Mutismus-Screening - Kindergarten}

Dieser Fragebogen wurde am

von ausgefüllt.

\section{Allgemeine Angaben zum Kind}

1. Name des Kindes:

2. Alter des Kindes: Jahre Monate

3. Geschlecht des Kindes: $\quad \square$ weiblich $\quad \square$ männlich

4. Wächst das Kind mehrsprachig auf? $\quad \square$ ja $\quad \square$ nein $\quad \square$ weiß nicht

5. Das Kind besucht seit Jahren Monate den Kindergarten.

6. Das Schweigen des Kindes im Kindergarten besteht bereits länger als 2 Monate.
$\square$ ja
$\square$ nein
$\square$ weiß nicht

\section{Beobachtbare Verhaltensweisen}

Es folgt nun eine Liste mit Aussagen zu Verhaltensweisen, welche Sie in typischen Kindergartensituationen beim Kind beobachten können. Bitte lesen Sie diese aufmerksam durch und beurteilen Sie auf einer Skala von 1 (trifft gar nicht zu) bis 5 (trifft genau zu), inwieweit Sie das jeweilige Verhalten innerhalb der letzten 4 Wochen beim Kind beobachten konnten. Es ist wichtig, dass Sie alle Aussagen vollständig bearbeiten und keine Antwort auslassen, auch wenn Ihnen einige Aussagen ähnlich erscheinen. Wählen Sie im Zweifelsfall (z. B. wenn das Kind ganz selten mit anderen Kindern spricht, in der Regel jedoch schweigt) die Antwortalternative, die am ehesten zutrifft. Machen Sie bitte alle Ihre Angaben möglichst spontan.

\begin{tabular}{|c|c|c|c|c|c|c|c|}
\hline & & $\begin{array}{l}\text { trifft gar } \\
\text { nicht zu }\end{array}$ & $\begin{array}{l}\text { trifft eher } \\
\text { nicht zu }\end{array}$ & teils/teils & $\begin{array}{c}\text { trifft } \\
\text { eher } \\
\text { zu }\end{array}$ & $\begin{array}{c}\text { trifft } \\
\text { genau } \\
\text { zu }\end{array}$ & $\begin{array}{l}\text { weiß } \\
\text { nicht }\end{array}$ \\
\hline 1 & Das Kind singt bei Liedern in der Gruppe mit. & 1 & 2 & 3 & 4 & 5 & $\square$ \\
\hline 2 & Auf dem Hof spielt das Kind alleine. & 1 & 2 & 3 & 4 & 5 & $\square$ \\
\hline 3 & $\begin{array}{l}\text { Das Kind äußert seine Bedürfnisse, z.B. Durst } \\
\text { oder Toilettengang, in irgendeiner Form (z.B. } \\
\text { sprachliche Äußerungen, nichtsprachlich über } \\
\text { Handzeichen). }\end{array}$ & 1 & 2 & 3 & 4 & 5 & $\square$ \\
\hline 4 & Ich habe das Kind schon weinen gesehen. & 1 & 2 & 3 & 4 & 5 & $\square$ \\
\hline 5 & $\begin{array}{l}\text { Bei Ansprache der Erzieherin „erstarrt“ das } \\
\text { Kind. }\end{array}$ & 1 & 2 & 3 & 4 & 5 & $\square$ \\
\hline
\end{tabular}




\begin{tabular}{|c|c|c|c|c|c|c|c|}
\hline & & $\begin{array}{l}\text { trifft gar } \\
\text { nicht zu }\end{array}$ & $\begin{array}{l}\text { trifft eher } \\
\text { nicht zu }\end{array}$ & teils/teils & $\begin{array}{c}\text { trifft } \\
\text { eher } \\
\text { zu }\end{array}$ & $\begin{array}{c}\text { trifft } \\
\text { genau } \\
\text { zu }\end{array}$ & $\begin{array}{l}\text { weiß } \\
\text { nicht }\end{array}$ \\
\hline 6 & $\begin{array}{l}\text { Das Kind holt sich keine Unterstützung von der } \\
\text { Erzieherin, wenn es Hilfe benötigt (z.B. beim } \\
\text { Schuhe binden, Jacke schließen). }\end{array}$ & 1 & 2 & 3 & 4 & 5 & $\square$ \\
\hline 7 & $\begin{array}{l}\text { Das Kind reagiert bei direkter Ansprache } \\
\text { nichtsprachlich (z.B. durch Gesten). }\end{array}$ & 1 & 2 & 3 & 4 & 5 & $\square$ \\
\hline 8 & $\begin{array}{l}\text { Das Kind entzieht sich Konfliktsituationen (z.B. } \\
\text { Streitereien). }\end{array}$ & 1 & 2 & 3 & 4 & 5 & $\square$ \\
\hline 9 & $\begin{array}{l}\text { Beim gemeinsamen Singen „singt“ das Kind } \\
\text { tonlos mit. }\end{array}$ & 1 & 2 & 3 & 4 & 5 & $\square$ \\
\hline 10 & $\begin{array}{l}\text { Bedürfnisse werden von dem Kind nicht } \\
\text { geäußert. }\end{array}$ & 1 & 2 & 3 & 4 & 5 & $\square$ \\
\hline 11 & $\begin{array}{l}\text { Bei Ansprache der Erzieherin geht das Kind } \\
\text { weg. }\end{array}$ & 1 & 2 & 3 & 4 & 5 & $\square$ \\
\hline 12 & $\begin{array}{l}\text { Das Kind flüstert, wenn es mir etwas mitteilen } \\
\text { möchte. }\end{array}$ & 1 & 2 & 3 & 4 & 5 & $\square$ \\
\hline 13 & $\begin{array}{l}\text { Das Kind äußert verbal, wenn es Hilfe } \\
\text { benötigt. }\end{array}$ & 1 & 2 & 3 & 4 & 5 & $\square$ \\
\hline 14 & $\begin{array}{l}\text { Möchte das Kind etwas haben, zeigt es auf die } \\
\text { Sache, z.B. Schere beim Basteln. }\end{array}$ & 1 & 2 & 3 & 4 & 5 & $\square$ \\
\hline 15 & $\begin{array}{l}\text { Das Kind spricht mit Erziehern aus anderen } \\
\text { Gruppen. }\end{array}$ & 1 & 2 & 3 & 4 & 5 & $\square$ \\
\hline 16 & $\begin{array}{l}\text { Das Kind bittet andere Kinder beim Basteln um } \\
\text { Materialien, an die es selbst nicht heran } \\
\text { kommt. }\end{array}$ & 1 & 2 & 3 & 4 & 5 & $\square$ \\
\hline 17 & $\begin{array}{l}\text { Das Kind beteiligt sich an angeleiteten } \\
\text { Spielangeboten der Großgruppe, z.B. im } \\
\text { Stuhlkreis, bei Kreisspielen, bei } \\
\text { Bewegungsspielen. }\end{array}$ & 1 & 2 & 3 & 4 & 5 & $\square$ \\
\hline 18 & $\begin{array}{l}\text { In lustigen Situationen bleibt das Kind } \\
\text { regungslos. }\end{array}$ & 1 & 2 & 3 & 4 & 5 & $\square$ \\
\hline 19 & $\begin{array}{l}\text { Das Kind spielt gemeinsam mit anderen } \\
\text { Kindern. }\end{array}$ & 1 & 2 & 3 & 4 & 5 & $\square$ \\
\hline 20 & $\begin{array}{l}\text { Das Kind spricht mit Mutter oder Vater, wenn } \\
\text { es gebracht oder abgeholt wird. }\end{array}$ & 1 & 2 & 3 & 4 & 5 & $\square$ \\
\hline 21 & $\begin{array}{l}\text { Das Kind schweigt auf dem Hof gegenüber } \\
\text { Erziehern. }\end{array}$ & 1 & 2 & 3 & 4 & 5 & $\square$ \\
\hline 22 & $\begin{array}{l}\text { An besonderen Feiern (z.B. Karneval, } \\
\text { Nikolaus) zieht sich das Kind in der Gruppe } \\
\text { zurück. }\end{array}$ & 1 & 2 & 3 & 4 & 5 & $\square$ \\
\hline 23 & Wenn sich das Kind wehgetan hat, weint es. & 1 & 2 & 3 & 4 & 5 & $\square$ \\
\hline 24 & $\begin{array}{l}\text { Das Kind holt sich Unterstützung von anderen } \\
\text { Kindern, wenn es Hilfe benötigt. }\end{array}$ & 1 & 2 & 3 & 4 & 5 & $\square$ \\
\hline 25 & $\begin{array}{l}\text { Ja-Nein-Fragen beantwortet das Kind über } \\
\text { Nicken oder Kopfschütteln. }\end{array}$ & 1 & 2 & 3 & 4 & 5 & $\square$ \\
\hline 26 & $\begin{array}{l}\text { Das Kind verhält sich still, wenn es von Mutter } \\
\text { oder Vater gebracht oder abgeholt wird. }\end{array}$ & 1 & 2 & 3 & 4 & 5 & $\square$ \\
\hline
\end{tabular}




\begin{tabular}{|c|c|c|c|c|c|c|c|}
\hline & & $\begin{array}{l}\text { trifft gar } \\
\text { nicht zu }\end{array}$ & $\begin{array}{l}\text { trifft eher } \\
\text { nicht zu }\end{array}$ & teils/teils & $\begin{array}{l}\text { trifft } \\
\text { eher } \\
\text { zu }\end{array}$ & $\begin{array}{c}\text { trifft } \\
\text { genau } \\
\text { zu }\end{array}$ & $\begin{array}{l}\text { weiß } \\
\text { nicht }\end{array}$ \\
\hline 27 & $\begin{array}{l}\text { Im Kindergarten isst und/oder trinkt das Kind } \\
\text { nicht. }\end{array}$ & 1 & 2 & 3 & 4 & 5 & $\square$ \\
\hline 28 & Das Kind spielt alleine. & 1 & 2 & 3 & 4 & 5 & $\square$ \\
\hline 29 & Ich habe das Kind schon laut lachen gehört. & 1 & 2 & 3 & 4 & 5 & $\square$ \\
\hline 30 & $\begin{array}{l}\text { Das Kind signalisiert gegenüber der } \\
\text { Erzieherin, dass es z.B. Durst hat. }\end{array}$ & 1 & 2 & 3 & 4 & 5 & $\square$ \\
\hline 31 & $\begin{array}{l}\text { Nach Aussagen der Eltern spricht das Kind in } \\
\text { Situationen außerhalb des Kindergartens (z.B. } \\
\text { Spielplatz, Großeltern) unbefangen. }\end{array}$ & 1 & 2 & 3 & 4 & 5 & $\square$ \\
\hline 32 & $\begin{array}{l}\text { Das Kind nimmt Blickkontakt zu einer } \\
\text { Erzieherin auf, wenn es Hilfe benötigt. }\end{array}$ & 1 & 2 & 3 & 4 & 5 & $\square$ \\
\hline 33 & $\begin{array}{l}\text { Das Kind reagiert mit Gesten oder anderen } \\
\text { nichtsprachlichen Mitteln auf die direkte } \\
\text { Ansprache der Erzieherin }\end{array}$ & 1 & 2 & 3 & 4 & 5 & $\square$ \\
\hline 34 & Das Kind spricht mit mir in Einzelsituationen. & 1 & 2 & 3 & 4 & 5 & $\square$ \\
\hline 35 & $\begin{array}{l}\text { Das Kind zeigt seine Freude in lustigen } \\
\text { Situationen (z.B. durch Lächeln). }\end{array}$ & 1 & 2 & 3 & 4 & 5 & $\square$ \\
\hline 36 & Das Kind hat einen festen Spielpartner. & 1 & 2 & 3 & 4 & 5 & $\square$ \\
\hline 37 & $\begin{array}{l}\text { Das Kind isst und/oder trinkt gemeinsam mit } \\
\text { anderen Kindern. }\end{array}$ & 1 & 2 & 3 & 4 & 5 & $\square$ \\
\hline 38 & $\begin{array}{l}\text { Bei Ansprache der Erzieherin schweigt das } \\
\text { Kind. }\end{array}$ & 1 & 2 & 3 & 4 & 5 & $\square$ \\
\hline 39 & $\begin{array}{l}\text { Das Kind flüstert, wenn es mit anderen } \\
\text { Kindern spricht. }\end{array}$ & 1 & 2 & 3 & 4 & 5 & $\square$ \\
\hline 40 & $\begin{array}{l}\text { Das Kind signalisiert gegenüber der } \\
\text { Erzieherin, dass es Unterstützung (z.B. Jacke } \\
\text { schließen) benötigt. }\end{array}$ & 1 & 2 & 3 & 4 & 5 & $\square$ \\
\hline 41 & $\begin{array}{l}\text { In Konfliktsituationen (z.B. Streitereien) } \\
\text { verteidigt sich das Kind. }\end{array}$ & 1 & 2 & 3 & 4 & 5 & $\square$ \\
\hline 42 & $\begin{array}{l}\text { Werden Lieder in der Gruppe gesungen, sitzt } \\
\text { das Kind „teilnahmslos“ dabei. }\end{array}$ & 1 & 2 & 3 & 4 & 5 & $\square$ \\
\hline 43 & $\begin{array}{l}\text { An besonderen Feiern (z.B. Karneval, } \\
\text { Nikolaus) nimmt das Kind teil. }\end{array}$ & 1 & 2 & 3 & 4 & 5 & $\square$ \\
\hline 44 & Ich habe das Kind schon sprechen gehört. & 1 & 2 & 3 & 4 & 5 & $\square$ \\
\hline 45 & $\begin{array}{l}\text { Im Kindergarten geht das Kind nicht zur } \\
\text { Toilette. }\end{array}$ & 1 & 2 & 3 & 4 & 5 & $\square$ \\
\hline 46 & $\begin{array}{l}\text { Das Kind nimmt an Gruppenspielen (z.B. } \\
\text { Spiele im Stuhlkreis) teil. }\end{array}$ & 1 & 2 & 3 & 4 & 5 & $\square$ \\
\hline 47 & $\begin{array}{l}\text { Nach Aussage der Eltern schweigt das Kind zu } \\
\text { Hause. }\end{array}$ & 1 & 2 & 3 & 4 & 5 & $\square$ \\
\hline 48 & $\begin{array}{l}\text { Benötigt das Kind Hilfe (z.B. beim Basteln), } \\
\text { holt es sich Unterstützung bei der Erzieherin. }\end{array}$ & 1 & 2 & 3 & 4 & 5 & $\square$ \\
\hline 49 & Das Kind nimmt keinen Blickkontakt auf. & 1 & 2 & 3 & 4 & 5 & $\square$ \\
\hline 50 & Das Kind schweigt im Stuhlkreis. & 1 & 2 & 3 & 4 & 5 & $\square$ \\
\hline
\end{tabular}




\begin{tabular}{|c|c|c|c|c|c|c|c|}
\hline & & $\begin{array}{l}\text { trifft gar } \\
\text { nicht zu }\end{array}$ & $\begin{array}{l}\text { trifft eher } \\
\text { nicht zu }\end{array}$ & teils/teils & $\begin{array}{c}\text { trifft } \\
\text { eher } \\
\mathrm{zu}\end{array}$ & $\begin{array}{c}\text { trifft } \\
\text { genau } \\
\text { zu }\end{array}$ & $\begin{array}{l}\text { weiß } \\
\text { nicht }\end{array}$ \\
\hline 51 & In der Gruppe schweigt das Kind. & 1 & 2 & 3 & 4 & 5 & $\square$ \\
\hline 52 & $\begin{array}{l}\text { Das Kind entzieht sich Spielen in der } \\
\text { Großgruppe. }\end{array}$ & 1 & 2 & 3 & 4 & 5 & $\square$ \\
\hline 53 & $\begin{array}{l}\text { In Konfliktsituationen (z.B. Streitereien) zeigt } \\
\text { das Kind Wut oder Ärger. }\end{array}$ & 1 & 2 & 3 & 4 & 5 & $\square$ \\
\hline 54 & $\begin{array}{l}\text { Wenn sich das Kind wehgetan hat, zieht es } \\
\text { sich zurück. }\end{array}$ & 1 & 2 & 3 & 4 & 5 & $\square$ \\
\hline 55 & $\begin{array}{l}\text { In der Gruppe nimmt das Kind die Rolle des } \\
\text { Zuschauers ein. }\end{array}$ & 1 & 2 & 3 & 4 & 5 & $\square$ \\
\hline 56 & Das Kind sucht sich selbstständig ein Spiel. & 1 & 2 & 3 & 4 & 5 & $\square$ \\
\hline 57 & $\begin{array}{l}\text { Das Kind spricht mit Kindern aus der eigenen } \\
\text { Gruppe. }\end{array}$ & 1 & 2 & 3 & 4 & 5 & $\square$ \\
\hline 58 & $\begin{array}{l}\text { Das Kind spricht mit Erziehern aus der } \\
\text { eigenen Gruppe. }\end{array}$ & 1 & 2 & 3 & 4 & 5 & $\square$ \\
\hline 59 & $\begin{array}{l}\text { Das Kind beschäftigt sich nicht selbstständig } \\
\text { mit Spielmaterial. }\end{array}$ & 1 & 2 & 3 & 4 & 5 & $\square$ \\
\hline 60 & $\begin{array}{l}\text { Das Kind nimmt an Rollenspielen (z.B. Mutter- } \\
\text { Vater-Kind) mit anderen Kindern teil. }\end{array}$ & 1 & 2 & 3 & 4 & 5 & $\square$ \\
\hline 61 & $\begin{array}{l}\text { Emotionen (z.B. Freude, Ärger) zeigt das Kind } \\
\text { nicht. }\end{array}$ & 1 & 2 & 3 & 4 & 5 & $\square$ \\
\hline 62 & $\begin{array}{l}\text { Nach Aussage der Eltern spricht das Kind zu } \\
\text { Hause unbefangen. }\end{array}$ & 1 & 2 & 3 & 4 & 5 & $\square$ \\
\hline 63 & $\begin{array}{l}\text { In Spielsituationen mit anderen Kindern } \\
\text { schweigt das Kind. }\end{array}$ & 1 & 2 & 3 & 4 & 5 & $\square$ \\
\hline 64 & $\begin{array}{l}\text { Auf dem Hof nimmt das Kind Kontakt mit } \\
\text { anderen Kindern auf. }\end{array}$ & 1 & 2 & 3 & 4 & 5 & $\square$ \\
\hline 65 & $\begin{array}{l}\text { Im Stuhlkreis vermeidet das Kind sprachliche } \\
\text { Äußerungen. }\end{array}$ & 1 & 2 & 3 & 4 & 5 & $\square$ \\
\hline 66 & $\begin{array}{l}\text { Das Kind schickt andere Kinder vor, um } \\
\text { Unterstützung von einer Erzieherin zu holen. }\end{array}$ & 1 & 2 & 3 & 4 & 5 & $\square$ \\
\hline 67 & $\begin{array}{l}\text { Bei Spielangeboten in der Großgruppe, z.B. im } \\
\text { Stuhlkreis, bei Kreisspielen, bei } \\
\text { Bewegungsspielen, bleibt das Kind wie } \\
\text { „angewurzelt" an einer Stelle stehen. }\end{array}$ & 1 & 2 & 3 & 4 & 5 & $\square$ \\
\hline 68 & $\begin{array}{l}\text { Bei Ansprache der Erzieherin verhält sich das } \\
\text { Kind still. }\end{array}$ & 1 & 2 & 3 & 4 & 5 & $\square$ \\
\hline 69 & $\begin{array}{l}\text { Das Kind zieht sich bei Spielen in der } \\
\text { Großgruppe zurück. }\end{array}$ & 1 & 2 & 3 & 4 & 5 & $\square$ \\
\hline 70 & $\begin{array}{l}\text { In Spielsituationen spricht das Kind mit } \\
\text { anderen Kindern. }\end{array}$ & 1 & 2 & 3 & 4 & 5 & $\square$ \\
\hline 71 & $\begin{array}{l}\text { Bedürfnisse (z.B. Durst, Toilettengang) werden } \\
\text { vom Kind geäußert. }\end{array}$ & 1 & 2 & 3 & 4 & 5 & $\square$ \\
\hline 72 & $\begin{array}{l}\text { In Spielsituationen auf dem Hof isoliert sich } \\
\text { das Kind von den anderen Kindern. }\end{array}$ & 1 & 2 & 3 & 4 & 5 & $\square$ \\
\hline
\end{tabular}




\begin{tabular}{|c|c|c|c|c|c|c|c|}
\hline & & $\begin{array}{l}\text { trifft gar } \\
\text { nicht zu }\end{array}$ & $\begin{array}{l}\text { trifft eher } \\
\text { nicht zu }\end{array}$ & teils/teils & $\begin{array}{l}\text { trifft } \\
\text { eher } \\
\text { zu }\end{array}$ & $\begin{array}{l}\text { trifft } \\
\text { genau } \\
\text { zu }\end{array}$ & $\begin{array}{l}\text { weiß } \\
\text { nicht }\end{array}$ \\
\hline 73 & $\begin{array}{l}\text { In Gesprächssituationen vermeidet das Kind } \\
\text { sprachliche Äußerungen. }\end{array}$ & 1 & 2 & 3 & 4 & 5 & $\square$ \\
\hline 74 & $\begin{array}{l}\text { Bei Ansprache der Erzieherin nimmt das Kind } \\
\text { Blickkontakt auf. }\end{array}$ & 1 & 2 & 3 & 4 & 5 & $\square$ \\
\hline 75 & Das Kind antwortet verbal auf Fragen. & 1 & 2 & 3 & 4 & 5 & $\square$ \\
\hline 76 & $\begin{array}{l}\text { In Spielsituationen spricht das Kind mit } \\
\text { anderen Kindern. }\end{array}$ & 1 & 2 & 3 & 4 & 5 & $\square$ \\
\hline 77 & $\begin{array}{l}\text { Nach Aussage der Eltern schweigt das Kind } \\
\text { außerhalb des Kindergartens. }\end{array}$ & 1 & 2 & 3 & 4 & 5 & $\square$ \\
\hline 78 & $\begin{array}{l}\text { Auf dem Hof spricht das Kind mit anderen } \\
\text { Kindern. }\end{array}$ & 1 & 2 & 3 & 4 & 5 & $\square$ \\
\hline 79 & $\begin{array}{l}\text { In Konfliktsituationen (z.B. Streitereien) } \\
\text { „erstarrt“ das Kind. }\end{array}$ & 1 & 2 & 3 & 4 & 5 & $\square$ \\
\hline 80 & $\begin{array}{l}\text { In der Gruppe nimmt das Kind Kontakt zu } \\
\text { anderen Kindern auf. }\end{array}$ & 1 & 2 & 3 & 4 & 5 & $\square$ \\
\hline
\end{tabular}


Anhang D: Beobachtungsbögen und Beobachtungsmanual 

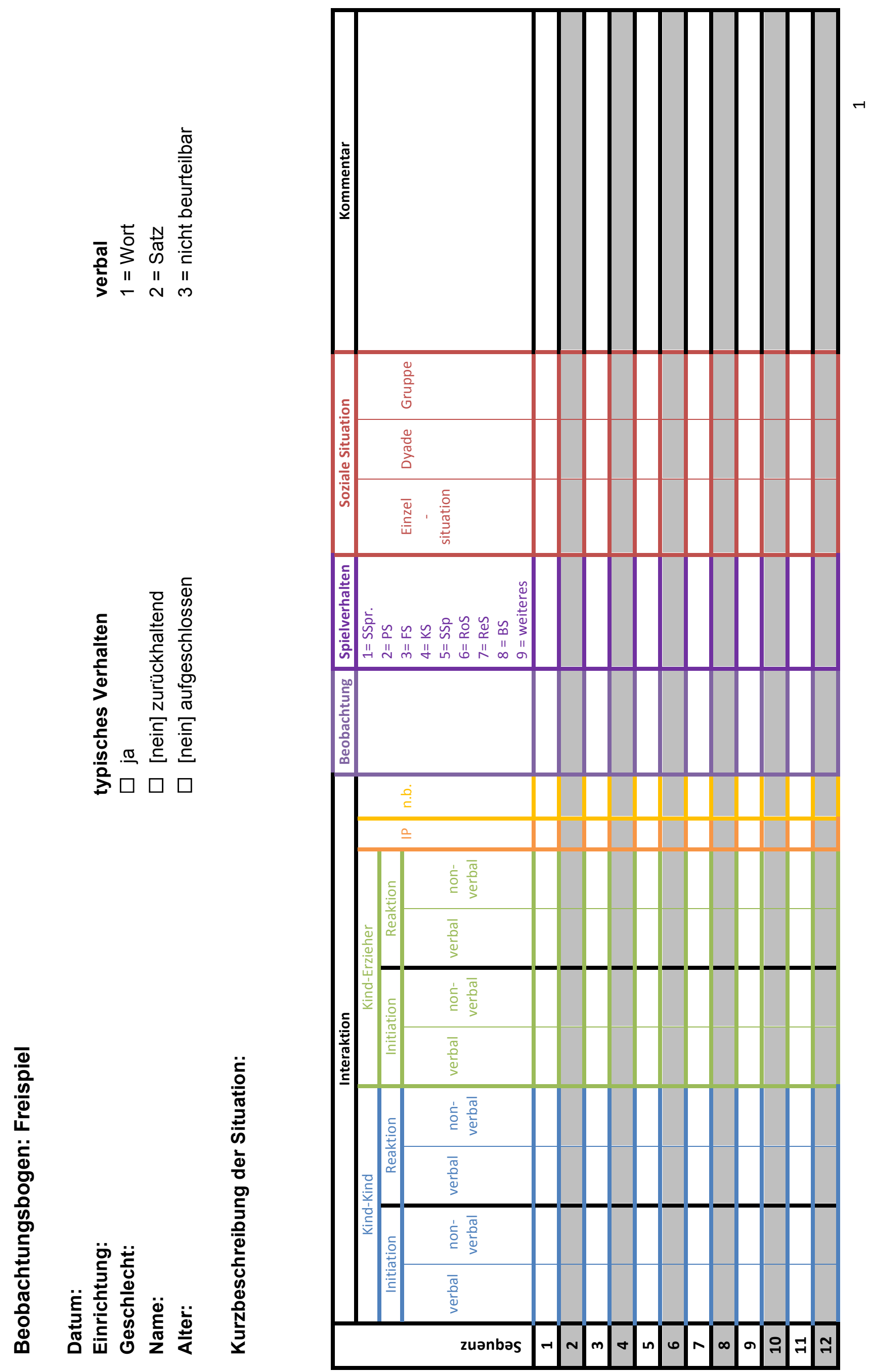


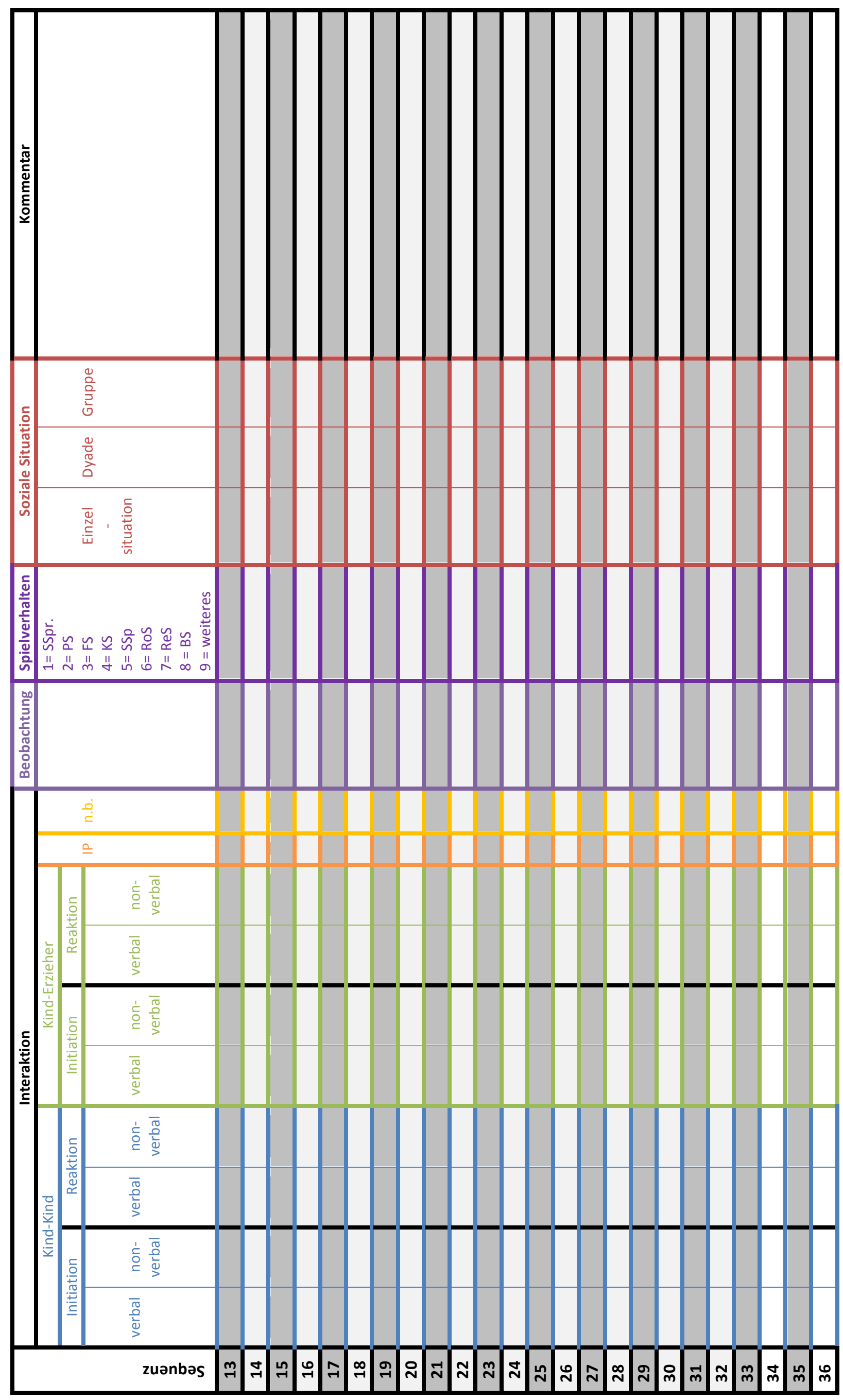




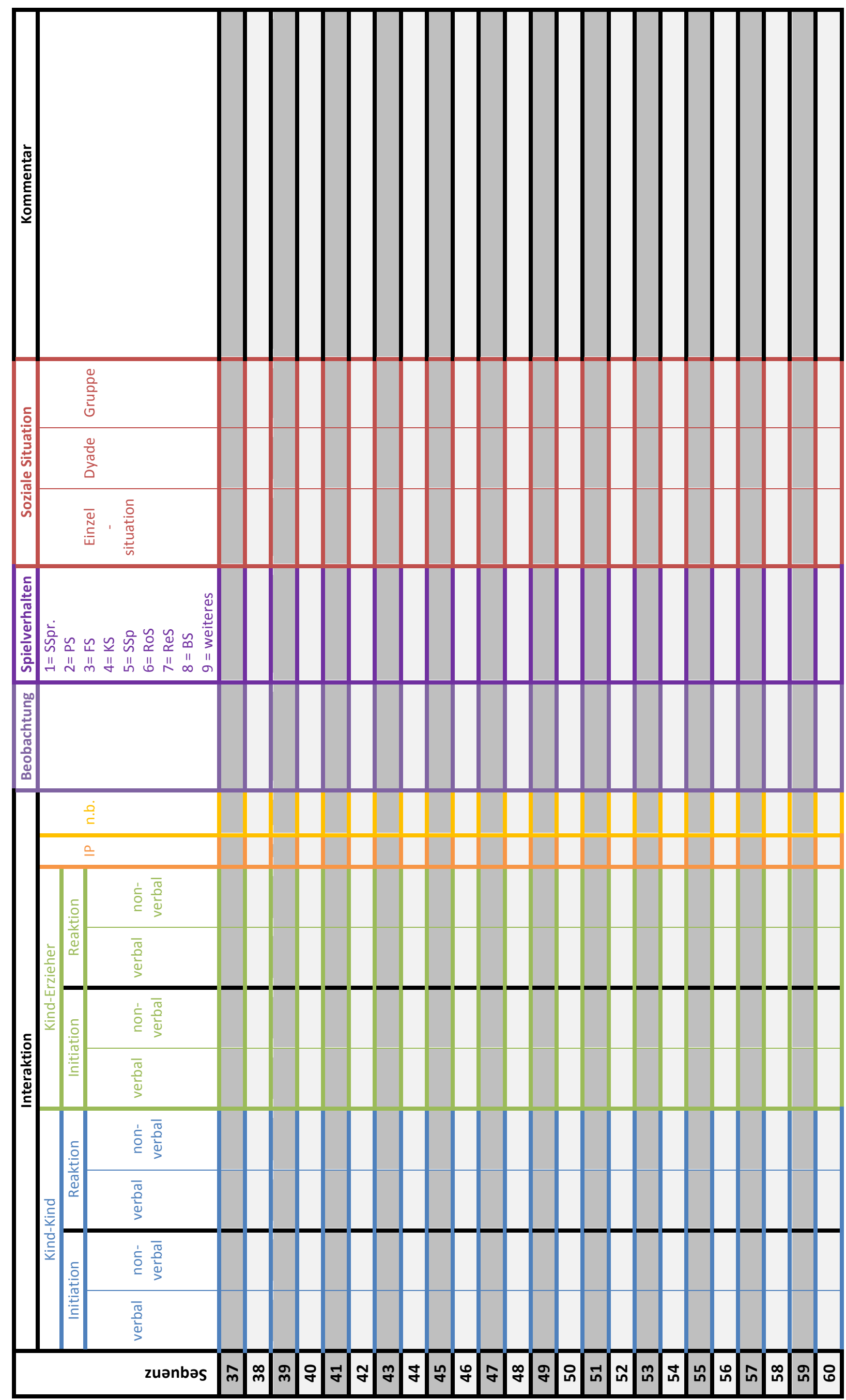




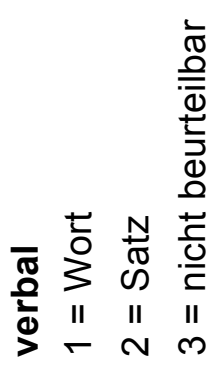

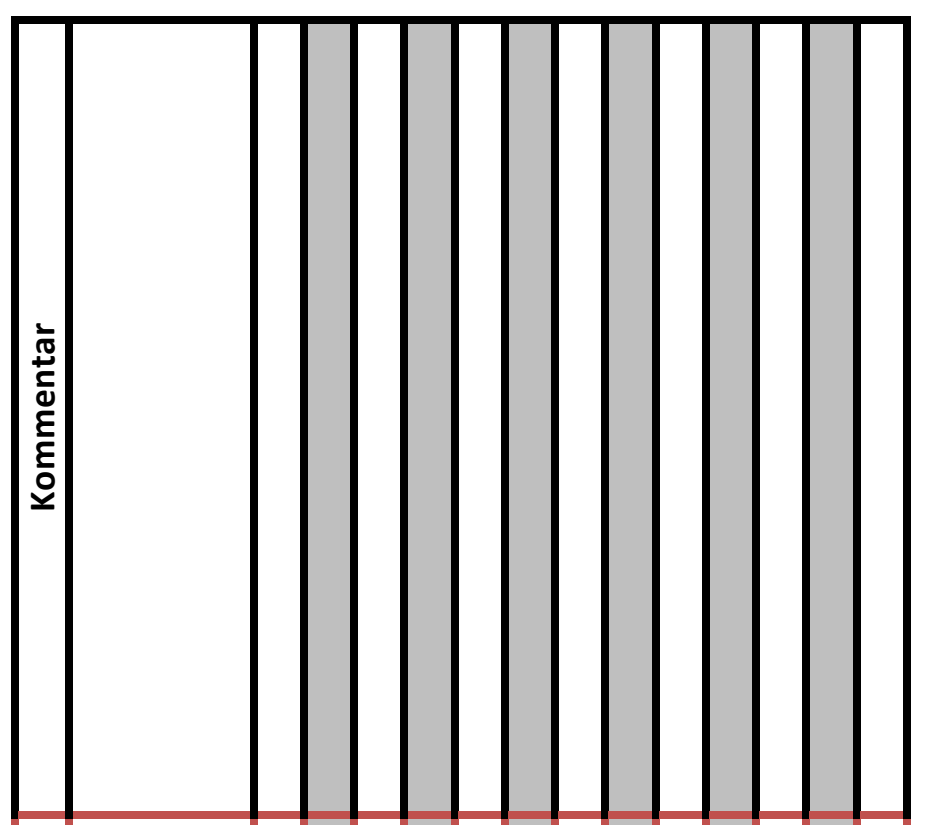

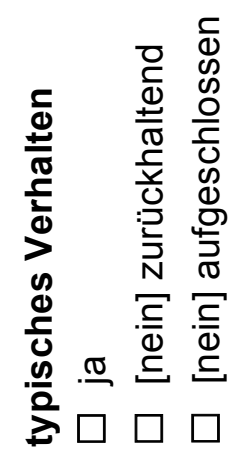

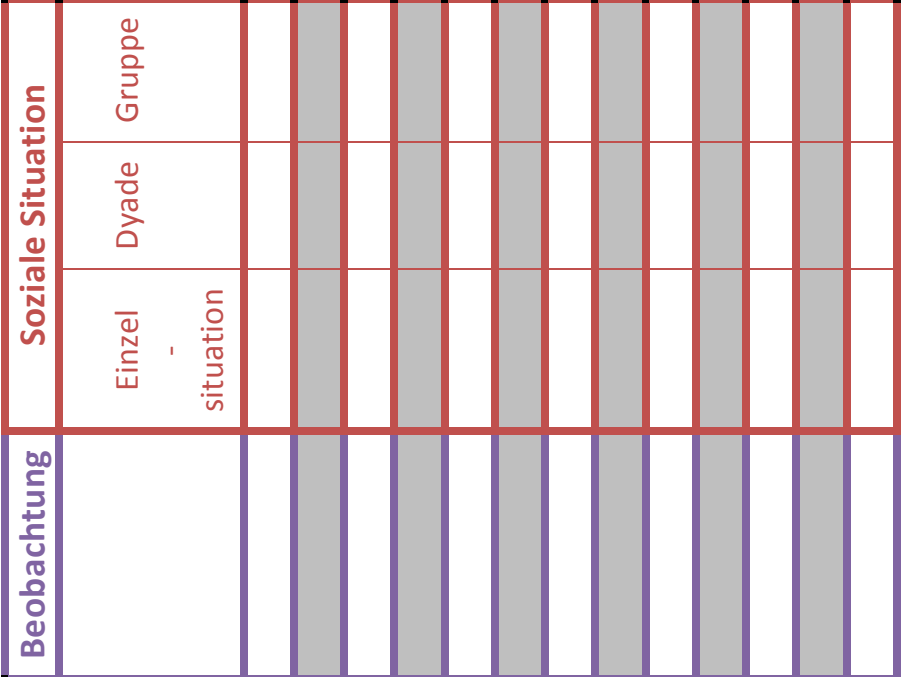

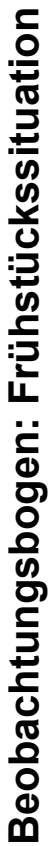

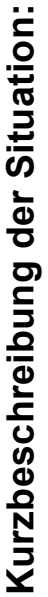

竎

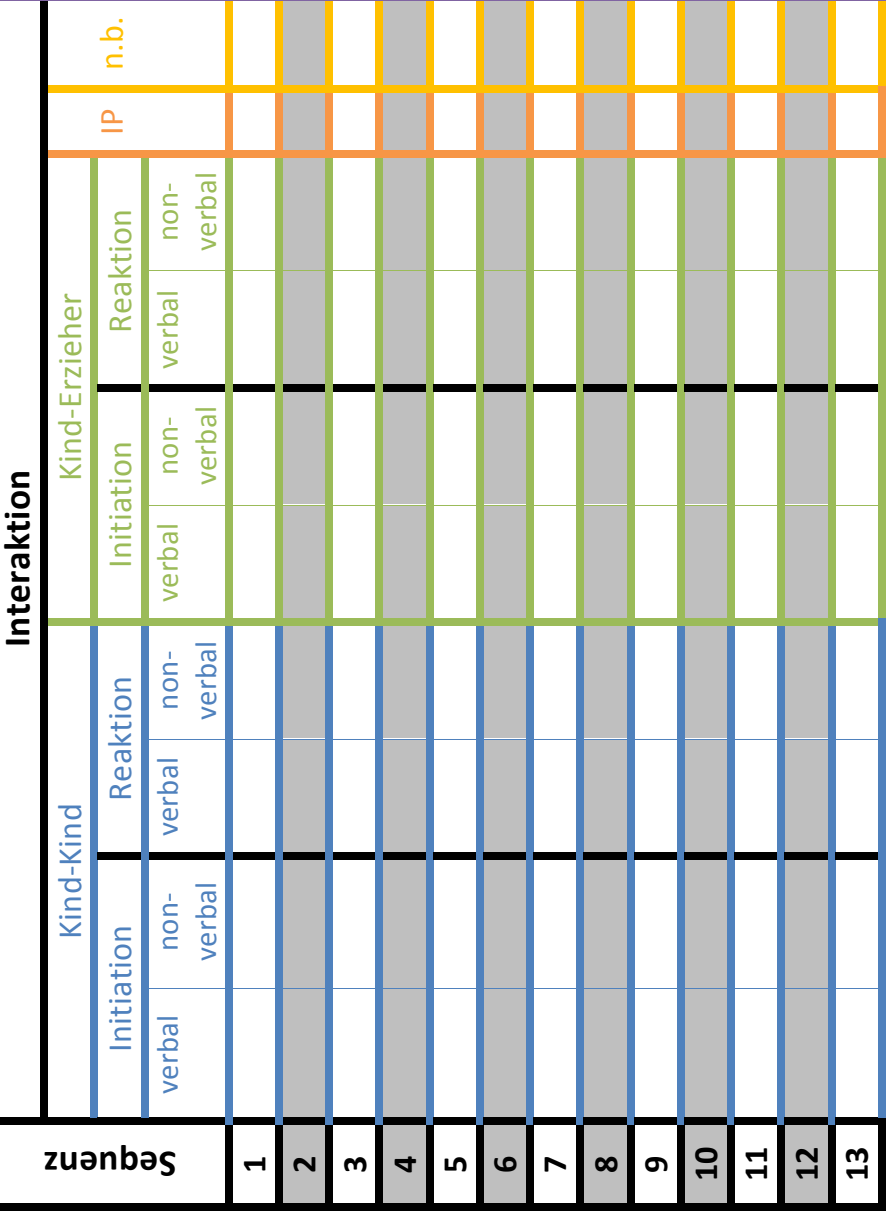




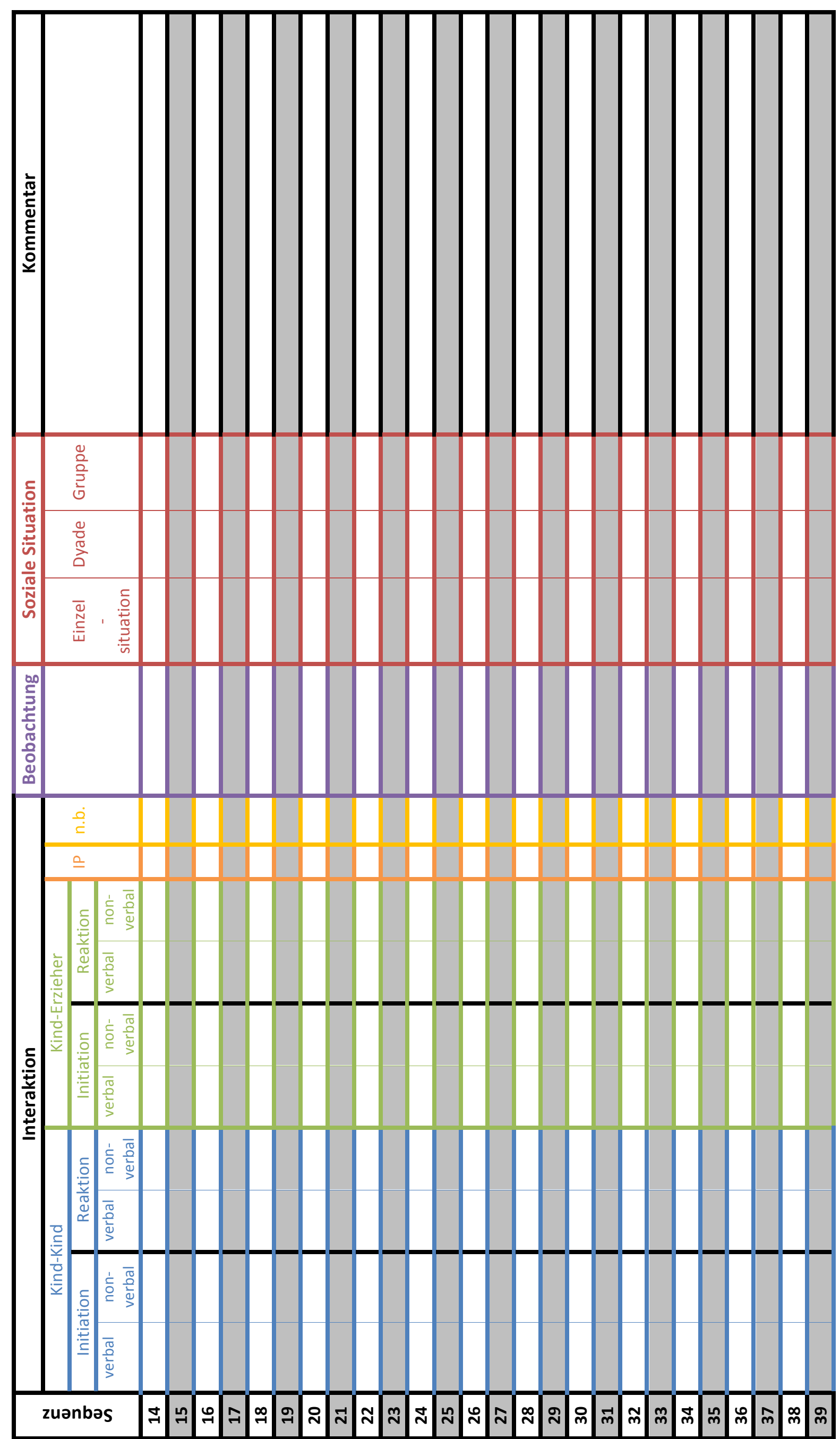




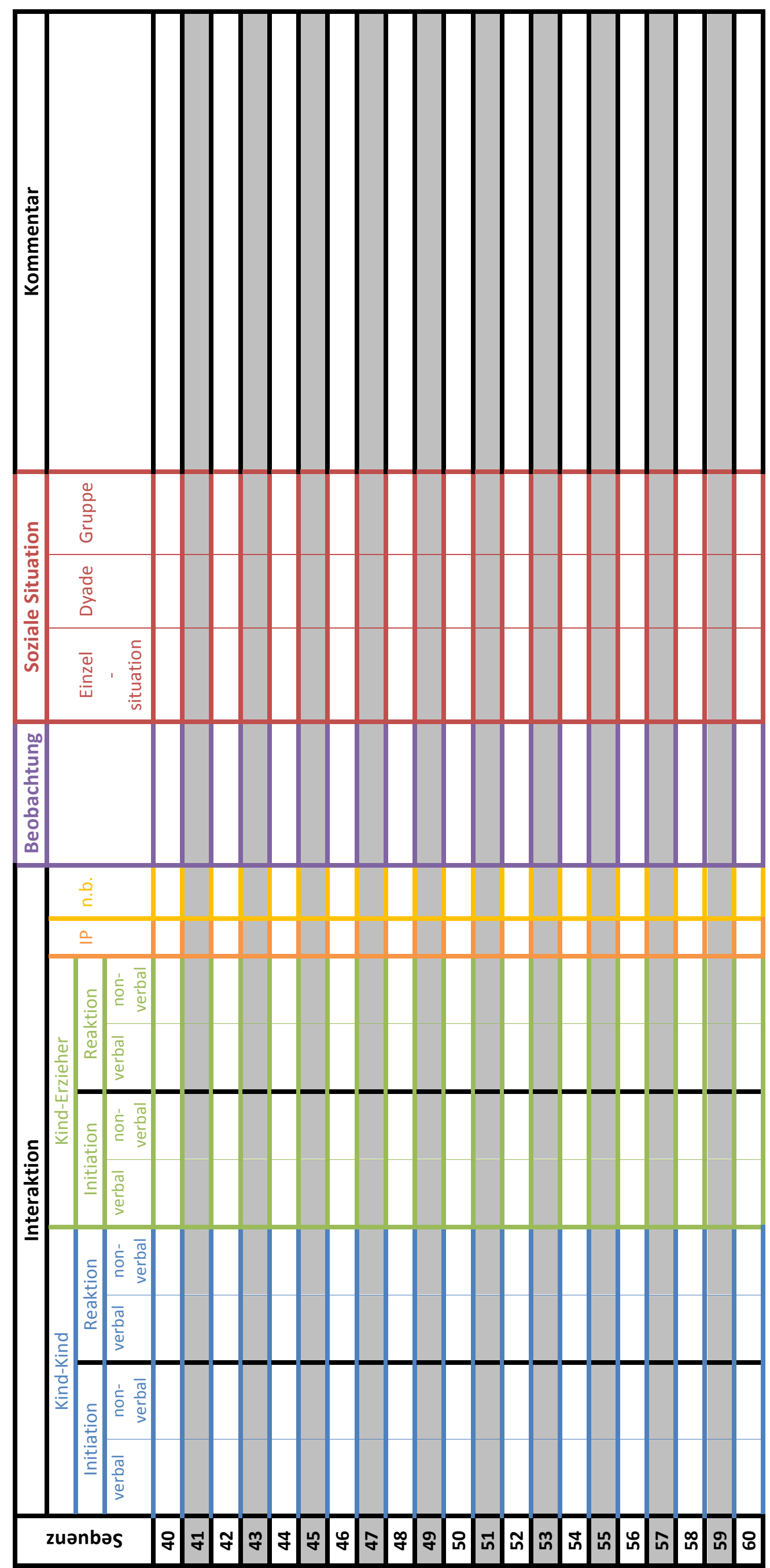




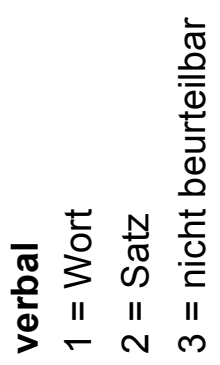
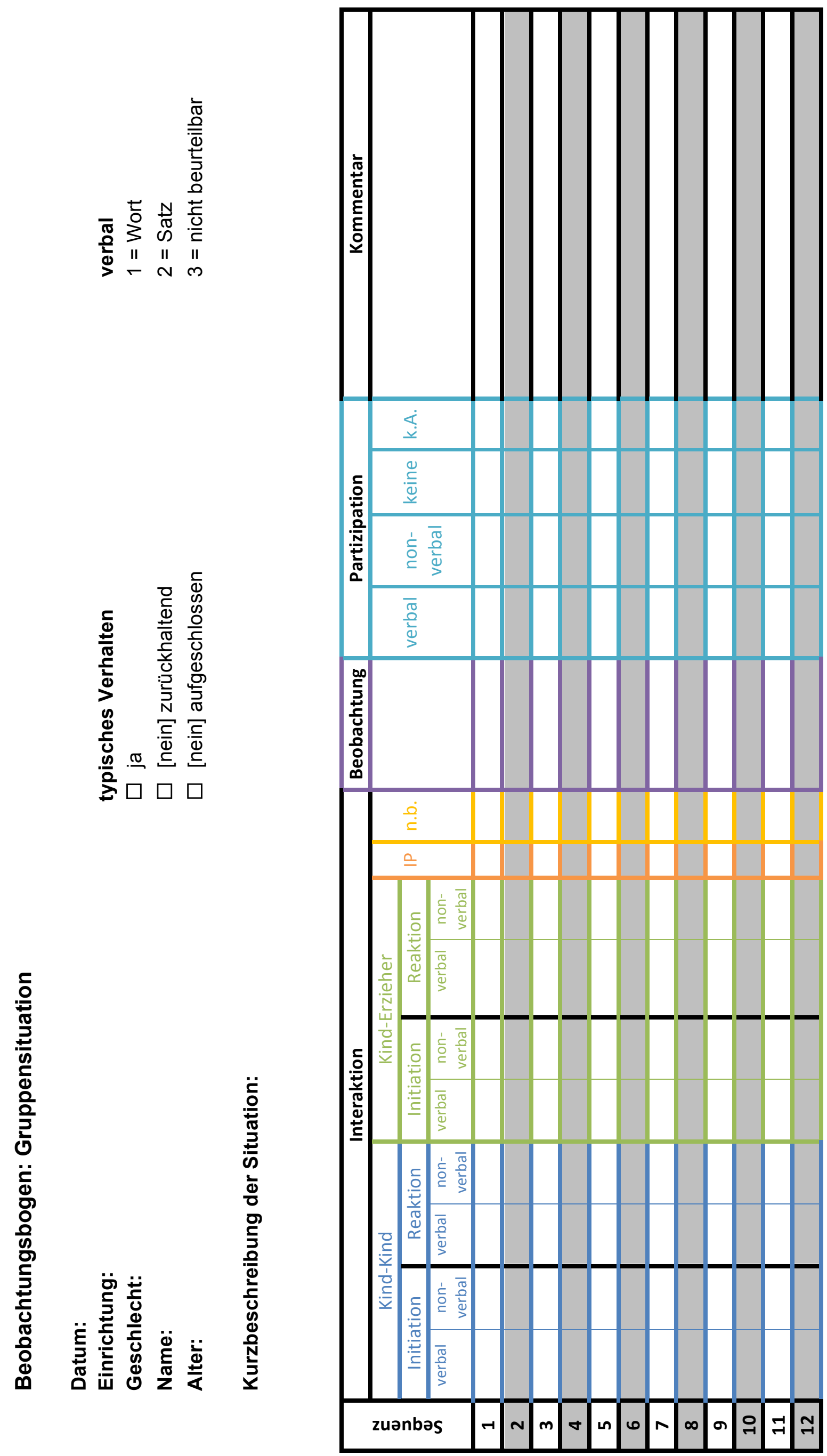


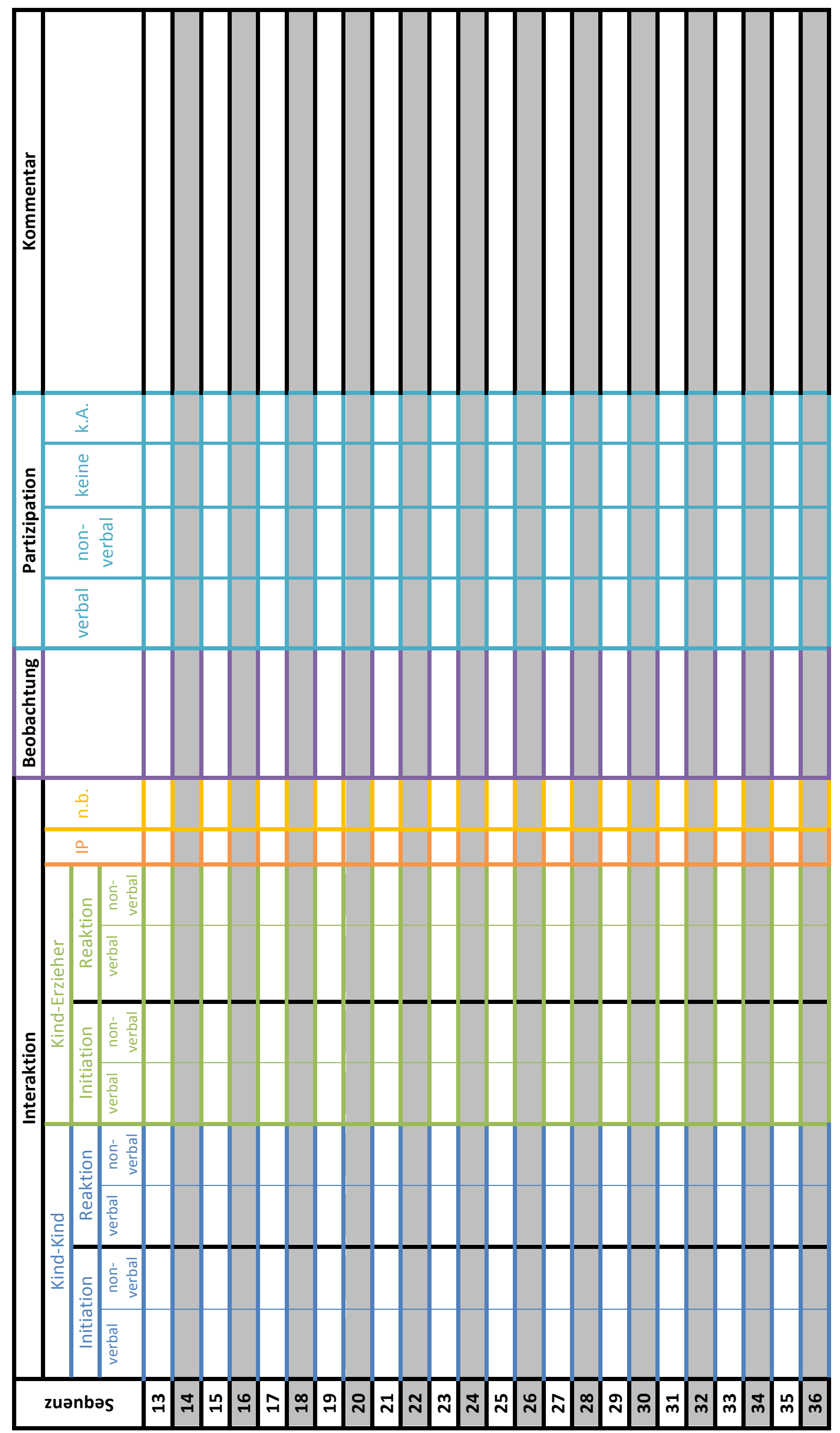



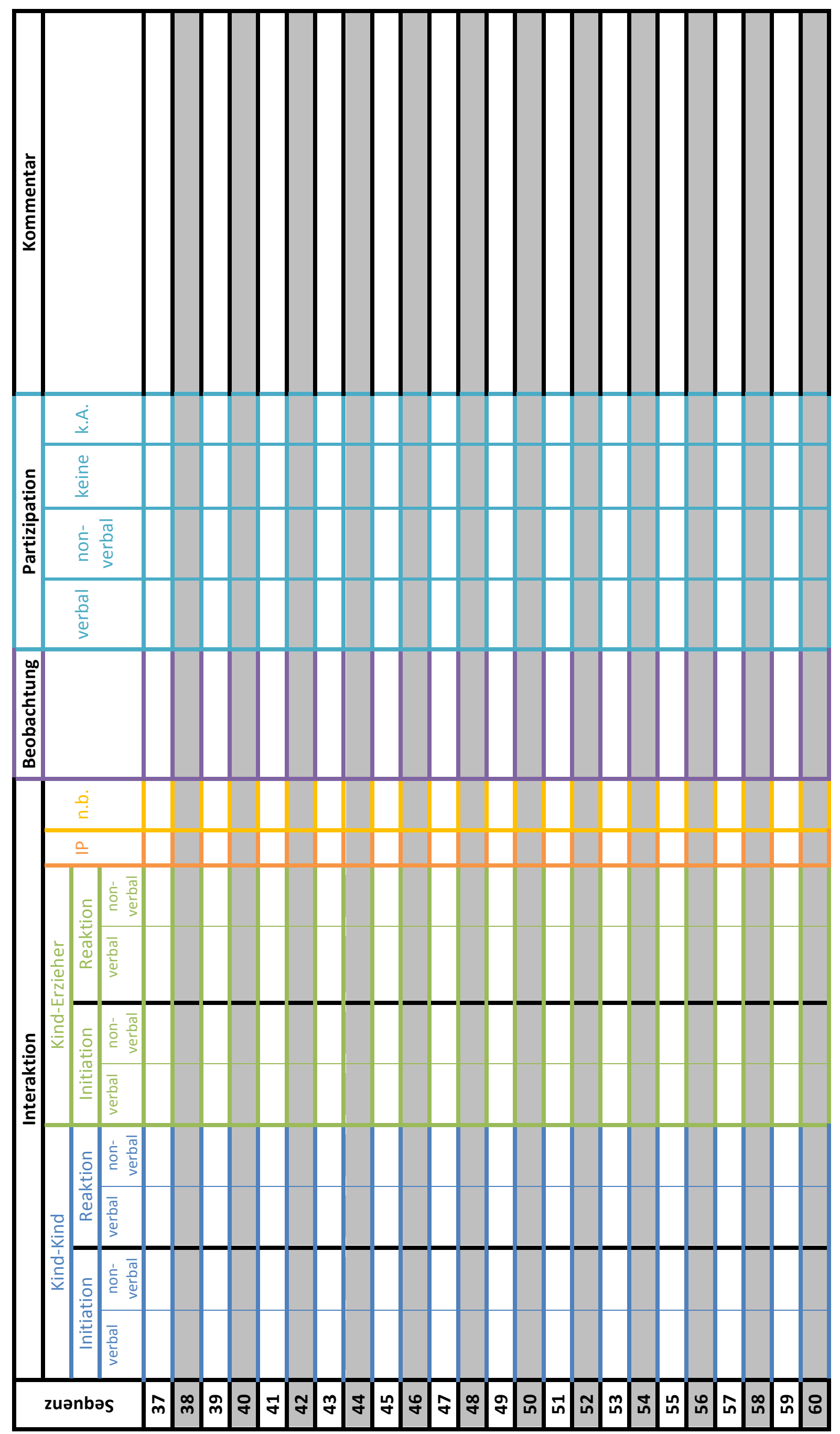


\section{MANUAL ZU DEN BEOBACHTUNGSBÖGEN}

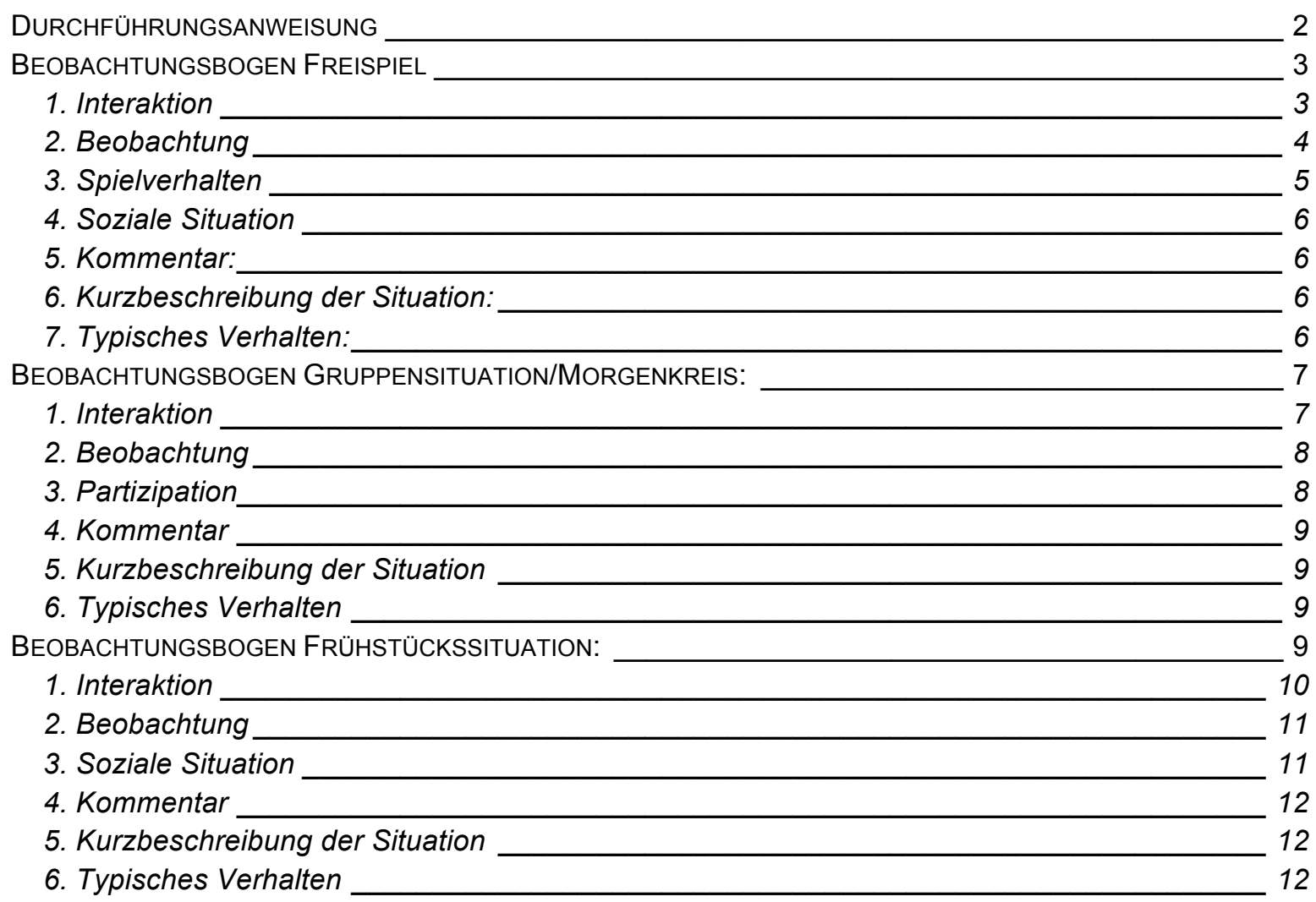




\section{Durchführungsanweisung}

Bei diesem Beobachtungsbogen handelt es sich um eine quantitative Datenerhebung.

Die Beobachtung des Interaktionsverhaltens 3-4jähriger Kinder mittels des vorgestellten Beobachtungsbogens sollte nur durch geschulte Beobachter erfolgen, um zu möglichst genauen und objektiven Ergebnissen zu gelangen. Nicht geschulte Beobachter bringen zu unterschiedliches Vorwissen und Erfahrungen in die Beobachtung ein, so dass eine Vergleichbarkeit der Ergebnisse nicht möglich ist.

Die Beobachtung des Interaktionsverhaltens findet während des Vormittags im Kindergarten statt. Für jede Situation gibt es einen eigenen Beobachtungsbogen mit teilweise unterschiedlichen Beobachtungskategorien. Für alle Situationen gilt, die Beobachter beobachten das jeweilige Kind so unauffällig wie möglich. Bei Ansprache von Kindern verhalten sich die Beobachter freundlich und zurückhaltend. Muss das Kind während der Beobachtung auf die Toilette, wird die Beobachtung für diese Zeit unterbrochen und die Unterbrechung im Kommentarfeld dokumentiert. Verschwindet das Kind in der Beobachtungszeit aus dem Blickfeld, wird zunächst versucht durch das Ändern der eigenen Position das Kind wieder sehen zu können. Ist dies nicht möglich (zum Beispiel wenn sich das Kind in einer Höhle versteckt hat), wird zwei Sequenzen weiter beobachtet und geschaut, ob das Kind wieder herauskommt. Wenn es über zwei Sequenzen verschwunden bleibt, wird die Beobachtung ebenfalls unterbrochen und wieder aufgenommen, wenn das Kind wieder im eigenen Blickfeld ist.

Tauchen innerhalb einer Beobachtungssituation in einer Kategorie (zum Beispiel „soziale Situation“) mehrere Ereignisse auf, entscheiden Sie sich immer für das bedeutsamere für den selektiven Mutismus.

Interaktion: Initiiert das Kind eine Interaktion mit einer Person sowohl nonverbal als auch verbal, kreuzen Sie nur verbal an. Gleiches gilt für die Reaktion. Reagiert das Kind auf eine Ja-Nein-Frage beispielsweise durch das Sagen des Wortes „Ja“ und Kopfnicken, kreuzen Sie nur verbal bei Reaktion an.

Zeigt das Kind in einer Sequenz sowohl eine Initiation als auch eine Reaktion wird beides unabhängig voneinander dokumentiert!

Spielverhalten: Zeigt das Kind in einer Sequenz mehrere Spielformen oder spielt es parallel zu einem Kind (siehe ausführliche Beschreibung bei Spielverhalten), werden alle Arten des Spiels eingetragen.

Soziale Situation: Befindet sich das Kind in einer Beobachtungssequenz in verschiedenen sozialen Situationen, wird nur die „höherwertige“ angegeben. Kommt beispielsweise innerhalb der Beobachtungssequenz ein Kind hinzu (Wechsel von Einzelsituation zu Dyade), wird nur Dyade angekreuzt. Gruppe ist die bedeutsamste soziale Situation, gefolgt von Dyade, gefolgt von Einzelsituation.

Partizipation: Ähnliches gilt für den Bereich der Partizipation. Beteiligt sich das Kind sowohl nonverbal als auch verbal am Gruppengeschehen, wird nur verbal angekreuzt. 


\section{Beobachtungsbogen Freispiel}

Die Beobachtung eines Kindes in der Freispielphase findet nach Beendigung der Bringzeit, in der zweiten Freispielphase statt.

Das Kind wird für insgesamt 30 Minuten beobachtet. Nach einer 20 Sekunden umfassenden Beobachtungsphase erfolgt eine 20 Sekunden umfassende Notationsphase. Bei der Beobachtung durch zwei Beobachter muss auf die Synchronität der Beobachtungsintervalle geachtet werden. (Als Zeitgeber empfehlen wird die App „iTimer“ oder eine Audiodatei mit einem wiederkehrenden Gong)

Die Beobachtung kann in dieser Kategorie unterbrochen werden, falls die äußeren Umstände dies erfordern.

\section{Interaktion}

Unter sozialer Interaktion verstehen wir eine wechselseitige Beeinflussung von Aktivitäten.

\section{Kind-Kind}

Eine Interaktion zwischen dem zu beobachtenden Kinder und einem anderen Kind bzw. anderen Kindern.

- Initiation: Das zu beobachtende Kind regt eine Interaktion mit einem anderen Kind oder anderen Kindern an.

- verbal: Das zu beobachtende Kind initiiert eine Interaktion mit einem anderen Kind oder anderen Kindern mittels Sprache. Die sprachlichen Äußerungen sollen folgendermaßen differenziert werden:

$>1=$ Wort

$>2$ = Satz (auch Satzabbrüche wie zum Beispiel "Ich hab“)

$>3=$ nicht beurteilbar (n.b.) [eine verbale Äußerung liegt vor, kann aber nicht differenziert werden]

- nonverbal: Das zu beobachtende Kind initiert eine Interaktion mit einem anderen Kind oder anderen Kindern mittels körperlichen Ausdrucks (z.B. Mimik, Gestik oder Berührungen).

- Reaktion: Das zu beobachtende Kind geht auf ein an es gerichtete Verhalten (verbal und/oder nonverbal) eines anderen Kindes oder anderer Kinder ein.

- verbal: Das zu beobachtende Kind reagiert mittels Sprache auf ein an es gerichtete Verhalten (verbal und/oder nonverbal) eines anderen Kindes oder anderer Kinder ein. Die sprachlichen Äußerungen sollen folgendermaßen differenziert werden:

$>1=$ Wort

$>2$ = Satz (auch Satzabbrüche wie zum Beispiel "Ich hab“)

$>3$ = nicht beurteilbar (n.b.) [eine verbale Äußerung liegt vor, kann aber nicht differenziert werden]

- nonverbal: Das zu beobachtende Kind reagiert mittels körperlichen Ausdrucks (z.B. Mimik, Gestik und Berührungen) auf ein an es gerichtetes Verhalten (verbal und/oder nonverbal) eines anderen Kindes oder anderer Kinder. 


\section{Kind-Erzieher}

Interaktion zwischen dem zu beobachtendem Kind und einer Erzieherin oder einem Erzieher bzw. betreuenden Erwachsenen in der Einrichtung (auch Praktikanten, Integrationskräfte, Therapeuten usw.)

- Initiation: Das zu beobachtende Kind beginnt eine Interaktion mit einer/einem Erzieherln.

- verbal: Das zu beobachtende Kind regt eine Interaktion mit einer/einem ErzieherIn mittels Sprache an. Die sprachlichen Äußerungen sollen folgendermaßen differenziert werden:

$>1=$ Wort

$>2$ = Satz (auch Satzabbrüche wie zum Beispiel „Ich hab“)

$>3=$ nicht beurteilbar (n.b.) [eine verbale Äußerung liegt vor, kann aber nicht differenziert werden]

- nonverbal: Das zu beobachtende Kind initiiert eine Interaktion mit einer/einem Erzieherln mittels körperlichem Ausdruck, wie Mimik, Gestik und Berührungen.

- Reaktion: Das zu beobachtende Kind geht auf ein an es gerichtetes Verhalten (verbal und/oder non-verbal) einer/eines Erzieherln ein.

- verbal: Das zu beobachtende Kind reagiert mittels Sprache auf ein an es gerichtete Verhalten (verbal und/oder nonverbal) einer/eines Erzieherln ein. Die sprachlichen Äußerungen sollen folgendermaßen differenziert werden:

$>1=$ Wort

$>2$ = Satz (auch Satzabbrüche wie zum Beispiel „Ich hab“)

> $3=$ nicht beurteilbar (n.b.) [eine verbale Äußerung liegt vor, kann aber nicht differenziert werden]

- nonverbal: Das zu beobachtende Kind reagiert mittels körperlichem Ausdruck, wie Mimik, Gestik und Berührungen auf ein an es gerichtetes Verhalten (verbal und/oder nonverbal) einer/eines Erzieherln.

\section{Wechsel von Interaktionspartnern (IP)}

Innerhalb des Beobachtungszeitraumes von 20 Sekunden findet ein Wechsel des Interaktionspartners statt. Das Kind wird von einer „neuen“ Person angesprochen oder spricht selbst eine neue Person an bzw. eine „neue“ Person kommt in das Gruppengeschehen herein und nimmt an der Gruppeninteraktion teil.

Beispiel: (1) Das Kind puzzelt gerade alleine am Tisch. Die Erzieherin kommt hinzu und fragt, ob das Kind etwas trinken möchte. (2) Das Kind baut gerade mit einem anderen Kind einen Turm aus Bauklötzen. Ein drittes Kind kommt hinzu und fragt, ob es mitbauen darf. Das zu beobachtende Kind schaut das neue Kind an. Das zweite Kind antwortet verbal, dass das dritte mitspielen darf.

\section{nicht beurteilbar (nb)}

Der Beobachter hat irgendeine Art der Interaktion identifizieren können. Eine Kategorisierung in Initiation oder Reaktion ist jedoch nicht eindeutig möglich.

\section{Beobachtung}

Das zu beobachtende Kind ist in keine Interaktion eingebunden. Das Kind befindet sich somit in einer Einzelsituation (siehe soziale Situation). Es beobachtet die anderen Kinder oder 
Erwachsenen bei ihren Tätigkeiten. Das Ankreuzen von Beobachtung schließt die sozialen Situationen Dyade und Gruppe aus sowie irgendeine Art von Interaktion.

Beispiel: Das Kind sitzt am Tisch und malt. Es schaut hoch und beobachtet eine Kindergruppe, die gerade gemeinsam einen Turm bauen.

\section{Spielverhalten}

- $\quad 1$ = Sympraktisches Sprechen: das eigene spielbegleitende Sprechen. Das Kind ist in einer Einzelsituation und spricht alleine für sich. Beispiel: Es spielt mit Autos und beschreibt, woher sie fahren, dass es einen Unfall gibt usw.

- $\quad \mathbf{2}$ = Parallelspiel: Kinder spielen nebeneinander, beschäftigen sich teilweise mit demselben Spielgegenstand und beobachten sich dabei gegenseitig. Sie greifen aber nicht in das Spiel des anderen ein (Renner, 2008/S.109 f). Das Kind befindet sich somit nur in einer Einzelsituation.

z.B. Zwei Kinder spielen im Sandkasten nebeneinander je mit einem Bagger und schaufeln Sand. Dabei beobachten sie einander. Zwei Kinder fahren nebeneinander auf einem Bobbycar und beobachten sich gegenseitig.

Die beiden Kategorien Sympraktisches Sprechen und Parallelspiel kommen immer in Kombination mit einer weiteren Spielform vor!

Für die oben stehenden Beispiele: 1. Symbolspiel und Sympraktisches Sprechen, 2.

Funktionsspiel und Parallelspiel, 3. Bewegungsspiel und Parallelspiel

- $\quad 3$ = Funktionsspiel: Hier wird das gegenstandsbezogene Funktionsspiel gemeint. Das heißt das Kind probiert die Funktion eines Gegenstandes aus. Zum Beispiel das Spiel mit einer Kugelbahn: die Kugeln werden immer wieder die Bahn heruntergerollt und dabei betrachtet. Oder das Ein- und Ausschütten von Eimern mit Sand. Der Eimer wird immer wieder mit Sand gefüllt und dann wieder ausgeschüttet.

- $\quad 4=$ Konstruktionsspiel: [Bauspiel] Mit Materialen (Bausteinen, LEGO, Steinen, Sand, Plastik, Holz etc.) werden planmäßig Bauwerke hergestellt. Auch Steckspiele wie Puzzle gehören dazu. z.B. das Bauen eines Turms mit Bauklötzen oder auch Kneten von Figuren.

- $\quad \mathbf{5}=$ Symbolspiel: [Als-ob-Spiel] Das Kind generalisiert in symbolischen Darstellungen und Handlungen konkrete Erfahrungen und Verhaltensweisen und stellt sie zum Schein dar (Renner, 2008, S.109). z.B. Das Kind füttert die Puppe von einem leeren Teller. Das Kind bezahlt mit Bauklötzen. Das Kind benutzt einen Bauklotz als Telefon.

- $\quad \mathbf{6}=$ Rollenspiel: Im Rollenspiel beziehen sich die Kinder auf einen gemeinsamen Gegenstand (Spielthema) und nehmen bezogen auf diesen eine fiktive Rolle ein, welche zur Richtschnur ihres Handelns wird. z.B. Einkaufen spielen (Verkäuferin und Kunde), Doktor spielen (Arzt und Patient), Piraten spielen (Anführer und Gefolgsleute), Polizist und Räuber.

- 7 = Regelspiel: Spiele, bei denen die Regel zum Spielinhalt wird oder zumindest stark im Vordergrund des Spiels steht (Renner, 2008, S.130).

z.B. Brettspiele, Kartenspiele

- $\quad \mathbf{8}=$ Bewegungsspiel: Spiele, bei denen die Lust an der Bewegung im Vordergrund steht. z.B. Fangenspiel, Balancieren, Schaukeln, Rutschen, Ballspiele, Rollerfahren

- 9 = weiteres: Restkategorie für alle nicht konkret spielerischen Handlungen aber „sinnvollen Beschäftigungen“ wie zum Beispiel Malen, Bilderbuch lesen, Weben, 
Basteln, Übernahmen von Aufgaben (helfen bei der Frühstückszubereitung). Die Handlung selbst sollte dann im Kommentarfeld kurz beschrieben werden.

\section{Literatur:}

Fritz, J. (2004) Das Spiel verstehen. Eine Einführung in Theorie und Bedeutung. Weinheim und München: Juventa Verlag

Renner, M. (2008). Spieltheorie und Spielpraxis. Eine Einführung für pädagogische Berufe (3. Neu bearbeitet Auflage). Freiburg im Breisgau: Lambertus-Verlag.

Oerter, R. (1993). Psychologie des Spiels. Ein handlungstheoretischer Ansatz. München: Quintessenz.

\section{Soziale Situation}

Grundsätzlich ist bei der Kategorie soziale Situation der Bezug zu einer anderen Person oder anderen Personen bedeutsam, nicht die reine Anwesenheit. Das heißt, die Kinder können zwar gemeinsam am Tisch sitzen, aber trotzdem für sich alleine spielen und nicht miteinander interagieren.

- Einzelsituation: Das zu beobachtende Kind ist für sich.

- Dyade: Das zu beobachtende Kind befindet sich in einer Interaktion mit einem weiteren Interaktionspartner (Kind/Erwachsener).

- Gruppe: Das zu beobachtende Kind befindet sich in einer Interaktion mit mehr als einem weiteren Interaktionspartner (Kind/Erwachsener).

\section{Kommentar:}

Vermerken von möglichen Besonderheiten, wie zum Beispiel Emotionen (lautes Lachen, Weinen etc.), sowie Faktoren, die die Beobachtungssituationen beeinflussen. Hier können außerdem Dinge vermerkt werden, welche vom Beobachter als wichtig wahrgenommen werden. Zum Beispiel auch Unterbrechung der Beobachtung aufgrund des Toilettenganges des Kindes; genauere Beschreibung des Spiels.

\section{Kurzbeschreibung der Situation:}

Darstellung der beobachteten Situation bezogen auf Räumlichkeiten, Verhaltensweisen/Emotionen des zu beobachtenden Kindes und das Spielthema. Möglichkeit für weitere Anmerkungen des Beobachters.

\section{Typisches Verhalten:}

Nach der Beobachtung wird die Erzieherin/ der Erzieher kurz danach gefragt, ob das Kind in den letzten 30 Minuten sein typisches Verhalten gezeigt hat. Wird das Verhalten als eher untypische kategorisiert, soll nach dem Unterschied gefragt werden. War das Kind zurückhaltender oder aufgeschlossener als sonst. 


\section{Beobachtungsbogen Gruppensituation/Morgenkreis:}

Das Kind wird während einer Gruppensituation (Morgenkreis) maximal 30 Minuten beobachtet. Nach einer 15 Sekunden umfassenden Beobachtungsphase erfolgt eine 15 Sekunden umfassende Notationsphase. Die Durchführung der Beobachtung erfolgt nach obigem Schema.

\section{Interaktion}

Unter sozialer Interaktion verstehen wir eine wechselseitige Beeinflussung von Aktivitäten.

\section{Kind-Kind}

Eine Interaktion zwischen dem zu beobachtenden Kinder und einem anderen Kind bzw. anderen Kindern.

- Initiation: Das zu beobachtende Kind regt eine Interaktion mit einem anderen Kind oder anderen Kindern an.

- verbal: Das zu beobachtende Kind initiiert eine Interaktion mit einem anderen Kind oder anderen Kindern mittels Sprache. Die sprachlichen Äußerungen sollen folgendermaßen differenziert werden:

$>1=$ Wort

$>2$ = Satz (auch Satzabbrüche wie zum Beispiel "Ich hab“)

$>3=$ nicht beurteilbar (n.b.) [eine verbale Äußerung liegt vor, kann aber nicht differenziert werden]

- nonverbal: Das zu beobachtende Kind initiiert eine Interaktion mit einem anderen Kind oder anderen Kindern mittels körperlichen Ausdrucks (z.B. Mimik, Gestik oder Berührungen).

- Reaktion: Das zu beobachtende Kind geht auf ein an es gerichtete Verhalten (verbal und/oder nonverbal) eines anderen Kindes oder anderer Kinder ein.

- verbal: Das zu beobachtende Kind reagiert mittels Sprache auf ein an es gerichtete Verhalten (verbal und/oder nonverbal) eines anderen Kindes oder anderer Kinder ein. Die sprachlichen Äußerungen sollen folgendermaßen differenziert werden:

$>1=$ Wort

$>2$ = Satz (auch Satzabbrüche wie zum Beispiel „Ich hab“)

$>3=$ nicht beurteilbar (n.b.) [eine verbale Äußerung liegt vor, kann aber nicht differenziert werden]

- nonverbal: Das zu beobachtende Kind reagiert mittels körperlichen Ausdrucks (z.B. Mimik, Gestik und Berührungen) auf ein an es gerichtetes Verhalten (verbal und/oder nonverbal) eines anderen Kindes oder anderer Kinder.

\section{Kind-Erzieher}

Interaktion zwischen dem zu beobachtendem Kind und einer Erzieherin oder einem Erzieher bzw. betreuenden Erwachsenen in der Einrichtung (auch Praktikanten, Integrationskräfte, Therapeuten usw.)

- Initiation: Das zu beobachtende Kind beginnt eine Interaktion mit einer/einem Erzieherln.

- verbal: Das zu beobachtende Kind regt eine Interaktion mit einer/einem Erzieherln mittels Sprache an. Die sprachlichen Äußerungen sollen folgendermaßen differenziert werden: 
$>1=$ Wort

$>2$ = Satz (auch Satzabbrüche wie zum Beispiel „Ich hab“)

$>3=$ nicht beurteilbar (n.b.) [eine verbale Äußerung liegt vor, kann aber nicht differenziert werden]

- nonverbal: Das zu beobachtende Kind initiiert eine Interaktion mit einer/einem Erzieherln mittels körperlichem Ausdruck, wie Mimik, Gestik und Berührungen.

- Reaktion: Das zu beobachtende Kind geht auf ein an es gerichtetes Verhalten (verbal und/oder non-verbal) einer/eines Erzieherln ein.

- verbal: Das zu beobachtende Kind reagiert mittels Sprache auf ein an es gerichtete Verhalten (verbal und/oder nonverbal) einer/eines Erzieherln ein. Die sprachlichen Äußerungen sollen folgendermaßen differenziert werden:

$>1=$ Wort

$>2$ = Satz (auch Satzabbrüche wie zum Beispiel „Ich hab“)

$>3=$ nicht beurteilbar (n.b.) [eine verbale Äußerung liegt vor, kann aber nicht differenziert werden]

- nonverbal: Das zu beobachtende Kind reagiert mittels körperlichem Ausdruck, wie Mimik, Gestik und Berührungen auf ein an es gerichtetes Verhalten (verbal und/oder nonverbal) einer/eines Erzieherln.

\section{Wechsel von Interaktionspartnern (IP)}

Innerhalb des Beobachtungszeitraumes von 20 Sekunden findet ein Wechsel des Interaktionspartners statt. Das Kind wird von einer „neuen“ Person angesprochen oder spricht selbst eine neue Person an bzw. eine „neue“ Person kommt in das Gruppengeschehen herein und nimmt an der Gruppeninteraktion teil.

Beispiel: (1) Das Kind puzzelt gerade alleine am Tisch. Die Erzieherin kommt hinzu und fragt, ob das Kind etwas trinken möchte. (2) Das Kind baut gerade mit einem anderen Kind einen Turm aus Bauklötzen. Ein drittes Kind kommt hinzu und fragt, ob es mitbauen darf. Das zu beobachtende Kind schaut das neue Kind an. Das zweite Kind antwortet verbal, dass das dritte mitspielen darf.

\section{nicht beurteilbar (nb)}

Der Beobachter hat irgendeine Art der Interaktion identifizieren können. Eine Kategorisierung in Initiation oder Reaktion ist jedoch nicht eindeutig möglich.

\section{Beobachtung}

Das zu beobachtende Kind ist in keine Interaktion eingebunden. Das Kind befindet sich somit in einer Einzelsituation (siehe soziale Situation). Es beobachtet die anderen Kinder oder Erwachsenen bei ihren Tätigkeiten. Das Ankreuzen von Beobachtung schließt die sozialen Situationen Dyade und Gruppe aus sowie irgendeine Art von Interaktion.

Beispiel: Das Kind sitzt zwar im Stuhlkreis, beteiligt sich aber offensichtlich nicht an den Aktivitäten und beobachtet lediglich das Geschehen. Oder das Kind sitzt außerhalb des Kreises und beobachtet das Geschehen der Gruppensituationen.

\section{Partizipation}

- verbal: Das zu beobachtende Kind beteiligt sich mittels Sprache an den Aktivitäten. Es erzählt beispielsweise ein Erlebnis vom Wochenende oder spricht/singt Sprech/Singspiele mit. 
- nonverbal: Das zu beobachtende Kind beteiligt sich mittels körperlichem Ausdruck (Gestik, Mimik und/oder Berührungen) an den Aktivitäten. Es klatscht zum Beispiel beim Singen mit.

- keine: Das zu beobachtende Kind beteiligt sich weder verbal noch nonverbal an den Aktivitäten.

- kein Angebot: Dem zu beobachtenden Kind wird kein Partizipationsangebot gemacht. Das heißt in der Beobachtungssituation gibt es trotz Stuhlkreis- oder Gruppensituation keine Gelegenheit für das Kind, sich in irgendeiner Art an den Handlungen zu beteiligen. Zum Beispiel erzählt nur ein Kind von seinem Wochenende, die anderen Kinder dürfen nicht mehr erzählen. Oder das Kind sitzt außerhalb der Gruppe und wird nicht zum Mitmachen in der Gruppe eingeladen.

- Im Kommentarfeld wird vermerkt, welche Art von Aktivität durchgeführt wurde. Die Aktivitäten sollen folgendermaßen differenziert werden:

- verbale Aktivität (singen, Reime mitsprechen usw.)

- ausschließlich nonverbale Aktivität (Bewegungsspiele usw.)

\section{Kommentar}

Vermerken von möglichen Besonderheiten, wie zum Beispiel Emotionen (lautes Lachen, Weinen etc.), sowie Faktoren, die die Beobachtungssituationen beeinflussen. Hier können außerdem Dinge vermerkt werden, welche vom Beobachter als wichtig wahrgenommen werden.

\section{Kurzbeschreibung der Situation}

Darstellung der beobachteten Situation bezogen auf Räumlichkeiten, Verhaltensweisen/Emotionen des zu beobachtenden Kindes und das Spielthema. Möglichkeit für weitere Anmerkungen des Beobachters.

\section{Typisches Verhalten}

Nach der Beobachtung wird die Erzieherin/ der Erzieher kurz danach gefragt, ob das Kind in den letzten 30Minuten sein typisches Verhalten gezeigt hat. Wird das Verhalten als eher untypische kategorisiert, soll nach dem Unterschied gefragt werden. War das Kind zurückhaltender oder aufgeschlossener als sonst.

\section{Beobachtungsbogen Frühstückssituation:}

Das Kind wird während des Frühstücks oder einer vergleichbaren Essenssituation maximal 30 Minuten beobachtet. Nach einer 20 Sekunden umfassenden Beobachtungsphase erfolgt eine 20 Sekunden umfassende Notationsphase. Die Durchführung der Beobachtung erfolgt nach obigem Schema. 


\section{Interaktion}

Unter sozialer Interaktion verstehen wir eine wechselseitige Beeinflussung von Aktivitäten.

\section{Kind-Kind}

Eine Interaktion zwischen dem zu beobachtenden Kinder und einem anderen Kind bzw. anderen Kindern.

- Initiation: Das zu beobachtende Kind regt eine Interaktion mit einem anderen Kind oder anderen Kindern an.

- verbal: Das zu beobachtende Kind initiiert eine Interaktion mit einem anderen Kind oder anderen Kindern mittels Sprache. Die sprachlichen Äußerungen sollen folgendermaßen differenziert werden:

$>1=$ Wort

$>2$ = Satz (auch Satzabbrüche wie zum Beispiel "Ich hab“)

$>3=$ nicht beurteilbar (n.b.) [eine verbale Äußerung liegt vor, kann aber nicht differenziert werden]

- nonverbal: Das zu beobachtende Kind initiiert eine Interaktion mit einem anderen Kind oder anderen Kindern mittels körperlichen Ausdrucks (z.B. Mimik, Gestik oder Berührungen).

- Reaktion: Das zu beobachtende Kind geht auf ein an es gerichtete Verhalten (verbal und/oder nonverbal) eines anderen Kindes oder anderer Kinder ein.

- verbal: Das zu beobachtende Kind reagiert mittels Sprache auf ein an es gerichtete Verhalten (verbal und/oder nonverbal) eines anderen Kindes oder anderer Kinder ein. Die sprachlichen Äußerungen sollen folgendermaßen differenziert werden:

$>1=$ Wort

$>2$ = Satz (auch Satzabbrüche wie zum Beispiel „Ich hab“)

$>3=$ nicht beurteilbar (n.b.) [eine verbale Äußerung liegt vor, kann aber nicht differenziert werden]

- nonverbal: Das zu beobachtende Kind reagiert mittels körperlichen Ausdrucks (z.B. Mimik, Gestik und Berührungen) auf ein an es gerichtetes Verhalten (verbal und/oder nonverbal) eines anderen Kindes oder anderer Kinder.

\section{Kind-Erzieher}

Interaktion zwischen dem zu beobachtendem Kind und einer Erzieherin oder einem Erzieher bzw. betreuenden Erwachsenen in der Einrichtung (auch Praktikanten, Integrationskräfte, Therapeuten usw.)

- Initiation: Das zu beobachtende Kind beginnt eine Interaktion mit einer/einem Erzieherln.

- verbal: Das zu beobachtende Kind regt eine Interaktion mit einer/einem Erzieherln mittels Sprache an. Die sprachlichen Äußerungen sollen folgendermaßen differenziert werden:

$>1=$ Wort

$>2$ = Satz (auch Satzabbrüche wie zum Beispiel „Ich hab“)

$>3=$ nicht beurteilbar (n.b.) [eine verbale Äußerung liegt vor, kann aber nicht differenziert werden]

- nonverbal: Das zu beobachtende Kind initiiert eine Interaktion mit einer/einem Erzieherln mittels körperlichem Ausdruck, wie Mimik, Gestik und Berührungen. 
- Reaktion: Das zu beobachtende Kind geht auf ein an es gerichtetes Verhalten (verbal und/oder non-verbal) einer/eines Erzieherln ein.

- verbal: Das zu beobachtende Kind reagiert mittels Sprache auf ein an es gerichtete Verhalten (verbal und/oder nonverbal) einer/eines Erzieherln ein. Die sprachlichen Äußerungen sollen folgendermaßen differenziert werden:

$>1=$ Wort

$>2$ = Satz (auch Satzabbrüche wie zum Beispiel „Ich hab“)

$>3=$ nicht beurteilbar (n.b.) [eine verbale Äußerung liegt vor, kann aber nicht differenziert werden]

- nonverbal: Das zu beobachtende Kind reagiert mittels körperlichem Ausdruck, wie Mimik, Gestik und Berührungen auf ein an es gerichtetes Verhalten (verbal und/oder nonverbal) einer/eines Erzieherln.

\section{Wechsel von Interaktionspartnern (IP)}

Innerhalb des Beobachtungszeitraumes von 20 Sekunden findet ein Wechsel des

Interaktionspartners statt. Das Kind wird von einer „neuen“ Person angesprochen oder spricht selbst eine neue Person an bzw. eine „neue“ Person kommt in das Gruppengeschehen herein und nimmt an der Gruppeninteraktion teil.

Beispiel: Das Kind sitzt zunächst alleine am Tisch und isst. Ein weiteres Kind kommt hinzu und spricht das Kind an. Oder eine Erzieherin kommt hinzu und fragt das Kind, ob es lieber Saft oder Kakao trinken möchte.

\section{nicht beurteilbar (nb)}

Der Beobachter hat irgendeine Art der Interaktion identifizieren können. Eine Kategorisierung in Initiation oder Reaktion ist jedoch nicht eindeutig möglich.

\section{Beobachtung}

Das zu beobachtende Kind ist in keine Interaktion eingebunden. Das Kind befindet sich somit in einer Einzelsituation (siehe soziale Situation). Es beobachtet die anderen Kinder oder Erwachsenen bei ihren Tätigkeiten. Das Ankreuzen von Beobachtung schließt die sozialen Situationen Dyade und Gruppe aus sowie irgendeine Art von Interaktion.

Beispiel: Das Kind sitzt alleine am Tisch und beobachtet die anderen Kinder am Nachbartisch.

\section{Soziale Situation}

Grundsätzlich ist bei der Kategorie soziale Situation der Bezug zu einer anderen Person oder anderen Personen bedeutsam, nicht die reine Anwesenheit. Das heißt, die Kinder können zwar gemeinsam am Tisch sitzen, aber trotzdem für sich alleine spielen und nicht miteinander interagieren.

- Einzelsituation: Das zu beobachtende Kind ist für sich.

- Dyade: Das zu beobachtende Kind befindet sich in einer Interaktion mit einem weiteren Interaktionspartner (Kind/Erwachsener).

- Gruppe: Das zu beobachtende Kind befindet sich in einer Interaktion mit mehr als einem weiteren Interaktionspartner (Kind/Erwachsener). 


\section{Kommentar}

Vermerken von möglichen Besonderheiten, wie zum Beispiel Emotionen (lautes Lachen, Weinen etc.), sowie Faktoren, die die Beobachtungssituationen beeinflussen. Hier können außerdem Dinge vermerkt werden, welche vom Beobachter als wichtig wahrgenommen werden.

\section{Kurzbeschreibung der Situation}

Darstellung der beobachteten Situation bezogen auf Räumlichkeiten,

Verhaltensweisen/Emotionen des zu beobachtenden Kindes und das Spielthema.

Möglichkeit für weitere Anmerkungen des Beobachters.

\section{Typisches Verhalten}

Nach der Beobachtung wird die Erzieherin/ der Erzieher kurz danach gefragt, ob das Kind in den letzten 30 Minuten sein typisches Verhalten gezeigt hat. Wird das Verhalten als eher untypische kategorisiert, soll nach dem Unterschied gefragt werden. War das Kind zurückhaltender oder aufgeschlossener als sonst. 
Anhang E: Beschreibung der Interaktionssettings 


\section{Ebenen der Interaktionssettings (Thema Zoo)}

\section{Materialien:}

Ebene 1

- Bild vom Seehund mit Ball auf der Nase

- Ball

- 11 Belohnungsfische

- 2 Sitzkissen

\section{Ebene 2}

- 4 Tierkäfige mit Bildern der jeweiligen Tiere

- 4 passende Tierarten (je 5 Stück): Nilpferde, Zebras, Giraffen, Eisbären (Schleich)

- Decke

Ebene 3

- Actionfigur

Reihenfolge der Ebenen:

1. Ebene $1+3$

2. Ebene 2

3. TROG-D

\section{Settingsaufbau:}

Vorbereitung:

Setting 2

Zebra, Eisbär, Giraffe Nilpferd

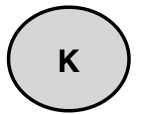

\section{$\mathbf{T}$}

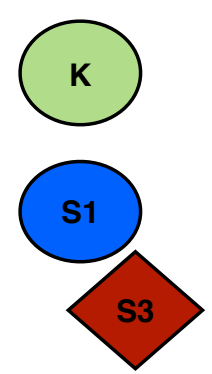

\section{$\mathrm{T}$}


- Seehundspiel aufbauen (Actionfigur aufstellen, Seehundbild, Ball und Fische bereitlegen)

- Zootiere-Spiel aufbauen (Käfige aufstellen, Tiere verteilen, Decke ausbreiten)

- von rechts nach links: Eisbär, Nilpferd, Giraffe, Zebras

\section{Beginn des Settings:}

Kind kommt mit Testleiter in den Raum

„Schön, dass du da bist. Ich habe mir etwas ganz Tolles überlegt: Wir spielen heute Zoo!

Wir können uns schon einmal hier auf den Boden setzen. Jeder hat hier seinen eigenen Platz. Du kannst dich auf den Teppich setzen und ich setze mich hier hin." (Platz von Ebene 1+3)

\section{Ebene (Reaktion) + 3. Ebene (Eigeninitiative des Kindes)}

\section{Teil}

"Schau mal. Ich habe hier ein Bild von einem Seehund. Die gibt es im Zoo und die können tolle Kunststücke mit einem Ball machen. Seehunde können einen Ball auf ihrer Nase balancieren oder auch mit ihrer Flosse schießen. Wir probieren jetzt auch einmal, ob wir den Ball mit unseren Händen schießen können, so wie ein Seehund mit seiner Flosse.

Oh, ich sehe gerade, der Elmo steht ja auch hier. Elmo, du kannst uns zugucken. So, ich stelle ihn mal beiseite."

Testleiter schaltet die Figur unauffällig ein.

"Oh, jetzt hab ich ihn aus Versehen angeschaltet. Der ist lustig, was? Er ist sehr kitzelig."

Nachdem die Figur wieder ruhig ist: "Ich stelle ihn mal hier hin. Da kann er uns gut sehen. Vielleicht können wir ja später noch einmal mit ihm spielen, wenn du möchtest."

Testleiter stellt die Figur auf die Kommode.

\begin{tabular}{|c|c|c|}
\hline $\begin{array}{l}\text { Kind zeigt auf die Figur/ macht } \\
\text { Gesten }\end{array}$ & $\begin{array}{l}\text { Kind lächelt nur/ sieht die Figur } \\
\text { an }\end{array}$ & $\begin{array}{l}\text { Kind reagiert nicht/ zeigt kein } \\
\text { Interesse an der Figur }\end{array}$ \\
\hline $\begin{array}{l}\text { "Oh, zeigst du auf Elmo? Möchtest } \\
\text { du ihn dir noch einmal } \\
\text { ansehen?" (Auf Reaktion warten) } \\
\text { "Ich hole ihn." }\end{array}$ & \begin{tabular}{|l|} 
"Lachst du wegen der lustigen \\
Figur? Gefällt sie dir? (Auf \\
Reaktion warten). Du möchtest sie \\
wohl haben, was? Soll ich sie noch \\
einmal holen?"
\end{tabular} & Fortfahren mit dem Setting \\
\hline Testleiter holt Figur & Testleiter holt die Figur & $\begin{array}{l}\text { Kind hat noch bis Ende des } \\
\text { Settings Zeit, auf die Actionfigur } \\
\text { zu reagieren. }\end{array}$ \\
\hline $\begin{array}{l}\text { "Schau mal. Elmo ist kitzelig. } \\
\text { Wenn du hier auf den Fuß } \text { drückst, } \\
\text { dann fängt er an zu lachen." }\end{array}$ & \begin{tabular}{|l|} 
"Schau mal. Elmo ist kitzelig. \\
Wenn du hier auf den Fuß drückst, \\
dann fängt er an zu lachen."
\end{tabular} & \\
\hline $\begin{array}{l}\text { Nach ein paar Minuten, in denen } \\
\text { das Kind mit der Figur spielen } \\
\text { durfte, wird mit dem Setting } \\
\text { fortgefahren. }\end{array}$ & $\begin{array}{l}\text { Nach ein paar Minuten, in denen } \\
\text { das Kind mit der Figur spielen } \\
\text { durfte, wird mit dem Setting } \\
\text { fortgefahren. }\end{array}$ & \\
\hline $\begin{array}{l}\text { "So, jetzt haben wir den Elmo } \\
\text { genug gekitzelt. Ich stelle ihn } \\
\text { wieder zurück auf die Kommode, } \\
\text { ok?" }\end{array}$ & $\begin{array}{l}\text { "So, jetzt haben wir den Elmo } \\
\text { genug gekitzelt. Ich stelle ihn } \\
\text { wieder zurück auf die Kommode, } \\
\text { ok?" }\end{array}$ & \\
\hline
\end{tabular}

\section{Teil:-}

„Und jetzt versuchen wir, uns gegenseitig den Ball zu zu rollen. Ich zeig dir gleich, wie es geht. Wenn es einer von uns schafft, den Ball gut zu rollen, bekommt er als Belohnung einen Fisch. Guck, so einen."

Testleiter zeigt dem Kind den Eimer mit den Fischen.

"Seehunde essen nämlich sehr gerne Fisch. So, ich zeige dir jetzt, wie man den Ball rollt." 
Testleiter rollt dem Kind den Ball entgegen.

"Hast du gesehen? Möchtest du es auch einmal versuchen?"

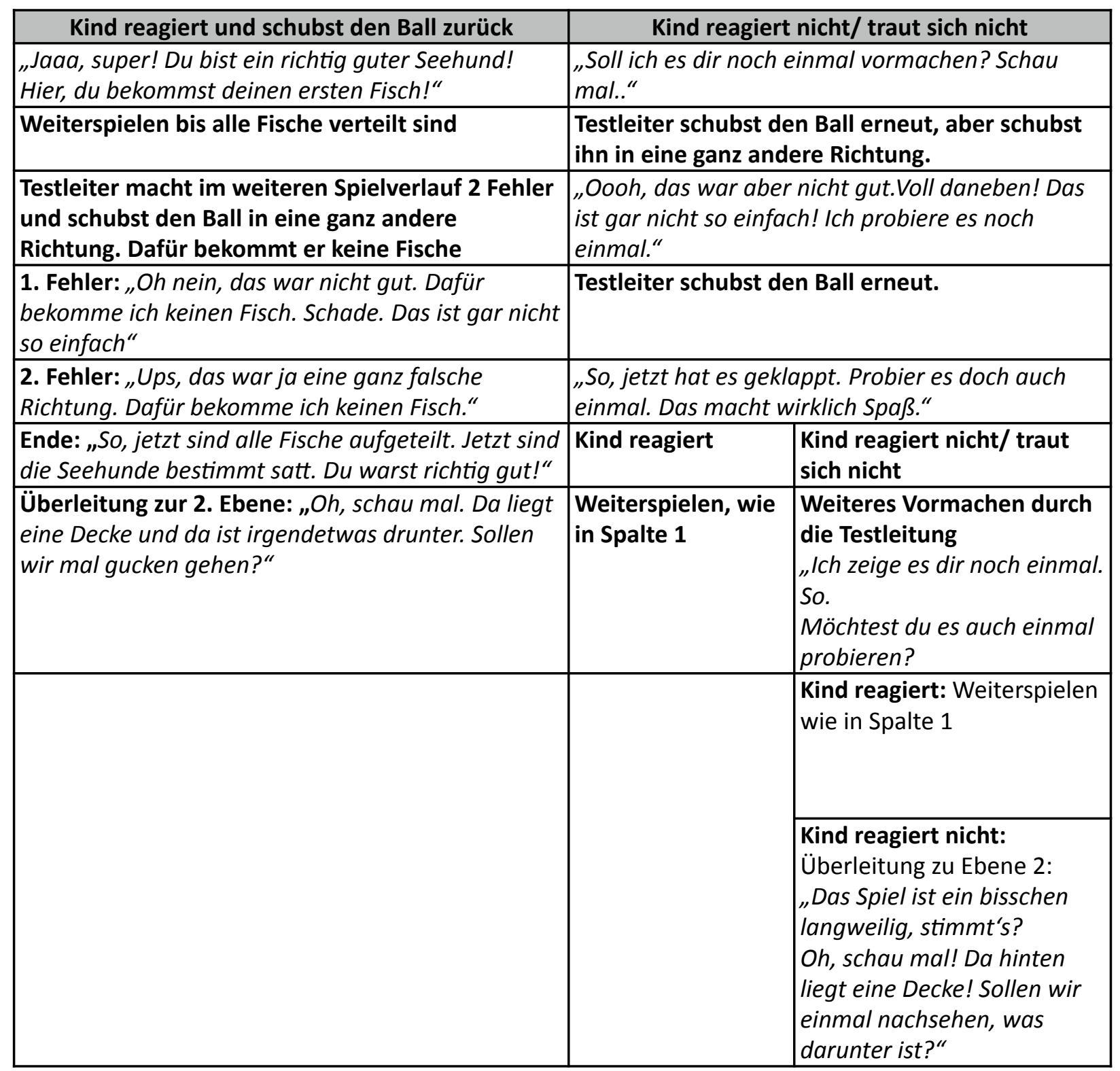

2. Ebene (Missverständnissituation)

Decke wird abgenommen:

„Oh, Nein! Hier laufen alle Tiere durcheinander! Sie sind aus ihren Käfigen ausgebrochen.Oje! Das ist aber nicht gut! Die vertragen sich doch gar nicht alle. Hilfst du mir, sie wieder in ihre Käfige zu bringen?"

Testleiter muss anhand der Bilder auf den Käfigen genau erklären, welche Tiere in welchen Käfig gehören (Dabei Käfige hochheben und gut zeigen).

„Auf diesem Käfig ist ein Zebra. Darin wohnen die Zebras. Die fressen gerne Heu.

Und auf dem Käfig hier ist ein Eisbär zu sehen. Dort wohnen die Eisbären. Die leben da, wo es sehr kalt ist. Hier auf diesem Käfig kannst du eine Giraffe sehen. Giraffen sind gaaanz groß und deshalb haben sie es gern, wenn ihr Futter hoch oben hängt, damit sie besser fressen können.

Und hier, das Nilpferd, das gehört in den Käfig mit dem Bild vom Nilpferd drauf. Da ist ganz viel Wasser drin, weil Nilpferde gerne schwimmen.

Wir müssen gleich gut aufpassen, dass wir die Tiere auch in die richtigen Käfige stellen, denn jedes Tier braucht bestimmte Dinge, die nur in seinem eigenen Käfig sind. 
3 Fehler beim Einsortieren durch den Testleiter:

1. Zebra in Eisbärenkäfig

2. Nilpferd in Giraffenkäfig

3. Giraffe in Nilpferdkäfig

"Das Zebra kommt hier hin" (dabei Blickkontakt zum Kind)

"Das Nilpferd stelle ich hier rein."

"Die Giraffe, die geht hier hinein." (Blickkontakt zum Kind)

\begin{tabular}{|c|c|c|}
\hline Kind klärt die Fehler auf & $\begin{array}{l}\text { Kind bemerkt den Fehler, zögert } \\
\text { aber }\end{array}$ & Kind reagiert nicht \\
\hline $\begin{array}{l}\text { Fehler 1: „Oh, ich hab das Zebra in } \\
\text { den Eisbärenkäfig gestellt. Oje. } \\
\text { Das darf mir aber nicht passieren. } \\
\text { Da ist es doch viel zu kalt für das } \\
\text { arme Zebra! Ich stelle es schnell in } \\
\text { seinen eigenen Käfig! Gut, dass du } \\
\text { so gut aufpasst!“ }\end{array}$ & $\begin{array}{l}\text { Fehler 1: „Du schaust so skeptisch. } \\
\text { Hab ich etwas falsch gemacht? } \\
\text { Oh, jetzt sehe ich es auch! Ich } \\
\text { habe das Zebra in den } \\
\text { Eisbärenkäfig gestellt! Da ist es } \\
\text { doch viel zu kalt für das arme } \\
\text { Zebra! Ich stelle es schnell in } \\
\text { seinen eigenen Käfig! Du hast das } \\
\text { sofort bemerkt, dass ich mich } \\
\text { vertan hab, stimmt's? Du musst } \\
\text { mir dann am besten sofort } \\
\text { Bescheid geben, wenn ich mich } \\
\text { vertue, damit die Tiere in die } \\
\text { richtigen Käfige kommen!" }\end{array}$ & $\begin{array}{l}\text { Fehler 1: „Oh, ups, Schau mal, ich } \\
\text { hab das Zebra in den Eisbärenkäfig } \\
\text { gestellt! Das ist aber nicht gut! Da } \\
\text { ist es doch viel zu kalt für das arme } \\
\text { Zebra! } \\
\text { Ich muss besser aufpassen! Und } \\
\text { wenn du siehst, dass ich einen } \\
\text { Fehler mache, kannst du mir das } \\
\text { zeigen. Ich vertu mich sonst." }\end{array}$ \\
\hline $\begin{array}{l}\text { Fehler 2: „Ups, ich hab schon } \\
\text { wieder nicht richtig aufgepasst! } \\
\text { Das Nilpferd kommt natürlich in } \\
\text { den Nilpferdkäfig!" }\end{array}$ & $\begin{array}{l}\text { Fehler 2: „Du schaust wieder so. } \\
\text { Hab ich schon wieder einen Fehler } \\
\text { gemacht? Ohje, ich sehe es } \\
\text { gerade! Das Nilpferd steht im } \\
\text { Giraffenkäfig! Das geht natürlich } \\
\text { nicht! Schnell in den Nilpferdkäfig } \\
\text { mit dem Nilpferd! Du hast das } \\
\text { sofort bemerkt, ne?" }\end{array}$ & $\begin{array}{l}\text { Fehler 2: „Oh nein, Guck mal hier! } \\
\text { Da steht ein Nilpferd im } \\
\text { Giraffenkäfig. Da habe ich mich ja } \\
\text { total vertan! Ich stelle es schnell in } \\
\text { seinen eigenen Käfig!“ }\end{array}$ \\
\hline $\begin{array}{l}\text { Fehler 3: "Oje, ich hab die Giraffe } \\
\text { zu den Nilpferden gestellt, aber die } \\
\text { kann doch gar nicht schwimmen! } \\
\text { Schnell in den Giraffenkäfig mit } \\
\text { ihr!" }\end{array}$ & $\begin{array}{l}\text { Fehler 3: „Oh, sieh mal! Ich habe } \\
\text { aus Versehen die Giraffe zu den } \\
\text { Nilpferden gestellt! Aber die kann } \\
\text { doch gar nicht schwimmen. } \\
\text { Schnell in den Giraffenkäfig mit } \\
\text { ihr!“ }\end{array}$ & $\begin{array}{l}\text { Fehler 3: } \\
\text { "Oh sieh mal. Die Giraffe ist falsch } \\
\text { abgebogen und ist aus Versehen } \\
\text { zu den Nilpferden gegangen. Aber } \\
\text { Giraffe, du kannst doch gar nicht } \\
\text { schwimmen! Los, lauf schnell } \\
\text { rüber in den Giraffenkäfig, damit } \\
\text { du fressen kannst!" }\end{array}$ \\
\hline
\end{tabular}

Ende:

„So, jetzt sind alle Tiere wieder in ihren eigenen Käfigen! Das ist gut! Danke für deine Hilfe!"

\section{Überleitung zum TROG-D:}

„Als letztes möchte ich mir noch ein Buch mit dir anschauen. Schau, das liegt noch da auf dem Schrank. Ich hol es mal. (TROG-D holen) Erstmal möchte ich wissen, welche Wörter du schon kannst. Ich sage dir jetzt immer ein Wort und du zeigst mir das passende Bild hier auf den Blättern. (Wörter überprüfen). Danach TROG-D nach Anweisung durchführen. 
Anhang F: Anleitung zur Anfertigung der Spontansprachaufnahme 


\section{Anleitung zur Umsetzung des Rollenspiels: Der Bär beim Arzt}

\section{Was soll gemacht werden?}

Mit Ihrer Hilfe möchten wir einige spontane Äußerungen Ihres Kindes aufnehmen. Über ein gemeinsames Spiel mit einem Arztkoffer und einem Teddybären soll Ihr Kind zum Sprechen angeregt werden.

\section{Welche Materialien benötigen Sie?}

- Plüschbär

- Arztkoffer mit Inhalt

- Aufnahmegerät

- Beispielvideo

\section{Vorbereitungen}

1. Schauen Sie sich das Beispielvideo an.

2. Achten Sie darauf, dass nur das Kind und die durchführende Person im Raum sind.

3. Stellen Sie sicher, dass Sie während der Umsetzung nicht gestört werden (z.B. durch Geschwisterkinder oder Haustiere).

4. Stellen Sie alle Störquellen aus (TV-Gerät, Radio, Computer etc.).

\section{Durchführung}

Zeichnen Sie ca. 10-15 Minuten des Spiels auf. Achten Sie darauf, dass das Aufnahmegerät in der Nähe des Kindes positioniert ist. Schalten Sie das Aufnahmegerät ein.

Beginnen Sie nun mit dem Spielen. Ihr Kind spielt den Arzt/die Ärztin. Der Bär hat Bauchschmerzen und soll untersucht werden. Begeben Sie sich bitte in die Rolle der Begleitperson, die den Bären zum Arzt bringt und inn dort betreut. Zusätzlich können Sie die Rolle des Bären einnehmen. Der Bär kann beispielsweise „Aua" sagen oder aber selbst Fragen stellen. Versuchen Sie so natürlich wie möglich mit Ihrem Kind zu spielen - also so, wie Sie es auch sonst mit Ihrem Kind tun. Um die Spielsituation einzuleiten können Sie folgenden Satz nutzen:

\section{Einleitungssatz}

Schau mal, das hier ist Bobo der Bär. Er hat mir erzählt, dass er ganz schlimme Bauchschmerzen hat. Er braucht dringend Hilfe. Kannst du den Bären mal untersuchen?

Ihr Kind darf den Bären nun ausgiebig untersuchen und verarzten.

Folgende Fragen bzw. Äußerungen können Sie während des Spiels nutzen:

\begin{tabular}{|l|l|}
\hline Das könnte die Begleitperson sagen: & Dies könnte der Bär sagen: \\
\hline Was machst du da gerade? & Aua, das tut weh! \\
\hline Was musst du jetzt alles untersuchen? & $\begin{array}{l}\text { Ich möchte keine Spritze (Pflaster, } \\
\text { Creme etc.) }\end{array}$ \\
\hline Warum machst du das jetzt? & Was machst du mit mir? \\
\hline $\begin{array}{l}\text { Warum braucht der Bär eine Spritze (Pflaster, } \\
\text { Creme etc.)? }\end{array}$ & Bäh, die Medizin schmeckt nicht! \\
\hline Warum hat der Bär Bauchschmerzen? & Wann kann ich wieder nach Hause? \\
\hline Worauf muss der Bär jetzt besonders achten? & Was muss ich jetzt machen? \\
\hline Was darf der Bär jetzt nicht mehr machen? & Sei aber vorsichtig! \\
\hline $\begin{array}{l}\text { Was muss ich zu Hause machen, damit es dem } \\
\text { Bären wieder besser geht? }\end{array}$ & Ich habe Angst! \\
\hline
\end{tabular}

Verwenden Sie lediglich die Materialien, die sich im Arztkoffer befindenden. Fügen Sie bitte nichts hinzu.

Falls Ihr Kind noch einen weiteren „Patienten“ behandeln möchte, spielen Sie einfach weiter. Das Kind darf gerne eines seiner Kuscheltiere oder Puppen auswählen.

Schalten Sie nach 10-15 Minuten das Aufnahmegerät aus. 
Anhang G: Informationen zur Studie für Kitas und Eltern 

„Selektiver Mutismus und Mehrsprachigkeit“

Sehr geehrte Leitung der Kindertageseinrichtung,

sehr geehrte Erzieherinnen und Erzieher,

in Ihrem Berufsalltag haben Sie sicher alle bereits mit schüchternen Kindern gearbeitet. Gerade zu Beginn des Kindergartenbesuches fällt es einigen Kindern schwer, sich in die Gruppe zu integrieren. Sie spielen lieber für sich alleine, sprechen nicht mit anderen Kindern oder ziehen sich bei Gruppenaktivitäten eher zurück. Einige von ihnen benötigen lediglich eine Eingewöhnungszeit. Sie werden zunehmend offener, finden Freunde und gliedern sich ins Gruppengeschehen ein. Andere bleiben jedoch schweigend und zurückgezogen. Bei ihnen liegt mit hoher Wahrscheinlichkeit ein so genannter selektiver Mutismus vor, der sich häufig erstmals bei Kindergarteneintritt mit ca. drei Jahren zeigt. Diese Kommunikationsstörung ist charakterisiert durch ein konsequentes Schweigen in ganz spezifischen sozialen Situationen, obwohl das betroffene Kind grundsätzlich fähig ist zu sprechen. Etwa ein Prozent der Kinder zeigt dieses Phänomen, wobei mehrsprachige Kinder deutlich häufiger betroffen sind als einsprachige Kinder. Bislang ist jedoch noch unklar, was mehrsprachige Kinder für dieses extreme Verhalten besonders anfällig macht.

Behalten Kinder das Schweigen längerfristig bei, ist mit negativen Folgen für die sozialemotionale, sprachliche und schulische Entwicklung zu rechnen. Durch den geringen Kontakt zu anderen Kindern und das Nicht-Sprechen in der Interaktion mit anderen, können Kinder wichtige soziale Regeln nicht üben. Gesprächsregeln und Strategien zum Meistern neuer Situationen können nicht erworben werden. Neben der nicht vorhandenen mündlichen Mitarbeit wirkt sich das Schweigen im schulischen Kontext zudem auf den Wissenserwerb aus. Kinder mit selektivem Mutismus fragen weniger nach, holen sich seltener Unterstützung bei Mitschülern und Lehrern und bleiben unter Umständen in ihrer schulischen Entwicklung zurück. Gerade deswegen sind eine frühe Identifizierung des selektiven Mutismus und eine entsprechende Intervention bereits im Kindergartenalter sehr bedeutsam. 


\section{Ziel des Projektes}

In unserem Projekt „Selektiver Mutismus und Mehrsprachigkeit“ interessiert uns, warum einige der schüchternen Kinder nach einer Eingewöhnungsphase im Kindergarten beginnen zu sprechen und andere eben nicht. Wir möchten deshalb der Frage nachgehen, welche Entwicklungsvoraussetzungen, Persönlichkeitseigenschaften oder Lebensbedingungen des Kindes die Ausbildung eines selektiven Mutismus begünstigen. Um die Aspekte Sprache und Kultur mit zu berücksichtigen, beziehen wir sowohl ein- als auch mehrsprachige Kinder in unser Projekt ein. Langfristig möchten wir so zu Verbesserung der frühen Identifikation der Kinder beitragen. Indem wir mehr über die Entwicklungsmechanismen des selektiven Mutismus wissen, können wir potenzielle Risikokinder früher entdecken und ihnen eine entsprechende Präventions- oder Interventionsmaßnahme zukommen lassen.

\section{Ablauf des Projektes}

In den kommenden 10 Monaten möchten wir dreijährige Kinder, die gerade in die Kindertageseinrichtung gekommen sind, in ihrem ersten Kindergartenjahr begleiten. Zu vier Zeitpunkten im Abstand von drei Monaten (3. Kitamonat, 6. Kitamonat, 9. und 12. Kitamonat) werden wir die aktuellen Fähigkeiten, das Verhalten des Kindes sowie verschiedene Aspekte in der Lebensumwelt über Eltern, ErzieherIn und Beobachtungen des Kindes erfassen. In der nachfolgenden Tabelle können Sie sehen, zu welchem Zeitpunkt welche Daten erhoben werden sollen.

\begin{tabular}{|c|c|c|c|c|}
\hline & Oktober/November 2012 & $\begin{array}{l}\text { Januar/ Feb- } \\
\text { ruar } 2013\end{array}$ & $\begin{array}{l}\text { April/Mai } \\
2013\end{array}$ & Juli/August 2013 \\
\hline Eltern & $\begin{array}{l}\text { zwei Termine an der TU } \\
\text { Dortmund } \\
\text { 1. Elterngespräch }\end{array}$ & \multicolumn{2}{|c|}{$\begin{array}{l}\text { Ausfüllen eines Fragebo- } \\
\text { gens } \\
(2 \mathrm{mal})\end{array}$} & $\begin{array}{l}\text { zwei Termine an der TU } \\
\text { Dortmund } \\
\text { 1. Elterngespräch }\end{array}$ \\
\hline $\begin{array}{l}\text { Eltern } \\
\text { und } \\
\text { Kind }\end{array}$ & $\begin{array}{l}\text { 2. Termin mit dem Kind } \\
\text { zuhause: Aufnahme von } \\
\text { Spontansprache des Kindes }\end{array}$ & & & $\begin{array}{l}\text { 2. Termin mit dem Kind } \\
\text { zuhause: Aufnahme von } \\
\text { Spontansprache des Kindes }\end{array}$ \\
\hline $\begin{array}{l}\text { in der } \\
\text { KiTa }\end{array}$ & \multicolumn{4}{|c|}{$\begin{array}{l}\text { Beobachtung des Spielverhalten (4 mal) } \\
\text { durch MitarbeiterInnen des Projektes }\end{array}$} \\
\hline $\begin{array}{l}\text { Erzie- } \\
\text { herln }\end{array}$ & \multicolumn{4}{|c|}{$\begin{array}{l}\text { Ausfüllen eines Fragebogens ( } 4 \text { mal) } \\
\text { Zeitaufwand: ca. } 20 \text { Minuten pro Kind }\end{array}$} \\
\hline
\end{tabular}

\section{Welche Funktion hat Ihre Einrichtung im Projekt? Was erwartet Sie?}

Die Kernsymptomatik des selektiven Mutismus ist ein Schweigen in spezifischen sozialen Situationen, insbesondere in wenig vertrauten Situationen außerhalb von zuhause. Kindergarten und Schule sind dabei die Kontexte, an denen das Schweigen am häufigsten auftritt. Deswegen kommt Ihnen eine ganz besonders bedeutsame Rolle zu. Nur Sie können beurteilen, wie sich ein Kind typischerweise in der KiTa verhält und ob es dort ein solch extremes Schweigen zeigt.

Im Projekt selbst werden Sie und Ihre MitarbeiterInnen somit zunächst potenzielle Kinder für unser Projekt auswählen. Für ein jedes „Projektkind“ wird die Bezugserzieherin im Verlauf des Projektes vier Mal einen Fragebogen zu seinem aktuellen (Sprech-)Verhalten ausfüllen. Zusätzlich wird das Kind in der KiTa innerhalb des Gruppengeschehens zu jedem der vier Zeitpunkte von Projektmitarbeiterinnen beobachtet, die für je einen Vormittag in Ihre Einrichtung kommen. 


\section{Welche Kinder können teilnehmen?}

Wir suchen extrem schüchterne dreijährige Kinder (ein- und mehrsprachig), die gerade in Ihre Einrichtung gekommen sind. Folgende Kriterien sollen erfüllt sein:

- Das Kind zeigt in der Gruppe extrem schüchternes Verhalten.

- Das Kind spricht nicht mit anderen Kindern.

- Das Kind spricht nicht mit Erwachsenen der Einrichtung.

- Sie wissen von den Eltern, dass das Kind zuhause ganz unbefangen spricht.

Sollte sich ein extrem schüchternes Kind in Ihrer Einrichtung befinden, suchen Sie bitte ein sehr kontaktfreudiges und offenes Kind gleichen Alters, gleichen Geschlechts und entsprechend einoder mehrsprachig aus. Folgende Kriterien sollen erfüllt sein:

- Das Kind spricht unbefangen mit anderen Kindern.

- Das Kind spricht unbefangen mit Erwachsenen der Einrichtung.

- Das Kind hat bereits Freunde gefunden.

Die beigelegte Checkliste soll Ihnen bei der Auswahl möglicher Kinder helfen. Die Angaben sind ausschließlich für Sie gedacht und werden nicht an uns weitergegeben. Sollten Sie anhand der Kriterien und der Checkliste Kinder ausgewählt haben, schicken wir Ihnen eine entsprechende Anzahl Elterninformationen über das Projekt zu.

\section{Nutzen und Risiken des Projektes}

Gerne kommt unsere Mitarbeiterin Anja Starke im Vorfeld oder im Verlauf der Studie in Ihre Einrichtung, um Sie und Ihre MitarbeiterInnen über das Phänomen „selektiver Mutismus“ zu informieren. Nach Abschluss der Studie lassen wir Ihnen zudem gerne Informationen zu den Ergebnissen und unseren Schlussfolgerungen zukommen.

Weder die Beobachtungen in Ihrer Einrichtung noch die Untersuchungen in den Räumen der Universität beinhalten ein Risiko für Sie, das Kind oder seine Familie. Wir bemühen uns alle Situationen so angenehm wie möglich zu gestalten. Sollte sich herausstellen, dass eines der Kinder tatsächlich einen selektiven Mutismus entwickelt, stehen wir mit dem Mutismus-Netzwerk des Sprachtherapeutischen Ambulatoriums Ihnen und den Eltern gerne beratend zur Verfügung.

\section{Vertraulichkeit}

Jegliche Informationen, die in dieser Studie erfasst werden, werden vertraulich behandelt. Veröffentlichungen zu dieser Studie werden keinerlei Informationen beinhalten, die Ihre Einrichtung, das Kind oder seine Familie identifizieren könnten.

\section{Wie geht es nun weiter?}

Unsere Mitarbeiterin Anja Starke wird sich im Verlauf der nächsten zwei Wochen bei Ihnen telefonisch melden, um sich nach möglichen Kindern für unser Projekt zu erkundigen und Sie über den genauen Ablauf des Projektes zu informieren. Wenn Sie vorab bereits Fragen haben, melden Sie sich gerne bei uns!

Wir danken Ihnen bereits im Voraus für Ihr Interesse und Ihre Unterstützung!

Mit freundlichen Grüßen,

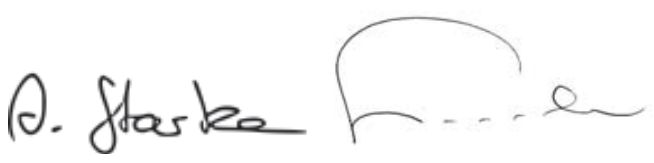

Anja Starke \& Katja Subellok 


\section{Checkliste zur Probandenauswahl}

Die Angaben in dieser Checkliste dienen ausschließlich als Unterstützung für Sie zur Auswahl möglicher Kinder. Sie werden zunächst nicht an uns weitergegeben. Sie sollen sich lediglich dadurch vergewissern, ob ein Kind in Ihrer Einrichtung für das Projekt in Frage kommt.

\section{Kind 1 - schüchtern}

Bitte beantworten Sie die nachfolgenden Fragen zunächst für das extrem schüchterne Kind!

Name:

Alter: Jahre;Monate

Geschlecht

weiblich

$\square$ männlich

einsprachig $\quad \square$ mehrsprachig, mit folgenden Sprachen

Das Kind besucht seit Sommer 2012 unsere Einrichtung.
$\square$ ja
$\square$ nein

\section{(Sprech-)Verhalten}

Das Kind spricht zuhause mit seinen Eltern und/oder Geschwistern.
$\square$ ja
$\square$ nein
$\square$ weiß nicht

Das Kind schweigt in der KiTa mit anderen Kindern.
$\square$ ja
$\square$ nein
$\square$ weiß nicht

Das Kind schweigt in der KiTa mit Erwachsenen.
$\square$ ja
$\square$ nein
$\square$ weiß nicht

Das Kind spielt in der KiTa häufig alleine.
$\square$ ja
$\square$ nein
$\square$ weiß nicht

Das Kind zieht sich bei Gruppenaktivitäten eher zurück.
$\square$ ja
nein
$\square$ weiß nicht

\section{Entwicklungsbeeinträchtigungen}

Liegt bei dem Kind eine Entwicklungsverzögerung irgendeiner Art vor (Sprache, Motorik, Kognition)?
$\square$ ja
nein
weiß nicht

Liegt bei dem Kind eine Behinderung irgendeiner Art vor?
$\square$ ja
nein
$\square$ weiß nicht

Wenn Sie die Fragen im Block „(Sprech-)Verhalten“ mit JA und die Fragen im Block „Entwicklungsbeeinträchtigungen“ mit NEIN beantworten konnten, ist das Kind ein potenzielles Kind für unser Projekt. 


\section{Kind 2 - kontaktfreudig}

Suchen Sie nun ein kontaktfreudiges Kind aus der Gruppe aus, welches das gleiche Geschlecht und in etwas das gleiche Alter wie das extrem schüchterne Kind hat sowie entsprechend ein- oder mehrsprachig ist.

Bitte beantworten Sie die nachfolgenden Fragen nun für das kontaktfreudige Kind!

Name:

Alter: Jahre;Monate

Geschlecht $\square$ weiblich $\square$ männlich

einsprachig $\quad \square$ mehrsprachig, mit folgenden Sprachen

Das Kind besucht seit Sommer 2012 unsere Einrichtung.
$\square$ ja
$\square$ nein

\section{(Sprech-)Verhalten}

Das Kind spricht zuhause mit seinen Eltern und/oder Geschwistern.
$\square$ ja
$\square$ nein
$\square$ weiß nicht

Das Kind spricht in der KiTa mit anderen Kindern.
$\square$ ja
nein
weiß nicht

Das Kind spricht in der KiTa mit Erwachsenen.
$\square$ ja
$\square$ nein
$\square$ weiß nicht

Das Kind spielt in der KiTa häufig mit anderen Kindern.
$\square$ ja
nein
$\square$ weiß nicht

Das Kind hat bereits Freunde in der KiTa gefunden.
$\square$ ja
$\square$ nein
$\square$ weiß nicht

\section{Entwicklungsbeeinträchtigungen}

Liegt bei dem Kind eine Entwicklungsverzögerung irgendeiner Art vor (Sprache, Motorik, Kognition)?
$\square$ ja
nein
$\square$ weiß nicht

Liegt bei dem Kind eine Behinderung irgendeiner Art vor?
$\square$ ja
$\square$ nein
$\square$ weiß nicht

Wenn Sie die Fragen im Block „(Sprech-)Verhalten“ mit JA und die Fragen im Block „Entwicklungsbeeinträchtigungen" mit NEIN beantworten konnten, ist das Kind ein potenzielles Kind für unser Projekt.

Bitte notieren Sie, wie viele Kinder in Ihrer Einrichtung für das Projekt in Frage kommen. Unsere Mitarbeiterin Anja Starke wird sich zeitnah telefonisch bei Ihnen melden, um nach der Anzahl zu fragen und Ihnen entsprechend Elterninformationen zukommen lassen. 


\section{Elterninformation}

Liebe Eltern, liebe Erziehungsberechtigten,

Ihr Kind ist gerade in den Kindergarten gekommen und startet nun mit einer wichtigen Entwicklungsphase. Alltäglich ist es nun mit gleichaltrigen Kindern zusammen. Es wird sich immer mehr an fremde Personen gewöhnen und lernen, mit ihnen in Kontakt zu treten, sie kennenzulernen, Freundschaften zu bilden, sich zu streiten oder Dinge auszuhandeln. Im Spiel mit anderen Kindern wird Ihr Kind dies nun im Kindergarten ausprobieren und seine Fähigkeiten weiter entwickeln.

Einigen Kindern fällt es jedoch schwer, mit anderen Kindern in Kontakt zu treten. Sie sind extrem schüchtern, halten sich zurück und spielen lieber für sich alleine. Manche von ihnen sprechen sogar nicht mit den anderen Kindern und manchmal auch nicht mit den Erziehern und Erzieherinnen. Vielen von diesen Kindern gelingt es jedoch sich einzugewöhnen. Sie beginnen nach wenigen Wochen mit anderen Kindern zu spielen und zu sprechen. Einige wenige behalten ihr Schweigen im Kindergarten aber bei. Zuhause reden sie mit ihren Eltern und Geschwistern meistens ganz normal. Bislang wissen wir noch nicht, warum diese Kinder im Kindergarten so extrem reagieren und sie nicht mit fremden Personen sprechen.

\section{Ziel unserer Studie}

Aus diesem Grund werden wir Kinder (im Alter von 3 Jahren) und ihre Familien in ihrem ersten Kindergartenjahr begleiten. Wir möchten herausfinden, welche Lebensbedingungen oder persönliche Eigenschaften des Kindes dazu beitragen können, dass es im Kindergarten nicht mit anderen Personen spricht. Wir suchen Familien, deren dreijährige Kinder in diesem Sommer 2012 in den Kindergarten aufgenommen wurden. Die Kinder sollen entweder sehr schüchtern oder im Kontrast dazu besonders kontaktfreudig sein.

\section{Ablauf der Studie}

Um ein möglichst genaues Bild von den Fähigkeiten Ihres Kindes zu erhalten, werden sowohl Sie als Eltern als auch die Erzieher und Erzieherinnen Ihres Kindes mit in die Studie einbezogen. Zusätzlich möchten wir Ihr Kind selbst kennenlernen und einige Spiele mit ihm durchführen. Wir werden Sie dazu zu Beginn und zum Ende unserer einjährigen Studie in die Räume der Technischen Universität Dortmund einladen. Außerdem werden Mit- 
arbeiter und Mitarbeiterinnen des Projektes insgesamt vier Mal in den Kindergarten Ihres Kindes kommen und Ihr Kind beim Spielen in der Gruppe beobachten. Den genauen Ablaufplan können Sie der Tabelle entnehmen:

\begin{tabular}{|c|c|c|c|c|}
\hline & $\begin{array}{l}\text { Oktober/ November } \\
2012\end{array}$ & $\begin{array}{c}\text { Januar/ } \\
\text { Februar } 2013\end{array}$ & April/ Mai 2013 & Juli/ August 2013 \\
\hline Eltern & $\begin{array}{l}\text { zwei Termine an der TU } \\
\text { Dortmund } \\
\text { 1. Elterngespräch }\end{array}$ & \multicolumn{2}{|c|}{$\begin{array}{l}\text { Ausfüllen eines Fragebogens } \\
\qquad(2 \mathrm{mal})\end{array}$} & $\begin{array}{l}\text { zwei Termine an der TU } \\
\text { Dortmund } \\
\text { 1. Termin mit dem Kind }\end{array}$ \\
\hline $\begin{array}{l}\text { Eltern } \\
\text { und } \\
\text { Kind }\end{array}$ & $\begin{array}{l}\text { 2. Termin mit dem Kind } \\
\text { zuhause: Aufnahme von } \\
\text { Spontansprache des } \\
\text { Kindes }\end{array}$ & & & $\begin{array}{l}\text { 2. Abschlussgespräch } \\
\text { zuhause: Aufnahme von } \\
\text { Spontansprache des } \\
\text { Kindes }\end{array}$ \\
\hline $\begin{array}{l}\text { in der } \\
\mathrm{KiTa}\end{array}$ & \multicolumn{4}{|c|}{ Beobachtung des Spielverhalten (4 mal) } \\
\hline $\begin{array}{l}\text { Erzie- } \\
\text { herln }\end{array}$ & \multicolumn{4}{|c|}{ Ausfüllen eines Fragebogens (4 mal) } \\
\hline
\end{tabular}

\section{Wie können Sie teilnehmen?}

Wenn Sie Interesse an der Teilnahme an unserer Studie haben, geben Sie bitte unten Ihre Kontaktdaten an und den Abschnitt an Ihre Kindertageseinrichtung zurück. Wir werden Sie dann zeitnah telefonisch kontaktieren, um einen individuellen Termin mit Ihnen auszumachen. In einem ersten Gespräch informieren wir Sie gerne noch einmal über den Ablauf des Projektes und beantworten all Ihre Fragen.

Wir möchten uns bereits im Voraus für Ihr Interesse bedanken. Auch möchten wir Ihnen versichern, dass das Wohl Ihres Kindes (und das von Ihnen) unserer größtes Interesse ist. Die geplanten Spiel- und Erhebungssituationen mit ihrem Kind sind ausgesprochen kindgemäß gestaltet und werden es nicht belasten. Selbstverständlich garantieren wir auch, dass alle Angaben und Daten streng vertraulich behandelt und ausschließlich zu Forschungszwecken verwendet werden.

Wenn Sie vorab Fragen haben, dürfen Sie uns jederzeit gerne ansprechen.

Mit freundlichen Grüßen,

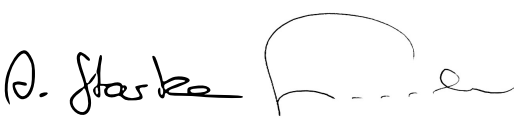

Anja Starke \& Katja Subellok

Name des Kindes:

Alter des Kindes:

Kindertageseinrichtung:

Ihr Name:

Ihre Telefonnummer:

Ihre Emailadresse: 


\section{Einwilligung der Eltern zur Teilnahme Ihres Kindes an der Längsschnittstudie „Selektiver Mutismus und Mehrsprachigkeit"“}

Liebe Eltern, liebe Erziehungsberechtigten, in unserem Projekt „Selektiver Mutismus und Mehrsprachigkeit“ möchten wir die Entwicklung von ein- und mehrsprachigen Kindern im ersten Kindergartenjahr untersuchen. Sie wurden als mögliche Teilnehmer/in ausgewählt, da lhr Kind gerade in den Kindergarten gekommen ist und sich dort als sehr kontaktfreudig oder als zurückhaltend gezeigt hat. Die Studie wird an der Technischen Universität Dortmund von Anja Starke unter der Leitung von Priv.-Doz. Dr. Katja Subellok und Prof. Dr. Christoph Käppler im Zeitraum von Oktober 2012 bis August 2013 durchgeführt. Anja Starke ist akademische Sprachtherapeutin und hat bereits viel Erfahrung in der Arbeit mit schüchternen Kindern. Wir bitten Sie, diese Information aufmerksam zu lesen und Fragen zu stellen, damit Sie prüfen können, ob Sie und Ihr Kind an der Studie teilnehmen möchten.

\section{Ziel unserer Studie}

Wir möchten beobachten, wie sich extrem schüchterne im Vergleich zu sehr kontaktfreudigen Kindern in ihrem ersten Kindergartenjahr entwickeln. Es geht uns darum herauszufinden, warum einige schüchterne Kinder trotz einer langen Eingewöhnungsphase in der Kindertageseinrichtung zurückhaltend bleiben und dort nicht sprechen, andere es nach einiger Zeit aber tun. Das Phänomen des Schweigens in ganz bestimmten sozialen Situationen nennt man selektiven Mutismus. Betroffene Kinder schweigen zum Beispiel im Kindergarten, sprechen zuhause jedoch ganz normal.

In unserem Projekt möchten wir mehrsprachige Kinder mit einbeziehen. Viele mehrsprachige Kinder lernen im ersten Kindergartenjahr eine neue Sprache hinzu. Es ist verständlich, dass einige von ihnen in der neuen Umgebung erst einmal nicht reden. Sie können vielleicht noch nicht ausreichend Deutsch sprechen, um sich mitzuteilen. Wir wollen gerade bei diesen Kindern herausfinden, welche von ihnen noch mehr Unterstützung benötigen, um sich in dieser neuen Welt zurechtzufinden. Deshalb möchten wir die Entwicklung Ihres Kindes in diesem ersten Kindergartenjahr jeweils im Abstand von drei Monaten beobachten.

\section{Beschreibung der Studie}

Die Studie wird insgesamt zehn Monate dauern und während des ersten Kindergartenjahres Ihres Kindes durchgeführt. Wir werden zu vier verschiedenen Zeitpunkten, jeweils im Abstand von drei Monaten (3., 6., 9. und 12. Monat), Ihr Kind beobachten und zu Beginn und am Ende der Studie mit Ihnen Gespräche führen.

Zu Beginn laden wir Sie und Ihr Kind zu insgesamt zwei Terminen in die Räume der Technischen Universität Dortmund ein. Beim ersten Termin findet ein Gespräch mit Ihnen statt, um Sie über den Ablauf des Projektes zu informieren. Wir bitten Sie dann einige Fragebögen zu Ihnen und Ihrem Kind auszufüllen. Die Beantwortung der Fragen ist selbstverständlich freiwillig. Zusätzlich werden wir Sie bitten, zuhause einige sprachliche Äußerungen Ihres Kindes in der deutschen Sprache aufzunehmen. Im gemeinsamen Spiel mit einem Familienmitglied zeigen Kinder meist ihre tatsächlichen sprachlichen Fähigkeiten. Dazu geben wir Ihnen einige Spielmaterialien mit nach Hause. Sie erhalten von uns zudem eine genaue Anweisung, wie Sie das gemeinsame Spiel mit Ihrem Kind gestalten können. Gemeinsam werden wir dann einen zweiten Termin an der TU Dortmund vereinbaren, an dem wir mit Ihrem Kind spielen möchten. Während des zweiten Termins wird eine Mitarbeiterin des Projektes Ihrem Kind Spielangebote machen. Ihr Kind darf dabei immer entscheiden, ob es darauf reagieren möchte oder nicht. Es wird zu keinerlei Handlung gezwungen. Sie dürfen Ihr Kind selbstverständlich begleiten. Wir möchten Sie jedoch bitten, nicht in die Untersuchungssituation einzugreifen oder Ihr Kind zu irgendeiner Handlung aufzufordern. Wir sind daran interessiert, wie Ihr Kind gegenüber einer fremden Person üblicherweise reagiert. Das gemeinsame Spiel mit Ihrem Kind wird auf Video aufgezeichnet und im Nachhinein ausgewertet. 
Zusätzlich werden Mitarbeiterinnen des Projektes zu allen vier Zeitpunkten Ihr Kind im Kindergarten besuchen und beobachten, wie und mit wem Ihr Kind spielt und spricht. Sie und die Erzieherin/ der Erzieher werden zu jedem der vier Zeitpunkte gebeten, einen Fragebogen zum aktuellen Verhalten Ihres Kindes auszufüllen. Damit möchten wir die Entwicklung Ihres Kindes sowohl im Kindergarten als auch Zuhause erfassen.

Zum Abschluss der Studie möchten wir Sie und Ihr Kind nochmals in die Räume der TU Dortmund einladen. An einem ersten Termin möchten wir Sie über die Entwicklung Ihres Kindes im Verlauf der Studie informieren. Anschließend bitten wir Sie erneut, einige sprachliche Äußerungen in der deutschen Sprache zuhause aufzunehmen. An einem zweiten Termin an der TU Dortmund möchten wir erneut mit Ihrem Kind spielen.

\section{Nutzen, Risiko und Unannehmlichkeiten}

Sollte sich herausstellen, dass Ihr Kind zu den extrem schüchternen und schweigenden Kindern gehört, beraten wir Sie gerne hinsichtlich Therapie- und Fördermöglichkeiten. Wir verfügen über ein großes Netzwerk von Sprachtherapeutinnen, die Erfahrungen mit extrem schüchternen Kindern haben. Weder die Untersuchungen in den Räumen der Universität noch die Beobachtungen in der Kindertageseinrichtung beinhalten ein Risiko für Sie und Ihr Kind. Wir bemühen uns alle Situationen so angenehm wie möglich für Sie und Ihr Kind zu gestalten. Sie können die Teilnahme an der Studie jederzeit vorübergehend oder ganz abbrechen, ohne dass für Sie ein Nachteil entsteht.

\section{Vertraulichkeit}

Jegliche Informationen aus dieser Studie, die Sie oder Ihr Kind betrifft, werden vertraulich behandelt. Veröffentlichungen zu dieser Studie werden keinerlei Informationen beinhalten, die Sie oder Ihr Kind identifizieren könnten. Mit Ihrem Einverständnis werden von Ihnen und Ihrem Kind während der Spielsituationen in den Räumen der TU Dortmund Videoaufnahmen gemacht. Diese ermöglichen es uns, das Verhalten Ihres Kindes genauer zu analysieren. Die Videoaufzeichnungen werden in einem verschlossenen Raum in unseren Räumlichkeiten aufbewahrt, zu dem nur die MitarbeiterInnen unseres Projektes Zugang haben. Die Daten werden im Wesentlichen von diesem Projektteam bearbeitet. Unter Umständen wird deren Arbeit durch ForschungsassistentInnen unterstützt, die einen Teil der Daten bearbeiten und auswerten. Diese ForschungsassistentInnen haben jedoch keinen Zugriff zu Ihrem vollständigen Namen.

Wenn Sie zustimmen, können die Audio- und Videoaufnahmen von Ihrem Kind zu Trainings- und Lehrzwecken verwendet werden, d.h. dass Ausschnitte aus einer Situation mit Ihnen und Ihrem Kind Studierenden oder Kollegen aus der Forschung gezeigt werden. Jedoch werden alle Informationen über Ihre und die Identität Ihres Kindes ausgeblendet. Bitte beachten Sie, dass jemand, der Sie und/oder Ihr Kind kennt, Sie und/oder Ihr Kind auf Audio- und Videoaufnahmen wiedererkennen könnte. Sie können uns zu jeder Zeit kontaktieren und verlangen, dass wir Ihre Videoaufzeichnungen aus dem Archiv entfernen.

\section{Freiwilligkeit}

Die Teilnahme an dieser Studie und das Ausfüllen der Fragebögen sind freiwillig. Ihre Entscheidung über die Teilnahme wird Ihre zukünftigen Kontakte zur TU Dortmund in keiner Weise beeinflussen. Wenn Sie sich zu einer Teilnahme entschließen, steht es Ihnen jederzeit frei, Ihr Einverständnis zurückzuziehen und Ihre Teilnahme zu beenden, ohne einen Nachteil deswegen befürchten zu müssen.

\section{Fragen zur Studie}

Falls Sie jetzt Fragen zum Forschungsprojekt haben, stellen Sie diese gerne. Wenn Sie später Fragen zur Forschung und/oder zu damit verbundenen Themen haben, kontaktieren Sie bitte

Frau Anja Starke unter der Nummer 0231/755-8093

per E-Mail anja.starke@tu-dortmund.de oder

persönlich: Emil-Figge-Str. 50, Raum 5.409, 44227 Dortmund

Wir freuen uns, Ihre Fragen zu beantworten! 


\section{Kopie der Einverständniserklärung zur Teilnahme an der Studie „Selektiver Mutismus und Mehrsprachigkeit“ \\ (bitte behalten Sie diese Seite!)}

Mit Ihrer Unterschrift bestätigen Sie, dass Sie die Informationen in dieser Einverständniserklärung gelesen haben und dass Sie Gelegenheit hatten, schriftlich, telefonisch und/oder persönlich Fragen zur Studie zu stellen. Des Weiteren bestätigen Sie mit Ihrer Unterschrift, dass Sie einverstanden sind und auch Ihrem Kind erlauben, an der Studie teilzunehmen. Das heißt, dass von Ihnen und Ihrem Kind Audio- und Videoaufnahmen während der Studie gemacht werden, die eine anschließende Analyse des Verhaltens Ihres Kindes zu Forschungszwecken ermöglichen.

Sie wurden weiterhin darüber informiert, dass Sie jederzeit Ihre Meinung ändern und Ihr Einverständnis zurücknehmen können.

Bitte behalten Sie die Kopie über diese Informationen und Vereinbarung!

Name des Kindes

Unterschrift des Elternteils

Unterschrift der Versuchsleiterin
Name des Elternteils

Ort, Datum

Ort, Datum

\section{Einverständniserklärung für die Nutzung der Audio- und Videoaufzeichnungen zu Lehr-, Trainings-, Vortrags- oder Forschungszwecken}

Auch wenn Sie nicht einverstanden sind, dass die Aufzeichnungen zu Lehr-, Trainings-, Vortrags- oder späteren Forschungszwecken genutzt werden, können Sie an der Studie teilnehmen.

Ich stimme zu, dass die Audio- und Videoaufnahmen von meinem Kind zu Lehr-, Trainings-, Vortrags- oder späteren Forschungszwecken benutzt werden. (bitte kreuzen Sie an!)
$\square \mathrm{Ja}$
$\square$ Nein

Unterschrift:

Datum: 


\section{Einverständniserklärung zur Teilnahme an der Studie „Selektiver Mutismus und Mehrsprachigkeit“}

Mit Ihrer Unterschrift bestätigen Sie, dass Sie die Informationen in dieser Einverständniserklärung gelesen haben und dass Sie Gelegenheit hatten, schriftlich, telefonisch und/oder persönlich Fragen zur Studie zu stellen. Des Weiteren bestätigen Sie mit Ihrer Unterschrift, dass Sie einverstanden sind und auch Ihrem Kind erlauben, an der Studie teilzunehmen. Das heißt, dass von Ihnen und Ihrem Kind Audio- und Videoaufnahmen während der Studie gemacht werden, die eine anschließende Analyse des Verhaltens Ihres Kindes zu Forschungszwecken ermöglichen.

Sie wurden weiterhin darüber informiert, dass Sie jederzeit Ihre Meinung ändern und Ihr Einverständnis zurücknehmen können.

Name des Kindes

Unterschrift des Elternteils

Unterschrift der Versuchsleiterin
Name des Elternteils

Datum

Datum

\section{Einverständniserklärung für die Nutzung der Audio- und Videoaufzeichnungen zu Lehr-, Trainings-, Vortrags- oder Forschungszwecken}

Auch wenn Sie nicht einverstanden sind, dass die Aufzeichnungen zu Lehr-, Trainings-, Vortrags- oder späteren Forschungszwecken genutzt werden, können Sie an der Studie teilnehmen.

Ich stimme zu, dass die Audio- und Videoaufnahmen von meinem Kind zu Lehr-, Trainings-, Vortrags- oder späteren Forschungszwecken benutzt werden. (bitte kreuzen Sie an!)

$\square$ Ja $\quad \square$ Nein

Unterschrift: Datum: 
Anhang H: Erstinformation für die Kitas 

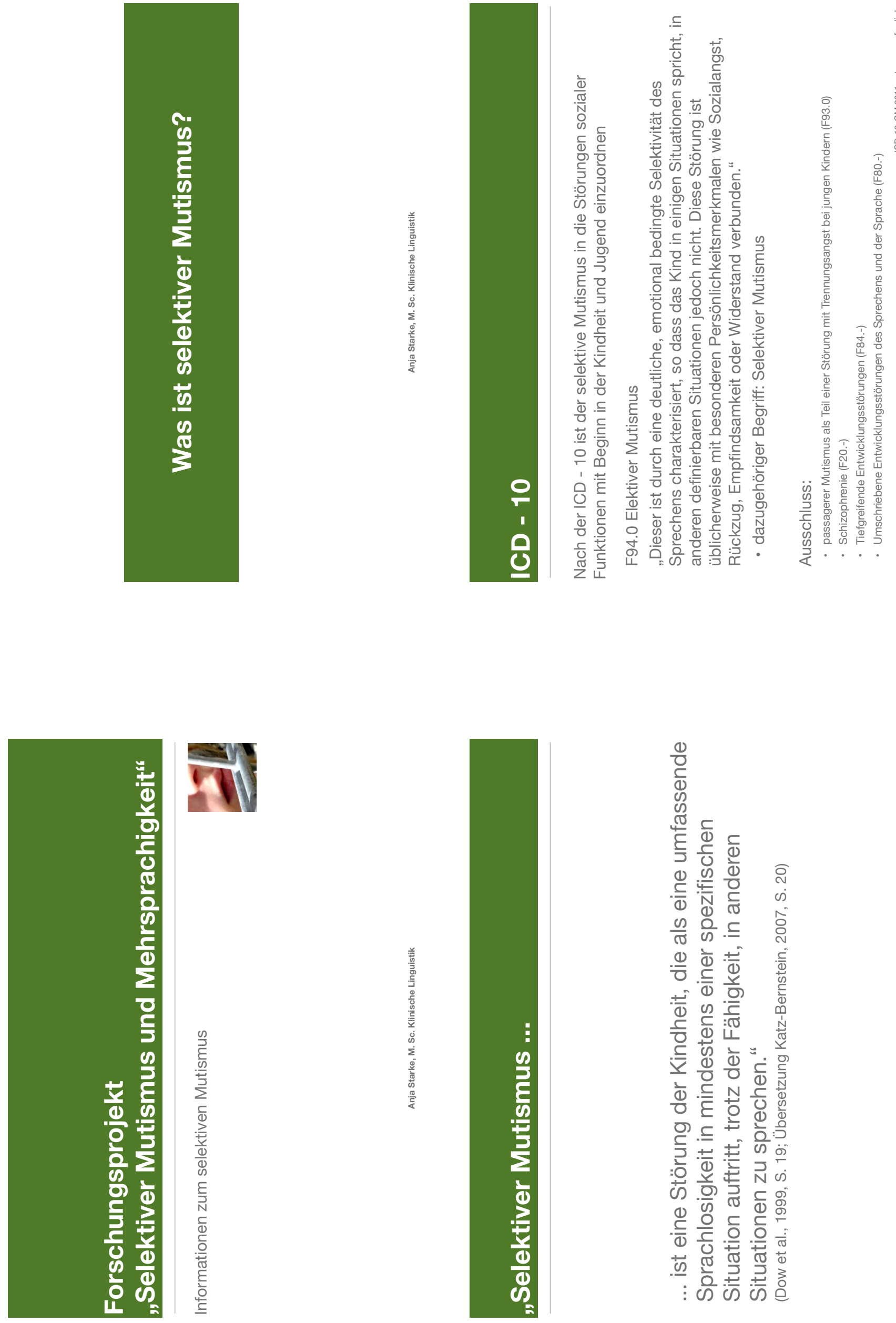

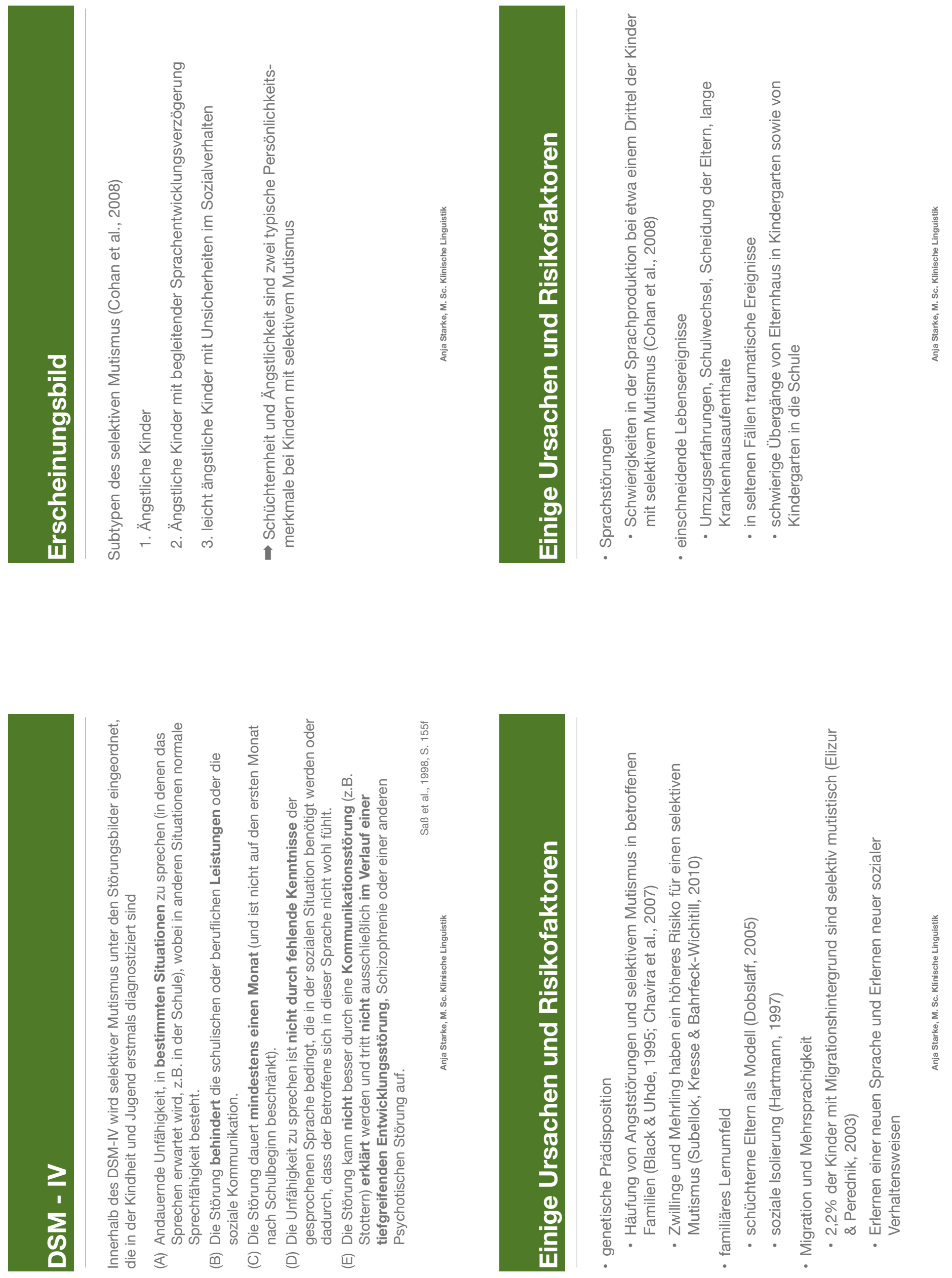

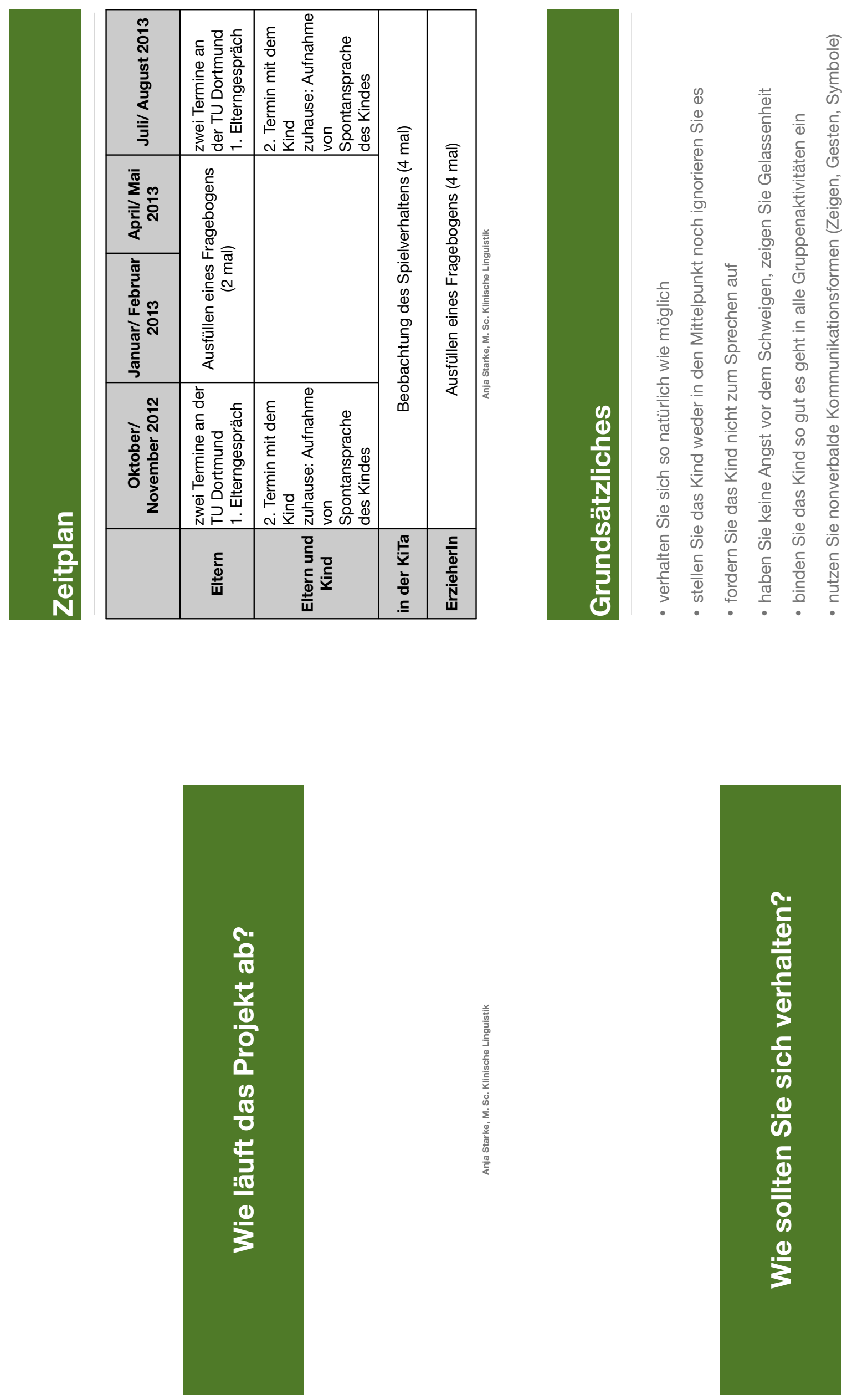


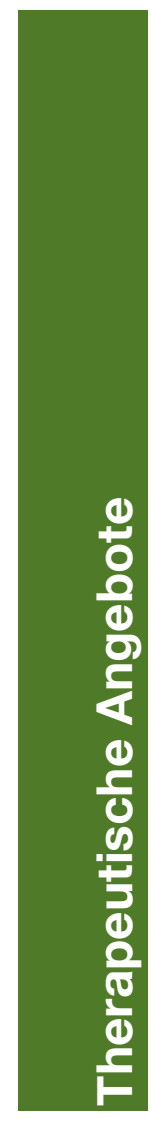

\begin{tabular}{|c|c|c|c|c|}
\hline 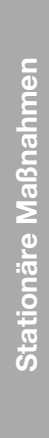 & \multicolumn{2}{|c|}{ 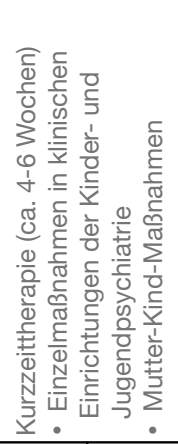 } & 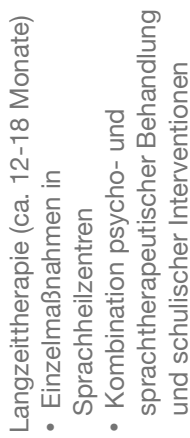 & 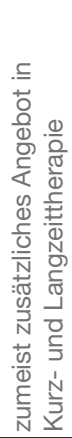 \\
\hline \multirow[t]{2}{*}{ 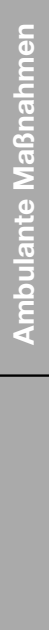 } & 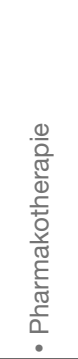 & 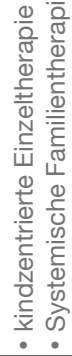 & 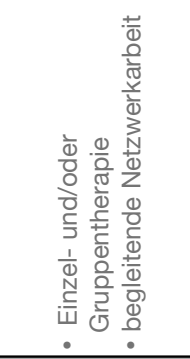 & 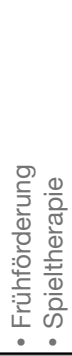 \\
\hline & 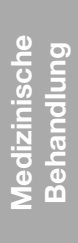 & $\begin{array}{l}\frac{0}{0} \\
\frac{0}{\frac{0}{0}} \\
\frac{0}{0} \\
\frac{5}{0} \\
\frac{0}{0} \\
\frac{0}{0} \\
\frac{0}{0}\end{array}$ & 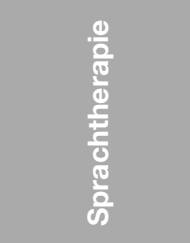 & 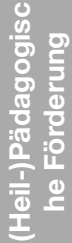 \\
\hline
\end{tabular}
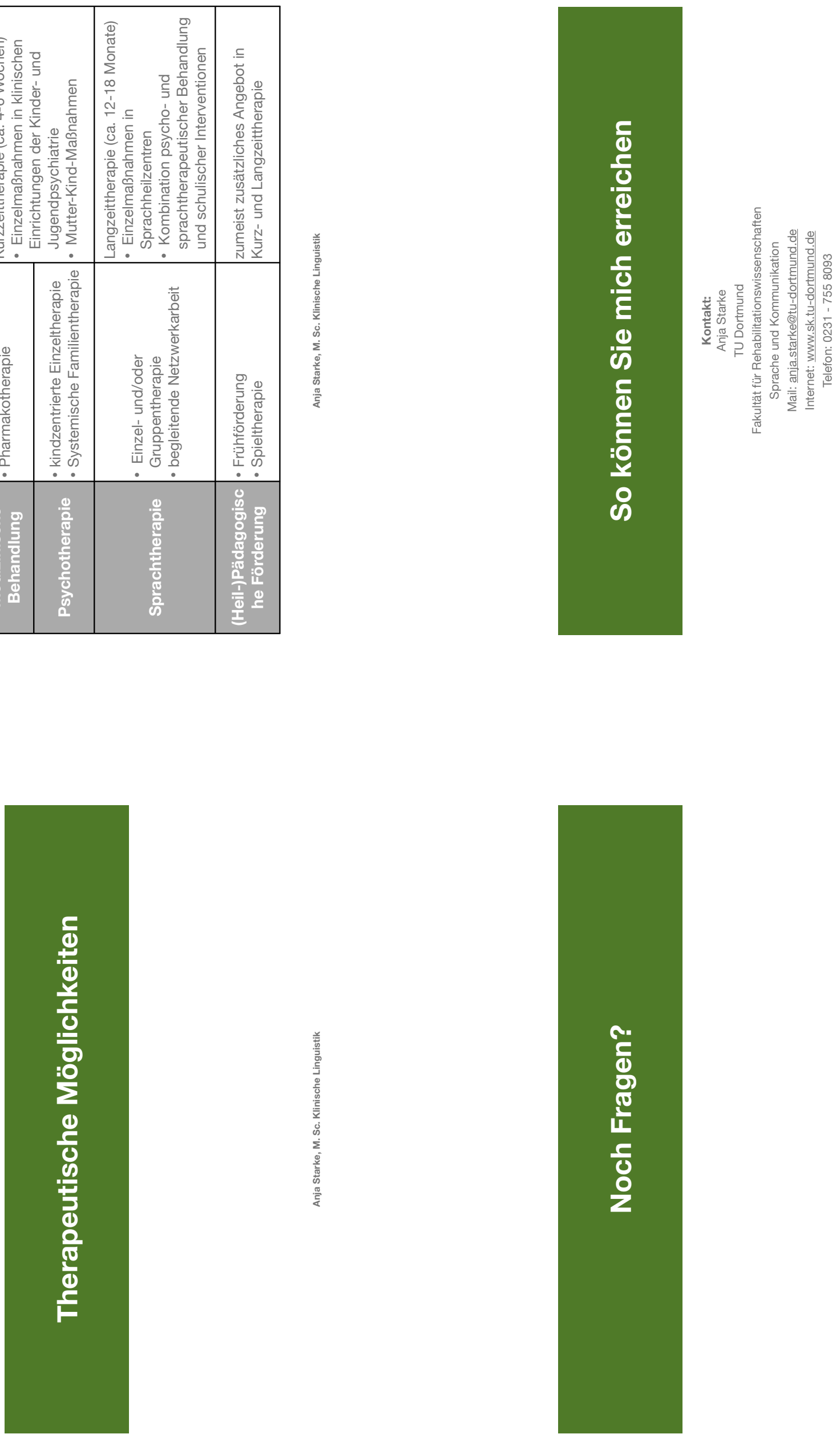
Anhang I: Kodierschema Spontansprache 
Manual zur Transkription und Analyse der Spontansprachaufnahmen

\section{Inhaltsverzeichnis}

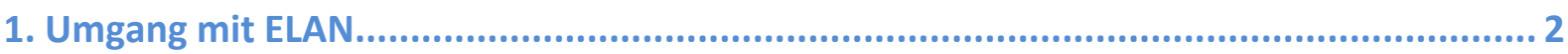

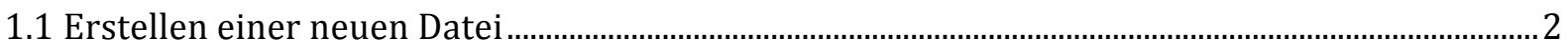

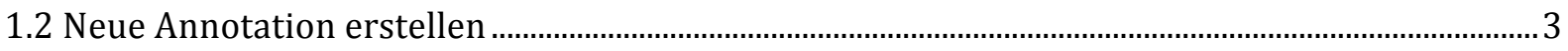

2. Transkription der Spontansprache...................................................................4

3. Analyse der transkribierten Åußerungen ........................................................... 5

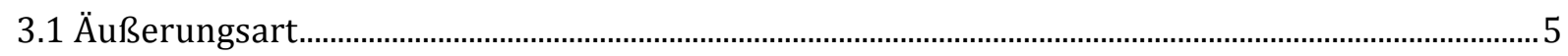

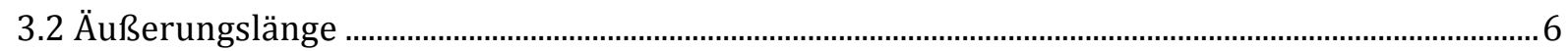

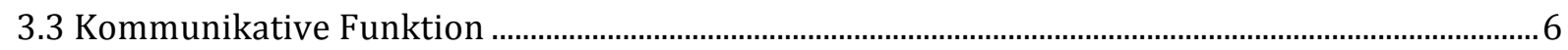

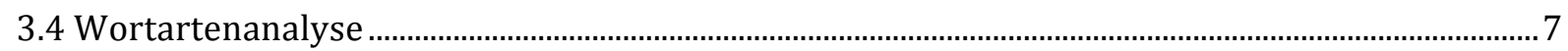

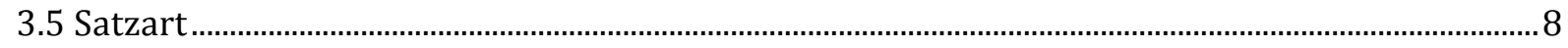

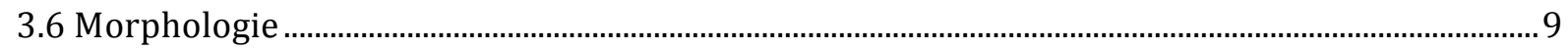




\section{Umgang mit ELAN}

ELAN ist ein Tool zur Analyse von Audio- und Videoaufnahmen. Das Programm kann kostenlos unter folgendem Link runtergeladen werden: http://tla.mpi.nl/tools/tla-tools/elan/

Auf der Homepage von Elan finden Sie zudem zahlreiche Anleitungen sowie Foren, in denen Sie sich über das Programm selbst und seine Funktionen informieren können.

\subsection{Erstellen einer neuen Datei}

Zum Erstellen einer neuen Datei gehen Sie auf "Datei“ => „Neu“. Wählen Sie dann die zu transkribierende Audiodatei über die Funktion „Medien“ sowie die Vorlagendatei „Spontansprache_V2.etf“ unter „Vorlage“ aus. Drücken Sie dann ok => Sie haben eine neue Datei erstellt.

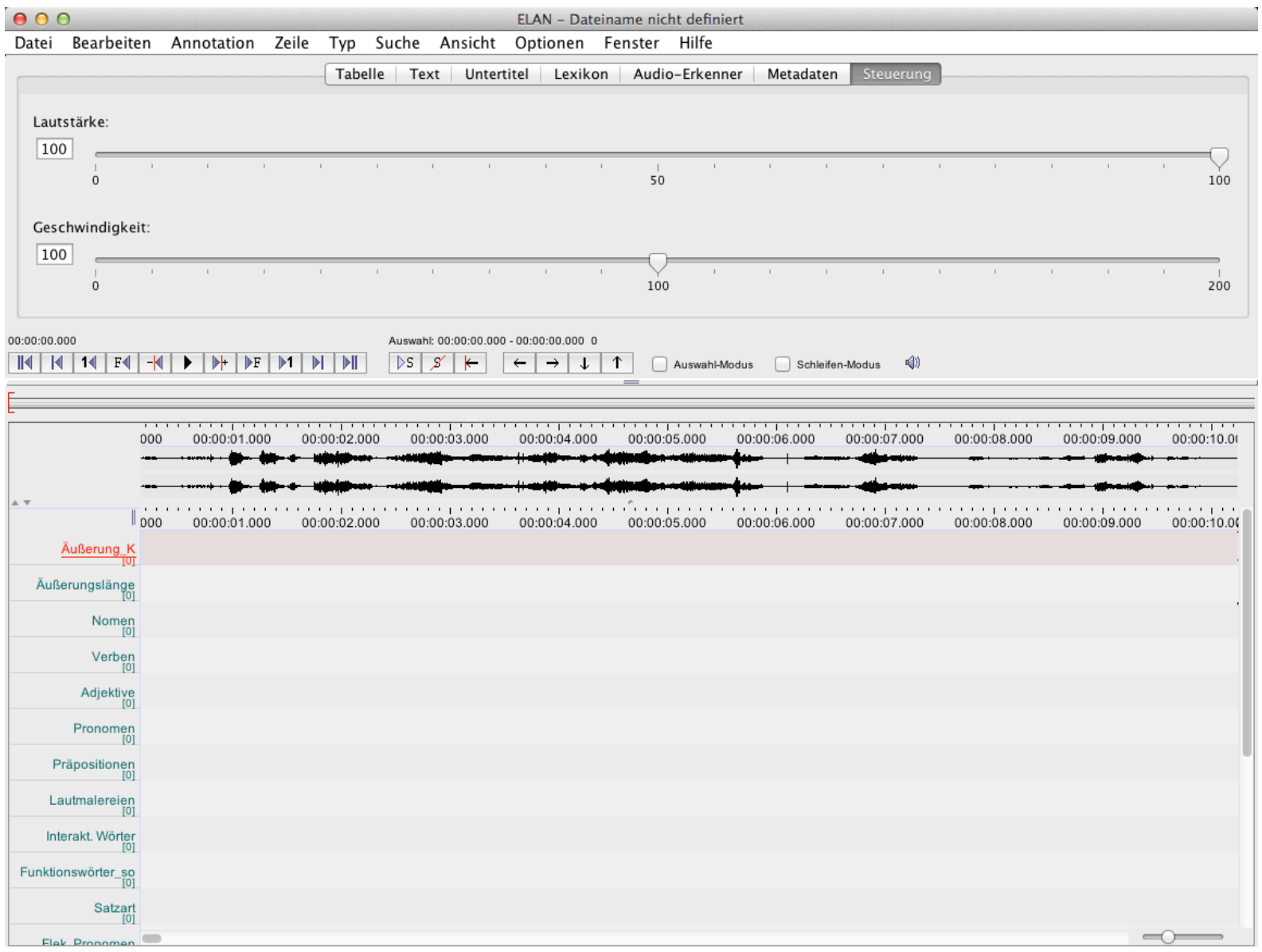

Geben Sie der Datei anschließend einen Namen und speichern Sie sie ab (Datei => Speichern unter).

Der Name der Datei setzt sich folgendermaßen zusammen:

Code des Kindes_Messzeitpunkt_Initialen des Annotatoren

Bsp.: S1_T1_AS => Code: S1 Messzeitpunkt: T1 (Ende 2012) Annotator: AS (Anja Starke) 


\subsection{Neue Annotation erstellen}

Zum Annotieren (Aufschreiben/ Transkribieren) eines Abschnittes markieren Sie diesen. Orientieren Sie sich zum Annotieren am besten an den „Wellen“ der Audiodatei. Durch einen Doppelklick in der markierten Stellen können Sie die Annotation bearbeiten. Zum Speichern des Geschriebenen müssen Sie Strg+Enter (Windows) bzw. cmd+Enter (Mac) drücken. Tun Sie dies nicht, wird nur der markierte Bereich gespeichert, nicht aber das Geschriebene!

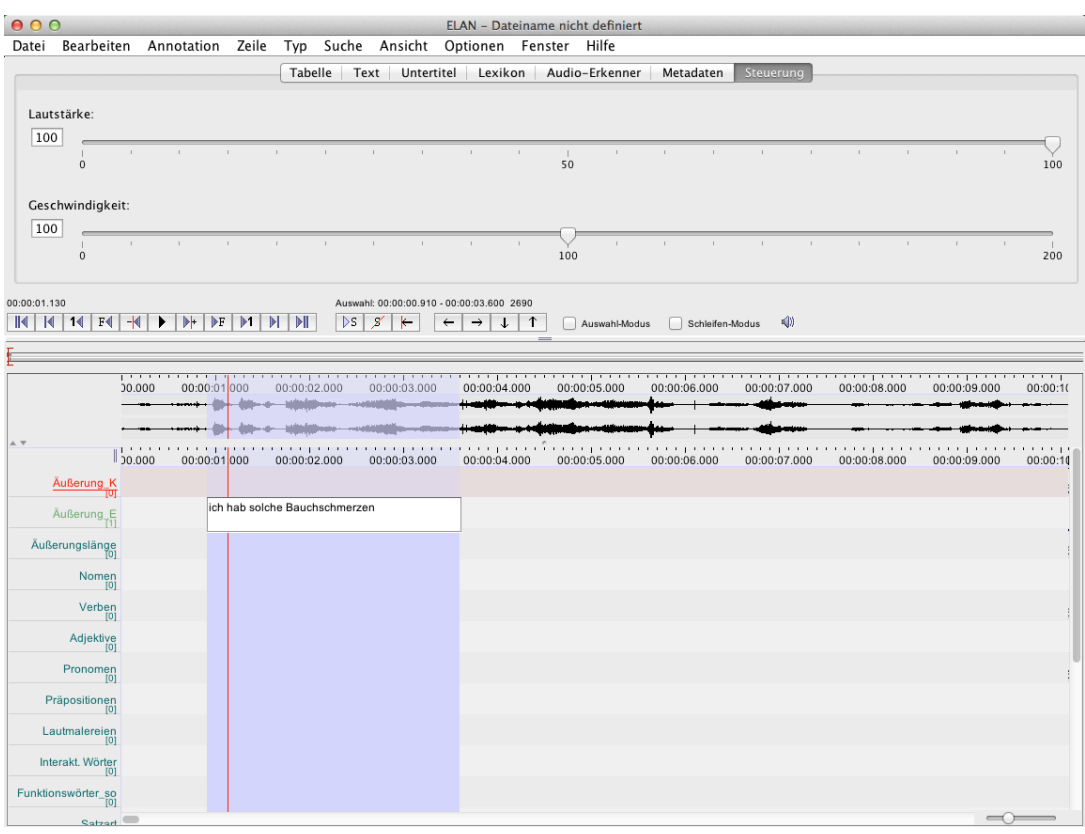

In Zeilen, die ein Wörterbuch hinterlegt haben, können Sie nichts selbst annotieren, sondern müssen aus den vorgegebenen Kategorien die Passende durch Anklicken auswählen und mit Enter bestätigen.

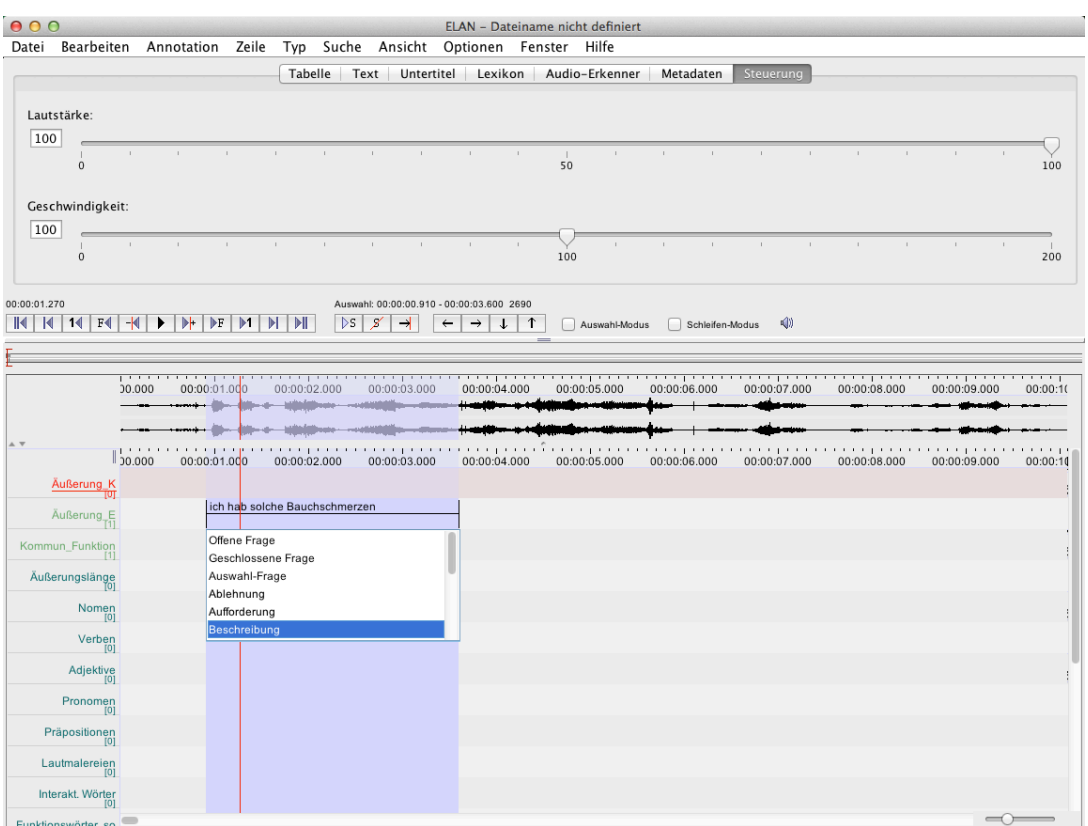




\section{Transkription der Spontansprache}

Zur reinen Transkription der Spontansprachäußerungen sind zwei Zeilen in der Elan-Vorlage vorgesehen - (1) Äußerung_K (Äußerung Kind) und Äußerung_E (Äußerung Erwachsener). In diesen beiden Zeilen wird zunächst das festgehalten, was die jeweilige Person sagt. Es wird äußerungsweise vorgegangen. Die Einteilung der Spontansprache in Äußerungen erfolgt möglichst nach dem Kriterium Syntax. Eine Äußerung enthält ein finites Verb oder einen satzwertigen Infinitiv.

Bsp.: [Ich hab solche Bauchschmerzen] [kannst du mir helfen?]

[Bär Medizin nehmen] [und schlafen]

Kann die Spontansprache nicht über das Kriterium der Syntax eingeteilt werden (z.B. aufgrund von Unverständlichkeit der Sprache), wird als nächstes das Kriterium der Intonation und Pausenstruktur angelegt.

Intonation

( $\rightarrow$ gleichbleibende Intonation; $\downarrow$ fallende Intonation)

Bsp.: [Mama da soll (?) da nich]

\section{Pausenstruktur}

Bsp.: [Mama da] ... [Bär krank]

Die Transkription der Spontansprache erfolgt in orthografischer Umschrift nach folgenden Regeln:

- alle Wörter werden in Kleinschrift notiert, um eine vorherige Interpretation der Sprache bei der Transkription zu vermeiden.

- Füllwörter und Interjektionen werden nach den folgenden Konventionen transkribiert: äh, ähm, mhm (bejahend), mh (überlegend), hmhm (verneinend), nee (verneinend), ne

- Umgangssprachliche, dialektale oder andere lautliche Variationen werden so gut wie möglich orthografisch realisiert, um ggf. Ausspracheprobleme des Kindes feststellen zu können. Ersetzt ein Kind /sch/ durch /s/ wird dies in den Graphemkombinationen <st> und <sp> durch "szt" und "szp“ dokumentiert.

- Zu Wörtern werden allgemein nur Wörter Inhalts- und Funktionswörter gezählt. Interjektionen, unverständliche Äußerungen und Neologismen werden nicht als Wörter gewertet.

- Besonderheiten in den Äußerungen können über die folgenden Transkriptionszeichen markiert werden: 


\begin{tabular}{|l|l|l|}
\hline Zeichen & Inhalt & Beispiel \\
\hline$(\ldots)$ & schlecht verständliche Äußerung & (geh) \\
\hline$(?)$ & unverständliches Wort & {$[$ is will (?) habn] } \\
\hline $\begin{array}{l}\mathrm{x} / \text { initial } \\
\mathrm{x} / \text { medial } \\
/ \mathrm{x} \text { final }\end{array}$ & unverständlicher Wortteil & $\begin{array}{l}\mathrm{x} / \mathrm{nane} \\
\mathrm{ba} / \mathrm{x} / \mathrm{ne} \\
\text { bana/x }\end{array}$ \\
\hline$\cdot$ & Kennzeichnung von Stocken bzw. getrennter & t.t.tonne \\
\hline$\ldots$ & Artikulation von Phonemen & \\
\hline$?$ & Kennzeichnung einer Pause & {$[$ mit ... Sahne] } \\
\hline & Anhebung der Intonation bei einer Frage & {$[$ kommst du?] } \\
\hline
\end{tabular}

\section{Analyse der transkribierten Äußerungen}

\section{1 Äußerungsart}

Nach der Transkription der Äußerungen werden die Äußerungen des Kindes in analysierbare und nicht analysierbare Äußerungen eingeteilt. Dazu steht die Zeile „Äußerungsart“ mit einem vordefinierten Wörterbuch zur Verfügung.

Folgende Äußerungstypen gelten als nicht analysierbar:

- unverständliche Äußerungen: Äußerung wird aufgrund akustischer Störfaktoren verdeckt oder lautliche Fehlbildung führt zu Unverständlichkeit

- einfache Antwort: vor allem "ja“ und „nein“

- Imitation/Echolalie: wortwörtliche Wiederholung von Teilen oder einer gesamten Äußerung des Erwachsenen (E: [der Bär bekommt eine Spritze] K: [eine Spritze]) ABER: Wenn das Kind eine Umstellung der Satzteile vornimmt, z.B. durch Topikalisierung, gilt das Aufgreifen einer Äußerung des Erwachsenen als analysierbar; E: [der Bär bekommt eine Spritze] K: [eine Spritze bekommt der Bär]

- Floskeln/Stereotypien: Anredeformen wie „Hallo“, Redefloskeln wie „Guten Tag“ oder Äußerungen, die in unveränderter Form immer wieder in unterschiedlichen Situationen „stereotyp“ verwendet werden, z.B. „weiß nicht“ 


\section{2 Äußerungslänge}

Für alle analysierbaren Äußerungen wird die Äußerungslänge bestimmt. Das heißt alle Wörter der Äußerung, auch Lautmalereinen wie „brumm brumm“, Partikel wie „so“ oder Eigennamen werden mitgezählt. In die Zeile „Äußerungslänge“ wird dann die entsprechend Wortanzahl eingetragen.

Bei Wiederholungen von Wörtern oder Konstituenten (z.B. durch Selbstkorrektur) werden die Wörter nicht doppelt gezählt. Bsp.: [die bär ... der bär schläft] Äußerungslänge: 3

Konstituentenverbindende Konjunktionen werden mitgezählt. Bsp.: [der hund und der bär müssen schlafen] Äußerungslänge: 7

Satzverbindende Konjunktionen werden nicht gezählt. Bsp.: [und der bär muss jetzt schlafen] Äußerungslänge: 5

\subsection{Kommunikative Funktion}

Als nächstes wird die kommunikative Funktion der Äußerungen von Kind und Erwachsenem bestimmt. In den beiden entsprechenden Zeilen „Kommunikative Funktion_K“ und „Kommunikative Funktion_E“ wird für jede analysierbare Äußerung eine entsprechende Funktion aus dem Wörterbuch ausgewählt. Folgende Kategorien werden unterschieden:

\begin{tabular}{|l|l|}
\hline $\begin{array}{l}\text { Begriffe der } \\
\text { Wörterbücher }\end{array}$ & \multicolumn{1}{|c|}{ Erläuterung } \\
\hline Aufforderung & $\begin{array}{l}\text { Handlungsvorschrift, Instruktion, Aufforderung etwas zu tun } \\
\text { Beispiele: "Du kannst einen Fisch nehmen", "Achtung" } \\
\text { Auch das Ansprechen des Partners mit seinem Namen wird als Aufforderung } \\
\text { gewertet, da die Aufmerksamkeit des Anderen eingefordert wird. }\end{array}$ \\
\hline Zustimmung & Bejahung, Einverständnis \\
\hline Ablehnung & ablehnende Antwort, Verweigerung, Zurückweisung \\
\hline Bitte & an jemanden gerichteter Wunsch \\
\hline Beschreibung & Beschreiben eines Ereignisses/ einer Tat/ eines Zustandes \\
\hline Kommentar & $\begin{array}{l}\text { Erläutern mit kritischen Anmerkungen/ kritische Stellungnahme zu einem Ereignis } \\
\text { oder Thema/ persönliche Anmerkungen } \\
\text {-> wertend } \\
\text { Beispiele: "Genau", "Toll gemacht", jede Form von Lob }\end{array}$ \\
\hline Offene Frage & $\begin{array}{l}\text { breites Spektrum an Antwortmöglichkeiten, Gesprächsinitiative wird an } \\
\text { Gesprächspartner abgegeben } \\
\text { Beispiele: „Wie alt bist du?" } \\
\text { "Welches Tier findest du am schönsten?“ }\end{array}$ \\
\hline
\end{tabular}




\begin{tabular}{|l|l|}
\hline $\begin{array}{l}\text { Begriffe der } \\
\text { Wörterbücher }\end{array}$ & \multicolumn{1}{|c|}{ Erläuterung } \\
\hline Ja-Nein-Frage & $\begin{array}{l}\text { Die Antwortmöglichkeiten sind durch „ja“ und "nein“ vorgegeben } \\
\text { Beispiele: „Hast du Geschwister?" } \\
\text { "Warst du schon einmal im Zoo?" }\end{array}$ \\
\hline Auswahlfrage & $\begin{array}{l}\text { Kombination von Antwortmöglichkeiten (nicht ja oder nein) } \\
\text { Beispiele: „Möchtest du mit dem Teddy oder der Puppe spielen?" } \\
\text { "Magst du lieber Nudeln oder Kartoffeln?" }\end{array}$ \\
\hline Repetition & Wiederholung des Gesagten \\
\hline Expansion & Erweiterung/ Modellierung des Gesagten \\
\hline
\end{tabular}

\subsection{Wortartenanalyse}

Die kindlichen Äußerungen werden noch weitergehend analysiert.

Für alle analysierbaren Äußerungen werden die Wortarten für jedes einzelne enthaltene Wort bestimmt. Zusätzlich werden interaktive Wörter (siehe unten) in den nicht analysierbaren Äußerungen mit dokumentiert. Folgende Wortarten werden unterschieden:

\begin{tabular}{|l|l|l|}
\hline Wortart & Beschreibung & Beispiel \\
\hline Nomen & $\begin{array}{l}\text { deklinierbare Wörter, die in Verbindung mit einem } \\
\text { Artikel verwendet werden; auch substantivierte } \\
\text { Adjektive oder Verben }\end{array}$ & $\begin{array}{l}\text { das Haus } \\
\text { das Malen } \\
\text { der Blaue }\end{array}$ \\
\hline Verben & $\begin{array}{l}\text { konjugierbare Wörter, die einen Zustand, einen } \\
\text { Vousdrücken; auch Partikel- und Hilfsverben }\end{array}$ & $\begin{array}{l}\text { schlafen, sitzen, nehmen } \\
\text { aufmachen, zumachen } \\
\text { haben, sein, dürfen }\end{array}$ \\
\hline Adjektive & $\begin{array}{l}\text { deklinier- und steigerbare Wörter, die eine } \\
\text { Eigenschaft oder ein Merkmal bezeichnen }\end{array}$ & $\begin{array}{l}\text { kranke Bär } \\
\text { hohes Fieber }\end{array}$ \\
\hline Adverbien & $\begin{array}{l}\text { unflektierbare Wörter, die lokale, temporale, } \\
\text { modale oder kausale Umstände angeben }\end{array}$ & $\begin{array}{l}\text { dort gefällt es mir gut } \\
\text { gut sehen } \\
\text { sehr oft }\end{array}$ \\
\hline Pronomen & $\begin{array}{l}\text { Wörter, die stellvertretend für ein Nomen stehen } \\
\text { hier auch pronominale Elemente (Artikel und } \\
\text { Demonstrativpronomen) }\end{array}$ & $\begin{array}{l}\text { ich, du, mir, dich, er } \\
\text { ein, eine, der, die, das } \\
\text { dieser, dieses }\end{array}$ \\
\hline Präpositionen & $\begin{array}{l}\text { unflektierbare Wörter, die Beziehungen oder } \\
\text { Verhältnisse zwischen Wörtern kennzeichnen }\end{array}$ & $\begin{array}{l}\text { auf, unter, neben } \\
\end{array}$
\end{tabular}




\begin{tabular}{|l|l|l|}
\hline Lautmalereien & Nachahmung eines Geräusches & brumm brumm \\
\hline interaktive & $\begin{array}{l}\text { Wörter, die nur in der Interaktion eine Funktion } \\
\text { haben, z.B. Grußwörter }\end{array}$ & $\begin{array}{l}\text { Hallo, Tschüß } \\
\text { ja, nein }\end{array}$ \\
\hline $\begin{array}{l}\text { sonstige } \\
\text { Funktionswörter }\end{array}$ & $\begin{array}{l}\text { in diese Kategorien fallen alle Wörter, die bislang } \\
\text { noch nicht einsortiert werden konnten. Z.B. auch } \\
\text { Eigennamen, Konjunktionen }\end{array}$ & \\
\hline
\end{tabular}

Das einzelne Wort wird unflektiert, das heißt in seiner Grundform, in die entsprechende Zeile eingetragen.

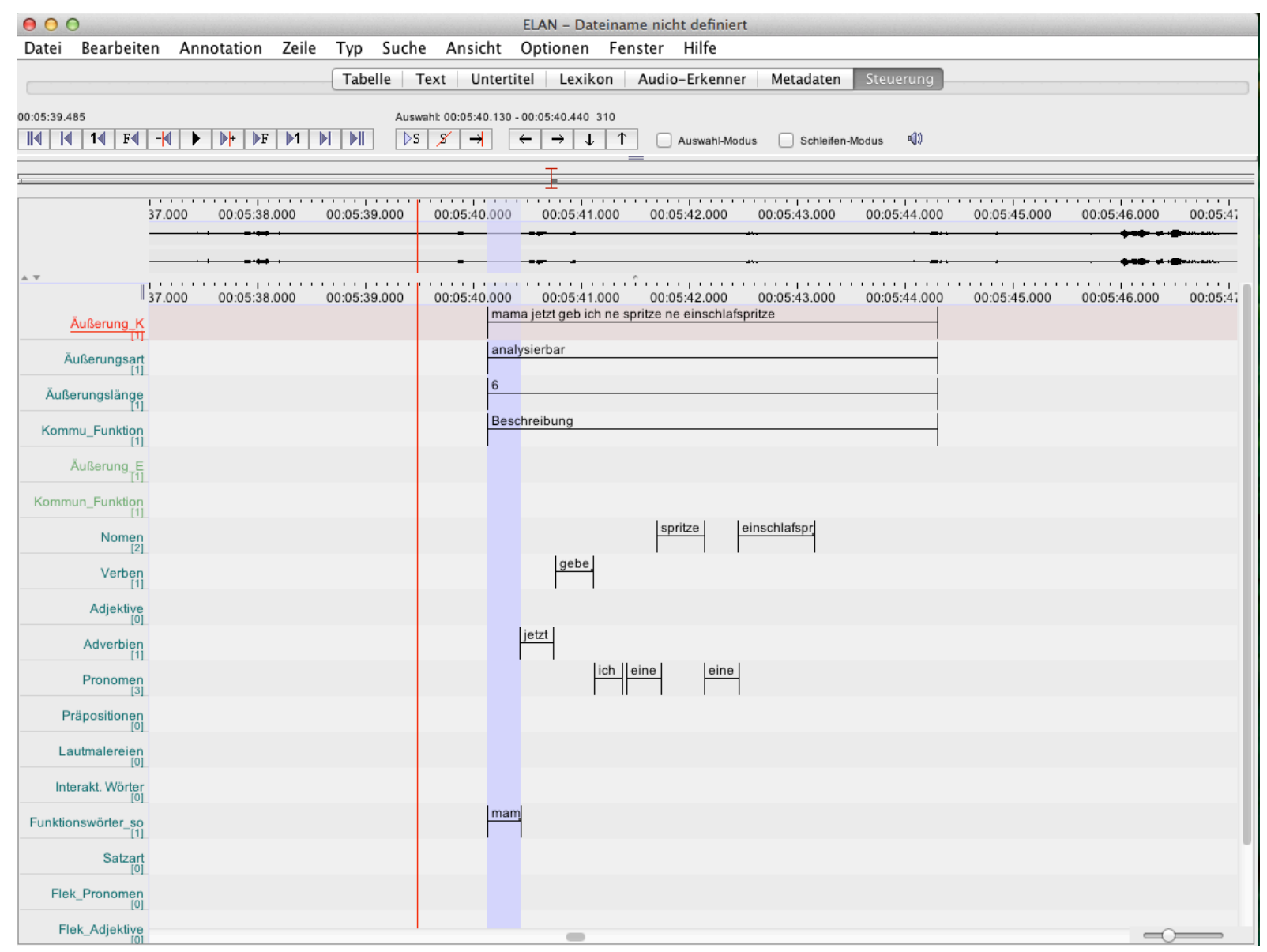

\subsection{Satzart}

In der Zeile "Satzart" wird analysiert, ob die Äußerung des Kindes satzwertig ist (d.h. Haupt- oder Nebensatz) oder ob diese eine Ellipse ist. Zur Kategorisierung steht wiederum ein Wörterbuch zur Verfügung.

Ellipsen sind Äußerungen, die aufgrund ihres Kontextes, in dem sie geäußert werden, als Teilwortsätze interpretiert werden können. Das heißt die Äußerung eines vollständigen Satzes ist in diesem Kontext gar nicht notwendig.

Bsp.: [wohin kommt denn die spritze?] [in den bauch] 
Satzwertige Äußerungen sind solche, die

- $\quad$ einen vollständigen Satz

[der bär muss schlafen]

- einen Nebensatz

[weil er krank ist]

- eine als Satz angelegte, grammatisch nicht korrekte Äußerung [bär schlafen muss]

- $\quad$ oder einen Imperativ repräsentieren.

[leg dich hin]

\subsection{Morphologie}

Als letztes wird analysiert, ob das Kind Nomen, Verben, Adjektive und Pronomen korrekt flektiert.

Dazu steht wiederum ein Wörterbuch mit den beiden Kategorien „korrekt" und „inkorrekt“ zur Verfügung.

Nomen

Bsp.: korrekt [der bär muss zwei tabletten nehmen]

inkorrekt [der bär muss zwei tablettens nehmen]

Verben

Bsp.: korrekt [der bär schläft]

inkorrekt [der bär schlafen]

Adjektive

Bsp.: $\quad$ korrekt [der bär hat hohes fieber]

inkorrekt [der bär hat hohen fieber]

\section{Pronomen}

Bsp.: $\quad$ korrekt [ich geb dir eine spritze]

inkorrekt [ich geb dich eine spritze] 
Quellen:

Manual basiert maßgeblich auf den Transkriptions- und Analyserichtlinien des ASAS (Aachener Screeningverfahren zur Analyse von Spontansprache).

Schrey-Dern, D. (2006). Sprachentwicklungsstörungen. Logopädische Diagnostik und Therapieplanung. Stuttgart: Thieme.

sowie den Transkriptionsrichtlichen der ASPA (Aachener Sprachanalyse)

Huber, W., Springer, L., Grande, M. \& Biniek, R. (2002). Aachener Sprachanalyse.

Transkriptionsrichtlinien. In 2. Jahrestagung der Gesellschaft für Aphasieforschung und -behandlung, Oberhausen. 